\title{
cancers
}

Volume 2

Role of Medical

Imaging in Cancers

Edited by

Stefano Fanti and Laura Evangelista Printed Edition of the Special Issue Published in Cancers 


\section{Role of Medical Imaging in Cancers}





\section{Role of Medical Imaging in Cancers}

\section{Volume 2}

Editors

Stefano Fanti

Laura Evangelista 
Editors

Stefano Fanti

University of Bologna

Italy
Laura Evangelista

Veneto Institute of Oncology IOV_-IRCCS

Italy

Editorial Office

MDPI

St. Alban-Anlage 66

4052 Basel, Switzerland

This is a reprint of articles from the Special Issue published online in the open access journal Cancers (ISSN 2072-6694) (available at: https://www.mdpi.com/journal/cancers/special_issues/ imaging_cancer).

For citation purposes, cite each article independently as indicated on the article page online and as indicated below:

LastName, A.A.; LastName, B.B.; LastName, C.C. Article Title. Journal Name Year, Volume Number, Page Range.

Volume 2

ISBN 978-3-0365-0206-9 (Hbk)

ISBN 978-3-0365-0207-6 (PDF)
Volume 1-2

ISBN 978-3-0365-0208-3 (Hbk)

ISBN 978-3-0365-0209-0 (PDF)

(C) 2021 by the authors. Articles in this book are Open Access and distributed under the Creative Commons Attribution (CC BY) license, which allows users to download, copy and build upon published articles, as long as the author and publisher are properly credited, which ensures maximum dissemination and a wider impact of our publications.

The book as a whole is distributed by MDPI under the terms and conditions of the Creative Commons license CC BY-NC-ND. 


\section{Contents}

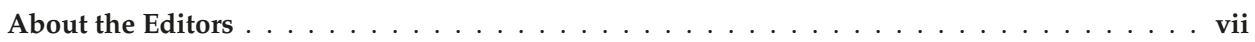

Preface to "Role of Medical Imaging in Cancers" $\ldots \ldots \ldots \ldots \ldots \ldots$ ix

Christos Sachpekidis, Ioannis Karampinis, Jens Jakob, Bernd Kasper, Kai Nowak,

Lothar Pilz, Ulrike Attenberger, Timo Gaiser, Hans-G ünter Derigs, Matthias Schwarzbach,

Peter Hohenberger, Antonia Dimitrakopoulou-Strauss and Ulrich Ronellenfitsch

Neoadjuvant Pazopanib Treatment in High-Risk Soft Tissue Sarcoma: A Quantitative Dynamic

${ }^{18}$ F-FDG PET/CT Study of the German Interdisciplinary Sarcoma Group

Reprinted from: Cancers 2019, 11, 790, doi:10.3390/cancers11060790 . . . . . . . . . . . . .

Anna Myriam Perrone, Giulia Dondi, Giacomo Maria Lima, Paolo Castellucci, Marco Tesei, Sara Coluccelli, Giuseppe Gasparre, Anna Maria Porcelli, Cristina Nanni, Stefano Fanti and Pierandrea De Iaco

Potential Prognostic Role of ${ }^{18}$ F-FDG PET/CT in Invasive Epithelial Ovarian Cancer Relapse.

A Preliminary Study

Reprinted from: Cancers 2019, 11, 713, doi:10.3390/cancers11050713 . . . . . . . . . . . . .

Fabio Zattoni, Elena Incerti, Fabrizio Dal Moro, Marco Moschini, Paolo Castellucci, Stefano Panareo, Maria Picchio, Federico Fallanca, Alberto Briganti, Andrea Gallina, Stefano Fanti, Riccardo Schiavina, Eugenio Brunocilla, Ilaria Rambaldi, Val Lowe, R. Jeffrey Karnes and Laura Evangelista

${ }^{18}$ F-FDG PET/CT and Urothelial Carcinoma: Impact on Management and Prognosis-A Multicenter Retrospective Study

Reprinted from: Cancers 2019, 11, 700, doi:10.3390/cancers11050700 . . . . . . . . . . . . .

Alexey Surov, Hans Jonas Meyer, Anne-Kathrin Höhn, Andreas Wienke, Osama Sabri and Sandra Purz

${ }^{18}$ F-FDG-PET Can Predict Microvessel Density in Head and Neck Squamous Cell Carcinoma Reprinted from: Cancers 2019, 11, 543, doi:10.3390/cancers11040543 . . . . . . . . . . . . . .

Stefano Frega, Alessandro Dal Maso, Giulia Pasello, Lea Cuppari, Laura Bonanno, PierFranco Conte and Laura Evangelista

Novel Nuclear Medicine Imaging Applications in Immuno-Oncology

Reprinted from: Cancers 2020, 12, 1303, doi:10.3390/cancers12051303

Simone Serafini, Cosimo Sperti, Alessandra Rosalba Brazzale, Diego Cecchin, Pietro Zucchetta, Elisa Sefora Pierobon, Alberto Ponzoni, Michele Valmasoni and Lucia Moletta The Role of Positron Emission Tomography in Clinical Management of Intraductal Papillary Mucinous Neoplasms of the Pancreas

Reprinted from: Cancers 2020, 12, 807, doi:10.3390/cancers12040807 . . . . . . . . . . . . . . .

Conrad-Amadeus Voltin, Jasmin Mettler, Jirka Grosse, Markus Dietlein, Christian Baues, Christine Schmitz, Peter Borchmann, Carsten Kobe and Dirk Hellwig

FDG-PET Imaging for Hodgkin and Diffuse Large B-Cell Lymphoma-An Updated Overview Reprinted from: Cancers 2020, 12, 601, doi:10.3390/cancers12030601 . . . . . . . . . . . . 83

Pierre Decazes and Pierre Bohn

Immunotherapy by Immune Checkpoint Inhibitors and Nuclear Medicine Imaging: Current and Future Applications

Reprinted from: Cancers 2020, 12, 371, doi:10.3390/cancers12020371 . . . . . . . . . . . . . 99 
Barbara Salvatore, Maria Grazia Caprio, Billy Samuel Hill, Annachiara Sarnella, Giovanni Nicola Roviello and Antonella Zannetti

Recent Advances in Nuclear Imaging of Receptor Expression to Guide Targeted Therapies in Breast Cancer

Reprinted from: Cancers 2019, 11, 1614, doi:10.3390/cancers11101614 . . . . . . . . . . . . 121

Riccardo Laudicella, Domenico Albano, Salvatore Annunziata, Diletta Calabrò,

Giovanni Argiroffi, Elisabetta Abenavoli, Flavia Linguanti, Domenico Albano,

Antonio Vento, Antonio Bruno, Pierpaolo Alongi and Matteo Bauckneht

Theragnostic Use of Radiolabelled Dota-Peptides in Meningioma: From Clinical Demand to

Future Applications

Reprinted from: Cancers 2019, 11, 1412, doi:10.3390/cancers11101412 . . . . . . . . . . . . 143

Riccardo Laudicella, Domenico Albano, Pierpaolo Alongi, Giovanni Argiroffi, Matteo Bauckneht, Sergio Baldari, Francesco Bertagna, Michele Boero, Giuseppe De Vincentis, Angelo Del Sole, Giuseppe Rubini, Lorenzo Fantechi, Viviana Frantellizzi, Gloria Ganduscio, Priscilla Guglielmo, Anna Giulia Nappi, Laura Evangelista and on the behalf of Young AIMN Working Group

${ }^{18}$ F-Facbc in Prostate Cancer: A Systematic Review and Meta-Analysis

Reprinted from: Cancers 2019, 11, 1348, doi:10.3390/cancers11091348

Giorgio Treglia, Salvatore Annunziata, Daniele A. Pizzuto, Luca Giovanella, John O. Prior and Luca Ceriani

Detection Rate of ${ }^{18} \mathrm{~F}$-Labeled PSMA PET/CT in Biochemical Recurrent Prostate Cancer:

A Systematic Review and a Meta-Analysis

Reprinted from: Cancers 2019, 11, 710, doi:10.3390/cancers11050710 . . . . . . . . . . . . 181

Christopher Montemagno, Shamir Cassim, Dimitry Trichanh, Clara Savary, Jacques Pouyssegur, Gilles Pagès, Daniel Fagret, Alexis Broisat and Catherine Ghezzi

${ }^{99 \mathrm{~m}} \mathrm{Tc}-\mathrm{A} 1$ as a Novel Imaging Agent Targeting Mesothelin-Expressing Pancreatic Ductal Adenocarcinoma

Reprinted from: Cancers 2019, 11, 1531, doi:10.3390/cancers11101531 . . . . . . . . . . . . . 195

Malene Grubbe Hildebrandt, Jeppe Faurholdt Lauridsen, Marianne Vogsen, Jorun Holm, Mie Holm Vilstrup, Poul-Erik Braad, Oke Gerke, Mads Thomassen, Marianne Ewertz, Poul Flemming Høilund-Carlsen and The Centre for Personalized Response Monitoring in Oncology (PREMIO)

FDG-PET/CT for Response Monitoring in Metastatic Breast Cancer: Today, Tomorrow, and Beyond

Reprinted from: Cancers 2019, 11, 1190, doi:10.3390/cancers11081190 . . . . . . . . . . . 203 


\section{About the Editors}

Stefano Fanti Fanti is born in Bologna (Italy) where completed all his studies and finally graduated at University of Bologna. Started working in conventional nuclear medicine in the '90, then involved in the project of PET Unit at S.Orsola Policlinic Hospital, participating to site planning then appointed as medical director in 2002. Last year the PET Unit carried out more than 14000 exams, resulting one of the most active in Europe; in particular the PET Center in Bologna is known for the nonFDG scans, in both clinical routine and research area. At present he is full time employed in Nuclear Medicine as Full Professor of Diagnostic Imaging as well as Director of Nuclear Medicine Division and of PET Unit at the S.Orsola Policlinic Hospital. Author of more than 1000 papers, including 450 full articles published in peer reviewed international journals (more than 18000 citations and H-index of 73 on Google Scholar, 60 on Scopus), dozen of books and chapters; invited lecturer at more than 200 national and international meetings.

Laura Evangelista is a researcher of Diagnostic Imaging at University of Padova (Italy), Vice-Director of the Nuclear Medicine residency program since 2019. Dr. Evangelista received an undergraduate degree in medicine in 2004 and the Nuclear Medicine degree in 2009, both from University of Napoli (Italy). Author of more than 250 publications, including about 200 original papers in international peer-reviewed journals, more than 300 abstracts, 15 books or chapters; H-index: 18; invited speakers in more than 50 international meetings. Dr. Evangelista's current research is currently focused on PET/MRI imaging in oncology and non-oncology settings. She is currently focused on the evaluation of the additional value of hybrid PET/MRI scanners in the management of oncological patients. 



\section{Preface to "Role of Medical Imaging in Cancers"}

Medical imaging comprises a huge amount of imaging techniques, from ultrasound and computed tomography (CT) to molecular imaging comprising magnetic resonance imaging (MRI) and positron emission tomography (PET).

Molecular imaging allows for the remote, noninvasive sensing and measurement of cellular and molecular processes in living subjects. It provides a window into the biology of cancer from the sub-cellular level to the patient undergoing a new, experimental therapy. Conventional imaging, and mainly CT, remains critical to the management of patients with cancer, while molecular imaging provides more specific information, such as early detection of changes with therapy, identification of patient-specific cellular and metabolic characteristics, that have a considerable impact on morbidity and mortality.

Molecular imaging has developed rapidly in the last years, particularly in the oncological field. The development of new hybrid scanners, like digital PET/CT, PET/MRI and single photon emission tomography (SPET)/CT, has significantly improved the detection of tumors, in all phase of disease (from the initial staging to the evaluation of response to therapy). Moreover, the introduction of various radiopharmaceutical agents has opened new scenarios for the in-vivo molecular characterization of cancer.

This Special Issue will highlight the role of medical imaging, with particular interest to molecular imaging in cancer management, covering some important aspects by focalizing the attention of new discoveries for the big killers, like prostate cancer, lung cancer, breast cancer and colon cancer.

Stefano Fanti, Laura Evangelista Editors 

Article

\title{
Neoadjuvant Pazopanib Treatment in High-Risk Soft Tissue Sarcoma: A Quantitative Dynamic ${ }^{18}$ F-FDG PET/CT Study of the German Interdisciplinary Sarcoma Group
}

\author{
Christos Sachpekidis 1,* Ioannis Karampinis ${ }^{2}$, Jens Jakob ${ }^{2,3}$, Bernd Kasper ${ }^{4}$, Kai Nowak ${ }^{2,5}$, \\ Lothar Pilz ${ }^{6}$, Ulrike Attenberger ${ }^{7}$, Timo Gaiser ${ }^{8}$, Hans-Günter Derigs ${ }^{9}$, \\ Matthias Schwarzbach ${ }^{10}$, Peter Hohenberger ${ }^{2}$, Antonia Dimitrakopoulou-Strauss ${ }^{1,+}$ \\ and Ulrich Ronellenfitsch $2,11,+$ \\ 1 Clinical Cooperation Unit Nuclear Medicine, German Cancer Research Center, 69120 Heidelberg, Germany; \\ ads@ads-net.de \\ 2 Division of Surgical Oncology and Thoracic Surgery, University Medical Center Mannheim, \\ 68167 Mannheim, Germany; Ioannis.Karampinis@umm.de (I.K.); jens.jakob@med.uni-goettingen.de (J.J.); \\ nowakk@me.com (K.N.); peter.hohenberger@umm.de (P.H.); Ulrich.Ronellenfitsch@uk-halle.de (U.R.) \\ 3 Department of General, Visceral and Child Surgery, University Medical Center Göttingen, \\ 37075 Göttingen, Germany \\ 4 Interdisciplinary Tumor Center Mannheim, Sarcoma Unit, Mannheim University Medical Center, \\ 68167 Mannheim, Germany; bernd.kasper@umm.de \\ 5 Department of Abdominal, Vascular and Thoracic Surgery, Romed Klinikum, 83022 Rosenheim, Germany \\ 6 Medical Faculty Mannheim, University of Heidelberg, 68167 Mannheim, Germany; \\ Lothar.Pilz@medma.uni-heidelberg.de \\ 7 Institute of Clinical Radiology and Nuclear Medicine, University Medical Center Mannheim, \\ 68167 Mannheim, Germany; Ulrike.Attenberger@medma.uni-heidelberg.de \\ 8 Institute of Pathology, University Medical Center Mannheim, 68167 Mannheim, Germany; \\ Timo.Gaiser@umm.de \\ 9 Department of Hematology and Oncology, Klinikum Frankfurt-Hoechst, \\ 65929 Frankfurt am Main, Germany; derigs@klinikumfrankfurt.de \\ 10 Department of Surgery, Klinikum Frankfurt-Hoechst, 65929 Frankfurt am Main, Germany; \\ Matthias.Schwarzbach@KlinikumFrankfurt.de \\ 11 Department of Abdominal, Vascular, and Endocrine Surgery, University Hospital Halle, \\ 06120 Halle (Saale), Germany \\ * Correspondence: christos_saxpe@yahoo.gr; Tel.: +49-6221-42-2500; Fax: +49-6221-42-2476 \\ + These authors share joint senior authorship.
}

Received: 8 May 2019; Accepted: 6 June 2019; Published: 8 June 2019

\begin{abstract}
The outcome of high-risk soft tissue sarcoma (STS) is poor with radical surgery being the only potentially curative modality. Pazopanib is a multikinase inhibitor approved for the treatment of metastatic STS. Herein, in terms of the German Interdisciplinary Sarcoma Group (GISG-04/NOPASS) trial, we evaluate the potential role of kinetic analysis of fludeoxyglucose F-18 $\left({ }^{18} \mathrm{~F}-\mathrm{FDG}\right)$ data derived from the application of dynamic positron emission tomography/computed tomography (PET/CT) in response assessment to pazopanib of STS patients scheduled for surgical resection. Sixteen STS patients treated with pazopanib as neoadjuvant therapy before surgery were enrolled in the analysis. All patients underwent dynamic PET/CT prior to and after pazopanib treatment. Data analysis consisted of visual (qualitative) analysis of the PET/CT scans, semi-quantitative evaluation based on standardized uptake value (SUV) calculations, and quantitative analysis of the dynamic ${ }^{18}$ F-FDG PET data, based on two-tissue compartment modeling. Resection specimens were histopathologically assessed and the percentage of regression grade was recorded in 14/16 patients. Time to tumor relapse/progression was also calculated. In the follow-up, $12 / 16$ patients $(75 \%)$ were alive without relapse, while four patients (25\%) relapsed, among them one patient died. Median histopathological
\end{abstract}


regression was $20 \%$ (mean $26 \%$, range $5-70 \%$ ). The studied population was dichotomized using a histopathological regression grade of $20 \%$ as cut-off. Based on this threshold, $10 / 14$ patients $(71 \%)$ showed partial remission (PR), while stable disease (SD) was seen in the rest 4 evaluable patients $(29 \%)$. Semi-quantitative evaluation showed no statistically significant change in the widely used PET parameters, $\mathrm{SUV}_{\text {average }}$ and $\mathrm{SUV}_{\max }$. On the other hand, ${ }^{18} \mathrm{~F}-\mathrm{FDG}$ kinetic analysis revealed a significant decrease in the perfusion-related parameter $K_{1}$, which reflects the carrier-mediated transport of ${ }^{18} \mathrm{~F}-\mathrm{FDG}$ from plasma to tumor. This decrease can be considered as a marker in response to pazopanib in STS and could be due to the anti-angiogenic effect of the therapeutic agent.

Keywords: soft tissue sarcoma (STS); pazopanib; dynamic ${ }^{18} \mathrm{~F}-\mathrm{FDG}$ PET/CT; SUV; two-tissue compartment model

\section{Introduction}

The outcome of high-risk soft tissue sarcoma (STS) is poor. Radical surgery, usually in combination with radiotherapy, is the mainstay of treatment and the only potentially curative modality [1]. Surgical removal can, however, be cumbersome due to the large tumor size with infiltration of adjacent structures, and extensive tumor vasculature [2]. In this context, the development and application of a fast acting, preoperative STS therapy that would facilitate tumor resection and at the same time have low toxicity would be of high significance.

Pazopanib is a multikinase inhibitor, approved for the treatment of metastatic STS based on a phase III trial in patients with non-adipocytic STS, who had progressed on at least one prior chemotherapy regimen [3]. Given this proof of efficacy, and its favorable safety profile [4], the German Interdisciplinary Sarcoma Group has very recently published the first results of a single arm phase II trial of preoperative pazopanib therapy in 21 patients with high-risk STS (GISG-04/NOPASS) [5]. Using the metabolic response rate-defined as $\geq 50 \%$ reduction of mean standardized uptake value $\left(\mathrm{SUV}_{\text {mean }}\right)$ in post- vs. pretreatment fludeoxyglucose F-18 positron emission tomography/computed tomography $\left({ }^{18} \mathrm{~F}\right.$-FDG PET/CT) —as the primary endpoint, this window-of-opportunity trial showed that preoperative pazopanib is not effective for unselected high-risk STS patients. Nevertheless, metabolic response was observed in a single patient.

In the GISG-04/NOPASS trial, metabolic response was based on estimations of SUV, a semiquantitative parameter whose calculation requires only static imaging when the tracer ${ }^{18} \mathrm{~F}$-FDG has reached equilibrium. SUV represents the tissue activity within a region of interest (ROI) corrected for injected activity and body weight and is the most widely used PET parameter. Nevertheless, the generally accepted method for accurate analysis of ${ }^{18} \mathrm{~F}-\mathrm{FDG}$ metabolism and kinetics is a two-tissue compartment model [6]. A prerequisite for this is the performance of full dynamic PET studies for at least $60 \mathrm{~min}$. In patients with STS, dynamic ${ }^{18} \mathrm{~F}$-FDG PET/CT has been prospectively validated as a strong predictor of histopathological response and progression-free survival (PFS), for both neoadjuvant and palliative chemotherapy [7-9].

The present analysis is part of the GISG-04/NOPASS trial evaluating the role of neoadjuvant pazopanib in high-risk STS. Herein, we assess the potential role of kinetic analysis of ${ }^{18} \mathrm{~F}$-FDG data derived from the application of dynamic PET/CT in response assessment to pazopanib in STS patients scheduled for surgical resection.

\section{Materials and Methods}

\subsection{Patients-Treatment}

Out of the 21 patients enrolled in the GISG-04/NOPASS trial [5], sixteen STS patients (eight female; median age 67.8 years, range $46.3-88.5$ years) had evaluable dynamic PET/CT both pre- and post 
treatment, and were thus enrolled in the analysis. Patients with metastases at presentation were excluded from the study. Treatment consisted of pazopanib $800 \mathrm{mg}$ daily for 21 days as a neoadjuvant therapy before surgery. An interval of 7-14 days between pazopanib therapy completion and surgery was allowed in order to minimize potential perioperative complications due to pazopanib. The herein presented patient cohort has already been studied, and patient data derived from a different analysis have been published elsewhere [5]. The histopathological classification as well as the localization of the sarcomas is presented in Table 1.

Table 1. Histopathological characteristics and localization of the soft tissue sarcoma (STS) of the 16 studied patients.

\begin{tabular}{ccc}
\hline Histology & No. & $\%$ \\
\hline Dedifferentiated liposarcoma & 8 & $50 \%$ \\
Undifferentiated pleomorphic sarcoma & 2 & $12.5 \%$ \\
Fibrohistiocytic sarcoma & 1 & $6.25 \%$ \\
Leiomyosarcoma & 1 & $6.25 \%$ \\
Malignant peripheral nerve sheath tumor & 1 & $6.25 \%$ \\
Myxoid liposarcoma & 1 & $6.25 \%$ \\
Pleomorphic liposarcoma & 1 & $6.25 \%$ \\
Synovial sarcoma & 1 & $6.25 \%$ \\
\hline Localization of the primary & No. & $\%$ \\
\hline Retroperitoneal & 6 & $37.5 \%$ \\
Left thigh & 4 & $25 \%$ \\
Right shank & 2 & $12.5 \%$ \\
Left gluteal & 1 & $6.25 \%$ \\
Left inguinal & 1 & $6.25 \%$ \\
Pelvis & 1 & $6.25 \%$ \\
Right middle abdomen & 1 & $6.25 \%$ \\
\hline
\end{tabular}

\subsection{PET/CT}

\subsubsection{Data Acquisition}

All patients underwent PET/CT 14 days prior to and 1-7 days (median 6 days) after pazopanib treatment. A dedicated PET/CT system (Biograph mCT, S128, Siemens Co., Erlangen, Germany) with TruePoint and TrueV, operated in a three-dimensional mode was used. Data acquisition consisted of the dynamic PET/CT (dPET/CT studies) and the static part (whole body PET/CT). dPET/CT studies were performed over the STS area after intravenous administration of ${ }^{18} \mathrm{~F}-\mathrm{FDG}$ for $60 \mathrm{~min}$ using a multistep dynamic acquisition over two-bed positions with a total field of view of $(21.6 \times 2) 43.2 \mathrm{~cm}$. The data were acquired in list mode. A 24-frame protocol (10 frames of $30 \mathrm{~s}, 5$ frames of $60 \mathrm{~s}, 5$ frames of $120 \mathrm{~s}$, and 4 frames of $600 \mathrm{~s}$ ) was applied. After the end of the dynamic acquisition, whole body, static imaging was performed in all patients with image duration of $2 \mathrm{~min}$ per bed position. A low-dose attenuation CT $(120 \mathrm{kV}, 30 \mathrm{~mA})$ was used for attenuation correction of the dynamic emission PET data and for image fusion. All PET images were attenuation corrected and an image matrix of $400 \times 400$ pixels was used for iterative image reconstruction. Iterative images reconstruction was based on the ordered subset expectation maximization (OSEM) algorithm with two iterations and 21 subsets as well as time of flight (TOF).

\subsubsection{Data Analysis}

Data analysis consisted of visual (qualitative) analysis of the PET/CT scans, semi-quantitative evaluation based on SUV calculations, and quantitative analysis of the dynamic ${ }^{18} \mathrm{~F}$-FDG PET data. The assessment of PET/CT scans was performed by two nuclear medicine physicians (Christos Sachpekidis, Antonia Dimitrakopoulou-Strauss). 
Qualitative analysis was based on the identification of the STS lesions as sites of increased ${ }^{18} \mathrm{~F}$-FDG uptake greater than the background or liver activity.

Semi-quantitative evaluation was based on volumes of interest (VOIs) and on subsequent calculation of $\mathrm{SUV}_{\text {average }}$ and $\mathrm{SUV}_{\max }$. VOIs were drawn with an isocontour mode (pseudo-snake) and were placed over STS lesions [10]. SUV measurements were performed at the 60-min post injection frames.

Quantitative evaluation of the dynamic ${ }^{18} \mathrm{~F}-\mathrm{FDG}$ PET/CT data of the STS lesions was performed using a dedicated software and based on a two-tissue compartment model with a blood component $\left(V_{B}\right)$, with methods already reported by our group [11,12]. One problem in patients is the accurate measurement of input function, which theoretically requires arterial blood sampling. It has been shown however, that input function can be accurately retrieved from image data [13]. For the input function, the mean value of the VOI data from a large arterial vessel (e.g., aorta or common iliac artery) was used. A vessel VOI consisted of at least seven ROIs in sequential PET images. The recovery coefficient was 0.85 for a diameter of $8 \mathrm{~mm}$. Partial volume correction was performed for small vessels with diameter $<8 \mathrm{~mm}$, based on the phantom measurements of the recovery function using dedicated software [14].

The application of a two-tissue compartment model leads to the extraction of the kinetic parameters $\mathrm{K}_{1}, \mathrm{k}_{2}, \mathrm{k}_{3}$, and $\mathrm{k}_{4}$ as well as the influx $\left(\mathrm{K}_{\mathrm{i}}\right)$ that describes specific molecular processes: $\mathrm{K}_{1}$ reflects the carrier-mediated transport of ${ }^{18} \mathrm{~F}$-FDG from plasma to tissue, while $\mathrm{k}_{2}$ reflects its transport back from the tissue to plasma, and $k_{3}$ represents the phosphorylation rate, while $k_{4}$ the dephosphorylation rate of the glucose analog. The model parameters were accepted when $K_{1}, k_{2}, k_{3}$, and $k_{4}$ were less than 1 and $V_{B}$ exceeded zero. The unit for the rate constants $K_{1}, k_{2}, k_{3}$, and $k_{4}$ is $1 / \mathrm{min}$, while $V_{B}$ reflects the fraction of blood within the VOI. Tracer influx $\left(K_{i}\right)$ is derived from the equation $=\left(K_{1} \times k_{3}\right) /\left(k_{2}+k_{3}\right)$. The two-tissue compartment model we applied is a modification of the one proposed by Sokoloff et al., which did not take into account the parameters $\mathrm{k}_{4}$ and $\mathrm{V}_{\mathrm{B}}$ [15]. This lack of $\mathrm{k}_{4}$ and $\mathrm{V}_{\mathrm{B}}$ however, leads to different values of the parameters $k_{1}$ and $k_{3}$, since $k_{1}$ is dependent on $V_{B}$ and $k_{3}$ on $k_{4}$.

Apart from performing compartment analysis, a non-compartment model based on the fractal dimension (FD) for the time activity data was applied. FD is a parameter of heterogeneity of tracer kinetics based on the box counting procedure of chaos theory and was calculated in each individual voxel of a VOI. The values of FD vary from 0 to 2 showing the more deterministic or chaotic distribution of the tracer activity via time, respectively [16].

In addition to the previous analysis, parametric images of the slope and the intercept were calculated based on the dynamic PET (dPET) data by fitting a linear regression function to the time activity data and for each pixel using the PMOD software (PMOD Technologies, Zurich, Switzerland). Parametric imaging is a method for feature extraction, enabling the visualization of single parameters of tracer kinetics, with images of the slope reflecting primarily the trapping of ${ }^{18} \mathrm{~F}-\mathrm{FDG}$ and images of the intercept reflecting the distribution volume of the tracer. These images may be used for the delineation of the malignant lesions and the VOIs placement due to potentially high contrast in the surrounding tissue. Details of this method have been described elsewhere $[17,18]$. Nevertheless, parametric imaging analysis was not the focus of the present work.

\subsection{Histological Response}

Resection specimens were histopathologically assessed. The percentage of regression grade was recorded as well as data regarding tumor size, resection status (free margins, smallest distance between margin and vital tumor, microscopic and macroscopic infiltration), histological subtype, grade (G1-3), and most prevalent type of regression (hyalinous necrosis, apoptosis, scar tissue, hemorrhagic necrosis) [5] were also recorded. In the present analysis, potential associations between the percentage of regression grade and PET parameters' changes in response to treatment were assessed. 


\subsection{Statistical Analysis}

For continuous variables the mean, standard deviation, 95\% confidence interval for the mean, skewness and excess, p-value of the d'Agostino-Pearson test (test for normal distribution), median, range, $95 \%$ confidence interval for the median, quartile difference, and lower and upper quartile were given. For dichotomous and ordinal variables, the absolute and relative frequencies, as well as the $95 \%$ confidence interval for proportions (Wald method) was used. To compare the values of the PET variables before/after pazopanib treatment the Wilcoxon rank sum test (paired) was performed with the exact p-value, the median difference, and the $95 \%$ confidence interval. For the survival analysis, Kaplan-Meier plots were used. Since there were only a few events (deaths or recurrences/relapses), standard statistics was also applied to describe survival. Time to progression (TTP) was measured from first date of PET imaging (entry into the study) to the date of event of local and/or distant relapse. Non-events were censored with the last recorded follow-up date. The same procedure was used for overall survival with the event of death. Univariate comparisons between response groups were performed with unpaired $t$-tests and rank-sum tests (inclusive the estimated mean/median differences and the $95 \%$ confidence intervals of these differences, respectively).

The level of statistical significance was set to $\alpha=0.05$. The statistical tool used was SAS 9.4 (SAS Institute Inc., Cary, North Carolina USA). Graphical data were used in Microsoft Excel.

\section{Results}

\subsection{Follow-up Status}

Median overall survival (OS) was 3.14 years with a 95\% confidence interval of 1.71-3.43 years. In the follow-up, $12 / 16$ patients (75\%) were alive without a relapse. Four patients $(25 \%)$ relapsed, and one patient among them died (Table 2).

Table 2. Follow up status of the 16 studied patients treated with pazopanib.

\begin{tabular}{ccc}
\hline Follow Up Status & No. & $\%$ \\
\hline Alive without relapse & 12 & $75 \%$ \\
Alive with relapse & 3 & $18.75 \%$ \\
Dead & 1 & $6.25 \%$ \\
\hline
\end{tabular}

Regarding the four patients with relapse, three patients showed local relapse and distant metastases, while one of them had only distant metastases. Mean time to progression (TTP) after pazopanib treatment was 1.23 years $( \pm 0.60$ years) and median TTP was 1.46 years with a range 0.31 to 1.70 years.

\subsection{Histopathological Regression}

In total, 14 patients were evaluable after pazopanib treatment in terms of histopathology. Histopathological regression was in the mean $26 \% \pm 17 \%$ with a median of $20 \%$ and a range of $5-70 \%$. The studied population was dichotomized according to the percentage of histopathological regression after pazopanib. Using the $20 \%$ regression as threshold, $10 / 14$ patients $(71 \%)$ showed partial remission (PR; responders), while stable disease (SD; non-responders) was seen in the rest of the 4 evaluable patients $(21 \%)$. These results are shown in a waterfall plot (Figure 1). 


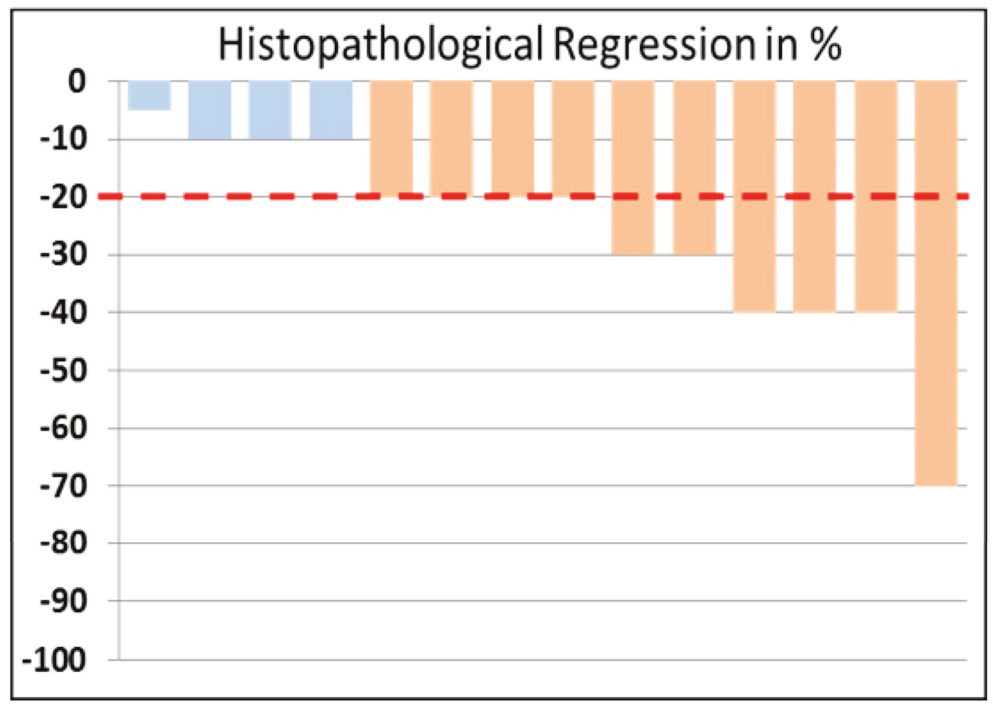

Figure 1. Waterfall plot of the grade of histopathological regression, available for 14 patients. Ten patients had a reduction in vital tumor tissue of $>20 \%$, while four patients demonstrated a reduction of less than $20 \%$.

\subsection{PET/CT Analysis}

The results of dPET/CT evaluations before and after pazopanib treatment are presented in Table 3. No statistically significant change of the semi-quantitative parameters $\mathrm{SUV}_{\text {average }}$ and $\mathrm{SUV}_{\max }$ was demonstrated. Regarding ${ }^{18}$ F-FDG kinetic analysis, $\mathrm{K}_{1}$ was the only parameter that significantly decreased after therapy. All other kinetic parameters did not show any significant change in response to pazopanib.

Table 3. Descriptive statistics of mean and median values prior and after pazopanib therapy for the ${ }^{18}$ F-FDG semi-quantitative and quantitative parameters in STS. The values of parameters $K_{1}, k_{2}, k_{3}, k_{4}$ and influx are $1 / \mathrm{min}$. SUV values, blood component $\left(\mathrm{V}_{\mathrm{B}}\right)$, and fractal dimension (FD) have no units.

\begin{tabular}{ccccc}
\hline Parameter & Mean Prior & Median Prior & Mean After & Median After \\
\hline SUV $_{\text {average }}$ & 5.7 & 3.8 & 5.0 & 4.1 \\
SUV $_{\max }$ & 10.2 & 7.3 & 8.0 & 6.5 \\
$\mathrm{~V}_{\mathrm{B}}$ & 0.10 & 0.06 & 0.07 & 0.03 \\
$\mathrm{~K}_{1}{ }^{(1 / \mathrm{min})}$ & 0.26 & 0.19 & 0.16 & 0.12 \\
$\mathrm{k}_{2}(1 / \mathrm{min})$ & 0.35 & 0.33 & 0.31 & 0.25 \\
$\mathrm{k}_{3}(1 / \mathrm{min})$ & 0.11 & 0.10 & 0.13 & 0.14 \\
$\mathrm{k}_{4}(1 / \mathrm{min})$ & 0.03 & 0.03 & 0.05 & 0.03 \\
Influx $(1 / \mathrm{min})$ & 0.06 & 0.04 & 0.04 & 1.16 \\
$\mathrm{FD}$ & 1.18 & 1.16 & 1.17 & \\
\hline
\end{tabular}

* Significant probabilities $(p<0.05)$. SUV, standardized uptake value; FD, fractal dimension. 
Figures 2 and 3 demonstrate an example of a metabolic responder patient after application of conventional, static PET/CT (Figure 2) as well after dynamic PET acquisition involving SUV and parametric images (Figure 3). Figure 4 depicts a metabolic non-responder to pazopanib.

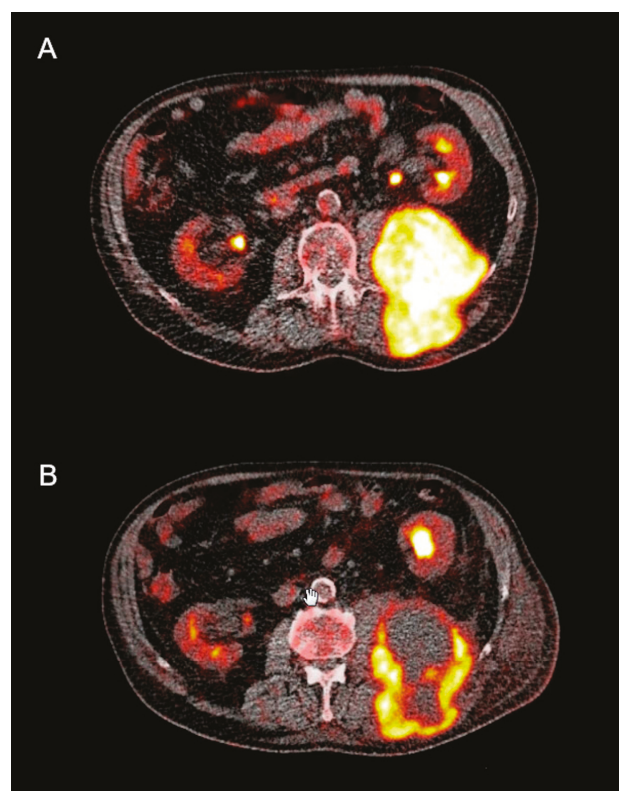

Figure 2. Transaxial fludeoxyglucose F-18 positron emission tomography/computed tomography $\left({ }^{18} \mathrm{~F}\right.$-FDG PET/CT) of an 80-year old male patient with retroperitoneal sarcoma infiltrating the back muscles before (A) and after pazopanib therapy (B). Clear metabolic remission of the initially intense metabolic lesion with areas of central necrosis in response to pazopanib. 


\section{Prior pazopanib}

\section{After pazopanib}

\section{SUV images}
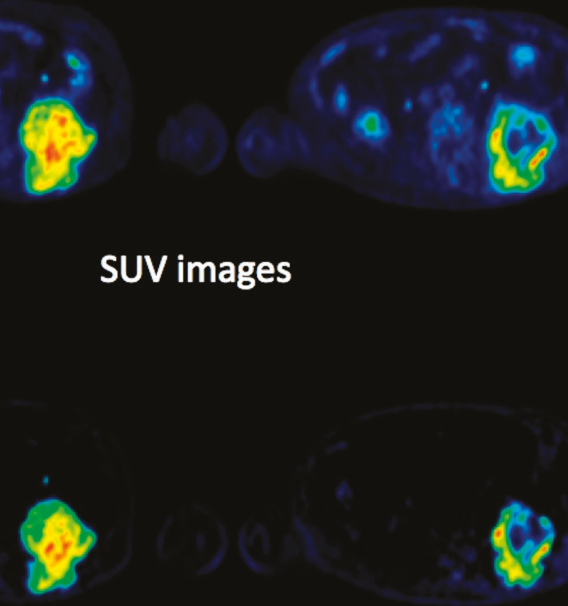

\section{slope images}

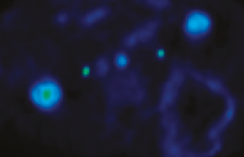

\section{intercept images}

Figure 3. Transaxial fludeoxyglucose F-18 positron emission tomography/computed tomography $\left({ }^{18} \mathrm{~F}-\mathrm{FDG}\right.$ PET/CT) of the same patient as in Figure 2 before (left) and after pazopanib therapy (right). Standardized uptake value (SUV) images acquired after $60 \mathrm{~min}$ of dynamic PET acquisition show a clear metabolic remission of the intense metabolic lesion with central necrosis in response to pazopanib (upper row). Slope parametric images also show initially intense uptake in the area of the tumor, which responds with an essential decrease after therapy due to a decrease in the phosphorylation (middle row). Intercept parametric images demonstrate the tumor very faintly due to the low distribution volume (lower row). 


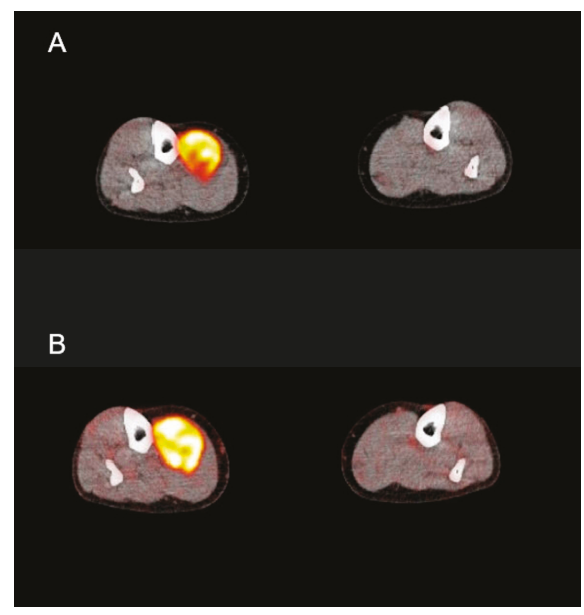

Figure 4. Transaxial fludeoxyglucose F-18 positron emission tomography/computed tomography $\left({ }^{18} \mathrm{~F}-\mathrm{FDG}\right.$ PET/CT) of a 70-year old female patient with sarcoma of the leg before (A) and after pazopanib therapy (B). Persistent metabolic activity in the tumor after pazopanib treatment.

Figure 5 depicts the time-activity curve (TAC) of ${ }^{18}$ F-FDG in a STS before and after treatment.
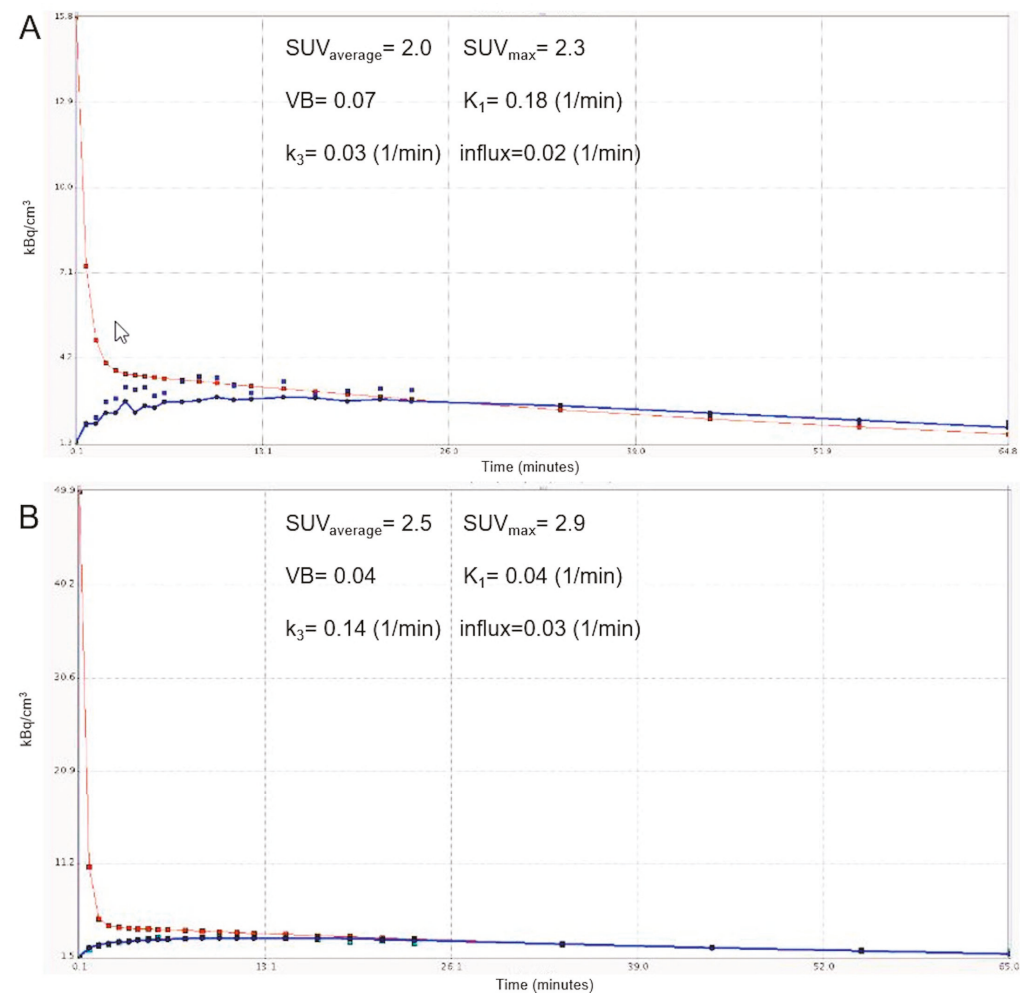

Figure 5. Time-activity curves (TACs) derived from dynamic positron emission tomography/computed tomography (PET/CT)studies of a retroperitoneal soft tissue sarcoma (STS) before (A) and after (B) 
pazopanib therapy (y-axis: $\mathrm{kBq} / \mathrm{cm} 3$; $\mathrm{x}$-axis: minutes). The TACs are derived from volumes of interest (VOIs) corresponding to the tumor (blue curve) and the descending aorta (red curve). The tumor curves show an increase in the fludeoxyglucose F-18 ( ${ }^{18}$ F-FDG) accumulation in the tumor VOI during the 60 min of dynamic PET acquisition (reflected by an increase in standardized uptake value-SUV values), but at the same time a decrease in the carrier-mediated transport of the tracer from plasma to the tumor (reflected by a decrease in $\mathrm{K}_{1}$ ) in response to pazopanib. VB: blood volume.

We further performed comparisons of the PET parameters between the groups of responders (PR) and non-responders (SD), according to the histopathological criteria applied in the study. Unpaired test procedures (t-test/rank-sum Wilcoxon test as appropriate) showed no statistically significant differences between the two groups in response to the treatment. No significant correlation between histopathological regression and ${ }^{18}$ F-FDG kinetics response was observed.

Finally, TTP data were also studied in association with ${ }^{18}$ F-FDG PET/CT data. Similarly to histopathological regression, no association between tracer kinetics and time to tumor relapse was observed.

\section{Discussion}

The current study was designed to analyze the potential role of both the semi-quantitative (SUV) and quantitative/kinetic evaluation of ${ }^{18}$ F-FDG PET for response assessment of neoadjuvant pazopanib treatment in STS patients. This analysis took place in the framework of a phase II window-of-opportunity trial of the German Interdisciplinary Sarcoma Group (GISG-04/NOPASS).

No statistically significant changes of the semi-quantitative parameters $S U V_{\text {average }}$ and $S U V_{\max }$ in response to pazopanib could be demonstrated. This finding is expected, given the recently published results of our group, which revealed a mean decrease of $6 \%$ in SUV average of post- vs. pretreatment PET/CT in the same patient cohort [2]. The primary endpoint of that analysis was based on a definition of metabolic response as $>50 \%$ decrease in $\mathrm{SUV}_{\text {average, }}$ leading to the characterization of only one subject as the metabolic responder. It could be argued that the applied SUV threshold of $50 \%$ was rather high, and the application of a lower one would have led to a higher metabolic response rate. Nevertheless, a reduction of the threshold to $40 \%$ - as suggested by Schuetze et al. [19]—would have yielded the same metabolic response rate. Respectively, the application of the thresholds suggested by the two most widely used PET criteria (positron emission tomography response criteria in solid tumors-PERCIST and European Organisation for Research and Treatment of Cancer-EORTC) for definition of partial metabolic response would not have led to essentially different results. In particular, the usage of a 30\% decrease of SUV-according to the PERCIST criteria [20] — would also classify only one patient as the metabolic responder, while a reduction of the SUV threshold to $25 \%$ —according to the EORTC criteria for PET [21]—would have resulted in three metabolic responders and thus still in a formally negative trial result.

SUV is the most widely used method for quantification of PET data, since its calculation requires only static imaging usually $60 \mathrm{~min}$ p.i. However, the ${ }^{18} \mathrm{~F}$-FDG uptake $60 \mathrm{~min}$ after tracer injection is the result of a dynamic process. One important aspect of PET is the possibility of performing accurate, noninvasive quantitative measurements of tracer concentration in patients. The generally accepted method for accurate analysis of ${ }^{18}$ F-FDG metabolism and pharmacokinetics is a two-tissue compartment model [6], which requires, however, the performance of a dynamic PET (dPET) study with duration of $60 \mathrm{~min}$, in addition to the regular static PET/CT scan. This is of course more time consuming, both for the patient and the institution, and thus is mainly limited to research centers, as ours. Particularly in STS, the application of dPET and the subsequent acquisition of ${ }^{18}$ F-FDG kinetic information have been shown useful in early prediction of chemosensitivity in the neoadjuvant context [8].

${ }^{18}$ F-FDG kinetic analysis before and after pazopanib treatment revealed a significant decrease of the parameter $K_{1}$, which reflects the carrier-mediated transport of the tracer from plasma to tumor, 
in response to therapy. Although not in line with the results of semi-quantitative (SUV) analysis, this decrease in $\mathrm{K}_{1}$ could be partly explained by the mechanism of action of pazopanib. Pazopanib exerts its action through inhibition of growth factor receptors associated with angiogenesis and tumor cell proliferation [22]. Given that $K_{1}$ is a perfusion-related parameter [23], it could be suggested that the anti-angiogenic effect of pazopanib is responsible for this finding. Moreover, this result is in accordance with that published by Dimitrakopoulou-Strauss et al. in a group of 31 STS patients receiving neoadjuvant chemotherapy consisting of etoposide, ifosfamide, and doxorubicin, who were also followed by dPET; the patients in that study also showed a significant decline in $\mathrm{K}_{1}$ after two chemotherapeutic cycles [8]. Based on these results, the parameter $K_{1}$ could be potentially used as an early response marker of the anti-angiogenic effect of pazopanib with changes of this parameter being used for therapeutic management decisions, of course after taking into account other tumor-related parameters. However, long-term follow-up is required to confirm the herein presented results.

On the other hand, the phosphorylation rate of ${ }^{18} \mathrm{~F}-\mathrm{FDG}\left(\mathrm{k}_{3}\right)$, its influx $\left(\mathrm{K}_{\mathrm{i}}\right)$, and the fractal dimension (FD) did not show any significant change. This is in line with the lack of significant tracer uptake (SUV) decrease in response to treatment. Although the reason for this lacking response is unknown, apart from the small sample size, a targeted anti-angiogenic action of pazopanib without a pronounced and fast effect on tumor glucose metabolism could be hypothesized.

Since there is no uniformly accepted gold standard to define histopathological regression, a threshold of $20 \%$-based on the calculated median histopathological regression grade and in line with the first published GISG-04/NOPASS study [5]—was applied in order to dichotomize the studied population into responders and non-responders after pazopanib. The comparison of the PET parameters between these groups showed no statistically significant differences both at baseline and as response to treatment. Moreover, no significant correlation between histopathological regression and ${ }^{18} \mathrm{~F}-\mathrm{FDG}$ kinetics response was observed, which, however may be due to the small sample size.

Although the follow-up time was rather short, TTP data were also utilized in the present analysis to search for potential associations between ${ }^{18} \mathrm{~F}$-FDG kinetics and disease relapse. Four of the studied patients $(25 \%)$ relapsed with a mean TTP of 1.23 years; three of them showed local relapse and distant metastases, while one of them showed only distant metastases. In line with the previously presented results, no correlation between tracer kinetics and TTP was observed, which could be attributed to the small sample size and the limited number of progression events.

The main limitation of our analysis is the small number of patients evaluated, rendering a more robust statistical evaluation difficult. This is because patient enrolment in the trial was stopped based on the result of a futility analysis presented in the first results published by our group [5].

\section{Conclusions}

The results of the present study show that the widely used PET parameter SUV may not be the adequate measure to assess the response to neoadjuvant pazopanib treatment in STS patients. On the other hand, the perfusion-related, kinetic parameter $\mathrm{K}_{1}$-reflecting the carrier-mediated transport of ${ }^{18}$ F-FDG from plasma to tumor-significantly decreased during pazopanib treatment. This finding could be due to the anti-angiogenic effect of the agent. Based on these findings, $K_{1}$ could be potentially used as an early response marker of the pazopanib treatment efficacy, with changes of this parameter being used for therapeutic management decisions. However, long-term follow-up is required to confirm the herein presented results.

Author Contributions: Conceptualization, C.S., A.D.-S. and U.R.; methodology, C.S., A.D.-S. and U.R.; software, C.S. and A.D.-S.; validation, C.S., A.D.-S. and U.R.; formal analysis, C.S., L.P., U.A., T.G., H.-G.D., A.D.-S. and U.R.; investigation, C.S., L.P., A.D.-S. and U.R.; resources, I.K., J.J., B.K., K.N., M.S., P.H. and U.R.; data curation, C.S., L.P., A.D.-S. and U.R.; writing-original draft preparation, C.S., L.P., A.D.-S. and U.R.; statistical analysis, L.P.; writing-review and editing, C.S., L.P., A.D.-S. and U.R.; visualization, C.S., T.U.A., T.G. and A.D.-S.; supervision, A.D.-S. and U.R.; project administration, C.S.

Funding: This study was partially funded by GlaxoSmithKline Oncology/Novartis. 
Conflicts of Interest: The authors declare no conflict of interest.

\section{References}

1. Casali, P.; Abecassis, N.; Bauer, S.; Bauer, S.; Biagini, R.; Bielack, S.; Bonvalot, S.; Boukovinas, I.; Bovee, J.V.M.G.; Brodowicz, T.; et al. ESMO guidelines committee and EURACAN. Soft tissue and visceral sarcomas: ESMO-EURACAN clinical practice guidelines for diagnosis, treatment and follow-up. Ann. Onco. 2018, 29, iv268-iv269. [CrossRef] [PubMed]

2. Ronellenfitsch, U.; Dimitrakopoulou-Strauss, A.; Jakob, J.; Kasper, B.; Nowak, K.; Pilz, L.R.; Attenberger, U.; Gaiser, T.; Egerer, G.; Fröhling, S.; et al. Preoperative therapy with pazopanib in high-risk soft tissue sarcoma: A phase II window-of-opportunity study by the German Interdisciplinary Sarcoma Group (GISG-04/NOPASS). BMJ Open 2016, 6, e009558. [CrossRef] [PubMed]

3. Van der Graaf, W.T.; Blay, J.Y.; Chawla, S.P.; Kim, D.W.; Bui-Nguyen, B.; Casali, P.G.; Schöffski, P.; Aglietta, M.; Staddon, A.P.; Beppu, Y.; et al. EORTC soft tissue and bone sarcoma group; PALETTE study group. Pazopanib for metastatic soft-tissue sarcoma (PALETTE): A randomised, double-blind, placebo-controlled phase 3 trial. Lancet 2012, 379, 1879-1886. [CrossRef]

4. Hamberg, P.; Verweij, J.; Sleijfer, S. (Pre-)clinical pharmacology and activity of pazopanib, a novel multikinase angiogenesis inhibitor. Oncologist 2010, 15, 539-547. [CrossRef] [PubMed]

5. Ronellenfitsch, U.; Karampinis, I.; Dimitrakopoulou-Strauss, A.; Sachpekidis, C.; Jakob, J.; Kasper, B.; Nowak, K.; Pilz, L.; Attenberger, U.; Gaiser, T.; et al. Preoperative pazopanib in high-risk soft tissue sarcoma (STS): Phase II window-of opportunity study of the german interdisciplinary sarcoma group (NOPASS/GISG-04). Ann. Surg. Onco. 2019, 26, 1332-1339. [CrossRef] [PubMed]

6. Phelps, M.E.; Huang, S.C.; Hoffman, E.J.; Selin, C.; Sokoloff, L.; Kuhl, D.E. Tomographic measurement of local cerebral glucose metabolic rate in humans with (F-18)2-fluoro-2-deoxy-d-glucose: Validation of method. Ann. Neurol. 1979, 6, 371-388. [CrossRef] [PubMed]

7. Kasper, B.; Dietrich, S.; Dimitrakopoulou-Strauss, A.; Strauss, L.G.; Haberkorn, U.; Ho, A.D.; Egerer, G. Early prediction of therapy outcome in patients with high-risk soft tissue sarcoma using positron emission tomography. Onkologie 2008, 31, 107-112. [CrossRef] [PubMed]

8. Dimitrakopoulou-Strauss, A.; Strauss, L.G.; Egerer, G.; Vasamiliette, J.; Mechtersheimer, G.; Schmitt, T.; Lehner, B.; Haberkorn, U.; Stroebel, P.; Kasper, B. Impact of dynamic 18F-FDG PET on the early prediction of therapy outcome in patients with high-risk soft-tissue sarcomas after neoadjuvant chemotherapy: A feasibility study. J. Nucl. Med. 2010, 51, 551-558. [CrossRef] [PubMed]

9. Dimitrakopoulou-Strauss, A.; Strauss, L.G.; Egerer, G.; Vasamiliette, J.; Schmitt, T.; Haberkorn, U.; Kasper, B. Prediction of chemotherapy outcome in patients with metastatic soft tissue sarcomas based on dynamic FDG PET (dPET) and a multiparameter analysis. Eur. J. Nucl. Med. Mol. Imaging 2010, 37, 1481-1489. [CrossRef] [PubMed]

10. PMOD Technologies. Available online: http://www.pmod.com/files/download/v31/doc/pbas/4729.htm (accessed on 26 December 2018).

11. Sachpekidis, C.; Thieke, C.; Askoxylakis, V.; Nicolay, N.H.; Huber, P.E.; Thomas, M.; Dimitrakopoulou, G.; Debus, J.; Haberkorn, U.; Dimitrakopoulou-Strauss, A. Combined use of (18)F-FDG and (18)F-FMISO in unresectable non-small cell lung cancer patients planned for radiotherapy: A dynamic PET/CT study. Am. J. Nucl. Med. Mol. Imaging 2015, 5, 127-142. [PubMed]

12. Sachpekidis, C.; Goldschmidt, H.; Kopka, K.; Kopp-Schneider, A.; Dimitrakopoulou-Strauss, A. Assessment of glucose metabolism and cellular proliferation in multiple myeloma: A first report on combined 18F-FDG and 18F-FLT PET/CT imaging. EJNMMI Res. 2018, 8, 28. [CrossRef] [PubMed]

13. Ohtake, T.; Kosaka, N.; Watanabe, T.; Yokoyama, I.; Moritan, T.; Masuo, M.; Iizuka, M.; Kozeni, K.; Momose, T.; $\mathrm{Oku}, \mathrm{S}$.; et al. Noninvasive method to obtain input function for measuring tissue glucose utilization of thoracic and abdominal organs. J. Nucl. Med. 1991, 32, 1432-1438. [PubMed]

14. Burger, C.; Buck, A. Requirements and implementations of a flexible kinetic modeling tool. J. Nucl. Med. 1997, 38, 1818-1823. [PubMed]

15. Sokoloff, L.; Smith, C.B. Basic principles underlying radioisotopic methods for assay of biochemical processes in vivo. In The Metabolism of the Human Brain Studied with Positron Emission Tomography; Greitz, T., Ingvar, D.H., Widén, L., Eds.; Raven Press: New York, NY, USA, 1983; pp. 123-148. 
16. Dimitrakopoulou-Strauss, A.; Strauss, L.G.; Mikolajczyk, K.; Burger, C.; Lehnert, T.; Bernd, L.; Ewerbeck, V. On the fractal nature of positron emission tomography (PET) studies. World J. Nucl. Med. 2003, 4, 306-313.

17. Dimitrakopoulou-Strauss, A.; Pan, L.; Strauss, L.G. Quantitative approaches of dynamic FDG-PET and PET/CT studies (dPET/CT) for the evaluation of oncological patients. Cancer Imaging 2012, 12, $283-289$. [CrossRef] [PubMed]

18. Sachpekidis, C.; Pan, L.; Hadaschik, B.A.; Kopka, K.; Haberkorn, U.; Dimitrakopoulou-Strauss, A. 68Ga-PSMA-11 PET/CT in prostate cancer local recurrence: Impact of early images and parametric analysis. Am. J. Nucl. Med. Mol. Imaging 2018, 8, 351-359.

19. Schuetze, S.M.; Rubin, B.P.; Vernon, C.; Hawkins, D.S.; Bruckner, J.D.; Conrad, E.U., 3rd; Eary, J.F. Use of positron emission tomography in localized extremity soft tissue sarcoma treated with neoadjuvant chemotherapy. Cancer 2005, 103, 339-348. [CrossRef] [PubMed]

20. Wahl, R.L.; Jacene, H.; Kasamon, Y.; Lodge, M.A. From RECIST to PERCIST: Evolving Considerations for PET response criteria in solid tumors. J. Nucl. Med. 2009, 50, 122S-150S. [CrossRef]

21. Young, H.; Baum, R.; Cremerius, U.; Herholz, K.; Hoekstra, O.; Lammertsma, A.A.; Pruim, J.; Price, P. Measurement of clinical and subclinical tumour response using [18F]-fluorodeoxyglucose and positron emission tomography: Review and 1999 EORTC recommendations. European Organization for Research and Treatment of Cancer (EORTC) PET Study Group. Eur. J. Cancer 1999, 35, 1773-1782. [CrossRef]

22. Gotink, K.J.; Verheul, H.M. Anti-angiogenic tyrosine kinase inhibitors: What is their mechanism of action? Angiogenesis 2010, 13, 1-14. [CrossRef]

23. Kristian, A.; Revheim, M.E.; Qu, H.; Mælandsmo, G.M.; Engebråten, O.; Seierstad, T.; Malinen, E. Dynamic (18)F-FDG-PET for monitoring treatment effect following anti angiogenic therapy in triple-negative breast cancer xenografts. Acta Oncol. 2013, 52, 1566-1572. [CrossRef] [PubMed]

(C) 2019 by the authors. Licensee MDPI, Basel, Switzerland. This article is an open access article distributed under the terms and conditions of the Creative Commons Attribution (CC BY) license (http://creativecommons.org/licenses/by/4.0/). 

Article

\title{
Potential Prognostic Role of ${ }^{18}$ F-FDG PET/CT in Invasive Epithelial Ovarian Cancer Relapse. A Preliminary Study
}

\author{
Anna Myriam Perrone ${ }^{1, *,+}$, Giulia Dondi ${ }^{1}$, Giacomo Maria Lima ${ }^{2}$, Paolo Castellucci ${ }^{2}$, \\ Marco Tesei ${ }^{1}$, Sara Coluccelli ${ }^{1}$, Giuseppe Gasparre ${ }^{1,3}$, Anna Maria Porcelli ${ }^{4}$, Cristina Nanni ${ }^{2}$, \\ Stefano Fanti ${ }^{2}$ and Pierandrea De Iaco ${ }^{1}$ \\ 1 Department of Medical and Surgical Sciences (DIMEC), Unit of Gynecologic Oncology, University of \\ Bologna, 40138, Bologna, Italy; giulia.dondi@gmail.com (G.D.); marco.tesei2@gmail.com (M.T.); \\ sara.coluccelli2@unibo.it (S.C.); giuseppe.gasparre@gmail.com (G.G.); pierandrea.deiaco@unibo.it (P.D.I.) \\ 2 Nuclear Medicine Unit, University of Bologna, S. Orsola-Malpighi Hospital Bologna, University of Bologna, \\ 40138 Bologna, Italy; giacomo.maria.lima@gmail.com (G.M.L.); paolo.castellucci@aosp.bo.it (P.C.); \\ cristina.nanni@aosp.bo.it (C.N.); stefano.fanti@aosp.bo.it (S.F.) \\ 3 Center for Applied Biomedical Research (CRBA), University of Bologna-S. Orsola Hospital, \\ 40138 Bologna, Italy \\ 4 Department of Pharmacy and Biotechnology (FABIT), University of Bologna, 40126 Bologna, Italy; \\ annamaria.porcelli@unibo.it \\ * Correspondence: myriam.perrone@aosp.bo.it; Tel.: +39-23498359048 \\ † Anna Myriam Perrone: Unit of Gynecologic Oncology, S.Orsola Hospital, 40138 Bologna, Italy.
}

Received: 25 April 2019; Accepted: 21 May 2019; Published: 23 May 2019

\begin{abstract}
Epithelial ovarian cancer (EOC) is the most lethal gynecological malignancy, with relapse occurring in about $70 \%$ of advanced cases with poor prognosis. Fluorine-18-2-fluoro-2-deoxy-d-glucose PET/CT ( ${ }^{18} \mathrm{~F}$-FDGPET/CT) is the most specific radiological imaging used to assess recurrence. Some intensity-based and volume-based PET parameters, maximum standardized uptake values $\left(\mathrm{SUV}_{\max }\right)$, metabolic tumor volume (MTV) and total lesion glycolysis (TLG), are indicated to have a correlation with treatment response. The aim of our study is to correlate these parameters with post relapse survival (PRS) and overall survival (OS) in Epithelial Ovarian Cancer (EOC) relapse. The study included 50 patients affected by EOC relapse who underwent ${ }^{18}$ F-FDGPET/CT before surgery. All imaging was reviewed and SUV $\max , M T V$ and TLG were calculated and correlated to PRS and OS. PRS and OS were obtained from the first relapse and from the first diagnosis to the last follow up or death, respectively. SUV $\max$, MTV and TLG were tested in a univariate logistic regression analysis, only $S U V_{\max }$ demonstrated to be significantly associated to PRS and OS ( $p=0.005$ and $p=0.024$ respectively). Multivariate analysis confirmed the results. We found a cut-off of SUV max of 13 that defined worse or better survival $(p=0.003)$. In the first relapse of EOC, SUV max is correlated to PRS and OS, and when $\mathrm{SUV}_{\max }$ is greater than 13, it is an unfavorable prognostic factor.
\end{abstract}

Keywords: ovarian cancer; $\mathrm{PET} / \mathrm{CT}$; relapse; $\mathrm{SUV}_{\max }$; targeted therapy; prognosis

\section{Introduction}

Epithelial ovarian cancers (EOC) are the most lethal and silent gynecological tumors with a diagnosis in advanced stages (III-IV) in about $62 \%$ of cases [1,2]. The standard approach for treating EOC is surgery and chemotherapy [3-7]. Despite optimal surgery and appropriate first-line chemotherapy, about $70-80 \%$ of patients with EOC will develop disease recurrence. Recurrence occurs in $23 \%$ of patients during or within 6 months after end of primary chemotherapy, and $60 \%$ after six 
months [8]. Ultrasound, contrast-enhanced tomography (CT), fluorine-18-2-fluoro-2-deoxy-d-glucose PET/CT ( ${ }^{18}$ F-FDG-PET/CT) and the periodic evaluation of CA 125 levels are the most used methods during the follow up to detect cancer recurrence, even if the correct modalities of follow up are not well defined [9]. A leading option for the treatment of recurrent ovarian cancer is chemotherapy, however in selected cases, resection of the tumor may be considered [10]. The role of surgery in recurrence of EOC is still debated, surgery represents a good option when an absent residual disease (CC0) is present, as demonstrated by the Arbeitsgemeinschaft Gynaekologische Onkologie (AGO) Group DESKTOP OVAR I trial (DESKTOP I) [11], and more recently by the preliminary data of the DESKTOP III from the last ASCO meeting. Data demonstrated a benefit of secondary cytoreductive surgery and chemotherapy, as opposed to chemotherapy alone exclusively in patients with complete resection with a progression-free survival of 5-6 months [12,13]. Good predictive factors of CC0 were macroscopically complete resection at first surgery, good performance status, and the absence of ascites greater than $500 \mathrm{~mL}$. The role of Imaging has become increasingly important, allowing to properly monitor patients, distinguishing the different relapse patterns, thus guiding correct management and therapy. If compared with $\mathrm{CT},{ }^{18} \mathrm{~F}-\mathrm{FDG} \mathrm{PET} / \mathrm{CT}$ is able to identify recurrence earlier because, in most cases of recurrent, the tissue is characterized by a high consumption of glucose, and therefore in an increased uptake of ${ }^{18} \mathrm{~F}$-fluoro-2-deoxyglucose [14]. Recently, the prognostic role of ${ }^{18} \mathrm{~F}$-FDG PET/CT through its metabolic parameters has been studied and PET imaging techniques could be used to explore the biological behaviour of tumors during therapy, however there is no consensus on their use [15]. In cervical cancer, our group found that the assessment of the response to therapy based on ${ }^{18} \mathrm{~F}$-FDG PET/CT predicts survival in patients with locally advanced cervical cancer treated with concomitant chemo-radiotherapy [16]. In ovarian cancer, some studies proposed that ${ }^{18} \mathrm{~F}$-FDG-PET/CT is useful for defining treatment response (Positron Emission Tomography Response Criteria in Solid Tumors-PERCIST criteria) $[17,18]$ to neoadjuvant chemotherapy (NACT) and the method could be a potential predictor of prognosis in NACT and relapse [19]. Based on these premises, the role of ${ }^{18} \mathrm{~F}-\mathrm{FDG}-\mathrm{PET} / \mathrm{CT}$ as a biological parameter of a tumour to predict prognosis appears promising. The aim of the study is to test the prognostic value of the ${ }^{18}$ F-FDG PET/CT parameters ( $\mathrm{SUV}_{\max }$, MTV and TLG) as prognostic factors in patients with first EOC recurrence.

\section{Materials and Methods}

\subsection{Population and Protocol}

This is a retrospective study. The clinical data of all patients referred to the Ovarian Cancer Center of Bologna, Italy, from January 2008 to May 2016 were analysed. Among these, we selected patients at first relapse who underwent surgery before chemotherapy. Inclusion criteria were: a) histologically confirmed diagnosis of EOC according to the WHO criteria [20]; b) standard first-line treatment based on cytoreductive surgery and combined platinum-based chemotherapy (carboplatin and paclitaxel $6-9$ cycles) c) diagnosis of recurrent EOC confirmed by ${ }^{18}$ F-FDG PET/CT available and performed at our Institute; d) secondary surgery performed in our institution, and e) adequate follow-up over 12 months. The exclusion criteria were: a) borderline and non-EOC; b) patients not evaluated with ${ }^{18} \mathrm{~F}-\mathrm{FDG}$ PET/CT at the time of the first relapse, and c) patients with inadequate information about primary treatment and secondary surgery.

All clinical and pathological data were recovered and examined, including age, body mass index (BMI), histological subtype divided in type I and type II [21], International Federation of Gynecology and Obstetrics (FIGO) stage [22], serum CA 125 levels at the first diagnosis and relapse, chemotherapy schedules and number, surgical information including score of surgical complexity measured with the Aletti's score [23] and residual disease was divided in the absence of (CC-0) $0.1-0.5 \mathrm{~cm}$, (CC-1) $0.6-1.0 \mathrm{~cm},(\mathrm{CC}-2)>1 \mathrm{~cm}$, and (CC3) [24]. Periodic clinical and radiological control data were recovered. In our institute, follow-up was performed as follows: CA 125 examination and assessment every four months for the first two years and then every 6 months for five years, CT scan every six months. ${ }^{18}$ F-FDG 
PET/CT was prescribed whenever there was a clinical suspicion of relapse or as confirmation of another instrumental examination such as CT. According to inclusion criteria, patients with disease relapse are submitted to surgery in case of platinum sensible disease (>12 months to the last chemotherapy) $[25,26]$, single or multiple recurrence amenable to complete surgical removal, the absence of extra-abdominal metastasis, a low level or absence of ascites, low levels of CA125 ( $\leq 500 \mathrm{U} / \mathrm{mL})$, and if they were fit for surgery. Otherwise, patients were submitted for chemotherapy without surgery. Progression free survival (PFS) was calculated from the first diagnosis to recurrence, post-relapse survival (PRS) and overall survival (OS) was obtained from the first relapse and from the first diagnosis to the last follow up or death. The study is a part of a larger study that was approved by Comitato Etico indipendente Ospedaliero Universitaria di Bologna on 11th November 2011 (EC number 107/2011/U/Tess). Consent to analyse the data was obtained from the local ethics committee, and informed consent forms were signed by patients and collected.

\subsection{Radiopharmaceuticals, Imaging Protocol and Images Analysis}

Whole-body ${ }^{18}$ F-FDG PET/CT scans were carried out following standard procedures. Following a 6-h fast, $3 \mathrm{MBq} / \mathrm{kg}$ of ${ }^{18} \mathrm{~F}-\mathrm{FDG}$ was intravenously injected in patients. The uptake time was $60 \mathrm{~min}$ in all patients on a 3D tomography (Discovery STE; GE) for 2 min per bed position. Cross-calibration was performed using an image quality NEMA phantom. A low-dose CT scan (120 kV, $80 \mathrm{~mA})$ was performed both for attenuation correction and as an anatomical map. PET/CT scans were evaluated by two nuclear medicine physicians experienced in oncology reviewing transverse, coronal and sagittal planes. For each scan, maximum and mean standardized uptake values (SUV $\mathrm{max}_{\text {and }} \mathrm{SUV}_{\text {mean }}$ ), metabolic tumor volume (MTV) and total lesion glycolysis (TLG) were measured. MTV measurement was calculated on PET/CT images using a semi-quantitative analysis (40\% threshold). SUV $\mathrm{Smax}_{\text {and }}$ $\mathrm{SUV}_{\text {mean }}$ normalized to body weight were measured within the MTV defined as above. TLG values were calculated as the product of MTV and SUV mean [27]. For each scan, the number of 18 F-FDG avid lesions was also measured.

\subsection{Statistical Analysis}

Statistical analysis was performed using SPSS version 24 (IBM Corp., Armonk, NY). The association between the PET parameters (SUV max $_{1}, \mathrm{MTV}$ and TLG), the PRS and OS were investigated by performing a univariate and multivariate analysis (Cox proportional hazard model). An ROC analysis was performed on those PET parameters showing an association with OS in order to carry out a cut-off value useful to predict the risk of mortality. Thereafter, patients were divided into two categories using the cut-off value suggested by ROC analysis. A Kaplan-Meier analysis was performed to show possible different overall survival between these two groups.

\section{Results}

\subsection{Population and Clinical Data}

The flow cart of the recruitment is shown in Figure 1. Characteristics of the 50 patients are reported in Table 1; the majority of patients present type II (82\%) diseases and were in an advanced stage $(78 \%)$ with about $34 \%$ of patients undergoing neoadjuvant therapy before surgery. The suspicion of recurrence was represented by clinical symptoms (intestinal discomfort and abdominal pain) in $4 / 50(8 \%)$, increased blood levels of CA 125 in 14/50 (28\%), ultrasound 4/50 (8\%), CT scan 24/50 (28\%) and ${ }^{18}$ F-FDG PET/CT 4/50 (8\%). Recurrence occurred after a disease-free survival (DSF) of $36.3 \pm 40.13$ months (mean \pm SD—standard deviation) and the levels of CA 125 were significantly lower $(p=0.001)$ in the relapse than in the first diagnosis. 


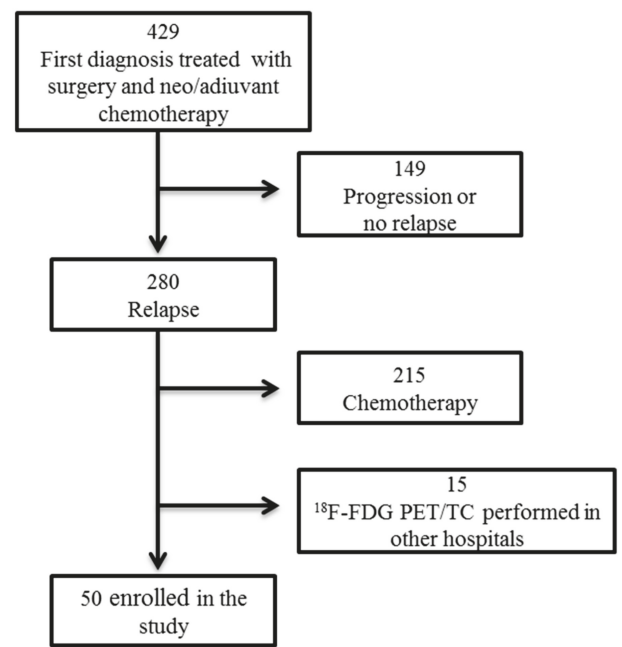

Figure 1. Flow chart of the study. Patient's selection from our database of patients with ovarian cancer.

\subsection{Surgical Data}

At first diagnosis, optimal residual disease (CC-0) was achieved in $90 \%$ of cases. Surgical complexity was significantly lower $(p=0.001)$ in the relapse than in primary surgery. Six patients $(12 \%)$ who underwent surgery were judged not optimal cytoreducible for disease extension; 44 patients $(88 \%)$ received optimal debulking surgery $(\mathrm{CC} 0)$. The Aletti's score in secondary surgery was lower in relapse than primary surgery $(p=0.009)$ (Table 1$)$.

Table 1. Clinical and surgical parameters in our patients at first diagnosis and relapse.

\begin{tabular}{lccc}
\hline & First Diagnosis & Relapse & $p$ \\
\hline $\begin{array}{l}\text { Age } \\
\text { (Mean } \pm \text { SD) }\end{array}$ & $53.0 \pm 9.2$ & $55.7 \pm 9.5$ & $n s$ \\
\hline $\begin{array}{l}\text { Body mass Index (BMI) } \\
\text { (Mean } \pm \text { SD) }\end{array}$ & $24.2 \pm 6.7$ & $25 \pm 5.6$ & ns \\
\hline Histological parameters & $9(18 \%)$ & & \\
Type 1 & $41(82 \%)$ & & \\
Type 2 & $35(70 \%)$ & & \\
Serous & $1(2 \%)$ & \\
Mucinous & $11(22 \%)$ & \\
Endometrioid & $3(6 \%)$ & \\
Clear cell & & & \\
\hline Tumor Grading & $2(4 \%)$ & \\
G1 & $6(12 \%)$ & \\
G2 & $42(84 \%)$ & \\
G3 & & \\
\hline FIGO stage & $5(10 \%)$ & \\
I & $5(10 \%)$ & \\
II & $37(74 \%)$ & \\
III & $3(6 \%)$ & \\
IV & & \\
\hline
\end{tabular}


Table 1. Cont.

\begin{tabular}{|c|c|c|c|}
\hline & First Diagnosis & Relapse & $p$ \\
\hline \multicolumn{4}{|l|}{ Genetic mutations } \\
\hline BRCA 1 & $4(8 \%)$ & & \\
\hline BRCA 2 & $2(4 \%)$ & & \\
\hline Missmatch repair (MMR) & $1(2 \%)$ & & \\
\hline No mutations & $43(86 \%)$ & & \\
\hline \multicolumn{4}{|l|}{ Bevacizumab } \\
\hline Yes & $6(12 \%)$ & & \\
\hline No & $44(88 \%)$ & & \\
\hline \multicolumn{4}{|l|}{ SUVmax } \\
\hline$($ Mean \pm SD) & & $11 \pm 5.6$ & \\
\hline \multicolumn{4}{|l|}{ TLG } \\
\hline$($ Mean \pm SD) & & $\begin{array}{c}250.9 \pm \\
946\end{array}$ & \\
\hline \multicolumn{4}{|l|}{ MTV } \\
\hline$($ Mean \pm SD) & & $\begin{array}{c}34 \pm \\
105.63\end{array}$ & \\
\hline \multicolumn{4}{|l|}{ CA $125(\mathrm{U} / \mathrm{mL})$} \\
\hline $0-34$ & $3(6 \%)$ & $20(40 \%)$ & \\
\hline $35-499$ & $18(36 \%)$ & $25(50 \%)$ & \\
\hline $500-999$ & $9(18 \%)$ & $1(2 \%)$ & \\
\hline$\geq 1000$ & $14(28 \%)$ & $2(4 \%)$ & \\
\hline not available & $6(12 \%)$ & $2(4 \%)$ & 0.001 \\
\hline \multicolumn{4}{|l|}{ ALETTI SCORE } \\
\hline Low complexity & $13(26 \%)$ & $27(54 \%)$ & \\
\hline Mediun complexity & $26(52 \%)$ & $19(38 \%)$ & \\
\hline High complexity & $11(22 \%)$ & $4(8 \%)$ & 0.001 \\
\hline \multicolumn{4}{|l|}{ RESIDUAL DISEASE } \\
\hline $\mathrm{CCO}$ & $45(90 \%)$ & $44(88 \%)$ & \\
\hline CC1 & $4(8 \%)$ & $0(0 \%)$ & \\
\hline $\mathrm{CC} 2$ & $1(2 \%)$ & $2(4 \%)$ & \\
\hline $\mathrm{CC} 3$ & $0(0 \%)$ & $4(8 \%)$ & $n s$ \\
\hline $\begin{array}{l}\text { Time between first } \\
\text { relapse and death } \\
(\text { Mean } \pm S D)\end{array}$ & & $27.8 \pm 14.3$ & \\
\hline
\end{tabular}

Legend: FIGO stage: International Federation of Gynecology and Obstetics, SUVmax: maximum standardized uptake values, MTV: metabolic tumor volume, TGL: total lesion glycolysis, SD: standard deviation.

\subsection{Follow Up Data}

During the follow-up period, 6 patients died after $44.1 \pm 18.7$ months (mean \pm SD) and 13 relapsed after $21 \pm 7.7$ (mean $\pm \mathrm{SD}$ ) months and received subsequent lines of chemotherapy. The mean follow-up was $70.2 \pm 48.3$ months, the 5 -year OS was $87 \%$ (Figure 2). 


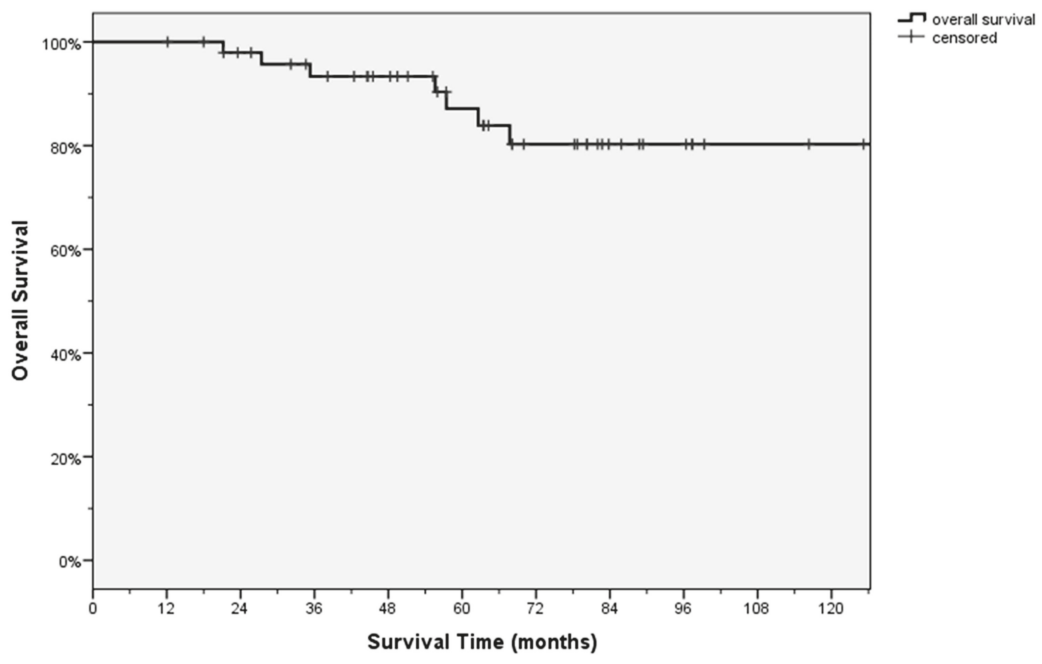

Figure 2. Kaplan-Mayer-Analysis of Overall Survival (OS) of the 50 patients enrolled in the study.

\subsection{PET's Data Analysis}

According to PET data analysis, ${ }^{18}$ F-FDG PET/CT showed a single positive lesion in 19/50 (38\%) of cases, multifocal disease in 23/50 (46\%) and diffuse (carcinomatosis) in $8 / 50(16 \%)$. The average number of lesions identified by PET was $3.4 \pm 3.6$ (mean $\pm \mathrm{SD}$ ) (range 1-6). The correspondence between ${ }^{18} \mathrm{~F}-\mathrm{FDG}-\mathrm{PET} / \mathrm{TC}$ and surgical evaluation was observed in $94 \%$ of cases. The SUV $\max$, MTV and TLG values were $11 \pm 5.6,33.4 \pm 10.5,246.1 \pm 946.7($ mean $\pm S D)$, respectively.

The univariate Cox analysis showed a correlation between $\mathrm{SUV}_{\max }$ values and PRS $(p=0.005)$ with an odds ratio $(\mathrm{OR})=1,244(95 \% \mathrm{CI}=1068-1447)$ and $\mathrm{OS}(p=0.024)$ with an odds ratio $(\mathrm{OR})=1177$ (95\% CI $=1021-1356)$. No correlation was observed between MTV and TLG with PRS ( $p=0.316$ and $p=0.074$, respectively) and OS (respectively $p=0.162$ and $p=0.106$ ).

The multivariate Cox analysis was performed by testing the following variables SUVmax, TLG and MTV with the Wald backward method (Table 2). The analysis showed that the best model predicting the OS was the $\mathrm{SUV}_{\max }$ variable alone. The ROC analysis showed that the best cut-off for $\mathrm{SUV}_{\max }$, in this cohort of patients, was 13 (Figure 3).

Table 2. Associations with PET to Overall Survival.

\begin{tabular}{clccc}
\hline & \multicolumn{1}{c}{ PET Parameters } & p-value & Odds Ratio & 95\% CI \\
\hline \multirow{2}{*}{ Step 1 } & Standardized Uptake Values (SUVmax) & 0.257 & 1.103 & $0.931-1.306$ \\
& Metabolic Tumor Volume MTV & 0.273 & 0.928 & $0.812-1.060$ \\
& Total Lesion Glycolysis (TLG) & 0.180 & 1.010 & $0.996-1.024$ \\
\hline \multirow{2}{*}{ Step 2 } & Standardized Uptake Values (SUVmax) & 0.201 & 1.125 & $0.939-1.347$ \\
& Total Lesion Glycolysis (TLG) & 0.446 & 1.002 & $0.997-1.006$ \\
\hline \multirow{2}{*}{ Step 3 } & Standardized Uptake Values (SUVmax) & 0.024 & 1.177 & $1.021-1.356$ \\
\hline
\end{tabular}




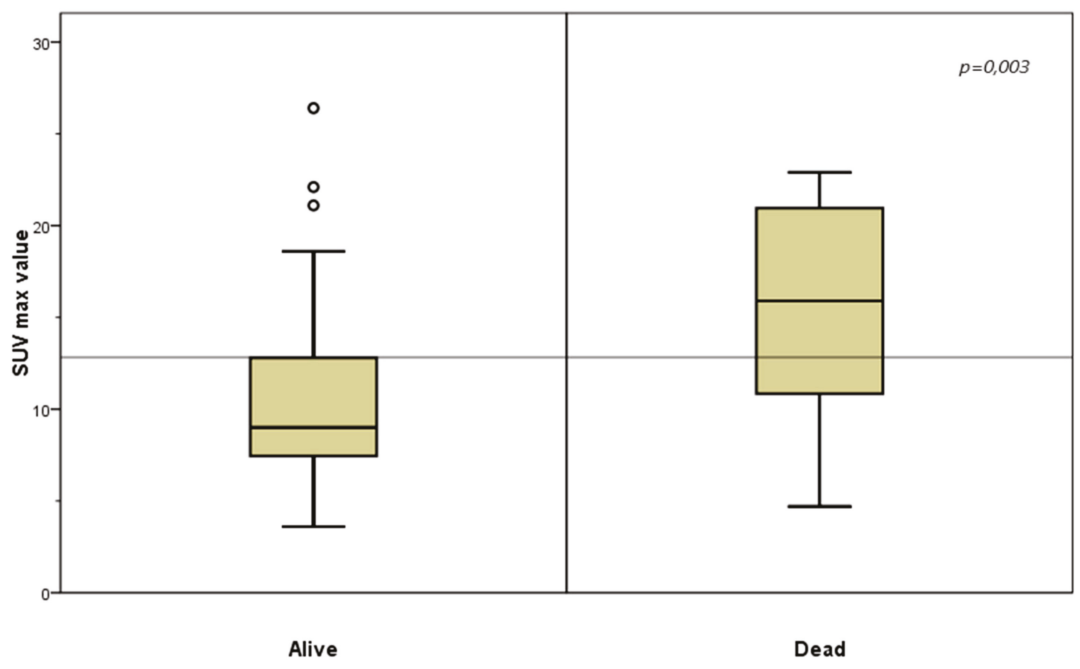

Figure 3. Standardized Uptake Values $\left(\mathrm{SUV}_{\max }\right)$ value and Overall Survival. $\mathrm{SUV}_{\max }$ greater than 13 represents a poor prognostic factor.

Therefore, patients were divided into two groups by using this value. A Kaplan-Meier performed between these two groups showed patients with a SUV $V_{\max }$ value lower than or equal to 13 had a significantly better OS ( $p=0.003)$ (Figure 4$)$.
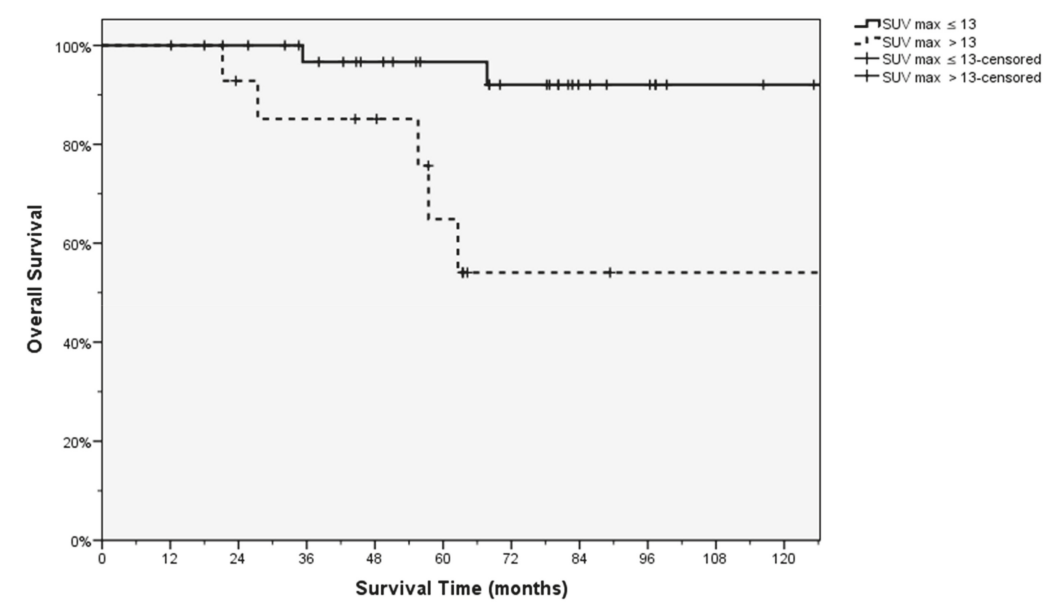

Figure 4. Different Overall Survival (OS) of the patients divided on the basis of the SUVmax value.

Moreover, it was investigated the association between $\mathrm{SUV}_{\max }$ and $\mathrm{CA} 125$ values at relapse by using the Pearson correlation test; no statistical correlation was found between these two variables $(p=0.264)$.

\section{Discussion}

This pilot study, performed in a selected population of EOC relapse patients, lays out the clinical foundation to investigate the PET parameters, such as SUV $\max$, MTV and TLG as prognostic factors in addition to the existing ones during EOC relapse. Particularly, we found that one of these parameters, 
SUV $_{\text {max }}$, is correlated with PRS and OS. To the best of our knowledge, this is the second study of its kind to explore the possible prognostic role of ${ }^{18} \mathrm{~F}$-FDG PET/TC in EOC relapse.

Although our series is a selected group of patients, it can be representative of a larger group of relapsing patients undergoing surgery, taking into account some parameters: the high incidence of recurrence found in the initial population $(80 \%)$ and the high number of patients selected for chemotherapy (215 patients) compared to those undergoing surgery (65 patients) as described in the literature $[10,11,13,28]$. Our data showed a good selection of patients suitable for surgery, as evidenced by the high percentage of $\mathrm{CC} 0(82 \%)$ and the high overall survival of the population at 5 years $(87 \%)$. We chose to study patients submitted to surgical procedures because surgery represents the gold standard to confirm the diagnosis of relapse, and to compare the characteristics found with PET. In our study, we observed a good agreement between the two assessments in $94 \%$ of cases. Moreover, ${ }^{18} \mathrm{~F}-\mathrm{FDG}$-PET/TC combines the best features of PET with CT and has been shown to have a sensibility and specificity of $91 \%$ and $88 \%$, respectively, and a predictive positive value (PPV) of $94 \%$. PET/CT in EOC relapse is more accurate than other imaging methods in detecting small carcinomatosis implants, lymph node involvement, as well as chest and bone metastasis [29].

The literature data demonstrate that when EOC recurs, it should be considered a chronic and lethal disease with poor prognosis [11]. In these cases, different therapeutic options should be proposed, in particular clinical studies and new therapeutic strategies that should be different from case to case basis. The well-known intertumoral and intratumoral heterogeneity of ovarian cancer excludes the likelihood of finding a single therapy that can be curative for most patients, and therefore requires the development of tools that can instead lead to individualized therapy [30].

At the time of the first relapse, recent studies have reported that the surgical approach with no residual disease $(\mathrm{CC} 0)$ associated with chemotherapy has led to a better prognosis than chemotherapy alone [31]. To obtain these results, it is important to select patients who will initially benefit from surgical treatment. The DESKTOP studies $[10,11,13]$ have tried to define the profile of suitable candidates for surgery, taking into account the patient's performance status, biological tumour aggressiveness (from stage and residual tumour to first diagnosis) and actual diffusion of the disease (presence of ascites). None of these parameters, however, consider the intratumoral and intertumoral heterogeneity of recurrence, which is a "hot spot" in ovarian cancer therapy. In our study, we selected patients for surgical treatment according to the current guidelines, but also attempted to understand tumour biodiversity using PET parameters.

Despite the potential ability of ${ }^{18} \mathrm{~F}$-FDG PET to study tumour metabolism in vivo, this issue is poorly investigated. The possible role of PET's parameters based on volume and uptake intensity,

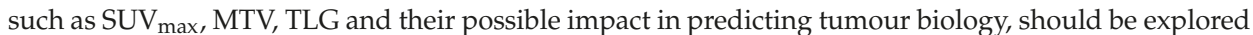
with different intentions: to monitor therapy response, study heterogeneity of the tumour and for the early identification of patients who are candidates for surgery or chemotherapy [32]. ${ }^{18}$ F-FDG is a glucose analogue that is preferentially taken up by metabolically active cells (normal and neoplastic cells). Neoplastic cells tend to show high levels of uptake, due to their greater dependence on glucose [33]. Aggressive tumours, and in particular their metastases, increase glycolysis and suppress oxidative phosphorylation, suggesting that an increase in glycolysis preference may be a hallmark of the metastatic and aggressive phenotype. A high glucose uptake could be compatible with an aggressive tumour as a sign of a glycolytic tumour and this can probably be exploited for the imaging of metabolically active tumours using ${ }^{18}$ F-FDG PET/CT $[15,16]$. Based on these assumptions, we tried to correlate ${ }^{18} \mathrm{~F}$-FDG PET/CT metabolic parameters to OS and we found that $\mathrm{SUV}_{\max }$ represents a prognostic factor $(p=0.024)$ of aggressiveness and the cut-off 13 represents a marker of poor prognosis (Figure 3). No correlations between prognosis and TGL and MTV were found. Our data are supported by a follow up longer than five years (Figure 2).

In a previous retrospective study, Kim et al. [19] evaluated the prognostic value of quantitative metabolic parameters of ${ }^{18} \mathrm{~F}-\mathrm{FDG}$-PET/CT at the time of the first relapse in patients with EOC relapse. Results of this study showed that quantitative metabolic parameters measured with ${ }^{18} \mathrm{~F}$-FDG-PET/CT 
at the time of first relapse were significant predictors of prognosis. Univariate and multivariate analyses demonstrated that whole-body metabolic tumor volume and whole-body total lesion glycolysis were independent predictors of prognosis. However, $\mathrm{SUV}_{\max }$ analyzed as continuous variable, had no correlation with prognosis, however the same authors found that a cut-off higher than 14 in the SUV $\max$ defines a worse course of the disease.

In the literature, prognostic factors and predictive response to therapy of the PET parameters were explored in several tumours and the most extensive studies have been performed on the lung and o oesophagus with conflicting results. With regard to lung cancer, 21 retrospective studies, including 2637 patients with stages I to IV non squamous cellular lung cancer (NSCLC), found that a high $\mathrm{SUV}_{\max }$ was associated with poor prognosis [34], and a second meta-analysis, limited to patients with stage I NSCLC, found that a lower FDG uptake was associated with a better prognosis $[35,36]$. In a meta-analysis of seven studies in oesophageal cancer that evaluated the impact of $\mathrm{SUV}_{\max }$ on overall survival, a high SUV predicted a worse survival [37], but data were not confirmed in a large retrospective series [38]. The results suggested a better response to preoperative chemoradiotherapy in the group with high $\mathrm{SUV}_{\max }$.

The main limitations of the study included the small number of patients enrolled and the retrospective analysis which could constitute a bias; data should be confirmed in a larger and prospective series of patients, probably including EOC relapse in chemo-sensible and chemo-insensible patients.

\section{Conclusions}

In conclusion, ${ }^{18} \mathrm{~F}$-FDG PET/CT is a diagnostic method that combines anatomical imaging with molecular behaviour of cancer cells. The uptake of ${ }^{18} \mathrm{~F}$-FDG reveals the heterogeneity of tumours and if associated to clinical, surgical and pathological parameters, could contribute to the development of a therapeutic choice tailored on a patient-by-patient basis. Indeed, ${ }^{18} \mathrm{~F}$-FDG PET/CT may represent an alternative approach to characterize relapsed ovarian tumours. In the future, clinicians should consider the metabolic information provided by ${ }^{18} \mathrm{~F}-\mathrm{FDG} \mathrm{PET} / \mathrm{CT}$ in the therapeutic choices for their patients.

Author Contributions: Conceptualization, A.M.P. and C.N.; Data curation, G.M.L. and M.T.; Formal analysis, P.C. and S.C.; Investigation, G.D.; Methodology, G.G. and A.M.P.; Supervision, S.F. and P.d.I.

Funding: This work was partly funded by the Italian Association for Cancer Reasearch grant IG17387 to A.M.P.

Acknowledgments: The authors are grateful to Stefano Friso for data collection and statistical analysis.

Conflicts of Interest: The authors declare no conflict of interest.

\section{References}

1. Howlader, N.; Noone, A.M.; Krapcho, M.; Neyman, N.; Aminou, R.; Waldron, W. SEER Cancer Statistics Review, 1975-2008, National Cancer Institute. Available online: http://seer.cancer.gov/csr/1975_2008 (accessed on 20 October 2011).

2. Perrone, A.M.; Girolimetti, G.; Procaccini, M.; Marchio, L.; Livi, A.; Borghese, G.; Porcelli, A.M.; De Iaco, P.; Gasparre, G. Potential for mitochondrial DNA sequencing in the differential diagnosis of gynaecological malignancies. Int. J. Mol. Sci. 2018, 19, 48. [CrossRef]

3. Girolimetti, G.; Perrone, A.M.; Santini, D.; Barbieri, E.; Guerra, F.; Ferrari, S.; Zamagni, C.; De Iaco, P.; Gasparre, G.; Turchetti, D. BRCA-Associated ovarian cancer: From molecular genetics to risk management. Biomed. Res. Int. 2014. [CrossRef] [PubMed]

4. Bristow, R.E.; Tomacruz, R.S.; Armstrong, D.K.; Trimble, E.L.; Montz, F.J. Survival effect of maximal cytoreductive surgery for advanced ovarian carcinoma during the platinum era: A meta-analysis. J. Clin. Oncol. 2002, 20, 1248-1259. [CrossRef]

5. Ozols, R.F.; Bundy, B.N.; Greer, B.E.; Fowler, J.M.; Clarke-Pearson, D.; Burger, R.A.; Mannel, R.S.; DeGeest, K.; Hartenbach , E.M.; Baergen, R. Phase III trial of carboplatin and paclitaxel compared with cisplatin and paclitaxel in patients with optimally resected stage III ovarian cancer: A Gynecologic Oncology Group study. J. Clin. Oncol. 2003, 21, 3194-3200. [CrossRef] [PubMed] 
6. Girolimetti, G.; Guerra, F.; Iommarini, L.; Kurelac, I.; Vergara, D.; Maffia, M.; Vidone, M.; Amato, L.B.; Leone, G.; Dusi, S.; et al. Platinum-induced mitochondrial DNA mutations confer lower sensitivity to paclitaxel by impairing tubulin cytoskeletal organization. Hum. Mol. Genet. 2017, 26, 2961-2974. [CrossRef] [PubMed]

7. Du Bois, A.; Luck, H.J.; Meier, W.; Adams, H.P.; Mobus, V.; Costa, S.; Bauknecth, T.; Richter, B.; Warm, M.; Schroder, W.; et al. A randomized clinical trial of cisplatin/paclitaxel versus carboplatin/paclitaxel as first-line treatment of ovarian cancer. J. Natl. Cancer Inst. 2003, 95, 1320-1329. [CrossRef]

8. Du Bois, A.; Reuss, A.; Pujade-Lauraine, E.; Harter, P.; Ray-Coquard, I.; Pfisterer, J. Role of surgical outcome as prognostic factor in advanced epithelial ovarian cancer: A combined exploratory analysis of 3 prospectively randomized phase 3 multicenter trials: By the Arbeitsgemeinschaft Gynaekologische Onkologie Studiengruppe Ovarialkarzinom (AGO-OVAR) and the Groupe. Cancer 2009, 115, 1234-1244. [CrossRef] [PubMed]

9. Krell, D.; Said Battistino, F.; Benafif, S.; Ganegoda, L.; Hall, M.; Rustin, G.J.S. Audit of CA125 follow-up after first-line therapy for ovarian cancer. Int. J. Gynecol. Cancer 2017, 27, 1118-1122. [CrossRef] [PubMed]

10. Harter, P.; Sehouli, J.; Reuss, A.; Hasenburg, A.; Scambia, G.; Cibula, D.; Mahner, S.; Vergote, I.; Reinthaller, A.; Burges, A.; et al. Prospective validation study of a predictive score for operability of recurrent ovarian cancer: The Multicenter Intergroup Study DESKTOP II. A project of the AGO Kommission OVAR, AGO Study Group, NOGGO, AGO-Austria, and MITO. Int. J. Gynecol. Cancer 2011, 21, 289-295. [CrossRef] [PubMed]

11. Harter, P.; Du Bois, A.; Hahmann, M.; Hasenburg, A.; Burges, A.; Loibl, S.; Gropp, M.; Huober, J.; Fink, D.; Schröder, W.; et al. Surgery in recurrent ovarian cancer: The Arbeitsgemeinschaft Gynaekologische Onkologie (AGO) DESKTOP OVAR trial. Ann. Surg. Oncol. 2006, 13, 1702-1710. [CrossRef]

12. Guerra, F.; Paiano, A.; Migoni, D.; Girolimetti, G.; Perrone, A.M.; De Iaco, P.; Fanizzi, F.P.; Gasparre, G.; Bucci, C. Modulation of RAB7A Protein expression determines resistance to cisplatin through late endocytic pathway impairment and extracellular vesicular secretion. Cancers 2019, 11, 52. [CrossRef] [PubMed]

13. Du Bois, A.; Vergote, I.; Ferron, G.; Reuss, A.; Meier, W.; Greggi, S.; Jensen, P.T.; Selle, F.; Guyon, F.; Pomel, C.; et al. A randomized controlled phase III study evaluating the impact of secondary cytoreductive surgery in recur- rent ovarian cancer: AGO DESKTOP III/ENGOT ov20. J. Clin. Oncol. 2017, 35, 5501. [CrossRef]

14. Rubello, D.; Marzola, M.C.; Colletti, P.M. The prognostic value of 18F-FDG PET/CT in monitoring chemotherapy in ovarian cancer both at initial diagnosis and at recurrent disease. Clin. Nucl. Med. 2018, 43, 735-738. [CrossRef] [PubMed]

15. Li, Q.; Zhang, J.; Cheng, W.; Zhu, C.; Chen, L.; Xia, F.; Wang, M.; Yang, F.; Ma, X. Prognostic value of maximum standard uptake value, metabolic tumor volume, and total lesion glycolysis of positron emission tomography/computed tomography in patients with nasopharyngeal carcinoma: A systematic review and meta-analysis. Medicine (Baltimore) 2017, 96, 8084. [CrossRef]

16. Lima, G.M.; Matti, A.; Vara, G.; Dondi, G.; Naselli, N.; De Crescenzo, E.M.; Morganti, A.G.; Perrone, A.M.; De Iaco, P.; Nanni, C.; et al. Prognostic value of posttreatment 18F-FDG PET/CT and predictors of metabolic response to therapy in patients with locally advanced cervical cancer treated with concomitant chemoradiation therapy: An analysis of intensity- and volume-based PET parameters. Eur. J. Nucl. Med. Mol. Imaging 2018, 45, 2139-2146. [CrossRef]

17. Wahl, R.L.; Jacene, H.; Kasamon, Y.; Lodge, M.A. From RECIST to PERCIST: Evolving considerations for PET response criteria in solid tumors. J. Nucl. Med. 2009, 50, 122S-150S. [CrossRef]

18. Vallius, T.; Hynninen, J.; Kemppainen, J.; Alves, V.; Auranen, K.; Matomäki, J.; Oksa, S.; Virtanen, J.; Grénman, S.; Auranen, A.; et al. 18F-FDG-PET/CT based total metabolic tumor volume change during neoadjuvant chemotherapy predicts outcome in advanced epithelial ovarian cancer. Eur. J. Nucl. Med. Mol. Imaging 2018, 45, 1224-1232. [CrossRef]

19. Kim, C.Y.; Jeong, S.Y.; Chong, G.O.; Son, S.H.; Jung, J.H.; Kim, D.H.; Lee, S.W.; Ahn, B.C.; Lee, J. Quantitative metabolic parameters measured on F-18 FDG PET/CT predict survival after relapse in patients with relapsed epithelial ovarian cancer. Gynecol. Oncol. 2015, 136, 498-504. [CrossRef] [PubMed]

20. Kurman, R.J.; Carcangiu, M.L.; Herrington, C.S.; Young, R.H. WHO Classification of Tumours of Female Reproductive Organs, 4th ed.; IARC: Lyon, France, 2014.

21. Shih, I.M.; Kurman, R.J. Ovarian tumorigenesis: A proposed model based on morphological and molecular genetic analysis. Am. J. Pathol. 2004, 164, 1511-1518. [CrossRef] 
22. Zeppernick, F.; Meinhold-Heerlein, I. The new FIGO staging system for ovarian, fallopian tube, and primary peritoneal cancer. Arch. Gynecol. Obstet. 2014, 290, 839-842. [CrossRef] [PubMed]

23. Aletti, G.D.; Santillan, A.; Eisenhauer, E.L.; Hu, J.; Aletti, G.; Podratz, K.C.; Bristow, R.E.; Chi, D.S.; Cliby, W.A. A new frontier for quality of care in gynecologic oncology surgery: Multi-institutional assessment of short-term outcomes for ovarian cancer using a risk-adjusted model. Gynecol. Oncol. 2007, 107, 99-106. [CrossRef]

24. Jacquet, P.; Sugarbaker, P.H. Clinical research methodologies in diagnosis and staging of patients with peritoneal carcinomatosis. Cancer. Treat. Res. 1996, 82, 359-374.

25. Petrillo, M.; De Iaco, P.; Cianci, S.; Perrone, M.; Costantini, B.; Ronsini, C.; Scambia, G.; Fagotti, A. Long-term survival for platinum-sensitive recurrent ovarian cancer patients treated with secondary cytoreductive surgery plus hyperthermic intraperitoneal chemotherapy (HIPEC). Ann. Surg. Oncol. 2016, 23, 1660-1665. [CrossRef]

26. Friedlander, M.; Trimble, E.; Tinker, A.; Alberts, D.; Avall-Lundqvist, E.; Brady, M.; Harter, P.; Pignata, S.; Pujade-Lauraine, E.; Sehouli, J.; et al. Clinical trials in recurrent ovarian cancer. Int. J. Gynecol. Cancer 2011, 21, 771-775. [CrossRef] [PubMed]

27. Lee, J.A. Segmentation of positron emission tomography images: Some recommendations for target delineation in radiation oncology. Radiother. Oncol. 2010, 96, 302-307. [CrossRef]

28. Heitz, F.; Du Bois, A.; Kurzeder, C.; Pfisterer, J.; Barinoff, J.; Grabowski, J.; Hilpert, F.; Mahner, S.; Harter, P. Surgery for recurrent ovarian cancer. Womens Health (Lond.) 2011, 7, 529-535. [CrossRef]

29. De Gaetano, A.M.; Calcagni, M.L.; Rufini, V.; Valenza, V.; Giordano, A.; Bonomo, L. Imaging of peritoneal carcinomatosis with FDG PET-CT: Diagnostic patterns, case examples and pitfalls. Abdom. Imaging 2009, 34, 391-402. [CrossRef] [PubMed]

30. Kroeger, P.T., Jr.; Drapkin, R. Pathogenesis and heterogeneity of ovarian cancer. Curr. Opin. Obstet. Gynecol. 2017, 29, 26-34. [CrossRef]

31. Giudice, M.T.; D’Indinosante, M.; Cappuccio, S.; Gallotta, V.; Fagotti, A.; Scambia, G.; Petrillo, M. Secondary cytoreduction in ovarian cancer: Who really benefits? Arch. Gynecol. Obstet. 2018, 298, 873-879. [CrossRef]

32. Chong, G.O.; Jeong, S.Y.; Lee, Y.H.; Lee, H.J.; Lee, S.W.; Han, H.S.; Hong, D.G.; Lee, Y.S. The ability of whole-body SUVmax in F-18 FDG PET/CT to predict suboptimal cytoreduction during primary debulking surgery for advanced ovarian cancer. J. Ovarian Res. 2019, 12, 12. [CrossRef]

33. Griffin, L.R.; Thamm, D.H.; Selmic, L.E.; Ehrhart, E.J.; Randall, E. Pilot study utilizing Fluorine-18 fluorodeoxyglucose-positron emission tomography/computed tomography for glycolytic phenotyping of canine mast cell tumors. Vet. Radiol. Ultrasound 2018, 59, 461-468. [CrossRef]

34. Paesmans, M.; Berghmans, T.; Dusart, M.; Garcia, C.; Hossein-Foucher, C.; Lafitte, J.J.; Mascaux, C.; Meert, A.P.; Roelandts, M.; Scherpereel, A.; et al. Primary tumor standardized uptake value measured on fluorodeoxyglucose positron emission tomography is of prognostic value for survival in non-small cell lung cancer: Update of a systematic review and meta-analysis by the European Lung Cancer Working Party for the International Association for the Study of Lung Cancer Staging Project. J. Thorac. Oncol. 2010, 5, 612. [CrossRef] [PubMed]

35. Paesmans, M.; Garcia, C.; Wong, C.Y.O.; Patz, E.F., Jr.; Komaki, R.; Eschmann, S.; Govindan, R.; Vansteenkiste, J.; Meert, A.P.; De Jong, W.K.; et al. Primary tumour standardised uptake value is prognostic in nonsmall cell lung cancer: A multivariate pooled analysis of individual data. Eur. Respir. J. 2015, 46, 1751-1761. [CrossRef]

36. Nair, V.S.; Krupitskaya, Y.; Gould, M.K. Positron emission tomography 18F-fluorodeoxyglucose uptake and prognosis in patients with surgically treated, stage I non-small cell lung cancer: A systematic review. J. Thorac. Oncol. 2009, 4, 1473. [CrossRef]

37. Pan, L.; Gu, P.; Huang, G. Prognostic significance of SUV on PET/CT in patients with esophageal cancer: A systematic review and meta-analysis. Eur. J. Gastroenterol. Hepatol. 2009, 21, 1008. [CrossRef]

38. Rizk, N.P.; Tang, L.; Adusumilli, P.S.; Bains, M.S.; Akhurst, T.J.; Ilson, D.; Goodman, K.; Rusch, V.W. Predictive value of initial PET-SUVmax in patients with locally advanced esophageal and gastroesophageal junction adenocarcinoma. J. Thorac. Oncol. 2009, 4, 875. [CrossRef]

(C) 2019 by the authors. Licensee MDPI, Basel, Switzerland. This article is an open access article distributed under the terms and conditions of the Creative Commons Attribution (CC BY) license (http://creativecommons.org/licenses/by/4.0/). 

Article

\title{
${ }^{18}$ F-FDG PET/CT and Urothelial Carcinoma: Impact on Management and Prognosis-A Multicenter Retrospective Study
}

Fabio Zattoni ${ }^{1,2}, *$, Elena Incerti ${ }^{3}$, Fabrizio Dal Moro ${ }^{1,2}$, Marco Moschini ${ }^{4}$, Paolo Castellucci ${ }^{5}$, Stefano Panareo ${ }^{6}$, Maria Picchio ${ }^{3}$, Federico Fallanca ${ }^{3}$, Alberto Briganti ${ }^{4,7}$, Andrea Gallina ${ }^{4}$, Stefano Fanti ${ }^{5}$, Riccardo Schiavina ${ }^{8}$, Eugenio Brunocilla ${ }^{8}$, Ilaria Rambaldi ${ }^{6}$, Val Lowe ${ }^{9}$, R. Jeffrey Karnes ${ }^{10}$ and Laura Evangelista ${ }^{11}$

1 Department of Surgery, Oncology and Gastroenterology, University of Padua, 35128 Padua, Italy; fabrizio.dalmoro@gmail.com

2 Urology Unit, Academical Medical Centre Hospital, 33100 Udine, Italy

3 Nuclear Medicine Department, IRCCS San Raffaele Scientific Institute, 20132 Milan, Italy; incerti.elena@hsr.it (E.I.); picchio.maria@hsr.it (M.P.); fallanca.federico@hsr.it (F.F.)

4 Department of Urology, IRCCS San Raffaele Scientific Institute, 20132 Milan, Italy; marco.moschini87@gmail.com (M.M.); briganti.alberto@hsr.it (A.B.); gallina.andrea@hsr.it (A.G.)

5 Department of Nuclear Medicine, Sant'Orsola-Malpighi Hospital, 40138 Bologna, Italy; paolo.castellucci@aosp.bo.it (P.C.); stefano.fanti@aosp.bo.it (S.F.)

6 Nuclear Medicine Unit, Diagnostic Imaging e Laboratory Medicine Department, University Hospital of Ferrara, 44121 Ferrara, Italy; s.panareo@ospfe.it (S.P.); i.rambaldi@ospfe.it (I.R.)

7 Vita-Salute San Raffaele University, 20132 Milan, Italy

8 Department of Urology, Sant'Orsola-Malpighi Hospital, 40138 Bologna, Italy; rschiavina@yahoo.it (R.S.); eugenio.brunocilla@aosp.bo.it (E.B.)

9 Division of Nuclear Medicine, Mayo Clinic, Rochester, MN 55905, USA; vlowe@mayo.edu

10 Department of Urology, Mayo Clinic, Rochester, MN 55905, USA; Karnes.R@mayo.edu

11 Nuclear Medicine and Molecular Imaging Unit, Veneto Institute of Oncology IOV-IRCCS, 35128 Padua, Italy; laura.evangelista@iov.veneto.it

* Correspondence: fabiozattoni@gmail.com

Received: 11 April 2019; Accepted: 16 May 2019; Published: 20 May 2019

\begin{abstract}
Objectives: To evaluate the ability of ${ }^{18} \mathrm{~F}$-labeled fluoro-2-deoxyglucose positron emission tomography/computed tomography $\left({ }^{18} \mathrm{~F}-\mathrm{FDG} \mathrm{PET} / \mathrm{CT}\right)$ to predict survivorship of patients with bladder cancer (BC) and/or upper urinary tract carcinoma (UUTC). Materials: Data from patients who underwent FDG PET/CT for suspicion of recurrent urothelial carcinoma (UC) between 2007 and 2015 were retrospectively collected in a multicenter study. Disease management after the introduction of FDG PET/CT in the diagnostic algorithm was assessed in all patients. Kaplan-Meier and log-rank analysis were computed for survival assessment. A Cox regression analysis was used to identify predictors of recurrence and death, for BC, UUTC, and concomitant BC and UUTC. Results: Data from 286 patients were collected. Of these, 212 had a history of BC, 38 of UUTC and 36 of concomitant BC and UUTC. Patient management was changed in 114/286 (40\%) UC patients with the inclusion of FDG PET/CT, particularly in those with BC, reaching $74 \%(n=90 / 122)$. After a mean follow-up period of 21 months (Interquartile range: 4-28 mo.), 136 patients (47.4\%) had recurrence/progression of disease. Moreover, 131 subjects (45.6\%) died. At Kaplan-Meier analyses, patients with BC and positive PET/CT had a worse overall survival than those with a negative scan (log-rank $<0.001$ ). Furthermore, a negative PET/CT scan was associated with a lower recurrence rate than a positive examination, independently from the primary tumor site. At multivariate analysis, in patients with BC and UUTC, a positive FDG PET/CT resulted an independent predictor of disease-free and overall survival $(p<0,01)$. Conclusions: FDG PET/CT has the potential to change patient management, particularly for patients with BC. Furthermore, it can be considered a valid survival prediction tool
\end{abstract}


after primary treatment in patients with recurrent UC. However, a firm recommendation cannot be made yet. Further prospective studies are necessary to confirm our findings.

Keywords: PET/CT; urothelial carcinoma; bladder cancer; upper tract urothelial carcinoma; survival

\section{Introduction}

Bladder carcinoma (BC) is the fourth most common tumor in men, with an incidence of 146,650 new cases in the US every year. It accounts for $90-95 \%$ of urothelial carcinomas (UC) and it is the most common malignancy of the urinary tract [1]. Two percent of all cancer deaths in the United States are due to BC [1]. For $35 \%$ of patients with invasive BC diagnosed at a localized stage, the 5 -year survival rate is $70 \%$. However, survivorship drops from $81 \%$ to $47 \%$ for BC with non-muscle-invasive and with muscle-invasive disease [2]. In contrast, upper urinary tract carcinoma (UUTC) is uncommon and accounts for only 5-10\% of UCs [3]. Upper tract urothelial carcinomas that invade the muscle wall usually have a poor prognosis. The 5-year specific survival rate is $<50 \%$ for patients with $\mathrm{pT} 2 / \mathrm{pT} 3$ tumors and $<10 \%$ for those with pT $4[4,5]$.

Half of the patients with muscle-invasive UC relapse after surgery, depending on the pathological stage of the primary tumor and nodal status. Local recurrence accounts for $30 \%$ of relapses, whereas distant metastases are more common. Ten to fifteen percent of patients are already metastatic at diagnosis [6]. Before the development of effective chemotherapy, patients with metastatic urothelial cancer rarely had a median survival that exceeded 3-6 months [7].

Unfortunately, more than $50 \%$ of metastases are diagnosed after the appearance of symptoms despite the periodic monitoring with advanced imaging, including Computed Tomography (CT) and Magnetic Resonance Imaging (MRI) [8,9]. This is because recurrence patterns of UC after primary treatment are poorly predictable $[10,11]$. Thus, a major concern of follow up strategies after primary treatment is whether imaging can lead to the diagnosis of disease recurrence and, if so, how this may affect long-term survival [12]. These uncertainties during follow up may be justified by several factors, including:

(1) Available salvage treatments may be ineffective. Adjuvant chemotherapy after RC for patients with pT3/4 and/or lymph node positive $(\mathrm{N}+)$ disease without clinically detectable metastases (M0) is under debate [13] and is still infrequently used [14]. There is limited evidence from adequately conducted and accrued randomized phase III trials in favor of the routine use of adjuvant chemotherapy. From the currently available evidence, it is still unclear whether immediate adjuvant chemotherapy or chemotherapy at the time of relapse is superior, or if the two approaches are equivalent with respect to the endpoint of overall survival. Cisplatin-based combination chemotherapy results in long-term disease free survival, even in metastatic disease, mainly in patients with lymph node metastases only and with a good performance status [15]. Radiation therapy or salvage surgery are currently not an option for treatment of recurrence, but only for palliation.

(2) Existing biomarkers and conventional imaging accuracy may be insufficient in the assessment of lymph node involvement and distant metastasis. Indeed, guidelines do not recommend the use of biomarkers in daily clinical practice since they have no impact on predicting outcome, making treatment decisions, or monitoring therapy.

As a consequence, it is unclear what the best follow-up schedule on UC is and what the best imaging modalities are to diagnose disease recurrence and progression [12]. Preliminary studies on accuracy showed that positron emission tomography/ computed tomography (PET/CT) is a useful tool for restaging suspected UC relapse, especially in the assessment of lymphonodes (LN) or distant metastases [16,17]. PET/CT accuracy of these retrospective studies are comparable with an overall good performance of PET/CT at patient-based analysis [17]. 
Interestingly, only a few studies have evaluated the role of ${ }^{18} \mathrm{~F}$-labeled fluoro-2-deoxyglucose positron emission tomography/computed tomography $\left({ }^{18} \mathrm{~F}-\mathrm{FDG} \mathrm{PET} / \mathrm{CT}\right)$ as a predictive tool for UC progression [18], while how PET/CT may change a patient's surgical and medical treatment is still underreported. This has been shown in other urological cancers, such as prostate cancer [19]. PET/CT has a consolidated role in restaging after primary treatment of prostate cancer $[20,21]$ and has also been introduced as a guide for salvage surgery (salvage lymph node dissection) [19]. Therefore, based on the above-mentioned limitations, the aim of this study was to evaluate the role of ${ }^{18} \mathrm{~F}$-FDG PET/CT both in the management and in the survival prediction of patients with UC.

\section{Materials}

\subsection{Study Approval and Patient Population}

The study protocol was approved at IOV Institute on April 2016 (approval nr. 005275). Major US and European urological centers with experience in BC and PET/CT were asked to participate in the study. The centers that accepted and had available cases were provided with a dedicated Microsoft Excel file created for the purpose of the study. A computerized databank was generated to transfer data of anonymized patients. A retrospective database was then built with all patients who fit the inclusion and exclusion criteria of the study. After combining the data sets, reports were generated for each variable to identify data inconsistencies and other data integrity problems. Through regular communication with all sites, resolution of all identified anomalies was achieved before analysis. The database was then frozen, and the final data set was produced for the current analysis.

\subsection{Patient Population}

FDG PET/CT scans of 286 patients with suspected recurrent UC, collected by San Raffaele Hospital in Milan (Italy), Mayo Clinic in Rochester (MN, USA), Veneto Institute of Oncology IOV-IRCCS in Padua (Italy), Sant'Orsola Malpighi Hospital in Bologna (Italy), and Hospital of Ferrara (Italy), were retrospectively reviewed from 2005 to 2015 . The same population was already included in another study assessing FDG PET/CT accuracy compared to conventional imaging [17]; however, a few patients were excluded because they did not met the inclusion criteria.

Inclusion criteria for the study were: (1) a known history of BC and/or in UUTC; (2) at least one FDG PET/CT for disease restaging (suspicion of recurrent disease or doubtful conventional imaging findings) after primary treatment (independently from the type of therapy); (3) the availability of images from conventional imaging modalities (abdominal ceCT or MRI, or total body ceCT, and chest X-ray), and (4) the availability of information on mid-long term follow-up after PET/CT imaging. Exclusion criteria were other abdominal tumors and chemotherapy administration concomitant to imaging and non-UC variants. For each patient, the following variables were collected: demographic data (age, sex, body mass index-BMI), clinical data (history of bladder cancer, last clinical stage, history of radical cystectomy, pTNM stage, history of UUTC, UUTC location in the upper tract: pelvis, ureter, multifocal), UUTC treatment (nephroureterectomy, endourology, other conservative surgery), and use and type of neoadjuvant treatments.

\subsection{PET/CT Equipment and Image Acquisition Protocol}

A standard comparable protocol was used in all centers for PET/CT image acquisition. All patients fasted for at least $6 \mathrm{~h}$ prior to imaging, and blood glucose levels were $<180 \mathrm{mg} / \mathrm{dL}$ at the time of tracer injection. To minimize FDG uptake in skeletal muscles, all patients were instructed to avoid talking, chewing or any other muscular activity before undergoing PET/CT scan. PET/CT studies were acquired with integrated PET/CT systems, according to different study protocols in accordance with each participating Institution. PET data of the whole-body tracer distribution were then acquired (3 min per bed) in 3-D mode starting 60 min after i.v. administration of FDG. Attenuation correction was performed using CT images. CT and PET images were matched and fused into transaxial, coronal, 
and sagittal images. A positive PET scan was defined in the presence of pathological FDG uptake outside the areas of physiological biodistribution, later confirmed by co-registered CT abnormalities. The definition of true positive (TP), true negative (TN), false positive, and false negative are described elsewhere [15].

\subsection{Change in Management}

A change in patient management was defined as a modification of treatment or follow-up modalities after PET/CT scan compared to conventional imaging. For example, a shift from local therapies (surgery or radiotherapy) to systemic therapy or from systemic therapy to an observational period/palliation after PET/CT scan were both considered a modification of patient strategy. The number of patients whose management was changed after PET/CT scan were assessed separately for BC and UUTC. Change in management was based on the data available at the time of PET/CT, including the original interpretation, and was determined by the authors with a review of electronic medical records.

\subsection{Follow Up}

Patients were generally observed according to standard protocols at each institution: every three to six months for the first year after surgery, and annually thereafter. Follow-up consisted of a history, physical examination, routine blood work and serum chemistry studies, chest and abdominal conventional imaging. The follow-up data of selected patients were obtained from clinical charts. To determine follow-up time, the date of the last examination or consultation was used. Progression free survival (PFS) was defined as the length of time between imaging and disease progression. Cancer Specific Survival (CSS) was defined as the time from PET/CT to cancer specific death. Finally, Overall Survival (OS) was defined as the length of time between PET/CT and all-cause mortality. Death was confirmed by retrieval of death certificate.

\subsection{Statistical Analysis}

Categorical variables were reported as frequency, while continuous variables were reported as mean and standard deviation (SD) for variables with a normal distribution, and as median and interquartile range (IQR) for variables with a non-normal distribution. The Student's T-test and the Mann-Whitney U-test were used to compare the locations of continuous variables, as appropriate. The Pearson chi-test was used to compare categorical variables. The survival intervals were defined as the time elapsed from surgery to the last clinical evaluation, the occurrence of UC recurrence, or patient death. PFS, CSS and OS were evaluated using Kaplan-Meier analysis. The log-rank test was used for comparison of the survival curves. PFS, CCS, and OS were compared for the entire population and then separately according to the site of the primary tumor and for the administration of adjuvant treatments.

Univariate and multivariate COX regression analyses were used to assess predictors of recurrence and survival. Two models were performed: in the first, data entry into the multivariable analysis was performed with a stepwise method when no variables were eligible to enter the model; only variables set at a $p$ value less than 0.05 in the univariable analysis were included. As a second model, we included the most common clinical pathological variables such as age, sex (Male vs. Female), pT stage (pT $\leq 2$ vs. pT3-4) pN stage (positive vs. negative), and PET/CT findings (positive vs. negative). A measure of goodness-of-fit was performed with a ROC analysis to evaluate the fit of all Cox regressions performed.

For some analyses, BC and UTCC were grouped together because they originate from transitional cells (which cover the entire urinary system) and they have the same risk factors, and finally, from epidemiological point of view, because UTUC often presents after BC. Analyses were performed using SPSS version 20 (IBM, Armonk, NY, USA). 


\section{Results}

\subsection{Clinical Characteristics}

Of the 286 patients, $222(77.6 \%)$ were male and $64(22.4 \%)$ female. Mean age was 69 years $( \pm$ SD: 10 years). The majority had BC only $(n=212 ; 86.8 \%), 38(13.3 \%)$ had UUTC only and $36(12.6 \%)$ had both BC and UUTC. Table 1 shows the characteristics of the patient population. Median follow up after primary tumor treatment and after PET/CT was 13 (5-28) and seven months (2-23), respectively.

Table 1. Characteristics of patients with only bladder cancer (212/286), only UTUC (38/286) and concomitant bladder cancer and UTUC (36/286).

\begin{tabular}{|c|c|c|c|}
\hline Variables & $\begin{array}{c}\text { Only Bladder Cancer } \\
(212 / 286)\end{array}$ & $\begin{array}{l}\text { Only UTUC } \\
(38 / 286)\end{array}$ & $\begin{array}{c}\text { Concomitant Bladder } \\
\text { Cancer and UTUC }(36 / 286)\end{array}$ \\
\hline \multicolumn{4}{|l|}{ Tumor grade } \\
\hline Low Grade & $15 / 212(7.0)$ & $1 / 38(2.6)$ & $2 / 36(5.5)$ \\
\hline High grade & $197 / 212(91.0)$ & $27 / 38(96.4)$ & $30 / 36(83.3)$ \\
\hline N/A & $4 / 212(1.8)$ & & $4 / 36(11.1)$ \\
\hline \multicolumn{4}{|l|}{ pT of bladder cancer, n (\%) } \\
\hline $\mathrm{pT} \leq 2$ & $82 / 212(40.0)$ & & $21 / 36(58.3)$ \\
\hline pT 3-4 & $126 / 212(59.4)$ & & $11 / 36(30.5)$ \\
\hline NA & $4 / 212(1.8)$ & & $4 / 36(11.1)$ \\
\hline \multicolumn{4}{|l|}{$\mathrm{pN}$ of bladder cancer, $\mathrm{n}(\%)$} \\
\hline $\mathrm{pNx}$ & $3 / 212(1.4)$ & & $1 / 36(2.8)$ \\
\hline pNO & $125 / 212(59)$ & & $22 / 36(61.1)$ \\
\hline pN1 & 29/212 (13.7) & & $4 / 36(11.1)$ \\
\hline pN2 & $43 / 212(20.3)$ & & $4 / 36(11.1)$ \\
\hline pN3 & $9 / 212(4.2)$ & & $1 / 36(2.8)$ \\
\hline NA & $3 / 212(1.4)$ & & $4 / 36(11.1)$ \\
\hline \multicolumn{4}{|l|}{ pT of UUTC $(*), n(\%)$} \\
\hline $\mathrm{pT} \leq 2$ & & $13 / 38(34.2)$ & $17 / 36(47.2)$ \\
\hline pT 3-4 & & $16 / 38(42.1)$ & $12 / 36(33.3)$ \\
\hline NA & & $9 / 38(23.7)$ & $7 / 36(19.4)$ \\
\hline \multicolumn{4}{|l|}{ pN of UUTC $\left({ }^{*}\right), \mathrm{n}(\%)$} \\
\hline $\mathrm{pNx}$ & & $19 / 38(50.0)$ & $31 / 36(86.1)$ \\
\hline pNO & & $14 / 38(36.8)$ & $4 / 36(11.1)$ \\
\hline pN1 & & $2 / 38(5.2)$ & $1 / 36(2.8)$ \\
\hline $\mathrm{pN} 2$ & & $3 / 38(7.9)$ & \\
\hline $\begin{array}{l}\text { Neoadjuvant treatments, } \mathrm{n} \\
(\%)\end{array}$ & 25/212 (11.8) & $5 / 38(13.1)$ & $5 / 36(13.9)$ \\
\hline Adjuvant treatments, $\mathrm{n}(\%)$ & $93 / 212(43.9)$ & $8 / 38(21.1)$ & $10 / 36(27.8)$ \\
\hline \multicolumn{4}{|l|}{$\begin{array}{l}\text { Type of adjuvant } \\
\text { treatments, } \mathrm{n}(\%)\end{array}$} \\
\hline No & $119 / 212(56.1)$ & $30 / 38$ (72.2) & $26 / 36(72.2)$ \\
\hline Chemotherapy & $75 / 212(35.4)$ & $7 / 38(18.4)$ & $7 / 36(19.4)$ \\
\hline Radiotherapy & $17 / 212(8)$ & 0 & $2 / 36(5.6)$ \\
\hline Combination & $1 / 212(0.5)$ & $1 / 38(2.6)$ & $1 / 36(2.8)$ \\
\hline
\end{tabular}

UUTC: upper urinary tract carcinoma.

\subsection{Patient Management}

PET/CT was positive in 224 patients (175 with BC only and 49 with UUTC only or UUTC plus $\mathrm{BC})$. PET/CT findings were compatible with local, lymph node, skeletal, lung and liver recurrence in $58(25.9 \%), 125(55.8 \%), 57(25.4 \%), 53(23.7 \%)$ and $26(11.6 \%)$ cases, respectively. Patient management was changed in 114/286 (40\%) cases by the inclusion of FDG PET/CT in the restage setting done with 
conventional imaging. In particular, a change in management was made in 90/122 pts (73.7\%) with BC, $10 / 28(35.7 \%)$ for UUTC and 14/22 (63.6\%) for those with BC and UUTC. Of these patients, $33(29 \%)$ received local therapies (surgery or radiotherapy), 43 (38\%) chemotherapy, 33 (29\%) a combination of local and systemic therapy and finally, five $(4 \%)$ patients were observed without any therapy. Figures 1 and 2 report two illustrative cases.

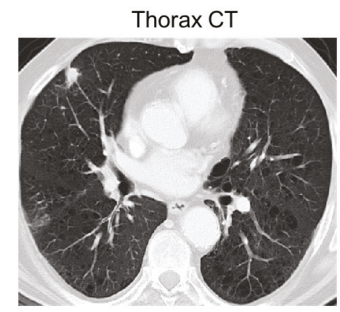

May 2009

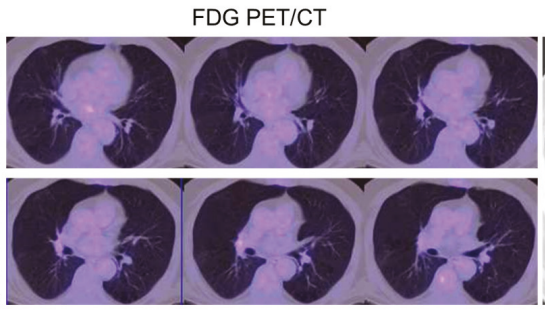

July 2009
Thorax CT

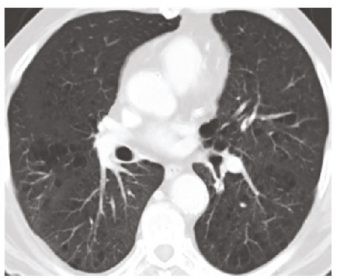

May 2010

Figure 1. A 74 year-old man with a history of bladder cancer underwent thorax and abdominal contrast-enhanced CT (ceCT) scan, which showed suspected lung recurrence. Later, positron emission tomography/computed tomography (PET/CT) was negative for any recurrence, particularly in the lung. A further thorax-abdominal ceCT scan, performed after 1 year later, revealed the disappearance of the lung nodule. Therefore, PET/CT was able to change therapeutic management, from a potential curative intent to an observational strategy.
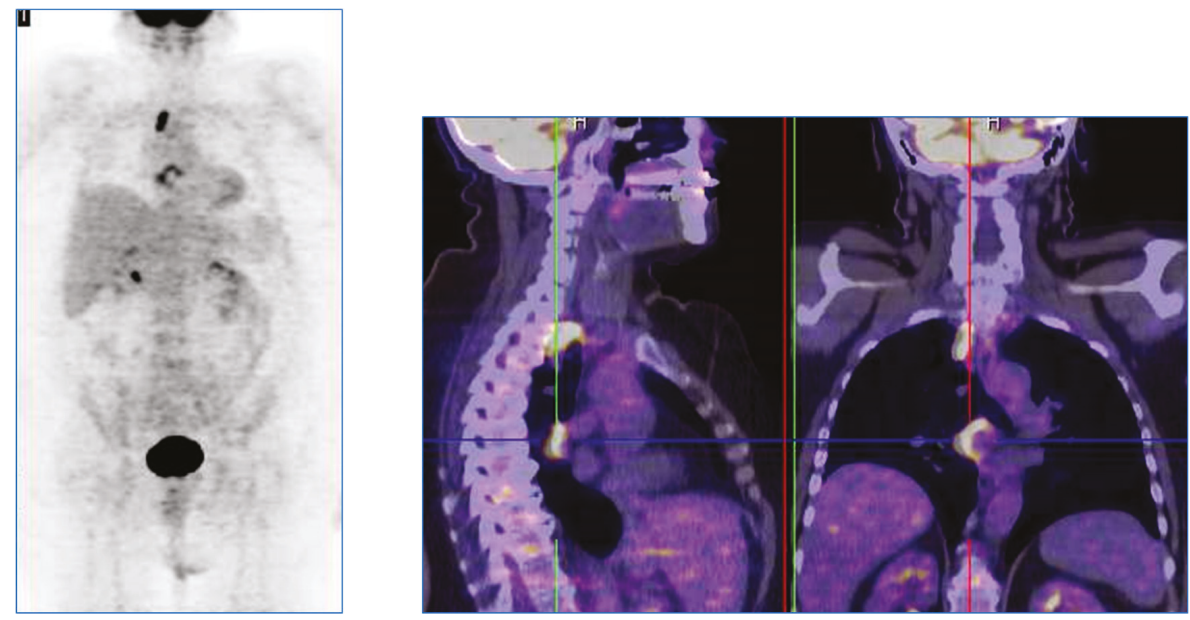

Figure 2. A 59 year-old woman with bladder cancer underwent abdominal contrast-enhanced $\mathrm{CT}$ (ceCT) for restaging of disease. CT images were considered completely negative. A positron emission tomography/computed tomography (PET/CT) performed after 3 months, for the persistence of suspicious symptomatology, showed a significant fluoro deoxyglucose uptake in the abdominal lymph nodes and in the liver, compatible with the presence of disease recurrence. The patient was later treated with systemic chemotherapy.

\subsection{Follow Up}

After a mean follow-up period of 21 months (IQR: 4-28 months) from primary treatment, 136 patients (47.4\%) had recurrence/progression of disease and of these, 87 (63.9\%) died. In total, 131 (45.8\%) patients died, of whom 104 (79.4\%) had a cancer-related death. A comparison of oncological outcomes among FDG PET/CT findings are reported in Table 2. 
Table 2. Comparison of oncological outcomes between fluoro-2-deoxyglucose positron emission tomography/computed tomography (FDG PET/CT) findings.

\begin{tabular}{cccc}
\hline & Negative PET/CT & Positive PET/CT & $p$ Value * \\
\hline $\begin{array}{c}\text { Disease recurrence/progression, } \\
\text { n (\%) }\end{array}$ & & & \\
No & $47(75.8)$ & $103(46)$ & $<0.001$ \\
Yes & $15(24.2)$ & $121(54)$ & \\
\hline All-cause mortality, n (\%) & $47(75.8)$ & $108(48.2)$ & $<0.001$ \\
No & $15(24.2)$ & $116(51.8)$ & \\
Yes & $52(83.9)$ & & $<0.001$ \\
Cancer-related mortality, n (\%) & $10(16.1)$ & $94(58)$ & \\
No & ${ }^{*}$ Chi-square test. &
\end{tabular}

In all 286 patients, 5-year OS, CSS, and PFS estimates were $29 \%, 36 \%$, and $28 \%$ in patients with a positive $\mathrm{PET} / \mathrm{CT}$, and $69 \%, 80 \%$, and $71 \%$ in patients with a negative PET/CT, respectively (all log rank; $p$ value $p<0.01$; Figure 3). In patients with BC only, the 5-year OS, CSS and RFS estimates were $37 \%, 29 \%$, and $29 \%$ in positive PET/CT group, and $90 \%, 75 \%$, and $68 \%$ in the negative PET/CT group, respectively (log-rank, $p$ value $p<0.01$; Figure 4 a).
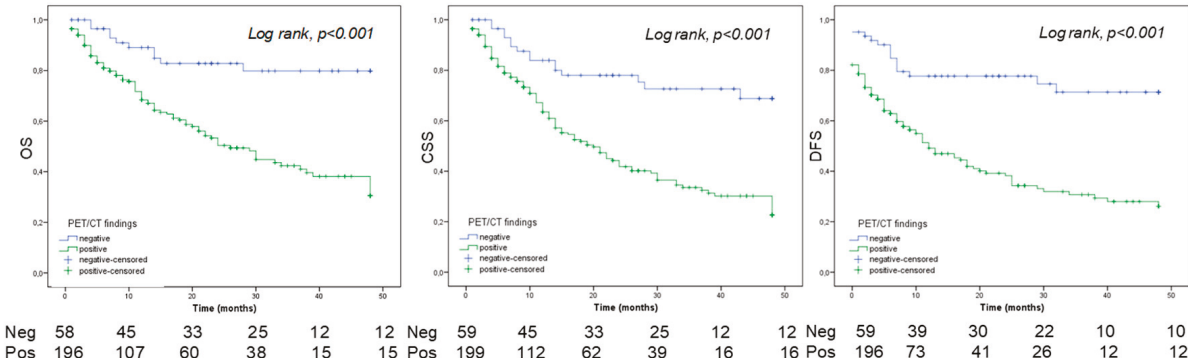

Figure 3. Overall survival (OS), cancer specific survival (CSS) and disease-free survival (DFS) for all patients $(\mathrm{n}=286)$.
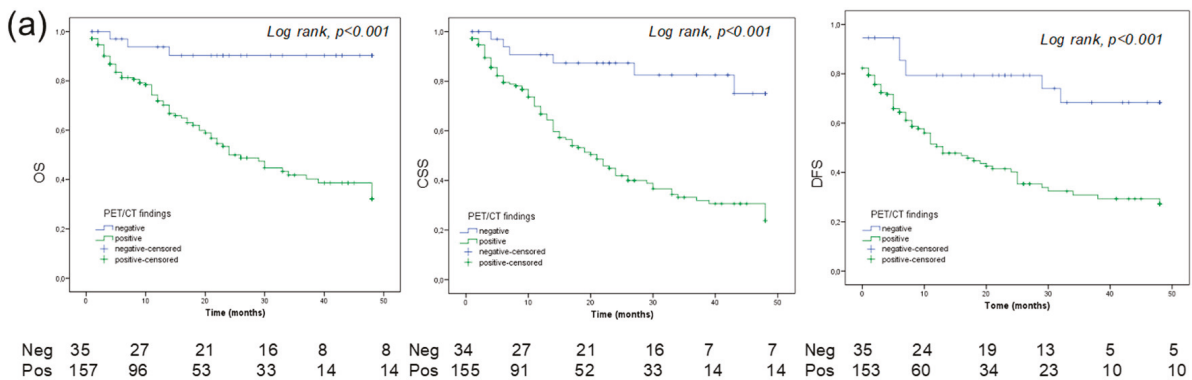

Figure 4. Cont. 
(b)

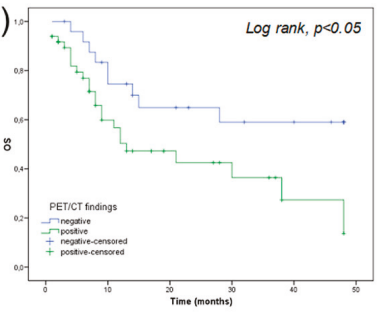

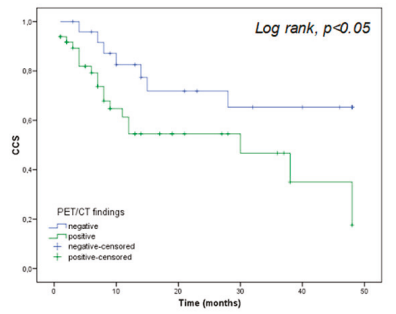

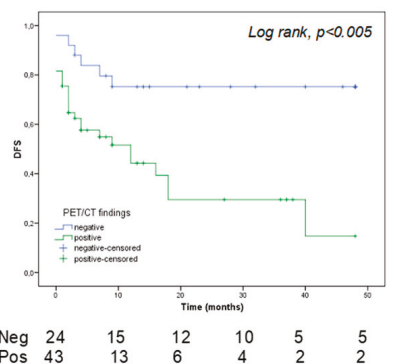

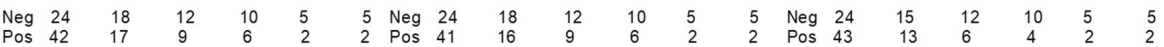

Figure 4. Overall survival (OS), cancer specific survival (CSS) and disease-free survival (DFS) for bladder cancer only $(\mathrm{n}=212)$ (a) and for all patients with UTUC/UTUC and BC $(\mathrm{n}=74)(\mathbf{b})$.

In patients with UUTC and both UUTC and BC, the 5-year OS, CSS and RFS estimates were $14 \%, 18 \%$, and $15 \%$ in positive PET/CT patients and $59 \%, 59 \%$, and $75 \%$ in negative PET/CT subjects, respectively (Log rank, $p$ value $p<0.05$; Figure $4 b$ ).

The 5-year OS, CSS, and RFS were significantly worse in patients with a positive PET/CT than the counterpart regardless of the use of adjuvant/salvage treatments in follow up (Table 3).

Table 3. 5-year survival rates in all patient population stratified according to the use of adjuvant/salvage treatments during follow up, regardless the use of adjuvant/salvage treatments the 5-year OS, CSS and RFS were significantly worse in patients with a positive PET/CT.

\begin{tabular}{llccc}
\hline & & Negative PET/CT & Positive PET/CT & $p$ Value * \\
\hline \multirow{2}{*}{ No adjuvant/salvage treatments $(\mathrm{n}=175$ pts) } & OS & 0.669 & 0.258 & $<0.001$ \\
& CSS & 0.805 & 0.318 & $<0.001$ \\
& RFS & 0.631 & 0.210 & $<0.001$ \\
\hline \multirow{2}{*}{ Adjuvant/salvage treatments $(\mathrm{n}=111 \mathrm{pts})$} & OSS & 0.743 & 0.300 & $<0.005$ \\
& CSS & 0.805 & 0.398 & $<0.01$ \\
& RFS & 0.614 & 0.224 & $<0.05$ \\
\hline
\end{tabular}

OS: overall survival; CSS: cancer specific survival; RFS: recurrence free survival, ${ }^{*}$ Log-Rank test.

Interestingly, PET/CT was able to stratify patients with a FP and FN findings at conventional imaging with survival analysis assessing OS and RFS ( $p<0.01)$ (Figures S1 and S2).

\subsection{PET/CT as a Predictive Tool}

Tables 4-6 summarize univariable and multivariable Cox regression analyses assessing predictors of OS, CSS and PFS for BC, UUTC, and UUTC plus BC.

In a first model, using only the variables set at a $p$ value $<0.05$ in the univariable analysis (Tables 4, 6 and 7), a positive FDG PET/CT, together with the presence of pT3-pT4, was an independent predictor of PFS, OS and CCS $(p<0.01)$ in patients with BC. In patients with either UUTC or concomitant BC and UUTC, a positive FDG PET/CT was a significant predictor of PFS, CCS, and OS only at univariate analysis.

In a second model, corrected for the most common clinical pathological variables (Tables 5, 8 and 9), a positive FDG PET/CT in patients with BC was an independent predictor of PFS, OS, and CCS $(p<0.01)$. In the same model for patients with UUTC and those with concomitant BC and UUTC, a positive FDG $\mathrm{PET} / \mathrm{CT}$ was an independent predictor of PFS $(p<0.01)$.

In the multivariate models, the inclusion of PET/CT increases the AUCs of the models (Figures S3-S7). 


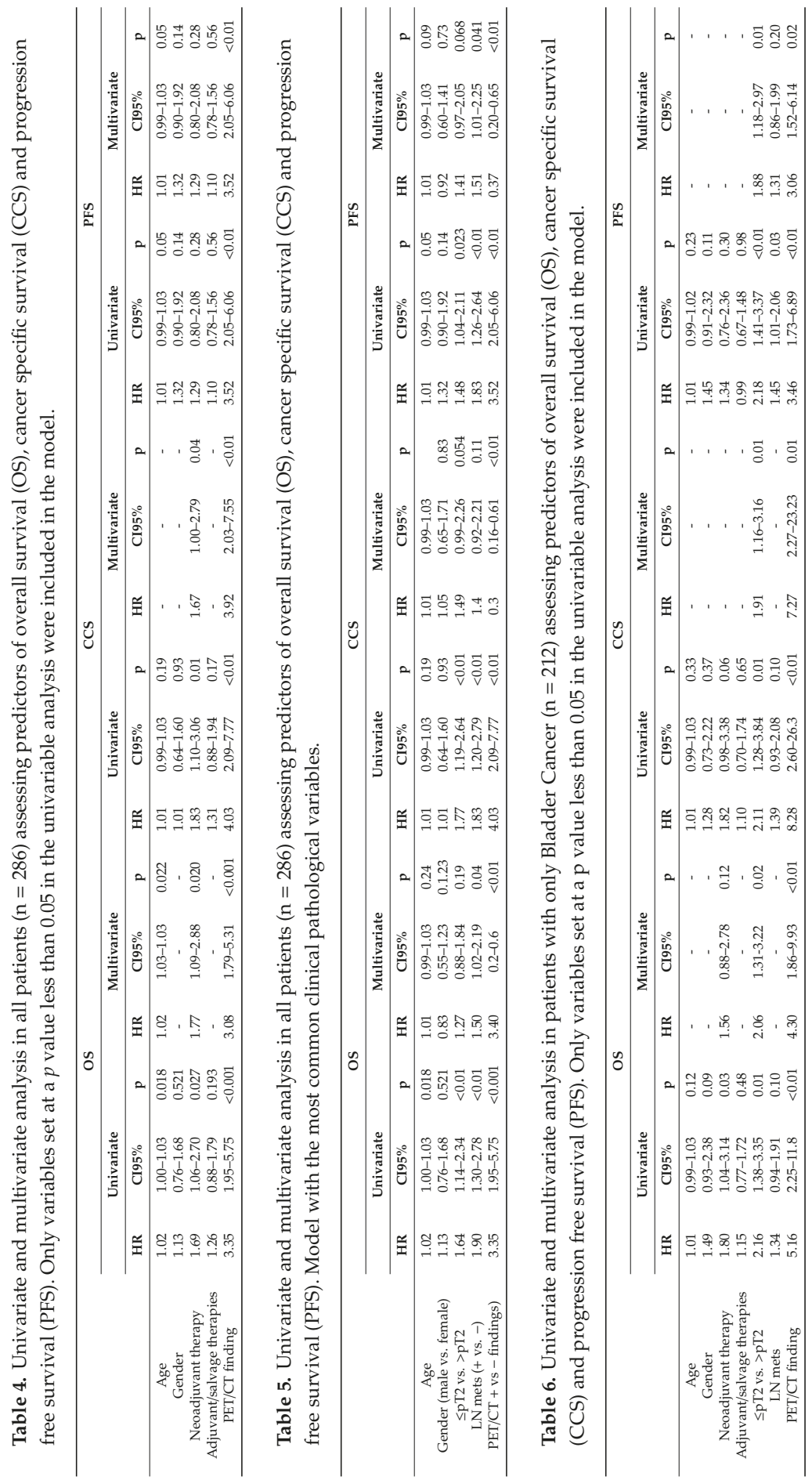




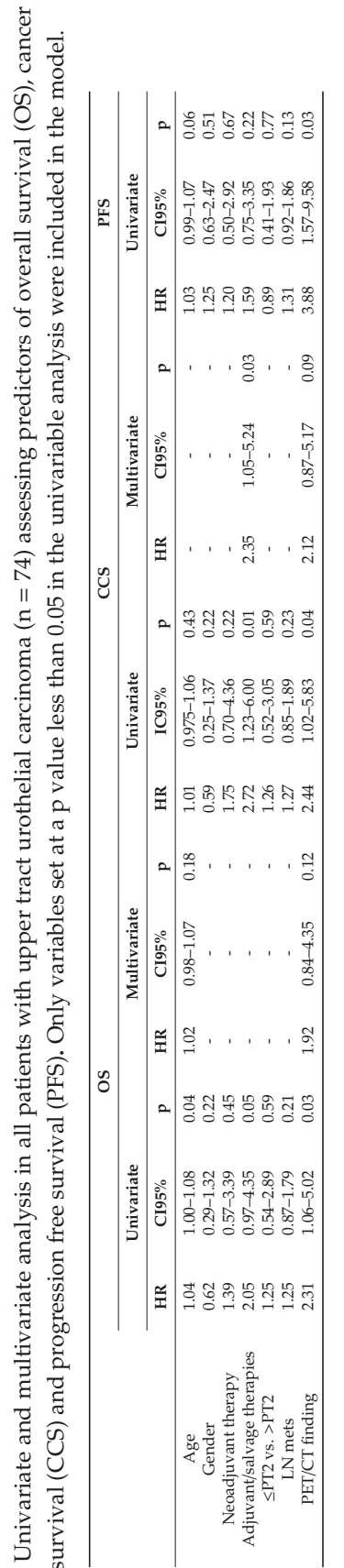

告
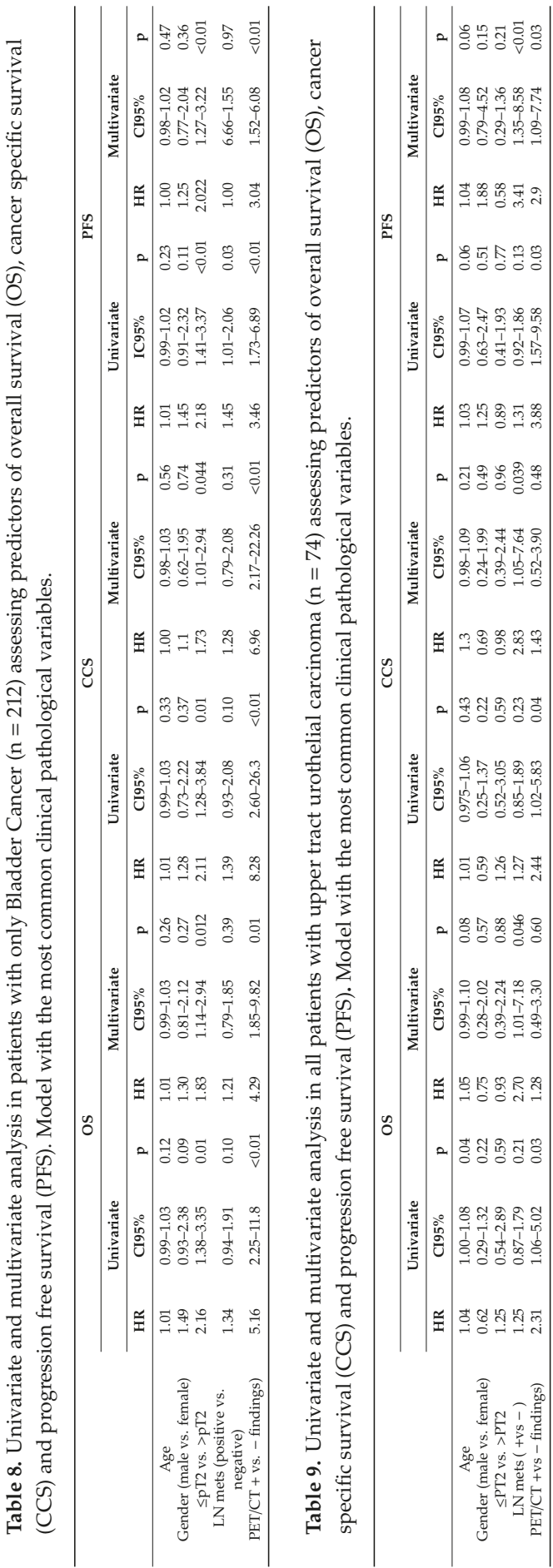


\section{Discussion}

PET/CT with FDG has been extensively used in solid cancers, but few papers are available on its use in urological cancer patients. FDG is highly expressed in aggressive tumors and its uptake is directly correlated with Glucose transporter 1 (GLUT1) expression. Some biological studies have demonstrated the relationship between glucose metabolism (i.e., GLUT 1 expression and others) and the proliferation of urothelial cancer [22]. Moreover, undifferentiated cancers, which are aggressive and associated with poor prognosis, are highly avid for glucose. These premises are essential for the employment of FDG PET/CT in this type of cancer. Our study finds two additional roles for PET/CT in patients with recurrent UC. First, PET/CT may predict the prognosis of recurrent UC patients. Second, PET/CT is able to change patient management in a high percentage of patients ( $40 \%$ ) due to the detection of lymph node and distant metastases in recurrent disease.

The clinical impact of these two conclusions are reflected in the possibility to personalize treatment for patients with a positive vs. negative PET scan. In particular, patients with a negative scan may benefit from further follow up while those with a positive scan may benefit from further treatment to improve survival (both disease-free and overall survival). Furthermore, PET/CT may also play a role after systemic treatment for the evaluation of response to therapy, particularly in patients with doubtful findings on conventional imaging or those with a mismatch between clinical and radiological findings. We believe that the inclusion of FDG PET/CT in the restaging phase of patients with urothelial cancer will have a profound impact on the management of the disease. Furthermore, the sensitivity of FDG PET/CT was higher than $80 \%$ for patients affected by bladder cancer and UUTC, as demonstrated by our previous paper involving the same patient population [15]. Moreover, the diagnostic accuracy of FDG PET/CT resulted higher than CI in both subgroups of patients (91\% vs. $81 \%$ in bladder cancer and $84 \%$ vs. $76 \%$ in UUTC, respectively for PET/CT and conventional Imaging.

To date, few studies with a limited number of enrolled patients have assessed the impact of FDG $\mathrm{PET} / \mathrm{CT}$ on the therapeutic management in BC. Alongi et al. found, in a study involving 41 patients, a change in treatment after FDG PET/CT in about $40 \%$ of cases, the same as the present study [18]. In another study involving 57 patients, among which $72 \%$ underwent PET/CT during restaging, management was changed in $68 \%$ of cases [23], supporting a substantial impact of FDG PET/CT in the suspicion of recurrence.

Furthermore, a positive PET is a predictor of OS and PFS for both BC and UUTC. From our study, it emerged that a positive PET/CT is associated with a significantly higher rate of disease progression and a significantly reduced OS. On the other hand, a negative scan predicts a more favorable outcome. Interestingly, a 5-year OS was significantly favorable in patients with BC and a negative PET/CT compared to those with a positive scan, showing a gap of $53 \%$ (90\% vs. $37 \%$, respectively; see Figure 4a). Conversely, patients with UUTC have a worse 5-year OS survival, particularly when PET/CT was positive (gap: $45 \%$ ). Moreover, in the predictive models, the inclusion of PET/CT increases the Area Under the ROC Curve (AUC), reaching a value of 0.83 in patients with UUTC. Furthermore, a positive PET/CT scan yielded the highest HR compared to other clinical pathological variables (see Tables 4 and 5). These results are interesting because their implications may provide clinicians with what is still needed in the re-stage setting of UC: a decisional tool for active treatment (i.e., adjuvant chemotherapy) vs. follow up. Indeed, the identification of asymptomatic recurrence in patients who may benefit from salvage chemotherapy could significantly influence patient survival $[24,25]$. Nowadays, clinicians mainly suggest adjuvant therapy based on the well-known pathologic characteristics of the tumor, such as pT stage, grade, pathological lymph nodes, and comorbidities (i.e., Karnofsky performance status). Nomograms on CSS following radical cystectomy have been developed; however, their use is still not recommended until further data becomes available $[26,27]$. Interestingly, all these models try to predict survival in patients with disease recurrence following radical cystectomy without the use of imaging, which could have an essential role in the early evaluation of recurrence and in the prediction of disease outcome after primary treatment. It is now recognized that biomarker panels reflecting the biological heterogeneity of UCs can identify patients who may need aggressive therapies [28,29]. However, such panels have not 
been clinically implemented due to several shortcomings, including underpowered and clinically heterogeneous cohorts, short follow-up, use of non-disease-specific endpoints, and limited clinical validation. As a consequence, the development of clinically useful biomarkers to determine optimal treatment for patients remains somewhat elusive. Despite this, targeted therapy has demonstrated an OS benefit in the treatment of metastatic UC. As a new frontier, antibodies radiolabeled with positron-emitting radionuclides may allow Immuno-PET imaging with an in vivo quantification of the efficacy of targeted therapies. Thus, antibody targeted PET/CT may play a role in the selection of patient therapy, the prediction of response to systemic medications and an in vivo assessment of the efficacy of therapies (theragnostic) [30,31].

As any retrospective study, limitations are inherent. Different surgical treatments and follow up schedules may have been provided to patients at the different institutions during the study period. In the assessment of time dependent outcomes, we included patients treated with adjuvant therapies. Although this variable was adjusted in the multivariable analysis, patient imbalance and selection bias cannot be excluded. In some analyses, patients with BC and UUTUC were considered as one group. Although tumors differed, stage and grade were concordant in almost all cases. A selection bias could not be excluded considering the selected nature of the population and the use of the imaging test only in the event of suspicion of recurrence. Finally, images were not centrally reviewed, although all the radiologists at the time had at least 5 years of experience in genito-urinary imaging and FDG PET/CT.

\section{Conclusions}

This study assesses a promising role of FDG-PET/CT as a predictor of UC outcomes. Patient management was changed after FDG PET/CT in $40 \%$ of patients with UC. This may suggest a role for FDG-PET/CT in post-surgical therapeutic decision-making, although firm recommendations cannot yet be made. In particular, whether the present results are translated into better oncological outcomes still needs to be confirmed. It is crucial to prospectively investigate the clinical value of FDG-PET/CT in the assessment of UC recurrence.

Supplementary Materials: The following are available online at http://www.mdpi.com/2072-6694/11/5/700/s1, Table S1a. Characteristics of patients with only bladder cancer (212/286); Table S1b. Characteristics of patients with only UTUC (38/286); Table S1c. Characteristics of patients with concomitant bladder cancer and UTUC (36/286); Figure S1. ROC curve assessing the accuracy of the multivariabile COX model all patients with and without PET/CT (model with only variables set at a p value less than 0.05 an univariate analysis); Figure S2. ROC curve assessing the accuracy of the multivariabile COX model all patients with and without PET/CT (model with the most common clinical-pathological variables); Figure S3. ROC curve assessing the accuracy of the multivariabile COX model for bladder cancer with and without PET/CT (model with only variables set at a p value less than 0.05 an univariate analysis); Figure S4. ROC curve assessing the accuracy of the multivariabile COX model for bladder cancer with and without PET/CT (model with the most common clinical-pathological variables); Figure S5. ROC curve assessing the accuracy of the multivariabile COX model for upper tract tumors with and without PET/CT (model with the most common clinical-pathological variables); Figure S6. PET/CT is able to stratify recurrence free survival for those patients with a FP and FN at conventional imaging; Figure S7. PET/CT is able to stratify overall survival for those patients with a FP and FN conventional imaging

Author Contributions: F.Z. and L.E.-Protocol; F.Z., L.E. and M.P.-Project development; P.C., S.P. F.F., S.F. and I.R.-Data collection or management; F.Z., L.E. and M.M.-Data analysis; F.Z., L.E., V.L., J.R.K. and R.S.-Manuscript writing/editing; A.B., A.G., E.B. and F.D.M.-Supervision.

Funding: No funding was received.

Acknowledgments: We thanks Christina Drace for the English revision.

Conflicts of Interest: The authors declare that they have no conflict of interest.

\section{References}

1. Siegel, R.L.; Miller, K.D.; Jemal, A. Cancer Statistics, 2017. CA Cancer J. Clin. 2017, 67, 7-30. [CrossRef] [PubMed]

2. Miller, K.D.; Siegel, R.L.; Lin, C.C.; Mariotto, A.B.; Kramer, J.L.; Rowland, J.H.; Stein, K.D.; Alteri, R.; Jemal, A. Cancer treatment and survivorship statistics, 2016. CA Cancer J. Clin. 2016, 66, 271-289. [CrossRef] [PubMed]

3. Munoz, J.J.; Ellison, L.M. Upper tract urothelial neoplasms: Incidence and survival during the last 2 decades. J. Urol. 2000, 164, 1523-1525. [CrossRef] 
4. Jeldres, C.; Sun, M.; Isbarn, H.; Lughezzani, G.; Budaus, L.; Alasker, A.; Shariat, S.F.; Lattouf, J.B.; Widmer, H.; Pharand, D.; et al. A population-based assessment of perioperative mortality after nephroureterectomy for upper-tract urothelial carcinoma. Urology. 2010, 75, 315-320. [CrossRef] [PubMed]

5. Abouassaly, R.; Alibhai, S.M.; Shah, N.; Timilshina, N.; Fleshner, N.; Finelli, A. Troubling outcomes from population-level analysis of surgery for upper tract urothelial carcinoma. Urology. 2010, 76, 895-901. [CrossRef]

6. Rosenberg, J.E.; Carroll, P.R.; Small, E.J. Update on chemotherapy for advanced bladder cancer. J. Urol. 2005, $174,14-20$.

7. Sternberg, C.N.; Vogelzang, N.J. Gemcitabine, paclitaxel, pemetrexed and other newer agents in urothelial and kidney cancers. Crit. Rev. Oncol. Hematol. 2003, 46, 105-115. [CrossRef]

8. Umbreit, E.C.; Crispen, P.L.; Shimko, M.S.; Farmer, S.A.; Blute, M.L.; Frank, I. Multifactorial, site-specific recurrence model after radical cystectomy for urothelial carcinoma. Cancer. 2010, 116, 3399-3407. [CrossRef] [PubMed]

9. Volkmer, B.G.; Kuefer, R.; Bartsch, G.C.; Gust, J.K.; Hautmann, R.E. Oncological followup after radical cystectomy for bladder cancer-is there any benefit? J. Urol. 2009, 181, 1587-1593. [CrossRef]

10. Donat, S.M. Staged based directed surveillance of invasive bladder cancer following radical cystectomy: Valuable and effective? World J. Urol. 2006, 24, 557-564.

11. Bochner, B.H.; Montie, J.E.; Lee, C.T. Follow-up strategies and management of recurrence in urologic oncology bladder cancer: Invasive bladder cancer. Urol. Clin. North. Am. 2003, 30, 777-789. [CrossRef]

12. Soukup, V.; Babjuk, M.; Bellmunt, J.; Dalbagni, G.; Giannarini, G.; Hakenberg, O.W.; Herr, H.; Lechevallier, E.; Ribal, M.J. Follow-up after surgical treatment of bladder cancer: A critical analysis of the literature. Eur. Urol. 2012, 62, 290-302. [CrossRef] [PubMed]

13. Cohen, S.M.; Goel, A.; Phillips, J.; Ennis, R.D.; Grossbard, M.L. The role of perioperative chemotherapy in the treatment of urothelial cancer. Oncologist. 2006, 11, 630-640. [CrossRef] [PubMed]

14. David, K.A.; Milowsky, M.I.; Ritchey, J.; Carroll, P.R.; Nanus, D.M. Low incidence of perioperative chemotherapy for stage III bladder cancer 1998 to 2003: A report from the National Cancer Data Base. J. Urol. 2007, 178, 451-454. [CrossRef] [PubMed]

15. Sternberg, C.N. Perioperative chemotherapy in muscle-invasive bladder cancer to enhance survival and/or as a strategy for bladder preservation. Semin. Oncol. 2007, 34, 122-128. [CrossRef]

16. Graziani, T.; Ceci, F.; Lopes, F.L.; Chichero, J.; Castellucci, P.; Schiavina, R.; Bianchi, L.; Chondrogiannis, S.; Colletti, P.M.; Costa, S.; et al. 11C-choline PET/CT for restaging of bladder cancer. Clin. Nucl. Med. 2015, 40, 1-5. [CrossRef]

17. Zattoni, F.; Incerti, E.; Colicchia, M.; Castellucci, P.; Panareo, S.; Picchio, M.; Fallanca, F.; Briganti, A.; Moschini, M.; Gallina, A.; et al. Comparison between the diagnostic accuracies of 18F-fluorodeoxyglucose positron emission tomography/computed tomography and conventional imaging in recurrent urothelial carcinomas: A retrospective, multicenter study. Abdom. Radiol. 2018, 1-9. [CrossRef]

18. Alongi, P.; Caobelli, F.; Gentile, R.; Stefano, A.; Russo, G.; Albano, D.; Baldari, S.; Gilardi, M.C.; Midiri, M. Recurrent bladder carcinoma: Clinical prognostic role of 18, F.-F.D.G.P.E.T./C.T. Eur. J. Nucl. Med. Mol. Imaging. 2017, 44, 224-233. [CrossRef] [PubMed]

19. Zattoni, F.; Nehra, A.; Murphy, C.R.; Rangel, L.; Mynderse, L.; Lowe, V.; Kwon, E.; Karnes, R.J. Mid-term Outcomes Following Salvage Lymph Node Dissection for Prostate Cancer Nodal Recurrence Status Post-radical Prostatectomy. Eur. Urol. Focus. 2016, 2, 522-531. [CrossRef]

20. Kitajima, K.; Yamamoto, S.; Fukushima, K.; Minamimoto, R.; Kamai, T.; Jadvar, H. Update on advances in molecular PET in urological oncology. Jpn. J. Radiol. 2016, 34, 470-485. [CrossRef]

21. Bouchelouche, K.; Choyke, P.L. PET/Computed Tomography in Renal, Bladder, and Testicular Cancer. PET Clin. 2015, 10, 361-374. [CrossRef]

22. Massari, F.; Ciccarese, C.; Santoni, M.; Iacovelli, R.; Mazzucchelli, R.; Piva, F.; Scarpellid, M.; Berardic, R.; Tortorab, G.; Lopez-Beltran, A.; et al. Metabolic phenotype of bladder cancer. Cancer Treat. Rev. 2016, 45, 46-57. [CrossRef]

23. Apolo, A.B.; Riches, J.; Schoder, H.; Akin, O.; Trout, A.; Milowsky, M.I.; Bajorin, D.F. Clinical value of fluorine-18 2-fluoro-2-deoxy-D-glucose positron emission tomography/computed tomography in bladder cancer. J. Clin. Oncol. 2010, 28, 3973-3978. [CrossRef] 
24. Boorjian, S.A.; Tollefson, M.K.; Cheville, J.C.; Costello, B.A.; Thapa, P.; Frank, I. Detection of asymptomatic recurrence during routine oncological followup after radical cystectomy is associated with improved patient survival. J. Urol. 2011, 186, 1796-1802. [CrossRef]

25. Giannarini, G.; Kessler, T.M.; Thoeny, H.C.; Nguyen, D.P.; Meissner, C.; Studer, U.E. Do patients benefit from routine follow-up to detect recurrences after radical cystectomy and ileal orthotopic bladder substitution? Eur. Urol. 2010, 58, 486-494. [CrossRef]

26. Zaak, D.; Burger, M.; Otto, W.; Bastian, P.J.; Denzinger, S.; Stief, C.G.; Buchner, H.; Hartmann, A.; Wieland, F.W.; Shariat, F.S.; et al. Predicting individual outcomes after radical cystectomy: An external validation of current nomograms. BJU Int. 2010, 106, 342-348. [CrossRef]

27. Karakiewicz, P.I.; Shariat, S.F.; Palapattu, G.S.; Gilad, A.E.; Lotan, Y.; Rogers, C.G.; Vazina, A.; Gupta, A.; Bastian, P.J.; Perrotte, P.; et al. Nomogram for predicting disease recurrence after radical cystectomy for transitional cell carcinoma of the bladder. J. Urol. 2006, 176, 1354-1361. [CrossRef]

28. Kamat, A.M.; Hegarty, P.K.; Gee, J.R.; Clark, P.E.; Svatek, R.S.; Hegarty, N.; Shariat, S.F.; Xylinas, E.; Schmitz-Dräger, B.J.; Lotan, Y.; et al. ICUD-EAU International Consultation on Bladder Cancer 2012: Screening, diagnosis, and molecular markers. Eur Urol. 2013, 63, 4-15. [CrossRef]

29. Grubmuller, B.; Roupret, M.; Briganti, A.; Shariat, S.F. The Use of Biomarkers for Bladder Cancer Diagnosis and Surveillance. Mini Rev. Med. Chem. 2016, 16, 1444-1449. [CrossRef]

30. Carmon, K.S.; Azhdarinia, A. Application of Immuno-PET in Antibody-Drug Conjugate Development. Mol. Imaging 2018, 17. [CrossRef]

31. Ramirez-Fort, M.K.; Mahase, S.S.; Osborne, J.R.; Lange, C.S. Theragnostic Target, Prostate-Specific Membrane Antigen-Also Specific for Nonprostatic Malignancies. Int. J. Radiat. Oncol. Biol. Phys. 2018, 101, 646-649. [CrossRef]

(C) 2019 by the authors. Licensee MDPI, Basel, Switzerland. This article is an open access article distributed under the terms and conditions of the Creative Commons Attribution (CC BY) license (http://creativecommons.org/licenses/by/4.0/). 


\title{
Article \\ ${ }^{18}$ F-FDG-PET Can Predict Microvessel Density in Head and Neck Squamous Cell Carcinoma
}

\author{
Alexey Surov ${ }^{1, *}$, Hans Jonas Meyer ${ }^{1}$, Anne-Kathrin Höhn ${ }^{2}$, Andreas Wienke ${ }^{3}$, Osama Sabri ${ }^{4}$ \\ and Sandra Purz 4 \\ 1 Department of Diagnostic and Interventional Radiology, University Hospital of Leipzig, Liebigstrasse 20, \\ 04103 Leipzig, Germany; hans-jonas.meyer@medizin.uni-leipzig.de \\ 2 Department of Pathology, University Hospital of Leipzig, Liebigstrasse 20, 04103 Leipzig, Germany; \\ Annekathrin.hoehn@medizin.uni-leipzig.de \\ 3 Institute of Medical Epidemiology, Biostatistics, and Informatics, Martin-Luther-University \\ Halle-Wittenberg, Magdeburger Str. 8, 06097 Halle, Germany; andreas.wienke@uk-halle.de \\ 4 Department of Nuclear Medicine, University Hospital of Leipzig, Liebigstrasse 18, 04103 Leipzig, Germany; \\ Osama.Sabri@medizin.uni-leipzig.de (O.S.); sandra.purz@medizin.uni-leipzig.de (S.P.) \\ * Correspondence: Alexey.Surov@medizin.uni-leipzig.de
}

Received: 11 March 2019; Accepted: 11 April 2019; Published: 15 April 2019

\begin{abstract}
Aim: Positron emission tomography (PET) with ${ }^{18}$ F-fluordeoxyglucose $\left({ }^{18} \mathrm{~F}-\mathrm{FDG}\right)$ plays an essential role in the staging and tumor monitoring of head and neck squamous cell carcinoma (HNSCC). Microvessel density (MVD) is one of the clinically important histopathological features in HNSCC. The purpose of this study was to analyze possible associations between ${ }^{18}$ F-FDG-PET findings and MVD parameters in HNSCC. Materials and Methods: Overall, 22 patients with a mean age of $55.2 \pm 11.0$ and with different HNSCC were acquired. In all cases, whole-body ${ }^{18}$ F-FDG-PET was performed. For each tumor, the maximum and mean standardized uptake values $\left(\mathrm{SUV}_{\max }\right.$; $\mathrm{SUV}_{\text {mean }}$ ) were determined. The MVD, including stained vessel area and total number of vessels, was estimated on CD105 stained specimens. All specimens were digitalized and analyzed by using ImageJ software $1.48 \mathrm{v}$. Spearman's correlation coefficient $(r)$ was used to analyze associations between investigated parameters. $p$-values of $<0.05$ were taken to indicate statistical significance. Results: $\mathrm{SUV}_{\max }$ correlated with vessel area $(r=0.532, p=0.011)$ and vessel count $(r=0.434, p=0.043)$. Receiver operating characteristic analysis identified a threshold SUV $\max$ of 15 to predict tumors with high MVD with a sensitivity of $72.7 \%$ and specificity of $81.8 \%$, with an area under the curve of $82.6 \%$. Conclusion: ${ }^{8}$ F-FDG-PET parameters correlate statistically significantly with MVD in HNSCC. SUV $\max$ may be used for discrimination of tumors with high tumor-related MVD.
\end{abstract}

Keywords: positron emission tomography; head and neck neoplasms; neovascularization; pathologic

\section{Introduction}

Radiological imaging, especially positron emission tomography (PET) with ${ }^{18} \mathrm{~F}$-fluorodeoxyglucose $\left({ }^{18} \mathrm{~F}\right.$-FDG), plays an essential role in characterization head and neck squamous cell carcinoma (HNSCC). ${ }^{18} \mathrm{~F}$-FDG-PET is increasingly used for the staging and treatment monitoring of HNSCC [1-3]. As reported previously, metabolic tumor activity measured via PET parameters such as maximum or mean standardized uptake values $\left(\mathrm{SUV}_{\max }\right.$ or $\left.\mathrm{SUV}_{\text {mean }}\right)$ correlates well with tumor stage and grade [2,3]. Advanced T stage tumors show higher PET parameters like $\mathrm{SUV}_{\text {max }}$ and $\mathrm{SUV}_{\text {mean }}$ in comparison to T1/T2 tumors [2,3]. Similarly, poorly differentiated (G3) tumors have higher $\mathrm{SUV}_{\max }$ than do low-grade (G1 or G2) lesions [3]. Furthermore, the metabolic tumor burden is associated with the clinical outcome of HNSCC: patients with high metabolic tumor burden have been associated with higher distant metastasis rates, translating into worse survival $[4,5]$. 
In addition, ${ }^{18} \mathrm{~F}$-FDG-PET can also predict treatment success in HNSCC. It has been shown that SUV values can be used as biomarkers in predicting the therapy response in HNSCC [6,7]. Kitagawa et al. reported that the SUV of pre-treatment ${ }^{18} \mathrm{~F}$-FDG-PET is useful in predicting the response to treatment, and post-treatment ${ }^{18} \mathrm{~F}$-FDG-PET is valuable in predicting residual viable tumors. It was also mentioned that a lower SUV $(<4)$ of post-treatment ${ }^{18}$ F-FDG-PET was significantly correlated with good histological results after therapy [6].

Some reports indicated that ${ }^{18} \mathrm{~F}-\mathrm{FDG}$-PET can reflect several clinically relevant histopathological features in HNSCC. So far, Jacob et al. found that $\mathrm{SUV}_{\max }$ correlated statistically significantly with the proliferation index KI $67(r=0.78)$ and proliferating cell nuclear antigen $(r=0.66)$ [8]. Grönroos et al. showed that $\mathrm{SUV}_{\max }$ tended to correlate with the expression of tumor suppressor protein p53 ( $p=0.47$, $p=0.078$ [ [9]. Furthermore, $\mathrm{SUV}_{\max }$ also correlated well with the expression of hypoxia-inducible factor HIF-1 $\alpha$ [10]. It has also been shown that p16-positive tumors had lower $\mathrm{SUV}_{\max }$ in comparison to p16-negative carcinomas [11,12].

According to the literature, microvessel density (MVD) also plays a significant role in HNSCC [13]. For example, MVD estimated from CD105 immunoexpression predicts a poor outcome in oral squamous cell carcinoma [13,14]. Like VEGF (vascular endothelial growth factor), CD105 (endoglin) is a hypoxia-inducible transmembrane glycoprotein, and its expression is up-regulated in actively proliferating endothelial cells. Endoglin has been described as a marker for tumor-related angiogenesis and neovascularization with potential in tumor diagnosis, prognosis, and therapy [15]. Xia et al. found that MVD can predict lymph node metastases and prognosis in HNSCC [16]. We assume that the parameters of ${ }^{18} \mathrm{~F}-\mathrm{FDG}-\mathrm{PET}$ might also reflect MVD in HNSCC. However, no previous study has investigated the relationships between ${ }^{18} \mathrm{~F}-\mathrm{FDG}$-PET and tumor MVD in HNSCC.

Therefore, the purpose of the present study was to analyze possible associations between ${ }^{18} \mathrm{~F}$-FDG-PET parameters and MVD in HNSCC.

\section{Methods}

This prospective study was approved by the institutional review board (Ethics Committee of the University of Leipzig, study codes 180-2007, 201-10-12072010, and 341-15-05102015). All methods were performed in accordance with the relevant guidelines and regulations. All patients gave their written informed consent.

\subsection{Patients}

For this study, patients with histologically proven HNSCC and available histopathological specimens and who underwent ${ }^{18} \mathrm{~F}$-FDG-PET/CT examinations at our institution were selected. Overall, there were 22 patients, $6(26.1 \%)$ women and $16(73.9 \%)$ men, with a mean age of $55.2 \pm 11.0$ years, age range of 24-77 years, and different HNSCC. Low-grade (G1/2) tumors were diagnosed in 10 cases (45.5\%) and high-grade (G3) tumors in the remaining 12 (54.5\%) patients.

\subsection{Imaging}

\section{${ }^{18}$ F-FDG-PET/CT}

In all 22 patients, an ${ }^{18}$ F-FDG-PET/CT (Siemens Biograph 16, Siemens Medical Solutions, Erlangen, Germany) was performed from the skull to the upper thigh after a fasting period of at least $6 \mathrm{~h}$. Application of ${ }^{18} \mathrm{~F}$-FDG was performed intravenously with a body-weight-adapted dose $(4 \mathrm{MBq} / \mathrm{kg}$, range: $168-427 \mathrm{MBq}$, mean $\pm \mathrm{SD}: 281 \pm 62.2 \mathrm{MBq}$ ). PET/CT image acquisition started on average 76 min (range 60-90 min) after ${ }^{18} \mathrm{~F}-\mathrm{FDG}$ application. Low-dose CT was used for attenuation correction of the PET data.

The acquired PET/CT datasets were evaluated by a board-certified nuclear medicine practitioner and a board-certified radiologist with substantial PET/CT experience in oncological image interpretation. PET/CT image analysis was performed on a dedicated workstation at Hermes Medical Solutions, 
Sweden. For each tumor, the maximum and mean SUV ( $\left.\mathrm{SUV}_{\text {max }} ; \mathrm{SUV}_{\text {mean }}\right)$ were determined from PET images (Figure 1). Prior to this, the tumor margins of the HNSCC were identified on CT images and fused PET/CT images, and a polygonal volume of interest (VOI) that include the entire lesion in the axial, sagittal, and coronal planes was placed in the PET dataset (SUV $\max$ threshold $40 \%$ ).

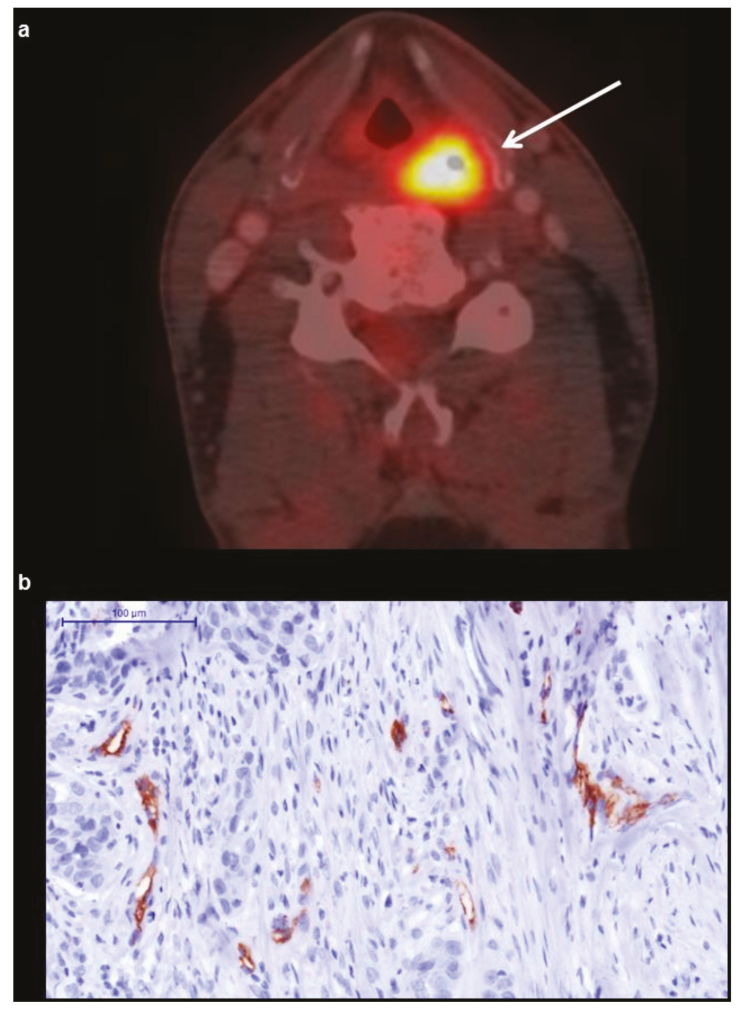

Figure 1. (a) ${ }^{18} \mathrm{~F}$-fluordeoxyglucose $\left({ }^{18} \mathrm{~F}\right.$-FDG)-PET/CT shows a metabolically active hypopharyngeal lesion. The acquired ${ }^{18}$ F-FDG-PET parameters of the lesion are as follows: maximum and mean standardized uptake values $\mathrm{SUV}_{\max }=22.07$ and $\mathrm{SUV}_{\text {mean }}=13.92$. magnification: 200x. (b) Histopathological findings (CD 105 stained specimen). Vessel area is $1.2 \%$, vessel count is 11 .

\subsection{Microvessel Density}

In all cases, the diagnosis was confirmed histopathologically by tumor biopsy before any form of treatment. For the present study, the biopsy specimens were deparaffinized, rehydrated, and cut into $5 \mu \mathrm{m}$ slices. The specimens were stained with CD 105 antigen (Abcamplc, 330 Cambridge Science Park, Cambridge, CB4 OFL, UK). Furthermore, all stained specimens were digitalized by using a Pannoramic microscope scanner (Pannoramic SCAN, 3DHISTECH Ltd., Budapest, Hungary) with Carl Zeiss objectives up to $41 \times$ bright field magnification by default. In the used bottom-up approach, the whole sample was acquired at high resolution. The digital slides (magnification of 200×) were evaluated using Pannoramic Viewer 1.15.4 (open source software, 3D HISTECH Ltd., Budapest, Hungary).

Thereafter, the digitalized histopathological images were analyzed using ImageJ software $1.48 \mathrm{v}$ (National Institutes of Health Image program) with a Windows system [17,18]. The microvessel density included the following parameters: stained vessel area (vessel area, \% per high-power field), calculated as the CD105 positive area divided by the total area of the analyzed histological specimens, and the total number of vessels (vessel count) according to Weidner et al. [19]. 


\subsection{Statistical Analysis}

Statistical analysis was performed using the SPSS package (IBM SPSS Statistics for Windows, version 22.0, Armonk, NY, USA: IBM corporation). The collected data were evaluated by means of descriptive statistics. The statistical data included means and medians with corresponding standard deviations and ranges of the acquired ${ }^{18}$ F-FDG-PET and histopathological parameters.

Spearman's correlation coefficient $(r)$ was used to analyze associations between the investigated variables. $p$-values of $<0.05$ were taken to indicate statistical significance. Furthermore, the sensitivity, specificity, negative and positive predictive values, accuracy, and area under the receiver operating characteristic curve (AUC) value were calculated for the diagnostic procedures. Thresholds were chosen to maximize the Youden index.

\section{Results}

Information regarding tumor localization, stage, and grade of the enrolled patients is given in Table 1. Table 2 shows a complete overview of the acquired ${ }^{18}$ F-FDG-PET and histopathological parameters including mean values, standard deviations, and ranges.

Table 1. Clinical characteristics of the patients enrolled in the study.

\begin{tabular}{cccccccc}
\hline No. & Sex & Age & Tumor Site & T Stage & N Stage & M Stage & Grading \\
\hline 1 & female & 33 & Oral cavity & 3 & 0 & 0 & 2 \\
2 & male & 62 & Larynx & 3 & 3 & 0 & 3 \\
3 & male & 55 & Oropharynx & 3 & 2 & 0 & 3 \\
4 & male & 56 & Hypopharynx & 3 & 1 & 0 & 3 \\
5 & female & 58 & Oropharynx & 1 & 2 & 0 & 3 \\
6 & male & 24 & Oral cavity & 4 & 2 & 0 & 2 \\
7 & male & 64 & Oral cavity & 2 & 1 & 0 & 3 \\
8 & male & 57 & Oropharynx & 2 & 2 & 0 & 3 \\
9 & male & 44 & Larynx & 4 & 0 & 0 & 3 \\
10 & female & 77 & Epipharynx & 4 & 1 & 1 & 3 \\
11 & male & 59 & Oropharynx & 3 & 1 & 0 & 2 \\
12 & male & 53 & Larynx & 4 & 2 & 0 & 3 \\
13 & male & 64 & Hypopharynx & 4 & 2 & 0 & 2 \\
14 & male & 61 & Oropharynx & 4 & 2 & 0 & 2 \\
15 & male & 58 & Oropharynx & 2 & 2 & 0 & 2 \\
16 & female & 60 & Oropharynx & 4 & 2 & 0 & 4 \\
17 & male & 55 & Oropharynx & 3 & 2 & 0 & 2 \\
18 & male & 54 & Oral cavity & 4 & 2 & 0 & 2 \\
19 & female & 65 & Oropharynx & 2 & 2 & 0 & 3 \\
20 & male & 50 & Oropharynx & 2 & 2 & 0 & 3 \\
21 & male & 48 & Hypopharynx & 2 & 2 & 0 & 2 \\
22 & female & 58 & Oral cavity & 4 & 2 & 0 & 1 \\
\hline
\end{tabular}

Table 2. Estimated ${ }^{18}$ F-FDG-PET and microvessel density (MVD) parameters of head and neck squamous cell carcinoma (HNSCC).

\begin{tabular}{cccc}
\hline Parameters & $\mathbf{M} \pm \mathbf{S D}$ & Median & Range \\
\hline $\mathrm{SUV}_{\max }$ & $14.34 \pm 5.05$ & 14.79 & $5.9-24.1$ \\
$\mathrm{SUV}_{\operatorname{mea}}$ & $8.40 \pm 3.11$ & 8.28 & $3.63-14.87$ \\
Vessel Area & $1.97 \pm 1.15$ & 1.76 & $0.4-4.56$ \\
Vessel Count & $11.64 \pm 4.97$ & 10 & $5-25$ \\
\hline
\end{tabular}

$\mathrm{SUV}_{\max }$ correlated statistically significantly with vessel area $(r=0.532, p=0.011)$ and vessel count

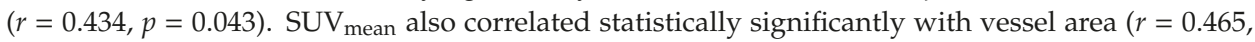
$p=0.029$ ). In the next step, receiver operating characteristic (ROC) analysis was performed to predict tumors with high microvessel density (vessel area $>1.76 \%$ as a result of median split) using SUV max. 
The Youden index identified a threshold $S U V_{\max }$ of 15 with a sensitivity of $72.7 \%$ and specificity of $81.8 \%$ (Figure 2). The positive predictive value was $80 \%$, the negative predictive value was $75 \%$, and the accuracy was $77.3 \%$. The area under the curve was $82.6 \%$.

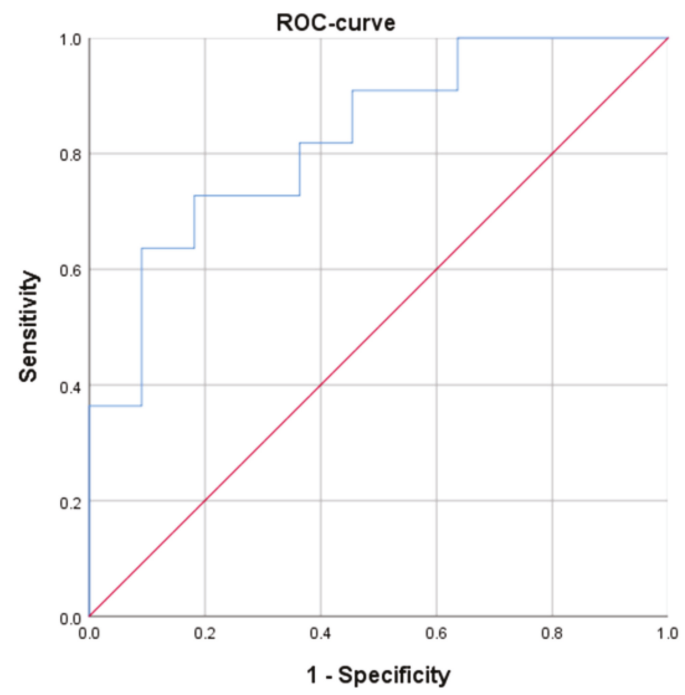

Figure 2. The receiver operating characteristic (ROC) curve using $S U V_{\max }$ for distinguishing tumors with high microvessel density from lesions with low microvascularization. The optimal threshold $\mathrm{SUV}_{\max }$ is 15 , resulting in a sensitivity of $70.0 \%$ and a specificity of $75.0 \%$. The area under the curve is $82.6 \%$.

\section{Discussion}

The present study identified significant associations between parameters of ${ }^{18} \mathrm{~F}$-FDG-PET and MVD in HNSCC. According to the literature, MVD is a very important histopathological feature in different malignancies. There are different immunohistochemical markers for the estimation of MVD. Some authors used pan-endothelial markers, namely, CD34, CD31, and von Willebrand factor [19-21]. However, it is well known that these markers have low sensitivity and specificity and are not always expressed in all intratumoral vessels [21]. In contrast to the non-specific pan-endothelial markers, CD105 or endoglin is upregulated in angiogenic vessels and accumulates preferentially in tumors $[22,23]$. Therefore, CD105 is a marker of tumor-related MVD.

Previously, numerous studies showed that CD105 correlated with tumor aggressiveness in lung cancer [24,25], breast carcinomas [26], colonic cancer [27], and endometrial carcinoma [28]. MVD is also one of the clinically relevant features in HNSCC [29-32]. According to the literature, tumor size/T stage correlated directly with the MVD/CD 105 ratio in oral cancers [31]. Furthermore, some reports indicated that high MVD/CD105 values were associated with the presence of lymph node metastasis in HNSCC $[29,32,33]$. Finally, high MVD/expression of CD105 was also associated with recurrence of disease or occurrence of distant metastasis and poorer 5-year survival [33-37]. Therefore, MVD can be applied as an important independent prognostic factor in HNSCC.

The possibility to predict MVD from imaging is very important. Previously, relationships between ${ }^{18}$ F-FDG-PET findings and MVD were analyzed in several malignancies. Remarkably, different correlation coefficients were observed between the investigated parameters. Furthermore, different markers for MVD were used. Han et al. used CD34 marker and did not observe statistically significant correlations between $\mathrm{SUV}_{\max }$ and MVD in lung cancer [38]. However, Xing et al. also estimated MVD from CD 34 stained specimens and reported a very strong correlation between tumor MVD 
and SUV in lung cancer $(r=0.915, p<0.01)$ [39]. In esophageal cancer, no significant correlations were detected between PET parameters and MVD measured from CD31 stained specimens [40]. Only few studies have analyzed the associations between PET and tumor-related MVD based on CD 105 expression. In the study by Groves et al., $\mathrm{SUV}_{\max }$ correlated statistically significantly with MVD $(r=0.6, p=0.005)$ in breast cancer [41]. Cochet et al. also investigated patients with breast cancer but could not identify statistically significant correlations between $\mathrm{SUV}_{\max }$ and the expression of CD105 or CD34 [42]. However, interestingly, in the same study, $\mathrm{SUV}_{\max }$ was associated with expression of CD105 in a non-triple-negative tumor subgroup $(r=0.5, p=0.005)$ [42]. Finally, in colorectal cancer, no significant correlations between metabolic parameters $\left(\mathrm{SUV}_{\max }\right.$ or $\left.\mathrm{SUV}_{\text {mean }}\right)$ and $\mathrm{CD} 105$ expression were found [43].

In HNSCC, there have been no previous studies about associations between ${ }^{18} \mathrm{~F}-\mathrm{FDG}-\mathrm{PET}$ and tumor-related MVD. The present study showed that $\mathrm{SUV}_{\max }$ may predict MVD in HNSCC. Moreover, $\mathrm{SUV}_{\max }$ may be used for discrimination of tumors with higher expression of CD 105, i.e., high-risk lesions. This finding is very important. It may help us to identify patients suitable for targeted therapy with anti-angiogenetic antibodies (e.g., anti-endoglin) in an oncological therapeutic setting. However, in agreement with the results by Groves et al. [41], the identified correlations were moderate. Also, the calculated negative and positive predictive values and the area under the curve and accuracy were relatively low. These facts limit the use of the present data to make a clinical decision.

Our study also identified another interesting aspect. The calculated correlation coefficients between $\mathrm{SUV}_{\max }$ and MVD are comparable with those for specific perfusion parameters. In fact, as reported previously, one of the parameters of dynamic contrast-enhanced MRI, namely Kep, correlated with vessel area $(r=0.51, p=0.041)$ in HNSCC [44]. Blood volume, a parameter of CT perfusion, correlated with vessel count $(r=0.59, p=0.035)$ [45]. Furthermore, in the present study, we analyzed important tumor-related MVD estimated from CD 105 expression. Previous reports, however, investigated MVD using the expression of CD 31 or CD 34 [29,30]. According to the literature, these markers are not tumor-specific and do not play a clinical role in HNSCC [14,27]. The present study is limited due to a small number of patients. Clearly, further investigations with more cases are needed to verify our results.

\section{Conclusions}

${ }^{18}$ F-FDG-PET parameters correlated statistically significantly with MVD in HNSCC. SUV $\max$ may be used for discrimination of tumors with high tumor-related MVD.

Author Contributions: Conceptualization, A.S. and S.P.; methodology, S.P.; software, S.P., H.J.M. and A.-K.H.; validation, A.S., S.P. and O.S.; formal analysis, A.S.; investigation, S.P. and A.-K.H.; resources, H.J.M.; data curation, A.S.; writing — original draft preparation, A.S.; statistical analysis, A.W., writing-review and editing, S.P., O.S., A.W., H.J.M. and A.-K.H.; visualization, A.-K.H., H.J.M. and A.W.; supervision, S.P.; project administration, A.S.

Funding: This research received no external funding.

Conflicts of Interest: The authors declare no conflict of interest.

\section{Abbreviations}

$\begin{array}{ll}{ }^{18} \text { F-FDG } & { }^{18} \text { F-fluorodeoxyglucose } \\ \text { HNSCC } & \text { head and neck squamous cell carcinoma } \\ \text { MVD } & \text { micro vessel density } \\ \text { PET } & \text { positron emission tomography } \\ \text { ROC } & \text { receiver operating characteristic } \\ \text { SUV } & \text { standardized uptake values }\end{array}$




\section{References}

1. Varoquaux, A.; Rager, O.; Poncet, A.; Delattre, B.M.; Ratib, O.; Becker, C.D.; Dulguerov, P.; Dulguerov, N.; Zaidi, H.; Becker, M. Detection and quantification of focal uptake in head and neck tumours: (18)F-FDG PET/MR versus PET/CT. Eur. J. Nucl. Med. Mol. Imaging 2014, 41, 462-475. [CrossRef]

2. Haerle, S.K.; Huber, G.F.; Hany, T.F.; Ahmad, N.; Schmid, D.T. Is there a correlation between 18F-FDG-PET standardized uptake value, T-classification, histological grading and the anatomic subsites in newly diagnosed squamous cell carcinoma of the head and neck? Eur. Arch. Otorhinolaryngol. 2010, 267, 1635-1640. [CrossRef] [PubMed]

3. Li, S.J.; Guo, W.; Ren, G.X.; Huang, G.; Chen, T.; Song, S.L. Expression of Glut-1 in primary and recurrent head and neck squamous cell carcinomas, and compared with 2-[18F]fluoro-2-deoxy-D-glucose accumulation in positron emission tomography. Br. J. Oral Maxillofac. Surg. 2008, 46, 180-186. [CrossRef]

4. Kim, S.Y.; Roh, J.L.; Kim, J.S.; Ryu, C.H.; Lee, J.H.; Cho, K.J.; Choi, S.H.; Nam, S.Y. Utility of FDG PET in patients with squamous cell carcinomas of the oral cavity. Eur. J. Surg. Oncol. 2008, 34, 208-215. [CrossRef] [PubMed]

5. Abgral, R.; Keromnes, N.; Robin, P.; Le Roux, P.Y.; Bourhis, D.; Palard, X.; Rousset, J.; Valette, G.; Marianowski, R.; Salaün, P.Y. Prognostic value of volumetric parameters measured by 18F-FDG PET/CT in patients with head and neck squamous cell carcinoma. Eur. J. Nucl. Med. Mol. Imaging 2014, 41, 659-667. [CrossRef] [PubMed]

6. Kitagawa, Y.; Sano, K.; Nishizawa, S.; Nakamura, M.; Ogasawara, T.; Sadato, N.; Yonekura, Y. FDG-PET for prediction of tumour aggressiveness and response to intra-arterial chemotherapy and radiotherapy in head and neck cancer. Eur. J. Nucl. Med. Mol. Imaging 2003, 30, 63-71. [PubMed]

7. Wong, K.H.; Panek, R.; Welsh, L.; Mcquaid, D.; Dunlop, A.; Riddell, A.; Murray, I.; Du, Y.; Chua, S.; Koh, D.M.; et al. The Predictive Value of Early Assessment After 1 Cycle of Induction Chemotherapy with 18F-FDG PET/CT and Diffusion-Weighted MRI for Response to Radical Chemoradiotherapy in Head and Neck Squamous Cell Carcinoma. J. Nucl. Med. 2016, 57, 1843-1850. [CrossRef] [PubMed]

8. Jacob, R.; Welkoborsky, H.J.; Mann, W.J.; Jauch, M.; Amedee, R. [Fluorine-18] Fluorodeoxyglucose Positron Emission Tomography, DNA Ploidy and Growth Fraction in Squamous-Cell Carcinomas of the Head and Neck. ORL J. Otorhinolaryngol. Relat. Spec. 2001, 63, 307-313. [CrossRef]

9. Grönroos, T.J.; Lehtiö, K.; Söderström, K.O.; Kronqvist, P.; Laine, J.; Eskola, O.; Viljanen, T.; Grénman, R.; Solin, O.; Minn, H. Hypoxia, blood flow and metabolism in squamous-cell carcinoma of the head and neck: Correlations between multiple immunohistochemical parameters and PET. BMC Cancer 2014, 14, 876. [CrossRef]

10. Zhao, K.; Yang, S.Y.; Zhou, S.H.; Dong, M.J.; Bao, Y.Y.; Yao, H.T. Fluorodeoxyglucose uptake in laryngeal carcinoma is associated with the expression of glucose transporter 1 and hypoxia inducible factor $1 \alpha$ and the phosphoinositide 3 kinase/protein kinase B pathway. Oncol. Lett. 2014, 7, 984-990. [CrossRef]

11. Surov, A.; Meyer, H.J.; Höhn, A.K.; Winter, K.; Sabri, O.; Purz, S. Associations Between [18F]FDG-PET and Complex Histopathological Parameters Including Tumor Cell Count and Expression of KI 67, EGFR, VEGF, HIF-1 $\alpha$, and p53 in Head and Neck Squamous Cell Carcinoma. Mol. Imaging Biol. 2019, 21, 368-374. [CrossRef]

12. Rasmussen, G.B.; Vogelius, I.R.; Rasmussen, J.H.; Schumaker, L.; Ioffe, O.; Cullen, K.; Fischer, B.M.; Therkildsen, M.H.; Specht, L.; Bentzen, S.M. Immunohistochemical biomarkers and FDG uptake on PET/CT in head and neck squamous cell carcinoma. Acta Oncol. 2015, 54, 1408-1415. [CrossRef]

13. Szafarowski, T.; Sierdzinski, J.; Szczepanski, M.J.; Whiteside, T.L.; Ludwig, N.; Krzeski, A. Microvessel density in head and neck squamous cell carcinoma. Eur. Arch. Otorhinolaryngol. 2018, 275, 1845-1851. [CrossRef]

14. Patil, B.R.; Bhat, K.; Somannavar, P.; Hosmani, J.; Kotrashetti, V.; Nayak, R. Comparison of immunohistochemical expression of vascular endothelial growth factor and CD105 in oral squamous cell carcinoma: Its correlation with prognosis. J. Cancer Res. Ther. 2018, 14, 421-427.

15. Nassiri, F.; Cusimano, M.D.; Scheithauer, B.W.; Rotondo, F.; Fazio, A.; Yousef, G.M.; Syro, L.V.; Kovacs, K.; Lloyd, R.V. Endoglin (CD105): A review of its role in angiogenesis and tumor diagnosis, progression and therapy. Anticancer Res. 2011, 31, 2283-2290. 
16. Xia, X.; Du, R.; Zhao, L.; Sun, W.; Wang, X. Expression of AEG-1 and microvessel density correlates with metastasis and prognosis of oral squamous cell carcinoma. Hum. Pathol. 2014, 45, 858-865. [CrossRef]

17. Surov, A.; Stumpp, P.; Meyer, H.J.; Gawlitza, M.; Höhn, A.K.; Boehm, A.; Sabri, O.; Kahn, T.; Purz, S. Simultaneous 18F-FDG-PET/MRI: Associations between diffusion, glucose metabolism and histopathological parameters in patients with head and neck squamous cell carcinoma. Oral Oncol. 2016, 58, 14-20. [CrossRef]

18. Surov, A.; Meyer, H.J.; Winter, K.; Richter, C.; Hoehn, A.K. Histogram analysis parameters of apparent diffusion coefficient reflect tumor cellularity and proliferation activity in head and neck squamous cell carcinoma. Oncotarget 2018, 9, 23599-23607. [CrossRef]

19. Weidner, N.; Semple, J.P.; Welch, W.R.; Folkman, J. Tumor angiogenesis and metastasis—Correlation in invasive breast carcinoma. N. Engl. J. Med. 1991, 324, 1-8. [CrossRef]

20. Saad, R.S.; Liu, Y.L.; Nathan, G.; Celebrezze, J.; Medich, D.; Silverman, J.F. Endoglin (CD105) and vascular endothelial growth factor as prognostic markers in colorectal cancer. Mod. Pathol. 2004, 17, 197-203. [CrossRef]

21. Wang, J.M.; Kumar, S.; Pye, D.; Haboubi, N.; al-Nakib, L. Breast carcinoma: Comparative study of tumor vasculature using two endothelial cell markers. J. Natl. Cancer Inst. 1994, 86, 386-388. [CrossRef]

22. Duff, S.E.; Li, C.; Garland, J.M.; Kumar, S. CD105 is important for angiogenesis: Evidence and potential applications. FASEB J. 2003, 17, 984-992. [CrossRef]

23. Fonsatti, E.; Altomonte, M.; Nicotra, M.R.; Natali, P.G.; Maio, M. Endoglin (CD105): A powerful therapeutic target on tumor-associated angiogenetic blood vessels. Oncogene 2003, 22, 6557-6563. [CrossRef]

24. Brattstrom, D.; Bergqvist, M.; Wester, K.; Hesselius, P.; Ren, Z.P.; Scheibenpflug, L.; Wagenius, G.; Brodin, O. Endothelial markers and circulating angiogenic factors and p53 may be potential markers for recurrence in surgically resected nonsmall cell lung cancer patients. Med. Sci. Monit. 2004, 10, 331-338.

25. Mineo, T.C.; Ambrogi, V.; Baldi, A.; Rabitti, C.; Bollero, P.; Vincenzi, B.; Tonini, G. Prognostic impact of VEGF, CD31, CD34, and CD105 expression and tumour vessel invasion after radical surgery for IB-IIA non-small cell lung cancer. J. Clin. Pathol. 2004, 57, 591-597. [CrossRef]

26. Kumar, S.; Ghellal, A.; Li, C.; Byrne, G.; Haboubi, N.; Wang, J.M.; Bundred, N. Breast carcinoma: Vascular density determined using CD105 antibody correlates with tumor prognosis. Cancer Res. 1999, 59, 856-861.

27. Li, C.; Gardy, R.; Seon, B.K.; Duff, S.E.; Abdalla, S.; Renehan, A.; O’Dwyer, S.T.; Haboubi, N.; Kumar, S. Both high intratumoral microvessel density determined using CD105 antibody and elevated plasma levels of CD105 in colorectal cancer patients correlate with poor prognosis. Br. J. Cancer 2003, 88, 1424-1431. [CrossRef]

28. Salvesen, H.B.; Gulluoglu, M.G.; Stefansson, I.; Akslen, L.A. Significance of CD 105 expression for tumour angiogenesis and prognosis in endometrial carcinomas. APMIS 2003, 111, 1011-1018. [CrossRef]

29. Kyzas, P.A.; Agnantis, N.J.; Stefanou, D. Endoglin (CD105) as a prognostic factor in head and neck squamous cell carcinoma. Virchows Arch. 2006, 448, 768-775. [CrossRef]

30. Zvrko, E.; Mikic, A.; Vuckovic, L.; Djukic, V.; Knezevic, M. Prognostic relevance of CD105-assessed microvessel density in laryngeal carcinoma. Otolaryngol. Head. Neck Surg. 2009, 141, 478-483. [CrossRef]

31. Schimming, R.; Marmé, D. Endoglin (CD105) expression in squamous cell carcinoma of the oral cavity. Head. Neck 2002, 24, 151-156. [CrossRef] [PubMed]

32. Chien, C.Y.; Su, C.Y.; Hwang, C.F.; Chuang, H.C.; Chen, C.M.; Huang, C.C. High expressions of CD105 and VEGF in early oral cancer predict potential cervical metastasis. J. Surg. Oncol. 2006, 94, 413-417. [CrossRef] [PubMed]

33. Marioni, G.; Marino, F.; Giacomelli, L.; Staffieri, C.; Mariuzzi, M.L.; Violino, E.; De Filippis, C. Endoglin expression is associated with poor oncologic outcome in oral and oropharyngeal carcinoma. Acta Otolaryngol. 2006, 126, 633-639. [CrossRef]

34. Marioni, G.; Staffieri, A.; Fasanaro, E.; Stramare, R.; Giacomelli, L.; Bernardi, E.; Val, M.; Stellini, E.; de Filippis, C.; Blandamura, S. The role of angiogenin in pT1-T2 tongue carcinoma neo-angiogenesis and cell proliferation: An exploratory study. J. Oral Pathol. Med. 2013, 42, 606-611. [CrossRef] [PubMed]

35. Martone, T.; Rosso, P.; Albera, R.; Migliaretti, G.; Fraire, F.; Pignataro, L.; Pruneri, G.; Bellone, G.; Cortesina, G. Prognostic relevance of CD105+ microvessel density in HNSCC patient outcome. Oral Oncol. 2005, 41, 147-155. [CrossRef] [PubMed]

36. Lionello, M.; Staffieri, A.; Marioni, G. Potential prognostic and therapeutic role for angiogenesis markers in laryngeal carcinoma. Acta Otolaryngol. 2012, 132, 574-582. [CrossRef] 
37. Lovato, A.; Marioni, G.; Manzato, E.; Staffieri, C.; Giacomelli, L.; Ralli, G.; Staffieri, A.; Blandamura, S. Elderly patients at higher risk of laryngeal carcinoma recurrence could be identified by a panel of two biomarkers (nm23-H1 and CD105) and pN+ status. Eur. Arch. Otorhinolaryngol. 2015, 272, 3417-3424. [CrossRef]

38. Han, B.; Lin, S.; Yu, L.J.; Wang, R.Z.; Wang, Y.Y. Correlation of ${ }^{18}$ F-FDG PET activity with expressions of survivin, Ki67, and CD34 in non-small-cell lung cancer. Nucl. Med. Commun. 2009, 30, 831-837. [CrossRef]

39. Xing, N.; Cai, Z.L.; Zhao, S.H.; Yang, L.; Xu, B.X.; Wang, F.L. The Use of CT Perfusion to Determine Microvessel Density in Lung Cancer: Comparison with FDG-PET and Pathology. Chin. J. Cancer Res. 2011, 23, 118-122. [CrossRef]

40. Westerterp, M.; Sloof, G.W.; Hoekstra, O.S.; Ten Kate, F.J.; Meijer, G.A.; Reitsma, J.B.; Boellaard, R.; van Lanschot, J.J.; Molthoff, C.F. 18FDG uptake in oesophageal adenocarcinoma: Linking biology and outcome. J. Cancer Res. Clin. Oncol. 2008, 134, 227-236. [CrossRef]

41. Groves, A.M.; Shastry, M.; Rodriguez-Justo, M.; Malhotra, A.; Endozo, R.; Davidson, T.; Kelleher, T.; Miles, K.A.; Ell, P.J.; Keshtgar, M.R. 18F-FDG PET and biomarkers for tumour angiogenesis in early breast cancer. Eur. J. Nucl. Med. Mol. Imaging 2011, 38, 46-52. [CrossRef]

42. Cochet, A.; Pigeonnat, S.; Khoury, B.; Vrigneaud, J.M.; Touzery, C.; Berriolo-Riedinger, A.; Dygai-Cochet, I.; Toubeau, M.; Humbert, O.; Coudert, B.; et al. Evaluation of breast tumor blood flow with dynamic first-pass 18F-FDG PET/CT: Comparison with angiogenesis markers and prognostic factors. J. Nucl. Med. 2012, 53, 512-520. [CrossRef]

43. Goh, V.; Rodriguez-Justo, M.; Engledow, A.; Shastry, M.; Endozo, R.; Peck, J.; Meagher, M.; Taylor, S.A.; Halligan, S.; Groves, A.M. Assessment of the metabolic flow phenotype of primary colorectal cancer: Correlations with microvessel density are influenced by the histological scoring method. Eur. Radiol. 2012, 22, 1687-1692. [CrossRef]

44. Surov, A.; Meyer, H.J.; Gawlitza, M.; Höhn, A.K.; Boehm, A.; Kahn, T.; Stumpp, P. Correlations Between DCE MRI and Histopathological Parameters in Head and Neck Squamous Cell Carcinoma. Transl. Oncol. 2017, 10, 17-21. [CrossRef]

45. Ash, L.; Teknos, T.N.; Gandhi, D.; Patel, S.; Mukherji, S.K. Head and neck squamous cell carcinoma: CT perfusion can help noninvasively predict intratumoral microvessel density. Radiology 2009, 251, 422-428. [CrossRef]

(C) 2019 by the authors. Licensee MDPI, Basel, Switzerland. This article is an open access article distributed under the terms and conditions of the Creative Commons Attribution (CC BY) license (http://creativecommons.org/licenses/by/4.0/). 



\title{
Novel Nuclear Medicine Imaging Applications in Immuno-Oncology
}

\author{
Stefano Frega ${ }^{1, *}$, Alessandro Dal Maso ${ }^{2}$, Giulia Pasello ${ }^{1}$, Lea Cuppari ${ }^{3}$, Laura Bonanno ${ }^{1}$, \\ PierFranco Conte ${ }^{1,2}$ and Laura Evangelista ${ }^{4}$ \\ 1 Oncology 2 Unit, Department of Medical Oncology, Veneto Institute of Oncology IOV - IRCCS, 35128 Padova, \\ Italy; giulia.pasello@iov.veneto.it (G.P.); laura.bonanno@iov.veneto.it (L.B.); pierfranco.conte@unipd.it (P.C.) \\ 2 Department of Surgery, Oncology and Gastroenterology, University of Padua, 35128 Padova, Italy; \\ alessandro.dalmaso@iov.veneto.it \\ 3 Nuclear Medicine Unit, Veneto Institute of Oncology IOV-IRCCS, University of Padua, 35128 Padova, Italy; \\ lea.cuppari@gmail.com \\ 4 Nuclear Medicine Unit, Department of Medicine, University of Padua, 35128 Padova, Italy; \\ laura.evangelista@aopd.veneto.it \\ * Correspondence: stefano.frega@iov.veneto.it; Tel.: +39-049-8215931
}

Received: 19 April 2020; Accepted: 19 May 2020; Published: 21 May 2020

\begin{abstract}
The global immuno-oncology pipeline has grown progressively in recent years, leading cancer immunotherapy to become one of the main issues of the healthcare industry. Despite their success in the treatment of several malignancies, immune checkpoint inhibitors (ICIs) perform poorly in others. Again, ICIs action depends on such a multitude of clinico-pathological features, that the attempt to predict responders/long-responders with ad-hoc built immunograms revealed to be quite complex. In this landscape, the role of nuclear medicine might be crucial, with first interesting evidences coming from small case series and pre-clinical studies. Positron-emission tomography (PET) techniques provide functional information having a predictive and/or prognostic value in patients treated with ICIs or adoptive T-cell therapy. Recently, a characterization of the tumor immune microenvironment (TiME) pattern itself has been shown to be feasible through the use of different radioactive tracers or image algorithms, thus adding knowledge about tumor heterogeneity. Finally, nuclear medicine exams permit an early detection of immune-related adverse events (irAEs), with on-going clinical trials investigating their correlation with patients' outcome. This review depicts the recent advances in molecular imaging both in terms of non-invasive diagnosis of TiME properties and benefit prediction from immunotherapeutic agents.
\end{abstract}

Keywords: nuclear medicine; positron-emission tomography; single-photon emission computed tomography; immunotherapy; immune checkpoint inhibitors

\section{Immuno-Oncology (I-O)}

The host immune system interacts with tumor cells through the activation of innate and adaptive immune mechanisms. Initially, transformed cells are eliminated by a competent immune system. Nevertheless, sporadic tumor cells manage to survive immune destruction and may enter an equilibrium phase during which editing occurs. Finally, immunologically sculpted tumors begin to grow progressively, establish an immunosuppressive tumor microenvironment and become clinically apparent [1]. The immune system-tumor interaction is targeted by immunotherapy, with the aim of stimulating the immune system to direct immune-mediated responses against the tumor.

The global I-O pipeline has grown progressively in last years. The administration of interleukin-2 (IL-2) and the adoptive transfer of antitumor T cells grown in IL-2 represented the first effective immunotherapies for cancer in humans [2]. Toll-like receptor agonist imiquimod has been used to treat 
a variety of skin cancers including basal cell cancer, squamous cell cancer, lentigo maligna melanoma, and cutaneous T-cell lymphoma [3]. Recombinant interferon $\alpha-2 b$ is indicated in hairy cell leukemia, chronic myelogenous leukemia, multiple myeloma, follicular lymphoma, and as adjuvant therapy in malignant melanoma [4]. Calmette-Guerin bacillus is the gold standard adjuvant treatment of high-risk non-muscle invasive bladder cancer [5].

Moreover, the development of immune checkpoint inhibitors (ICIs) is a revolutionary milestone in the field of I-O. ICIs reinvigorate antitumor immune responses by interrupting co-inhibitory signaling pathways and promote immune-mediated elimination of tumor cells. Anti-programmed-death 1 (PD-1) nivolumab and pembrolizumab, anti-programmed death-ligand 1 (PD-L1) atezolizumab, durvalumab and avelumab, and anti-cytotoxic T-lymphocyte antigen 4 (CTLA-4) ipilimumab are the standard of care in adjuvant treatment or advanced disease treatment for many solid tumors (Table 1).

Table 1. Immune checkpoint inhibitor for solid tumors (European Medicines Agency and Food and Drugs Administration approval).

\begin{tabular}{|c|c|c|}
\hline Immune Checkpoint Inhibitor & Solid Tumor & Reference \\
\hline \multicolumn{3}{|l|}{ Adjuvant Setting } \\
\hline Ipilimumab & Malignant melanoma & [6] \\
\hline Nivolumab & Malignant melanoma & [7] \\
\hline Pembrolizumab & Malignant melanoma & [8] \\
\hline \multicolumn{3}{|l|}{ Advanced Disease Setting } \\
\hline Ipilimumab & Malignant melanoma & [9] \\
\hline \multirow[t]{8}{*}{ Nivolumab } & Malignant melanoma & [10] \\
\hline & Non-small cell lung cancer & {$[11,12]$} \\
\hline & Renal cell carcinoma & [13] \\
\hline & Hodgkin's lymphoma & {$[14]$} \\
\hline & Head and neck cancer & [15] \\
\hline & Urothelial carcinoma & {$[16]$} \\
\hline & $\begin{array}{l}\text { Mismatch-repair deficient/Microsatellite } \\
\text { instability-high colorectal carcinoma }{ }^{1}\end{array}$ & {$[17]$} \\
\hline & Hepatocellular carcinoma ${ }^{1}$ & {$[18]$} \\
\hline \multirow[t]{3}{*}{ Nivolumab plus Ipilimumab } & Malignant melanoma & [19] \\
\hline & Renal cell carcinoma & [20] \\
\hline & $\begin{array}{l}\text { Mismatch-repair deficient/Microsatellite } \\
\text { instability-high colorectal carcinoma }{ }^{1}\end{array}$ & {$[21]$} \\
\hline \multirow[t]{12}{*}{ Pembrolizumab } & Malignant melanoma & [22] \\
\hline & $\begin{array}{l}\text { Non-small cell lung cancer (with or } \\
\text { without chemotherapy) }\end{array}$ & [23-25] \\
\hline & Renal cell carcinoma (with axitinib) & [26] \\
\hline & Hodgkin's lymphoma & [27] \\
\hline & Head and neck cancer ${ }^{1}$ & [28] \\
\hline & Urothelial carcinoma & {$[29,30]$} \\
\hline & Hepatocellular carcinoma ${ }^{1}$ & [31] \\
\hline & Gastric cancer ${ }^{1}$ & [32] \\
\hline & Esophageal cancer ${ }^{1}$ & [33] \\
\hline & Cervical cancer ${ }^{1}$ & [34] \\
\hline & Merkel cell carcinoma ${ }^{1}$ & [35] \\
\hline & Small cell lung cancer ${ }^{1}$ & {$[36]$} \\
\hline \multirow[t]{4}{*}{ Atezolizumab } & Urothelial carcinoma & {$[37,38]$} \\
\hline & $\begin{array}{l}\text { Non-small cell lung cancer (with or without } \\
\text { chemotherapy and bevacizumab) }\end{array}$ & {$[39-41]$} \\
\hline & $\begin{array}{l}\text { Small cell lung cancer (with carboplatin } \\
\text { and etoposide) }\end{array}$ & [42] \\
\hline & Triple-negative breast cancer (with nab-paclitaxel) ${ }^{1}$ & [43] \\
\hline
\end{tabular}


Table 1. Cont.

\begin{tabular}{ccc}
\hline Immune Checkpoint Inhibitor & Solid Tumor & Reference \\
\hline Avelumab & Merkel cell carcinoma & {$[44]$} \\
& Urothelial carcinoma $^{1}$ & {$[45]$} \\
& Renal cell carcinoma (with axitinib) $^{1}$ & {$[46]$} \\
\hline Durvalumab & Non-small cell lung cancer & {$[47]$} \\
& Urothelial carcinoma $^{1}$ & {$[48]$} \\
\hline
\end{tabular}

${ }^{1}$ Food and Drugs Administration approval only.

However, despite these substantial advances, only a subset of patients receiving ICIs derive clinical benefit. It is then crucial to identify and to develop predictive biomarkers of ICIs response. PD-L1 expression and tumor mutation burden are the only predictive factors validated in phase III clinical trials, but are still imperfect since there are ICI non-responders expressing high biomarkers levels and ICI responders with low biomarkers levels. New determinants of response are being investigated, with strategies encompassing multiple biomarkers [49,50].

\section{Cancer, Nuclear Medicine (NM) and Response to I-O}

\subsection{Nuclear Medicine}

Nuclear medicine is a branch of medicine using radionuclides in the diagnosis and treatment of diseases. Diagnostic applications consist in "functional imaging" and are based on the ability of radiopharmaceutical agents to concentrate in pathological tissues and to emit radiations that are revealed by external detectors and then recorded. Therapeutic approaches exploit selective concentration of radiopharmaceutical agents in pathological tissues and the use of the radiations to destroy them. Techniques of nuclear medicine imaging include two-dimensional scintigraphy, three-dimensional single-photon emission computed tomography (SPECT) and positron emission tomography (PET), and hybrid techniques combining computed tomography (CT) or magnetic resonance imaging (MRI) scans with SPECT or PET [51].

2- $\left[{ }^{18} \mathrm{~F}\right]$ fluoro-2-deoxy-D-glucose $\left({ }^{18} \mathrm{~F}\right.$-FDG or FDG) PET/CT has become an established standard nuclear imaging modality in oncology [52] based on the finding that tumor hypoxic cells have increased glucose demand and, consequently, FDG uptake and accumulation [53]. FDG-PET/CT is thus widely used in cancer diagnosis, staging, and response to treatment evaluation [54-56].

However, some drawbacks have to be mentioned [57]. Infection and inflammation can lead to false positive findings. Another limitation is that some organs display a higher physiological FDG uptake (i.e., brain, myocardial tissue, brown fat) and therefore are not studied with this modality. Moreover, FDG-PET/CT can result false negative for lesions or $<8-10 \mathrm{~mm}$ and for several histotypes. Hyperglycemia has to be correctly managed to convey a valid exam. Furthermore, FDG-PET/CT is nonspecific for tumor subtypes. Finally, risk-benefit considerations should minimize radiation exposure. For all these reasons, clinical information exchange between oncologists and nuclear medicine specialists is crucial for patients' care and outcomes [54-56].

\subsection{Assessment of Tumor Response}

Assessment of response represents a measure of antitumor drug activity. Radiological overall response rate is the primary endpoint of about $70 \%$ of phase II trials. Thus, positive trials are the basis of accelerated approval in refractory advanced disease, a setting where there is no available therapy [58]. Furthermore, response assessment is a cornerstone of daily clinical practice.

Practically, response assessment starts with summing the size of tumor lesions in a baseline CT scan before beginning a new therapy. After a specified time, the relative change of the sum of lesion sizes is registered. Finally, a threshold on this relative change is applied to decide whether treatment should be administered or discontinued. Historically, the WHO Response Criteria were first published 
in 1981 and were based on bidimensional measurements [59]. As CT scans were more easily available, the WHO Criteria were simplified and harmonized by the Response Evaluation Criteria in Solid Tumors (RECIST) in which unidimensional measurements were considered [60]. A further update was provided by the RECIST 1.1 version, including revised specifications on measurable disease, nodal disease, and progressive disease [61].

Nevertheless, less than half of the studies that met their response endpoint measured with RECIST criteria in a phase II clinical trial are successful in phase III clinical trials [62-64]. It has been proposed that response assessment with FDG-PET could be more sensitive and specific for the detection of metastatic disease. In particular, bone disease is not evaluable by RECIST criteria. Moreover, stable disease as defined by RECIST criteria could represent on the one hand a consequence of tumor growth inhibition by effective therapy, on the other hand a result of an indolent tumor biology, whereas it is more likely that growth inhibition results in a decrease of tumor FDG uptake [65].

Two metabolic response criteria were thus developed. European Organization for Research and Treatment of Cancer (EORTC) PET response criteria were based on standalone PET imaging and focused on evaluation of only one lesion [66]. PET Response Criteria in Solid Tumors (PERCIST) were applied to PET/CT scans and deal with multiple lesions evaluation $[67,68]$. Moreover, main differences include the use of standardized uptake value (SUV) mean in EORTC criteria compared to the SUV peak normalized by lean body mass (SULpeak) used in PERCIST criteria as the quantitative parameter, finally a different threshold for progressive metabolic disease is used. Robust clinical validation is still awaited.

\subsection{Assessment of Tumor Response to Immunotherapy}

Response assessment for I-O must deal with a unique challenge, pseudoprogression, that is a temporary increase in tumor size, meeting the criteria for progressive disease based on RECIST, which is lately not confirmed. Pseudoprogression is caused by immune cells infiltrating tumor lesions. Its prevalence is up to $10 \%$, and it has been characterized in malignant melanoma studies $[69,70]$.

Four new response criteria have been proposed to solve this issue and all of these require a confirmation of progressive disease [71]. Immune-related response criteria (irRC) are based on WHO criteria, and therefore on bidimensional measurements [72]. Immune-related response evaluation criteria in solid tumors (irRECIST) combine irRC with RECIST 1.1 criteria, thus rely on unidimensional measurements [73]. Immune response evaluation criteria in solid tumors (iRECIST) are based on RECIST 1.1, too, but differ from irRECIST because they do not include measurements of the new lesions on tumor burden [74]. Immune-modified response evaluation criteria in solid tumors (imRECIST) are similar to irRECIST [75].

IrRC and irRECIST were clinically validated in patients with advanced malignant melanoma treated with ipilimumab, imRECIST in patients with advanced NSCLC and metastatic urothelial carcinoma treated with atezolizumab, whereas iRECIST are based on a consensus statement. The RECIST working group recommends that recent trials should use RECIST 1.1 to define the primary and secondary efficacy-based endpoints and reserve irRC or irRECIST for exploratory endpoints [74].

The role of already published metabolic criteria was investigated in patients treated with immunotherapy. EORTC criteria applied to PET/CT evaluation after two cycles of ipilimumab on 22 advanced melanoma patients were found to be predictive of final treatment outcome [76]. Retrospective baseline and 1-year PET/CT analysis with EORTC criteria of 104 metastatic melanoma patients treated with nivolumab or nivolumab and ipilimumab showed that patients with complete metabolic responses at 1 year had ongoing responses to therapy thereafter [77]. PERCIST criteria were used to evaluate response on a PET/CT scan 1 month after the start of nivolumab in 24 advanced NSCLC patients, metabolic responses were closely associated with therapeutic response and survival [78].

Likewise, semi quantitative parameters were found to be predictive of outcome in patients treated with immunotherapy. This could be the case for SUV, metabolic tumor volume (MTV), and tumor total lesion glycolysis (TLG). These three variables define the entire metabolic tumor burden (MTB), that may represent a reliable value to predict treatment success, being known that I-O work better in a context 
of low tumor load [79]. Variations in MTV and TLG in FDG-PET/CT scans taken before and after 4 cycles of nivolumab in 20 patients with metastatic NSCLC correlated with objective response by CT scan [80]. Baseline SUVmax and SUVmean identified progression after 8 weeks of therapy in 27 NSCLC patients treated with nivolumab or pembrolizumab [81]. Increased SUVmax and SULpeak of the most FDG-avid lesion at interim PET/CT identified non-responders in 30 advanced melanoma patients treated with pembrolizumab [82]. Whole-body maximum SUV (SUVmaxwb) in 32 advanced NSCLC patients treated with nivolumab correlated with response [83]. Moreover, an associated flare response on FDG PET/CT imaging at 1 week post-therapy defined as $>100 \%$ increase in tumor SUVmax between baseline and post-therapy scans was found to be predictive of a complete response to immunotherapy, and this correlated usually with evidence of $\mathrm{T}$ cell reinvigoration in the blood at 1 week post therapy [84].

In parallel, several I-O-adapted metabolic criteria have been proposed. PET/CT Criteria for Early Prediction of Response to Immune Checkpoint Inhibitor Therapy (PECRIT) are based on RECIST 1.1 to define complete response, partial response and progressive disease, whereas percent change in SULpeak by PERCIST criteria is used to distinguish patients with stable disease per RECIST 1.1 and clinical benefit from those with stable disease and no clinical benefit. The comparison of baseline PET/CT scan with a second PET/CT scan at 3-4 weeks after the start of immunotherapy based on PECRIT was able to predict the best overall response at 4 months in 20 patients with advanced melanoma patients treated with nivolumab, ipilimumab or BMS-936559 [85].

Conversely, the number of new FDG-avid lesions was considered in PET Response Evaluation Criteria for Immunotherapy (PERCIMT). Four newly emerged avid lesions on PET/CT scan after 3 months of therapy was found to be predictive of treatment failure in 41 melanoma patients treated with ipilimumab, with the functional size of the new lesions playing an important role in predicting the clinical response [86]. PERCIMT evaluation was applied to PET/CT scan after two cycles of immunotherapy on the same cohort of patients, showing higher sensitivity compared to EORTC criteria in predicting clinical benefit, but specificity in predicting no clinical benefit was not significantly higher [87].

Inflammatory response caused by immunotherapy may confound the ability of FDG-PET to identify patients with a favorable response. Thus, similarly to iRECIST, immune PET Response Criteria in Solid Tumors (iPERCIST) included the concept of disease progression confirmation in PERCIST. iPERCIST were retrospectively applied to a cohort of 28 NSCLC patients treated with nivolumab. All patients underwent a baseline PET/CT scan and a second scan after 2 months of treatment, which was evaluated with the proposed criteria. A metabolic unconfirmed progressive disease was re-evaluated with a third PET/CT scan after 4 weeks. The 1-year survival rates were significantly higher in iPERCIST responders compared with non-responders [88]. Beyond the above seen parameters, even spleen to liver ratio (SLR) has been found to correlate with ICIs efficacy, and thus may represent a novel PET biomarker to evaluate patients' baseline immune state prior to immunotherapy [89]. All these criteria require further validation.

Figure 1 depicts an overview of CT-based and PET-based response criteria for solid tumors.

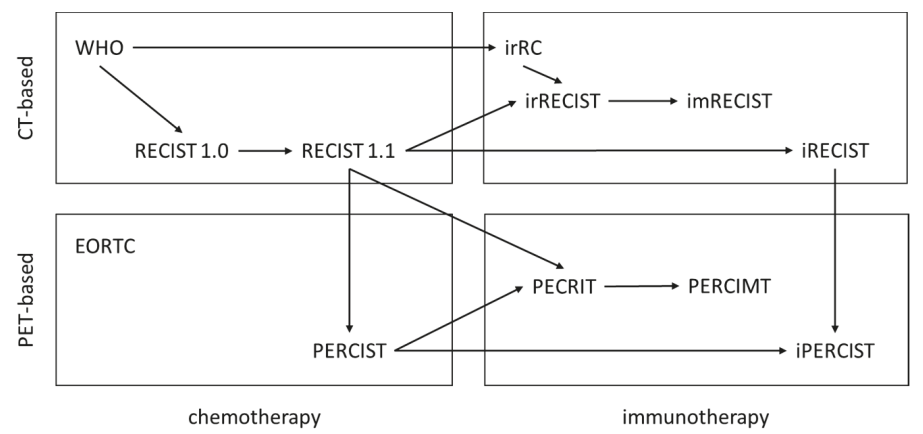

Figure 1. Overview of computed tomography (CT)-based (upper panel) and positron emission tomography 
(PET)-based (lower panel) response criteria for solid tumors. On the left side are grouped response criteria designed for chemotherapy, on the right side those specifically designed for immunotherapy. RECIST: Response Evaluation Criteria in Solid Tumors, irRC: Immune Related Response Criteria, irRECIST: Immune-related Response Evaluation Criteria in Solid Tumors, imRECIST: Immune-modified response evaluation criteria in solid tumors, iRECIST: Immune response evaluation criteria in solid tumors. EORTC: European Organization for Research and Treatment of Cancer criteria, PERCIST: PET Response Criteria in Solid Tumors, PERCIMT: PET Response Evaluation Criteria for Immunotherapy, PECRIT: PET/CT Criteria for Early Prediction of Response to Immune Checkpoint Inhibitor Therapy, iPERCIST: immune PET Response Criteria in Solid Tumors.

\section{NM and Tumor Immune-Microenvironment (TiME) Analysis}

The survival benefit from using ICIs varies considerably from patient to patient. ICIs manage to slow down cancer progression, changing completely long-term survival perspective for a subgroup of patients, that goes from $10 \%$ to $30 \%$, depending from the tumor types and drug used [90,91]. The reverse of the medal, of course, is that ICIs own poor or no efficacy in a considerable proportion of treated subjects.

Last year, scientific community efforts in oncology were greatly conveyed to unhinge the reasons of this imbalance, trying to recognize the ideal candidate for immunotherapy; the final aim is to sharpen and potentiate ICIs tools [92]. ICIs activity is influenced by several clinical features, tumor histology, and also tumor genes' expression [93].

The first studied marker has been PD-L1, a protein variously expressed by tumor cells, that for some cancer types correlate well with benefits from specific anti-PD-L1 agents [94]; in fact, some of these drugs have received approval on the basis of tumor PD-L1 expression [95]. For this reason, PD-L1 is to date the most used determinant in patients treated with ICIs worldwide, though it remains an imperfect predictive biomarker.

Evaluating US Food and Drug Administration (FDA) approvals of 45 ICIs agents across 15 tumor types, PD-L1 was found to be predictive in less than a third of them [94]. This phenomenon can be explained by multiple factors. First, PD-L1 expression in individual patients could be heterogeneous possibly being different in different tumor sites but also in a single tumor site, and influenced by the sample itself [96]. Secondarily, ICIs action can be affected by the huge amount of other tumor immune-microenvironment (TiME) properties [97]. B7-H3, a member of the B7 superfamily, is expressed on tumor cells and potentially circumvents CD8+-T-cell-mediated immune surveillance. Anti-B7-H3 immunotherapy combination with anti-PD-1/PD-L1 antibody therapy is a promising approach for B7-H3-expressing cancers [98]. Tumor-infiltrating T-cells (TILs) co-receptors (PD-1, CTLA-4, Lymphocyte-activation gene 3 (LAG-3), etc.) have great importance in the context of TiME, by interacting with tumor cells, becoming new target for a series of new ICIs, some of which already approved. Finally, factors related to the host immune system such as blood biomarkers (peripheral blood mononuclear cells (PBMCs), etc.) could affect ICIs activity [99].

Thus, it would be of extreme importance to consider TiME globally in patients eligible for ICIs. Analyses of TiME on tissue could be affected both by tumor tissue sample itself in terms of quantity and/or quality and by tumor heterogeneity [100]. NM imaging can bypass these two tissue intrinsic limits. Through specific radiotracer-labeled antibody, NM proved to characterize a series of TiME properties, and with good concordance with tissue data. Indeed, surface markers of PBMCss, TILs and tumor cells could be selectively targeted, leading to a successful definition of TiME composition with preclinical animal models, but also in human adults with cancer (Table 2). This constitutes a unique opportunity to overcome the intrinsic limits of TiME analyses on tissue, that results to be moreover both cost and time consuming. 


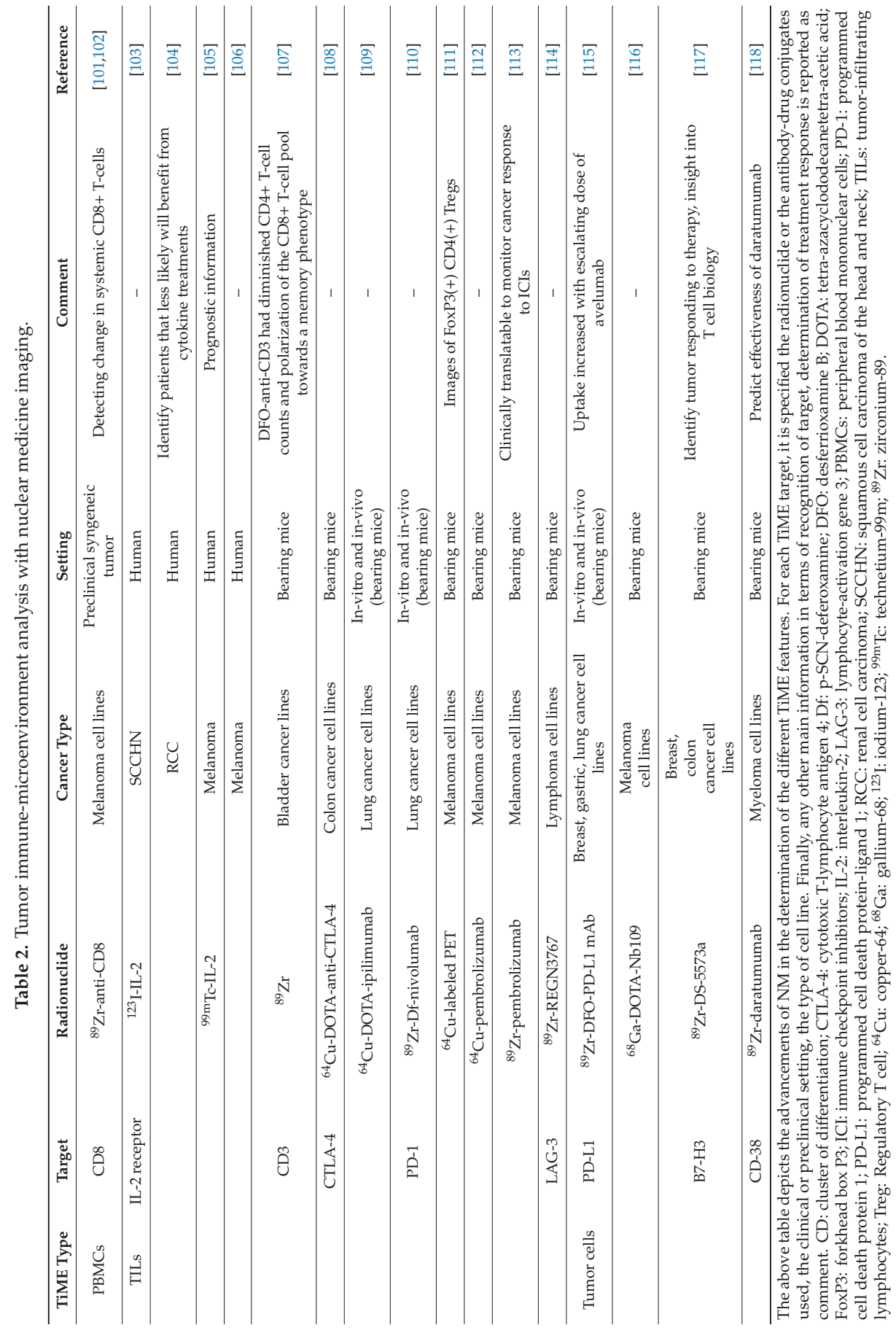


Recently, imaging of TiME by PET has gained an excellent progress by the introduction of different tracers targeting fibroblast activation protein (FAP), among which ${ }^{68} \mathrm{Gallium}$-labeled chelator-linked FAP inhibitors (FAPIs) and ${ }^{18}$ F-labeled glycosylated FAPIs [119]. FAP is highly expressed by cancer-associated fibroblasts (CAFs), that are generally associated with poor prognosis and represent for some cancer types one of the main reason of resistance to immunotherapy [120].

NM imaging can provide a static baseline representation of the identified marker, so having a predictive value, possibly bringing our therapeutic choices to be as careful as possible. Otherwise, these techniques can be used in a more dynamic way, by repeating NM imaging in different disease time-points in patients treated with ICIs. The aim will be to monitor treatment response, by using labeled-radiotracers conjugated with immunotherapeutic agents, with first brilliant results in this sense.

\subsection{PBMCs-Targeted Imaging}

The generation of anti-CD8 ${ }^{89} \mathrm{Zr}$-desferrioxamine-labeled cys-diabody permitted a noninvasive immuno-PET tracking of endogenous CD8+ T cells in melanoma immunotherapy models, including anti-PD-L1 agents, antigen-specific adoptive T-cell transfer and agonistic antibody therapy.

The great power of such imaging consists in its ability to acquire dynamic information regarding therapy-induced alterations of both PBMCs and TILs component [101].

\subsection{TILs-Targeted Imaging}

Similarly, with an immuno-PET with Zirconium- $89\left({ }^{89} \mathrm{Zr}\right)$-labeled PEGylated single-domain antibody fragments specific for $\mathrm{CD} 8$, Rashidian et al. demonstrated the ability to longitudinally detect TILs in melanoma models and to distinguish responding tumors from those that do not respond to anti-CTLA4 therapy [102].

${ }^{123}$ I-interleukin-2 (IL-2) single-photon emission computed tomography (SPECT) well depicted TILs in cancer patients, also giving a preliminary response to cytokine treatment $[103,104]$, while TILs targeting through ${ }^{99 \mathrm{~m}} \mathrm{Tc}-\mathrm{IL}-2$ in melanoma patients treated with I-O permits to acquire information about pseudo-progression and prognosis $[105,106]$. Prognosis impact has also found to be appraisable by quantifying TILs in animal models of bladder cancer trough anti-CD3 monoclonal antibody (mAb) modified with desferrioxamine (DFO) and radiolabeled with ${ }^{89} \mathrm{Zr}$ [107]. Recognition of CTLA-4 TILs receptor has been realized by a specific ${ }^{64} \mathrm{Cu}$-DOTA-anti-CTLA-4 $\mathrm{mAb}[108,109]$, while anti-PD-1 agents labeled with ${ }^{64} \mathrm{Cu}$ or ${ }^{89} \mathrm{Zr}$ can detect very precisely PD-1-expressing TILs [110-113]. Moreover, the fully human anti-LAG3 antibody REGN3767, radiolabeled with ${ }^{89} \mathrm{Zr}$ using the bifunctional chelator p-SCN-Bn-Deferoxamine (DFO) led to an immune-PET detection of LAG-3 expressing TILs [114]. NM individuation of PD-1, CTLA-4 and LAG-3 expressed by TILs could assist in patient stratification, disease diagnostics, and monitoring response to ICIs.

\subsection{Tumor Cell-Targeted Imaging}

A similar usefulness may derive from PET-imaging of some proteins expressed directly by tumor cells. High affinity to PD-L1 has been guaranteed by specifically synthetized ${ }^{68} \mathrm{Ga}$-labeled single-domain antibody tracer called ${ }^{68} \mathrm{Ga}-\mathrm{NOTA}-\mathrm{Nb} 109$, and ${ }^{89} \mathrm{Zr}$-DFO-PD-L1 monoclonal antibody $[115,116]$. Moreover, saturation of B7-H3 tumor cell protein by anti-B7-H3 humanized monoclonal antibody DS-5573a labeled with ${ }^{89} \mathrm{Zr}$ was demonstrated in tumors responding to DS-5573a therapy [117]. ${ }^{89} \mathrm{Zr}$-DFO-daratumumab provides successful whole-body PET visualization of myeloma, and predicts the effectiveness of daratumumab therapy [118].

All these applications represent in a certain way an evolution of the most widely used FDG-PET, already proved as seen to acquire response to ICIs earlier than through the classical CT scan imaging [72,78,79].

\subsection{Tumor Heterogeneity}

The therapeutic approach cannot ignore inter-patients and intra-patient cancer heterogeneity. The latter depends on a complex phenotypic evolution that involve (epi)genetic transformations, 
surface markers selections and cell-cycle modifications in response to environmental stimuli [121]. What is more, not only tumor cells but also TiME features might become different [122]. This is quite challenging because prognosis and response to treatments could be thus tightly influenced.

NM may be helpful to visualize tumor heterogeneity, with interesting applications in the field of I-O. Quantitative analyses of canonical ${ }^{18}$ F-FDG PET imaging can point out the presence of aggressive tumor foci, which may divert patients' prognosis [123]; CAFs have found to contribute to the intratumor heterogeneity of ${ }^{18}$ F-FDG PET and to enhance tumor uptake [123].

The recognition of TiME and cancer cells markers heterogeneity, through specific tracers, could guide in the future daily therapeutic choices. PD-L1 is the forerunner marker in this sense, with different contributes about its possible discordant expression in different tumor lesions [124-126], but the same concept could be applied to other markers.

\section{NM and Immune-Adverse Events (irAEs) Analysis}

Immune-related adverse events (irAEs) are unique adverse events caused by activation of an immune response against healthy tissues due to immunotherapy. IrAEs occur in about two thirds of patients treated with IO, of which $14 \%$ is grade 3 or superior [127]. Timely detection and correct management of irAEs according to their grading, with suspension of IO or eventually immunosuppressive medication, are crucial [128,129]. If not treated, irAEs could require hospitalization and may lead to life-threatening complications. Therefore, predictive markers for irAEs are an unmet clinical need [130].

Diagnosis of irAEs is made through clinical, biohumoral, and radiological investigations. Early radiological detection of irAEs could improve their correct management. In a recent study, medical imaging detected $74 \%$ of irAE in patients treated with anti-PD1. Among typical irAEs, interstitial lung disease is mainly diagnosed with CT scans, as well as enterocolitis and pancreatitis, whereas thyroiditis with ultrasound and hypophysitis with magnetic resonance imaging [131].

Beyond tumor lesions, FDG uptake can be induced by the inflammatory reaction related to irAEs. Thus, sarcoid-like reaction [132,133] and enterocolitis [134] are easily detected by PET/CT scans. PET/CT could also be useful in detecting thyroiditis, hypophysitis, pancreatitis, gastroduodenitis, and cholangitis [131,135-137]. In another study with 35 patients with malignant melanoma receiving nivolumab and ipilimumab combination, 39\% of irAEs were diagnosed subsequent to FDG-PET/TC and $11 \%$ remained clinically occult [138].

Furthermore, irAEs could predict response to immunotherapy [139]. A retrospective study enrolled 40 patients with malignant melanoma, malignant lymphoma or renal cell carcinoma; all patients with an increase of SUVmax in the thyroid of more than 1.5 on the first restaging scan compared to baseline scan had a complete response to I-O in 1 year [140]. Overt thyroid irAEs could be predicted by baseline thyroid uptake of FDG in another study that enrolled 200 patients treated with nivolumab. In the same study, thyroid irAEs were related to good prognosis in patients diagnosed with lung cancer [141]. As regards to enterocolitis, increased FDG uptake correlated with clinical symptoms but did not predict clinical outcome to ipilimumab in 100 advanced melanoma patients [142]. In a study with 41 patients with malignant melanoma treated with ipilimumab, 4 out of 31 patients having disease control were diagnosed sarcoid-like reaction in the follow-up PET/TC, while none of the patients with progressive disease [143].

\section{Conclusions and Future Applications}

NM imaging has historically contributed in a decisive way to expand the knowledge about cancer management and cancer response to treatment, providing several functional data. In the context of modern I-O, this could become quite challenging, because each day millions of cancer patients worldwide are treated with I-O therapies, and lots of crucial aspects remain to be clarified. Last decade scientific reports added progressively important pieces to the definition of the complex immune-cancer 
cycle, finding out favorable clinical and pathological patterns of response to I-O drugs, and even creating complex predictive algorithms.

Main open questions include the optimal candidate for I-O therapy, the determination of response and appropriate duration of treatments, the mechanisms that underlie primary and secondary resistance to I-O. The goals are I-O empowerment in terms of efficacy and healthcare resources optimization.

As seen, PET-CT scans has shown to predict responders and non-responders to I-O earlier than the conventional CT scan, through the acquisition of both anatomical and metabolic data. Moreover, several PET-CT scan parameters, acquired at baseline or during treatment, have been found to be predictive of response to I-O. NM applications could well depict several predictive TiME features by using different radiotracers and/or drug-radiotracer conjugates, and also precociously recognize drug-induced irAEs usually correlated with I-O benefit. This would permit ideally to change in the near future the approach of cancer care, by selecting the right I-O drug for the right patient, revealing the immunologic reasons of innate and acquired resistance to I-O, and laying the foundations to get around them.

In the last few years, I-O imaging field has been enriched by radiomics, a set of methods used to extract a large amount of quantitative data from biomedical images—called features-aiming to personalize medicine even more. The typical radiomics workflow is a multi-step process, that starts with the acquisition of measurable features and, through different phases of data processing, concludes with data analysis [144]. Radiomics can acquire and deal with traditional radiology imaging (CT, magnetic resonance), but also with PET imaging, building-up mathematical algorithms in order to study tumor characteristics and clinical outcomes. PD-L1 expression of patients affected by head and neck carcinoma $(n=53)$ has found to be correlated to textural features derived from FDG-PET organization of tumor pixels [145]. PET/CT-based radiomic signature could have both a prognostic value for NSCLC patients that underwent to surgery [146] and a predictive role to identify those patients most likely to benefit from immunotherapy. A multiparametric radiomics signature, built up combining different radiomics features, have shown in fact to well predict durable clinical benefit from immunotherapy in advanced NSCLC patients $(n=99)$ [147], with other experiences confirming this potentiality [148]. Moreover, radiomics could improve tumor heterogeneity characterization and prognosis prediction, in order to stratify patients to receive therapeutic regimens [123]. Despite its great premises, some concerns exist about applicability of NM radiomics in daily clinical practice [149], and further studies investigating its potential in I-O field are urgently warranted.

Author Contributions: Conceptualization by S.F.; L.E.; A.D.M.; writing—original draft preparation by S.F.; L.E.; A.D.M.; writing-review by L.E.; G.P.; L.C.; L.B.; P.C.; editing by S.F.; A.D.M. All authors have read and agreed to the published version of the manuscript.

Funding: This research did not receive external funding.

Conflicts of Interest: The authors declare no conflict of interest.

\section{References}

1. Mittal, D.; Gubin, M.M.; Schreiber, R.D.; Smyth, M.J. New insights into cancer immunoediting and its three component phases-Elimination, equilibrium and escape. Curr. Opin. Immunol. 2014, 27, 16-25. [CrossRef] [PubMed]

2. Rosenberg, S.A. IL-2: The first effective immunotherapy for human cancer. J. Immunol. 2014, 192, 5451-5458. [CrossRef] [PubMed]

3. Huen, A.O.; Rook, A.H. Toll receptor agonist therapy of skin cancer and cutaneous T-cell lymphoma. Curr. Opin. Oncol. 2014, 26, 237-244. [CrossRef]

4. Borden, E.C. Interferons $\alpha$ and $\beta$ in cancer: Therapeutic opportunities from new insights. Nat. Rev. Drug Discov. 2019, 18, 219-234. [CrossRef] [PubMed]

5. Herr, H.W.; Morales, A. History of bacillus Calmette-Guerin and bladder cancer: An immunotherapy success story. J. Urol. 2008, 179, 53-56. [CrossRef] 
6. Eggermont, A.M.M.; Chiarion-sileni, V.; Grob, J.J.; Dummer, R.; Wolchok, J.D.; Schmidt, H.; Hamid, O.; Robert, C.; Ascierto, P.A.; Richards, J.M.; et al. Prolonged survival in stage III melanoma with ipilimumab adjuvant therapy. N. Engl. J. Med. 2016, 375, 1845-1855. [CrossRef]

7. Weber, J.; Mandala, M.; Del Vecchio, M.; Gogas, H.J.; Arance, A.M.; Cowey, C.L.; Dalle, S.; Schenker, M.; Chiarion-Sileni, V.; Marquez-Rodas, I.; et al. Adjuvant Nivolumab versus Ipilimumab in Resected Stage III or IV Melanoma. N. Engl. J. Med. 2017, 377, 1824-1835. [CrossRef]

8. $\quad$ Eggermont, A.M.M.; Blank, C.U.; Mandala, M.; Long, G.V.; Atkinson, V.; Dalle, S.; Haydon, A.; Lichinitser, M.; Khattak, A.; Carlino, M.S.; et al. Adjuvant pembrolizumab versus placebo in resected stage III melanoma. N. Engl. J. Med. 2018, 378, 1789-1801. [CrossRef]

9. Hodi, F.S.; O’Day, S.J.; McDermott, D.F.; Weber, R.W.; Sosman, J.A.; Haanen, J.B.; Gonzalez, R.; Robert, C.; Schadendorf, D.; Hassel, J.C.; et al. Improved survival with ipilimumab in patients with metastatic melanoma. N. Engl. J. Med. 2010, 363, 711-723. [CrossRef]

10. Robert, C.; Long, G.V.; Brady, B.; Dutriaux, C.; Maio, M.; Mortier, L.; Hassel, J.C.; Rutkowski, P.; McNeil, C.; Kalinka-Warzocha, E.; et al. Nivolumab in previously untreated melanoma without BRAF mutation. N. Engl. J. Med. 2015, 372, 320-330. [CrossRef]

11. Brahmer, J.; Reckamp, K.L.; Baas, P.; Crinò, L.; Eberhardt, W.E.E.; Poddubskaya, E.; Antonia, S.; Pluzanski, A.; Vokes, E.E.; Holgado, E.; et al. Nivolumab versus docetaxel in advanced squamous-cell non-small-cell lung cancer. N. Engl. J. Med. 2015, 373, 123-135. [CrossRef] [PubMed]

12. Borghaei, H.; Paz-Ares, L.; Horn, L.; Spigel, D.R.; Steins, M.; Ready, N.E.; Chow, L.Q.; Vokes, E.E.; Felip, E.; Holgado, E.; et al. Nivolumab versus docetaxel in advanced nonsquamous non-small-cell lung cancer. N. Engl. J. Med. 2015, 373, 1627-1639. [CrossRef] [PubMed]

13. Motzer, R.J.; Escudier, B.; McDermott, D.F.; George, S.; Hammers, H.J.; Srinivas, S.; Tykodi, S.S.; Sosman, J.A.; Procopio, G.; Plimack, E.R.; et al. Nivolumab versus everolimus in advanced renal-cell carcinoma. N. Engl. J. Med. 2015, 373, 1803-1813. [CrossRef]

14. Younes, A.; Santoro, A.; Shipp, M.; Zinzani, P.L.; Timmerman, J.M.; Ansell, S.; Armand, P.; Fanale, M.; Ratanatharathorn, V.; Kuruvilla, J.; et al. Nivolumab for classical Hodgkin's lymphoma after failure of both autologous stem-cell transplantation and brentuximab vedotin: A multicentre, multicohort, single-arm phase 2 trial. Lancet Oncol. 2016, 17, 1283-1294. [CrossRef]

15. Ferris, R.L.; Blumenschein, G.; Fayette, J.; Guigay, J.; Colevas, A.D.; Licitra, L.; Harrington, K.; Kasper, S.; Vokes, E.E.; Even, C.; et al. Nivolumab for recurrent squamous-cell carcinoma of the head and neck. N. Engl. J. Med. 2016, 375, 1856-1867. [CrossRef] [PubMed]

16. Sharma, P.; Retz, M.; Siefker-Radtke, A.; Baron, A.; Necchi, A.; Bedke, J.; Plimack, E.R.; Vaena, D.; Grimm, M.O.; Bracarda, S.; et al. Nivolumab in metastatic urothelial carcinoma after platinum therapy (CheckMate 275): A multicentre, single-arm, phase 2 trial. Lancet Oncol. 2017, 18, 312-322. [CrossRef]

17. Overman, M.J.; McDermott, R.; Leach, J.L.; Lonardi, S.; Lenz, H.J.; Morse, M.A.; Desai, J.; Hill, A.; Axelson, M.; Moss, R.A.; et al. Nivolumab in patients with metastatic DNA mismatch repair-deficient or microsatellite instability-high colorectal cancer (CheckMate 142): An open-label, multicentre, phase 2 study. Lancet Oncol. 2017, 18, 1182-1191. [CrossRef]

18. El-Khoueiry, A.B.; Sangro, B.; Yau, T.; Crocenzi, T.S.; Kudo, M.; Hsu, C.; Kim, T.Y.; Choo, S.P.; Trojan, J.; Welling, T.H.; et al. Nivolumab in patients with advanced hepatocellular carcinoma (CheckMate 040): An open-label, non-comparative, phase 1/2 dose escalation and expansion trial. Lancet 2017, 389, 2492-2502. [CrossRef]

19. Larkin, J.; Chiarion-Sileni, V.; Gonzalez, R.; Grob, J.J.; Cowey, C.L.; Lao, C.D.; Schadendorf, D.; Dummer, R.; Smylie, M.; Rutkowski, P.; et al. Combined nivolumab and ipilimumab or monotherapy in untreated Melanoma. N. Engl. J. Med. 2015, 373, 23-34. [CrossRef]

20. Motzer, R.J.; Tannir, N.M.; McDermott, D.F.; Arén Frontera, O.; Melichar, B.; Choueiri, T.K.; Plimack, E.R.; Barthélémy, P.; Porta, C.; George, S.; et al. Nivolumab plus Ipilimumab versus Sunitinib in advanced renal-cell carcinoma. N. Engl. J. Med. 2018, 378, 1277-1290. [CrossRef]

21. Overman, M.J.; Lonardi, S.; Wong, K.Y.M.; Lenz, H.J.; Gelsomino, F.; Aglietta, M.; Morse, M.A.; Van Cutsem, E.; McDermott, R.; Hill, A.; et al. Durable clinical benefit with nivolumab plus ipilimumab in DNA mismatch repair-deficient/microsatellite instability-high metastatic colorectal cancer. J. Clin. Oncol. 2018, 36, 773-779. [CrossRef] [PubMed] 
22. Robert, C.; Schachter, J.; Long, G.V.; Arance, A.; Grob, J.J.; Mortier, L.; Daud, A.; Carlino, M.S.; McNeil, C.; Lotem, M.; et al. Pembrolizumab versus ipilimumab in advanced melanoma. N. Engl. J. Med. 2015, 372, 2521-2532. [CrossRef] [PubMed]

23. Herbst, R.S.; Baas, P.; Kim, D.W.; Felip, E.; Pérez-Gracia, J.L.; Han, J.Y.; Molina, J.; Kim, J.H.; Arvis, C.D.; Ahn, M.J.; et al. Pembrolizumab versus docetaxel for previously treated, PD-L1-positive, advanced non-small-cell lung cancer (KEYNOTE-010): A randomised controlled trial. Lancet 2016, 387, 1540-1550. [CrossRef]

24. Reck, M.; Rodriguez-Abreu, D.; Robinson, A.G.; Hui, R.; Csöszi, T.; Fülöp, A.; Gottfried, M.; Peled, N.; Tafreshi, A.; Cuffe, S.; et al. Pembrolizumab versus Chemotherapy for PD-L1-Positive Non-Small-Cell Lung Cancer. N. Engl. J. Med. 2016, 375, 1823-1833. [CrossRef] [PubMed]

25. Gandhi, L.; Rodríguez-Abreu, D.; Gadgeel, S.; Esteban, E.; Felip, E.; De Angelis, F.; Domine, M.; Clingan, P.; Hochmair, M.J.; Powell, S.F.; et al. Pembrolizumab plus chemotherapy in metastatic non-small-cell lung cancer. N. Engl. J. Med. 2018, 378, 2078-2092. [CrossRef] [PubMed]

26. Rini, B.I.; Plimack, E.R.; Stus, V.; Gafanov, R.; Hawkins, R.; Nosov, D.; Pouliot, F.; Alekseev, B.; Soulières, D.; Melichar, B.; et al. Pembrolizumab plus axitinib versus sunitinib for advanced renal-cell carcinoma. N. Engl. J. Med. 2019, 380, 1116-1127. [CrossRef]

27. Chen, R.; Zinzani, P.L.; Fanale, M.A.; Armand, P.; Johnson, N.A.; Brice, P.; Radford, J.; Ribrag, V.; Molin, D.; Vassilakopoulos, T.P.; et al. Phase II study of the efficacy and safety of pembrolizumab for relapsed/refractory classic Hodgkin Lymphoma. J. Clin. Oncol. 2017, 35, 2125-2132. [CrossRef]

28. Cohen, E.E.W.; Soulières, D.; Le Tourneau, C.; Dinis, J.; Licitra, L.; Ahn, M.J.; Soria, A.; Machiels, J.P.; Mach, N.; Mehra, R.; et al. Pembrolizumab versus methotrexate, docetaxel, or cetuximab for recurrent or metastatic head-and-neck squamous cell carcinoma (KEYNOTE-040): A randomised, open-label, phase 3 study. Lancet 2019, 393, 156-167. [CrossRef]

29. Bellmunt, J.; de Wit, R.; Vaughn, D.J.; Fradet, Y.; Lee, J.-L.; Fong, L.; Vogelzang, N.J.; Climent, M.A.; Petrylak, D.P.; Choueiri, T.K.; et al. Pembrolizumab as Second-Line Therapy for Advanced Urothelial Carcinoma. N. Engl. J. Med. 2017, 376, 1015-1026. [CrossRef]

30. Balar, A.V.; Castellano, D.; O’Donnell, P.H.; Grivas, P.; Vuky, J.; Powles, T.; Plimack, E.R.; Hahn, N.M.; de Wit, R.; Pang, L.; et al. First-line pembrolizumab in cisplatin-ineligible patients with locally advanced and unresectable or metastatic urothelial cancer (KEYNOTE-052): A multicentre, single-arm, phase 2 study. Lancet Oncol. 2017, 18, 1483-1492. [CrossRef]

31. Zhu, A.X.; Finn, R.S.; Edeline, J.; Cattan, S.; Ogasawara, S.; Palmer, D.; Verslype, C.; Zagonel, V.; Fartoux, L.; Vogel, A.; et al. Pembrolizumab in patients with advanced hepatocellular carcinoma previously treated with sorafenib (KEYNOTE-224): A non-randomised, open-label phase 2 trial. Lancet Oncol. 2018, 19, 940-952. [CrossRef]

32. Fuchs, C.S.; Doi, T.; Jang, R.W.; Muro, K.; Satoh, T.; Machado, M.; Sun, W.; Jalal, S.I.; Shah, M.A.; Metges, J.P.; et al. Safety and efficacy of pembrolizumab monotherapy in patients with previously treated advanced gastric and gastroesophageal junction cancer: Phase 2 clinical KEYNOTE-059 trial. JAMA Oncol. 2018, 4, e180013. [CrossRef] [PubMed]

33. Shah, M.A.; Kojima, T.; Hochhauser, D.; Enzinger, P.; Raimbourg, J.; Hollebecque, A.; Lordick, F.; Kim, S.-B.; Tajika, M.; Kim, H.T.; et al. Efficacy and Safety of Pembrolizumab for Heavily Pretreated Patients With Advanced, Metastatic Adenocarcinoma or Squamous Cell Carcinoma of the Esophagus: The Phase 2 KEYNOTE-180 Study. JAMA Oncol. 2019, 5, 546-550. [CrossRef]

34. Chung, H.C.; Ros, W.; Delord, J.-P.; Perets, R.; Italiano, A.; Shapira-Frommer, R.; Manzuk, L.; Piha-Paul, S.A.; Xu, L.; Zeigenfuss, S.; et al. Efficacy and Safety of Pembrolizumab in Previously Treated Advanced Cervical Cancer: Results From the Phase II KEYNOTE-158 Study. J. Clin. Oncol. 2019, 37, 1470-1478. [CrossRef] [PubMed]

35. Nghiem, P.; Bhatia, S.; Lipson, E.J.; Sharfman, W.H.; Kudchadkar, R.R.; Brohl, A.S.; Friedlander, P.A.; Daud, A.; Kluger, H.M.; Reddy, S.A.; et al. Durable Tumor Regression and Overall Survival in Patients With Advanced Merkel Cell Carcinoma Receiving Pembrolizumab as First-Line Therapy. J. Clin. Oncol. 2019, 37, 693-702. [CrossRef] [PubMed]

36. Chung, H.C.; Lopez-Martin, J.A.; Kao, S.C.-H.; Miller, W.H.; Ros, W.; Gao, B.; Marabelle, A.; Gottfried, M.; Zer, A.; Delord, J.-P.; et al. Phase 2 study of pembrolizumab in advanced small-cell lung cancer (SCLC): KEYNOTE-158. J. Clin. Oncol. 2018, 36, 8506. [CrossRef] 
37. Rosenberg, J.E.; Hoffman-Censits, J.; Powles, T.; Van Der Heijden, M.S.; Balar, A.V.; Necchi, A.; Dawson, N.; O'Donnell, P.H.; Balmanoukian, A.; Loriot, Y.; et al. Atezolizumab in patients with locally advanced and metastatic urothelial carcinoma who have progressed following treatment with platinum-based chemotherapy: A single-arm, multicentre, phase 2 trial. Lancet 2016, 387, 1909-1920. [CrossRef]

38. Powles, T.; Durán, I.; van der Heijden, M.S.; Loriot, Y.; Vogelzang, N.J.; De Giorgi, U.; Oudard, S.; Retz, M.M.; Castellano, D.; Bamias, A.; et al. Atezolizumab versus chemotherapy in patients with platinum-treated locally advanced or metastatic urothelial carcinoma (IMvigor211): A multicentre, open-label, phase 3 randomised controlled trial. Lancet 2018, 391, 748-757. [CrossRef]

39. Rittmeyer, A.; Barlesi, F.; Waterkamp, D.; Park, K.; Ciardiello, F.; von Pawel, J.; Gadgeel, S.M.; Hida, T.; Kowalski, D.M.; Dols, M.C.; et al. Atezolizumab versus docetaxel in patients with previously treated non-small-cell lung cancer (OAK): A phase 3, open-label, multicentre randomised controlled trial. Lancet 2017, 389, 255-265. [CrossRef]

40. Socinski, M.A.; Jotte, R.M.; Cappuzzo, F.; Orlandi, F.; Stroyakovskiy, D.; Nogami, N.; Rodriguez-Abreu, D.; Moro-Sibilot, D.; Thomas, C.A.; Barlesi, F.; et al. Atezolizumab for first-line treatment of metastatic nonsquamous NSCLC. N. Engl. J. Med. 2018, 378, 2288-2301. [CrossRef]

41. Reck, M.; Mok, T.S.K.; Nishio, M.; Jotte, R.M.; Cappuzzo, F.; Orlandi, F.; Stroyakovskiy, D.; Nogami, N.; Rodríguez-Abreu, D.; Moro-Sibilot, D.; et al. Atezolizumab plus bevacizumab and chemotherapy in non-small-cell lung cancer (IMpower150): Key subgroup analyses of patients with EGFR mutations or baseline liver metastases in a randomised, open-label phase 3 trial. Lancet Respir. Med. 2019, 7, 387-401. [CrossRef]

42. Horn, L.; Mansfield, A.S.; Szczęsna, A.; Havel, L.; Krzakowski, M.; Hochmair, M.J.; Huemer, F.; Losonczy, G.; Johnson, M.L.; Nishio, M.; et al. First-line atezolizumab plus chemotherapy in extensive-stage small-cell lung cancer. N. Engl. J. Med. 2018, 379, 2220-2229. [CrossRef] [PubMed]

43. Schmid, P.; Adams, S.; Rugo, H.S.; Schneeweiss, A.; Barrios, C.H.; Iwata, H.; Dieras, V.; Hegg, R.; Im, S.A.; Shaw Wright, G.; et al. Atezolizumab and nab-paclitaxel in advanced triple-negative breast cancer. N. Engl. J. Med. 2018, 379, 2108-2121. [CrossRef] [PubMed]

44. Kaufman, H.L.; Russell, J.; Hamid, O.; Bhatia, S.; Terheyden, P.; D’Angelo, S.P.; Shih, K.C.; Lebbé, C.; Linette, G.P.; Milella, M.; et al. Avelumab in patients with chemotherapy-refractory metastatic Merkel cell carcinoma: A multicentre, single-group, open-label, phase 2 trial. Lancet Oncol. 2016, 17, 1374-1385. [CrossRef]

45. Patel, M.R.; Ellerton, J.; Infante, J.R.; Agrawal, M.; Gordon, M.; Aljumaily, R.; Britten, C.D.; Dirix, L.; Lee, K.-W.; Taylor, M.; et al. Avelumab in metastatic urothelial carcinoma after platinum failure (JAVELIN Solid Tumor): Pooled results from two expansion cohorts of an open-label, phase 1 trial. Lancet Oncol. 2018, 19, 51-64. [CrossRef]

46. Motzer, R.J.; Penkov, K.; Haanen, J.; Rini, B.; Albiges, L.; Campbell, M.T.; Venugopal, B.; Kollmannsberger, C.; Negrier, S.; Uemura, M.; et al. Avelumab plus axitinib versus sunitinib for advanced renal-cell carcinoma. N. Engl. J. Med. 2019, 380, 1103-1115. [CrossRef]

47. Antonia, S.J.; Villegas, A.; Daniel, D.; Vicente, D.; Murakami, S.; Hui, R.; Kurata, T.; Chiappori, A.; Lee, K.H.; De Wit, M.; et al. Overall survival with durvalumab after chemoradiotherapy in stage III NSCLC. N. Engl. J. Med. 2018, 379, 2342-2350. [CrossRef]

48. Massard, C.; Gordon, M.S.; Sharma, S.; Rafii, S.; Wainberg, Z.A.; Luke, J.; Curiel, T.J.; Colon-Otero, G.; Hamid, O.; Sanborn, R.E.; et al. Safety and Efficacy of Durvalumab (MEDI4736), an Anti-Programmed Cell Death Ligand-1 Immune Checkpoint Inhibitor, in Patients With Advanced Urothelial Bladder Cancer. J. Clin. Oncol. 2016, 34, 3119-3125. [CrossRef]

49. Havel, J.J.; Chowell, D.; Chan, T.A. The evolving landscape of biomarkers for checkpoint inhibitor immunotherapy. Nat. Rev. Cancer 2019, 19, 133-150. [CrossRef]

50. Camidge, D.R.; Doebele, R.C.; Kerr, K.M. Comparing and contrasting predictive biomarkers for immunotherapy and targeted therapy of NSCLC. Nat. Rev. Clin. Oncol. 2019, 16, 341-355. [CrossRef]

51. McCready, V.R. Milestones in nuclear medicine. Eur. J. Nucl. Med. 2000, 27, S49-S79. [CrossRef] [PubMed]

52. Hricak, H.; Choi, B.I.; Scott, A.M.; Sugimura, K.; Muellner, A.; Von Schulthess, G.K.; Reiser, M.F.; Graham, M.M.; Dunnick, N.R.; Larson, S.M. Global trends in hybrid imaging. Radiology 2010, 257, 498-506. [CrossRef] [PubMed] 
53. Shen, B.; Huang, T.; Sun, Y.; Jin, Z.; Li, X.F. Revisit 18F-fluorodeoxyglucose oncology positron emission tomography: "systems molecular imaging" of glucose metabolism. Oncotarget 2017, 8, 43536-43542. [CrossRef] [PubMed]

54. Delbeke, D.; Coleman, R.E.; Guiberteau, M.J.; Brown, M.L.; Royal, H.D.; Siegel, B.A.; Townsend, D.W.; Berland, L.L.; Parker, J.A.; Hubner, K.; et al. Procedure guideline for tumor imaging with 18F-FDG PET/CT 1.0. J. Nucl. Med. 2006, 47, 885-895.

55. Boellaard, R.; O’Doherty, M.J.; Weber, W.A.; Mottaghy, F.M.; Lonsdale, M.N.; Stroobants, S.G.; Oyen, W.J.G.; Kotzerke, J.; Hoekstra, O.S.; Pruim, J.; et al. FDG PET and PET/CT: EANM procedure guidelines for tumour PET imaging: Version 1.0. Eur. J. Nucl. Med. Mol. Imaging 2010, 37, 181-200. [CrossRef]

56. Boellaard, R.; Delgado-Bolton, R.; Oyen, W.J.G.; Giammarile, F.; Tatsch, K.; Eschner, W.; Verzijlbergen, F.J.; Barrington, S.F.; Pike, L.C.; Weber, W.A.; et al. FDG PET/CT: EANM procedure guidelines for tumour imaging: Version 2.0. Eur. J. Nucl. Med. Mol. Imaging 2015, 42, 328-354. [CrossRef]

57. Griffeth, L.K. Use of Pet/Ct Scanning in Cancer Patients: Technical and Practical Considerations. Baylor Univ. Med. Cent. Proc. 2005, 18, 321-330. [CrossRef]

58. Blumenthal, G.M.; Pazdur, R. Response rate as an approval end point in oncology: Back to the future. JAMA Oncol. 2016, 2, 780-781. [CrossRef]

59. Miller, A.B.; Hoogstraten, B.; Staquet, M.; Winkler, A. Reporting results of cancer treatment. Cancer 1981, 47, 207-214. [CrossRef]

60. Therasse, P.; Arbuck, S.G.; Eisenhauer, E.A.; Wanders, J.; Kaplan, R.S.; Rubinstein, L.; Verweij, J.; Van Glabbeke, M.; van Oosterom, A.T.; Christian, M.C.; et al. New guidelines to evaluate the response to treatment in solid tumors. European Organization for Research and Treatment of Cancer, National Cancer Institute of the United States, National Cancer Institute of Canada. J. Natl. Cancer Inst. 2000, 92, 205-216. [CrossRef]

61. Eisenhauer, E.A.; Therasse, P.; Bogaerts, J.; Schwartz, L.H.; Sargent, D.; Ford, R.; Dancey, J.; Arbuck, S.; Gwyther, S.; Mooney, M.; et al. New response evaluation criteria in solid tumours: Revised RECIST guideline (version 1.1). Eur. J. Cancer 2009, 45, 228-247. [CrossRef] [PubMed]

62. Monzon, J.G.; Hay, A.E.; McDonald, G.T.; Pater, J.L.; Meyer, R.M.; Chen, E.; Chen, B.E.; Dancey, J.E. Correlation of single arm versus randomised phase 2 oncology trial characteristics with phase 3 outcome. Eur. J. Cancer 2015, 51, 2501-2507. [CrossRef] [PubMed]

63. Vickers, A.J.; Ballen, V.; Scher, H.I. Setting the bar in phase II trials: The use of historical data for determining "go/no go" decision for definitive phase III testing. Clin. Cancer Res. 2007, 13, 972-976. [CrossRef] [PubMed]

64. Gan, H.K.; You, B.; Pond, G.R.; Chen, E.X. Assumptions of expected benefits in randomized phase III trials evaluating systemic treatments for cancer. J. Natl. Cancer Inst. 2012, 104, 590-598. [CrossRef] [PubMed]

65. Pinker, K.; Riedl, C.; Weber, W.A. Evaluating tumor response with FDG PET: Updates on PERCIST, comparison with EORTC criteria and clues to future developments. Eur. J. Nucl. Med. Mol. Imaging 2017, 44, 55-66. [CrossRef] [PubMed]

66. Young, H.; Baum, R.; Cremerius, U.; Herholz, K.; Hoekstra, O.; Lammertsma, A.A.; Pruim, J.; Price, P. Measurement of clinical and subclinical tumour response using [18F]- fluorodeoxyglucose and positron emission tomography: Review and 1999 EORTC recommendations. Eur. J. Cancer 1999, 35, 1773-1782. [CrossRef]

67. Wahl, R.L.; Jacene, H.; Kasamon, Y.; Lodge, M.A. From RECIST to PERCIST: Evolving considerations for PET response criteria in solid tumors. J. Nucl. Med. 2009, 50, 122S-150S. [CrossRef]

68. O, J.H.; Lodge, M.A.; Wahl, R.L. Practical PERCIST: A Simplified Guide to PET Response Criteria in Solid Tumors 1.0. Radiology 2016, 280, 576-584. [CrossRef]

69. Di Giacomo, A.M.; Danielli, R.; Guidoboni, M.; Calabrò, L.; Carlucci, D.; Miracco, C.; Volterrani, L.; Mazzei, M.A.; Biagioli, M.; Altomonte, M.; et al. Therapeutic efficacy of ipilimumab, an anti-CTLA-4 monoclonal antibody, in patients with metastatic melanoma unresponsive to prior systemic treatments: Clinical and immunological evidence from three patient cases. Cancer Immunol. Immunother. 2009, 58, 1297-1306. [CrossRef]

70. Chiou, V.L.; Burotto, M. Pseudoprogression and immune-related response in solid tumors. J. Clin. Oncol. 2015, 33, 3541-3543. [CrossRef]

71. Kataoka, Y.; Hirano, K. Which criteria should we use to evaluate the efficacy of immune-checkpoint inhibitors? Ann. Transl. Med. 2018, 6, 222. [CrossRef] [PubMed] 
72. Wolchok, J.D.; Hoos, A.; O’Day, S.; Weber, J.S.; Hamid, O.; Lebbé, C.; Maio, M.; Binder, M.; Bohnsack, O.; Nichol, G.; et al. Guidelines for the evaluation of immune therapy activity in solid tumors: Immune-related response criteria. Clin. Cancer Res. 2009, 15, 7412-7420. [CrossRef] [PubMed]

73. Nishino, M.; Giobbie-Hurder, A.; Gargano, M.; Suda, M.; Ramaiya, N.H.; Hodi, F.S. Developing a common language for tumor response to immunotherapy: Immune-related response criteria using unidimensional measurements. Clin. Cancer Res. 2013, 19, 3936-3943. [CrossRef] [PubMed]

74. Seymour, L.; Bogaerts, J.; Perrone, A.; Ford, R.; Schwartz, L.H.; Mandrekar, S.; Lin, N.U.; Litière, S.; Dancey, J.; Chen, A.; et al. iRECIST: Guidelines for response criteria for use in trials testing immunotherapeutics. Lancet Oncol. 2017, 18, e143-e152. [CrossRef]

75. Hodi, F.S.; Ballinger, M.; Lyons, B.; Soria, J.C.; Nishino, M.; Tabernero, J.; Powles, T.; Smith, D.; Hoos, A.; McKenna, C.; et al. Immune-modified response evaluation criteria in solid tumors (imrecist): Refining guidelines to assess the clinical benefit of cancer immunotherapy. J. Clin. Oncol. 2018, 36, 850-858. [CrossRef]

76. Sachpekidis, C.; Larribere, L.; Pan, L.; Haberkorn, U.; Dimitrakopoulou-Strauss, A.; Hassel, J.C. Predictive value of early 18F-FDG PET/CT studies for treatment response evaluation to ipilimumab in metastatic melanoma: Preliminary results of an ongoing study. Eur. J. Nucl. Med. Mol. Imaging 2015, 42, 386-396. [CrossRef]

77. Tan, A.C.; Emmett, L.; Lo, S.; Liu, V.; Kapoor, R.; Carlino, M.S.; Guminski, A.D.; Long, G.V.; Menzies, A.M. FDG-PET response and outcome from anti-PD-1 therapy in metastatic melanoma. Ann. Oncol. 2018, 29, 2115-2120. [CrossRef]

78. Kaira, K.; Higuchi, T.; Naruse, I.; Arisaka, Y.; Tokue, A.; Altan, B.; Suda, S.; Mogi, A.; Shimizu, K.; Sunaga, N.; et al. Metabolic activity by $18 \mathrm{~F}-\mathrm{FDG}-\mathrm{PET} / \mathrm{CT}$ is predictive of early response after nivolumab in previously treated NSCLC. Eur. J. Nucl. Med. Mol. Imaging 2018, 45, 56-66. [CrossRef]

79. Pai, C.C.S.; Huang, J.T.; Lu, X.; Simons, D.M.; Park, C.; Chang, A.; Tamaki, W.; Liu, E.; Roybal, K.T.; Seagal, J.; et al. Clonal Deletion of Tumor-Specific T Cells by Interferon- $\gamma$ Confers Therapeutic Resistance to Combination Immune Checkpoint Blockade. Immunity 2019, 50, 477-492. [CrossRef]

80. Bellevre, D.; Petyt, G.; Collet, G.; Hossein-foucher, C. [18F]-FDG-PET/CT early response to nivolumab in NSCLC. J. Nucl. Med. 2019, 57, 135. [CrossRef]

81. Grizzi, F.; Castello, A.; Lopci, E. Is it time to change our vision of tumor metabolism prior to immunotherapy? Eur. J. Nucl. Med. Mol. Imaging 2018, 45, 1072-1075. [CrossRef] [PubMed]

82. Fuser, D.; Hernandez-Aya, L.; Mhlanga, J.; Crandall, J.; Ash, L.; Wahl, R.; Chen, D. Evaluation of 18F-FDG-PET/CT for Response Assessment in Patients with Advanced Melanoma Treated with Pembrolizumab Checkpoint Inhibitor Monotherapy. J. Nucl. Med. 2018, 59, 133.

83. Evangelista, L.; Cuppari, L.; Menis, J.; Bonanno, L.; Reccia, P.; Frega, S.; Pasello, G. 18F-FDG PET/CT in non-small-cell lung cancer patients. Nucl. Med. Commun. 2019, 40, 802-807. [CrossRef] [PubMed]

84. Chang, B.; Huang, A.; Shang, C.; Palmer, K.; Schubert, E.; Xu, W.; Amaravadi, R.; Schuchter, L.; Mitchell, T.; Wherry, E.; et al. Evaluation of the anti-PD-1 flare response in patients with advanced melanoma using FDG PET/CT imaging and hematologic biomarkers. J. Nucl. Med. 2019, 60, 2019-2020.

85. Cho, S.Y.; Lipson, E.J.; Im, H.J.; Rowe, S.P.; Gonzalez, E.M.; Blackford, A.; Chirindel, A.; Pardoll, D.M.; Topalian, S.L.; Wahl, R.L. Prediction of response to immune checkpoint inhibitor therapy using early-time-point 18F-FDG PET/CT imaging in patients with advanced melanoma. J. Nucl. Med. 2017, 58, 1421-1428. [CrossRef] [PubMed]

86. Anwar,H.; Sachpekidis, C.; Winkler, J.; Kopp-Schneider, A.; Haberkorn, U.; Hassel, J.C.; Dimitrakopoulou-Strauss, A . Absolute number of new lesions on 18F-FDG PET/CT is more predictive of clinical response than SUV changes in metastatic melanoma patients receiving ipilimumab. Eur. J. Nucl. Med. Mol. Imaging 2018, 45, 376-383. [CrossRef]

87. Sachpekidis, C.; Anwar, H.; Winkler, J.; Kopp-Schneider, A.; Larribere, L.; Haberkorn, U.; Hassel, J.C.; Dimitrakopoulou-Strauss, A. The role of interim 18 F-FDG PET/CT in prediction of response to ipilimumab treatment in metastatic melanoma. Eur. J. Nucl. Med. Mol. Imaging 2018, 45, 1289-1296. [CrossRef]

88. Goldfarb, L.; Duchemann, B.; Chouahnia, K.; Zelek, L.; Soussan, M. Monitoring anti-PD-1-based immunotherapy in non-small cell lung cancer with FDG PET: Introduction of iPERCIST. EJNMMI Res. 2019, 9, 1-10. [CrossRef]

89. Aide, N.; Hicks, R.J.; Le Tourneau, C.; Lheureux, S.; Fanti, S.; Lopci, E. FDG PET/CT for assessing tumour response to immunotherapy: Report on the EANM symposium on immune modulation and recent review of the literature. Eur. J. Nucl. Med. Mol. Imaging 2019, 46, 238-250. [CrossRef] 
90. Garon, E.B.; Hellmann, M.D.; Rizvi, N.A.; Carcereny, E.; Leighl, N.B.; Ahn, M.J.; Eder, J.P.; Balmanoukian, A.S.; Aggarwal, C.; Horn, L.; et al. Five-year overall survival for patients with advanced non-small-cell lung cancer treated with pembrolizumab: Results from the phase I KEYNOTE-001 study. J. Clin. Oncol. 2019, 37, 2518-2527. [CrossRef]

91. Larkin, J.; Chiarion-Sileni, V.; Gonzalez, R.; Grob, J.J.; Rutkowski, P.; Lao, C.D.; Cowey, C.L.; Schadendorf, D.; Wagstaff, J.; Dummer, R.; et al. Five-year survival with combined nivolumab and ipilimumab in advanced melanoma. N. Engl. J. Med. 2019, 381, 1535-1546. [CrossRef] [PubMed]

92. Otoshi, T.; Nagano, T.; Tachihara, M.; Nishimura, Y. Possible biomarkers for cancer immunotherapy. Cancers 2019, 11, 935. [CrossRef] [PubMed]

93. Hwang, S.; Kwon, A.Y.; Jeong, J.Y.; Kim, S.; Kang, H.; Park, J.; Kim, J.H.; Han, O.J.; Lim, S.M.; An, H.J. Immune gene signatures for predicting durable clinical benefit of anti-PD-1 immunotherapy in patients with non-small cell lung cancer. Sci. Rep. 2020, 10, 1-10. [CrossRef] [PubMed]

94. Davis, A.A.; Patel, V.G. The role of PD-L1 expression as a predictive biomarker: An analysis of all US food and drug administration (FDA) approvals of immune checkpoint inhibitors. J. Immunother. Cancer 2019, 7, 278. [CrossRef]

95. Smit, E.F.; de Langen, A.J. Pembrolizumab for all PD-L1-positive NSCLC. Lancet 2019, 393, 1776-1778. [CrossRef]

96. Munari, E.; Zamboni, G.; Lunardi, G.; Marchionni, L.; Marconi, M.; Sommaggio, M.; Brunelli, M.; Martignoni, G.; Netto, G.J.; Hoque, M.O.; et al. PD-L1 Expression Heterogeneity in Non-Small Cell Lung Cancer: Defining Criteria for Harmonization between Biopsy Specimens and Whole Sections. J. Thorac. Oncol. 2018, 13, 1113-1120. [CrossRef]

97. Chen, D.S.; Mellman, I. Oncology meets immunology: The cancer-immunity cycle. Immunity 2013, 39, 1-10. [CrossRef]

98. Yonesaka, K.; Haratani, K.; Takamura, S.; Sakai, H.; Kato, R.; Takegawa, N.; Takahama, T.; Tanaka, K.; Hayashi, H.; Takeda, M.; et al. B7-h3 negatively modulates ctl-mediated cancer immunity. Clin. Cancer Res. 2018, 24, 2653-2664. [CrossRef]

99. Nebhan, C.A.; Johnson, D.B. Predictive biomarkers of response to immune checkpoint inhibitors in melanoma. Expert Rev. Anticancer Ther. 2020, 8049, 2019-2020. [CrossRef]

100. Petitprez, F.; Sun, C.-M.; Lacroix, L.; Sautès-Fridman, C.; de Reyniès, A.; Fridman, W.H. Quantitative Analyses of the Tumor Microenvironment Composition and Orientation in the Era of Precision Medicine. Front. Oncol. 2018, 8, 390. [CrossRef]

101. Tavaré, R.; Escuin-Ordinas, H.; Mok, S.; McCracken, M.N.; Zettlitz, K.A.; Salazar, F.B.; Witte, O.N.; Ribas, A.; Wu, A.M. An Effective Immuno-PET Imaging Method to Monitor CD8-Dependent Responses to Immunotherapy. Cancer Res. 2016, 76, 73-82. [CrossRef] [PubMed]

102. Rashidian, M.; Ingram, J.R.; Dougan, M.; Dongre, A.; Whang, K.A.; LeGall, C.; Cragnolini, J.J.; Bierie, B.; Gostissa, M.; Gorman, J.; et al. Predicting the response to CTLA-4 blockade by longitudinal noninvasive monitoring of CD8 T cells. J. Exp. Med. 2017, 214, 2243-2255. [CrossRef] [PubMed]

103. Loose, D.; Signore, A.; Staelens, L.; Vanden Bulcke, K.; Vermeersch, H.; Dierckx, R.A.; Bonanno, E.; Van De Wiele, C. 123I-Interleukin-2 uptake in squamous cell carcinoma of the head and neck carcinoma. Eur. J. Nucl. Med. Mol. Imaging 2008, 35, 281-286. [CrossRef]

104. Renard, V.; Staelens, L.; Signore, A.; Van Belle, S.; Dierckx, R.A.; Van De Wiele, C. Iodine-123-interleukin-2 scintigraphy in metastatic hypernephroma: A pilot study. Q. J. Nucl. Med. Mol. Imaging 2007, 51, 352-356. [PubMed]

105. Signore, A.; Lauri, C.; Auletta, S.; Anzola, K.; Galli, F.; Casali, M.; Versari, A.; Glaudemans, A.W.J.M. Immuno-Imaging to Predict Treatment Response in Infection, Inflammation and Oncology. J. Clin. Med. 2019, 8, 681. [CrossRef] [PubMed]

106. Markovic, S.N.; Galli, F.; Suman, V.J.; Nevala, W.K.; Paulsen, A.M.; Hung, J.C.; Gansen, D.N.; Erickson, L.A.; Marchetti, P.; Wiseman, G.A.; et al. Non-invasive visualization of tumor infiltrating lymphocytes in patients with metastatic melanoma undergoing immune checkpoint inhibitor therapy: A pilot study. Oncotarget 2018, 9, 30268-30278. [CrossRef] 
107. Beckford Vera, D.R.; Smith, C.C.; Bixby, L.M.; Glatt, D.M.; Dunn, S.S.; Saito, R.; Kim, W.Y.; Serody, J.S.; Vincent, B.G.; Parrott, M.C. Immuno-PET imaging of tumor-infiltrating lymphocytes using zirconium-89 radiolabeled anti-CD3 antibody in immune-competent mice bearing syngeneic tumors. PLoS ONE 2018, 13, e0193832. [CrossRef]

108. Higashikawa, K.; Yagi, K.; Watanabe, K.; Kamino, S.; Ueda, M.; Hiromura, M.; Enomoto, S. 64Cu-DOTA-anti-CTLA-4 mAb enabled PET visualization of CTLA-4 on the T-cell infiltrating tumor tissues. PLoS ONE 2014, 9, e109866. [CrossRef]

109. Ehlerding, E.B.; England, C.G.; Majewski, R.L.; Valdovinos, H.F.; Jiang, D.; Liu, G.; McNeel, D.G.; Nickles, R.J.; Cai, W. ImmunoPET Imaging of CTLA-4 Expression in Mouse Models of Non-small Cell Lung Cancer. Mol. Pharm. 2017, 14, 1782-1789. [CrossRef]

110. England, C.G.; Jiang, D.; Ehlerding, E.B.; Rekoske, B.T.; Ellison, P.A.; Hernandez, R.; Barnhart, T.E.; McNeel, D.G.; Huang, P.; Cai, W. 89 Zr-labeled nivolumab for imaging of T-cell infiltration in a humanized murine model of lung cancer. Eur. J. Nucl. Med. Mol. Imaging 2018, 45, 110-120. [CrossRef]

111. Natarajan, A.; Mayer, A.T.; Xu, L.; Reeves, R.E.; Gano, J.; Gambhir, S.S. Novel Radiotracer for ImmunoPET Imaging of PD-1 Checkpoint Expression on Tumor Infiltrating Lymphocytes. Bioconjug. Chem. 2015, 26, 2062-2069. [CrossRef] [PubMed]

112. Natarajan, A.; Patel, C.B.; Habte, F.; Gambhir, S.S. Dosimetry Prediction for Clinical Translation of 64Cu-Pembrolizumab ImmunoPET Targeting Human PD-1 Expression. Sci. Rep. 2018, 8, 633. [CrossRef] [PubMed]

113. Natarajan, A.; Mayer, A.T.; Reeves, R.E.; Nagamine, C.M.; Gambhir, S.S. Development of Novel ImmunoPET Tracers to Image Human PD-1 Checkpoint Expression on Tumor-Infiltrating Lymphocytes in a Humanized Mouse Model. Mol. Imaging Biol. 2017, 19, 903-914. [CrossRef]

114. Kelly, M.P.; Tavare, R.; Giurleo, J.T.; Makonnen, S.; Hickey, C.; Danton, M.A.; Arnold, T.C.; Ma, D.; Dai, J.; Pei, J.; et al. Abstract 3033: Immuno-PET detection of LAG-3 expressing intratumoral lymphocytes using the zirconium-89 radiolabeled fully human anti-LAG-3 antibody REGN3767. In Proceedings of the Cancer Research; American Association for Cancer Research (AACR): Philadelphia, PA, USA, 2018; Volume 78, p. 3033.

115. Jagoda, E.M.; Vasalatiy, O.; Basuli, F.; Opina, A.C.L.; Williams, M.R.; Wong, K.; Lane, K.C.; Adler, S.; Ton, A.T.; Szajek, L.P.; et al. Immuno-PET Imaging of the Programmed Cell Death-1 Ligand (PD-L1) Using a Zirconium-89 Labeled Therapeutic Antibody, Avelumab. Mol. Imaging 2019, 18. [CrossRef]

116. Lv, G.; Sun, X.; Qiu, L.; Sun, Y.; Li, K.; Liu, Q.; Zhao, Q.; Qin, S.; Lin, J. PET Imaging of Tumor PD-L1 Expression with a Highly Specific Nonblocking Single-Domain Antibody. J. Nucl. Med. 2020, 61, 117-122. [CrossRef] [PubMed]

117. Burvenich, I.J.G.; Parakh, S.; Lee, F.T.; Guo, N.; Liu, Z.; Gan, H.K.; Rigopoulos, A.; O’Keefe, G.J.; Gong, S.J.; Goh, Y.W.; et al. Molecular imaging of T cell co-regulator factor B7-H3 with 89Zr-DS-5573a. Theranostics 2018, 8, 4199-4209. [CrossRef] [PubMed]

118. Ulaner, G.; Sobol, N.; O’Donoghue, J.; Burnazi, E.; Staton, K.; Weber, W.; Lyashchenko, S.; Lewis, J.; Landgren, C.O. Preclinical development and First-in-human imaging of 89Zr-Daratumumab for CD38 targeted imaging of myeloma. J. Nucl. Med. 2019, 60, 203.

119. Toms, J.; Kogler, J.; Maschauer, S.; Daniel, C.; Schmidkonz, C.; Kuwert, T.; Prante, O. Targeting Fibroblast Activation Protein: Radiosynthesis and Preclinical Evaluation of an ${ }^{18}$ F-labeled FAP Inhibitor. J. Nucl. Med. 2020. [CrossRef]

120. Loktev, A.; Lindner, T.; Mier, W.; Debus, J.; Altmann, A.; Jäger, D.; Giesel, F.; Kratochwil, C.; Barthe, P.; Roumestand, C.; et al. A tumor-imaging method targeting cancer-associated fibroblasts. J. Nucl. Med. 2018, 59, 1423-1429. [CrossRef]

121. Prasetyanti, P.R.; Medema, J.P. Intra-tumor heterogeneity from a cancer stem cell perspective. Mol. Cancer 2017, 16, 41. [CrossRef]

122. Bu, L.; Baba, H.; Yoshida, N.; Miyake, K.; Yasuda, T.; Uchihara, T.; Tan, P.; Ishimoto, T. Biological heterogeneity and versatility of cancer- associated fibroblasts in the tumor. Oncogene 2018, 38, 4887-4901. [CrossRef]

123. Bailly, C.; Bodet-Milin, C.; Bourgeois, M.; Gouard, S.; Ansquer, C.; Barbaud, M.; Sébille, J.C.; Chérel, M.; Kraeber-Bodéré, F.; Carlier, T. Exploring tumor heterogeneity using PET imaging: The big picture. Cancers 2019, 11, 1282. [CrossRef] [PubMed] 
124. Bensch, F.; van der Veen, E.; Lub-de Hooge, M.; Jorritsma-Smit, A. 89Zr-atezolizumab imaging as a non-invasive approach to assess clinical response to PD-L1. Nat. Med. 2018, 24, 1852-1858. [CrossRef] [PubMed]

125. Niemeijer, A.N.; Leung, D.; Huisman, M.C.; Bahce, I.; Hoekstra, O.S.; van Dongen, G.A.M.S.; Boellaard, R.; $\mathrm{Du}, \mathrm{S}$.; Hayes, W.; Smith, R.; et al. Whole body PD-1 and PD-L1 positron emission tomography in patients with non-small-cell lung cancer. Nat. Commun. 2018, 9, 4664. [CrossRef] [PubMed]

126. Xing, Y.; Chand, G.; Liu, C.; Cook, G.J.R.; O’Doherty, J.; Zhao, L.; Wong, N.C.L.; Meszaros, L.K.; Ting, H.H.; Zhao, J. Early phase I study of a 99mTc-labeled anti-programmed death ligand-1 (PD-L1) single-domain antibody in SPECT/CT assessment of PD-L1 expression in non-small cell lung cancer. J. Nucl. Med. 2019, 60, 1213-1220. [CrossRef]

127. Wang, Y.; Zhou, S.; Yang, F.; Qi, X.; Wang, X.; Guan, X.; Shen, C.; Duma, N.; Vera Aguilera, J.; Chintakuntlawar, A.; et al. Treatment-Related Adverse Events of PD-1 and PD-L1 Inhibitors in Clinical Trials: A Systematic Review and Meta-analysis. JAMA Oncol. 2019, 5, 1008-1019. [CrossRef]

128. Haanen, J.B.A.G.; Carbonnel, F.; Robert, C.; Kerr, K.M.; Peters, S.; Larkin, J.; Jordan, K. ESMO Guidelines Committee Management of toxicities from immunotherapy: ESMO Clinical Practice Guidelines for diagnosis, treatment and follow-up. Ann. Oncol. Off. J. Eur. Soc. Med. Oncol. 2017, 28, iv119-iv142. [CrossRef]

129. Brahmer, J.R.; Lacchetti, C.; Schneider, B.J.; Atkins, M.B.; Brassil, K.J.; Caterino, J.M.; Chau, I.; Ernstoff, M.S.; Gardner, J.M.; Ginex, P.; et al. Management of immune-related adverse events in patients treated with immune checkpoint inhibitor therapy: American society of clinical oncology clinical practice guideline. J. Clin. Oncol. 2018, 36, 1714-1768. [CrossRef]

130. Martins, F.; Sofiya, L.; Sykiotis, G.P.; Lamine, F.; Maillard, M.; Fraga, M.; Shabafrouz, K.; Ribi, C.; Cairoli, A.; Guex-Crosier, Y.; et al. Adverse effects of immune-checkpoint inhibitors: Epidemiology, management and surveillance. Nat. Rev. Clin. Oncol. 2019, 16, 563-580. [CrossRef]

131. Mekki, A.; Dercle, L.; Lichtenstein, P.; Marabelle, A.; Michot, J.M.; Lambotte, O.; Le Pavec, J.; De Martin, E.; Balleyguier, C.; Champiat, S.; et al. Detection of immune-related adverse events by medical imaging in patients treated with anti-programmed cell death 1. Eur. J. Cancer 2018, 96, 91-104. [CrossRef]

132. Nishino, M.; Sholl, L.M.; Awad, M.M.; Hatabu, H.; Armand, P.; Hodi, F.S. Sarcoid-Like Granulomatosis of the Lung Related to Immune-Checkpoint Inhibitors: Distinct Clinical and Imaging Features of a Unique Immune-Related Adverse Event. Cancer Immunol. Res. 2018, 6, 630-635. [CrossRef] [PubMed]

133. Van Willigen, W.W.; Gerritsen, W.R.; Aarntzen, E.H.J.G. 18F-FDG PET/CT of Multiorgan Sarcoid-Like Reaction during Anti-PD-1 Treatment for Melanoma. Clin. Nucl. Med. 2019, 44, 905-906. [CrossRef] [PubMed]

134. Lyall, A.; Vargas, H.A.; Carvajal, R.D.; Ulaner, G. Ipilimumab-induced colitis on FDG PET/CT. Clin. Nucl. Med. 2012, 37, 629-630. [CrossRef]

135. Alabed, Y.Z.; Aghayev, A.; Sakellis, C.; Van den Abbeele, A.D. Pancreatitis Secondary to Anti-Programmed Death Receptor 1 Immunotherapy Diagnosed by FDG PET/CT. Clin. Nucl. Med. 2015, 40, e528-e529. [CrossRef] [PubMed]

136. Wachsmann, J.W.; Ganti, R.; Peng, F. Immune-mediated Disease in Ipilimumab Immunotherapy of Melanoma with FDG PET-CT. Acad. Radiol. 2017, 24, 111-115. [CrossRef] [PubMed]

137. Călugăreanu, A.; Rompteaux, P.; Bohelay, G.; Goldfarb, L.; Barrau, V.; Cucherousset, N.; Heidelberger, V.; Nault, J.-C.; Ziol, M.; Caux, F.; et al. Late onset of nivolumab-induced severe gastroduodenitis and cholangitis in a patient with stage IV melanoma. Immunotherapy 2019, 11, 1005-1013. [CrossRef] [PubMed]

138. Iravani, A.; Osman, M.; Hofman, M.; Weppler, A. Response assessment and immune- related adverse events as detected by FDG PET/CT in patients treated with combined CTLA-4 and PD-1 checkpoint inhibitors as first-line therapy in advanced melanoma. J. Nucl. Med. 2019, 60, 647.

139. Haratani, K.; Hayashi, H.; Chiba, Y.; Kudo, K.; Yonesaka, K.; Kato, R.; Kaneda, H.; Hasegawa, Y.; Tanaka, K.; Takeda, M.; et al. Association of immune-related adverse events with nivolumab efficacy in non-small cell lung cancer. JAMA Oncol. 2018, 4, 374-378. [CrossRef]

140. Nobashi, T.; Baratto, L.; Reddy, S.A.; Srinivas, S.; Toriihara, A.; Hatami, N.; Yohannan, T.K.; Mittra, E. Predicting Response to Immunotherapy by Evaluating Tumors, Lymphoid Cell-Rich Organs, and Immune-Related Adverse Events Using FDG-PET/CT. Clin. Nucl. Med. 2019, 44, e272-e279. [CrossRef]

141. Yamauchi, I.; Yasoda, A.; Matsumoto, S.; Sakamori, Y.; Kim, Y.H.; Nomura, M.; Otsuka, A.; Yamasaki, T.; Saito, R.; Kitamura, M.; et al. Incidence, features, and prognosis of immune-related adverse events involving the thyroid gland induced by nivolumab. PLOS ONE 2019, 14, e0216954. [CrossRef] 
142. Lang, N.; Dick, J.; Slynko, A.; Schulz, C.; Dimitrakopoulou-Strauss, A.; Sachpekidis, C.; Enk, A.H.; Hassel, J.C. Clinical significance of signs of autoimmune colitis in ${ }^{18} \mathrm{~F}$-fluorodeoxyglucose positron emission tomography-computed tomography of 100 stage-IV melanoma patients. Immunotherapy 2019, 11, 667-676. [CrossRef] [PubMed]

143. Sachpekidis, C.; Larribère, L.; Kopp-Schneider, A.; Hassel, J.C.; Dimitrakopoulou-Strauss, A. Can benign lymphoid tissue changes in 18F-FDG PET/CT predict response to immunotherapy in metastatic melanoma? Cancer Immunol. Immunother. 2019, 68, 297-303. [CrossRef] [PubMed]

144. Bianconi, F.; Palumbo, I.; Spanu, A.; Nuvoli, S.; Fravolini, M.L.; Palumbo, B. PET/CT Radiomics in Lung Cancer: An Overview. Appl. Sci. 2020, 10, 1718. [CrossRef]

145. Chen, R.Y.; Lin, Y.C.; Shen, W.C.; Hsieh, T.C.; Yen, K.Y.; Chen, S.W.; Kao, C.H. Associations of Tumor PD-1 Ligands, Immunohistochemical Studies, and Textural Features in ${ }^{18}$ F-FDG PET in Squamous Cell Carcinoma of the Head and Neck. Sci. Rep. 2018, 8, 105. [CrossRef]

146. Kirienko, M.; Cozzi, L.; Antunovic, L.; Lozza, L.; Fogliata, A.; Voulaz, E.; Rossi, A.; Chiti, A.; Sollini, M. Prediction of disease-free survival by the PET/CT radiomic signature in non-small cell lung cancer patients undergoing surgery. Eur. J. Nucl. Med. Mol. Imaging 2018, 45, 207-217. [CrossRef]

147. $\mathrm{Mu}, \mathrm{W}$.; Tunali, I. Radiomics of ${ }^{18}$ F-FDG PET/CT images predicts clinical benefit of advanced NSCLC patients to checkpoint blockade immunotherapy. Eur. J. Nucl. Med. Mol. Imaging 2020, 47, 1168-1182. [CrossRef]

148. Nardone, V.; Tini, P.; Pastina, P.; Botta, C.; Reginelli, A.; Carbone, S.F.; Giannicola, R.; Calabrese, G.; Tebala, C.; Guida, C.; et al. Radiomics predicts survival of patients with advanced non-small cell lung cancer undergoing PD-1 blockade using Nivolumab. Oncol. Lett. 2020, 19, 1559-1566. [CrossRef]

149. Id, T.K.; Everitt, S.; La Fontaine, M.D.; Van De Kamer, J.B.; Macmanus, M.P.; Vogel, W.V.; Callahan, J.; Sonke, J. F-fluorodeoxyglucose positron emission tomography radiomics features in non-small cell lung cancer: Are there any? PLoS ONE 2020, 15, e0228793.

(C) 2020 by the authors. Licensee MDPI, Basel, Switzerland. This article is an open access article distributed under the terms and conditions of the Creative Commons Attribution (CC BY) license (http://creativecommons.org/licenses/by/4.0/). 



\title{
The Role of Positron Emission Tomography in Clinical Management of Intraductal Papillary Mucinous Neoplasms of the Pancreas
}

\author{
Simone Serafini ${ }^{1}$, Cosimo Sperti ${ }^{1, *}$, Alessandra Rosalba Brazzale ${ }^{2}$, Diego Cecchin ${ }^{3}$, \\ Pietro Zucchetta ${ }^{3}$, Elisa Sefora Pierobon ${ }^{1}$, Alberto Ponzoni ${ }^{4}$, Michele Valmasoni ${ }^{1}$ and \\ Lucia Moletta ${ }^{1}$ \\ 1 Department of Surgery, Oncology and Gastroenterology, 3rd Surgical Clinic, University of Padua, \\ 35122 Padua, Italy; simone.serafini@ymail.com (S.S.); elisaseforapierobon@gmail.com (E.S.P.); \\ michele.valmasoni@gmail.com (M.V.); lmoletta@libero.it (L.M.) \\ 2 Department of Statistical Sciences, University of Padua, 35122 Padua, Italy; alessandra.brazzale@unipd.it \\ 3 Department of Medicine, Nuclear Medicine Unit, University of Padua, 35122 Padua, Italy; \\ diego.cecchin@unipd.it (D.C.); pietro.zucchetta@unipd.it (P.Z.) \\ 4 Department of Radiology, Padua General Hospital, 35121 Padua, Italy; alberto.ponzoni@aopd.veneto.it \\ * Correspondence: csperti@libero.it; Tel.: +39-049-821-8845; Fax: +39-049-821-8821
}

Received: 9 March 2020; Accepted: 23 March 2020; Published: 27 March 2020

\begin{abstract}
Intraductal papillary mucinous neoplasms (IPMNs) of the pancreas represent a heterogeneous group of tumors, increasingly diagnosed in clinical practice. An early differential diagnosis between malignant and benign lesions is crucial to patient management and the choice of surgery or observation. The therapeutic approach is currently based on a patient's clinical, biochemical, and morphological characteristics. The latest published International Consensus Guidelines (ICG) make no mention of the role of metabolic assessments of IPMNs. The aim of this study was to review the current literature, examining the role of 18-fluorodeoxyglucose (FDG) positron emission tomography (PET) in IPMN management. An extensive literature review was conducted according to the 2009 Preferred Reporting Items for Systematic Reviews and Meta-Analyses (PRISMA) guidelines, and 10 articles were analyzed in detail, focusing on the value of PET as opposed to other standard imaging criteria. Data were retrieved on 419 patients. The 18-FDG-PET proved more sensitive, specific, and accurate than the ICG criteria in detecting malignant IPMNs (reaching $80 \%$, 95\%, and $87 \%$ vs. $67 \%, 58 \%$, and $63 \%$, respectively). Metabolic assessments may be used as an additional tool for the appropriate management of patients with doubtful imaging findings.
\end{abstract}

Keywords: cystic tumor; International Consensus Guidelines; intraductal papillary mucinous neoplasms; pancreatic neoplasms; positron emission tomography

\section{Introduction}

Intraductal papillary mucinous neoplasms (IPMN) of the pancreas are papillary, mucin hyper-secreting lesions that originate from the epithelium of pancreatic ducts $[1,2]$. They account for $0.5 \%$ to $10 \%$ of all pancreatic exocrine neoplasms [3,4], but their real incidence is difficult to calculate because most of them are detected incidentally during imaging procedures performed for other medical reasons. Because of their tendency to become malignant, IPMNs are considered as precursors of pancreatic ductal adenocarcinomas. At the time of their diagnosis, they may appear as low-, medium-, high-grade dysplasia adenoma or invasive carcinoma [5]. The differential diagnosis between benign and malignant IPMN is crucial to patient management, particularly for the purpose of scheduling surgery appropriately. In fact, surgery offers an opportunity to prevent and potentially cure 
pancreatic adenocarcinoma, but it may represent an overtreatment in many cases, with a considerable morbidity and mortality even at experienced centers. Several international guidelines have been published on this topic: they classify IPMNs on the basis of the expected risk of malignancy and indicate which lesions should be resected and which ones should only be kept under observation [6-8]. Several clinical, biochemical, and radiological criteria are considered to establish the likelihood of IPMNs being malignant, and any consequent indication for surgery. The features that should prompt a surgical procedure in fit patients are termed "high-risk stigmata" [9]. "Worrisome features" are characteristics that warrant further investigation with endoscopic ultrasonography (EUS) to better stratify the associated risk [10,11]. The latest revision of the International Consensus Guidelines (ICG) [8] defines the presence of obstructive jaundice in patients with cystic lesions of the pancreatic head, enhanced mural nodules $\geq 5 \mathrm{~mm}$ in diameter, and a main pancreatic duct (MPD) size of $\geq 10 \mathrm{~mm}$ as "high risk stigmata". "Worrisome features" include cysts $\geq 3 \mathrm{~cm}$ in size, enhancing mural nodules $<5 \mathrm{~mm}$, thickened and enhanced cyst walls, a MPD 5-9 $\mathrm{mm}$ in size, abrupt changes in MPD caliber with distal pancreatic atrophy, lymphadenopathy, high serum levels of carbohydrate antigen (CA)19-9, and a rapid rate of cyst growth ( $>5 \mathrm{~mm}$ in 2 years) [8]. The ICG do not consider metabolic assessment of the lesions among the criteria for the risk stratification of IPMNs. Imaging with positron emission tomography using 18-fluorodeoxyglucose positron emission tomography (18-FDG PET) is based on the increased glucose uptake of neoplastic cells, which overexpress the main cell membrane glucose transporter GLUT-1, resulting in higher uptake of 18-FDG [12]. It is part of the standard diagnostic work-up in many neoplasms, but its role in the management of IPMN is not yet defined. Nonetheless, a growing number of studies focusing on the role of 18-FDG PET in the preoperative assessment of IPMNs have been published over the years. The aim of this review was to examine the recent English literature to clarify the value of 18-FDG PET in IPMN management.

\section{Materials and Methods}

An extensive review of the English literature was conducted according to the 2009 Preferred Reporting Items for Systematic Reviews and Meta-Analyses (PRISMA) guidelines [13]. A systematic search was performed in PubMed and Scopus on articles published from 1 January, 2000 to 31 October, 2019, using the terms: "pancreatic cyst", "intraductal papillary mucinous neoplasms", "pancreatic neoplasms", and "positron emission tomography". The related articles function was used to expand the search, and all related abstracts, studies, and citations were analyzed. Three authors screened all titles and abstracts for suitable articles, based on a set of eligibility criteria (Table 1).

Table 1. Summary of inclusion and exclusion criteria adopted. PET: positron emission tomography; IPMN: intraductal papillary mucinous neoplasms.

\begin{tabular}{ll}
\hline \multicolumn{1}{c}{ Inclusion Criteria } & \multicolumn{1}{c}{ Exclusion Criteria } \\
\hline Articles published from 01/01/2000 to 10/31/2019 & Case report or small case series $(<5$ patients $)$ \\
\hline Written in English & No PET data available \\
\hline Study in humans $>18$ years old & No radiological imaging data available \\
\hline & No histopathological proven IPMN \\
\hline
\end{tabular}

Only human studies written in English were considered. Case reports or small series ( $<5$ patients) were disregarded. The full text of the selected studies was obtained. The manuscripts were examined and the related references were checked for potentially relevant papers not identified by the initial screening process. Articles were rejected if imaging, PET data, or histological diagnoses were not clearly reported, or if it proved impossible to extract the data of interest from the text. The studies included in our review were analyzed in terms of: first author's name, year of publication, number of patients, histology, and malignant or benign preoperative diagnosis. The cut-off for the maximum 
standardized uptake value (SUV max) and the different radiological criteria adopted in each study were examined.

The sensitivity, specificity, positive predictive value (PPV), negative predictive value (NPV), and accuracy of 18-FDG PET, and radiological imaging in differentiating between malignant and benign IPMNs were calculated according to the following formulas: sensitivity $=$ true positive $(\mathrm{TP}) /[\mathrm{TP}+$ false negative $(\mathrm{FN})]$; specificity $=$ true negative $(\mathrm{TN}) /[\mathrm{TN}+$ false positive $(\mathrm{FP})] ; \mathrm{PPV}=\mathrm{TP} /[\mathrm{TP}+\mathrm{FP}]$; $\mathrm{NPV}=\mathrm{TN} /[\mathrm{TN}+\mathrm{FN}]$ and accuracy $=[\mathrm{TP}+\mathrm{TN}] /[\mathrm{TP}+\mathrm{TN}+\mathrm{FP}+\mathrm{FN}]$. The total numbers of TP, TN, $\mathrm{FP}$, and FN cases were collected patient by patient from each case series, when reported by the authors. In the event of different SUV max cut-offs or radiological criteria being adopted to discriminate benign from malignant lesions preoperatively, the cut-offs or criteria with the best accuracy were recorded and analyzed. A statistical analysis was performed to compare the summary measures (sensitivity, specificity, accuracy) of the two diagnostic tests (18-FDG PET and ICG criteria). The individual $95 \%$ confidence intervals of sensitivity and specificity for each diagnostic test were calculated using the Wilson score method. Pearson's chi-squared test was used to compare the sensitivities and specificities of 18-FDG PET and of the ICG. $p$-values $<0.05$ were considered statistically significant. The $95 \%$ confidence intervals for the difference and the ratio between the two sensitivities/specificities were calculated using the Gart and Nam score methods with skewness correction. Statistical analysis was performed using the NCSS 2019 Statistical Software [14].

\section{Results}

Overall, 986 records were identified. After screening the titles and abstracts according to our inclusion and exclusion criteria, 100 full-text articles were assessed for eligibility and 10 studies [15-24] were ultimately included in our critical appraisal (Figure 1). Patients' data were collected and analyzed on the basis of their radiological or metabolic diagnostic findings.

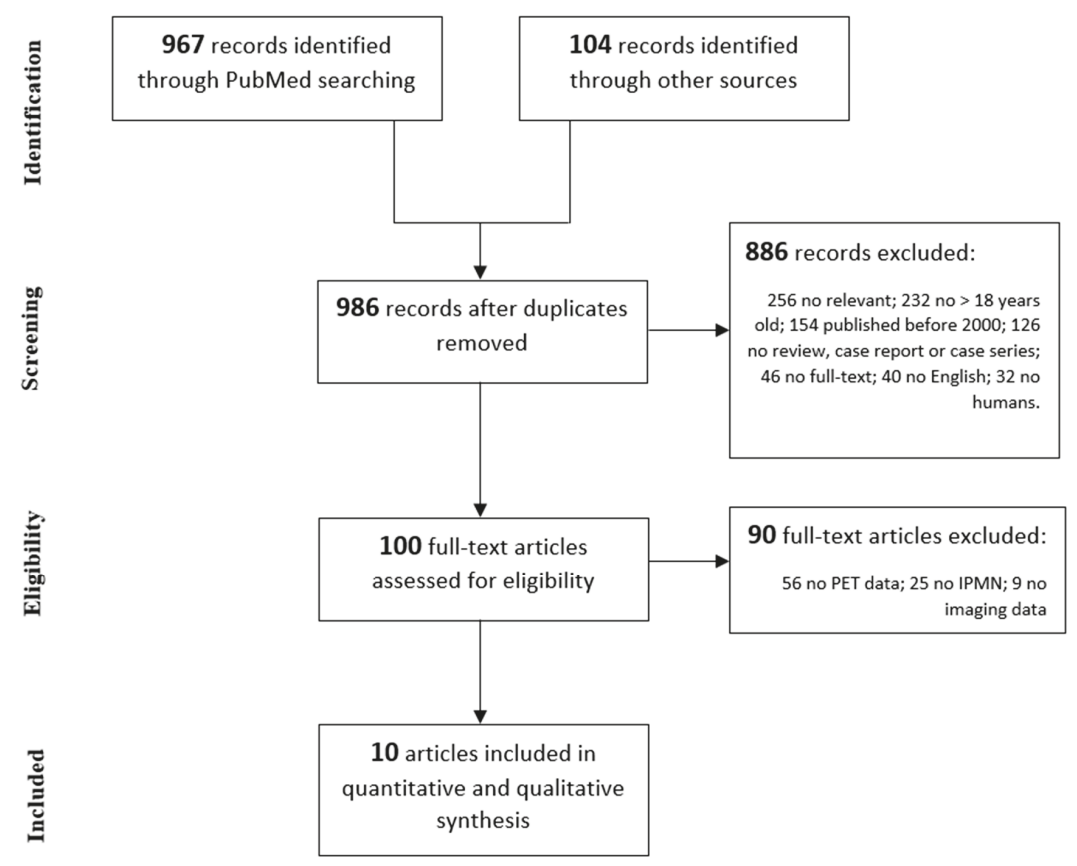

Figure 1. Literature review according to the 2009 Preferred Reporting Items for Systematic Reviews and Meta-Analyses PRISMA guidelines. 


\subsection{Diagnostic Accuracy of 18-FDG PET Scan to Identify Malignant IPMN}

All 10 studies examined the role of 18-FDG PET in the preoperative assessment of IPMNs. A preoperative 18-FDG PET was performed for a total of 419 patients with a histological diagnosis of IPMN; 210 of these neoplasms were benign and 209 were malignant. The findings of the single studies are listed in Table 2. The SUV max cut-offs used in the different studies to differentiate benign from malignant lesions ranged from 1.3 to 3.0 , with a mean value of 2.3 . Several authors $[15,16,18,19,24]$ used a SUV max of 2.5 as the cut-off for malignancy on the strength of previous reports in the literature. Takanami et al. [17] adopted a value of 2.3, based on a receiver operating characteristic curve (ROC) analysis in the cohort study balancing the sensitivity and specificity of different cut-offs. Saito et al. [20] chose a SUV max cut-off of 2.0 based on the number of patients diagnosed with high-grade dysplasia as opposed to invasive carcinoma. Furthermore, these authors acquired a late scan (120 min post-injection) to calculate a Retention Index $\left(\mathrm{RI}=\left(\mathrm{SUV}_{\text {delayed }}-\mathrm{SUV}_{\text {early }}\right) \times 100 / \mathrm{SUV}_{\text {early }}\right)$, which further increased the specificity of 18-FDG-PET from $88 \%$ to $94 \%$, without any loss of sensitivity. Roch et al. [21] considered as malignant any lesion with a SUV max higher than 3. Yamashita et al. [23] defined as malignant a focal uptake of radiotracer with a SUV $\max \geq 1$.3. Ohta et al. [22] did not specify a SUV max cut-off, but considered IPMNs as malignant if they had a greater metabolic uptake than the remnant gland.

Table 2. Summary of 18-FDG-PET results for Intraductal Papillary Mucinous Neoplasm. Tot: number of patients analyzed; Histo: number of patients with histopathological diagnosis available; SUV: Standardized Uptake Value Max; †: SUV max lesion > SUV max normal pancreas.

\begin{tabular}{|c|c|c|c|c|c|c|c|c|}
\hline Author & Year & $\begin{array}{l}\text { Patients/ } \\
\text { Histo }\end{array}$ & $\begin{array}{c}\text { Benign } \\
(n)\end{array}$ & $\begin{array}{c}\text { Malignant } \\
\text { (n) }\end{array}$ & $\begin{array}{c}\text { Sensitivity } \\
(\%)\end{array}$ & $\begin{array}{c}\text { Specificity } \\
(\%)\end{array}$ & $\begin{array}{c}\text { Accuracy } \\
(\%)\end{array}$ & $\begin{array}{c}\text { SUV } \\
\text { Cut-Of }\end{array}$ \\
\hline Hong et al. [15] & 2010 & $31 / 31$ & 15 & 16 & 100 & 87 & 94 & 2.5 \\
\hline Takanami et al. [17] & 2011 & $59 / 16$ & 7 & 9 & 78 & 100 & 88 & 2.3 \\
\hline Pedrazzoli et al. [18] & 2011 & $145 / 69$ & 33 & 36 & 83 & 100 & 91 & 2.5 \\
\hline Saito et al. [20] & 2013 & $48 / 48$ & 16 & 32 & 88 & 94 & 90 & 2.0 \\
\hline Roch et al. [21] & 2015 & $50 / 50$ & 37 & 13 & 62 & 95 & 86 & 3.0 \\
\hline Ohta et al. [22] & 2017 & $29 / 29$ & 9 & 20 & 90 & 78 & 86 & + \\
\hline
\end{tabular}

Overall, PET scanning identified 168 TP, 198 TN, 10 FP, 43 FN cases, resulting in a sensitivity, specificity, and accuracy of 79.6\% (73.7-84.5), 95.2\% (91.4-97.4) and 87.4\% (84.2-90.6), respectively. The PPV was 94.4\% (90.0-96.9), and the NPV 82.2\% (76.8-86.5). The Positive Likelihood Ratio (LR+) was 16.6 (9.0-30.4), and the Negative Likelihood Ratio (LR-) was $0.2(0.2-0.3)$.

\subsection{Diagnostic Accuracy of International Guidelines in Identifying Malignant IPMN}

Data on the diagnostic accuracy of the ICG criteria were available for a total of 412 patients with histological diagnosis of IPMN (207 benign and 196 malignant lesions). The findings of the single studies are given in Table 3. The radiological criteria used to differentiate benign from malignant IPMNs in the preoperative work-up varied. Hong et al. [15] identified as malignant a lesion with any of the following features: main duct type, marked dilation of the MPD ( $\geq 10 \mathrm{~mm}$ ), large mural nodules $(\geq 1 \mathrm{~cm})$, large cyst size $(\geq 3 \mathrm{~cm})$, irregular or septate cyst, calcifications, or patulous duodenal papilla. Tomimaru et al. [16] analyzed different radiological aspects (cyst size $\geq 3 \mathrm{~cm}$, dilation of MPD $\geq 7 \mathrm{~mm}$, presence/absence of mural nodules); in their series, the presence of mural nodules was the most accurate indicator (86\%) of malignancy, regardless of their size. Takanami et al. [17] and Saito et al. [20] 
respectively identified a dilation of the MPD $>5 \mathrm{~mm}$ and $\geq 7 \mathrm{~mm}$ as the most accurate radiological criterion of malignancy. Other authors [18,19,21,22,24] classified malignant IPMN according to ICG criteria [6,7], while Yamashita et al. [23] chose MPD $\geq 10 \mathrm{~mm}$ as the cut-off for distinguishing between benign and malignant lesions after performing a ROC analysis. The ICG criteria identified $152 \mathrm{TP}$, $108 \mathrm{TN}, 78 \mathrm{FP}$, and $74 \mathrm{FN}$ in all, resulting in a sensitivity, specificity, and accuracy of $67.3 \%$ (60.9-73.0), $58.1 \%$ (50.9-64.9), and 63.1\% (58.4-67.8), respectively. The PPV was 66.1\% (59.7-71.9), and the NPV $59.3 \%$ (52.1-66.2). The LR+ was $1.6(1.3-1.9)$, and the LR- was $0.6(0.5-0.7)$.

Table 3. Summary of diagnostic performance of International Guidelines. Tot: number of patients analyzed; Histo: number of patients with histopathological diagnosis available; $t$ : main duct-type, marked dilatation of the main pancreatic duct $(\geq 10 \mathrm{~mm})$, large mural nodule $(\geq 1 \mathrm{~cm})$, large cyst size $(\geq 3 \mathrm{~cm}$ ), irregular or septate cyst, calcification, or patulous duodenal papilla; MPD: Main Pancreatic Duct; SCG: Sendai Consensus guidelines; FCG: Fukuoka Consensus guidelines.

\begin{tabular}{|c|c|c|c|c|c|c|c|c|}
\hline Author & Year & Tot/Histo & $\begin{array}{c}\text { Benign } \\
\left(n^{\circ}\right)\end{array}$ & $\begin{array}{c}\text { Malignant } \\
\left(n^{\circ}\right)\end{array}$ & $\begin{array}{c}\text { Sensitivity } \\
\%\end{array}$ & $\begin{array}{c}\text { Specificity } \\
(\%)\end{array}$ & $\begin{array}{c}\text { Accuracy } \\
(\%)\end{array}$ & $\begin{array}{c}\text { Diagnostic } \\
\text { Criteria }\end{array}$ \\
\hline Hong et al. [15] & 2010 & $31 / 31$ & 15 & 16 & 93 & 60 & 77 & + \\
\hline Tomimaru et al. [16] & 2010 & $72 / 29$ & 15 & 14 & 93 & 80 & 86 & Mural nodule \\
\hline Takanami et al. [17] & 2011 & $59 / 16$ & 7 & 9 & 44 & 83 & 63 & $\mathrm{MPD}>5 \mathrm{~mm}$ \\
\hline Pedrazzoli et al. [18] & 2011 & $145 / 80$ & 36 & 44 & 93 & 22 & 61 & SCG \\
\hline Baiocchi et al. [19] & 2012 & $44 / 42$ & 30 & 12 & 100 & 22 & 43 & SCG \\
\hline Saito et al. [20] & 2013 & $32 / 32$ & 13 & 19 & 47 & 100 & 69 & $\mathrm{MPD} \geq 7 \mathrm{~mm}$ \\
\hline Roch et al. [21] & 2015 & $50 / 50$ & 37 & 13 & 92 & 27 & 44 & FCG \\
\hline Ohta et al. [22] & 2017 & $29 / 29$ & 9 & 20 & 50 & 67 & 55 & FCG \\
\hline Yamashita et al. [23] & 2019 & $79 / 38$ & 18 & 20 & 43 & 71 & 58 & $\mathrm{MPD} \geq 10 \mathrm{~mm}$ \\
\hline Hayashi et al. [24] & 2019 & $65 / 65$ & 28 & 37 & 71 & 85 & 77 & FCG \\
\hline Total & & $622 / 412$ & 208 & 204 & 67 & 58 & 63 & \\
\hline
\end{tabular}

3.3. Diagnostic Accuracy Comparison of 18-FDG PET and International Consensus Guidelines in Identifying Malignant IPMN

The 18-FDG PET shows an overall better performance than ICG criteria in identifying malignant IPMN. The sensitivity of 18-FG PET exceeds by about $18.4 \%$ (5.8-33.1) the sensitivity of the ICG criteria. This value increases to $63.9 \%(45.7-87.6)$ if the specificity is concerned. The two sensitivities differ by $12.4 \%$ (4.1-20.5), and the two specificities by 37.1 (29.5-44.8). Both differences are statistically significant at the $5 \%$ level $(p$-value $=0.004$ and $p$-value $<0.001)$. The overall accuracy differs by $24.3 \%(18.7-29.9)$. The better performance carries also over to the PPV (94.4\% vs. 66.1\%) and the NPV (82.2\% vs. 59.3\%). The positive and negative likelihood ratios of 18-FDG PET (16.6 and 0.2) highlight a much stronger association of 18-FDG PET with the presence/absence of the disease than ICG criteria (1.6 and 0.6).

\subsection{Statistical Analyses}

Reported in the Supplementary Materials (Text S1).

\section{Discussion}

IPMNs are intraductal tumors characterized by papillary proliferations of pancreatic mucin-producing epithelial cells $[25,26]$. They are classified according to their site of origin as: main duct (MD) type; branch duct (BD) type; and mixed type (MT) [27]. IPMNs can be divided histologically into four subtypes: intestinal, pancreatobiliary, oncocytic, and gastric $[3,28]$. Their prognosis varies greatly, depending on their site of origin and histological characteristics: MD-IPMNs carry the highest risk of malignant degeneration (28-81\%), followed by MT-IPMN (20-65\%), and BD-IPMN (7-42\%) [29-34]. Appropriate management of these lesions is still a controversial issue. In 2006, an international conference established the International Consensus Guidelines (ICG), proposing criteria 
for choosing between surgical and surveillance strategies [6]. These guidelines were subsequently revised in 2012, and again in 2017 [7,8]. Other guidelines were published by the American College of Gastroenterology (ACG) [35], the American Gastroenterological Association (AGA) [36], and the European Study Group on Cystic Tumors of the Pancreas [37] (Table 4). The criteria adopted by these latter guidelines have been examined in a few studies [38,39], but not as extensively as the ICG, which remain the standard reference all over the world.

Table 4. Surgical indication for branch duct (BD), main duct (MD), and mixed type (MT)-IPMN according to most recent guidelines. IPMN: Intraductal papillary mucinous neoplasms; ICG: International Consensus Guidelines; EU: European Study Group on Cystic Tumors of the Pancreas; ACG, American College of Gastroenterology; AGA, American Gastroenterological Association; MPD: main pancreatic duct; CA 19-9: cancer antigen 19-9; DM: diabetes mellitus.

\begin{tabular}{|c|c|c|c|c|}
\hline IPMN Type & ICG Guidelines (2017) [8] & EU Guidelines (2018) [37] & $\begin{array}{c}\text { ACG Guidelines } \\
\text { (2018) [35] }\end{array}$ & $\begin{array}{c}\text { AGA Guidelines } \\
\text { (2015) [36] }\end{array}$ \\
\hline & $\begin{array}{l}\text { High-risk stigmata: } \\
\text { Enhancing mural nodule } \\
\geq 5 \mathrm{~mm} \\
\text { MPD } \geq 10 \mathrm{~mm} \\
\text { Jaundice }\end{array}$ & $\begin{array}{l}\text { Absolute indications: } \\
\text { Solid mass } \\
\text { Enhancing mural nodule } \\
\geq 5 \mathrm{~mm} \\
\text { MPD } \geq 10 \mathrm{~mm} \\
\text { Jaundice }\end{array}$ & \multirow{2}{*}{$\begin{array}{l}\text { High-risk features: } \\
\text { Mural nodule/solid } \\
\text { component } \\
\text { MPD } \geq 5 \mathrm{~mm} \\
\text { MPD caliber change } \\
\text { and atrophy } \\
\text { Cyst size } \geq 3 \mathrm{~cm} \\
\text { Cyst growth } \\
\geq 3 \mathrm{~mm} / \text { year } \\
\text { Jaundice } \\
\text { Acute pancreatitis } \\
\text { Elevated serum CA } \\
19-9 \\
\text { New-onset DM }\end{array}$} & \multirow[b]{2}{*}{$\begin{array}{l}\text { High-risk } \\
\text { features: } \\
\text { Solid component } \\
\text { Dilated MPD } \\
\text { Cyst size } \geq 3 \mathrm{~cm}\end{array}$} \\
\hline BD-IPMN & $\begin{array}{l}\text { Worrisome features: } \\
\text { Growth } \geq 5 \mathrm{~mm} / 2 \text { years } \\
\text { Cyst size } \geq 3 \mathrm{~cm} \\
\text { Enhancing mural } \\
\text { nodule } \geq 5 \mathrm{~mm} \\
\text { Enhancing thickened } \\
\text { cyst wall } \\
\text { MPD } 5-9 \mathrm{~mm} \\
\text { MPD diameter change } \\
\text { Elevated serum CA 19-9 } \\
\text { Acute pancreatitis }\end{array}$ & $\begin{array}{l}\text { Relative indications: } \\
\text { Cyst growth } \geq 5 \mathrm{~mm} / \text { year } \\
\text { Cyst size } \geq 4 \mathrm{~cm} \\
\text { Enhancing mural nodule } \\
\geq 5 \mathrm{~mm} \\
\text { MPD 5-9.9 mm } \\
\text { Elevated serum CA 19-9 } \\
\text { New-onset DM } \\
\text { Acute pancreatitis }\end{array}$ & & \\
\hline MD/MT-IPMN & $\geq 1$ High-risk stigmata & All & Not mentioned & Not mentioned \\
\hline
\end{tabular}

The standard radiological work-up proposed in the ICG includes computed tomography (CT), magnetic resonance (MR), and magnetic resonance cholangiopancreatography (MRCP), which are very sensitive in differentiating between benign and malignant lesions, but suffer from a suboptimal specificity $[33,40,41]$. MRCP is more accurate than CT because it can reveal the anatomy of the pancreatic duct, its connections to the cysts in the branch duct, and any parietal nodules or filling defects $[18,42]$. Hence, it is considered the gold standard diagnostic procedure. The ICG recommend resection for fit patients with one or more high-risk stigmata. In patients with worrisome features, endoscopic ultrasound (EUS) or contrast-enhanced EUS are recommended for a more accurate detection of any malignant characteristics, such as mural nodules and intracystic structures. EUS also enables the fine needle aspiration of cystic fluid and biopsy for biochemical, genetic, and histological analyses [43,44]. Some drawbacks remain despite improvements in radiological imaging and accuracy of international guidelines. A recent literature review reported that up to $14 \%$ of IPMNs without worrisome features or high-risk stigmata were ultimately found to harbor high-grade dysplasia or malignancy on final histology [45]. The question is whether or not surgery is always the best solution for patients meeting the ICG criteria. Uehara et al. [34] described a case series of MD-IPMN patients with a low likelihood of malignancy that prompted them to challenge the recommendation for surgery, whereas three large retrospective studies validated the role of surgery for BD-IPMN [46-48]. Not all IPMNs presenting with worrisome features according to the ICG are actually malignant, as well not all small, asymptomatic BD-IPMN are benign [49-51]. In other words, we need better predictors, especially in elderly patients, or those unfit for prophylactic surgery. Preoperative metabolic characterization using 18-FDG-PET might improve patients' risk stratification and management. Moreover, 18-FDG-PET can help in the differential diagnosis of benign and malignant lesions regardless of an IPMN's morphological subtype. 
In the present literature review, PET achieved an overall sensitivity, specificity, and accuracy of $80 \%, 94 \%$, and $87 \%$, respectively. Unlike those of the ICG criteria, these values have remained constant over the years, and in the various studies reviewed, whatever the time of publication and standard radiological criteria adopted (Figure 2). The better performance of PET is also supported by a superior PPV (94.4\% vs. $66.1 \%)$ and NPV ( $82.2 \%$ vs. $59.3 \%)$. The positive and negative likelihood ratios of 18-FDG PET (16.6 and 0.2) highlight a much stronger association of 18-FDG PET with the presence/absence of the disease than ICG criteria (1.6 and 0.6).

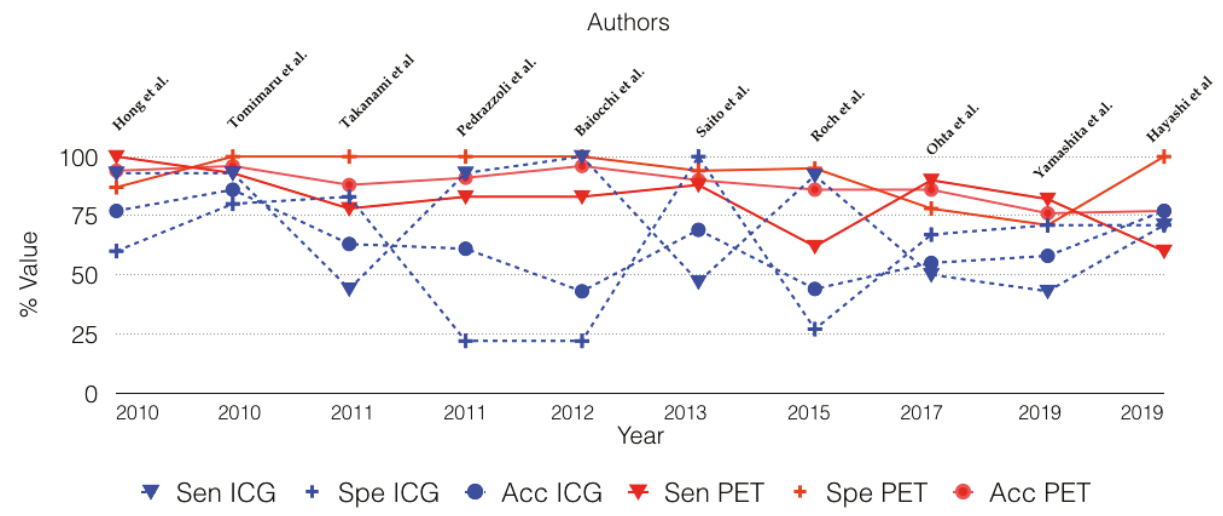

Figure 2. Changes in the sensitivity (SEN), specificity (SPE), and accuracy (Acc) of 18-FDG-PET (PET) and International Consensus Guidelines (ICG) over time.

Pedrazzoli et al. [18], and Baiocchi et al. [19] compared the diagnostic efficacy of 18-FDG PET with that of the Sendai consensus guidelines [7]. Pedrazzoli et al. [18] found that, with a SUV cut-off of 2.5, the sensitivity, specificity and accuracy of PET in detecting histologically-confirmed malignancies were $83.3 \%, 100 \%$, and $91.36 \%$ as opposed to $93.2 \%, 22.2 \%$, and $61.2 \%$ of the ICG criteria. Baiocchi et al. [19] reported similar results, with PET achieving a sensitivity, specificity and accuracy of $83 \%, 100 \%$, and $96 \%$ vs. $100 \%, 22 \%$, and $43 \%$ of the ICG. When Roch et al. [21] compared the diagnostic performance of PET with that of the Fukuoka consensus guidelines [8], the former reached a sensitivity and specificity of $61.5 \%$ and $94.6 \%$, while for the latter, the sensitivity and specificity were $92.3 \%$ and $27 \%$, respectively. When the ICG criteria were used in combination with PET/CT, this association resulted in a $77.8 \%$ sensitivity and $100 \%$ specificity for the diagnosis of malignant IPMN [21].

It is important to mention that 18-FDG PET can be liable to false-positive results due to inflammatory disease, as in pancreatitis. Dual time-points acquisition [20] could be useful in this context, because the SUV of malignant cells most often increases over time, whereas inflammatory lesions do not show an increasing uptake in late images. As seen in previous experiences [18,21], PET is an effective procedure for the differential diagnosis of benign and malignant lesions, but less useful than the ICG criteria in predicting the risk of benign IPMNs harboring malignancy. Metabolic assessments of IPMN may be used as an additional tool for the appropriate management of patients with doubtful imaging findings. In this subset of patients, the use of the 18-FDG PET in association with ICG criteria, should improve preoperative diagnostic accuracy for malignant lesions. However, the real usefulness of PET in clinical practice remains controversial because it seems clear that PET/CT improves accuracy in the diagnostic work-up of patients with IPMN, but it also increases the related costs, even if no cost-effectiveness comparisons have been published to date. Moreover, it is difficult to compare the related cost increase with the benefit offered by better patient stratification. Therefore, it seems reasonable to suggest that IPMN management be calibrated according to a patient's individual surgical risk: the high sensitivity and specificity of PET would be especially important when considering surgery for elderly, comorbid, and/or high-risk patients, when we need to strike the right balance between surgical risk and long-term 
benefit. It would be less useful for patients fit for surgery in whom resection is warranted according to the ICG criteria. The timing of PET in patients under surveillance remains to be established, but it might reasonably be performed when lesions reveal morphological changes or patients become symptomatic.

The clinical availability of integrated PET/MR scanners opens new perspectives in this field. The offline fusion of PET and MR images has proven already useful in the diagnosis of pancreatic tumors [52], and a pilot study in pancreatic cancer [53] proved the usefulness of combining various imaging biomarkers (Apparent Diffusion Coefficient, SUVmax, etc.), suggesting a possible role for 18-FDG-PET/MR in the management of IPMN.

\section{Conclusions}

The limited number of published experiences, the retrospective nature of most studies, the different definitions of SUV cut-offs, and the different guidelines considered all interfere with the interpretation of the results of our review. PET, nonetheless, appears to be a useful diagnostic tool in association with standard clinical and radiological criteria, especially when considering older comorbid patients at high surgical risk. The usefulness of PET in the management of IPMNs should be investigated in larger prospective studies.

Supplementary Materials: The following are available online at http://www.mdpi.com/2072-6694/12/4/807/s1, Text S1. Report of statistical analysis performed for two sample binary diagnostic tests. 18-FDG PET (Test 1) and ICG (Test 2).

Author Contributions: S.S., L.M., C.S. conceived the study and drafted the manuscript; A.R.B. performed statistical analysis; D.C., P.Z. contributed to implementation of the research and to the writing of the manuscript; E.S.P., A.P., M.V. conducted the literature review, data extraction, and analysis. All authors have read and agreed to the published version of the manuscript.

Funding: This research received no external funding.

Conflicts of Interest: The authors declare no conflict of interest.

\section{References}

1. Visser, B.C.; Muthusamay, V.R.; Mulvihill, S.J.; Coakley, F. Diagnostic Imaging of Cystic Pancreatic Neoplasms. Surg. Oncol. 2004, 13, 27-39. [CrossRef] [PubMed]

2. Yeoh, K.G. Cystic Neoplasms of the Pancreas. Ital. J. Gastroenterol. Hepatol. 1999, 31, 183-185. [PubMed]

3. Kloppel, G.; Solcia, E.; Longnecker, D.S.; Capella, C. Histological Typing of Tumours of the Exocrine Pancreas, 2nd ed.; Springer: Berlin, Germany, 1996.

4. Roggin, K.K.; Chennat, J.; Oto, A.; Noffsinger, A.; Briggs, A.; Matthews, J.B. Pancreatic Cystic Neoplasm. Curr. Probl. Surg. 2010, 47, 459-510. [CrossRef] [PubMed]

5. Demos, T.C.; Posniak, H.V.; Harmath, C.; Olson, M.C.; Aranha, G. Cystic Lesions of the Pancreas. Am. J. Roentgenol. 2002, 179, 1375-1388. [CrossRef] [PubMed]

6. Tanaka, M.; Chari, S.; Adsay, V.; Fernandez-del Castillo, C.; Falconi, M.; Shimizu, M.; Yamaguchi, K.; Yamao, K.; Matsuno, S. International Association of Pancreatology. International Consensus Guidelines for Management of Intraductal Papillary Mucinous Neoplasms and Mucinous Cystic Neoplasms of the Pancreas. Pancreatology 2006, 6, 17-32. [CrossRef]

7. Tanaka, M.; Fernández-del Castillo, C.; Adsay, V.; Chari, S.; Falconi, M.; Jang, J.-Y.; Kimura, W.; Levy, P.; Pitman, M.B.; Schmidt, C.M.; et al. International Consensus Guidelines 2012 for the Management of IPMN and MCN of the Pancreas. Pancreatology 2012, 12, 183-197. [CrossRef]

8. Tanaka, M.; Fernández-Del Castillo, C.; Kamisawa, T.; Jang, J.Y.; Levy, P.; Ohtsuka, T.; Salvia, R.; Shimizu, Y.; Tada, M.; Wolfgang, C.L. Revisions of International Consensus Fukuoka Guidelines for the Management of IPMN of the Pancreas. Pancreatology 2017, 17, 738-753. [CrossRef]

9. Pham, K.H.; Ramaswamy, M.R.; Hawkins, R.A. Advances in Positron Emission Tomography Imaging for the GI Tract. Gastrointest. Endosc. 2002, 55, S53-S63. [CrossRef]

10. Kim, T.H.; Song, T.J.; Hwang, J.; Yoo, K.; Lee, W.; Lee, K.; Dong, S.; Park, C.; Park, E.; Moon, J.; et al. Predictors of Malignancy in Pure Branch Duct Type Intraductal Papillary Mucinous Neoplasm of the Pancreas: A Nationwide Multicenter Study. Pancreatology 2015, 15, 405-410. [CrossRef] 
11. Ohno, E.; Hirooka, Y.; Itoh, A.; Ishigami, M.; Katano, Y.; Ohmiya, N.; Niwa, Y.; Goto, H. Intraductal Papillary Mucinous Neoplasms of the Pancreas: Differentiation of Malignant and Benign Tumors by Endoscopic Ultrasound Findings of Mural Nodules. Ann. Surg. 2009, 249, 628-634. [CrossRef]

12. Kitano, M.; Sakamoto, H.; Komaki, T.; Kudo, M. New Techniques and Future Perspective of EUS for The Differential Diagnosis Of Pancreatic Malignancies: Contrast Harmonic Imaging: Contrast Harmonic EUS In Pancreas. Dig. Endosc. 2011, 23, 46-50. [CrossRef] [PubMed]

13. Moher, D.; Liberati, A.; Tetzlaff, J.; Altman, D.G.; PRISMA Group. Preferred Reporting Items for Systematic Reviews and Meta-Analyses: The PRISMA Statement. BMJ 2009, 339, b2535. [CrossRef] [PubMed]

14. NCSS 2019 Statistical Software; NCSS, LLC: Kaysville, UT, USA, 2019.

15. Hong, H.-S.; Yun, M.; Cho, A.; Choi, J.-Y.; Kim, M.-J.; Kim, K.W.; Choi, Y.J.; Lee, J.D. The Utility of F-18 FDG PET/CT in the Evaluation of Pancreatic Intraductal Papillary Mucinous Neoplasm. Clin. Nucl. Med. 2010, 35, 776-779. [CrossRef] [PubMed]

16. Tomimaru, Y.; Takeda, Y.; Tatsumi, M.; Kim, T.; Kobayashi, S.; Marubashi, S.; Eguchi, H.; Tanemura, M.; Kitagawa, T.; Nagano, H.; et al. Utility of 2-[18F] Fluoro-2-Deoxy-D-Glucose Positron Emission Tomography in Differential Diagnosis of Benign and Malignant Intraductal Papillary-Mucinous Neoplasm of the Pancreas. Oncol. Rep. 2010, 24, 613-620. [CrossRef] [PubMed]

17. Takanami, K.; Hiraide, T.; Tsuda, M.; Nakamura, Y.; Kaneta, T.; Takase, K.; Fukuda, H.; Takahashi, S. Additional Value of FDG PET/CT to Contrast-Enhanced CT in the Differentiation between Benign and Malignant Intraductal Papillary Mucinous Neoplasms of the Pancreas with Mural Nodules. Ann. Nucl. Med. 2011, 25, 501-510. [CrossRef]

18. Pedrazzoli, S.; Sperti, C.; Pasquali, C.; Bissoli, S.; Chierichetti, F. Comparison of International Consensus Guidelines versus 18-FDG PET in Detecting Malignancy of Intraductal Papillary Mucinous Neoplasms of the Pancreas. Ann. Surg. 2011, 254, 971-976. [CrossRef]

19. Baiocchi, G.L.; Bertagna, F.; Gheza, F.; Grazioli, L.; Calanducci, D.; Giubbini, R.; Portolani, N.; Giulini, S.M. Searching for Indicators of Malignancy in Pancreatic Intraductal Papillary Mucinous Neoplasms: The Value of 18FDG-PET Confirmed. Ann. Surg. Oncol. 2012, 19, 3574-3580. [CrossRef]

20. Saito, M.; Ishihara, T.; Tada, M.; Tsuyuguchi, T.; Mikata, R.; Sakai, Y.; Tawada, K.; Sugiyama, H.; Kurosawa, J.; Otsuka, M.; et al. Use of F-18 Fluorodeoxyglucose Positron Emission Tomography with Dual-Phase Imaging to Identify Intraductal Papillary Mucinous Neoplasm. Clin. Gastroenterol. Hepatol. 2013, 11, 181-186. [CrossRef]

21. Roch, A.M.; Barron, M.R.; Tann, M.; Sandrasegar, K.; Hannaford, K.N.; Ceppa, E.P.; House, M.G.; Zyromski, N.J.; Nakeeb, A.; Schmidt, C.M. Does PET with CT Have Clinical Utility in the Management of Patients with Intraductal Papillary Mucinous Neoplasm? J. Am. Coll. Surg. 2015, 221, 48-56. [CrossRef]

22. Ohta, K.; Tanada, M.; Sugawara, Y.; Teramoto, N.; Iguchi, H. Usefulness of Positron Emission Tomography (PET)/Contrast-Enhanced Computed Tomography (CE-CT) in Discriminating between Malignant and Benign Intraductal Papillary Mucinous Neoplasms (IPMNs). Pancreatology 2017, 17, 911-919. [CrossRef]

23. Yamashita, Y.-I.; Okabe, H.; Hayashi, H.; Imai, K.; Nakagawa, S.; Nakao, Y.; Yusa, T.; Itoyama, R.; Yama, T.; Umesaki, N.; et al. Usefulness of 18-FDG PET/CT in Detecting Malignancy in Intraductal Papillary Mucinous Neoplasms of the Pancreas. Anticancer Res. 2019, 39, 2493-2499. [CrossRef] [PubMed]

24. Hayashi, M.; Mikata, R.; Horikoshi, T.; Senoo, J.; Kusakabe, Y.; Ohyama, H.; Yasui, S.; Uchida, Y.; Uchiyama, K.; Kishimoto, T.; et al. Diffusion-Weighted Magnetic Resonance Imaging and 18-Fluorodeoxglucose Positron Emission Tomography With Computed Tomography for Evaluating Malignancy of Branch Duct and Mixed Type Intraductal Papillary Mucinous Neoplasms of the Pancreas. Pancreas 2019, 48, e43-e45. [CrossRef] [PubMed]

25. Adsay, N.V. Cystic Neoplasia of the Pancreas: Pathology and Biology. J. Gastrointest. Surg. 2008, 12, 401-404. [CrossRef] [PubMed]

26. Talamini, M.A.; Pitt, H.A.; Hruban, R.H.; Boitnott, J.K.; Coleman, J.; Cameron, J.L. Spectrum of Cystic Tumors of the Pancreas. Am. J. Surg. 1992, 163, 117-123; discussion 123-124. [CrossRef]

27. Yang, E.Y.; Joehl, R.J.; Talamonti, M.S. Cystic Neoplasms of the Pancreas. J. Am. Coll. Surg. 1994, 179, 747-757.

28. Grützmann, R.; Niedergethmann, M.; Pilarsky, C.; Klöppel, G.; Saeger, H.D. Intraductal Papillary Mucinous Tumors of the Pancreas: Biology, Diagnosis, and Treatment. Oncologist 2010, 15, 1294-1309. [CrossRef]

29. Van Dam, J. EUS in Cystic Lesions of the Pancreas. Gastrointest. Endosc. 2002, 56 (Suppl. S4), S91-S93. [CrossRef] 
30. Sand, J.A.; Hyoty, M.K.; Mattila, J.; Dagorn, J.C.; Nordback, I.H. Clinical Assessment Compared with Cyst Fluid Analysis in the Differential Diagnosis of Cystic Lesions in the Pancreas. Surgery 1996, 119, 275-280. [CrossRef]

31. Kobari, M.; Egawa, S.; Shibuya, K.; Shimamura, H.; Sunamura, M.; Takeda, K.; Matsuno, S.; Furukawa, T. Intraductal Papillary Mucinous Tumors of the Pancreas Comprise 2 Clinical Subtypes: Differences in Clinical Characteristics and Surgical Management. Arch. Surg. 1999, 134, 1131-1136. [CrossRef]

32. Sakorafas, G.H.; Sarr, M.G. Cystic Neoplasms of the Pancreas; What a Clinician Should Know. Cancer Treat. Rev. 2005, 31, 507-535. [CrossRef]

33. Carbognin, G.; Zamboni, G.; Pinali, L.; Chiara, E.D.; Girardi, V.; Salvia, R.; Mucelli, R.P. Branch Duct IPMTs: Value of Cross-Sectional Imaging in the Assessment of Biological Behavior and Follow-Up. Abdom. Imaging 2006, 31, 320-325. [CrossRef] [PubMed]

34. Uehara, H.; Ishikawa, O.; Ikezawa, K.; Kawada, N.; Inoue, T.; Takakura, R.; Takano, Y.; Tanaka, S.; Takenaka, A. A Natural Course of Main Duct Intraductal Papillary Mucinous Neoplasm of the Pancreas with Lower Likelihood of Malignancy. Pancreas 2010, 39, 653-657. [CrossRef] [PubMed]

35. Elta, G.H.; Enestvedt, B.K.; Sauer, B.G.; Lennon, A.M. ACG Clinical Guideline: Diagnosis and Management of Pancreatic Cysts. Am. J. Gastroenterol. 2018, 113, 464-479. [CrossRef] [PubMed]

36. Vege, S.S.; Ziring, B.; Jain, R.; Moayyedi, P.; Adams, M.A.; Dorn, S.D.; Dudley-Brown, S.L.; Flamm, S.L.; Gellad, Z.F.; Gruss, C.B.; et al. American Gastroenterological Association Institute Guideline on the Diagnosis and Management of Asymptomatic Neoplastic Pancreatic Cysts. Gastroenterology 2015, 148, 819-822. [CrossRef] [PubMed]

37. European Study Group on Cystic Tumours of the Pancreas. European Evidence-Based Guidelines on Pancreatic Cystic Neoplasms. Gut 2018, 67, 789-804. [CrossRef]

38. Lekkerkerker, S.J.; Besselink, M.G.; Busch, O.R.; Verheij, J.; Engelbrecht, M.R.; Rauws, E.A.; Fockens, P.; van Hooft, J.E. Comparing 3 Guidelines on the Management of Surgically Removed Pancreatic Cysts with Regard to Pathological Outcome. Gastrointest. Endosc. 2017, 85, 1025-1031. [CrossRef]

39. Imbe, K.; Nagata, N.; Hisada, Y.; Takasaki, Y.; Sekine, K.; Mishima, S.; Kawazoe, A.; Tajima, T.; Shimbo, T.; Yanase, M.; et al. Validation of the American Gastroenterological Association Guidelines on Management of Intraductal Papillary Mucinous Neoplasms: More than 5 Years of Follow-Up. Eur. Radiol. 2018, 28, 170-178. [CrossRef]

40. Kalra, M.K.; Maher, M.M.; Sahani, D.V.; Digmurthy, S.; Saini, S. Current Status of Imaging in Pancreatic Diseases. J. Comput. Assist. Tomogr. 2002, 26, 661-675. [CrossRef]

41. Kauhanen, S.; Rinta-Kiikka, I.; Kemppainen, J.; Grönroos, J.; Kajander, S.; Seppänen, M.; Alanen, K.; Gullichsen, R.; Nuutila, P.; Ovaska, J. Accuracy of 18F-FDG PET/CT, Multidetector CT, and MR Imaging in the Diagnosis of Pancreatic Cysts: A Prospective Single-Center Study. J. Nucl. Med. 2015, 56, 1163-1168. [CrossRef]

42. Uribarri-Gonzalez, L.; Keane, M.G.; Pereira, S.P.; Iglesias-García, J.; Dominguez-Muñoz, J.E.; Lariño-Noia, J. Agreement among Magnetic Resonance Imaging/Magnetic Resonance Cholangiopancreatography (MRI-MRCP) and Endoscopic Ultrasound (EUS) in the Evaluation of Morphological Features of Branch Duct Intraductal Papillary Mucinous Neoplasm (BD-IPMN). Pancreatology 2018, 18, 170-175. [CrossRef]

43. Lewandrowski, K.; Lee, J.; Southern, J.; Centeno, B.; Warshaw, A. Cyst Fluid Analysis in the Differential Diagnosis of Pancreatic Cysts: A New Approach to the Preoperative Assessment of Pancreatic Cystic Lesions. Am. J. Roentgenol. 1995, 164, 815-819. [CrossRef] [PubMed]

44. Sperti, C.; Pasquali, C.; Guolo, P.; Polverosi, R.; Liessi, G.; Pedrazzoli, S. Serum Tumor Markers and Cyst Fluid Analysis Are Useful for the Diagnosis of Pancreatic Cystic Tumors. Cancer 1996, 78, 237-243. [CrossRef]

45. Hernandez, L.V.; Mishra, G.; Forsmark, C.; Draganov, P.V.; Petersen, J.M.; Hochwald, S.N.; Vogel, S.B.; Bhutani, M.S. Role of Endoscopic Ultrasound (EUS) and EUS-Guided Fine Needle Aspiration in the Diagnosis and Treatment of Cystic Lesions of the Pancreas. Pancreas 2002, 25, 222-228. [CrossRef] [PubMed]

46. Pelaez-Luna, M.; Chari, S.T.; Smyrk, T.C.; Takahashi, N.; Clain, J.E.; Levy, M.J.; Pearson, R.K.; Petersen, B.T.; Topazian, M.D.; Vege, S.S.; et al. Do Consensus Indications for Resection in Branch Duct Intraductal Papillary Mucinous Neoplasm Predict Malignancy? A Study of 147 Patients. Am. J. Gastroenterol. 2007, 102, 1759-1764. [CrossRef] 
47. Rodriguez, J.R.; Salvia, R.; Crippa, S.; Warshaw, A.L.; Bassi, C.; Falconi, M.; Thayer, S.P.; Lauwers, G.Y.; Capelli, P.; Mino-Kenudson, M.; et al. Branch-Duct Intraductal Papillary Mucinous Neoplasms: Observations in 145 Patients Who Underwent Resection. Gastroenterology 2007, 133, 72-79; quiz 309-310. [CrossRef] [PubMed]

48. Tang, R.S.; Weinberg, B.; Dawson, D.W.; Reber, H.; Hines, O.J.; Tomlinson, J.S.; Chaudhari, V.; Raman, S.; Farrell, J.J. Evaluation of the Guidelines for Management of Pancreatic Branch-Duct Intraductal Papillary Mucinous Neoplasm. Clin. Gastroenterol. Hepatol. 2008, 6, 815-819; quiz 719. [CrossRef]

49. Srinivasan, N.; Teo, J.-Y.; Chin, Y.-K.; Hennedige, T.; Tan, D.M.; Low, A.S.; Thng, C.H.; Goh, B.K.P. Systematic Review of the Clinical Utility and Validity of the Sendai and Fukuoka Consensus Guidelines for the Management of Intraductal Papillary Mucinous Neoplasms of the Pancreas. HPB 2018, 20, 497-504. [CrossRef]

50. Sperti, C.; Pasquali, C.; Chierichetti, F.; Liessi, G.; Ferlin, G.; Pedrazzoli, S. Value of 18-Fluorodeoxyglucose Positron Emission Tomography in the Management of Patients with Cystic Tumors of the Pancreas. Ann. Surg. 2001, 234, 675-680. [CrossRef]

51. Sperti, C.; Pasquali, C.; Decet, G.; Chierichetti, F.; Liessi, G.; Pedrazzoli, S. F-18-Fluorodeoxyglucose Positron Emission Tomography in Differentiating Malignant from Benign Pancreatic Cysts: A Prospective Study. J. Gastrointest. Surg. 2005, 9, 22-28; discussion 28-29. [CrossRef]

52. Nagamachi, S.; Nishii, R.; Wakamatsu, H.; Mizutani, Y.; Kiyohara, S.; Fujita, S.; Futami, S.; Sakae, T.; Furukoji, E.; Tamura, S.; et al. The Usefulness of 18F-FDG PET/MRI Fusion Image in Diagnosing Pancreatic Tumor: Comparison with 18F-FDG PET/CT. Ann. Nucl. Med. 2013, 27, 554-563. [CrossRef]

53. Chen, B.-B.; Tien, Y.-W.; Chang, M.-C.; Cheng, M.-F.; Chang, Y.-T.; Wu, C.-H.; Chen, X.-J.; Kuo, T.-C.; Yang, S.-H.; Shih, I.-L.; et al. PET/MRI in Pancreatic and Periampullary Cancer: Correlating Diffusion-Weighted Imaging, MR Spectroscopy and Glucose Metabolic Activity with Clinical Stage and Prognosis. Eur. J. Nucl. Med. Mol. Imaging 2016, 43, 1753-1764. [CrossRef] [PubMed]

(C) 2020 by the authors. Licensee MDPI, Basel, Switzerland. This article is an open access article distributed under the terms and conditions of the Creative Commons Attribution (CC BY) license (http://creativecommons.org/licenses/by/4.0/). 



\title{
FDG-PET Imaging for Hodgkin and Diffuse Large B-Cell Lymphoma-An Updated Overview
}

\author{
Conrad-Amadeus Voltin ${ }^{1, *}$, Jasmin Mettler ${ }^{1}$, Jirka Grosse ${ }^{2}$, Markus Dietlein ${ }^{1}$, \\ Christian Baues ${ }^{3}$, Christine Schmitz ${ }^{4}$, Peter Borchmann ${ }^{5}$, Carsten Kobe ${ }^{1}$ and Dirk Hellwig ${ }^{2}$ \\ 1 Department of Nuclear Medicine, Faculty of Medicine and University Hospital Cologne, University of \\ Cologne, 50937 Cologne, Germany; jasmin.mettler@uk-koeln.de (J.M.); markus.dietlein@uk-koeln.de (M.D.); \\ carsten.kobe@uk-koeln.de (C.K.) \\ 2 Department of Nuclear Medicine, University Hospital Regensburg, 93053 Regensburg, Germany; \\ jirka.grosse@ukr.de (J.G.); dirk.hellwig@ukr.de (D.H.) \\ 3 Department of Radiation Oncology and Cyberknife Center, Faculty of Medicine and University Hospital \\ Cologne, University of Cologne, 50937 Cologne, Germany; christian.baues@uk-koeln.de \\ 4 Department of Hematology, West German Cancer Center (WTZ), University Hospital Essen, University of \\ Duisburg-Essen, 45147 Essen, Germany; christine.schmitz@uk-essen.de \\ 5 Department of Internal Medicine I, Center for Integrated Oncology Aachen-Bonn-Cologne-Dusseldorf (CIO \\ ABCD), Faculty of Medicine and University Hospital Cologne, University of Cologne, \\ 50937 Cologne, Germany; peter.borchmann@uk-koeln.de \\ * Correspondence: conrad-amadeus.voltin@uk-koeln.de; Tel.: +49-221-478-7534
}

Received: 14 January 2020; Accepted: 3 March 2020; Published: 5 March 2020

\begin{abstract}
Since the mid-1990s, ${ }^{18}$ F-fluorodeoxglucose (FDG)-positron emission tomography (PET) in combination with computed tomography has come to play a prominent role in the management of malignant lymphomas. One of the first PET applications in oncology was the detection of lymphoma manifestations at staging, where it has shown high sensitivity. Nowadays, this imaging modality is also used during treatment to evaluate the individual chemosensitivity and adapt further therapy accordingly. If the end-of-treatment PET is negative, irradiation in advanced-stage Hodgkin lymphoma patients can be safely omitted after highly effective chemotherapy. Thus far, lymphoma response assessment has mainly been performed using visual criteria, such as the Deauville five-point scale, which became the international standard in 2014. However, novel measures such as metabolic tumor volume or total lesion glycolysis have recently been recognized by several working groups and may further increase the diagnostic and prognostic value of FDG-PET in the future.
\end{abstract}

Keywords: Hodgkin lymphoma; diffuse large B-cell lymphoma; positron emission tomography; staging; response assessment

\section{Introduction}

Hodgkin lymphoma is a hematologic malignancy with one of the best long-term outcomes after first-line treatment. Ongoing efforts to improve therapy effectiveness have resulted in a 5 -year relative survival rate of about $90 \%$ for patients diagnosed between the ages of 20 and 64 years [1]. Treatment is selected depending on individual factors and consists of chemotherapy alone or chemotherapy followed by irradiation [2]. Over the years, ${ }^{18} \mathrm{~F}$-fluorodeoxyglucose (FDG)-positron emission tomography (PET) in combination with computed tomography (CT) has proved a valuable tool for detecting manifestations of lymphoma at diagnosis, and is nowadays considered state-of-the-art. On the basis of stage and other clinical risk indicators, Hodgkin lymphoma can be divided into three different risk-groups, these being early-favorable, early-unfavorable, and advanced disease. While the early stages commonly respond well to a combination of doxorubicin, bleomycin, vinblastine, 
and dacarbazine (ABVD), advanced Hodgkin lymphoma is more effectively treated with bleomycin, etoposide, doxorubicin, cyclophosphamide, vincristine, procarbazine, and prednisone (BEACOPP), for instance in its dose-escalated form (eBEACOPP) [3-7]. However, genotoxic treatment can lead to several complications and might be associated with increased long-term morbidity, including secondary malignancies and cardiovascular disease. A large survey among 1149 Hodgkin lymphoma survivors showed that optimal lymphoma control and primary cure are of utmost importance from the patient's perspective [8]. In advanced stage, these goals can be most effectively achieved through an intensive chemotherapy regimen. However, as the group of patients with advanced Hodgkin lymphoma includes individuals with very different risk profiles, a substantial proportion of them, around $60 \%$ to $70 \%$, would presumably be over-treated by eBEACOPP [9]. Biomarkers to assess the tumor under treatment are therefore of great importance for identifying those patients who respond very well to chemotherapy and in whom treatment intensity can be reduced without compromising the primary cure rate. On the other hand, individuals showing poor response might require escalation of therapy. A growing body of evidence strongly supports the use of FDG-PET for assessing changes in tumor metabolism during Hodgkin lymphoma treatment. As shown by large randomized trials, decisions regarding escalation [4] or de-escalation of therapy [3,7] can safely be based on PET results. The outstanding performance of FDG-PET in Hodgkin lymphoma most probably arises from its ratio of neoplastic to reactive microenvironment cells, which differs significantly from other lymphoma subtypes $[10,11]$.

Diffuse large B-cell lymphoma (DLBCL) represents the most common subtype of non-Hodgkin lymphoma, accounting for 30\% to $40 \%$ of all newly diagnosed cases [12]. Despite significant advances in the management of this malignancy, cure rates are still generally lower than for Hodgkin lymphoma, with approximately one-third of all individuals failing front-line therapy. Due to its superior sensitivity in the detection of nodal and extra-nodal lymphoma manifestations, FDG-PET is strongly recommended for staging patients with DLBCL [13]. Moreover, several international trials have examined whether PET imaging may be used early during treatment to separate individuals needing therapy intensification from good responders, that is, candidates for de-escalation [14-18]. As standard treatment, patients receive six chemotherapy cycles of cyclophosphamide, doxorubicin, vincristine, and prednisone alongside the monoclonal antibody rituximab (R-CHOP). Unlike early-stage disease, which can be considered for brief chemotherapy [19], advanced DLBCL with high risk, as defined by the International Prognostic Index, may require intensified treatment, including central nervous system prophylaxis [20,21]. The role of radiotherapy is still under discussion in DLBCL. Large randomized trials have indicated that subsequent consolidative irradiation of former bulky sites in advanced-stage patients may have a positive effect on outcome [22,23].

This article presents an overview of current PET applications in Hodgkin lymphoma and DLBCL. Furthermore, recent developments regarding imaging-directed therapy concepts for these individuals as well as future directions are discussed.

\section{Initial Staging}

Accurate and reproducible staging is crucial to determine the most appropriate treatment in both Hodgkin lymphoma and DLBCL. With high sensitivity for involved lymph nodes and extra-nodal disease, FDG-PET has become established as the standard of care for assessing all FDG-avid lymphomas, and is recommended by the 2014 Lugano criteria [24], which updated the established Ann Arbor classification [25].

\subsection{Hodgkin Lymphoma}

In the randomized multi-center U.K. RATHL trial [26], FDG-PET upstaged 14\% of Hodgkin lymphoma patients, mainly due to bone marrow involvements, which had not been identified by biopsy. Downstaging occurred in $6 \%$ of cases, particularly as a result of splenomegaly with normal FDG uptake or enlarged but PET-negative lymph nodes. Several studies on Hodgkin lymphoma demonstrated that FDG-PET has a sensitivity even higher than biopsy for detecting bone marrow 
involvement. While bone marrow biopsy diagnoses about $5 \%$ of involvements, focal skeletal FDG lesions are seen in up to $20 \%$ of newly diagnosed patients [27-31]. The largest head-to-head comparison to date showed a sensitivity and negative predictive value (NPV) of $95 \%$ and $99.9 \%$, respectively, for FDG-PET when taking positive biopsy as the reference standard [32]. Moreover, Pedersen et al. reported a significantly inferior progression-free survival (PFS) for individuals with focal bone lesions, independent of chemotherapy type [33]. Based on the high level of scientific evidence, there is a broad consensus that bone marrow biopsy can be abandoned in Hodgkin lymphoma patients staged by FDG-PET. Importantly, diffusely increased skeletal FDG uptake is rarely associated with positive biopsy results, and should therefore not be considered a sign of lymphoma [34].

In the vast majority of patients with Hodgkin lymphoma, PET-detected skeletal lesions do not influence therapy selection, as only $<1 \%$ of otherwise early-stage individuals were found to have bone marrow involvement on FDG-PET [32]. In a study of Picardi et al., PET-based staging at least resulted in an improved event-free survival compared to a historical cohort. However, an improved overall survival (OS) could not be shown by a randomized prospective trial [35].

\section{2. $D L B C L$}

A retrospective analysis on DLBCL by Fuertes et al. reported upstaging in 15\% of patients after FDG-PET [36]. Further studies focusing on subjects with DLBCL found similar results for non-Hodgkin lymphoma and rarely observed PET-based downstaging $[37,38]$. It should be taken into consideration that FDG-PET has proven to be less sensitive for identifying bone marrow involvements in aggressive non-Hodgkin lymphoma as compared to Hodgkin lymphoma [39,40]. On the basis of data from one Canadian and two Danish centers, Alzahrani et al. determined a sensitivity and NPV of $60 \%$ and $91 \%$, respectively [41]. Similar findings emerged from a pooled analysis of the PETAL and OPTIMAL $>60$ study cohorts [42]. In this context, the limited sensitivity of PET can most likely be explained by the observation that positive bone marrow biopsy results of DLBCL patients are quite frequently associated with diffusely increased skeletal FDG uptake. On the other hand, the use of biopsy seems justified only when results could have a direct impact on treatment selection, for example in patients with limited-stage disease and lack of further risk factors. Importantly, bone marrow biopsy should be omitted in all individuals where involvement has already been proven with FDG-PET. Figure 1 shows a patient with multiple FDG-avid bone marrow lesions, which were missed by undirected biopsy.

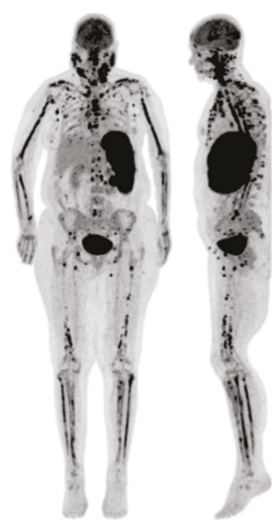

(a)

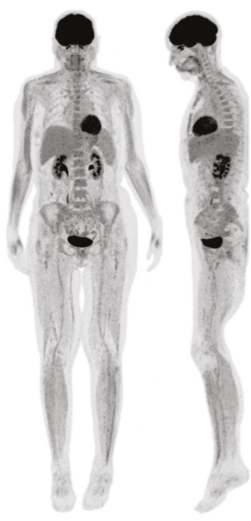

(b)

Figure 1. Female diffuse large B-cell lymphoma (DLBCL) patient with multifocal bone marrow involvement at baseline ${ }^{18} \mathrm{~F}$-fluorodeoxyglucose (FDG)-positron emission tomography (PET) (a), which was missed by undirected bone marrow biopsy and remitted completely after six cycles of chemoimmunotherapy (b). 


\section{Early Response Assessment}

In 2009, the so-called Deauville score was introduced to meet the growing need for simple and reproducible PET interpretation in the setting of early response assessment [43]. Based on a visual comparison of lesional FDG uptake with that in the reference regions mediastinal blood pool and liver, it classifies residual tissue from 1 to 5 (Table 1). Several trials have shown superior accuracy and inter-observer agreement of PET-based lymphoma response assessment when using the Deauville criteria and contributed to a rapid integration of the proposed method into reporting routine. Importantly, graded assessment made interpretation more flexible, as this approach allows adjustment of the cut-off between positive and negative results depending on the clinical context.

Table 1. Deauville five-point scale for therapy stratification in patients with FDG-avid lymphomas.

\begin{tabular}{|c|c|c|}
\hline Score & Criteria & Interpretation * \\
\hline 1 & No FDG uptake & \multirow{3}{*}{ CR } \\
\hline 2 & FDG uptake lower than or equal to the mediastinal blood pool & \\
\hline 3 & FDG uptake higher than the mediastinal blood pool but lower or equal to liver & \\
\hline 4 & FDG uptake moderately increased compared to the liver & \multirow[b]{2}{*}{$\mathrm{PR} / \mathrm{SD} / \mathrm{PD}$} \\
\hline 5 & $\begin{array}{l}\text { FDG uptake markedly increased compared to the liver and/or new sites } \\
\text { of disease }\end{array}$ & \\
\hline
\end{tabular}

* Response to treatment as defined by the Lugano classification [24]. CR, complete metabolic response; PR, partial metabolic response; $\mathrm{SD}$, stable disease; $\mathrm{PD}$, progressive disease.

\subsection{Hodgkin Lymphoma}

The key question of studies on early-stage Hodgkin lymphoma has been whether irradiation can be abandoned in the case of complete metabolic response after chemotherapy. In the U.K. RAPID trial [44], 602 stage IA and IIA patients without bulky mediastinal disease underwent PET imaging after three cycles of ABVD. Individuals with a negative PET scan, defined by Deauville scores 1 and 2, were randomly assigned to no further treatment or $30 \mathrm{~Gy}$ of involved-field irradiation. At a median follow-up of 60 months, patients receiving radiotherapy had a 3-year PFS of $94.6 \%$ compared to $90.8 \%$ in the non-irradiated group. The study did not prove non-inferiority of chemotherapy alone, as it was designed to exclude a difference in 3-year PFS of more than 7\%. Thus, based on the data available thus far, we would not generally recommend omitting consolidative radiotherapy in PET-negative individuals after three courses of ABVD.

The H10 trial randomized 1950 patients with limited-stage favorable and unfavorable Hodgkin lymphoma to PET-guided or standard treatment with three to four cycles of ABVD plus involved-node radiotherapy [4]. In the experimental arm, PET scans were performed after two courses of chemotherapy (PET-2) and evaluated using the mediastinal blood pool as cut-off. Individuals with positive PET-2 received two cycles of eBEACOPP followed by involved-node radiotherapy, which resulted in a significantly improved 5-year PFS (90.6\%) compared to standard treatment (77.4\%). After a second randomization, PET-negative individuals either underwent combined-modality treatment or continued with ABVD alone. The latter arm had to be terminated after preplanned interim analysis due to a high number of relapses with the strategy omitting irradiation. Here again, the omission of radiotherapy in PET-negative patients appeared questionable.

Finally, the German Hodgkin Study Group (GHSG) conducted HD16 as a PET-driven trial for early-stage Hodgkin lymphoma [45]. In this study, patients either received standard combined-modality treatment with two courses of ABVD plus $20 \mathrm{~Gy}$ of involved-field radiotherapy or were assigned to the experimental arm where PET-negative individuals underwent no further treatment after chemotherapy. Any residual tissue showing an FDG uptake greater than the mediastinal blood pool was rated PET-positive. At a median follow-up of 45 months, PET-negative patients after combined-modality treatment had a 5 -year PFS of $93.4 \%$ versus $86.1 \%$ with ABVD alone. Since PET-guided omission of 
irradiation resulted in worse disease control, the primary study objective was not achieved. However, among the 693 subjects receiving combined-modality treatment, PET-2 was predictive for therapy failure with a 5-year PFS of $93.2 \%$ in PET-negative patients versus $88.4 \%$ in the PET-positive group.

Based on the published data, radiotherapy should be generally recommended in limited-stage favorable Hodgkin lymphoma after treatment with two cycles of ABVD. Moreover, as revealed by the H10 trial, an intensification of therapy to eBEACOPP must be considered in patients with positive PET results. For intermediate-stage disease, the question for or against irradiation after two cycles of eBEACOPP and two courses ABVD has not yet been resolved. Here, we excitedly await the final results of the GHSG HD17 trial.

In advanced-stage Hodgkin lymphoma, several large studies strongly support the use of FDG-PET for tailoring treatment intensity. The RATHL trial included 1214 patients with stage IIB to IV or high-risk stage IIA disease and tested omission of bleomycin in ABVD cycles three to six after negative PET-2 using the liver as cut-off for PET interpretation [6]. Although results fell short of the prespecified non-inferiority margin, it should be noted that de-escalation of chemotherapy reduced the incidence of pulmonary side-effects without significant loss in efficacy. Individuals with Deauville scores 4 and 5 received either an accelerated BEACOPP version or eBEACOPP and had a 3-year PFS of $67.5 \%$. Quite similar results emerged from the Italian phase III HD 0607 study, where treatment was escalated to BEACOPP in the case of positive PET-2. For these patients, the investigators reported a 3-year PFS of 57\% [46]. The Lymphoma Study Association AHL2011 study examined de-escalation to ABVD in PET-negative patients after two cycles of BEACOPP and reported a 5-year PFS of $85.7 \%$ in the PET-driven arm versus $86.2 \%$ for continued treatment with BEACOPP $(p=0.65)$ [47]. Therefore, Casasnovas et al. concluded that FDG-PET after two cycles of induction BEACOPP can safely guide therapy in advanced-stage Hodgkin lymphoma and allows for the switching to ABVD in early responders without impairment of disease control.

Another de-escalation strategy was examined in the GHSG HD18 trial on advanced-stage Hodgkin lymphoma [7]. In patients receiving eBEACOPP from the beginning, negative PET-2 allowed shortening of treatment from six or eight to only four courses eBEACOPP, with a 5-year PFS of $90.8 \%$ and $92.2 \%$, respectively. Importantly, severe infections and organ toxicities occurred significantly less often in subjects receiving four cycles of eBEACOPP. To avoid undertreatment, patients in the HD18 trial were randomized, rating a Deauville score of 3 as PET-positive. However, a further analysis revealed that only Deauville scores of 4 or higher signify a relevant risk regarding survival for individuals treated with eBEACOPP upfront, whereas Deauville scores of 1, 2, and 3 should be considered PET-negative [48].

\section{2. $D L B C L$}

In aggressive non-Hodgkin lymphoma, there are only few randomized studies investigating the role of interim PET as an early biomarker for treatment success or failure. The vast majority of trials on DLBCL have examined whether PET-positive patients benefit from therapy escalation. Depending on the timing of PET imaging, treatment was adapted after two to four therapy courses. However, differences in image evaluation and the absence of a control group made it difficult to draw definitive conclusions regarding potential benefits of PET-guided treatment for this lymphoma subset.

In their congress abstract on a study comprising 65 patients with early-stage DLBCL [49], Canadian researchers reported about the value of FDG-PET after three courses R-CHOP. Individuals with positive PET results received a fourth chemoimmunotherapy cycle instead of standard irradiation and were observed to have a superior 3 -year PFS of $92 \%$ versus $60 \%$ when using radiotherapy alone. The German PETAL trial calculated the percentage change in maximum standardized uptake value $\left(\mathrm{SUV}_{\max }\right)$ between baseline and follow-up $\left(\Delta \mathrm{SUV}_{\max }\right)$ for response stratification after two courses of R-CHOP and demonstrated semi-quantitative interpretation to be superior as compared with visual assessment [17,18]. Most of the 812 non-Hodgkin lymphoma patients recruited had DLBCL. All individuals with CD20-positive lymphoma and negative PET-2 were randomly assigned to four more cycles of R-CHOP either with or without two additional rituximab doses. Because the latter did 
not influence clinical outcome, therapy can be limited to six cycles of R-CHOP in the case of negative PET-2 without a loss of efficacy. In contrast, PET positivity was an independent biomarker for poor outcome that could not, however, be improved by treatment intensification.

Another prospective study on DLBCL, the PET-guided GAINED trial, compared obinutuzumab to rituximab plus chemotherapy in treatment-naïve patients younger than 60 years [50]. Individuals showing an early good response received the scheduled immunochemotherapy according to initial randomization, whereas slowly responding patients were treated with two courses of high-dose methotrexate followed by autologous stem-cell transplantation. Non-responders underwent salvage treatment according to local investigators. Based on the exploratory results of the LNH2007-3B study [51], PET positivity was defined as a decline of SUV $\max$ in an FDG-avid target lesion of at least $66 \%$ or $70 \%$ from baseline to PET after 2 and 4 cycles of chemotherapy, respectively. This trial aimed to validate the $\triangle \mathrm{SUV}_{\max }$-driven consolidation in young patients with high-risk disease.

Finally, a recent analysis including 1977 DLBCL patients from different trials showed that FDG-PET is able to clearly discriminate between responding and non-responding individuals after two, three, or four chemotherapy cycles, while the optimal timing for identification of responders is after two courses [52]. Moreover, the authors recommended PET scanning after four cycles to identify poorly responding patients. Therefore, FDG-PET following chemotherapy courses two and four appears most suitable for future response-adapted trials and therapy regimens in aggressive non-Hodgkin lymphoma.

\section{Late Response Evaluation}

Before the era of FDG-PET, CT imaging was the standard modality for end-of-treatment stratification after chemotherapy. However, CT evaluation suffered from the inability of distinguishing between fibrotic masses and active residual lymphoma tissue, causing high rates of unconfirmed response [53]. Since the late 1990s, a number of studies have consistently demonstrated the high NPV of end-of-treatment PET and underlined its value for this application, particularly in Hodgkin lymphoma [54-60]. Spanish researchers examined 37 subjects with follicular lymphoma, as well as 72 Hodgkin lymphoma and 72 DLBCL patients, in order to compare the diagnostic performance of $\mathrm{CT}$ and FDG-PET/CT [13]. While CT was concordant with the defined reference methods in only $78 \%$ of cases, FDG-PET/CT showed an excellent agreement rate of $97.8 \%$. In addition, PET imaging may be used during follow-up in certain situations. Here, it should be kept in mind that the value of both negative and positive results is limited. Even though FDG-PET might be able to detect relapses earlier than conventional imaging, it has been reported that only one- to two-thirds of positive scans actually indicate relapse and that that there is a significant number of false-negative results in this setting [60-63]. Moreover, the cost-effectiveness of routine PET scanning during follow-up could not be demonstrated [64]. This is why we recommend restricting the use of FDG-PET to selected cases, such as suspected relapse.

\subsection{Hodgkin Lymphoma}

In the GHSG HD15 trial on advanced-stage Hodgkin lymphoma, FDG-PET showed a NPV of $94.1 \%$ for tumor recurrence after BEACOPP chemotherapy [59]. The 4-year PFS of PET-negative patients who did not receive further treatment was $91.5 \%$ [65]. As a result of the HD15 study, radiotherapy is limited to PET-positive residual tissue in advanced-stage Hodgkin lymphoma after eBEACOPP therapy. Moreover, recently presented data have shown that consolidative irradiation might also be omitted in advanced-stage disease after treatment with ABVD [66]. Nevertheless, it is important to keep in mind that the positive predictive value of end-of-treatment PET is generally much lower than its NPV and that most PET-positive individuals stay disease-free after receiving additional radiotherapy, at least in early-stage Hodgkin lymphoma after ABVD and in advanced-stage disease treated with eBEACOPP. 


\section{2. $D L B C L$}

Importantly, PET positivity after completion of R-CHOP therapy has to be interpreted as a sign of unfavorable prognosis in all stages of DLBCL. The prospective Swiss Group for Clinical Cancer Research 38/07 trial reported a significantly lower event-free survival of $48 \%$ in PET-positive patients versus $74 \%$ for those with negative FDG-PET after six courses of dose-dense R-CHOP (R-CHOP-14) [14]. In a population-based Canadian study, irradiation of PET-positive residual tissue after chemoimmunotherapy was shown to substantially improve clinical outcome so that the prognosis of subjects with positive and negative end-of-treatment PET were on a comparable level [15]. These observations could be confirmed by the interim analysis of the prospective German OPTIMAL $>60$ trial for patients with aggressive CD20-positive non-Hodgkin lymphoma [16]. Because this study had no randomization regarding FDG-PET, historic data from the very similar RICOVER-60 study are used for comparison. The latter found that individuals older than 60 years benefit additionally from irradiating former lymphoma bulk after six cycles of (R-)CHOP-14. According to the OPTIMAL>60 interim analysis, consolidating radiotherapy in the case of PET-positive bulky disease seems to have a positive effect on survival. Moreover, the Canadian and German data suggest that irradiation can be omitted in $42 \%$ to $60 \%$ of patients with PET-negative bulk.

\section{Recent Advances and Future Directions}

The Deauville score has been applied for therapy stratification in several clinical trials and evolved as an established method for monitoring response to treatment. However, there are ongoing discussions on how to interpret FDG uptake. Barrington et al. recently conducted a subsidiary analysis of the U.K. RAPID study for Hodgkin lymphoma patients and reported that a Deauville score of 5 was associated with an inferior outcome in their trial, where score 5 was defined quantitatively as three or more times the maximum liver uptake [67]. In the GHSG trials HD16 and HD18, Deauville score 3 proved not to be prognostic for progression or relapse, whereas Deauville score 4 did. Even though the majority of individuals with a Deauville score of 4 are cured, these patients show significantly impaired PFS and OS. Similar findings were reported for DLBCL [52]. This is why we recommend interpreting Deauville scores 4 and 5 as positive whenever cure is the primary aim.

Several working groups have recognized quantitative measures as an important tool in staging and response assessment, for instance $S U V_{\max }$ or $\Delta S U V_{\max }$. Studies investigating the role of $\Delta S U V_{\max }$ demonstrated its usefulness for response stratification in both Hodgkin lymphoma and DLBCL [68,69]. Additionally, FDG-PET provides biomarkers such as the metabolic tumor volume (MTV) or total lesion glycolysis (TLG), which incorporate information concerning tumor burden and disease activity. Cottereau et al. reported that the MTV improves baseline risk stratification of patients with early-stage Hodgkin lymphoma as compared to currently used staging systems [70]. Interestingly, individuals with early-stage unfavorable disease can even be subdivided into low- and high-risk categories based on the MTV and TLG, as shown elsewhere [71]. A retrospective analysis including 310 patients identified that pretreatment MTV is also a predictive factor for early response to eBEACOPP after two cycles of chemotherapy in advanced-stage Hodgkin lymphoma [72]. Moreover, metabolic measures were reported to have a prognostic value in non-Hodgkin lymphoma. Mikhaeel et al. demonstrated that MTV at staging is an important prognostic factor for DLBCL and that combining MTV with results of early PET response assessment improves the predictive power [73]. Similar findings emerged from a recently published analysis including 510 DLBCL patients treated within the PET-guided therapy optimization trial PETAL [74]. Interestingly, it has been also shown that MTV is a valid prognosticator in elderly individuals with DLBCL receiving R-CHOP. A study by Vercellino and colleagues found high pretreatment MTV to be significantly associated with inferior PFS and OS in this group [75]. Including patients from the LNH073B study, a French working group additionally examined the role of radiomic features characterizing lesion dissemination and reported that combining them with baseline MTV further improves risk stratification in DLBCL patients [76]. However, MTV calculation was carried out rather inconsistently in different working groups, which used both adaptive and fixed thresholds 
(Figure 2, Table 2). A recent review on the increasing importance of MTV addressed the need for standardization and pointed out that further analyses are needed to set common criteria [77]. Up until now, tumor delineation has been significantly more time-consuming in advanced-stage disease than in patients with fewer lesions. The application of machine learning and artificial intelligence can be expected to optimize complex workflows and will open up new perspectives in the future.
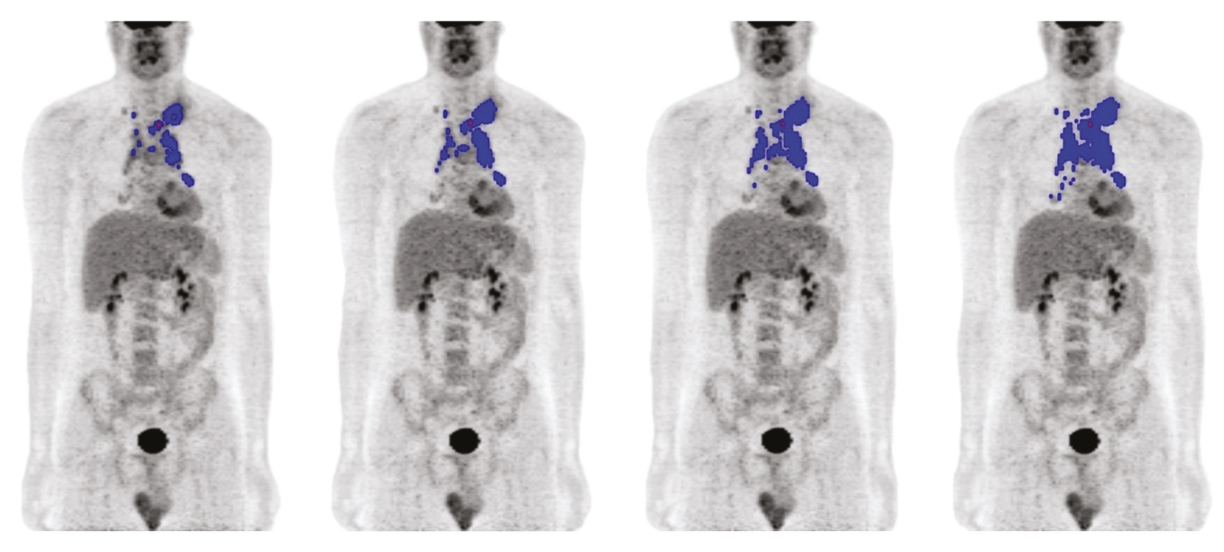

$41 \%$ of tumor $S U V_{\max }$

Liver SUV $\max$

SUV 2.5

Liver SUVmean

Figure 2. Metabolic tumor volume (MTV) measurement in a patient with advanced-stage Hodgkin lymphoma based on different thresholding methods. SUV, standardized uptake value; $\mathrm{SUV}_{\max }$, maximum SUV; SUV mean, mean SUV.

Table 2. Common approaches for MTV calculation with their main characteristics.

\begin{tabular}{|c|c|c|}
\hline Threshold & Advantages & Disadvantages \\
\hline \multirow{2}{*}{$\begin{array}{l}\text { Fixed absolute (e.g., SUV } 2.5 \\
\text { or } 4.0)\end{array}$} & High reproducibility & $\begin{array}{l}\text { Overestimation if tumor lies adjacent to areas of } \\
\text { high physiologic uptake }\end{array}$ \\
\hline & Observer-independence & $\begin{array}{l}\text { Underestimation in tumors that have many } \\
\text { voxels with an uptake less than the threshold }\end{array}$ \\
\hline \multirow{2}{*}{$\begin{array}{l}\text { Reference regions (e.g., liver } \\
\text { or mediastinum)* }\end{array}$} & \multirow{2}{*}{$\begin{array}{l}\text { Adjusted to patient } \\
\text { and scan }\end{array}$} & More time-consuming \\
\hline & & Low availability on commercial software \\
\hline \multirow{2}{*}{$\begin{array}{l}\text { Fixed relative (e.g., } 41 \% \text { of } \\
\text { tumor SUVmax) }\end{array}$} & \multirow{2}{*}{ Observer-independence } & $\begin{array}{l}\text { Overestimation in case of low } \\
\text { lesion-to-background ratio }\end{array}$ \\
\hline & & $\begin{array}{l}\text { Underestimation of tumors with heterogeneous } \\
\text { uptake and high SUVmax }\end{array}$ \\
\hline \multirow{2}{*}{$\begin{array}{c}\text { Adaptive (e.g., } \\
\text { signal-to-background ratio) }\end{array}$} & \multirow{2}{*}{$\begin{array}{l}\text { Adjusted to patient } \\
\text { and scan }\end{array}$} & More time-consuming \\
\hline & & Low availability on commercial software \\
\hline
\end{tabular}

* Thresholding method proposed by the PET Response Criteria in Solid Tumors (PERCIST) [78].

It needs to be kept in mind that the reliability of visual and quantitative response assessment can be impaired by inconsistent PET scanning protocols and image reconstruction methods [79]. Intravenous CT contrast media were found to affect PET images quantitatively due to an overestimation of attenuation factors in contrast-enhanced anatomic structure. This may have clinical consequences, particularly for response assessment, as the uptake values of lymphoma tissue rise less sharply than in the reference regions commonly used for PET interpretation [80]. Even though standardization 
is still lacking, quantitative FDG-PET has the potential to substantially improve prognostication in lymphoma. An integration of metabolic measures into clinical trials is currently planned by several working groups and will provide further evidence.

Over recent years, PET using FDG has brought many advances in the diagnosis and treatment of lymphoma patients, but there will most probably be a demand for more specific tracers other than FDG in future clinical practice. Indeed, the first successful trials with immune checkpoint inhibitors point to a major role for such substances in the first-line treatment of lymphomas [81]. However, formerly established PET response criteria may fail in these novel therapy regimens due to false-positive findings caused by inflammatory changes. Therefore, the so-called RECIL scoring system, a combination of single-dimension measurement and PET criteria, was introduced for response evaluation by consensus of an international working group [82]. Not only new drugs but also the RECIL evaluation need prospective studies to confirm their clinical relevance. In this context, it is worth mentioning that radiolabeled programmed cell death-ligand 1 (PD-L1) antibodies have recently proved to be useful for monitoring therapy-induced changes of PD-L1 expression in preclinical tumor models [83], and first clinical studies on solid malignancies have also demonstrated the feasibility of programmed cell death protein 1 (PD-1)/PD-L1 imaging [84]. Thus, highly specific tracers promise to improve our understanding of the dynamic tumor microenvironment and enable optimization of checkpoint inhibitor-based therapy strategies, not only in solid tumors but also in lymphoma patients. Imaging agents such as the chemokine receptor-targeting ${ }^{68} \mathrm{Ga}$-pentixafor [85] or the microenvironment-reflecting ${ }^{68} \mathrm{Ga}$-labeled fibroblast activation protein inhibitor (FAPI) [86] may offer further tools with which to elucidate the mechanisms of treatment and cure.

\section{Conclusions}

In FDG-avid lymphomas, PET has been established as the standard modality for both diagnosis and therapy monitoring (Table 3). PET-based staging is highly sensitive for detecting lymphoma lesions and therefore plays a crucial role in the context of contemporary treatment strategies. However, it should be noted that the positive predictive value of PET is rather moderate, with numerous non-malignant causes for FDG avidity. Importantly, undirected bone marrow biopsy can be safely omitted in Hodgkin lymphoma patients and individuals with DLBCL where involvement has already been proven by FDG-PET. As the overall predictive value of interim PET was found to be particularly high in Hodgkin lymphoma, it was established earlier in this disease than in other lymphoma subtypes. Personalized medicine is nowadays a reality, since escalation and de-escalation of chemo- and radiotherapy are adapted according to PET results. In aggressive non-Hodgkin lymphoma, baseline and interim PET imaging provide important information for disease prognosis as well. Moreover, end-of-treatment PET seems suitable to define whether a particular patient with DLBCL requires irradiation after chemotherapy or not.

Table 3. Evidence-based recommendations on the use of FDG-PET before, during, and after treatment.

\begin{tabular}{ccc}
\hline Indication & Hodgkin Lymphoma & DLBCL \\
\hline Staging & +++ & +++ \\
Early response assessment & ++ & ++ \\
End-of-treatment & ++ & ++ \\
Follow-up & $+/-$ & $+/-$ \\
\hline
\end{tabular}

$*+++$, standard modality; ++ , standard—depending on therapy protocol; $+/-$, optional—recommended in selected cases, e.g., suspected relapse.

Novel imaging-derived biomarkers such as the MTV or TLG may further individualize treatment of lymphomas. Hence, prospective trials are needed to validate the prognostic and predictive value of quantitative PET measures, given their great potential as guides for determining the future management of patients with lymphoma. 
Author Contributions: Initial planning and conceptualization of the review, C.A.V., C.K., and D.H.; writing of the original draft, all authors; proofreading and editing of the final manuscript, all authors. All authors have read and agreed to the published version of the manuscript.

Funding: The present work received no external funding.

Conflicts of Interest: There are no conflicts of interest to declare.

\section{References}

1. Howlader, N.; Noone, A.M.; Krapcho, M.; Miller, D.; Brest, A.; Yu, M.; Ruhl, J.; Tatalovich, Z.; Mariotto, A.; Lewis, D.R.; et al. SEER Cancer Statistics Review, 1975-2016; National Cancer Institute: Bethesda, MD, USA, 2019; Available online: https://seer.cancer.gov/csr/1975_2016/ (accessed on 19 November 2019).

2. Eichenauer, D.A.; Aleman, B.M.P.; André, M.P.E.; Federico, M.; Hutchings, M.; Illidge, T.; Engert, A.; Ladetto, M. Hodgkin lymphoma: ESMO clinical practice guidelines for diagnosis, treatment and follow-up. Ann. Oncol. 2018, 29, iv19-iv29. [CrossRef] [PubMed]

3. Engert, A.; Plütschow, A.; Eich, H.T.; Lohri, A.; Dörken, B.; Borchmann, P.; Berger, B.; Greil, R.; Willborn, K.C.; Wilhelm, M.; et al. Reduced treatment intensity in patients with early-stage Hodgkin's lymphoma. N. Engl. J. Med. 2010, 363, 640-652. [CrossRef] [PubMed]

4. André, M.P.E.; Girinsky, T.; Federico, M.; Reman, O.; Fortpied, C.; Gotti, M.; Casasnovas, O.; Brice, P.; van der Maazen, R.; Re, A.; et al. Early positron emission tomography response-adapted treatment in stage I and II Hodgkin lymphoma: Final results of the randomized EORTC/LYSA/FIL H10 trial. J. Clin. Oncol. 2017, 35, 1786-1794. [CrossRef]

5. Gordon, L.I.; Hong, F.; Fisher, R.I.; Bartlett, N.L.; Connors, J.M.; Gascoyne, R.D.; Wagner, H.; Stiff, P.J.; Cheson, B.D.; Gospodarowicz, M.; et al. Randomized phase III trial of ABVD versus Stanford V with or without radiation therapy in locally extensive and advanced-stage Hodgkin lymphoma: An intergroup study coordinated by the Eastern Cooperative Oncology Group (E2496). J. Clin. Oncol. 2013, 31, 684-691. [CrossRef]

6. Johnson, P.; Federico, M.; Kirkwood, A.; Fosså, A.; Berkahn, L.; Carella, A.; d'Amore, F.; Enblad, G.; Franceschetto, A.; Fulham, M.; et al. Adapted Treatment Guided by Interim PET-CT Scan in Advanced Hodgkin's Lymphoma. N. Engl. J. Med. 2016, 374, 2419-2429. [CrossRef] [PubMed]

7. Borchmann, P.; Goergen, H.; Kobe, C.; Lohri, A.; Greil, R.; Eichenauer, D.A.; Zijlstra, J.M.; Markova, J.; Meissner, J.; Feuring-Buske, M.; et al. PET-guided treatment in patients with advanced-stage Hodgkin's lymphoma (HD18): Final results of an open-label, international, randomised phase 3 trial by the German Hodgkin Study Group. Lancet 2018, 390, 2790-2802. [CrossRef]

8. Kreissl, S.; Goergen, H.; Müller, H.; Meissner, J.; Mehnert, A.; Bürkle, C.; Fuchs, M.; Engert, A.; Behringer, K.; Borchmann, P. Survivors' perspectives on risks and benefits of Hodgkin lymphoma treatment: Results of a survey by the German Hodgkin Study Group. Leuk. Lymphoma 2019, 60, 1389-1398. [CrossRef]

9. Skoetz, N.; Trelle, S.; Rancea, M.; Haverkamp, H.; Diehl, V.; Engert, A.; Borchmann, P. Effect of initial treatment strategy on survival of patients with advanced-stage Hodgkin's lymphoma: A systematic review and network meta-analysis. Lancet Oncol. 2013, 14, 943-952. [CrossRef]

10. Steidl, C.; Connors, J.M.; Gascoyne, R.D. Molecular pathogenesis of Hodgkin's lymphoma: Increasing evidence of the importance of the microenvironment. J. Clin. Oncol. 2011, 29, 1812-1826. [CrossRef]

11. Liu, W.R.; Shipp, M.A. Signaling pathways and immune evasion mechanisms in classical Hodgkin lymphoma. Hematol. Am. Soc. Hematol. Educ. Program 2017, 2017, 310-316. [CrossRef]

12. Swerdlow, S.H.; Campo, E.; Harris, N.L.; Jaffe, E.S.; Pileri, S.A.; Stein, H.; Thiele, J.; Arber, D.A.; Hasserjian, R.P.; Le Beau, M.M.; et al. WHO Classification of Tumours of Haematopoietic and Lymphoid Tissues, Review, 4th ed.; International Agency for Research on Cancer: Lyon, France, 2017.

13. Gómez León, N.; Delgado-Bolton, R.C.; Del Campo Del Val, L.; Cabezas, B.; Arranz, R.; García, M.; Cannata, J.; González Ortega, S.; Pérez Sáez, M.Á.; López-Botet, B.; et al. Multicenter comparison of contrast-enhanced FDG PET/CT and 64-slice multi-detector-row CT for initial staging and response evaluation at the end of treatment in patients with lymphoma. Clin. Nucl. Med. 2017, 42, 595-602. [CrossRef] [PubMed] 
14. Mamot, C.; Klingbiel, D.; Hitz, F.; Renner, C.; Pabst, T.; Driessen, C.; Mey, U.; Pless, M.; Bargetzi, M.; Krasniqi, F.; et al. Final results of a prospective evaluation of the predictive value of interim positron emission tomography in patients with diffuse large B-cell lymphoma treated with R-CHOP-14 (SAKK 38/07). J. Clin. Oncol. 2015, 33, 2523-2529. [CrossRef] [PubMed]

15. Freeman, C.L.; Savage, K.J.; Villa, D.; Scott, D.W.; Srour, L.; Gerrie, A.S.; Brown, M.J.; Slack, G.W.; Farinha, P.; Skinnider, B.; et al. Long-term results of PET-guided radiation therapy in patients with advanced-stage diffuse large B-cell lymphoma treated with R-CHOP in British Columbia. Blood 2017, 130, 823.

16. Pfreundschuh, M.; Christofyllakis, K.; Altmann, B.; Ziepert, M.; Haenel, M.; Viardot, A.; Neubauer, A.; Held, A.; Truemper, L.; Schmidt, C.; et al. Radiotherapy to bulky disease PET-negative after immunochemotherapy in elderly DLBCL patients: Results of a planned interim analysis of the first 187 patients with bulky disease treated in the OPTIMAL > 60 study of the DSHNHL. J. Clin. Oncol. 2017, 35, 7506. [CrossRef]

17. Dührsen, U.; Müller, S.; Hertenstein, B.; Thomssen, H.; Kotzerke, J.; Mesters, R.; Berdel, W.E.; Franzius, C.; Kroschinsky, F.; Weckesser, M.; et al. PETAL trial investigators. Positron emission tomography-guided therapy of aggressive non-Hodgkin lymphomas (PETAL): A multicenter, randomized phase III trial. J. Clin. Oncol. 2018, 36, 2024-2034. [CrossRef]

18. Hüttmann, A.; Rekowski, J.; Müller, S.P.; Hertenstein, B.; Franzius, C.; Mesters, R.; Weckesser, M.; Kroschinsky, F.; Kotzerke, J.; Ganser, A.; et al. Six versus eight doses of rituximab in patients with aggressive B cell lymphoma receiving six cycles of CHOP: Results from the "Positron Emission Tomography-Guided Therapy of Aggressive Non-Hodgkin Lymphomas" (PETAL) trial. Ann. Hematol. 2019, 98, 897-907. [CrossRef]

19. Poeschel, V.; Held, G.; Ziepert, M.; Witzens-Harig, M.; Holte, H.; Thurner, L.; Borchmann, P.; Viardot, A.; Soekler, M.; Keller, U.; et al. Four versus six cycles of CHOP chemotherapy in combination with six applications of rituximab in patients with aggressive B-cell lymphoma with favourable prognosis (FLYER): A randomised, phase 3, non-inferiority trial. Lancet 2020, 394, 2271-2281. [CrossRef]

20. The International Non-Hodgkin's Lymphoma Prognostic Factors Project. A predictive model for aggressive non-Hodgkin's lymphoma. N. Engl. J. Med. 1993, 329, 987-994. [CrossRef]

21. Tilly, H.; Gomes da Silva, M.; Vitolo, U.; Jack, A.; Meignan, M.; Lopez-Guillermo, A.; Walewski, J.; André, M.P.E.; Johnson, P.W.; Pfreundschuh, M.; et al. ESMO Guidelines Committee. Diffuse large B-cell lymphoma (DLBCL): ESMO Clinical Practice Guidelines for diagnosis, treatment and follow-up. Ann. Oncol. 2015, 26, v116-v125. [CrossRef]

22. Pfreundschuh, M.; Schubert, J.; Ziepert, M.; Schmits, R.; Mohren, M.; Lengfelder, E.; Reiser, M.; Nickenig, C.; Clemens, M.; Peter, N.; et al. German High-Grade Non-Hodgkin Lymphoma Study Group (DSHNHL). Six versus eight cycles of bi-weekly CHOP-14 with or without rituximab in elderly patients with aggressive CD20 + B-cell lymphomas: A randomised controlled trial (RICOVER-60). Lancet Oncol. 2008, 9, 105-116. [CrossRef]

23. Held, G.; Murawski, N.; Ziepert, M.; Fleckenstein, J.; Pöschel, V.; Zwick, C.; Bittenbring, J.; Hänel, M.; Wilhelm, S.; Schubert, J.; et al. Role of radiotherapy to bulky disease in elderly patients with aggressive B-cell lymphoma. J. Clin. Oncol. 2014, 32, 1112-1118. [CrossRef] [PubMed]

24. Cheson, B.D.; Fisher, R.I.; Barrington, S.F.; Cavalli, F.; Schwartz, L.H.; Zucca, E.; Lister, T.A. Recommendations for initial evaluation, staging, and response assessment of Hodgkin and non-Hodgkin lymphoma: The Lugano classification. J. Clin. Oncol. 2014, 32, 3059-3068. [CrossRef] [PubMed]

25. Carbone, P.P.; Kaplan, H.S.; Musshoff, K.; Smithers, D.W.; Tubiana, M. Report of the committee on Hodgkin's disease staging classification. Cancer Res. 1971, 31, 1860-1861. [PubMed]

26. Barrington, S.F.; Kirkwood, A.A.; Franceschetto, A.; Fulham, M.J.; Roberts, T.H.; Almquist, H.; Brun, E.; Hjorthaug, K.; Viney, Z.N.; Pike, L.C.; et al. PET-CT for staging and early response: Results from the response-adapted therapy in advanced Hodgkin lymphoma study. Blood 2016, 127, 1531-1538. [CrossRef]

27. Moulin-Romsee, G.; Hindié, E.; Cuenca, X.; Brice, P.; Decaudin, D.; Bénamor, M.; Brière, J.; Anitei, M.; Filmont, J.E.; Sibon, D.; et al. ${ }^{18}$ F-FDG PET/CT bone/bone marrow findings in Hodgkin's lymphoma may circumvent the use of bone marrow trephine biopsy at diagnosis staging. Eur. J. Nucl. Med. Mol. Imaging 2010, 37, 1095-1105. [CrossRef] 
28. El-Galaly, T.C.; d'Amore, F.; Mylam, K.J.; de Nully Brown, P.; Bøgsted, M.; Bukh, A.; Specht, L.; Loft, A.; Iyer, V.; Hjorthaug, K.; et al. Routine bone marrow biopsy has little or no therapeutic consequence for positron emission tomography/computed tomography-staged treatment-naïve patients with Hodgkin lymphoma. J. Clin. Oncol. 2012, 30, 4508-4514. [CrossRef]

29. Weiler-Sagie, M.; Kagna, O.; Dann, E.J.; Ben-Barak, A.; Israel, O. Characterizing bone marrow involvement in Hodgkin's lymphoma by FDG-PET/CT. Eur. J. Nucl. Med. Mol. Imaging 2014, 41, 1133-1140. [CrossRef]

30. Chen-Liang, T.H.; Martin-Santos, T.; Jerez, A.; Senent, L.; Orero, M.T.; Remigia, M.J.; Muiña, B.; Romera, M.; Fernandez-Muñoz, H.; Raya, J.M.; et al. The role of bone marrow biopsy and FDG-PET/CT in identifying bone marrow infiltration in the initial diagnosis of high grade non-Hodgkin B-cell lymphoma and Hodgkin lymphoma. Accuracy in a multicenter series of 372 patients. Am. J. Hematol. 2015, 90, 686-690. [CrossRef]

31. Purz, S.; Mauz-Körholz, C.; Körholz, D.; Hasenclever, D.; Krausse, A.; Sorge, I.; Ruschke, K.; Stiefel, M.; Amthauer, H.; Schober, O.; et al. $\left[{ }^{18} \mathrm{~F}\right]$ fluorodeoxyglucose positron emission tomography for detection of bone marrow involvement in children and adolescents with Hodgkin's lymphoma. J. Clin. Oncol. 2011, 29, 3523-3528. [CrossRef]

32. Voltin, C.A.; Goergen, H.; Baues, C.; Fuchs, M.; Mettler, J.; Kreissl, S.; Oertl, J.; Klaeser, B.; Moccia, A.; Drzezga, A.; et al. Value of bone marrow biopsy in Hodgkin lymphoma patients staged by FDG PET: Results from the German Hodgkin Study Group trials HD16, HD17, and HD18. Ann. Oncol. 2018, 29, 1926-1931. [CrossRef]

33. Pedersen, M.A.; Gormsen, L.C.; Kamper, P.; Wassberg, C.; Andersen, M.D.; d'Amore, A.L.; Barrington, S.F.; Johnson, P.; Hamilton-Dutoit, S.; Amini, R.M.; et al. Focal skeletal FDG uptake indicates poor prognosis in cHL regardless of extent and first-line chemotherapy. Br. J. Haematol. 2019, 186, 431-439. [CrossRef] [PubMed]

34. Adams, H.J.; de Klerk, J.M.; Fijnheer, R.; Heggelman, B.G.; Dubois, S.V.; Nievelstein, R.A.; Kwee, T.C. Variety in bone marrow ${ }^{18}$ F-FDG uptake in Hodgkin lymphoma patients without lymphomatous bone marrow involvement: Does it have an explanation? Nucl. Med. Commun. 2016, 37, 23-29. [CrossRef] [PubMed]

35. Picardi, M.; Soricelli, A.; Grimaldi, F.; Nicolai, E.; Gallamini, A.; Pane, F. Fused FDG-PET/contrast-enhanced CT detects occult subdiaphragmatic involvement of Hodgkin's lymphoma thereby identifying patients requiring six cycles of anthracycline-containing chemotherapy and consolidation radiation of spleen. Ann. Oncol. 2011, 22, 671-680. [CrossRef] [PubMed]

36. Fuertes, S.; Setoain, X.; López-Guillermo, A.; Montserrat, E.; Fuster, D.; Paredes, P.; Lomeña, F.; Pons, F. The value of positron emission tomography/computed tomography (PET/CT) in the staging of diffuse large B-cell lymphoma. Med. Clin. 2007, 129, 688-693. [CrossRef] [PubMed]

37. Raanani, P.; Shasha, Y.; Perry, C.; Metser, U.; Naparstek, E.; Apter, S.; Nagler, A.; Polliack, A.; Ben-Bassat, I.; Even-Sapir, E. Is CT scan still necessary for staging in Hodgkin and non-Hodgkin lymphoma patients in the PET/CT era? Ann. Oncol. 2006, 17, 117-122. [CrossRef]

38. Elstrom, R.L.; Leonard, J.P.; Coleman, M.; Brown, R.K. Combined PET and low-dose, noncontrast CT scanning obviates the need for additional diagnostic contrast-enhanced CT scans in patients undergoing staging or restaging for lymphoma. Ann. Oncol. 2008, 19, 1770-1773. [CrossRef]

39. Cerci, J.J.; Györke, T.; Fanti, S.; Paez, D.; Meneghetti, J.C.; Redondo, F.; Celli, M.; Auewarakul, C.; Rangarajan, V.; Gujral, S.; et al. Combined PET and biopsy evidence of marrow involvement improves prognostic prediction in diffuse large B-cell lymphoma. J. Nucl. Med. 2014, 55, 1591-1597. [CrossRef]

40. Khan, A.B.; Barrington, S.F.; Mikhaeel, N.G.; Hunt, A.A.; Cameron, L.; Morris, T.; Carr, R. PET-CT staging of DLBCL accurately identifies and provides new insight into the clinical significance of bone marrow involvement. Blood 2013, 122, 61-67. [CrossRef]

41. Alzahrani, M.; El-Galaly, T.C.; Hutchings, M.; Hansen, J.W.; Loft, A.; Johnsen, H.E.; Iyer, V.; Wilson, D.; Sehn, L.H.; Savage, K.J.; et al. The value of routine bone marrow biopsy in patients with diffuse large B-cell lymphoma staged with PET/CT: A Danish-Canadian study. Ann. Oncol. 2016, 27, 1095-1099. [CrossRef]

42. Kaddu-Mulindwa, D.; Altmann, B.; Held, G.; Ziepert, M.; Menhart, K.; Grosse, J.; Angel, S.; Stilgenbauer, S.; Pfreundschuh, M.; Herrmann, K.; et al. Role of FDG PET/CT to detect bone marrow involvement in the initial staging of aggressive non-Hodgkin lymphoma. Blood 2019, 134, 2892. [CrossRef]

43. Meignan, M.; Gallamini, A.; Haioun, C. Report on the First International Workshop on Interim-PET-Scan in Lymphoma. Leuk. Lymphoma 2009, 50, 1257-1260. [CrossRef] [PubMed] 
44. Radford, J.; Illidge, T.; Counsell, N.; Hancock, B.; Pettengell, R.; Johnson, P.; Wimperis, J.; Culligan, D.; Popova, B.; Smith, P.; et al. Results of a trial of PET-directed therapy for early-stage Hodgkin's lymphoma. N. Engl. J. Med. 2015, 372, 1598-1607. [CrossRef] [PubMed]

45. Fuchs, M.; Goergen, H.; Kobe, C.; Kuhnert, G.; Lohri, A.; Greil, R.; Sasse, S.; Topp, M.S.; Schäfer, E.; Hertenstein, B.; et al. Positron emission tomography-guided treatment in early-stage favorable hodgkin lymphoma: Final results of the international, randomized phase III HD16 trial by the German Hodgkin Study Group. J. Clin. Oncol. 2019, 37, 2835-2845. [CrossRef] [PubMed]

46. Gallamini, A.; Tarella, C.; Viviani, S.; Rossi, A.; Patti, C.; Mulé, A.; Picardi, M.; Romano, A.; Cantonetti, M.; La Nasa, G.; et al. Early chemotherapy intensification with escalated beacopp in patients with advanced-stage Hodgkin lymphoma with a positive interim positron emission tomography/computed tomography scan after two ABVD cycles: Long-term results of the GITIL/FIL HD 0607 trial. J. Clin. Oncol. 2018, 36, 454-462. [CrossRef] [PubMed]

47. Casasnovas, R.O.; Bouabdallah, R.; Brice, P.; Lazarovici, J.; Ghesquieres, H.; Stamatoullas, A.; Dupuis, J.; Gac, A.C.; Gastinne, T.; Joly, B.; et al. PET-adapted treatment for newly diagnosed advanced Hodgkin lymphoma (AHL2011): A randomised, multicentre, non-inferiority, phase 3 study. Lancet Oncol. 2019, 20, 202-215. [CrossRef]

48. Kobe, C.; Goergen, H.; Baues, C.; Kuhnert, G.; Voltin, C.A.; Zijlstra, J.; Hoekstra, O.; Mettler, J.; Drzezga, A.; Engert, A.; et al. Outcome-based interpretation of early interim PET in advanced-stage Hodgkin lymphoma. Blood 2018, 132, 2273-2279. [CrossRef]

49. Sehn, L.H.; Savage, K.J.; Hoskins, P.; Klasa, R.; Shenkier, T.; Gascoyne, R.D.; Voss, N.; Wilson, D.; Connors, J. Limited-stage DLBCL patients with a negative PET scan following three cycles of R-CHOP have an excellent outcome following abbreviated immuno-chemotherapy alone. Ann. Oncol. 2008, 19, 99.

50. Casasnovas, R.O.; Salles, G.A.; Oberic, L.; Bodet-Milin, C.; Briere, J.; Morschhauser, F.; Tilly, H.; Ribrag, V.; Lamy, T.; Thieblemont, C.; et al. Obinutuzumab versus rituximab in combination with ACVBP-14 or CHOP-14 following a PET-driven strategy in Aa-IPI 1-3 DLBCL patients (<60 years): Third planned interim and final analyses of the gained trial. Blood 2017, 626, 190.

51. Casasnovas, R.O.; Ysebaert, L.; Thieblemont, C.; Bachy, E.; Feugier, P.; Delmer, A.; Tricot, S.; Gabarre, J.; André, M.P.E.; Fruchart, C.; et al. FDG-PET-driven consolidation strategy in diffuse large B-cell lymphoma: Final results of a randomized phase 2 study. Blood 2017, 130, 1315-1326. [CrossRef]

52. Eertink, J.J.; Burggraaff, C.N.; Heymans, M.W.; Barrington, S.F.; Mikhaeel, G.; Dührsen, U.; Hüttmann, A.; Ceriani, L.; Zucca, E.; Carr, R.; et al. The optimal timing of interim ${ }^{18}$ F-FDG PET in diffuse large B-cell lymphoma: An individual patient data meta-analysis by the Petra consortium. Blood 2019, 134, 487. [CrossRef]

53. Canellos, G.P. Residual mass in lymphoma may not be residual disease. J. Clin. Oncol. 1988, 6, 931-933. [CrossRef] [PubMed]

54. Jerusalem, G.; Beguin, Y.; Fassotte, M.F.; Najjar, F.; Paulus, P.; Rigo, P.; Fillet, G. Whole-body positron emission tomography using ${ }^{18} \mathrm{~F}$-fluorodeoxyglucose for posttreatment evaluation in Hodgkin's disease and non-Hodgkin's lymphoma has higher diagnostic and prognostic value than classical computed tomography scan imaging. Blood 1999, 94, 429-433. [CrossRef] [PubMed]

55. De Wit, M.; Bohuslavizki, K.H.; Buchert, R.; Bumann, D.; Clausen, M.; Hossfeld, D.K. ${ }^{18}$ FDG-PET following treatment as valid predictor for disease-free survival in Hodgkin's lymphoma. Ann. Oncol. 2001, 12, 29-37. [CrossRef]

56. Spaepen, K.; Stroobants, S.; Dupont, P.; Thomas, J.; Vandenberghe, P.; Balzarini, J.; De Wolf-Peeters, C.; Mortelmans, L.; Verhoef, G. Can positron emission tomography with $\left[{ }^{18} \mathrm{~F}\right]$-fluorodeoxyglucose after first-line treatment distinguish Hodgkin's disease patients who need additional therapy from others in whom additional therapy would mean avoidable toxicity? Br. J. Haematol. 2001, 115, 272-278. [CrossRef] [PubMed]

57. Weihrauch, M.R.; Re, D.; Scheidhauer, K.; Ansén, S.; Dietlein, M.; Bischoff, S.; Bohlen, H.; Wolf, J.; Schicha, H.; Diehl, V.; et al. Thoracic positron emission tomography using ${ }^{18} \mathrm{~F}$-fluorodeoxyglucose for the evaluation of residual mediastinal Hodgkin disease. Blood 2001, 98, 2930-2934. [CrossRef]

58. Schaefer, N.G.; Taverna, C.; Strobel, K.; Wastl, C.; Kurrer, M.; Hany, T.F. Hodgkin disease: Diagnostic value of FDG PET/CT after first-line therapy-Is biopsy of FDG-avid lesions still needed? Radiology 2007, 244, 257-262. [CrossRef] [PubMed] 
59. Kobe, C.; Dietlein, M.; Franklin, J.; Markova, J.; Lohri, A.; Amthauer, H.; Klutmann, S.; Knapp, W.H.; Zijlstra, J.M.; Bockisch, A.; et al. Positron emission tomography has a high negative predictive value for progression or early relapse for patients with residual disease after first-line chemotherapy in advanced-stage Hodgkin lymphoma. Blood 2008, 112, 3989-3994. [CrossRef]

60. Markova, J.; Kahraman, D.; Kobe, C.; Skopalova, M.; Mocikova, H.; Klaskova, K.; Dedeckova, K.; Eich, H.T.; Böll, B.; Dietlein, M.; et al. Role of $\left[{ }^{18} \mathrm{~F}\right]$-fluoro-2-deoxy-D-glucose positron emission tomography in early and late therapy assessment of patients with advanced Hodgkin lymphoma treated with bleomycin, etoposide, adriamycin, cyclophosphamide, vincristine, procarbazine and prednisone. Leuk. Lymphoma 2012, 53, 64-70. [CrossRef]

61. Jerusalem, G.; Beguin, Y.; Fassotte, M.F.; Belhocine, T.; Hustinx, R.; Rigo, P.; Fillet, G. Early detection of relapse by whole-body positron emission tomography in the follow-up of patients with Hodgkin's disease. Ann. Oncol. 2003, 14, 123-130. [CrossRef]

62. Mocikova, H.; Obrtlikova, P.; Vackova, B.; Trneny, M. Positron emission tomography at the end of first-line therapy and during follow-up in patients with Hodgkin lymphoma: A retrospective study. Ann. Oncol. 2010, 21, 1222-1227. [CrossRef]

63. Zinzani, P.L.; Stefoni, V.; Tani, M.; Fanti, S.; Musuraca, G.; Castellucci, P.; Marchi, E.; Fina, M.; Ambrosini, V.; Pellegrini, C.; et al. Role of $\left[{ }^{18} \mathrm{~F}\right]$ fluorodeoxyglucose positron emission tomography scan in the follow-up of lymphoma. J. Clin. Oncol. 2009, 27, 1781-1787. [CrossRef] [PubMed]

64. Picardi, M.; Pugliese, N.; Cirillo, M.; Zeppa, P.; Cozzolino, I.; Ciancia, G.; Pettinato, G.; Salvatore, C.; Quintarelli, C.; Pane, F. Advanced-stage Hodgkin lymphoma: US/chest radiography for detection of relapse in patients in first complete remission-A randomized trial of routine surveillance imaging procedures. Radiology 2014, 272, 262-274. [CrossRef] [PubMed]

65. Kobe, C.; Kuhnert, G.; Kahraman, D.; Haverkamp, H.; Eich, H.T.; Franke, M.; Persigehl, T.; Klutmann, S.; Amthauer, H.; Bockisch, A.; et al. Assessment of tumor size reduction improves outcome prediction of positron emission tomography/computed tomography after chemotherapy in advanced-stage Hodgkin lymphoma. J. Clin. Oncol. 2014, 32, 1776-1781. [CrossRef] [PubMed]

66. Gallamini, A.; Rossi, A.; Patti, C.; Picardi, M.; Romano, A.; Cantonetti, M.; Oppi, S.; Viviani, S.; Bolis, S.; Trentin, L.; et al. Consolidation radiotherapy could be omitted in advanced hodgkin lymphoma with large nodal mass in complete metabolic response after ABVD. Final analysis of the randomized HD0607 trial. Hematol. Oncol. 2019, 37, 99. [CrossRef]

67. Barrington, S.F.; Phillips, E.H.; Counsell, N.; Hancock, B.; Pettengell, R.; Johnson, P.; Townsend, W.; Culligan, D.; Popova, B.; Clifton-Hadley, L.; et al. Positron emission tomography score has greater prognostic significance than pretreatment risk stratification in early-stage hodgkin lymphoma in the UK RAPID Study. J. Clin. Oncol. 2019, 37, 1732-1741. [CrossRef]

68. Itti, E.; Meignan, M.; Berriolo-Riedinger, A.; Biggi, A.; Cashen, A.F.; Véra, P.; Tilly, H.; Siegel, B.A.; Gallamini, A.; Casasnovas, R.O.; et al. An international confirmatory study of the prognostic value of early PET/CT in diffuse large B-cell lymphoma: Comparison between Deauville criteria and $\triangle$ SUVmax. Eur. J. Nucl. Med. Mol. Imaging 2013, 40, 1312-1320. [CrossRef]

69. Rossi, C.; Kanoun, S.; Berriolo-Riedinger, A.; Dygai-Cochet, I.; Humbert, O.; Legouge, C.; Chrétien, M.L.; Bastie, J.N.; Brunotte, F.; Casasnovas, R.O. Interim ${ }^{18}$ F-FDG PET SUVmax reduction is superior to visual analysis in predicting outcome early in Hodgkin lymphoma patients. J. Nucl. Med. 2014, 55, 569-573. [CrossRef]

70. Cottereau, A.S.; Versari, A.; Loft, A.; Casasnovas, O.; Bellei, M.; Ricci, R.; Bardet, S.; Castagnoli, A.; Brice, P.; Raemaekers, J.; et al. Prognostic value of baseline metabolic tumor volume in early-stage Hodgkin lymphoma in the standard arm of the H10 trial. Blood 2018, 131, 1456-1463. [CrossRef]

71. Akhtari, M.; Milgrom, S.A.; Pinnix, C.C.; Reddy, J.P.; Dong, W.; Smith, G.L.; Mawlawi, O.; Abou Yehia, Z.; Gunther, J.; Osborne, E.M.; et al. Reclassifying patients with early-stage Hodgkin lymphoma based on functional radiographic markers at presentation. Blood 2018, 131, 84-94. [CrossRef]

72. Mettler, J.; Müller, H.; Voltin, C.A.; Baues, C.; Klaeser, B.; Moccia, A.; Borchmann, P.; Engert, A.; Kuhnert, G.; Drzezga, A.; et al. Metabolic tumor volume for response prediction in advanced-stage hodgkin lymphoma. J. Nucl. Med. 2019, 60, 207-211. [CrossRef] 
73. Mikhaeel, N.G.; Smith, D.; Dunn, J.T.; Phillips, M.; Møller, H.; Fields, P.A.; Wrench, D.; Barrington, S.F. Combination of baseline metabolic tumour volume and early response on PET/CT improves progression-free survival prediction in DLBCL. Eur. J. Nucl. Med. Mol. Imaging 2016, 43, 1209-1219. [CrossRef] [PubMed]

74. Schmitz, C.; Hüttmann, A.; Müller, S.P.; Hanoun, M.; Boellaard, R.; Brinkmann, M.; Jöckel, K.H.; Dührsen, U.; Rekowski, J. Dynamic risk assessment based on positron emission tomography scanning in diffuse large B-cell lymphoma: Post-hoc analysis from the PETAL trial. Eur. J. Cancer 2019, 124, 25-36. [CrossRef] [PubMed]

75. Vercellino, L.; Cottereau, A.S.; Casasnovas, R.O.; Tilly, H.; Feugier, P.; Chartier, L.; Fruchart, C.; Roulin, L.; Obéric, L.; Pica, G.M.; et al. High total metabolic tumor volume at baseline allows discrimination of survival even in patients aged 60 to 80 years responding to R-CHOP. Blood 2020. [CrossRef] [PubMed]

76. Cottereau, A.S.; Nioche, C.; Dirand, A.S.; Clerc, J.; Morschhauser, F.; Casasnovas, O.; Meignan, M.A.; Buvat, I. ${ }^{18}$ F-FDG-PET dissemination features in diffuse large B cell lymphoma are prognostic of outcome. J. Nucl. Med. 2019, 61, 40-45. [CrossRef]

77. Barrington, S.F.; Meignan, M. Time to prepare for risk adaptation in lymphoma by standardizing measurement of metabolic tumor burden. J. Nucl. Med. 2019, 60, 1096-1102. [CrossRef]

78. Wahl, R.L.; Jacene, H.; Kasamon, Y.; Lodge, M.A. From RECIST to PERCIST: Evolving considerations for PET response criteria in solid tumors. J. Nucl. Med. 2009, 50, 122S-150S. [CrossRef]

79. Boellaard, R.; Sera, T.; Kaalep, A.; Hoekstra, O.S.; Barrington, S.F.; Zijlstra, J.M. Updating PET/CT performance standards and PET/CT interpretation criteria should go hand in hand. EJNMMI Res. 2019, 9, 95. [CrossRef]

80. Voltin, C.A.; Mettler, J.; Boellaard, R.; Kuhnert, G.; Dietlein, M.; Borchmann, P.; Drzezga, A.; Kobe, C. Quantitative assessment of ${ }^{18} \mathrm{~F}-\mathrm{FDG}$ PET in patients with Hodgkin lymphoma: Is it significantly affected by contrast-enhanced computed tomography attenuation correction? Nucl. Med. Commun. 2019, 40, 249-257. [CrossRef]

81. Bröckelmann, P.J.; Goergen, H.; Keller, U.; Meissner, J.; Ordemann, R.; Halbsguth, T.V.; Sasse, S.; Sökler, M.; Kerkhoff, A.; Mathas, S.; et al. Nivolumab and AVD for Early-Stage Unfavorable Hodgkin Lymphoma (NIVAHL). Blood 2019, 134, 236. [CrossRef]

82. Younes, A.; Hilden, P.; Coiffier, B.; Hagenbeek, A.; Salles, G.; Wilson, W.; Seymour, J.F.; Kelly, K.; Gribben, J.; Pfreunschuh, M.; et al. International Working Group consensus response evaluation criteria in lymphoma (RECIL 2017). Ann. Oncol. 2017, 28, 1436-1447. [CrossRef]

83. Verhoeff, S.R.; van den Heuvel, M.M.; van Herpen, C.M.L.; Piet, B.; Aarntzen, E.H.J.G.; Heskamp, S. Programmed cell death-1/Ligand-1 PET imaging: A novel tool to optimize immunotherapy? PET Clin. 2020, 15, 35-43. [CrossRef] [PubMed]

84. Heskamp, S.; Hobo, W.; Molkenboer-Kuenen, J.D.; Olive, D.; Oyen, W.J.; Dolstra, H.; Boerman, O.C. Noninvasive imaging of tumor PD-L1 expression using radiolabeled Anti-PD-L1 antibodies. Cancer Res. 2015, 75, 2928-2936. [CrossRef] [PubMed]

85. Wester, H.J.; Keller, U.; Schottelius, M.; Beer, A.; Philipp-Abbrederis, K.; Hoffmann, F.; Šimeček, J.; Gerngross, C.; Lassmann, M.; Herrmann, K.; et al. Disclosing the CXCR4 expression in lymphoproliferative diseases by targeted molecular imaging. Theranostics 2015, 5, 618-630. [CrossRef] [PubMed]

86. Kratochwil, C.; Flechsig, P.; Lindner, T.; Abderrahim, L.; Altmann, A.; Mier, W.; Adeberg, S.; Rathke, H.; Röhrich, M.; Winter, H.; et al. ${ }^{68}$ Ga-FAPI PET/CT: Tracer uptake in 28 different kinds of cancer. J. Nucl. Med. 2019, 60, 801-805. [CrossRef]

(C) 2020 by the authors. Licensee MDPI, Basel, Switzerland. This article is an open access article distributed under the terms and conditions of the Creative Commons Attribution (CC BY) license (http://creativecommons.org/licenses/by/4.0/). 

Review

\title{
Immunotherapy by Immune Checkpoint Inhibitors and Nuclear Medicine Imaging: Current and Future Applications
}

\author{
Pierre Decazes ${ }^{1,2, *}$ and Pierre Bohn ${ }^{1,2}$ \\ 1 Department of Nuclear Medicine, Henri Becquerel Cancer Center, 76000 Rouen, France; \\ pierre.bohn@chb.unicancer.fr \\ 2 LITIS-QuantIF-EA (Equipe d'Accueil) 4108, IRIB, Faculty of Medicine, University of Rouen, \\ 76000 Rouen, France \\ * Correspondence: pierre.decazes@chb.unicancer.fr
}

Received: 18 December 2019; Accepted: 4 February 2020; Published: 6 February 2020

\begin{abstract}
Immunotherapy by using immune checkpoint inhibitors is a revolutionary development in oncology. Medical imaging is also impacted by this new therapy, particularly nuclear medicine imaging (also called radionuclide imaging), which uses radioactive tracers to visualize metabolic functions. Our aim was to review the current applications of nuclear medicine imaging in immunotherapy, along with their limitations, and the perspectives offered by this imaging modality. Method: Articles describing the use of radionuclide imaging in immunotherapy were researched using PubMed by April 2019 and analyzed. Results: More than 5000 articles were analyzed, and nearly 100 of them were retained. Radionuclide imaging, notably ${ }^{18} \mathrm{~F}$-FDG PET/CT, already has a major role in many cancers for pre-therapeutic and therapeutic evaluation, diagnoses of adverse effects, called immune-related adverse events (IrAE), and end-of-treatment evaluations. However, these current applications can be hindered by immunotherapy, notably due to atypical response patterns such as pseudoprogression, which is defined as an increase in the size of lesions, or the visualization of new lesions, followed by a response, and hyperprogression, which is an accelerated tumor growth rate after starting treatment. To overcome these difficulties, new opportunities are offered, particularly therapeutic evaluation criteria adapted to immunotherapy and immuno-PET allowing us to predict responses to immunotherapy. Moreover, some new technological solutions are also promising, such as radiomic analyses and body composition on associated anatomical images. However, more research has to be done, notably for the diagnosis of hyperprogression and pseudoprogression. Conclusion: Immunotherapy, by its major impact on cancer and by the new patterns generated on images, is revolutionary in the field of medical images. Nuclear medicine imaging is already established and will be able to help meet new challenges through its plasticity.
\end{abstract}

Keywords: positron emission tomography; programmed cell death 1 receptor; diagnostic imaging; CTLA-4 Antigen; Immunotherapy; Adoptive; radioactive tracers; radionuclide imaging; CD8-Positive T-Lymphocytes

\section{Introduction}

Cancers are a proliferation of abnormal cells that can have the ability to disrupt the host's adaptive immune response to avoid a control by tumoricidal attack [1]. This process can be overcome by immunotherapy, which aims to stimulate the body's immune system against cancer cells.

Immunotherapy may be broken down into three main families. Firstly, passive cancer immunotherapy, which consists of the use of antibodies directed against tumor proteins, or adoptive T-cell therapy targeting a tumor receptor; secondly, active immunotherapy involving cytokines and 
vaccines; thirdly, immunomodulatory therapy including immune checkpoint inhibitors (ICI) and targeting, more specifically, the tumor microenvironment.

Immunotherapy using ICI-notably anti-CTLA-4 (cytotoxic T-lymphocyte antigen 4), anti-PD-1 (programmed cell death protein-1), and anti-PDL-1 (PD1 ligand) antibodies-is a recent successful therapeutic approach that reactivates the immune system against cancers [2,3]. In a meta-analysis combining 19 studies involving 11,640 patients treated by ICI or other drugs, a team observed that a durable response (i.e., a progression-free survival exceeding three times the median progression-free survival of the whole population) occurred for $25 \%$ of the patients treated by ICI, which is a far better rate when compared to other drug classes (11\%) [4]. However, as the therapeutic approach of ICI is different from usual cytotoxic approaches-notably by generating inflammations rather than direct lysis-medical imaging has to be interpreted differently than when using cytotoxic chemotherapy.

Thus, ICI introduces new challenges in medical imaging. Pre-therapeutic examinations have to identify prognostic factors-linked, for example, to the extent and the burden of the disease-as well as predictive factors of the response to immunotherapy, since it is known that not all patients will benefit from immunotherapy. New imaging techniques, called immunoimaging, target tumor or inflammatory cell and are promising for this indication. Concerning therapeutic evaluation, follow-up examinations must allow the detection of non-responders; in order to change the therapeutic line, this detection has to be done early, particularly in cases of hyperprogression-which is an acceleration of tumor growth rate sometimes observed after starting immunotherapy $[5,6]$. At the same time, to avoid the premature discontinuation of treatment beneficial to the patient, a real disease progression should not be confused with a pseudoprogression, which is an increase in the size of lesions, or the visualisation of new lesions, followed by a response [6,7]. Finally, end-of-treatment examinations should allow patients to safely stop immunotherapy in the event of durable response [6].

These challenges concern morphological and anatomical imaging, including computed tomography (CT), magnetic resonance imaging (MRI), and ultrasound. However, they also concern functional imaging, particularly nuclear medicine imaging.

To produce images, nuclear medicine uses radiotracers, which are a combination of a radioactive atom and a tracer targeting a metabolic function. These radionuclide images can be produced by detecting in vivo radiotracers with, in case of gamma $(\gamma)$ radioactivity, a gamma camera (used notably to create a 3D single photon emission computed tomography, alias SPECT), or with, in case of a positron $(\beta+)$ radioactivity, positron emission tomography (PET), which is usually coupled with a $C T$ to form a PET/CT.

As morphological modifications, functional modifications due to ICI are visible on nuclear medicine images and must be known not only by nuclear physicians but also by oncologists requesting examinations. In addition, nuclear medicine, by its high lability and sensitivity, can offer many solutions, now and in the future, for the new challenges raised by ICI.

The objective of this review is to conduct a systematic review of the articles concerning nuclear medicine and ICI, with a summary of the pathophysiology and a focus on changes already made in the current clinical routine and those to come.

\section{Materials and Methods}

A literature review was performed using Medline (PubMed). The research was conducted on 2 April 2019 to identify articles published between 2008 and 2019 with a search key combining date of publication and MeSH terms linked to immunotherapy, nuclear medicine and medical imaging.

The PubMed key search was: (“2008” [Date—Create]: “2020” [Date-Create]) AND ("programmed cell death 1 receptor" [MeSH Terms] OR "CTLA-4 Antigen" [MeSH Terms] OR "CD8-Positive T-Lymphocytes" [MeSH Terms] OR "Immunotherapy, Adoptive" [MeSH Terms] OR “CTLA-4 Antigen" [nm] OR "PDCD1 protein, human" [nm] OR nivolumab [nm] OR "pembrolizumab" [nm] OR "durvalumab" [nm] OR "ipilimumab" [nm] OR "avelumab" [nm] OR "atezolizumab" [nm] OR "immunotherapy" [All Fields] OR "PD-L1" [All Fields] OR "PD-1" [All Fields] OR "CTLA-4" [All 
Fields]) AND ("radioimmunotherapy" [MeSH Terms] OR "Alpha Particles" [MeSH Terms] OR "Beta Particles" [MeSH Terms] OR "radionuclide imaging" [MeSH Terms] OR "Diagnostic Imaging" [MeSH Terms] OR "radioactive tracers" [MeSH Terms] OR "imaging" [All Fields] OR "PET" [All Fields] OR "radiology" [All Fields] OR "nuclear medicine" [All Fields] or radioactiv* [All Fields])

Search results were judged for relevance using the title, abstract, and full text for inclusion in the analysis. Two researchers (one nuclear medicine physician and one radiopharmacist) performed the research and the critical analysis of the articles. A Preferred Reporting Items for Systematic Reviews and Meta-Analyses (PRISMA) flowchart [8] of the studies selection process is shown in Figure 1.

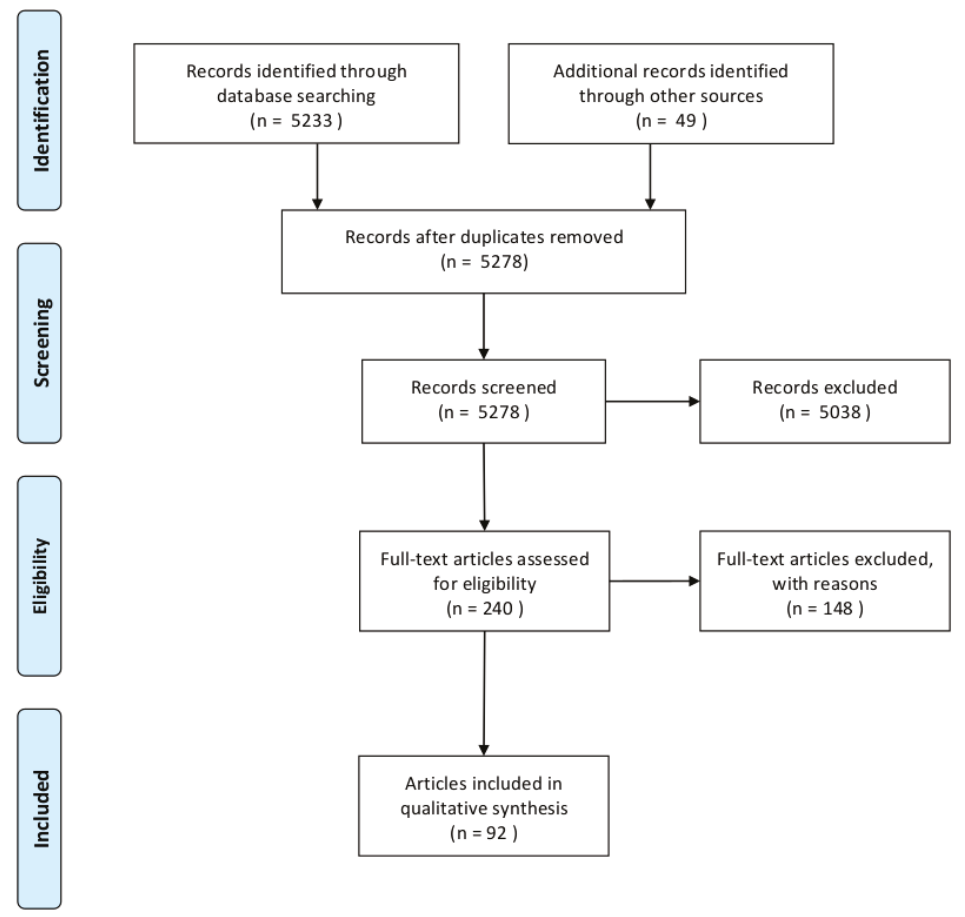

Figure 1. Preferred Reporting Items for Systematic Reviews and Meta-Analyses (PRISMA) flowchart for studies selection.

\section{Physiopathology}

${ }^{18}$ F-FDG, exploring glucose metabolism, is the main PET radiotracer used in nuclear medicine imaging. However, ${ }^{18}$ F-FDG is not specific to tumor cells and also targets immune cells. Since immunotherapy works by inducing an inflammatory response, it is therefore difficult to differentiate ${ }^{18}$ F-FDG uptakes related to tumor cells from those due to inflammation.

To overcome this limitation, an understanding of the mechanisms of ICI is helpful in identifying potential new targets for nuclear medicine imaging. The physiopathology is summarized below and shown in Figure 2. 


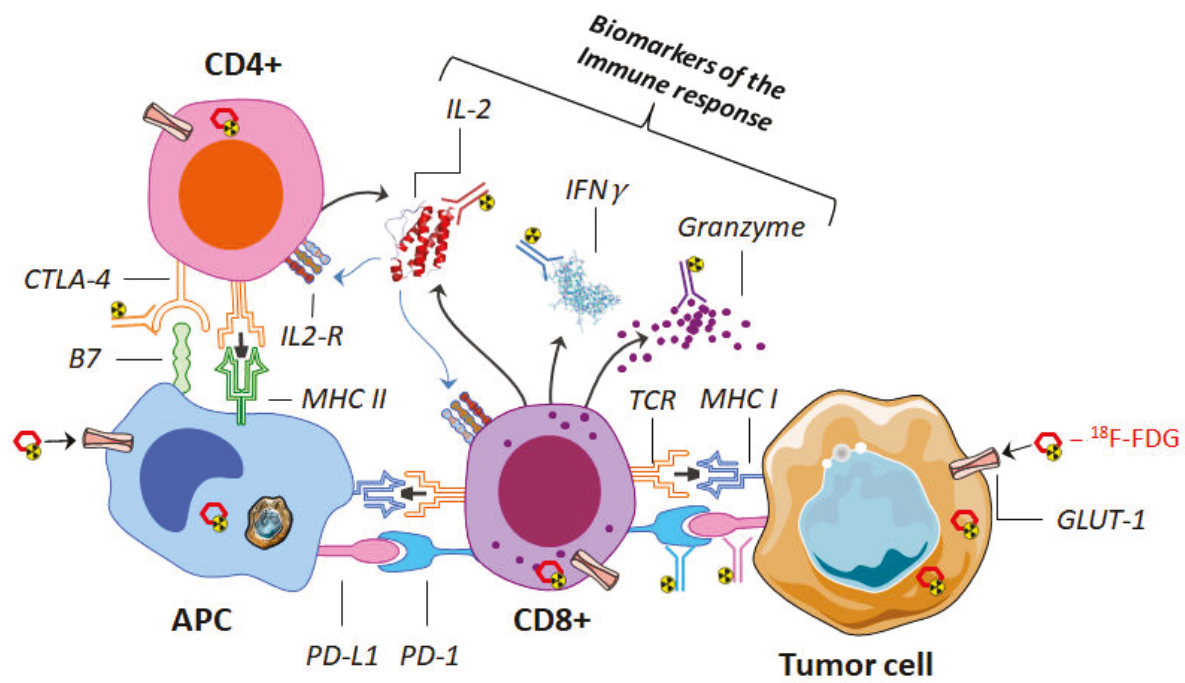

Figure 2. Representation of the interaction between CD4 and CD8 lymphocytes with an antigen-presenting cell (APC) (here a macrophage) and a tumor cell. Different targets can be imaged by use of several labeled antibodies (immuno-PET). These targets may be immune-checkpoints (such as cytotoxic T-lymphocyte antigen 4 (CTLA-4), PD-L1, and its receptor programmed cell death protein-1 (PD-1)) or biomarkers of the immune response (such as interferon $\gamma($ IFN $\gamma$ ), granzyme and interleukin-2 (IL2)). ${ }^{18}$ F-fluorodeoxyglucose (18F-FDG), on the other hand, makes it possible to assess the expression of the glucose transporter (GLUT-1); it can be incorporated indifferently, both in tumor and immune cells. PET: positron emission tomography.

Cell-mediated immunity is performed by immune cells independently from circulating antibodies. These cells are macrophages and natural killer cells from the innate system or $\mathrm{T}$ cells including helper, regulatory, and killer $\mathrm{T}$ cells from the adaptive immune system. Among them, cytotoxic $\mathrm{T}$ cells expressing CD8 as a co-receptor of T-cell receptor (TCR) cause the cell death of tumor cells after releasing serine proteases such as granzymes. However, the cytotoxic $\mathrm{T}$ cell activity is regulated and modulated by others cells such as myeloid-derived suppressor cells (MDSC) and regulatory T cells expressing CD4.

The cytotoxic $T$ cell allows an immune response against tumor cells. The specificity of the immune response is driven by the interaction between cells expressing major histocompatibility complex receptor (MHC) and TCR. Briefly, major histocompatibility complex class I (MHC-I), displaying an antigen from tumor cells, will be recognized by the TCR of a cytotoxic T cell. However, co-inhibitory and co-stimulatory signals regulate this immune response. Co-stimulatory signals such as interleukin-2 (IL-2) or interferon $\gamma$ (IFN $\gamma$ ) improve the immune response against foreign antigens. On the contrary, the co-inhibitory signals alleviate the immune response to allow self-tolerance. Thus, PD-L1 and PD-L2 expressed on the membrane surface of tumor cells are recognized by PD-1 of the cytotoxic T cell, and this leads to stopping the immune response. Moreover, if CTLA-4 expressed by regulatory T cells binds to B7 expressed by antigen-presenting cells (APCs), the immune response declines [9].

The signals shared between tumor cells, $\mathrm{T}$ cells, macrophages, and dendritic cells in the tumor microenvironment, due to the interaction of their ligand-receptor pairs, are known as immune checkpoints. The tumor cells that release and/or express mediators of immune suppression, such as PD-L1 and PD-L2, have a chance to proliferate by inhibiting the immune response.

The pharmacology of ICIs is based on the reactivation of the immune response against tumors rather than a direct effect on tumor cells $[10,11]$. The ICIs are only monoclonal antibodies targeting and blocking the co-inhibitory signals of either programmed cell death protein 1 (PD-1) or cytotoxic 
T-lymphocyte antigen-4 (CTLA-4) — which are cytotoxic T cell surface receptors-or a ligand of PD-1 (PD-L1) from tumor cells or antigen-presenting cells [12]. These molecules are used in the oncologic field and harness the immune system to help to fight tumor cells [10].

\section{Medical Imaging: Baseline Examination}

If the response to ICIs seems better compared to other drug classes [4], predictive biomarkers to determine which patients will respond to immune checkpoint inhibitors are useful in making the right treatment choice [13]. Medical imaging — particularly nuclear medicine- has an essential role to play in this field because it offers, in one examination, the possibility of characterizing tumors that are often multifocal and cannot therefore be all biopsied.

Compared to other medical imaging modalities such as CT or MRI, nuclear medicine imaging generally provides the advantage of a highly sensitive whole-body functional examination, the specificity depending on the radiotracer used. The specificity can be low for a multifunctional radiotracer (such as ${ }^{18} \mathrm{~F}-\mathrm{FDG}$, whose uptakes concern tumor and inflammatory cells) to very high for radiotracers targeting specifically an immune cell receptor or an active inflammatory signal (however, such radiotracers are still in development). Concerning the disadvantages, the spatial resolution of nuclear medicine imaging is worse than $\mathrm{CT}$ and MRI, and radiation protection must be taken into account. Many radiotracers are currently studied with a summary (potential interest, name, target, development phase) presented in Table 1.

Table 1. Radiotracers studied in the context of immunotherapy with potential interest, radiotracers, targets and development phase. GLUT-1: glucose transporter 1, NSCLC: non-small cell lung carcinoma.

\begin{tabular}{|c|c|c|c|}
\hline Potential Interest & Radiotracers & Targets & Development Phase \\
\hline $\begin{array}{l}\text { Imaging of tumor cells } \\
\text { and inflammation }\end{array}$ & $18 \mathrm{~F}-\mathrm{FDG}$ & GLUT-1 and hexokinase & Market Authorization \\
\hline \multirow[t]{7}{*}{$\begin{array}{l}\text { Imaging of checkpoints } \\
\text { inhibitors }\end{array}$} & 89Zr-Atezolizumab & PD-L1 & \multirow{7}{*}{$\begin{array}{c}\text { Phase I (Lymphoma, Breast } \\
\text { cancer, Renal cell carcinoma) } \\
\text { Preclinical (Rodent) } \\
\text { Phase I/II (Melanoma, } \\
\text { NSCLC, Oral cancer) } \\
\text { Phase I (NSCLC) } \\
\text { Preclinical (Rodent) } \\
\text { Preclinical (Primate) } \\
\text { Phase II (Metastatic } \\
\text { melanoma) }\end{array}$} \\
\hline & 18F-Adnectin & PD-L1 & \\
\hline & 18F-PD-L1 ([18F]BMS-986192) & PD-L1 & \\
\hline & 99mTc-anti-PD-L1(99m-Tc-NM-01) & PD-L1 & \\
\hline & 64Cu-WL12 & PD-L1 & \\
\hline & 89Zr-Nivolumab([89Zr]-BMS-936558) & PD-1 & \\
\hline & 89Zr-Ipilimumab & CTLA-4 & \\
\hline \multirow{4}{*}{$\begin{array}{l}\text { Imaging of biomarkers of } \\
\text { immune response }\end{array}$} & $89 \mathrm{Zr}-\mathrm{IFN} \gamma$ & $I F N \gamma$ & Preclinical (Rodent) \\
\hline & 68Ga-NOTA-GZP & Granzyme & \multirow{3}{*}{$\begin{array}{c}\text { Preclinical (Rodent) } \\
\text { Open label (metastatic } \\
\text { melanoma); Phase I (renal } \\
\text { transplant rejection) } \\
\text { Phase I (metastatic } \\
\text { melanoma) }\end{array}$} \\
\hline & 18F-IL2 ([18F]FB-IL2) & IL2 & \\
\hline & 99mTc-IL2 & IL2 & \\
\hline
\end{tabular}

It has to be noticed that the radionuclide used to label the radiotracer has to be chosen carefully. In particular, it must be able to chemically bind to the tracer molecule either by covalent or by coordinate bonds. The emitted radioactivity must also be adapted to the imaging method used with the gamma $(\gamma)$ ray emitter (Technetium ${ }^{99 \mathrm{~m}} \mathrm{Tc}$, Indium $\left.{ }^{111} \mathrm{In}\right)$, for the gamma camera and positron $(\beta+)$ emitter (Fluorine ${ }^{18} \mathrm{~F}$, Copper ${ }^{64} \mathrm{Cu}$, Gallium ${ }^{68} \mathrm{Ga}$, Zirconium ${ }^{89} \mathrm{Zr}$ ) for PET-the spatial resolution of the PET being inherently better than that of the gamma camera. Moreover, the radioactive half-life of the radionuclide has to be adapted to the physiological process being explored: for short processes (a few hours), ${ }^{99 \mathrm{~m}} \mathrm{Tc},{ }^{18} \mathrm{~F}$, and ${ }^{68} \mathrm{Ga}$ can be chosen, but for long processes (a few days) such as those observed with antibodies, ${ }^{111} \mathrm{In},{ }^{64} \mathrm{Cu}$, and ${ }^{89} \mathrm{Zr}$ with longer radioactive periods have to be used. Finally, the 
deposited energy dose, which varies according to the radionuclides and the tracers' clearance, must be taken into account to ensure that it is as low as possible.

\section{1. ${ }^{18} \mathrm{~F}-\mathrm{FDG}$ PET/CT}

${ }^{18} \mathrm{~F}$-fluorodeoxyglucose $\left({ }^{18} \mathrm{~F}-\mathrm{FDG}\right)$ is a multifunctional PET/CT radiotracer exploring glucose metabolism-including tumor metabolism and inflammation-and is a well-established nuclear medicine examination performed to explore cancer, notably for the extension assessment [14].

Beyond the extension assessment, baseline ${ }^{18} \mathrm{~F}$-FDG total metabolic tumor volume (TMTV), measured by segmenting all tumors on PET images, could be an interesting parameter, as it can be easily (semi)-automatically determined, and as it presents an important prognostic value in many cancers and treatments, including immunotherapy. Therefore, Ito et al. have shown for 142 patients with melanoma treated by ipilimumab that the median overall survival (OS) was significantly lower for patients with high TMTV (10.8 months 95\% CI 5.9-15.8 months) than with low TMTV (26.0 months $95 \%$ CI 3.0-49.2 months) [15]. In a multivariate analysis including TMTV and significant clinical parameters (including age and active brain metastases), TMVT was still a significant prognostic factor [15]. Similar results have been observed for non-small cell lung carcinoma (NSCLC) treated by ICI [16].

Beyond its role in the initial extension assessment, the ${ }^{18}$ F-FDG PET could also be useful in determining the PD-1/PD-L1 status, on which the response to immunotherapy depends. Effectively, PD-L1 promotes glycolytic metabolism in tumor cells, while this glucose consumption by tumors metabolically restricts T cells, notably by dampening their glycolytic capacity [17]. As a result, PD-L1 protein expression was significantly correlated to glucose transporter 1 (GLUT1) expression, which is the transporter of ${ }^{18} \mathrm{~F}-\mathrm{FDG}$, in lung adenocarcinoma [18,19] and squamous-cell carcinoma [20]. In a meta-analysis about lung cancer including three studies (718 patients), ${ }^{18}$ F-FDG PET SUVmax (maximal standardized uptake value reflecting tumor activity) and PD-L1 expression were slightly correlated (Spearman's correlation 0.36 (95\% CI: 0.22; 0.50)), although this low value does not allow the SUVmax to be used as a replacement for the PDL1 status in lung cancer [21]. For bladder cancer, a study including 63 patients has shown too that SUVmax was significantly higher in PD-1/PD-L1-positive patients but also that PD-1 and PD-L1 status could be predicted using a SUVmax cut-off value of 22.7, with quite good accuracies of $71.4 \%$ and $77.8 \%$, respectively [22]. It has to be added that ${ }^{18} \mathrm{~F}$-FDG SUV is not only significantly correlated to PD-1/PD-L1 status, but also to other biomarkers of interest when immune-checkpoint inhibitors are considered, including CD8 tumor infiltration by lymphocytes (TILs) [23].

${ }^{18} \mathrm{~F}-\mathrm{FDG}$ PET/CT could also be used to determine indirect predictive factors for response to immunotherapy treatment. Therefore, this examination could reflect the gut microbiota, whose analysis could be favorable to predict the treatment effect of immunotherapy, as gut microbiota seems linked to the therapeutic response [24]. Thus, in a retrospective pilot study involving 14 patients with metastatic melanoma treated with ipilimumab in the first line, a team observed that colonic, pre-therapeutic ${ }^{18} \mathrm{~F}$-FDG PET SUVmax seems lower for responding patients with mean SUVmax equal to $1.33 \pm 0.04,2.2 \pm 0.46$, and $3.33 \pm 2.67$ for individuals with complete response, partial response, and progressive disease, respectively [25]. This could be explained by an association between low bacterial load and higher physiologic colonic ${ }^{18}$ F-FDG uptake due to a shift in colonic metabolism from short-chain fatty acids, produced by colonic bacteria, to glycolysis when the bacterial load is low [26]. However, these promising preliminary results have to be confirmed.

Another indirect predictive factor is the determination of body composition parameters on the anatomical CT associated with the PET. Therefore, in a study including 55 patients with NSCLC treated by nivolumab, whole-body subcutaneous fat mass measured automatically on the CT of the PET was a significant prognostic factor with a better prognosis for fatty patients $(H R=0.75, p=0.006$ in multivariate analysis) $[27,28]$, possibly because adipocytes in the human obese subcutaneous fat mass release several pro-inflammatory cytokines and chemokines, which contribute to the establishment and maintenance of local and systemic inflammation [29]. 
Nevertheless, ${ }^{18} \mathrm{~F}$-FDG is not as tumor-specific as we might wish, notably for response assessment during immunotherapy. One of the main problems with ICIs is related to the inflammatory reactions that recall neutrophils and macrophages and activate $\mathrm{T}$ cells on the tumor site. This antineoplastic activation determines the high consumption of FDG by immune cells and, consequently, a loss of specificity for this radiotracer.

\subsection{Immunoimaging}

Despite the remarkable success of ICIs in clinical practice, the efficacy and responsiveness of these agents vary greatly among different tumor types and across individuals [30]. Biomarkers determined on tumor biopsy, including PD-1 and PD-L1 measured with immunohistochemistry (IHC), can help to select patients. However, these biomarkers are limited, as some patients can have a response without them, while others can have no response with them [31]. A possible explanation is that a single biopsy may not capture the heterogeneity across various tumor lesions in a patient or within a single lesion [31]. Therefore, the use of radiotracers to predict responses to immunotherapy is becoming increasingly important to better select patients, as they have the possibility to characterize the whole tumors in a single non-invasive examination. Among the possible methods, "immuno-PET", combining antibodies (or antibody fragments) with PET radionuclide (positron emitter), takes advantage of the specificity and affinity of antibodies and the sensitivity of PET [32].

For immuno-PET, targets can be general $\mathrm{T}$ cell markers (such as CD3, CD4, and CD8), immune checkpoints (such as PD-1, PD-L1, or CTLA-4) or biomarkers of the immune response (such as interferon- $\gamma$, interleukin-2, and granzyme B) $[33,34]$. The absence of impact on the functionality of in vivo T cells also needs to be considered [35]. Many radiotracers have been evaluated in preclinical models, with a large "immunoimaging toolbox" already available [36], but few of them have ever been tested in clinical trials [33,36,37]. A summary of the usable radiotracers and target is presented in Figure 2 and in Table 1.

Among the radiotracers tested in clinical trials, antibodies targeting checkpoints inhibitors, notably PD-1/PD-L1, have shown promising results. In a study including 22 patients with three tumor types (bladder cancer, NSCLC, or breast cancer) with pre-therapeutic PET/CT with ${ }^{89}$ Zirconium-labeled atezolizumab (anti-PD-L1), PET uptakes were heterogeneous and generally high in tumors [38]. Uptake was also high in lymphoid tissues and at sites of inflammation [38]. Interestingly, progression-free survival was better correlated with the pretreatment PET signal than with IHC-based predictive biomarkers, with, for example, a hazard ratio equal to 11.7 (95\% CI: 3.3-62.7) for PET versus 2.6 (95\% CI: 0.8-13.6) for an immunohistochemistry analysis of PD-L1 [38]. These results for the survival are possibly linked to the heterogeneity of the PD-L1 expression across different tumor localizations. Comparable results were obtained in another study evaluating two anti-PD-1/PD-L1 radiotracers ( ${ }^{18}{ }^{18}$ Fluor-labeled anti-PD-L1 Adnectin and an anti-PD-1, ${ }^{89}$ Zirconium-labeled nivolumab) on 13 patients with advanced NSCLC [39]. Other PD-L1-specific radiolabeled peptides exist. Notably, the so-called "[ $\left.{ }^{64} \mathrm{Cu}\right] \mathrm{WL12}$ ", which is a 14-amino acid circular peptide "WL12", with a binding interface on PD-L1 overlapping with PD-1 and PD-L1 therapeutics, radiolabeled with ${ }^{64} \mathrm{Cu}$, a positron $(\beta+)$ emitter [40]. A preclinical study on mice has shown that this radiotracer could generate highly contrasted images within 120 minutes after the injection [41], which is a usual time period for conducting nuclear medicine examinations. Moreover, this peptide could measure the PD-L1 occupancy and the pharmacokinetics of PD-L1 immune-checkpoint inhibitor non-invasively and independently of the type of antibody chosen for treatment [40].

Imaging of the biomarkers of immune response could also be interesting. Interferon- $\gamma$ (IFN $\gamma$ ) immuno-PET $\left({ }^{89} \mathrm{Zr}\right.$-anti-IFN- $\gamma$ ) offers the possibility to explore the activated lymphocytes inside tumors, including CD8+ cytotoxic T lymphocytes [42]. In a preclinical study, the tumor uptake with this radiotracer seemed more specific than CD3 immuno-PET $\left({ }^{89} \mathrm{Zr}\right.$-anti-CD3), which targets the general T-cell population localized in the tumor but also in other lymphoid tissues, and it could help to discern activated versus anergic/dysfunctional status [42]. Another interesting target of immune 
response could be the protease granzyme B (GZP), which is secreted by cytotoxic CD8+ and has a key role for immune-induced apoptosis [43]. In preclinical tumor models, a highly specific peptide PET imaging agent for GZP was predictive of the response to immune-checkpoint inhibitors, with high signal tumors responding to therapy and with $93 \%$ sensitivity and $94 \%$ negative predictive value [44]. Clinical studies have yet to be done.

Finally, a radiotracer for a gamma camera using monophotonic gamma radioactivity, which is different from the positron $(\beta+)$ radioactivity used for PET, is also possible. A study on 16 patients with NSCLC demonstrates that a ${ }^{99} \mathrm{~m}$ Tc-labeled anti-PD-L1-single domain antibody was feasible in humans safely and with acceptable dosimetry [45]. The image acquisition was also possible rather quickly after the injection at $2 \mathrm{~h}$ [45]. However, if the ratio between the tumor uptake and blood pool at $2 \mathrm{~h}$ was significantly correlated to the PD-L1 immunohistochemistry $(\rho=0.68, p=0.014)$ and the uptake ratio was lower in tumors with $\leq 1 \%$ PD-L1 expression (1.89 versus $2.49, p=0.048$ ), the results seem too limited to be used in clinical practice [45]. Another radiotracer for a gamma camera is ${ }^{99 \mathrm{~m}}$ Tc-interleukin-2 ( $\left.{ }^{99 \mathrm{~m}} \mathrm{Tc}-\mathrm{IL} 2\right)$, which could be used to detect TILs and distinguish between true progression and pseudoprogression [46]. A pilot study has demonstrated the safety and feasibility of ${ }^{99 \mathrm{~m}}$ Tc-IL2 imaging, but further studies are needed [46].

\subsection{Radiomics and Complex Quantitative Parameters}

Radiomics is a set of methods used to take advantage of medical imaging and extract quantitative features that can characterize the tumor phenotype. A very large number of features can be extracted (manually or automatically) from medical images (e.g., CT, MRI, PET) and then correlated to tumor characteristics and clinical outcomes using machine learning algorithms.

In a retrospective study using four independent cohorts of patients, Sun et al. have shown the usefulness of this method in determining the tumor infiltration by CD8 cells on contrast-enhanced $\mathrm{CT}$ images and with a signature combining eight features [47]. Despite the relatively low area under the curve of the score for this prediction (AUC $=0.67 ; 95 \%$ CI $0.57-0.77$ ), the signature was able to predict an objective response to anti-PD-1 and PD-L1 therapy, notably at 3 months ( $p=0.049)$, as well as overall survival in univariate (median overall survival was 24.3 months in the high radiomic score group versus 11.5 months in the low radiomic score group; $p=0.0081$ ) and multivariate analyses [47]. Another study explored the interest of radiomics as a non-invasive biomarker for responses to cancer immunotherapy on 1055 primary and metastatic lesions from 203 contrast-enhanced CTs from patients with advanced melanoma and NSCLC, undergoing anti-PD1 therapy [48]. They found on a lesion-based approach, reflecting the metastatic condition, that lesions with heterogeneous density and more compact and spherical (high volume/surface ratio) were associated with a better response [48]. Concerning ${ }^{18}$ F-FDG PET radiomic analysis and PD-1/PD-L1 expression, a team found in 53 oropharyngeal or hypopharyngeal cancer patients that several PET-derived textural features, describing the organization of tumor pixels, can provide information to determine tumor PD-L1 expression in head and neck carcinoma [49]. However, no clear and validated textural model distinguishing high and low PD-L1 expression is described, and more studies have yet to be done. Finally, delta-radiomics ( $\Delta$-radiomics), studying changes in radiomic features (e.g., texture within the nodule) on serial images could be useful to assess the effectiveness of therapy as well as predict early treatment response, but this domain as yet to be explored [50].

Complex quantitative parameters could be also interesting, such as compartmental parameters describing the kinetics of radiotracers more precisely than SUV. However, such parameters have not shown an added value compared to SUV in a population of 25 patients with metastatic melanoma treated with immunotherapy [51]; therefore, further studies are still needed.

\section{Therapeutic Evaluation}

${ }^{18} \mathrm{~F}$-FDG PET/CT is a routinely used for the examination of therapeutic evaluation of cancers. However, ICIs, treating cancer by inducing inflammation, question its interpretation and the time 
of execution, because this radiotracer presents an uptake in the case of active cancer but also of inflammation. Comparable challenges are observed for anatomical images. Different patterns of response according to the time of examination are shown in Figure 3.

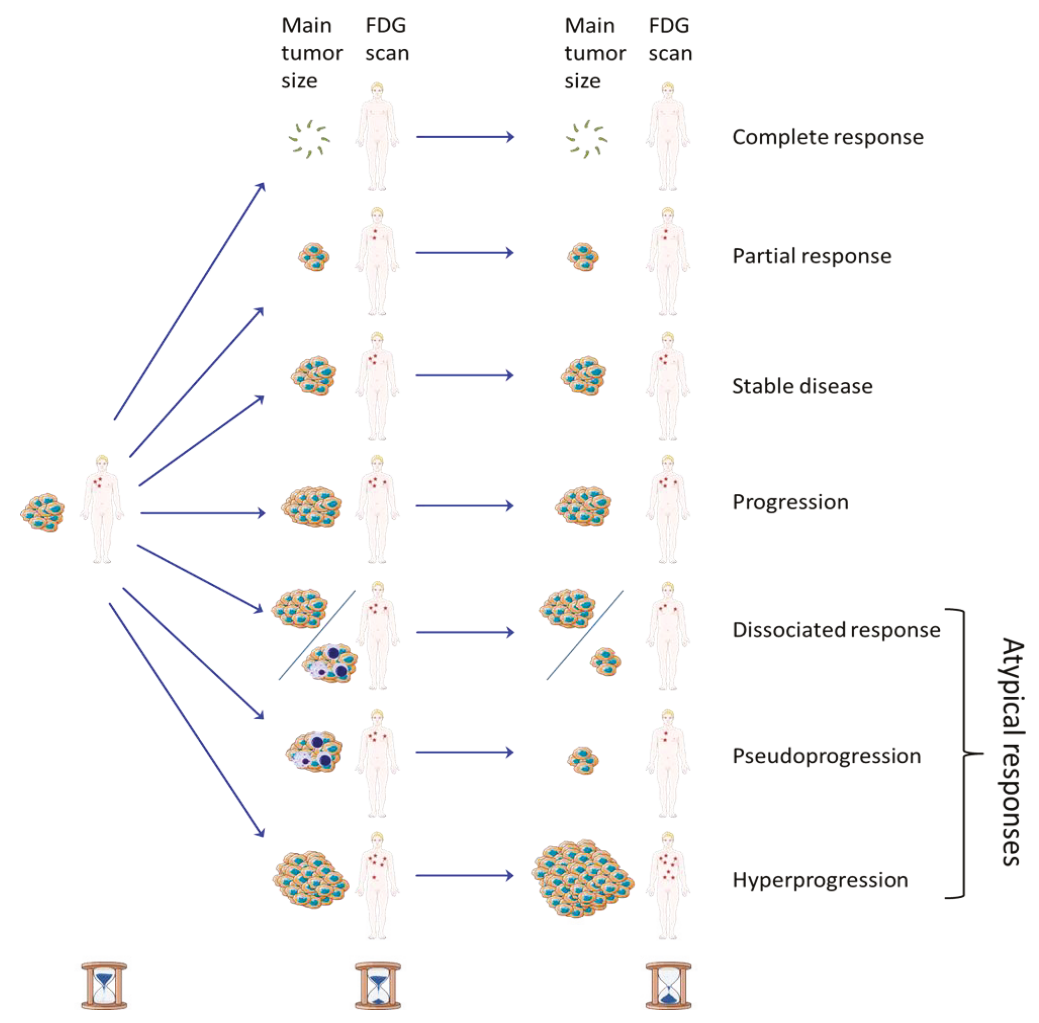

Figure 3. Patterns of response according to the size and number of the tumors and in function of time of examination. Complete response, partial response, stable disease, and progression are the four classical patterns of response in oncology. Concerning the atypical responses sometimes observed with immune-checkpoint inhibitors, a dissociated response corresponds to lesions shrinking and others growing; pseudoprogression is an initial increase in tumor size and/or number due to inflammation followed by a decrease, and hyperprogression is an accelerated tumor growth rate after starting treating.

\subsection{Standard Therapeutic Assessment Scales}

The therapeutic evaluation of cytotoxic chemotherapy in morphological imaging is based on the fact that increasing lesion size and/or the appearance of new lesions after treatment indicates progression and therapeutic failure [12]. Many therapeutic evaluation criteria exist for morphological imaging, including Response Evaluation Criteria in Solid Tumors (RECIST) 1.1, which uses unidimensional single-diameter measurements [52]. Compared to morphological imaging, such as CT, functional imaging including ${ }^{18} \mathrm{~F}$-FDG PET/CT can provide an earlier response assessment. One of the situations where therapeutic assessment by functional imaging by ${ }^{18}$ F-FDG PET may be much earlier than morphological imaging is gastrointestinal stromal tumors (GISTs) treated by imatinib. While measuring anatomical responses through morphological imaging (CT) often requires many months, 18F-FDG PET can predict responses within one day to one week [53]. Some therapeutic evaluation criteria exist in nuclear medicine, including the PET Response Criteria in Solid Tumors (PERCIST) 1.0 for solid tumors [54] and the Lugano classification for lymphomas [55]. 


\subsection{Limitations due to Atypical Tumor Response Patterns}

The therapeutic scales used to evaluate cytotoxic chemotherapies are generally based on a classification with four patterns of response: complete response, partial response, stable disease, or progression. However, "atypical" tumor response patterns that do not belong to these four categories can be observed with ICIs, which makes the therapeutic evaluation of these treatments a real challenge.

Pseudoprogression is an atypical tumor response pattern defined as an increase in the size of lesions or the visualization of new lesions, followed by a response [6,7]. This effect could be explained by the initial T-cell tumor infiltration, with an apparent increase in the tumor burden disconnected to the tumor cell proliferation [56]. In studies, rates of pseudoprogression never exceeded $10 \%$; therefore, they stayed rare compared to effective disease progression [6].

Dissociated responses, observed when some lesions shrink and others grow, are another atypical tumor response of ICIs. One study reported dissociated responses in 7.5\% of NSCLC patients treated with anti-PD1/PD-L1 agents with a better survival observed than true progressions [57]. Another study with 50 patients having NSCLC treated by ICI and followed by ${ }^{18}$ F-FDG PET showed that dissociated response occurred in $26 \%$ (five patients) of the 19 patients whose treatment was continued after initial progression with a clinical benefit for these patients [58].

Nevertheless, due to these atypical responses, classical criteria, such as RECIST or PERCIST, can be limited to the therapeutic assessment of immune checkpoint inhibitors; some diseases can be classified as "progressive", while this is the response to the treatment that occurs $[56,58]$.

\subsection{Updated Therapeutic Assessment Scales for Immunotherapy}

To avoid a misdiagnosis in therapeutic evaluation between progressive disease and inflammation, a modification of the scales has been proposed. In anatomical and morphological imaging, at least four modified criteria have already been proposed: irRC (immune-related response criteria) (2009) [56], irRECIST (immune-related RECIST) (2013) [59], iRECIST (immune RECIST) (2017) [7], and imRECIST (immune-modified RECIST) (2018) [60]. Some differences exist between these scales, but they have all in common the need to confirm a progressive disease on a new examination, which is usually at 4 weeks [61]. The interest of these updated scales has been highlighted in a study including 160 patients with NSCLC with follow up performed by computed tomography. Patients with atypical responses, according to the iRECIST and irRECIST, represented 11\% of the RECIST 1.1 so-called progressive patients, and they had superior overall survival compared to patients with the true disease progression [57].

If the increase in the size or appearance of new lesions due to pseudoprogression can mislead the therapeutic evaluation in radiological imaging, the ${ }^{18}$ F-FDG PET metabolic evaluation can also be misleading. Effectively, ${ }^{18} \mathrm{~F}-\mathrm{FDG}$ is not a tumor-specific radiotracer, and the inflammatory response, which displaces neutrophils, macrophages, and activated T cells to the tumor site, also leads to the uptake of ${ }^{18}$ F-FDG [62].

Therefore, an effort has been made in nuclear medicine with modified scales proposed, including, for solid tumors, PECRIT (PET/CT Criteria for the early prediction of Response to Immune checkpoint inhibitor Therapy) (2017) [63], PERCIMT (PET Response Evaluation Criteria for Immunotherapy) (2018) [64], imPERCIST5 (immunotherapy-modified PERCIST up to five lesions) (2019) [65], and iPERCIST (immune PERCIST) (2019) [66]. Table 2 resumes the proposed criteria for therapeutic evaluation by ${ }^{18}$ F-FDG PET of solid tumors treated by immune checkpoint inhibitor. 


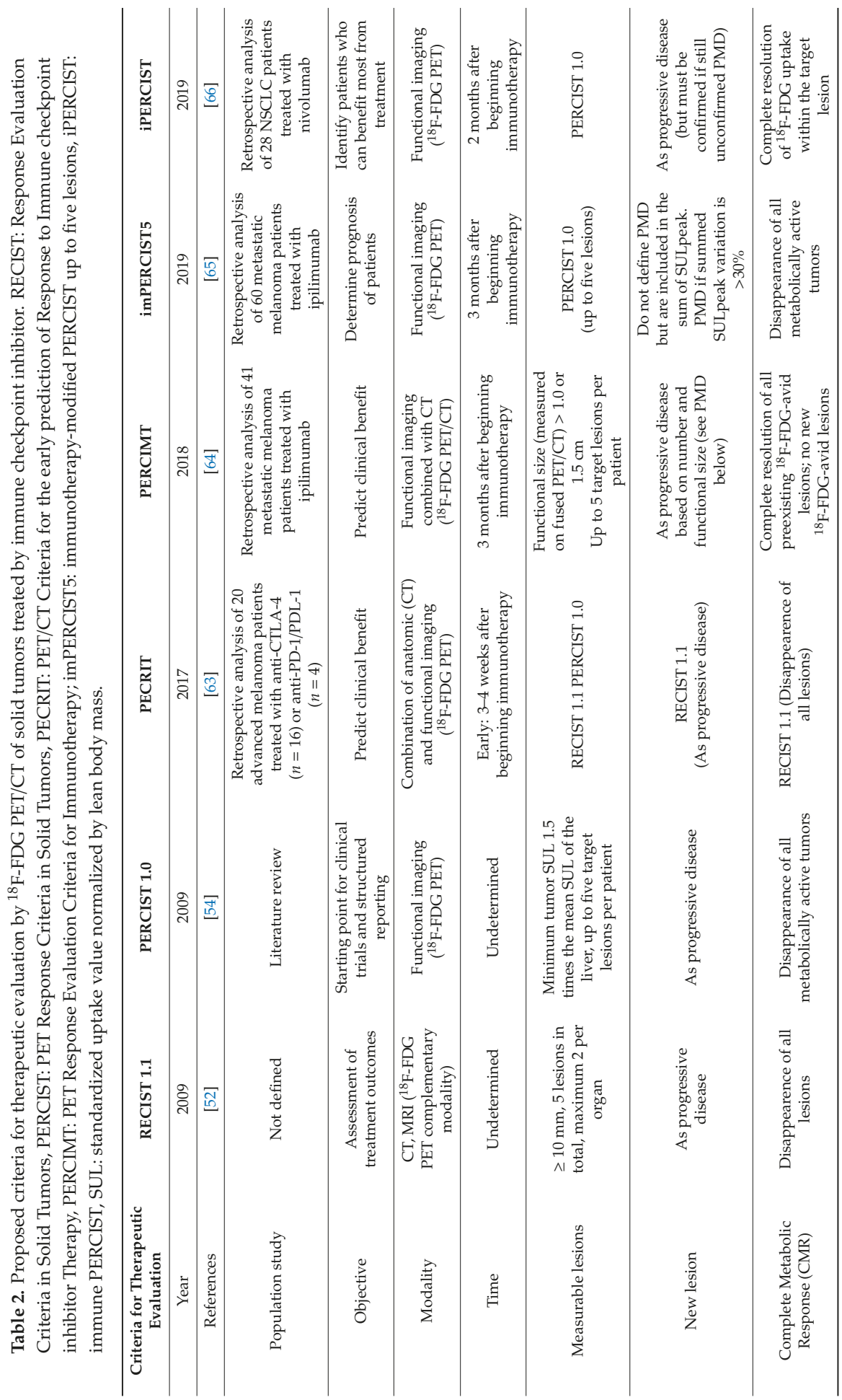


Cancers 2020, 12, 371

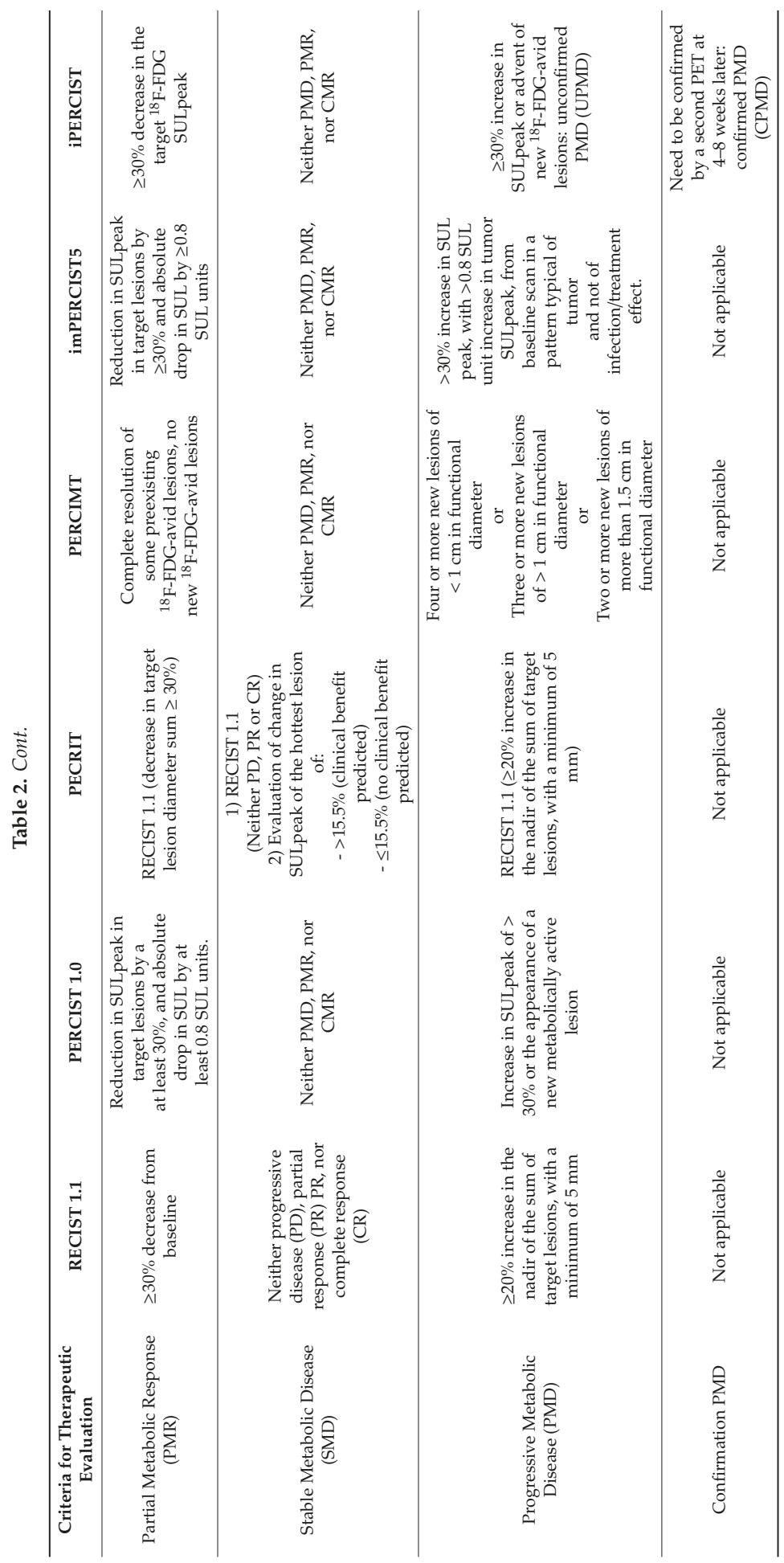


However, these nuclear medicine criteria are quite different, and therefore, standardization is needed [67]. It should be noticed that only iPERCIST introduces the need to confirm a progressive disease such as the updated radiological criteria [66]; however, such a control may limit the risk of a false positive PET scan. A dissociated response is also not considered in these scales, while it seems important as the patients experiencing these response patterns seems to have a clinical benefit of immunotherapy [58]. Moreover, the populations used to define these criteria were relatively small (from 20 patients for PERCRIT [63] to 60 patients for imPERCIST [65]), and studies with more patients (and, therefore, more cases of atypical tumor responses) are needed to validate these criteria. The localization of the uptake should also be considered, as an immune activation induced by checkpoint treatment can be observed in tumor-draining lymph nodes [68] that can induce an ${ }^{18}$ F-FDG uptake and be misinterpreted as progression, as seen in Figure 4.

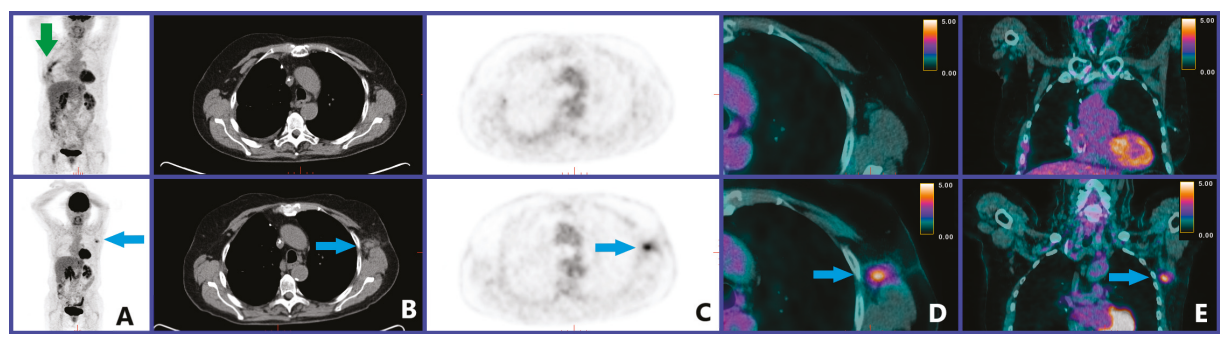

Figure 4. Clinical case of a patient with a previous history of treated left breast cancer and melanoma of the right leg with melanoma pulmonary metastasis recently operated. The images of the upper line show the PET/CT examination before the start of immune checkpoint inhibitor (pembrolizumab), and the images in the lower line show the PET/CT examination performed 9 months after the start of the treatment with (in A) a maximum intensity projection (MIP) PET image; in B, an axial CT slice; in C, an axial PET slice; in D, an axial PET/CT fused slice; in E, a frontal PET/CT fused slice. The green arrow on the pre-therapeutic PET MIP indicates the post-operative inflammation of melanoma pulmonary metastasis. The blue arrows on the follow-up PET/CT examination indicate that an intense left axillary lymph node uptake (SUVmax 4.7) appeared, which was considered suspicious of disease progression when it was found to be an inflammatory reactive lymph node in histological analysis.

Different scales for solid tumors and hematological tumors have to be considered as the response patterns appear to be different. For lymphomas, modified Lugano criteria have been proposed with the establishment of lymphoma response to immunomodulatory therapy criteria (LYRIC) [69]. Comparable to the category "unconfirmed progressive disease" used for solid tumors by iRECIST and iPERCIST $[7,66]$, LYRIC has introduced the category "indeterminate response" (IR) when an increase in tumor burden, new lesions, and/or increased ${ }^{18} \mathrm{~F}$-FDG uptake is observed with subsequent imaging within 12 weeks, which is recommended to confirm the true disease progression versus pseudoprogression [69]. However, concerning Hodgkin lymphoma, the contribution of LYRIC compared to the standard Lugano classification seems limited, because pseudoprogressions seem very rare in this disease. Thus, no pseudoprogression was reported in the two studies, including a total of 60 patients with refractory or relapsed Hodgkin lymphoma treated with immunotherapy and having an early therapeutic evaluation (2 to 3 months) by ${ }^{18}$ F-FDG PET/CT [70,71]. Moreover, in a retrospective multicentric study including 45 patients with Hodgkin lymphoma treated by nivolumab, the classification at the ${ }^{18}$ F-FDG PET performed at 2.0 months (interquartile range: $1.7-3.7$ months) was identical between Lugano criteria and LYRIC, since all 16 progression events on this examination classified as an indeterminate response per LYRIC were confirmed as progressive diseases on subsequent evaluations [72]. In most other types of lymphoma, including follicular lymphoma and diffuse large B cell lymphoma, ICIs have been rarely used to date, because they are less effective, and therefore, there is a lack of data regarding their therapeutic evaluation [73]. 


\subsection{Hyperprogression Disease and Early Therapeutic Response Evaluation}

Hyperprogression disease is an acceleration of the tumor growth rate (TGR) with a $\Delta$ TGR (variation of TGR per month) exceeding $50 \%$ at the first evaluation compared to pretreatment kinetics [74]. This phenomenon is quite common for patients treated with ICI, as it was observed in $7 \%$ (12 of 189) patients with solid tumors [5] and is associated with high metastatic burden and poor prognosis [74].

As hyperprogression disease drives toward early death, notably when it occurs in the first 6 weeks of treatment, the anticipated first radiological evaluation during PD-1/PD-L1 inhibitor treatment, including ${ }^{18} \mathrm{~F}-\mathrm{FDG}$ PET/CT, has to be discussed in order to identify them [74].

${ }^{18}$ F-FDG PET/CT performed early after the initiation of immunotherapy could not only detect hyperprogression but also participate in the therapeutic evaluation, notably by detecting early responders and non-responders more easily than with $\mathrm{CT}$. Therefore, in a study with 24 patients with NSCLC treated by nivolumab, metabolic responses determined by ${ }^{18} \mathrm{~F}-\mathrm{FDG}$ PET at 1 month (especially total lesion glycolysis, TLG, which is the product of the TMTV and the mean SUV) were closely associated with therapeutic response and survival, while it was difficult to distinguish between responders and non-responders on morphological changes on CT scans [75]. In another pilot prospective study of 10 patients with unresectable metastasized melanoma, the responding patients could be reliably identified as early as two weeks after the start of the therapy [76], which was consistent with the usual early metabolic response, compared to the generally later anatomical response.

Interim evaluation, performed after the first two cycles of immunotherapy, is also possible with interesting results, as was shown in a population of 41 patients with unresectable metastatic melanoma treated by ipilimumab. The PERCIMT classification showed a sensitivity of $93.6 \%$, a specificity of $70.0 \%$, and an accuracy of $87.8 \%$ to predict clinical benefit-including stable disease, partial response, and complete response (31 patients)—and those showing no clinical benefit including progressive disease (10 patients) according to the best clinical response of patients, which was assessed at a median of 21 months [77].

\subsection{Abscopal Effect}

Radiation therapy can induce the death of cancer cells and can activate the immune system [78]. The abscopal effect is a tumor regression observed in metastases distant from the local treatment site, such as the primary irradiation site [78]. The combination of radiotherapy and immunotherapy can have the advantage of controlling disease locally with potential systemic and lasting immune effects [78]. PET/CT can observe such responses, even if, until now, only case reports have been reported [79-81].

\section{Diagnosis of Side Effects in Nuclear Medicine Imaging}

The physiological objective of CTLA4 and PD-1/PD-L1 is the blockade of auto immune responses. When these pathways are inhibited, T-cell inflammatory responses can be triggered to target cancer; however, they also target some healthy tissues. The attacks on healthy tissue can generate immune-related adverse events (IrAE), the scope of which is large [82] and the gravity of which, though generally low, can be severe (around 10\% patients with anti-PD-1/PD-L1 experienced severe IrAEs [83]) and even potentially life threatening if not diagnosed and treated. Therefore, the permanent discontinuation of immune checkpoint inhibitors is advised in patients with high-grade ocular, hepatic, pancreatic and/or pulmonary IrAEs [82]. IrAEs are generally detected by clinicians, but medical imaging can help to find early signs of unknown IrAEs, with radiologically evident IrAEs reported in up to approximately $30 \%$ of patients [84]. Moreover, to avoid errors in therapeutic evaluation, these IrAEs have to be differentiated from active cancerous disease, notably from metastatic progression. Figure 5 resumes the discoverability by ${ }^{18}$ F-FDG PET/CT of different IrAEs. 


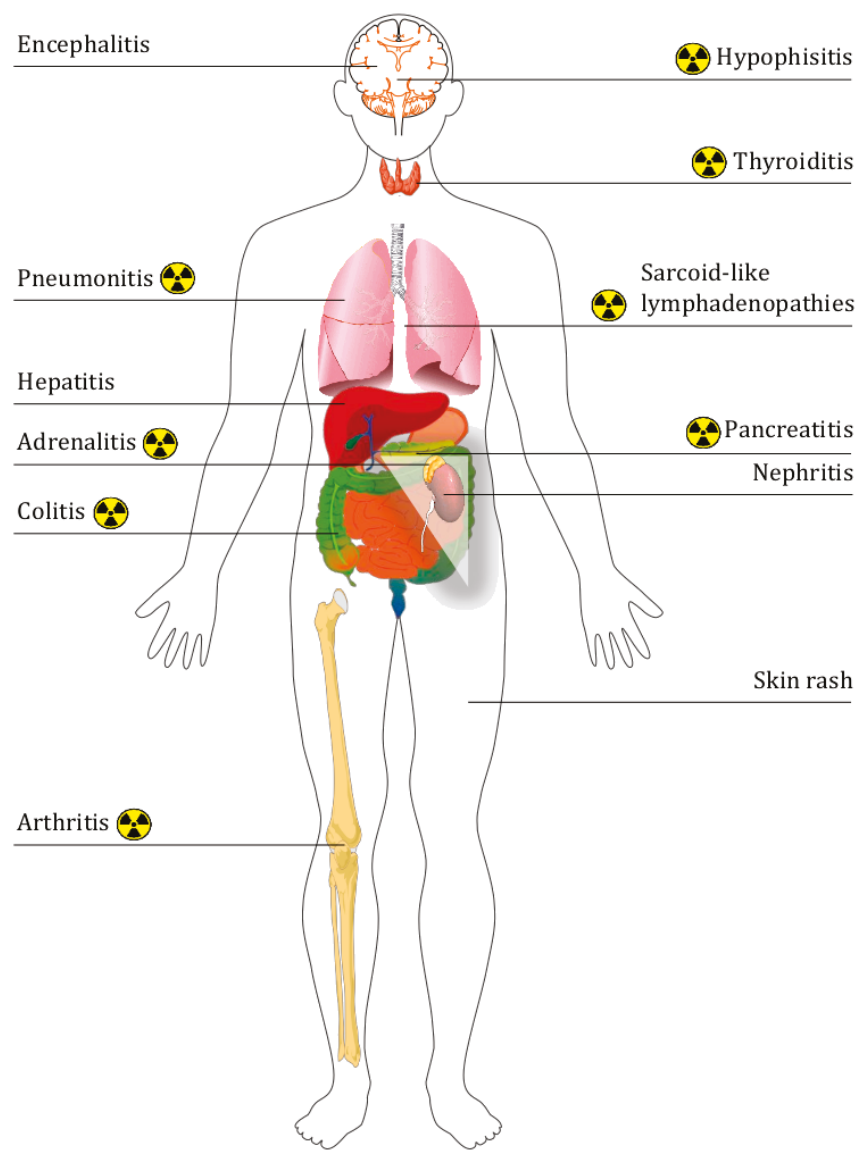

Figure 5. Summary diagram of the different immune-related adverse events. The nuclear symbol represents the side effects that can be visualized by ${ }^{18} \mathrm{~F}-\mathrm{FDG}$ PET/CT.

In a monocentric retrospective study with the objective of describing the IrAE characteristics on medical imaging and their detection rate, Mekki et al. found 39 (74\%) abnormal medical imaging findings on 53 patients who had a co-occurrence of irAEs and medical imaging (CT, PET/CT, MRI, US of radiography) within 15 days [85]. Among the 12 performed ${ }^{18}$ F-FDG PET/CT, 10 (83\%) were abnormal with thoracic sarcoid-like reaction, enterocolitis, thyroiditis, hypophysitis, and pancreatitis [85].

Regardless of their severity, the occurrence of an IrAE could also be linked to the therapeutic response by revealing the immune response. In a study of 41 patients with metastatic melanoma treated by anti-CTLA-4 (ipilimumab), four patients among the 31 having disease control had a sarcoid-like mediastino-hilar lymphadenopathy diagnosed on the follow-up ${ }^{18}$ FDG PET/CT, while this side effect was not observed on the 10 patients having progressive disease [86]. The same team performed a study on 16 patients with BRAF-mutation positive metastatic melanoma treated by a combination of vemurafenib and ipilimumab with longitudinal ${ }^{18} \mathrm{~F}$-FDG PET/CT for the follow-up. Seven patients developed imaging signs on PET/CT of at least one immune-related adverse event, with colitis and arthritis being the most frequent ones (five and four events, respectively), and these patients had a significantly longer progression-free survival than those without irAEs $(p=0.036)$ [87]. Another team found concordant results on a retrospective study of 40 patients with three types of cancer (malignant melanoma, malignant lymphoma, and renal cell carcinoma) treated by ICIs and followed 
by ${ }^{18}$ F-FDG PET/CT. They found that a PET-detectable immune-related adverse event indicated a favorable outcome (nine of 11 patients with IrAE had complete response at final evaluation) with in particular thyroiditis, which was seen earlier than other IRAE and could provide an early indicator of the efficacy of immunotherapy [88]. Therefore, immune-related inflammation has to be reported even if they are not necessarily associated with clinically significant IrAE [89]. Illustrations of ${ }^{18} \mathrm{~F}-\mathrm{FDG}$ PET-detectable immune-related adverse effects are presented in several articles $[85,88,90]$.

Another early sign of immune activity is an inversion of the liver-to-spleen ratio (normally $>1$ ) [89], possibly by reflecting the immune activation preceding $\mathrm{T}$ cell proliferation [91], but also the reactive nodes in the drainage basin of the primary tumor [89], which could be wrongly diagnosed as cancerous lymph nodes (cf Figure 4).

Finally, if immune checkpoint inhibitors do not promote infections, immune-related adverse effects often require immunosuppressive treatment, which in turn increases the risk of developing serious infections [92], and such infections can be observed on imaging.

\section{End-of-Treatment Assessment}

${ }^{18}$ F-FDG PET imaging may also help better predict long-term outcomes compared to standard computed tomography (CT) response criteria. Therefore, a team has shown in a retrospective study of 104 patients with melanoma, who received anti-PD-1 as monotherapy $(67 \%)$ or combined with ipilimumab (31\%), that while only a small proportion of patients had a complete response at 1 year on CT $(28 \%)$, most patients with a partial response on CT have a complete metabolic response on PET (68\% of the $66 \%$ having a partial response) [93]. Moreover, almost all patients with complete metabolic response on PET at 1 year had ongoing response to therapy thereafter (78\% had discontinued treatment and $96 \%$ had ongoing response) [93]. ${ }^{18}$ F-FDG PET could help to decide on the continuation/discontinuation of therapy [93], notably when durable response is observed [6].

\section{Conclusions}

In this systematic review, we have observed that immune checkpoint inhibitors have a major impact on nuclear medicine imaging with changes in its interpretation, including the consideration of induced inflammation by new therapeutic evaluation criteria.

New perspectives are also emerging, with a central role for nuclear medicine being the prediction of the response to immune checkpoint inhibitors, notably by the use of new radiotracers, such as immuno-PET, or new analysis techniques, including radiomics and body composition analyses.

$\begin{array}{ll}\text { Abbreviation } & \\ { }^{18} \text { F-fluorodeoxyglucose } & \\ \text { Antigen presenting cells } & { }^{18} \text { F-FDG } \\ \text { Complete Metabolic Response } & \text { APC } \\ \text { Complete response } & \mathrm{CMR} \\ \text { Computed tomography } & \mathrm{CR} \\ \text { Cytotoxic T cells } & \mathrm{CT} \\ \text { Cytotoxic T-lymphocyte antigen } 4 & \mathrm{CTLs} \\ \text { Gastrointestinal stromal tumors } & \mathrm{CTLA}-4 \\ \text { Glucose transporter 1 } & \text { GIST } \\ \text { Interferon } \gamma & \text { GLUT1 } \\ \text { Interleukine-2 } & \text { IFN } \gamma \\ \text { Immune checkpoint inhibitor } & \text { IL-2 } \\ \text { Immune-modified RECIST } & \text { ICI } \\ \text { Immune PERCIST } & \text { imRECIST } \\ \text { Immune RECIST } & \text { iPERCIST } \\ \text { Immune-related adverse effect } & \text { iRECIST } \\ & \text { IrAE }\end{array}$


Immune-related RECIST

irRECIST

Immune-related response criteria

irRC

Immunotherapy-modified PERCIST up to 5 lesions

imPERCIST5

Non-small cell lung cancer

NSCLC

Major histocompatibility complex

$\mathrm{MHC}$

Magnetic resonance imaging

MRI

Myeloid-derived suppressor cells

MDSC

Partial Metabolic Response

PMR

Partial Response

PET/CT Criteria for early prediction of Response to Immune checkpoint inhibitor Therapy

PET Response Criteria in Solid Tumors

PR

PET Response Evaluation Criteria for Immunotherapy

PECRIT

PERCIST

Programmed cell death protein-1

PERCIMT

Programmed cell death protein-1 ligand

PD-1

Progression disease

PDL-1

Positron emission tomography

Preferred Reporting Items for Systematic Reviews and Meta-Analyses

PD

PET

PRISMA

Response Evaluation Criteria in Solid Tumors

RECIST

Single photon emission computed tomography

SPECT

Stable disease

SD

Standardized uptake value

SUV

Standardized uptake value normalized par lean body mass

SUL

T-cell receptor

TCR

Total lesion glycolysis

TLG

Total metabolic tumor volume

TMTV

Tumor-infiltrating lymphocytes

TILs

Author Contributions: Conceptualization, P.D. and P.B.; methodology, P.D. and P.B.; formal analysis, P.D. and P.B.; investigation, P.D. and P.B.; resources, P.D. and P.B.; writing-original draft preparation, P.D. and P.B.; writing-review and editing, P.D. and P.B.; visualization, P.D. and P.B.; supervision, P.D. and P.B.; project administration, P.D. and P.B. All authors have read and agreed to the published version of the manuscript.

Funding: This research received no external funding.

Conflicts of Interest: The authors declare no conflict of interest.

\section{References}

1. Gonzalez, H.; Hagerling, C.; Werb, Z. Roles of the immune system in cancer: From tumor initiation to metastatic progression. Genes Dev. 2018, 32, 1267-1284. [CrossRef] [PubMed]

2. Egen, J.G.; Kuhns, M.S.; Allison, J.P. CTLA-4: New insights into its biological function and use in tumor immunotherapy. Nat. Immunol. 2002, 3, 611-618. [CrossRef] [PubMed]

3. Okazaki, T.; Chikuma, S.; Iwai, Y.; Fagarasan, S.; Honjo, T. A rheostat for immune responses: The unique properties of PD-1 and their advantages for clinical application. Nat. Immunol. 2013, 14, 1212-1218. [CrossRef] [PubMed]

4. Pons-Tostivint, E.; Latouche, A.; Vaflard, P.; Ricci, F.; Loirat, D.; Hescot, S.; Sablin, M.-P.; Rouzier, R.; Kamal, M.; Morel, C.; et al. Comparative Analysis of Durable Responses on Immune Checkpoint Inhibitors Versus Other Systemic Therapies: A Pooled Analysis of Phase III Trials. JCO Precis. Oncol. 2019, 3, 1-10. [CrossRef]

5. Kanjanapan, Y.; Day, D.; Wang, L.; Al-Sawaihey, H.; Abbas, E.; Namini, A.; Siu, L.L.; Hansen, A.; Razak, A.A.; Spreafico, A.; et al. Hyperprogressive disease in early-phase immunotherapy trials: Clinical predictors and association with immune-related toxicities. Cancer 2019, 125, 1341-1349. [CrossRef] [PubMed]

6. Borcoman, E.; Kanjanapan, Y.; Champiat, S.; Kato, S.; Servois, V.; Kurzrock, R.; Goel, S.; Bedard, P.; Le Tourneau, C. Novel patterns of response under immunotherapy. Ann. Oncol. 2019, 30, 385-396. [CrossRef]

7. Seymour, L.; Bogaerts, J.; Perrone, A.; Ford, R.; Schwartz, L.H.; Mandrekar, S.; Lin, N.U.; Litière, S.; Dancey, J.; Chen, A.; et al. iRECIST: Guidelines for response criteria for use in trials testing immunotherapeutics. Lancet Oncol. 2017, 18, e143-e152. [CrossRef] 
8. Moher, D.; Liberati, A.; Tetzlaff, J.; Altman, D.G.; Group, T.P. Preferred Reporting Items for Systematic Reviews and Meta-Analyses: The PRISMA Statement. PLOS Med. 2009, 6, e1000097. [CrossRef]

9. Somarouthu, B.; Lee, S.I.; Urban, T.; Sadow, C.A.; Harris, G.J.; Kambadakone, A. Immune-related tumour response assessment criteria: A comprehensive review. Br. J. Radiol. 2018, 91, 20170457. [CrossRef]

10. Pardoll, D.M. The blockade of immune checkpoints in cancer immunotherapy. Nat. Rev. Cancer 2012, 12, 252-264. [CrossRef]

11. Kwak, J.J.; Tirumani, S.H.; Van den Abbeele, A.D.; Koo, P.J.; Jacene, H.A. Cancer immunotherapy: Imaging assessment of novel treatment response patterns and immune-related adverse events. Radiographics 2015, 35, 424-437. [CrossRef] [PubMed]

12. Wang, G.X.; Kurra, V.; Gainor, J.F.; Sullivan, R.J.; Flaherty, K.T.; Lee, S.I.; Fintelmann, F.J. Immune Checkpoint Inhibitor Cancer Therapy: Spectrum of Imaging Findings. Radiographics 2017, 37, 2132-2144. [CrossRef]

13. Rossi, S.; Toschi, L.; Castello, A.; Grizzi, F.; Mansi, L.; Lopci, E. Clinical characteristics of patient selection and imaging predictors of outcome in solid tumors treated with checkpoint-inhibitors. Eur. J. Nucl. Med. Mol. Imaging 2017, 44, 2310-2325. [CrossRef] [PubMed]

14. Petersen, H.; Holdgaard, P.C.; Madsen, P.H.; Knudsen, L.M.; Gad, D.; Gravergaard, A.E.; Rohde, M.; Godballe, C.; Engelmann, B.E.; Bech, K.; et al. FDG PET/CT in cancer: Comparison of actual use with literature-based recommendations. Eur. J. Nucl. Med. Mol. Imaging 2016, 43, 695-706. [CrossRef]

15. Ito, K.; Schöder, H.; Teng, R.; Humm, J.L.; Ni, A.; Wolchok, J.D.; Weber, W.A. Prognostic value of baseline metabolic tumor volume measured on 18F-fluorodeoxyglucose positron emission tomography/computed tomography in melanoma patients treated with ipilimumab therapy. Eur. J. Nucl. Med. Mol. Imaging 2019, 46, 930-939. [CrossRef] [PubMed]

16. Seban, R.-D.; Mezquita, L.; Berenbaum, A.; Dercle, L.; Botticella, A.; Le Pechoux, C.; Caramella, C.; Deutsch, E.; Grimaldi, S.; Adam, J.; et al. Baseline metabolic tumor burden on FDG PET/CT scans predicts outcome in advanced NSCLC patients treated with immune checkpoint inhibitors. Eur. J. Nucl. Med. Mol. Imaging 2019, 1-11. [CrossRef] [PubMed]

17. Chang, C.-H.; Qiu, J.; O'Sullivan, D.; Buck, M.D.; Noguchi, T.; Curtis, J.D.; Chen, Q.; Gindin, M.; Gubin, M.M.; van der Windt, G.J.W.; et al. Metabolic Competition in the Tumor Microenvironment Is a Driver of Cancer Progression. Cell 2015, 162, 1229-1241. [CrossRef] [PubMed]

18. Koh, Y.W.; Lee, S.J.; Han, J.-H.; Haam, S.; Jung, J.; Lee, H.W. PD-L1 protein expression in non-small-cell lung cancer and its relationship with the hypoxia-related signaling pathways: A study based on immunohistochemistry and RNA sequencing data. Lung Cancer 2019, 129, 41-47. [CrossRef]

19. Kaira, K.; Shimizu, K.; Kitahara, S.; Yajima, T.; Atsumi, J.; Kosaka, T.; Ohtaki, Y.; Higuchi, T.; Oyama, T.; Asao, T.; et al. 2-Deoxy-2-[fluorine-18] fluoro-d-glucose uptake on positron emission tomography is associated with programmed death ligand-1 expression in patients with pulmonary adenocarcinoma. Eur. J. Cancer 2018, 101, 181-190. [CrossRef]

20. Kasahara, N.; Kaira, K.; Bao, P.; Higuchi, T.; Arisaka, Y.; Erkhem-Ochir, B.; Sunaga, N.; Ohtaki, Y.; Yajima, T.; Kosaka, T.; et al. Correlation of tumor-related immunity with 18F-FDG-PET in pulmonary squamous-cell carcinoma. Lung Cancer 2018, 119, 71-77. [CrossRef]

21. Surov, A.; Meyer, H.J.; Wienke, A. Standardized Uptake Values Derived from 18F-FDG PET May Predict Lung Cancer Microvessel Density and Expression of KI 67, VEGF, and HIF-1 $\alpha$ but Not Expression of Cyclin D1, PCNA, EGFR, PD L1, and p53. Contrast Media Mol. Imaging 2018, 2018, 9257929. [CrossRef] [PubMed]

22. Chen, R.; Zhou, X.; Liu, J.; Huang, G. Relationship between the expression of PD-1/PD-L1 and 18F-FDG uptake in bladder cancer. Eur. J. Nucl. Med. Mol. Imaging 2019, 46, 848-854. [CrossRef] [PubMed]

23. Lopci, E.; Toschi, L.; Grizzi, F.; Rahal, D.; Olivari, L.; Castino, G.F.; Marchetti, S.; Cortese, N.; Qehajaj, D.; Pistillo, D.; et al. Correlation of metabolic information on FDG-PET with tissue expression of immune markers in patients with non-small cell lung cancer (NSCLC) who are candidates for upfront surgery. Eur. J. Nucl. Med. Mol. Imaging 2016, 43, 1954-1961. [CrossRef] [PubMed]

24. Yi, M.; Jiao, D.; Xu, H.; Liu, Q.; Zhao, W.; Han, X.; Wu, K. Biomarkers for predicting efficacy of PD-1/PD-L1 inhibitors. Mol. Cancer 2018, 17, 129. [CrossRef]

25. Boursi, B.; Werner, T.J.; Gholami, S.; Margalit, O.; Baruch, E.; Markel, G.; Eshet, Y.; Houshmand, S.; Shacham-Shmueli, E.; Mitchell, T.C.; et al. Physiologic colonic fluorine-18-fluorodeoxyglucose uptake may predict response to immunotherapy in patients with metastatic melanoma. Melanoma Res. 2019, 29, 318-321. [CrossRef] 
26. Boursi, B.; Werner, T.J.; Gholami, S.; Houshmand, S.; Mamtani, R.; Lewis, J.D.; Wu, G.D.; Alavi, A.; Yang, Y.-X. Functional imaging of the interaction between gut microbiota and the human host: A proof-of-concept clinical study evaluating novel use for 18F-FDG PET-CT. PLoS ONE 2018, 13, e0192747. [CrossRef]

27. Popinat, G.; Cousse, S.; Goldfarb, L.; Becker, S.; Gardin, I.; Salaün, M.; Thureau, S.; Vera, P.; Guisier, F.; Decazes, P. Sub-cutaneous Fat Mass measured on multislice computed tomography of pretreatment PET/CT is a prognostic factor of stage IV non-small cell lung cancer treated by nivolumab. OncoImmunology 2019, 8, e1580128. [CrossRef]

28. Decazes, P.; Tonnelet, D.; Vera, P.; Gardin, I. Anthropometer3D: Automatic Multi-Slice Segmentation Software for the Measurement of Anthropometric Parameters from CT of PET/CT. J. Digit Imaging 2019, 32, 241-250. [CrossRef]

29. Frasca, D.; Diaz, A.; Romero, M.; Thaller, S.; Blomberg, B.B. Secretion of autoimmune antibodies in the human subcutaneous adipose tissue. PLoS ONE 2018, 13, e0197472. [CrossRef]

30. Teng, F.; Meng, X.; Kong, L.; Yu, J. Progress and challenges of predictive biomarkers of anti PD-1/PD-L1 immunotherapy: A systematic review. Cancer Lett. 2018, 414, 166-173. [CrossRef]

31. Van der Veen, E.L.; Bensch, F.; Glaudemans, A.W.J.M.; Lub-de Hooge, M.N.; de Vries, E.G.E. Molecular imaging to enlighten cancer immunotherapies and underlying involved processes. Cancer Treat. Rev. 2018, 70, 232-244. [CrossRef] [PubMed]

32. Tavaré, R.; McCracken, M.N.; Zettlitz, K.A.; Salazar, F.B.; Olafsen, T.; Witte, O.N.; Wu, A.M. Immuno-PET of Murine T Cell Reconstitution Postadoptive Stem Cell Transplantation Using Anti-CD4 and Anti-CD8 Cys-Diabodies. J. Nucl. Med. 2015, 56, 1258-1264. [CrossRef] [PubMed]

33. Wei, W.; Jiang, D.; Ehlerding, E.B.; Luo, Q.; Cai, W. Noninvasive PET Imaging of T cells. Trends Cancer 2018, 4, 359-373. [CrossRef] [PubMed]

34. Larimer, B.M.; Wehrenberg-Klee, E.; Caraballo, A.; Mahmood, U. Quantitative CD3 PET Imaging Predicts Tumor Growth Response to Anti-CTLA-4 Therapy. J. Nucl. Med. 2016, 57, 1607-1611. [CrossRef] [PubMed]

35. Mayer, K.E.; Mall, S.; Yusufi, N.; Gosmann, D.; Steiger, K.; Russelli, L.; de Oliviera Bianchi, H.; Audehm, S.; Wagner, R.; Bräunlein, E.; et al. T-cell functionality testing is highly relevant to developing novel immuno-tracers monitoring T cells in the context of immunotherapies and revealed CD7 as an attractive target. Theranostics 2018, 8, 6070-6087. [CrossRef]

36. Mayer, A.T.; Gambhir, S.S. The Immunoimaging Toolbox. J. Nucl. Med. 2018, 59, 1174-1182. [CrossRef]

37. Broos, K.; Lecocq, Q.; Raes, G.; Devoogdt, N.; Keyaerts, M.; Breckpot, K. Noninvasive imaging of the PD-1:PD-L1 immune checkpoint: Embracing nuclear medicine for the benefit of personalized immunotherapy. Theranostics 2018, 8, 3559-3570. [CrossRef]

38. Bensch, F.; van der Veen, E.L.; Lub-de Hooge, M.N.; Jorritsma-Smit, A.; Boellaard, R.; Kok, I.C.; Oosting, S.F.; Schröder, C.P.; Hiltermann, T.J.N.; van der Wekken, A.J.; et al. 89Zr-atezolizumab imaging as a non-invasive approach to assess clinical response to PD-L1 blockade in cancer. Nat. Med. 2018, 24, 1852-1858. [CrossRef]

39. Niemeijer, A.N.; Leung, D.; Huisman, M.C.; Bahce, I.; Hoekstra, O.S.; van Dongen, G.A.M.S.; Boellaard, R.; Du, S.; Hayes, W.; Smith, R.; et al. Whole body PD-1 and PD-L1 positron emission tomography in patients with non-small-cell lung cancer. Nat. Commun. 2018, 9, 4664. [CrossRef]

40. Kumar, D.; Lisok, A.; Dahmane, E.; McCoy, M.; Shelake, S.; Chatterjee, S.; Allaj, V.; Sysa-Shah, P.; Wharram, B.; Lesniak, W.G.; et al. Peptide-based PET quantifies target engagement of PD-L1 therapeutics. J. Clin. Invest. 2019, 129, 616-630. [CrossRef]

41. Chatterjee, S.; Lesniak, W.G.; Nimmagadda, S. Noninvasive Imaging of Immune Checkpoint Ligand PD-L1 in Tumors and Metastases for Guiding Immunotherapy. Mol. Imaging 2017, 16, 1536012117718459. [CrossRef] [PubMed]

42. Gibson, H.M.; McKnight, B.N.; Malysa, A.; Dyson, G.; Wiesend, W.N.; McCarthy, C.E.; Reyes, J.; Wei, W.-Z.; Viola-Villegas, N.T. IFN $\gamma$ PET Imaging as a Predictive Tool for Monitoring Response to Tumor Immunotherapy. Cancer Res. 2018, 78, 5706-5717. [CrossRef] [PubMed]

43. Costantini, A.; Takam Kamga, P.; Dumenil, C.; Chinet, T.; Emile, J.-F.; Giroux Leprieur, E. Plasma Biomarkers and Immune Checkpoint Inhibitors in Non-Small Cell Lung Cancer: New Tools for Better Patient Selection? Cancers 2019, 11, 1269. [CrossRef] [PubMed]

44. Larimer, B.M.; Bloch, E.; Nesti, S.; Austin, E.E.; Wehrenberg-Klee, E.; Boland, G.; Mahmood, U. The Effectiveness of Checkpoint Inhibitor Combinations and Administration Timing Can Be Measured by Granzyme B PET Imaging. Clin. Cancer Res. 2019, 25, 1196-1205. [CrossRef] 
45. Xing, Y.; Chand, G.; Liu, C.; Cook, G.J.R.; O' Doherty, J.; Zhao, L.; Wong, N.C.L.; Meszaros, L.K.; Ting, H.H.; Zhao, J. Early phase I study of a 99mTc labeled anti-PD-L1 single domain antibody in SPECT/CT assessment of programmed death ligand-1 expression in non-small cell lung cancer. J. Nucl. Med. 2019, 60, 1213-1220. [CrossRef]

46. Markovic, S.N.; Galli, F.; Suman, V.J.; Nevala, W.K.; Paulsen, A.M.; Hung, J.C.; Gansen, D.N.; Erickson, L.A.; Marchetti, P.; Wiseman, G.A.; et al. Non-invasive visualization of tumor infiltrating lymphocytes in patients with metastatic melanoma undergoing immune checkpoint inhibitor therapy: A pilot study. Oncotarget 2018, 9, 30268-30278. [CrossRef]

47. Sun, R.; Limkin, E.J.; Vakalopoulou, M.; Dercle, L.; Champiat, S.; Han, S.R.; Verlingue, L.; Brandao, D.; Lancia, A.; Ammari, S.; et al. A radiomics approach to assess tumour-infiltrating CD8 cells and response to anti-PD-1 or anti-PD-L1 immunotherapy: An imaging biomarker, retrospective multicohort study. Lancet Oncol. 2018, 19, 1180-1191. [CrossRef]

48. Trebeschi, S.; Drago, S.G.; Birkbak, N.J.; Kurilova, I.; Cǎlin, A.M.; Pizzi, A.D.; Lalezari, F.; Lambregts, D.M.J.; Rohaan, M.; Parmar, C.; et al. Predicting Response to Cancer Immunotherapy using Non-invasive Radiomic Biomarkers. Ann. Oncol. 2019, 30, 998-1004. [CrossRef]

49. Chen, R.-Y.; Lin, Y.-C.; Shen, W.-C.; Hsieh, T.-C.; Yen, K.-Y.; Chen, S.-W.; Kao, C.-H. Associations of Tumor PD-1 Ligands, Immunohistochemical Studies, and Textural Features in 18F-FDG PET in Squamous Cell Carcinoma of the Head and Neck. Sci. Rep. 2018, 8, 105. [CrossRef]

50. Bera, K.; Velcheti, V.; Madabhushi, A. Novel Quantitative Imaging for Predicting Response to Therapy: Techniques and Clinical Applications. Am. Soc. Clin. Oncol. Educ. Book 2018, 38, 1008-1018. [CrossRef]

51. Sachpekidis, C.; Anwar, H.; Winkler, J.K.; Kopp-Schneider, A.; Larribere, L.; Haberkorn, U.; Hassel, J.C.; Dimitrakopoulou-Strauss, A. Longitudinal studies of the 18F-FDG kinetics after ipilimumab treatment in metastatic melanoma patients based on dynamic FDG PET/CT. Cancer Immunol. Immunother. 2018, 67, 1261-1270. [CrossRef] [PubMed]

52. Eisenhauer, E.A.; Therasse, P.; Bogaerts, J.; Schwartz, L.H.; Sargent, D.; Ford, R.; Dancey, J.; Arbuck, S.; Gwyther, S.; Mooney, M.; et al. New response evaluation criteria in solid tumours: Revised RECIST guideline (version 1.1). Eur. J. Cancer 2009, 45, 228-247. [CrossRef] [PubMed]

53. Farag, S.; de Geus-Oei, L.-F.; van der Graaf, W.T.; van Coevorden, F.; Grunhagen, D.; Reyners, A.K.L.; Boonstra, P.A.; Desar, I.; Gelderblom, H.; Steeghs, N. Early Evaluation of Response Using 18F-FDG PET Influences Management in Gastrointestinal Stromal Tumor Patients Treated with Neoadjuvant Imatinib. J. Nucl. Med. 2018, 59, 194-196. [CrossRef] [PubMed]

54. Wahl, R.L.; Jacene, H.; Kasamon, Y.; Lodge, M.A. From RECIST to PERCIST: Evolving Considerations for PET response criteria in solid tumors. J. Nucl. Med. 2009, 50 (Suppl. 1), 122S-150S. [CrossRef]

55. Cheson, B.D.; Fisher, R.I.; Barrington, S.F.; Cavalli, F.; Schwartz, L.H.; Zucca, E.; Lister, T.A.; Alliance, Australasian Leukaemia and Lymphoma Group; Eastern Cooperative Oncology Group; European Mantle Cell Lymphoma Consortium; et al. Recommendations for initial evaluation, staging, and response assessment of Hodgkin and non-Hodgkin lymphoma: The Lugano classification. J. Clin. Oncol. 2014, 32, 3059-3068. [CrossRef]

56. Wolchok, J.D.; Hoos, A.; O’Day, S.; Weber, J.S.; Hamid, O.; Lebbé, C.; Maio, M.; Binder, M.; Bohnsack, O.; Nichol, G.; et al. Guidelines for the evaluation of immune therapy activity in solid tumors: Immune-related response criteria. Clin. Cancer Res. 2009, 15, 7412-7420. [CrossRef]

57. Tazdait, M.; Mezquita, L.; Lahmar, J.; Ferrara, R.; Bidault, F.; Ammari, S.; Balleyguier, C.; Planchard, D.; Gazzah, A.; Soria, J.C.; et al. Patterns of responses in metastatic NSCLC during PD-1 or PDL-1 inhibitor therapy: Comparison of RECIST 1.1, irRECIST and iRECIST criteria. Eur. J. Cancer 2018, 88, 38-47. [CrossRef]

58. Humbert, O.; Cadour, N.; Paquet, M.; Schiappa, R.; Poudenx, M.; Chardin, D.; Borchiellini, D.; Benisvy, D.; Ouvrier, M.J.; Zwarthoed, C.; et al. 18FDG PET/CT in the early assessment of non-small cell lung cancer response to immunotherapy: Frequency and clinical significance of atypical evolutive patterns. Eur. J. Nucl. Med. Mol. Imaging 2019, 1-10. [CrossRef]

59. Nishino, M.; Giobbie-Hurder, A.; Gargano, M.; Suda, M.; Ramaiya, N.H.; Hodi, F.S. Developing a Common Language for Tumor Response to Immunotherapy: Immune-related Response Criteria using Unidimentional measurements. Clin. Cancer Res. 2013, 19, 3936-3943. [CrossRef]

60. Hodi, F.S.; Ballinger, M.; Lyons, B.; Soria, J.-C.; Nishino, M.; Tabernero, J.; Powles, T.; Smith, D.; Hoos, A.; McKenna, C.; et al. Immune-Modified Response Evaluation Criteria In Solid Tumors (imRECIST): Refining Guidelines to Assess the Clinical Benefit of Cancer Immunotherapy. J. Clin. Oncol. 2018, 36, 850-858. [CrossRef] 
61. Kataoka, Y.; Hirano, K. Which criteria should we use to evaluate the efficacy of immune-checkpoint inhibitors? Ann. Transl. Med. 2018, 6, 222. [CrossRef] [PubMed]

62. Rossi, S.; Castello, A.; Toschi, L.; Lopci, E. Immunotherapy in non-small-cell lung cancer: Potential predictors of response and new strategies to assess activity. Immunotherapy 2018, 10, 797-805. [CrossRef] [PubMed]

63. Cho, S.Y.; Lipson, E.J.; Im, H.-J.; Rowe, S.P.; Gonzalez, E.M.; Blackford, A.; Chirindel, A.; Pardoll, D.M.; Topalian, S.L.; Wahl, R.L. Prediction of Response to Immune Checkpoint Inhibitor Therapy Using Early-Time-Point 18F-FDG PET/CT Imaging in Patients with Advanced Melanoma. J. Nucl. Med. 2017, 58, 1421-1428. [CrossRef] [PubMed]

64. Anwar, H.; Sachpekidis, C.; Winkler, J.; Kopp-Schneider, A.; Haberkorn, U.; Hassel, J.C.; Dimitrakopoulou-Strauss, A. Absolute number of new lesions on 18F-FDG PET/CT is more predictive of clinical response than SUV changes in metastatic melanoma patients receiving ipilimumab. Eur. J. Nucl. Med. Mol. Imaging 2018, 45, 376-383. [CrossRef] [PubMed]

65. Ito, K.; Teng, R.; Schöder, H.; Humm, J.L.; Ni, A.; Michaud, L.; Nakajima, R.; Yamashita, R.; Wolchok, J.D.; Weber, W.A. 18F-FDG PET/CT for Monitoring of Ipilimumab Therapy in Patients with Metastatic Melanoma. J. Nucl. Med. 2019, 60, 335-341. [CrossRef] [PubMed]

66. Goldfarb, L.; Duchemann, B.; Chouahnia, K.; Zelek, L.; Soussan, M. Monitoring anti-PD-1-based immunotherapy in non-small cell lung cancer with FDG PET: Introduction of iPERCIST. EJNMMI Res. 2019, 9, 8. [CrossRef] [PubMed]

67. Evangelista, L.; De Rimini, M.L.; Bianchi, A.; Schillaci, O. Immunotherapy and 18F-FDG PET/CT: Standardised procedures are needed. Clin. Transl. Imaging 2019, 7, 313-315. [CrossRef]

68. Fransen, M.F.; Schoonderwoerd, M.; Knopf, P.; Camps, M.G.; Hawinkels, L.J.; Kneilling, M.; van Hall, T.; Ossendorp, F. Tumor-draining lymph nodes are pivotal in PD-1/PD-L1 checkpoint therapy. JCI Insight 2018, 3, e124507. [CrossRef]

69. Cheson, B.D.; Ansell, S.; Schwartz, L.; Gordon, L.I.; Advani, R.; Jacene, H.A.; Hoos, A.; Barrington, S.F.; Armand, P. Refinement of the Lugano Classification lymphoma response criteria in the era of immunomodulatory therapy. Blood 2016, 128, 2489-2496. [CrossRef]

70. Dercle, L.; Seban, R.-D.; Lazarovici, J.; Schwartz, L.H.; Houot, R.; Ammari, S.; Danu, A.; Edeline, V.; Marabelle, A.; Ribrag, V.; et al. 18F-FDG PET and CT Scans Detect New Imaging Patterns of Response and Progression in Patients with Hodgkin Lymphoma Treated by Anti-Programmed Death 1 Immune Checkpoint Inhibitor. J. Nucl. Med. 2018, 59, 15-24. [CrossRef]

71. Castello, A.; Grizzi, F.; Qehajaj, D.; Rahal, D.; Lutman, F.; Lopci, E. 18F-FDG PET/CT for response assessment in Hodgkin lymphoma undergoing immunotherapy with checkpoint inhibitors. Leuk. Lymphoma 2019, 60, 367-375. [CrossRef] [PubMed]

72. Chen, A.; Mokrane, F.-Z.; Schwartz, L.; Morschhauser, F.; Stamatoullas, A.; Schiano de Colella, J.-M.; Vercellino, L.; Casasnovas, O.; Chauchet, A.; Delmer, A.; et al. Early 18F-FDG PET/CT response predicts survival in Relapsed/Refractory Hodgkin Lymphoma treated with Nivolumab. J. Nucl. Med 2019. eprint ahead of print. [CrossRef] [PubMed]

73. Kline, J.; Godfrey, J.; Ansell, S.M. The Immune Landscape and Response to Immune Checkpoint Blockade Therapy in Lymphoma. Blood 2019. eprint ahead of print. [CrossRef] [PubMed]

74. Ferrara, R.; Mezquita, L.; Texier, M.; Lahmar, J.; Audigier-Valette, C.; Tessonnier, L.; Mazieres, J.; Zalcman, G.; Brosseau, S.; Le Moulec, S.; et al. Hyperprogressive Disease in Patients With Advanced Non-Small Cell Lung Cancer Treated With PD-1/PD-L1 Inhibitors or With Single-Agent Chemotherapy. JAMA Oncol. 2018, 4, 1543-1552. [CrossRef] [PubMed]

75. Kaira, K.; Higuchi, T.; Naruse, I.; Arisaka, Y.; Tokue, A.; Altan, B.; Suda, S.; Mogi, A.; Shimizu, K.; Sunaga, N.; et al. Metabolic activity by 18 F-FDG-PET/CT is predictive of early response after nivolumab in previously treated NSCLC. Eur. J. Nucl. Med. Mol. Imaging 2018, 45, 56-66. [CrossRef] [PubMed]

76. Seith, F.; Forschner, A.; Schmidt, H.; Pfannenberg, C.; Gückel, B.; Nikolaou, K.; la Fougère, C.; Garbe, C.; Schwenzer, N. 18F-FDG-PET detects complete response to PD1-therapy in melanoma patients two weeks after therapy start. Eur. J. Nucl. Med. Mol. Imaging 2018, 45, 95-101. [CrossRef]

77. Sachpekidis, C.; Anwar, H.; Winkler, J.; Kopp-Schneider, A.; Larribere, L.; Haberkorn, U.; Hassel, J.C.; Dimitrakopoulou-Strauss, A. The role of interim 18F-FDG PET/CT in prediction of response to ipilimumab treatment in metastatic melanoma. Eur. J. Nucl. Med. Mol. Imaging 2018, 45, 1289-1296. [CrossRef] 
78. Hlavata, Z.; Solinas, C.; De Silva, P.; Porcu, M.; Saba, L.; Willard-Gallo, K.; Scartozzi, M. The Abscopal Effect in the Era of Cancer Immunotherapy: A Spontaneous Synergism Boosting Anti-tumor Immunity? Target Oncol. 2018, 13, 113-123. [CrossRef]

79. Azami, A.; Suzuki, N.; Azami, Y.; Seto, I.; Sato, A.; Takano, Y.; Abe, T.; Teranishi, Y.; Tachibana, K.; Ohtake, T. Abscopal effect following radiation monotherapy in breast cancer: A case report. Mol. Clin. Oncol. 2018, 9, 283-286. [CrossRef]

80. Britschgi, C.; Riesterer, O.; Burger, I.A.; Guckenberger, M.; Curioni-Fontecedro, A. Report of an abscopal effect induced by stereotactic body radiotherapy and nivolumab in a patient with metastatic non-small cell lung cancer. Radiat. Oncol. 2018, 13, 102. [CrossRef]

81. Golden, E.B.; Demaria, S.; Schiff, P.B.; Chachoua, A.; Formenti, S.C. An Abscopal Response to Radiation and Ipilimumab in a Patient with Metastatic Non-Small Cell Lung Cancer. Cancer Immunol. Res. 2013, 1, 365-372. [CrossRef] [PubMed]

82. Martins, F.; Sofiya, L.; Sykiotis, G.P.; Lamine, F.; Maillard, M.; Fraga, M.; Shabafrouz, K.; Ribi, C.; Cairoli, A.; Guex-Crosier, Y.; et al. Adverse effects of immune-checkpoint inhibitors: Epidemiology, management and surveillance. Nat. Rev. Clin. Oncol. 2019, 16, 563-580. [CrossRef] [PubMed]

83. Weber, J.S.; Hodi, F.S.; Wolchok, J.D.; Topalian, S.L.; Schadendorf, D.; Larkin, J.; Sznol, M.; Long, G.V.; Li, H.; Waxman, I.M.; et al. Safety Profile of Nivolumab Monotherapy: A Pooled Analysis of Patients with Advanced Melanoma. J. Clin. Oncol. 2017, 35, 785-792. [CrossRef] [PubMed]

84. Tirumani, S.H.; Ramaiya, N.H.; Keraliya, A.; Bailey, N.D.; Ott, P.A.; Hodi, F.S.; Nishino, M. Radiographic Profiling of Immune-Related Adverse Events in Advanced Melanoma Patients Treated with Ipilimumab. Cancer Immunol. Res. 2015, 3, 1185-1192. [CrossRef]

85. Mekki, A.; Dercle, L.; Lichtenstein, P.; Marabelle, A.; Michot, J.-M.; Lambotte, O.; Le Pavec, J.; De Martin, E.; Balleyguier, C.; Champiat, S.; et al. Detection of immune-related adverse events by medical imaging in patients treated with anti-programmed cell death 1. Eur. J. Cancer 2018, 96, 91-104. [CrossRef]

86. Sachpekidis, C.; Larribère, L.; Kopp-Schneider, A.; Hassel, J.C.; Dimitrakopoulou-Strauss, A. Can benign lymphoid tissue changes in 18F-FDG PET/CT predict response to immunotherapy in metastatic melanoma? Cancer Immunol. Immunother. 2019, 68, 297-303. [CrossRef]

87. Sachpekidis, C.; Kopp-Schneider, A.; Hakim-Meibodi, L.; Dimitrakopoulou-Strauss, A.; Hassel, J.C. 18F-FDG PET/CT longitudinal studies in patients with advanced metastatic melanoma for response evaluation of combination treatment with vemurafenib and ipilimumab. Melanoma Res. 2019, 29, 178-186. [CrossRef]

88. Nobashi, T.; Baratto, L.; Reddy, S.A.; Srinivas, S.; Toriihara, A.; Hatami, N.; Yohannan, T.K.; Mittra, E. Predicting Response to Immunotherapy by Evaluating Tumors, Lymphoid Cell-Rich Organs, and Immune-Related Adverse Events Using FDG-PET/CT. Clin. Nucl. Med. 2019, 44, e272-e279. [CrossRef]

89. Aide, N.; Hicks, R.J.; Le Tourneau, C.; Lheureux, S.; Fanti, S.; Lopci, E. FDG PET/CT for assessing tumour response to immunotherapy: Report on the EANM symposium on immune modulation and recent review of the literature. Eur. J. Nucl. Med. Mol. Imaging 2019, 46, 238-250. [CrossRef]

90. Carter, B.W.; Bhosale, P.R.; Yang, W.T. Immunotherapy and the role of imaging. Cancer 2018, 124, $2906-2922$. [CrossRef]

91. Pektor, S.; Hilscher, L.; Walzer, K.C.; Miederer, I.; Bausbacher, N.; Loquai, C.; Schreckenberger, M.; Sahin, U.; Diken, M.; Miederer, M. In vivo imaging of the immune response upon systemic RNA cancer vaccination by FDG-PET. EJNMMI Res. 2018, 8, 80. [CrossRef] [PubMed]

92. Maschmeyer, G.; De Greef, J.; Mellinghoff, S.C.; Nosari, A.; Thiebaut-Bertrand, A.; Bergeron, A.; Franquet, T.; Blijlevens, N.M.A.; Maertens, J.A.; European Conference on Infections in Leukemia (ECIL). Infections associated with immunotherapeutic and molecular targeted agents in hematology and oncology. A position paper by the European Conference on Infections in Leukemia (ECIL). Leukemia 2019, 33, 844-862. [CrossRef] [PubMed]

93. Tan, A.C.; Emmett, L.; Lo, S.; Liu, V.; Kapoor, R.; Carlino, M.S.; Guminski, A.D.; Long, G.V.; Menzies, A.M. FDG-PET response and outcome from anti-PD-1 therapy in metastatic melanoma. Ann. Oncol. 2018, 29, 2115-2120. [CrossRef] [PubMed]

(C) 2020 by the authors. Licensee MDPI, Basel, Switzerland. This article is an open access article distributed under the terms and conditions of the Creative Commons Attribution (CC BY) license (http://creativecommons.org/licenses/by/4.0/). 


\title{
Recent Advances in Nuclear Imaging of Receptor Expression to Guide Targeted Therapies in Breast Cancer
}

\author{
Barbara Salvatore ${ }^{\dagger}$, Maria Grazia Caprio ${ }^{\dagger}$, Billy Samuel Hill, Annachiara Sarnella, \\ Giovanni Nicola Roviello and Antonella Zannetti * \\ Istituto di Biostrutture e Bioimmagini-CNR, 80145 Naples, Italy; barbara.salvatore@cnr.it (B.S.); \\ mariagrazia.caprio@ibb.cnr.it (M.G.C.); billy.hill@ibb.cnr.it (B.S.H.); achiara.sarnella@gmail.com (A.S.); \\ giroviel@unina.it (G.N.R.) \\ * Correspondence: antonella.zannetti@cnr.it; Tel.: +39-3666115319 \\ + These authors contributed equally to this paper.
}

Received: 15 October 2019; Accepted: 18 October 2019; Published: 22 October 2019

\begin{abstract}
Breast cancer remains the most frequent cancer in women with different patterns of disease progression and response to treatments. The identification of specific biomarkers for different breast cancer subtypes has allowed the development of novel targeting agents for imaging and therapy. To date, patient management depends on immunohistochemistry analysis of receptor status on bioptic samples. This approach is too invasive, and in some cases, not entirely representative of the disease. Nuclear imaging using receptor tracers may provide whole-body information and detect any changes of receptor expression during disease progression. Therefore, imaging is useful to guide clinicians to select the best treatments for each patient and to evaluate early response thus reducing unnecessary therapies. In this review, we focused on the development of novel tracers that are ongoing in preclinical and/or clinical studies as promising tools to lead treatment decisions for breast cancer management.
\end{abstract}

Keywords: breast cancer; receptor status; molecular imaging

\section{Introduction}

Breast cancer is a malignant disease with the highest incidence in women worldwide [1]. There is a wide variation in patient prognosis and outcomes due to the high heterogeneity of this carcinoma. The three main biomarkers of interest in this cancer include estrogen receptor (ER), progesterone receptor (PR), and human epidermal growth factor receptor 2 (HER2). Nowadays, at least four molecular subtypes have been identified with different levels of expression of these receptors: luminal A, luminal B, HER2-enriched, and basal-like. Triple-negative breast cancer (TNBC), which is a part of the basal-like subgroup, is characterized by the lack of PR, ER, and HER2 expression, and by extremely high mortality due to metastatic and drug-resistance recurrent disease [2]. The ER is the major driver of breast cancer being expressed in $75 \%$ of patients and tamoxifen that binds ER was the first drug approved for metastatic breast cancer [3]. HER2 is overexpressed in approximately $15 \%$ to $25 \%$ of patients at initial diagnosis and is related with cancer aggressiveness and poor survival. The development of specific molecules, which target this receptor such as trastuzumab and pertuzumab or the tyrosine kinase inhibitor lapatinib, has significantly improved the outcome in HER2-positive breast cancer patients [3]. Both tumor heterogeneity and the occurrence of resistance to treatments may fundamentally affect receptor status and therefore response to therapy. To overcome this issue, recent investigations are focusing on the characterization of other well-known receptors involved in breast cancer progression as potential therapeutic and imaging targets such as tyrosine kinases receptors, 
integrin receptors, chemokine receptors, immune checkpoint receptors, somatostatin receptors, gastrin releasing peptide receptors, and neuropeptide $Y$ receptors. Over the past decades, many molecules specifically targeting them were developed: monoclonal antibodies, antibody fragments, nanobodies, small kinase inhibitors, peptides, and oligonucleotide aptamers. At present, several of these drugs are already in clinical trials while others are under investigation in pre-clinical models.

Current practice guidelines use biopsy and immunohistochemistry to define the receptors status in cancer tissues and to guide subsequent treatments. The integration of molecular imaging into breast cancer daily management could improve the standard of care, providing additional information on the heterogeneity of tumor lesions and facilitating early diagnosis, accurate staging, and personalized treatment planning. In particular, it could improve the management of metastatic breast carcinoma where receptor status can change during disease course and repeat biopsies may be too invasive for the patients [4]. Functional nuclear imaging using single photon emission computed tomography (SPECT) and positron emission tomography (PET) could allow the visualization of molecular changes in receptor status in primary tumor, metastases, and throughout treatments [5,6]. The main purpose of this review is to gain an overview of the relevant findings on the development of novel radiotracers targeting receptor status in breast cancer that are ongoing in preclinical studies for validation or that are already translated in clinical research (Table 1).

Table 1. Summary of tracers targeting receptors for breast cancer imaging.

\begin{tabular}{|c|c|c|c|c|c|}
\hline Receptor Type & Target & Tracer & Preclinical & Clinical & Reference \\
\hline \multirow{4}{*}{ Hormone Receptors } & \multirow{2}{*}{ ER } & {$\left[{ }^{18} \mathrm{~F}\right] \mathrm{FES}$} & & $\sqrt{ }$ & [7-16] \\
\hline & & {$\left[{ }^{18} \mathrm{~F}\right] 4 \mathrm{FMFES}$} & & $\sqrt{ }$ & {$[17,18]$} \\
\hline & \multirow{2}{*}{ PR } & {$\left[{ }^{18} \mathrm{~F}\right] \mathrm{FENP}$} & & $\sqrt{ }$ & [19-21] \\
\hline & & {$\left[{ }^{18} \mathrm{~F}\right] \mathrm{FFNP}$} & & $\sqrt{ }$ & [20-23] \\
\hline \multirow{14}{*}{ Tyrosine Kinase Receptors } & \multirow{8}{*}{ HER2 } & [ ${ }^{111}$ In] trastuzumab & & $\sqrt{ }$ & [24] \\
\hline & & {$\left[{ }^{89} \mathrm{Zr}\right]$ trastuzumab } & & $\sqrt{ }$ & [25-29] \\
\hline & & {$\left[{ }^{64} \mathrm{Cu}\right]$ DOTA-trastuzumab } & & $\sqrt{ }$ & {$[30,31]$} \\
\hline & & {$\left[{ }^{89} \mathrm{Zr}\right]$ Pertuzumab } & & $\sqrt{ }$ & {$[32,33]$} \\
\hline & & {$\left[{ }^{68} \mathrm{Ga}\right]$ HER2-Nanobody } & & $\sqrt{ }$ & [34] \\
\hline & & {$\left[{ }^{68} \mathrm{Ga}\right] \mathrm{ABY}-025$} & & $\sqrt{ }$ & [35] \\
\hline & & {$\left[{ }^{18} \mathrm{~F}\right]-5 \mathrm{~F} 7$} & $\sqrt{ }$ & & {$[36,37]$} \\
\hline & & {$\left[{ }^{18} \mathrm{~F}\right]-2 \mathrm{Rs} 15 \mathrm{~d}$} & $\sqrt{ }$ & & {$[36,37]$} \\
\hline & \multirow{2}{*}{ HER3 } & {$\left[{ }^{64} \mathrm{Cu}\right]$-patritumab } & & $\sqrt{ }$ & [38] \\
\hline & & {$\left[{ }^{64} \mathrm{Cu}\right]$ anti-HER3-F $\left(\mathrm{ab}^{\prime}\right) 2$} & $\sqrt{ }$ & & [39] \\
\hline & VEGFR & {$\left[{ }^{11} \mathrm{C}\right]-\mathrm{PAQ}$} & $\sqrt{ }$ & & [40] \\
\hline & c-Met & {$\left[{ }^{18} \mathrm{~F}\right] \mathrm{AH} 113804$} & $\sqrt{ }$ & & [41-43] \\
\hline & \multirow{2}{*}{ IGFR } & {$\left[{ }^{111} \mathrm{In}\right] /\left[{ }^{89} \mathrm{Zr}\right]-\mathrm{R} 1507$} & $\sqrt{ }$ & & [44] \\
\hline & & {$\left[{ }^{111} \operatorname{In}\right] \mathrm{F}\left(\mathrm{ab}^{\prime}\right) 2$} & $\sqrt{ }$ & & [45] \\
\hline \multirow{10}{*}{ Integrin Receptors } & \multirow{9}{*}{$\alpha v \beta 3$} & {$\left[{ }^{18} \mathrm{~F}\right]$ Galacto-RGD } & & $\sqrt{ }$ & [46] \\
\hline & & {$\left[{ }^{18} \mathrm{~F}\right] \mathrm{AH} 111585$} & & $\sqrt{ }$ & [47] \\
\hline & & {$\left[{ }^{99 \mathrm{~m}} \mathrm{Tc}\right] \mathrm{NC} 100692$} & & $\sqrt{ }$ & [48] \\
\hline & & {$\left[{ }^{18}\right.$ F]-FPPRGD2 } & & $\sqrt{ }$ & [49] \\
\hline & & {$\left[{ }^{99 \mathrm{~m}} \mathrm{Tc}\right] 3 P R G D 2$} & & $\sqrt{ }$ & {$[50,51]$} \\
\hline & & {$\left[{ }^{68} \mathrm{Ga}\right]$ RGD } & & $\sqrt{ }$ & [52] \\
\hline & & {$\left[{ }^{18} \mathrm{~F}\right]$ Alfatide II } & & $\sqrt{ }$ & [53] \\
\hline & & {$\left[{ }^{68} \mathrm{Ga}\right] \mathrm{BBN}-\mathrm{RGD}$} & & $\sqrt{ }$ & {$[54,55]$} \\
\hline & & {$\left[{ }^{18} \mathrm{~F}\right] \mathrm{RGD}$} & & $\sqrt{ }$ & [56] \\
\hline & $\alpha v \beta 6$ & {$\left[{ }^{18} \mathrm{~F}\right]-\alpha \mathrm{v} \beta 6-\mathrm{BP}$} & & $\sqrt{ }$ & [57] \\
\hline
\end{tabular}


Table 1. Cont.

\begin{tabular}{|c|c|c|c|c|c|}
\hline Receptor Type & Target & Tracer & Preclinical & Clinical & Reference \\
\hline \multirow{5}{*}{ Chemokine Receptors } & \multirow{4}{*}{ CXCR4 } & {$\left[{ }^{64} \mathrm{Cu}\right] \mathrm{AMD} 3100$} & $\sqrt{ }$ & & {$[58]$} \\
\hline & & {$\left[{ }^{64} \mathrm{Cu}\right] \mathrm{AuNCs}-\mathrm{AMD} 3100$} & $\sqrt{ }$ & & [59] \\
\hline & & {$\left[{ }^{68} \mathrm{Ga}\right]$ Pentixafor } & & $\sqrt{ }$ & {$[60,61]$} \\
\hline & & {$\left[{ }^{18} \mathrm{~F}\right] \mathrm{MCFB}$} & $\sqrt{ }$ & & [62] \\
\hline & CXCR7 & {$\left[{ }^{89} \mathrm{Zr}\right] \mathrm{ACKR} 3-\mathrm{mAb}$} & $\sqrt{ }$ & & [63] \\
\hline \multirow{7}{*}{ Immune checkpoints } & \multirow{7}{*}{ PD-L1 } & {$\left[{ }^{111}\right.$ In]PD-L1.3.1 } & $\sqrt{ }$ & & {$[64]$} \\
\hline & & $\begin{array}{c}{ }^{111} \text { In]- murine } \\
\text { anti-PD-L1 }\end{array}$ & $\sqrt{ }$ & & [65] \\
\hline & & {$\left[{ }^{64} \mathrm{Cu}\right]$ Atezolizumab } & $\sqrt{ }$ & & [66] \\
\hline & & {$\left[{ }^{111} \mathrm{In}\right]$ Atezolizumab } & $\sqrt{ }$ & & [67] \\
\hline & & [111 In]-PD-L1-mAb & $\sqrt{ }$ & & {$[68]$} \\
\hline & & {$\left[{ }^{64} \mathrm{Cu}\right] \mathrm{WL} 12$} & $\sqrt{ }$ & & [69] \\
\hline & & {$\left[{ }^{89} \mathrm{Zr}\right.$ ]atezolizumab } & & $\sqrt{ }$ & [70] \\
\hline \multirow{9}{*}{$\begin{array}{c}\text { Somatostatin, Gastrin Releasing } \\
\text { Peptide Receptors, } \\
\text { and Neuropeptide Y receptors }\end{array}$} & \multirow{3}{*}{ SSTR } & {$\left[{ }^{68} \mathrm{Ga}\right]$ DOTATOC } & $\sqrt{ }$ & & [71] \\
\hline & & $\begin{array}{c}{\left[{ }^{177} \mathrm{Lu}\right] \mathrm{DOTABASS}} \\
\text { and }\left[{ }^{177} \mathrm{Lu}\right] \text { DOTATATE }\end{array}$ & $\sqrt{ }$ & & [72] \\
\hline & & {$\left[{ }^{68}\right.$ Ga]NODAGA-JR11 } & $\sqrt{ }$ & & [73] \\
\hline & \multirow{4}{*}{ GRPR } & {$\left[{ }^{67} \mathrm{Ga}\right]-\mathrm{SB} 3$} & & $\sqrt{ }$ & [74] \\
\hline & & {$\left[{ }^{68} \mathrm{Ga}\right] \mathrm{RM} 2$} & & $\sqrt{ }$ & [75] \\
\hline & & {$\left[{ }^{68} \mathrm{Ga}\right]$ NOTA-RM26 } & & $\sqrt{ }$ & [76] \\
\hline & & {$\left[{ }^{67} \mathrm{Ga}\right]$ NeoBOMB1 } & $\sqrt{ }$ & & [77] \\
\hline & \multirow{2}{*}{ NPYR } & {$\left[{ }^{99 m} \mathrm{Tc}\right]$ NPYanalogue } & & $\sqrt{ }$ & [78] \\
\hline & & {$\left[{ }^{18} \mathrm{~F}\right]$ NPYanalogue } & & $\sqrt{ }$ & [79] \\
\hline
\end{tabular}

In this table are reported tracers currently used only in preclinical studies and tracers already translated in clinical research.

\section{Hormone Receptors}

The estrogen and progesterone receptors are overexpressed in around $75 \%$ of breast cancer either alone or together. They are considered important biomarkers to determine prognosis and predict the benefit of endocrine therapies in breast cancer patients [80,81]. Tumors with high levels of ER and PR are less aggressive and more indolent than negative tumors receptor [7].

Many studies report that PET imaging, using radiolabeled agents specifically targeting ER and PR, is able to detect receptor expression in tumor lesions [8].

Therefore, this non-invasive approach could allow the selection of eligible patients for endocrine therapies in a very early stage $[82,83] .16 \mathrm{a}-{ }^{18} \mathrm{~F}$-fluoro-17b-estradiol $\left(\left[{ }^{18} \mathrm{~F}\right] \mathrm{FES}\right)$, an estrogen derivative PET tracer, is the most frequently used probe for ER imaging applications. Clinical studies have revealed that $\left[{ }^{18} \mathrm{~F}\right] \mathrm{FES}$ is useful to detect positive lesions both in primary and metastatic breast cancer and in predicting response to anti-ER treatments with a good sensitivity (69-100\%) and high specificity (80-100\%) [9-12]. Therefore, $\left[{ }^{18} \mathrm{~F}\right] \mathrm{FES}-\mathrm{PET}$ is an appropriate imaging tool for the diagnosis, staging, and post-therapeutic evaluation of patients with ER+ breast cancer [7]. It has been reported that this tracer shows greater benefits than $\left[{ }^{18} \mathrm{~F}\right]-\mathrm{FDG}-\mathrm{PET}$ to distinguish flare reaction from a real disease progression [13]. Furthermore, a preoperative phase study by Gemignani et al. demonstrated that $\left[{ }^{18}\right.$ F]FES-PET Standardized Uptake Value (SUV) correlated with ER immunohistochemistry status in patients with early breast cancer and with size of the primary lesion [11].

Other findings have validated the association of $\left[{ }^{18} \mathrm{~F}\right] \mathrm{FES}$ uptake with ER concentration and the capability of this tracer to differentiate between positive and negative ER breast carcinomas [8]. At staging, a high uptake of $\left[{ }^{18} \mathrm{~F}\right] \mathrm{FES}$ is predictive of a good therapeutic response and an early detection 
of ER levels could increase treatment success. Furthermore, it has been described that $\left[{ }^{18} \mathrm{~F}\right]$ FES-PET may help in identifying patients with endocrine-resistant metastatic breast cancer that may paradoxically benefit from estradiol therapy [14]. Recently, Wang et al. reported that $\left[{ }^{18} \mathrm{~F}\right] \mathrm{FES}-\mathrm{PET} / \mathrm{CT}$ could be helpful to assess both the receptor status and the very early response to treatment with a novel ER antagonist GDC-0810 (Figure 1) [15]. The use of this tracer in a phase I dose escalation trial of GDC-0810 was capable to define the best dose of drug necessary to suppress ER thus reducing dose-related toxicities [15]. Importantly, several findings showed that PET imaging using ${ }^{18} \mathrm{~F}-\mathrm{FES}$ might be advantageous in patients with multiple lesions that are difficult to biopsy $[12,16]$. A novel estrogen PET tracer, 4-fluoro-11 $\beta$-methoxy-16 $\alpha$ - ${ }^{18}$ F-fluoroestradiol ( $\left.\left[{ }^{18} \mathrm{~F}\right] 4 \mathrm{FMFES}\right)$, was evaluated by Paquette et al. first in a murine model of breast cancer and then in a clinical study $[17,18]$. The authors compared the diagnostic potential of $\left[{ }^{18} \mathrm{~F}\right] 4 \mathrm{FMFES}$ with $\left[{ }^{18} \mathrm{~F}\right] \mathrm{FES}$ in a phase II clinical trial carried out in ER+ breast cancer patients. Here, they showed that $\left[{ }^{18} \mathrm{~F}\right] 4 \mathrm{FMFES}$ detected more lesions in comparison to $\left[{ }^{18} \mathrm{~F}\right] \mathrm{FES}$ with less background activity thus increasing diagnostic confidence and reduce false-negative diagnoses.

Many synthetic progestins with strong affinity for PR have been used as imaging agents. $21-\left[{ }^{18} \mathrm{~F}\right]$ Fluoro-16 $\alpha$-ethyl-19-norprogesterone $\left(\left[{ }^{18} \mathrm{~F}\right] \mathrm{FENP}\right)$ was the first radiolabeled progestin to be widely studied. Unfortunately, the high uptake of this tracer in breast adipose tissue caused its clinical application failure [19]. Nowadays, $21-\left[{ }^{18} \mathrm{~F}\right]-$ fluoro- $16 \alpha, 17 \alpha-\left[(\mathrm{R})-\left(1^{\prime}-\alpha\right.\right.$-furylmethylidene $)$ dioxy]-19-norpregn-4-ene-3,20-dione ( $\left.\left[{ }^{18} \mathrm{~F}\right] \mathrm{FFNP}\right)$ is the PET tracer most used for PR imaging $[20,21]$. Its dosimetry, safety, and correlation with PR status were evaluated in a first-in-human study performed in a small cohort of primary breast cancer patients [83]. Interestingly, a preclinical study carried out on endocrine-sensitive ER $\alpha+$ MCF7 human breast xenografts, showed that the reduction of $\left[{ }^{18} \mathrm{~F}\right] \mathrm{FFNP}$ uptake after estrogen-deprivation therapy, compared to baseline scan, predicted a positive tumor response [22]. Conversely, Salem K et al. observed in T47D breast tumor xenografts treated for only 3 days with $17 \beta$-estradiol, an increase of $\left[{ }^{18} \mathrm{~F}\right] \mathrm{FFNP}$ uptake in comparison to vehicle treated animals and a corresponding enhancement of PR levels [23]. Noteworthy, an advantage of $\left[{ }^{18} \mathrm{~F}\right] \mathrm{FFNP}$ over ${ }^{18} \mathrm{~F}-\mathrm{FES}$ is its ability to visualize lesions and measure PR status in patients treated with ER blocking agents, such as tamoxifen. Currently, in an ongoing clinical trial (NCT02455453; Clinicaltrials.gov) [ $\left.{ }^{18} \mathrm{~F}\right] \mathrm{FFNP}$ uptake is being assessed as a potential predictive biomarker of endocrine therapy response in ER+ metastatic breast cancer patients. Many studies are underway to validate the use of these tracers targeting hormone receptors alone or in combination to guide patient management towards the most efficient and least toxic therapies. An extensive and detailed review, focusing only on PET imaging of ER and PR, is beyond the scope of the present paper and we refer the reader to a recent and excellent review of this topic [8].

A

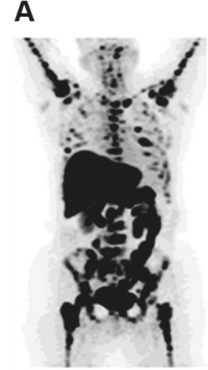

B

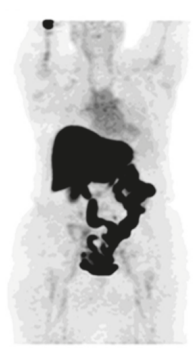

Figure 1. Complete suppression of $16 \mathrm{a}^{-18} \mathrm{~F}$-fluoro-17b-estradiol (FES)-avid lesions during GDC-0810 treatment. (A) Maximum intensity projection (MIP) FES- Positron Emission Tomography (PET) image before therapy showing pathologic uptake in multiple bone structures. Uptake in liver and bowel is physiologic. (B) MIP FES-PET image, after 2 cycles of GDC-0810 therapy, highlighted the drastic reduction of pathologic uptake (Figure adapted from Wang Y et al. (2017) [15], Permission number 4691821140364, (CAACR). 


\section{Receptor Tyrosine Kinases}

Receptor tyrosine kinases (RTKs) are often aberrantly activated during cancer progression and are involved in mediating signaling of all cancer hallmarks [84]. These receptors include an extracellular ligand-binding domain, a single transmembrane domain, a cytoplasmic kinase domain, and a carboxyl terminal tail. Generally, their activation is induced by specific ligands through dimerization and auto-phosphorylation. In many carcinomas, the gain-of-function mutations cause the constitutive activation of RTK and an amplification of intracellular signal pathways. Different classes of RTKs are deregulated in breast carcinoma including: the epidermal growth factor receptor family (EGFR/erbB1/HER1, Neu/erbB2/HER2, erbB3/HER-3, and erbB4/HER-4); vascular endothelial growth factor receptor (VEGFR); hepatocyte growth factor receptor (HGFR/c-Met); platelet-derived growth factor receptors (PDGFRs) and insulin-like growth factor-1 receptor (IGF-1R).

Many molecules, in particular antibodies, have been developed as imaging agents to detect HER2 expression in breast cancer lesions. Perik et al. showed that scintigraphy with ${ }^{111}$ Indium [ ${ }^{111}$ In]trastuzumab identified HER2-positive tumors but it was not useful in predicting trastuzumab-related cardiotoxicity in metastatic breast cancer patients [24]. Similarly, in a feasibility study on 14 patients with metastatic breast cancer, PET scan performed after administration of ${ }^{89}$ Zirconium $\left[{ }^{89} \mathrm{Zr}\right]$ trastuzumab allowed the visualization and quantification of the tracer uptake in HER2-positive lesions [25]. A very interesting study demonstrated that [ $\left.{ }^{89} \mathrm{Zr}\right]$ trastuzumab is able to detect the inter-lesion heterogeneity in advanced disease and select patients who would benefit from HER2-targeting therapy with antibody-drug conjugate trastuzumab emtansine (T-DM1) [26]. Furthermore, it was reported that this tracer could differentiate HER2-positive from HER2-negative and identify intra-patient heterogeneity in $20 \%$ of patients bearing numerous lesions [27]. Noteworthy, $\left[{ }^{89} \mathrm{Zr}\right]$ trastuzumab was capable of detecting HER2-positive metastases in a group of patients with HER2-negative primary breast cancer [28]. Furthermore, Bensch F et al. reported that $\left[{ }^{89} \mathrm{Zr}\right]$ trastuzumab PET could support clinical decision-making when standard approaches are not suitable to determinate HER2 status and repeat biopsies are not possible [29]. A representative image of [ ${ }^{89} \mathrm{Zr}$ ] trastuzumab tumor uptake in a HER2-positive breast cancer patient is reported in Figure 2. Similarly, [ $\left.{ }^{64} \mathrm{Cu}\right]$ DOTA-trastuzumab visualized HER2-positive metastatic breast cancer with high precision thus providing valuable information for selection of patients that could benefit by HER2-targeted treatments [30,31]. Recently, pertuzumab, another newer humanized HER2-targeting antibody, was labeled with ${ }^{89} \mathrm{Zr}$ and used for PET imaging of HER2-positive breast cancer patients. Since pertuzumab is able to bind HER2 at different sites and more efficiently compared to trastuzumab, the employment of this tracer could improve the detection of HER2-positive lesions that are not detected using trastuzumab [32]. This first-in-human trial demonstrated that [ $\left.{ }^{89} \mathrm{Zr}\right]$ pertuzumab PET/CT may be safely performed and it is able to evaluate the HER2 status and heterogeneity of lesions and detect unsuspected HER2-positive metastatic disease thus helping direct HER2-targeted therapy to appropriate patients [32]. Furthermore, pre-clinical findings showed that this tracer monitored early response to T-DM1 therapy of HER2-positive breast cancer xenograft-bearing mice and allowed precise detection of changes in tumor volume [33]. Many studies are ongoing to develop new HER2 targeting probes including antibody fragments, affibodies and nanobodies with superior characteristics appropriate for imaging, such as rapid targeting and quick blood clearance, high solubility, high stability, higher target-to-background ratios. The first-in-human application of ${ }^{68} \mathrm{Gallium}\left[{ }^{68} \mathrm{Ga}\right] \mathrm{HER} 2$-nanobody showed a favorable biodistribution, with the highest uptake in the kidneys, liver, and intestines and low background levels in other organs that generally harbor tumor lesions [34]. In a phase I/II study, the HER2-binding affibody ABY-025 was labeled with ${ }^{68} \mathrm{Ga}$ and used to measure HER2 levels in metastatic in breast cancer. SUV correlated with biopsy HER2-scores $(r=0.91, p<0.001)$ and PET correctly identified the conversion and mixed expression of HER2. These observations allowed the possibility to change the treatments in 3 of the 16 patients [35]. The tumor-targeting potential of the anti-HER2 nanobodies 5F7 and $2 \mathrm{Rs} 15 \mathrm{~d}$ labeled with ${ }^{18} \mathrm{~F}$ were assessed in a subcutaneous HER2-positive breast cancer murine model and in brain metastases [36,37]. Notably, it has been reported the involvement of HER3 in the development of the resistance to anti-HER2 therapies in breast cancer [85]. A specific monoclonal anti-HER3 antibody, 
patritumab, was labeled with ${ }^{64} \mathrm{Cu}$ and its safety, dosimetry, and binding were assessed in a group of patients with advance solid tumors including breast cancer [38]. Wehrenberg-Klee et al. demonstrated in a preclinical study that SUVmean of $\left[{ }^{64} \mathrm{Cu}\right] \mathrm{anti}-\mathrm{HER} 3-\mathrm{F}\left(\mathrm{ab}^{\prime}\right) 2$ increased in MDAMB468 xenografts treated with the AKT (Protein Kinase B) inhibitor GDC-0068 when compared to untreated control. The enhancement of tracer uptake in tumor correlated with HER3 levels and resistance to therapy [39].

The Vascular endothelial growth factor/Vascular endothelial growth factor receptor (VEGF/VEGFR) axis takes part in the regulation of angiogenesis in breast cancer, therefore, several molecules were developed to target it and then tested as anticancer agents and imaging probes [86]. 3-Piperidinylethoxy-anilinoquinazoline (PAQ) an analog to TKI vandetanib, with 40-fold stronger inhibitory properties for the VEGFR-2, was labeled with ${ }^{11} \mathrm{C}$ and used as PET probe for monitoring anticancer treatment in the MMTV-PyMT/FVB (PyMT) transgenic mouse breast cancer model [40]. Mice treated with vehicle, or the anti-VEGFA murine antibody B20-4.1.1, or paclitaxel (PTX) in combination or as single agents showed a SUVmax significantly reduced after 4 days in the B20-4.1.1/PTX combinational and B20-4.1.1 monotherapy groups ( $p<0.0005$ and $p<0.003$, respectively).

The Hepatocyte growth factor (HGF) receptor (c-Met) is overexpressed in basal-like phenotype of breast cancer that includes the aggressive TNBC sub-group [41,42]. PET imaging with [ $\left.{ }^{18} \mathrm{~F}\right] \mathrm{AH} 113804$ peptide, which has high affinity for human c-Met allowed the early recognition of locoregional tumor recurrence in a human basal-like murine breast cancer model [43]. The expression of the insulin-like growth factor 1 (IGF-1R) is assumed to be linked with the overall survival of breast cancer patients. Furthermore, phosphorylated IGF-1R appears to be an encouraging indicator for predicting clinical outcomes and may be an attractive target to improve antitumor treatment efficacy in patients with HER2-, ER+, and luminal B tumors. [87]. The antibody R1507 targeting IGF-1R and labeled with ${ }^{111}$ In for SPECT and ${ }^{89} \mathrm{Zr}$ for PET imaging, respectively, clearly visualized the subcutaneous TNBC SUM149 xenografts [44]. In the same animal model [111 $\mathrm{In}] \mathrm{F}\left(\mathrm{ab}^{\prime}\right) 2$ fragments showed an improved ability to target tumors expressing IGF-1R and a higher tumor-to-blood ratio in comparison to [111 In]R1507 [45]. Many fibrotic diseases and malignant tumors are associated with platelet-derived growth factor receptor beta (PDGFR $\beta$ ) overexpression and disproportionate signaling, making this receptor attractive for molecular targeting and imaging approaches. Recently, in a subgroup of mesenchymal TNBCs with invasive and stem-like phenotype, the role played by PDGFR $\beta$ as a suitable biomarker was investigated and the aptamer Gint4.T specifically targeting this receptor was proposed as a high effective tool for the imaging and suppression of TNBC lung metastases [88-90].

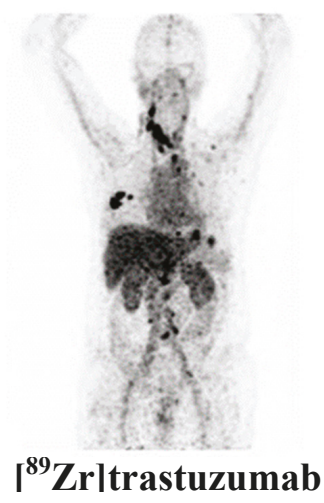

Figure 2. $\left[{ }^{89} \mathrm{Zr}\right]$ trastuzumab PET scan of a patient with HER2-positive breast cancer. (Figure adapted with permission from Bensch F et al. (2018) [29] Creative Commons Attribution 4.0 International License (http://creativecommons.org/licenses/by/4.0/). 


\section{Integrin Receptors}

The integrin families are heterodymeric cell adhesion receptors composed of non-covalently associated $\alpha$ and $\beta$ subunits that promote cell attachment and migration on the neighboring extracellular matrix (ECM). They regulate diverse cellular functions such as migration, invasion, proliferation, and survival, which are crucial to the initiation, progression, and metastasis of solid tumors [91].

In several tumor types, including breast cancer, the expression of specific integrins is associated with a worse patient prognosis and survival [91]. Integrins are capable of clustering together growth factors receptors in the membrane where they physically interact with each other to coordinate different pathways [92]. Interestingly, Stewart RL et al. observed a cross-talk between integrin $\alpha 6 \beta 4$ and HER2 in breast cancer [93]. Recently, it was reported that integrin $\alpha \mathrm{v} \beta 3$ is correlated with EGFR when TNBC cells were cultured on matrigel or injected subcutaneously in nude mice to form tumors, and this interaction was hampered by treatment with a specific EGFR aptamer CL4 both in vitro and in vivo [94]. This integrin is significantly up regulated on activated-endothelial cells and not on quiescent endothelial cells [95] and plays a crucial role in promoting tumor neo-angiogenesis and metastasis in aggressive tumors [91]. Therefore, labeled integrin antagonists could provide interesting diagnostic tools to assess the effectiveness of new anti-angiogenic and anti-tumoral therapies in breast cancer. Many molecules targeting $\alpha v \beta 3$ and harboring the same RGD (arginine-glycine-aspartic acid) sequence present in some ECM proteins, such as vitronectin and fibronectin, have been designed and developed as instruments for imaging and therapy [96-100]. ${ }^{18} \mathrm{~F}-$ Galacto-RGD was the first PET tracer applied in patients which successfully imaged $\alpha v \beta 3$ expression in human tumors including breast carcinomas with excellent tumor-background [46]. Several imaging studies using other RGD-containing radiolabeled peptides, such as $\left[{ }^{18} \mathrm{~F}\right] \mathrm{AH} 111585$ and $\left[{ }^{99 \mathrm{~m}} \mathrm{Tc}\right] \mathrm{NC} 100692$, were carried out to visualize malignant lesions in small cohorts of patients with breast cancer [47,48]. Moreover, variations in tracer design have been introduced to further improve the performance of $\alpha \mathrm{v} \beta 3$ imaging, e.g., the development of multimeric-RGD peptides able to recognize multivalent binding sites in RGD-containing ECM-proteins [101]. A pEGylated-dimeric-RGD (PEG3-E[c\{RGDyk\}]2) (FPPRGD2) labeled with $\left[{ }^{18} \mathrm{~F}\right]$ showed high and specific uptake in the primary tumor as well as in the metastases, with no safety issues in breast cancer patients. In addition, this probe showed higher average SUVmax when compared to monomeric RGD peptides [49]. [ $\left.{ }^{99 \mathrm{~m}} \mathrm{Tc}\right] 3 \mathrm{PRGD} 2$ tracer was evaluated for differential diagnosis of breast cancer and compared with traditional mammography [50]. When two methods were combined, the accuracy of diagnosis of breast cancer lesions enhanced greatly, especially for the dense mammary gland. Furthermore, $\left.{ }^{[99 \mathrm{~m}} \mathrm{Tc}\right] 3 \mathrm{PRGD} 2$ imaging had good performance for diagnosing primary breast tumor compared to $\left[{ }^{18} \mathrm{~F}\right]$ FDG PET/CT but showed lower sensitivity for identifying smaller metastatic lesions in the lymph nodes [51]. Moreover, the tracer uptake was related with integrin $\alpha v \beta 3$ expression $(r=0.582 ; p=0.001)$ and was greater in HER2-positive and stage III-IV patients $(p<0.05)$ [51]. A correlation between $\left[{ }^{18} \mathrm{~F}\right] \mathrm{FDG}$ and $\left[{ }^{68} \mathrm{Ga}\right] \mathrm{RGD}$ in patients with large or advanced invasive ductal breast cancer showed that SUVavg and SUVmax for $\left[{ }^{18} \mathrm{~F}\right]$ FDG was significantly higher in the ER negative and PR negative groups whereas these same parameters were significantly higher for $\left[{ }^{68} \mathrm{Ga}\right.$ ]RGD in the HER2-positive group [52]. Recently, a fluoride-aluminum complex, $\left[{ }^{18} \mathrm{~F}\right]$ Al-NOTA-PRGD2 (denoted as ${ }^{18} \mathrm{~F}$-Alfatide II), was developed and used in differentiating breast cancer from benign breast lesions [53]. Breast carcinoma showed relatively higher tracer uptake with a SUVmax of $3.77 \pm 1.78$ and SUVmean of $2.25 \pm 0.98$ compared to benign breast lesions. Furthermore, the authors showed that $\left[{ }^{18} \mathrm{~F}\right]$ Alfatide II had comparable diagnostic value to $\left[{ }^{18} \mathrm{~F}\right] \mathrm{FDG}$, but it was not superior in identifying breast cancer lesions. Interestingly, a heterodimeric peptide was synthesized from bombesin(7-14) (BBN) and c(RGDyK) and labeled with $\left[{ }^{68} \mathrm{Ga}\right],\left(\left[{ }^{68} \mathrm{Ga}\right] \mathrm{BBN}-\mathrm{RGD}\right)$, to target gastrin-releasing peptide receptor (GRPR) together with integrin $\alpha \mathrm{v} \beta 3[54,55]$. This tracer showed several advantages compared to the corresponding monomers because of the multivalency effects that result in an improved binding affinity and increased number of effective receptors $[54,55]$ (Figure 3). Significant correlations were identified between SUVmean determined by $\left[{ }^{68} \mathrm{Ga}\right] \mathrm{BBN}-\mathrm{RGD}$ PET and GRPR $\left(r^{2}=0.4791, p<0.0001\right)$ and integrin $\alpha \mathrm{V} \beta 3\left(r^{2}=0.3664, p<0.001\right)$. The correlation was 
greater when the sum of integrin $\alpha v \beta 3$ and GRPR was applied. [ ${ }^{68} \mathrm{Ga}$ ]BBN-RGD PET/CT was capable of visualizing primary breast cancer, axillary lymph nodes, and distant metastases. Recently, Li et al. provided pilot clinical evidence that tumor $\left[{ }^{18} \mathrm{~F}\right] \mathrm{RGD}$ uptake parameters at baseline are predictive for the therapeutic response to apatinib, an oral antiangiogenic drug, in breast cancer patients with local or metastatic lesions [56]. Patients whose lesions had a higher SUVmean responded better to treatment and showed a longer progression-free survival than those with a lower SUVmean.

Many reports have shown that tumors originating from the breast express elevated levels of $\alpha \mathrm{v} \beta 6$ integrin, therefore a specific peptide that targets this receptor has been developed and labeled with ${ }^{18} \mathrm{~F}$ for PET imaging. In a first-in-human study, the uptake of this probe was detected in primary breast lesions and lymph node metastases in stage IV invasive breast cancer [57].

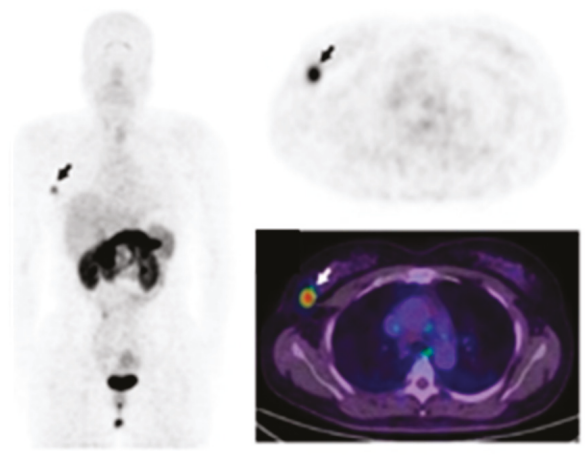

Figure 3. PET/CT imaging of gastrin-releasing peptide receptor and integrin $\alpha \mathrm{v} \beta 3$ expression using the heterodimeric tracer $\left[{ }^{68} \mathrm{Ga}\right] \mathrm{BBN}-\mathrm{RGD}$, in a patient with ER+, PR+, and HER2+ invasive ductal carcinoma. (Figure adapted with permission from Zhang J. et al. (2018) [55] Creative Commons Attribution (CC BY-NC) license (https://creativecommons.org/licenses/by-nc/4.0/).

\section{Chemokine Receptors}

Chemokines belong to a family of molecules that regulate many cellular functions by binding to G-protein-coupled receptors (GPCRs) with seven transmembrane domains. Upon binding to their specific ligand, they undergo a conformational change leading to the activation of intracellular signal pathways that evoke different cellular responses. The chemokine system comprises almost 50 chemokines and approximately 20 chemokine receptors. These receptors are classified as CC, CXC, CX3C, or XC based on the subfamily of their preferred ligands [102]. Interaction between chemokines and their specific receptors are critical to different biological processes in cancer progression such as tumor cell growth, tumor angiogenesis, organ-directed metastasis [103]. Particularly, chemokines and chemokine receptors such as CXCL12/CXCR4-CXCR7, CXCL16/CXCR6, CCL25/CCR9, CCL2/CCR2, and CCL5/CCR5 are found to play a crucial part in breast cancer progression [104].

The involvement of CXCL12/CXCR4 axis is well known in metastatic spreading, drug resistance, and overall worse diagnosis in breast carcinoma $[105,106]$. Furthermore, it facilitates cancer cell survival by allowing immune evasion and creating a favorable microenvironment for cancer cells to grow within the metastatic niche [107]. Promising molecules targeting CXCR4 have been developed for imaging and therapy $[108,109]$. A bicyclam-based antagonist AMD3100 (Plerixafor) was the first drug designed to block CXCR4 and to be used in humans [110]. Recently, Pernas et al. reported a phase 1 activity of balixafortide (CXCR4 antagonist) in combination with eribulin in heavily pretreated, relapsed metastatic breast cancer patients [111]. The tolerability and safety of this combination (balixafortide and eribulin) was comparable to that of each individual, and these two drugs together showed promising results in HER2 negative metastatic breast cancer patients. The application of molecular imaging with probes targeting CXCR4 could help to differentiate patients, monitor anti-CXCR4 therapy, and to clarify 
the intricate systemic effects. To visualize CXCR4 expressing tumor lesions, CXCR4 inhibitor AMD3100 was radiolabeled with $\left[{ }^{64} \mathrm{Cu}\right]$ and used for PET imaging. In preclinical studies, $\left[{ }^{64} \mathrm{Cu}\right] \mathrm{AMD} 3100$ was able to image breast cancer xenografts as well as lung metastases [58]. Similarly, tumor uptake of a ${ }^{64} \mathrm{Cu}$ doped gold nanoclusters conjugated with AMD3100 ([ $\left.{ }^{64} \mathrm{Cu}\right] \mathrm{AuNC}$-AMD3100) tracer correlated with CXCR4 expression in a 4T1 orthotopic mouse breast cancer. Furthermore, this probe was better than $\left[{ }^{64} \mathrm{Cu}\right] \mathrm{AMD} 3100$ alone in detecting initial-stage lesions and pre-metastatic niches in lungs [59]. A first pilot study with $\left[{ }^{68} \mathrm{Ga}\right.$ ]Pentixafor was carried out in a limited and heterogeneous group of patients with solid tumors including three patients with breast cancer [60]. Compared with $\left[{ }^{18}\right.$ F]FDG PET, this tracer showed a lower SUVmax in all measured malignant lesions. Afterwards, these observations were confirmed on a broader range of breast carcinomas with different histological features where $\left[{ }^{68} \mathrm{Ga}\right]$ Pentixafor uptake in tumor lesions was considerably lower compared to [ $\left.{ }^{18} \mathrm{~F}\right] \mathrm{FDG}$ [61] (Figure 4). Noteworthy, it has been reported that CXCR4 signaling could promote ER positive breast cancer to a therapy-resistant, estrogen-independent phenotype [112]. Therefore, imaging of CXCR4 in these circumstances may benefit from longitudinal spatio-temporal data on tumor development/metastasis during estrogen deprivation therapy. Recently, a cyclam-based small molecule radioprobe $\left[{ }^{18} \mathrm{~F}\right] \mathrm{MCFB}$ was developed and evaluated for imaging of CXCR4 expression in breast carcinoma xenografts [62]. The atypical chemokine receptor ACKR3 (CXCR7) is undetectable in normal breast tissues whereas is overexpressed in more than $30 \%$ of breast carcinomas [113]. Furthermore, the overexpression of this receptor is associated with poor overall survival and reduced lung metastasis-free survival in patients with invasive ductal carcinomas and with reduced relapse-free survival in patients with ER positive breast cancer [114]. In vivo [ ${ }^{89} \mathrm{Zr}$ ]ACKR3-mAb PET and ex vivo biodistribution studies performed in mice harboring TNBC tumors with graded ACKR3 expression levels, showed a direct correlation between tracer uptake and ACKR3 levels [63]. The signal specificity was verified through blocking studies performed in the presence of unlabeled ACKR3-mAb, which caused a significant reduction of tracer uptake in high-ACKR3-expressing tumors.
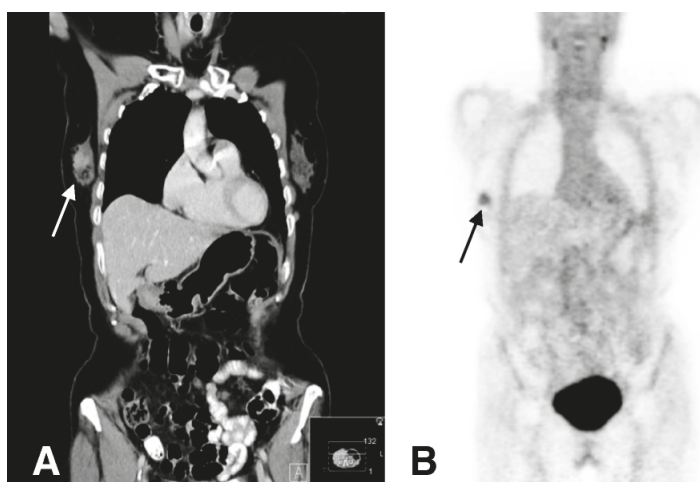

Figure 4. $\left[{ }^{68} \mathrm{Ga}\right]$ Pentixafor PET scan of a patient with invasive ductal primary breast carcinoma prior to treatment. (A) Coronal CT reconstruction shows contrast enhancement in a lesion in the right breast. (B) The tumor is visually detectable with $\left[{ }^{68} \mathrm{Ga}\right]$ Pentixafor. (Figure adapted with permission from Vag T. et al. (2018) [61] Creative Commons Attribution 4.0 International License (http://creativecommons. org/licenses/by/4.0/).

\section{Immune Checkpoint Receptors}

Tumors avoid the host immune system by activating biological systems, which in turn suppress antigen exposition and effector cells and create an immunosuppressive milieu in the cancer microenvironment [115]. Cancer cells activating inhibitory immune checkpoint proteins such as PD-1 (program death 1) receptor and PD-L1 (programmed cell death-ligand 1) evade and suppress immune responses [116]. PD-1 receptor is a trans-membrane receptor present on a variety of immune 
cells, such as monocytes, T cells, B cells, dendritic cells, and tumor-infiltrating lymphocytes (TILs). Conversely, PD-L1 is expressed by tumor and antigen presenting cells (APCs) and the binding of PD-L1 to PD-1 present on T cells leads to their dysfunction, exhaustion, and neutralization [117]. Therefore, PD-L1 overexpression protects tumor cells from T cell (CD8+) cytotoxicity [118]. In addition, B7-1 (CD80), another molecule expressed by T cells and APCs, is able to interact with PD-L1 promoting a negative regulation of T cell activation [119]. In this manner tumors create and uphold an immunosuppressive environment, which endorse cancer cell escape from immune surveillance. Immunotherapeutic approaches aim to trigger adaptive and native innate anti-tumor by reversing tumor-mediate immune suppression.

In breast carcinoma, PD-1/PD-L1 expression has been associated with large tumor size, high grade, high proliferation, estrogen receptor-negative status, and HER2-positive status [120] and it is inversely correlated with survival [121]. In metastatic breast cancer, initial clinical trials with immune checkpoint inhibitors have shown modest but interesting responses [122]. Treatment combinations with checkpoint blockade and chemotherapy in the neo-adjuvant setting caused an enhancement of pCR rates in comparison with chemotherapy alone [122]. Currently, checkpoint inhibitors in clinical trials for aggressive metastatic breast cancer, alone or together with conventional therapies, include anti-PD-1 antibodies such as pembrolizumab and nivolumab or anti-PD-L1 antibodies such as avelumab and atezolizumab [122]. At present, routine analysis of PD-1/PD-L1 expression is performed by immunohistochemistry on bioptic samples and used to guide immunotherapy. Interestingly, current studies have spotlighted the potential of molecular imaging to visualize the levels of these receptors non-invasively in whole tumor lesions and metastases. The longitudinal monitoring of PD-L1 levels could serve as a predictive biomarker to select patients for therapy with immune checkpoint inhibitors or as a tool to monitor PD-L1 expression during conventional anticancer treatments. For the first time, Heskamp et al. showed the feasibility of imaging PD-L1 expression in tumors noninvasively using a monoclonal antibody (PD-L1.3.1) radiolabeled with ${ }^{111}$ In [64]. In this study, they observed using SPECT/CT that $\left[{ }^{111}\right.$ In]PD-L1.3.1 accumulated in high PD-L1-expressing breast (MDA-MB-231 and SK-Br-3) cancer xenografts while no specific uptake was observed in breast tumors with no or undetectable levels of PD-L1 (SUM149, BT474, and MCF-7). Imaging and biodistribution studies in an immune-competent transgenic mouse model of breast carcinoma, reflecting the role of PD-L1 in the immune system, were performed using a murine anti-PD-L1 antibody conjugated to [111 In]DTPA [65]. Whole body SPECT images showed high signal intensity of [ ${ }^{111}$ In]DTPA-anti-PD-L1 in the tumors and in potentially cross-reactive organs such the thymus and the spleen [65]. Recently, an FDA (Food and Drug Administration) approved antibody atezolizumab (MPDL3280A) was used together with nab-paclitaxel for the first-line treatment of locally advanced unresectable or metastatic PD-L1-positive TNBC. This is a humanized monoclonal IgG1k antibody with high affinity for both human and mouse PD-L1. Lesniak et al. conjugated atezolizumab with DOTAGA and radiolabelled it with ${ }^{64} \mathrm{Cu}$ to perform PET/CT imaging on MDA-MB-231 and SUM149 breast carcinoma xenografts and on a syngeneic orthotopic breast cancer mouse model (4T1) [66]. They showed that the uptake of $\left[{ }^{64} \mathrm{Cu}\right]$ atezolizumab was higher in PD-L1 overexpressing tumors (MDA-MB-231) in comparison to xenografts that express low levels of this receptor (SUM149). Comparable results were obtained when the same breast cancer animal models underwent SPECT/CT with [ ${ }^{111}$ In]atezolizumab [67]. Recently, Heskamp et al. for the first time performed imaging studies using [ ${ }^{111}$ In]-PD-L1-mAb in a humanized breast carcinoma murine harboring PD-L1 expressing immune cells. They demonstrated that despite the presence of activated PD-L1-expressing human immune cells, the visualization of PD-L1 overexpressing tumors remained feasible [68]. Furthermore, they reported that $\left[{ }^{111} \mathrm{In}\right] \mathrm{anti}-\mathrm{mPD}-\mathrm{L} 1$ micro SPECT/CT could also sensitively monitor radiotherapy-related alteration of PD-L1 expression in tumors. Although, radiolabeled antibodies can offer data at specific doses, they have not been used to calculate the extent of PD-L1 drug engagement in patients, in part due to their prolonged circulation time. Notably, Kumar et al. reported that a high-affinity PD-L1-specific peptide (WL12) radiolabeled with ${ }^{64} \mathrm{Cu}$, was able to measure the PD-L1 engagement of each therapeutic antibody 
at the tumor, independently of its biophysical characteristics and in vivo kinetics [69]. These quantitative methods through mathematical modeling allow the calculation of antibody doses necessary to accomplish therapeutically effective occupancy (outlined $<90 \%$ ). Noteworthy, this study demonstrates the molecular imaging approach is able to predict the necessary dose to reach effective drug efficacy assessing the binding of novel molecules to target specific receptors.

In a first-in-human assessment of $\left[{ }^{89} \mathrm{Zr}\right.$ ]atezolizumab carried out in a group of 22 patients with bladder cancer ( 9 patients), NSCLC ( 9 patients), and TNBC (4 patients), prior to start atezolizumab therapy, tracer uptake appeared to be a good predictor of treatment response. Noteworthy, clinical responses in these patients were better correlated with pretreatment PET signal than with immunohistochemistry-or RNA-sequencing based predictive biomarkers [70] (Figure 5).

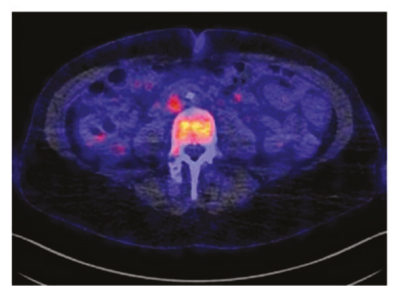

Figure 5. Representative PET/CT image of bone metastasis of a Triple-Negative Breast Cancer patient with [ ${ }^{89} \mathrm{Zr}$ ]atezolizumab on day 7 post injection (SUVmax 7.1). (Figure adapted from Bensch F et al. (2018) [70], Permission number 4691820293672, (CSpringer Nature).

\section{Somatostatin Receptors, Gastrin Releasing Peptide Receptors, and Neuropeptide Y Receptors}

It is well known that members of the somatostatin receptor family (SSTR) are overexpressed in neuroendocrine tumors whereas in breast carcinoma their role is not yet well clarified. Many years ago, Reubi et al. showed, through in vitro receptor autoradiography performed with a somatostatin analog labeled with $\left[{ }^{125} \mathrm{I}\right]$ on 342 breast cancer samples, that the levels of SSTR ranged from $21 \%$ in small tumor lesions to $46 \%$ in larger tumor lesions [123]. Furthermore, a low expression of subtype 2 SSTR was observed in normal breast tissue compared to malignant lesions [124]. A significant positive correlation between SSTR2 and ER expression was reported [125] as well as estrogen-mediated regulation of SSTR2 expression [126]. Similarly, Dalm et al. observed, in a cohort of 684 patients with LN-negative breast cancer, a strong correlation between ER, SSTR, and PR and suggested the possibility to use SSTR radiolabeled ligands to guide breast cancer therapy in estrogen-resistant patients [127]. Analogues of somatostatin such as octreotide and its derivatives, labeled with different radionuclides ${ }^{68} \mathrm{Ga},{ }^{90}$ Yttrium $\left[{ }^{90} \mathrm{Y}\right]$, and ${ }^{177}$ Lutetium $\left[{ }^{177} \mathrm{Lu}\right]$ are used in the imaging and therapy of neuroendrocrine tumors [128]. Recently, several investigations have occurred using these tracers in different tumor types including breast cancer [129]. Chereau and colleagues observed that $\left[{ }^{68} \mathrm{Ga}\right]$ DOTATOC (DOTA-DPhe1-Tyr3-Octreotide) was able to visualize breast cancer xenografts overexpressing SSTR2 that were undetected using [ ${ }^{18}$ F]FDG [71]. An autoradiographic study on an array of human breast cancer tissues showed that the antagonist $\left[{ }^{177} \mathrm{Lu}\right]$ DOTABASS had greater binding affinity to SSTR2 in comparison with the established agonist radioligand [ ${ }^{177}$ Lu]DOTATATE [72]. Conversely, Dude et al. reported in a murine model of human breast cancer, overexpressing this receptor that the agonists $\left[{ }^{68} \mathrm{Ga}\right]$ DOTATOC $(18.4 \pm 2.9 \% \mathrm{ID} / \mathrm{g})$ and $\left[{ }^{68} \mathrm{Ga}\right]$ DOTATATE $(15.2 \pm 2.2 \%$ ID $/ \mathrm{g}$, $\mathrm{ns})$ had higher tumor uptake compared to [ ${ }^{68} \mathrm{Ga}$ ]NODAGA-JR11 $(12.2 \pm 0.8 \%$ ID/g, $p<0.001)$ [73].

Gastrin releasing peptide receptor (GRPR) is a G-protein coupled receptor of the bombesin family expressed in most human cancers and represents an appealing biomarker for imaging and therapy of tumors. Recently, GRPR overexpression was found in $75.8 \%$ of breast carcinomas and was highly linked with ER positivity [130]. Maina et al. introduced a novel GRPR antagonist [ $\left.{ }^{68} \mathrm{Ga}\right] \mathrm{SB} 3$ for imaging studies and showed that it had high GRPR-affinity, good in vivo stability, and excellent targeting efficacy. In the first clinical PET/CT study carried out on a group of breast cancer patients, 
this tracer allowed the visualization of $50 \%$ of tumor lesions [74]. Another GRPR antagonist, RM2, was labeled with ${ }^{68} \mathrm{Ga}$ and used for the PET/CT pre-treatment staging of patients with primary breast carcinoma already confirmed by biopsy [75] (Figure 6). [ ${ }^{68} \mathrm{Ga}$ ]RM2 uptakes were strongly increased in $87 \%$ of tumors in comparison to healthy breast tissue and a strong association amongst GRPR binding and the ER and PR was observed. Noteworthy, this tracer was capable of detecting internal mammary lymph nodes and contralateral axillary lymph node metastasis (verified by biopsy) and bone metastases (undetected by bone scan and CT). Recently, a retrospective study performed with $\left[{ }^{68} \mathrm{Ga}\right] \mathrm{RM} 2$ and ${ }^{18} \mathrm{~F}$-FDG indicated that GRPR targeting imaging could be complementary or superior to $\left[{ }^{18} \mathrm{~F}\right] \mathrm{FDG}$ in ER-positive tumors with a low proliferation index [131]. A prospective pilot study was performed on 35 women with suspicion of breast cancer, based on mammography or ultrasonography, using $\left[{ }^{68} \mathrm{Ga}\right]$ NOTA-RM26 PET/CT [76]. This tracer uptake was high in tumor lesions and correlated significantly with ER+ expression. Interesting studies evaluated a novel radiolabeled GRPR antagonist, NeoBOMB1, radiolabeled with $\left[{ }^{67} / 68 \mathrm{Ga} /{ }^{111} \mathrm{In} /{ }^{177} \mathrm{Lu}\right]$ as a theranostic tool in a prostate cancer animal model [132] and in prostate cancer patients [133]. Recently, on the basis of these observations, the same authors investigated the potential of this molecule as an agent for imaging and therapy of GRPR-positive mammary carcinoma and reported the ability of $\left[{ }^{67} \mathrm{Ga}\right] \mathrm{NeoBOMB} 1$ to specifically localize in the tumors of mice bearing T-47D breast xenografts [77].
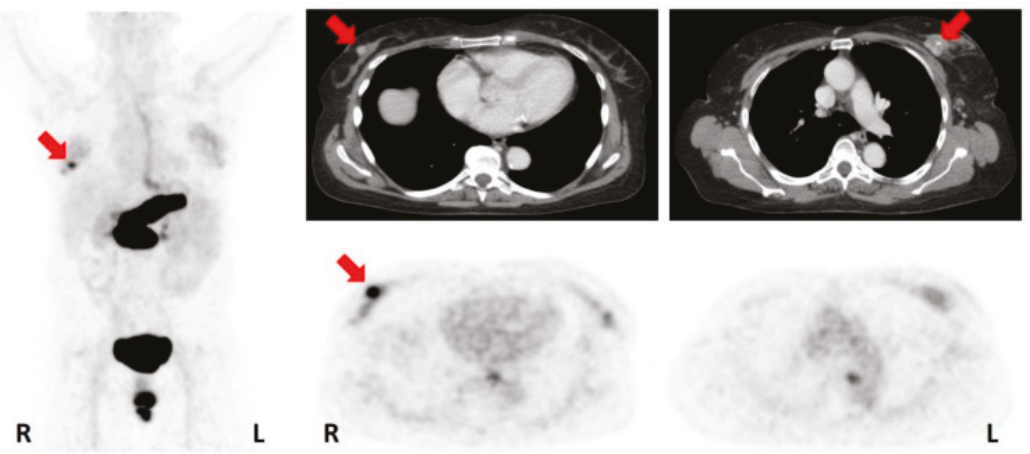

Figure 6. $\left[{ }^{68} \mathrm{Ga}\right] \mathrm{RM} 2-\mathrm{PET}$ in a patient with bilateral invasive ductal breast carcinoma; the tumor, on the right side was ER/PR positive and showed a strongly positive uptake, while the lesion on the left side was ER/PR negative and showed a weak uptake. Pancreas, esophagus, and rectum uptake is physiological. (Unmodified from Stoykow C. et al. (2016) [75]).

Notably, it has been observed that $85 \%$ of primary breast cancer and $100 \%$ of lymph node metastases express high levels of the sub-type 1 of the human neuropeptide $Y$ receptor family [134]. This is a G-protein coupled receptor family and includes, besides Y1R, other three isoforms (Y2R, Y4R, and Y5R) [135]. In addition, Reubi et al. reported a shift from Y2R expression observed in healthy tissue to Y1R during neoplastic transformation [132]. Based on these findings, Khan and colleagues developed an analogous of the neuropeptide $\mathrm{Y}$ (NPY) and, after labeling it with [ $\left.{ }^{99 \mathrm{~m}} \mathrm{TC}\right]$, they tested it for imaging of Y1R in four breast cancer patients showing different stages of disease [78]. They found that the tracer was able to visualize tumor and metastases whereas normal tissues showed only background radiation [78]. Interestingly, because it was observed in ER+ breast cancer cells (MCF-7) that estrogen induced up-regulation of Y1R mRNA [136], Memminger M et al. evaluated the potential of Y1R as a diagnostic target in ER+ breast cancer [137]. They investigated the effect of estradiol and tamoxifen treatment on the Y1R level in MCF-7 xenografts, through tissue autoradiography using the selective Y1R antagonist $\left[{ }^{3} \mathrm{H}\right]-$ UR-MK114. They found that the diagnostic value of the Y1R was compromised because anti-estrogen therapy caused receptor down-regulation. Although the majority of breast cancer tumors are ER+, in the very aggressive triple-negative forms lacking this receptor, Y1R could be an interesting imaging biomarker to investigate. Furthermore, a $\left[{ }^{18} \mathrm{~F}\right]$-labeled high-molecular-weight 
NPY glycopeptide tracer for PET imaging was developed and its ability to detect Y1R expression in vivo was tested and validated in MCF-7 tumor-bearing nude mice [79].

\section{Summary}

Nowadays, despite the well-known molecular heterogeneity of breast cancer and the identification of important mutations that characterize different subtypes, the only clinically relevant biomarkers and validated therapeutic targets are the hormone receptors ER, PR, and HER2. During disease progression and in response to conventional and non-conventional treatments, the receptor status can change and multiple biopsies are not always applicable. Therefore, the development of agents for nuclear imaging that allow the specific detection of receptor expression could contribute to improve patient management. In this review, we report some of the most recent studies focusing on the development and characterization of novel nuclear tracers targeting specific receptors that could be good candidates for innovative and strategic therapeutic approaches (Figure 7).

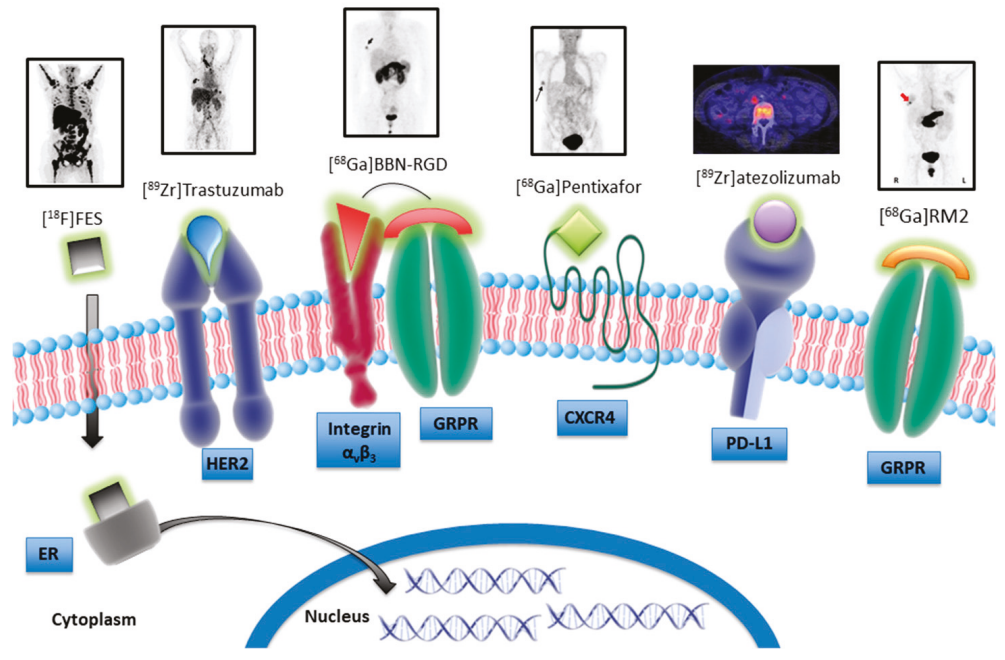

Figure 7. Schematic model illustrating nuclear tracers targeting specific receptors that are candidate for the theranostic approach.

There are many advantages that imaging techniques offer in comparison to traditional approaches that need bioptic samples. Firstly, they permit the non-invasive visualization and quantification of receptor patterning in whole-body, taking into account the inter- and intra-variability of lesions. Importantly, longitudinal studies carried out on the same patient can help clinical decision-making very early by following the evolution of receptor status. To date, $\left[{ }^{18} \mathrm{~F}\right] \mathrm{FES}$ and $\left[{ }^{18} \mathrm{~F}\right] 4 \mathrm{FMFES}$ are the main tracers used for ER detection in breast cancer. In addition, targeting PR using $\left[{ }^{18} \mathrm{~F}\right] \mathrm{FFNP}$ could be useful in-patient imaging when ER sites are saturated by tamoxifen treatment. The antibodies used in clinical trials for HER2 overexpressing tumors, trastuzumab and pertuzumab, labeled with positron radionuclides, are showing great potential as PET agents. Importantly, they are able to detect HER2-expressing metastases derived from primary negative breast cancer. To improve tissue penetration and increase signal-to-noise ratio of these tracers, smaller molecules such as antibody fragments, affibodies, and nanobodies have been developed for imaging applications and tested in pre-clinical and preliminary clinical studies. Several mechanisms are implicated in the emergence of resistance to hormone and HER2 targeting treatments, therefore some alternative targets are undergoing evaluation for the imaging and therapy of breast cancer. For example, probes targeting other RTKs besides HER2-such as HER3, VEGFR, and IGF-1R have been evaluated in breast cancer animal 
models. In the last decades, it has been clarified that $\alpha v \beta 3$ integrin expression is associated with a more aggressive phenotype of breast cancer given its role in both angiogenesis and metastatic processes. In 2008, Beer et al. reported [46], for the first time, the capability of [ $\left.{ }^{18} \mathrm{~F}\right]$ Galacto-RGD to visualize the expression of this receptor in breast cancer patients. Subsequently, many other tracers binding $\alpha v \beta 3$ with improved in vivo performance have been assessed in pre-clinical studies. A recent work by $\mathrm{Li}$ and colleagues [56] showed how $\left[{ }^{18} \mathrm{~F}\right]$-ALF-NOTA-PRGD2 detecting $\alpha \mathrm{v} \beta 3$ could predict a better response to anti-angiogenic therapy in many solid tumors including breast cancer. The application of dual targeting probes, such as $\left[{ }^{68} \mathrm{Ga}\right] B B N-R G D$, may have higher targeting efficacy aside from superior specificity than their corresponding mono-specific probes. Noteworthy, the initial results from a first-in-human study including TNBC patients showed that PET with $\left[{ }^{89} \mathrm{Zr}\right]$ atezolizumab was able to detect PD-L1 status and predict response to atezolizumab therapy. Furthermore, Kumar et al. showed that PET imaging with a peptide radiotracer targeting PD-L1, $\left[{ }^{64} \mathrm{Cu}\right] \mathrm{WL} 12$, may predict antibody dose needed to achieve therapeutically effective occupancy [69]. Moreover, other receptors such as SSTR, GRPR, and Y1R have been described to be also overexpressed in breast cancer, and radiotracers targeting them are used ongoing in preclinical and in-first-human clinical studies.

\section{Conclusions and Future Perspectives}

Many studies discussed in this review evaluated the same molecule both as an imaging agent and as an anti-cancer drug thus performing a theranostic approach (Table 2). This is an emerging field of medicine that by combining diagnostic tools and therapeutic strategies allows simultaneously or sequentially diagnosis, treatment, and monitoring drug response [138,139]. Considering that, with the exception of hormone receptors, most of the theranostic agents are currently evaluated in pre-clinical stages or in small groups of patients, further studies are still needed to translate them for clinical use. However, there are encouraging data suggesting that nuclear imaging may be a valid instrument to select breast cancer patients that could benefit from receptor targeted therapies and to monitor very early therapeutic response. Several studies are ongoing to validate promising probes that could help clinicians choose tailored treatments for each patient thus reducing therapeutic failures.

Table 2. Molecular Imaging for targeted therapy response assessment.

\begin{tabular}{lllll}
\hline Target & \multicolumn{1}{c}{ Tracer } & \multicolumn{1}{c}{ Therapy } & \multicolumn{1}{c}{ Type of Study } & Ref. \\
\hline ER & {$\left[{ }^{18} \mathrm{~F}\right]$ FES } & ER antagonist GDC-0810 & $\begin{array}{l}\text { patients with ER-positive } \\
\text { metastatic breast cancer }\end{array}$ & {$[15]$} \\
\hline HER2 & {$\left[{ }^{89} \mathrm{Zr}\right]$ trastuzumab } & $\begin{array}{l}\text { antibody-drug conjugate } \\
\text { trastuzumab emtansine (T-DM1) }\end{array}$ & $\begin{array}{l}\text { patients with advanced } \\
\text { HER2-positive breast cancer }\end{array}$ & {$[26]$} \\
\hline HER2 & {$\left[{ }^{89} \mathrm{Zr}\right]$ pertuzumab } & $\begin{array}{l}\text { antibody-drug conjugate } \\
\text { trastuzumab emtansine (T-DM1) }\end{array}$ & $\begin{array}{l}\text { mice bearing HER2+ breast cancer } \\
\text { xenografts (BT-474) }\end{array}$ & {$[33]$} \\
\hline HER3 & {$\left[{ }^{64} \mathrm{Cu}\right]$ anti-HER3-F(ab')2 } & AKT inhibitor GDC-0068 & $\begin{array}{l}\text { mice bearing breast cancer } \\
\text { xenografts (MDAMB468) }\end{array}$ & {$[39]$} \\
\hline VEGFR & $(\mathrm{R})-[11 \mathrm{C}]$ PAQ & $\begin{array}{l}\text { anti-VEGFA antibody (B20-4.1.1) } \\
\text { and/or paclitaxel (PTX) }\end{array}$ & $\begin{array}{l}\text { MMTV-PyMT/FVB (PyMT) } \\
\text { transgenic mouse breast cancer }\end{array}$ & {$[40]$} \\
\hline$\alpha v \beta 3$ & {$\left[{ }^{18} \mathrm{~F}\right]$ RGD } & apatinib & $\begin{array}{l}\text { breast cancer patients with local or } \\
\text { metastatic lesions }\end{array}$ & {$[56]$} \\
\hline PD-L1 & {$\left[{ }^{89} \mathrm{Zr}\right]$ atezolizumab } & atezolizumab & TNBC patients & [70] \\
\hline
\end{tabular}

Funding: This work was supported by the MIUR Progetti di Ricerca di Rilevante Interesse Nazionale (PRIN) Bando 2017-grant 2017MHJJ55; B.S.H was supported by INCIPIT International PhD program co-funded by Marie Sklodowska-Curie Actions.

Conflicts of Interest: The authors declare no conflict of interest. 


\section{References}

1. Harbeck, N.; Gnant, M. Breast cancer. Lancet 2017, 389, 1134-1150. [CrossRef]

2. Bianchini, G.; Balko, J.M.; Mayer, I.A.; Sanders, M.E.; Gianni, L. Triple-negative breast cancer: Challenges and opportunities of a heterogeneous disease. Nat. Rev. Clin. Oncol. 2016, 13, 674-690. [CrossRef] [PubMed]

3. Masoud, V.; Pagès, G. Targeted therapies in breast cancer: New challenges to fight against resistance. World J. Clin. Oncol. 2017, 8, 120-134. [CrossRef] [PubMed]

4. De Vries, E.G.E.; Kist de Ruijter, L.; Lub-de Hooge, M.N.; Dierckx, R.A.; Elias, S.G.; Oosting, S.F. Integrating molecular nuclear imaging in clinical research to improve anticancer therapy. Nat. Rev. Clin. Oncol. 2019, 16, 241-255. [CrossRef] [PubMed]

5. Basu, S.; Alavi, A. SPECT-CT and PET-CT in Oncology-An Overview. Curr. Med. Imaging Rev. 2011, 7, 202-209. [CrossRef]

6. Del Vecchio, S.; Zannetti, A.; Fonti, R.; Iommelli, F.; Pizzuti, L.M.; Lettieri, A.; Salvatore, M. PET/CT in cancer research: From preclinical to clinical applications. Contrast Media Mol. Imaging 2010, 5, 190-200. [CrossRef]

7. Lebron, L.; Greenspan, D.; Pandit-Taskar, N. PET Imaging of Breast Cancer: Role in Patient Management. PET Clin. 2015, 10, 159-195. [CrossRef]

8. Linden, H.M.; Peterson, L.M.; Fowler, A.M. Clinical Potential of Estrogen and Progesterone Receptor Imaging. PET Clin. 2018, 13, 415-422. [CrossRef]

9. Oude Munnink, T.H.; Nagengast, W.B.; Brouwers, A.H.; Schröder, C.P.; Hospers, G.A.; Lub-de Hooge, M.N.; van der Wall Evan Diest, P.J.; de Vries, E.G. Molecular imaging of breast cancer. Breast 2009, 18, S66-S73. [CrossRef]

10. Peterson, L.M.; Mankoff, D.A.; Lawton, T.; Yagle, K.; Schubert, E.K.; Stekhova, S.; Gown, A.; Link, J.M.; Tewson, T.; Krohn, K.A. Quantitative imaging of estrogen receptor expression in breast cancer with PET and ${ }^{18}$ F-fluoroestradiol. J. Nucl. Med. 2008, 49,367-374. [CrossRef]

11. Gemignani, M.L.; Patil, S.; Seshan, V.E.; Sampson, M.; Humm, J.L.; Lewis, J.S.; Brogi, E.; Larson, S.M.; Morrow, M.; Pandit-Taskar, N. Feasibility and predictability of perioperative PET and estrogen receptor ligand in patients with invasive breast cancer. J. Nucl. Med. 2013, 54, 1697-1702. [CrossRef] [PubMed]

12. Liu, H.; Chen, Y.; Wu, S.; Song, F.; Zhang, H.; Tian, M. Molecular imaging using PET and SPECT for identification of breast cancer subtypes. Nucl. Med. Commun. 2016, 37, 1116-1124. [CrossRef] [PubMed]

13. Dehdashti, F.; Mortimer, J.E.; Trinkaus, K.; Naughton, M.J.; Ellis, M.; Katzenellenbogen, J.A.; Welch, M.J.; Siegel, B.A. PET-based estradiol challenge as a predictive biomarker of response to endocrine therapy in women with estrogen-receptor-positive breast cancer. Breast Cancer Res. Treat. 2009, 113, 509-517. [CrossRef] [PubMed]

14. Van Kruchten, M.; Glaudemans, A.W.J.M.; de Vries, E.F.J.; Schröder, C.P.; de Vries, E.G.E.; Hospers, G.A.P. Positron emission tomography of tumor [(18)F]fluoroestradiol uptake in patients with acquired hormone-resistant metastatic breast cancer prior to oestradiol therapy. Eur. J. Nucl. Med. Mol. Imaging 2015, 42, 1674-1681. [CrossRef] [PubMed]

15. Wang, Y.; Ayres, K.L.; Goldman, D.A.; Dickler, M.N.; Bardia, A.; Mayer, I.A.; Winer, E.; Fredrickson, J.; Arteaga, C.L.; Baselga, J.; et al. ${ }^{18}$ F-Fluoroestradiol PET/CT Measurement of Estrogen Receptor Suppression during a Phase I Trial of the Novel Estrogen Receptor-Targeted Therapeutic GDC-0810: Using an Imaging Biomarker to Guide Drug Dosage in Subsequent Trials. Clin. Cancer Res. 2017, 23, 3053-3060. [CrossRef]

16. Kurihara, H.; Shimizu, C.; Miyakita, Y.; Yoshida, M.; Hamada, A.; Kanayama, Y.; Yonemori, K.; Hashimoto, J.; Tani, H.; Kodaira, M.; et al. Molecular imaging using PET for breast cancer. Breast Cancer 2016, 23, 24-32. [CrossRef]

17. Paquette, M.; Phoenix, S.; Ouellet, R.; Langlois, R.; van Lier, J.E.; Turcotte, E.E.; Bénard, F.; Lecomte, R. Assessment of the novel estrogen receptor PET tracer 4-fluoro-11 $\beta$-methoxy-16 $\alpha-\left[\left({ }^{18}\right)\right.$ F]fluoroestradiol (4FMFES) by PET imaging in a breast cancer murine model. Mol. Imaging Biol. 2013, 15, 625-632. [CrossRef]

18. Paquette, M.; Lavallée, É.; Phoenix, S.; Ouellet, R.; Senta, H.; van Lier, J.E.; Guérin, B.; Lecomte, R.; Turcotte, É.E. Improved Estrogen Receptor Assessment by PET Using the Novel Radiotracer ${ }^{18}$ F-4FMFES in Estrogen Receptor-Positive Breast Cancer Patients: An Ongoing Phase II Clinical Trial. J. Nucl. Med. 2018, 59, 197-203. [CrossRef] 
19. Cunha, S.; Gano, L.; Morais, G.R.; Thiemann, T.; Oliveira, M.C. Progesterone receptor targeting with radiolabelled steroids: An approach in predicting breast cancer response to therapy. J. Steroid Biochem. Mol. Biol. 2013, 137, 223-241. [CrossRef]

20. Fowler, A.M.; Clark, A.S.; Katzenellenbogen, J.A.; Linden, H.M.; Dehdashti, F. Imaging Diagnostic and Therapeutic Targets: Steroid Receptors in Breast Cancer. J. Nucl. Med. 2016, 57 (Suppl. 1), 75S-80S. [CrossRef]

21. Linden, H.M.; Dehdashti, F. Novel methods and tracers for breast cancer imaging. Semin. Nucl. Med. 2013, 43, 324-329. [CrossRef]

22. Chan, S.R.; Fowler, A.M.; Allen, J.A.; Zhou, D.; Dence, C.S.; Sharp, T.L.; Fettig, N.M.; Dehdashti, F.; Katzenellenbogen, J.A. Longitudinal noninvasive imaging of progesterone receptor as a predictive biomarker of tumor responsiveness to estrogen deprivation therapy. Clin. Cancer Res. 2015, 21, 1063-1070. [CrossRef]

23. Salem, K.; Kumar, M.; Yan, Y.; Jeffery, J.J.; Kloepping, K.C.; Michel, C.J.; Powers, G.L.; Mahajan, A.M.; Fowler, A.M. Sensitivity and Isoform Specificity of ${ }^{18}$ F-Fluorofuranylnorprogesterone for Measuring Progesterone Receptor Protein Response to Estradiol Challenge in Breast Cancer. J. Nucl. Med. 2019, 60, 220-226. [CrossRef]

24. Perik, P.J.; Lub-De Hooge, M.N.; Gietema, J.A.; van der Graaf, W.T.; de Korte, M.A.; Jonkman, S.; Kosterink, J.G.; van Veldhuisen, D.J.; Sleijfer, D.T.; Jager, P.L.; et al. Indium-111-labeled trastuzumab scintigraphy in patients with human epidermal growth factor receptor 2-positive metastatic breast cancer. J. Clin. Oncol. 2006, 24, 2276-2282. [CrossRef]

25. Dijkers, E.C.; Oude Munnink, T.H.; Kosterink, J.G.; Brouwers, A.H.; Jager, P.L.; de Jong, J.R.; van Dongen, G.A.; Schröder, C.P.; Lub-de Hooge, M.N.; de Vries, E.G. Biodistribution of ${ }^{89}$ Zr-trastuzumab and PET imaging of HER2-positive lesions in patients with metastatic breast cancer. Clin. Pharmacol. Ther. 2010, 87, 586-592. [CrossRef]

26. Gebhart, G.; Lamberts, L.E.; Wimana, Z.; Garcia, C.; Emonts, P.; Ameye, L.; Stroobants, S.; Huizing, M.; Aftimos, P.; Tol, J.; et al. Molecular imaging as a tool to investigate heterogeneity of advanced HER2-positive breast cancer and to predict patient outcome under trastuzumab emtansine (T-DM1): The ZEPHIR trial. Ann. Oncol. 2016, 27, 619-624. [CrossRef]

27. Dehdashti, F.; Wu, N.; Bose, R.; Naughton, M.J.; Ma, C.X.; Marquez-Nostra, B.V.; Diebolder, P.; Mpoy, C.; Rogers, B.E.; Lapi, S.E.; et al. Evaluation of $\left[{ }^{89} \mathrm{Zr}\right]$ trastuzumab-PET/CT in differentiating HER2-positive from HER2-negative breast cancer. Breast Cancer Res. Treat. 2018, 169, 523-530. [CrossRef]

28. Ulaner, G.A.; Hyman, D.M.; Lyashchenko, S.K.; Lewis, J.S.; Carrasquillo, J.A. ${ }^{89}$ Zr-Trastuzumab PET/CT for Detection of Human Epidermal Growth Factor Receptor 2-Positive Metastases in Patients With Human Epidermal Growth Factor Receptor 2-Negative Primary Breast Cancer. Clin. Nucl. Med. 2017, 42, 912-917. [CrossRef]

29. Bensch, F.; Brouwers, A.H.; Lub-de Hooge, M.N.; de Jong, J.R.; van der Vegt, B.; Sleijfer, S.; de Vries, E.G.E.; Schröder, C.P. ${ }^{89} \mathrm{Zr}$-trastuzumab PET supports clinical decision making in breast cancer patients, when HER2 status cannot be determined by standard work up. Eur. J. Nucl. Med. Mol. Imaging 2018, 45, 2300-2306. [CrossRef]

30. Mortimer, J.E.; Bading, J.R.; Colcher, D.M.; Conti, P.S.; Frankel, P.H.; Carroll, M.I.; Tong, S.; Poku, E.; Miles, J.K.; Shively, J.E.; et al. Functional imaging of human epidermal growth factor receptor 2-positive metastatic breast cancer using (64)Cu-DOTA-trastuzumab PET. J. Nucl. Med. 2014, 55, 23-29. [CrossRef]

31. Mortimer, J.E.; Bading, J.R.; Park, J.M.; Frankel, P.H.; Carroll, M.I.; Tran, T.T.; Poku, E.K.; Rockne, R.C.; Raubitschek, A.A.; Shively, J.E.; et al. Tumor Uptake of ${ }^{64} \mathrm{Cu}$-DOTA-Trastuzumab in Patients with Metastatic Breast Cancer. J. Nucl. Med. 2018, 59, 38-43. [CrossRef] [PubMed]

32. Ulaner, G.A.; Lyashchenko, S.K.; Riedl, C.; Ruan, S.; Zanzonico, P.B.; Lake, D.; Jhaveri, K.; Zeglis, B.; Lewis, J.S.; O'Donoghue, J.A. First-in-Human Human Epidermal Growth Factor Receptor 2-Targeted Imaging Using ${ }^{89} \mathrm{Zr}$-Pertuzumab PET/CT: Dosimetry and Clinical Application in Patients with Breast Cancer. J. Nucl. Med. 2018, 59, 900-906. [CrossRef] [PubMed]

33. Massicano, A.V.F.; Lee, S.; Crenshaw, B.K.; Aweda, T.A.; El Sayed, R.; Super, I.; Bose, R.; Marquez-Nostra, B.V.; Lapi, S.E. Imaging of HER2 with [ $\left.{ }^{89} \mathrm{Zr}\right]$ pertuzumab in Response to T-DM1 Therapy. Cancer Biother. Radiopharm. 2019, 34, 209-217. [CrossRef] 
34. Keyaerts, M.; Xavier, C.; Heemskerk, J.; Devoogdt, N.; Everaert, H.; Ackaert, C.; Vanhoeij, M.; Duhoux, F.P.; Gevaert, T.; Simon, P.; et al. Phase I Study of ${ }^{68}$ Ga-HER2-Nanobody for PET/CT Assessment of HER2 Expression in Breast Carcinoma. J. Nucl. Med. 2016, 57, 27-33. [CrossRef]

35. Sörensen, J.; Velikyan, I.; Sandberg, D.; Wennborg, A.; Feldwisch, J.; Tolmachev, V.; Orlova, A.; Sandström, M.; Lubberink, M.; Olofsson, H.; et al. Measuring HER2-Receptor Expression In Metastatic Breast Cancer Using [ $\left.{ }^{68} \mathrm{Ga}\right] \mathrm{ABY}-025$ Affibody PET/CT. Theranostics 2016, 6, 262-271. [CrossRef]

36. Vaidyanathan, G.; McDougald, D.; Choi, J.; Koumarianou, E.; Weitzel, D.; Osada, T.; Lyerly, H.K.; Zalutsky, M.R. Preclinical Evaluation of ${ }^{18}$ F-Labeled Anti-HER2 Nanobody Conjugates for Imaging HER2 Receptor Expression by Immuno-PET. J. Nucl. Med. 2016, 57, 967-973. [CrossRef]

37. Zhou, Z.; Vaidyanathan, G.; McDougald, D.; Kang, C.M.; Balyasnikova, I.; Devoogdt, N.; Ta, A.N.; McNaughton, B.R.; Zalutsky, M.R. Fluorine-18 Labeling of the HER2-Targeting Single-Domain Antibody 2Rs15d Using a Residualizing Label and Preclinical Evaluation. Mol. Imaging Biol. 2017, 19, 867-877. [CrossRef]

38. Lockhart, A.C.; Liu, Y.; Dehdashti, F.; Laforest, R.; Picus, J.; Frye, J.; Trull, L.; Belanger, S.; Desai, M.; Mahmood, S.; et al. Phase 1 Evaluation of [(64)Cu]DOTA-Patritumab to Assess Dosimetry, Apparent Receptor Occupancy, and Safety in Subjects with Advanced Solid Tumors. Mol. Imaging Biol. 2016, 18, 446-453. [CrossRef]

39. Wehrenberg-Klee, E.; Turker, N.S.; Heidari, P.; Larimer, B.; Juric, D.; Baselga, J.; Scaltriti, M.; Mahmood, U. Differential Receptor Tyrosine Kinase PET Imaging for Therapeutic Guidance. J. Nucl. Med. 2016, 57, 1413-1419. [CrossRef]

40. Tegnebratt, T.; Lu, L.; Eksborg, S.; Chireh, A.; Damberg, P.; Nikkhou-Aski, S.; Foukakis, T.; Rundqvist, H.; Holmin, S.; Kuiper, R.V.; et al. Treatment response assessment with (R)-[11CPAQ PET in the MMTV-PyMT mouse model of breast cancer. EJNMMI Res. 2018, 8, 25. [CrossRef]

41. Gastaldi, S.; Comoglio, P.M.; Trusolino, L. The Met oncogene and basal-like breast cancer: Another culprit to watch out for? Breast Cancer Res. 2010, 12, 208. [CrossRef]

42. Lowery, A.J.; Kell, M.R.; Glynn, R.W.; Kerin, M.J.; Sweeney, K.J. Locoregional recurrence after breast cancer surgery: A systematic review by receptor phenotype. Breast Cancer Res. Treat. 2012, 133, 831-841. [CrossRef]

43. Arulappu, A.; Battle, M.; Eisenblaetter, M.; McRobbie, G.; Khan, I.; Monypenny, J.; Weitsman, G.; Galazi, M.; Hoppmann, S.; Gazinska, P.; et al. c-Met PET Imaging Detects Early-Stage Locoregional Recurrence of Basal-Like Breast Cancer. J. Nucl. Med. 2016, 57, 765-770. [CrossRef]

44. Heskamp, S.; van Laarhoven, H.W.; Molkenboer-Kuenen, J.D.; Franssen, G.M.; Versleijen-Jonkers, Y.M.; Oyen, W.J.; van der Graaf, W.T.; Boerman, O.C. ImmunoSPECT and immunoPET of IGF-1R expression with the radiolabeled antibody R1507 in a triple-negative breast cancer model. J. Nucl. Med. 2010, 51, 1565-1572. [CrossRef]

45. Heskamp, S.; van Laarhoven, H.W.; Molkenboer-Kuenen, J.D.; Bouwman, W.H.; van der Graaf, W.T.; Oyen, W.J.; Boerman, O.C. Optimization of IGF-1R SPECT/CT imaging using 111In-labeled F(ab')2 and Fab fragments of the monoclonal antibody R1507. Mol. Pharm. 2012, 9, 2314-2321. [CrossRef]

46. Beer, A.J.; Niemeyer, M.; Carlsen, J.; Sarbia, M.; Nährig, J.; Watzlowik, P.; Wester, H.J.; Harbeck, N.; Schwaiger, M. Patterns of alphavbeta3 expression in primary and metastatic human breast cancer as shown by ${ }^{18}$ F-Galacto-RGD PET. J. Nucl. Med. 2008, 49, 255-259. [CrossRef]

47. Kenny, L.M.; Coombes, R.C.; Oulie, I.; Contractor, K.B.; Miller, M.; Spinks, T.J.; McParland, B.; Cohen, P.S.; Hui, A.M.; Palmieri, C.; et al. Phase I trial of the positron-emitting Arg-Gly-Asp (RGD) peptide radioligand ${ }^{18}$ F-AH111585 in breast cancer patients. J. Nucl. Med. 2008, 49, 879-886. [CrossRef]

48. Bach-Gansmo, T.; Danielsson, R.; Saracco, A.; Wilczek, B.; Bogsrud, T.V.; Fangberget, A.; Tangerud, A.; Tobin, D. Integrin receptor imaging of breast cancer: A proof-of-concept study to evaluate 99mTc-NC100692. J. Nucl. Med. 2006, 47, 1434-1439.

49. Iagaru, A.; Mosci, C.; Shen, B.; Chin, F.T.; Mittra, E.; Telli, M.L.; Gambhir, S.S. ${ }^{18}$ F-FPPRGD2 PET/CT: Pilot phase evaluation of breast cancer patients. Radiology 2014, 273, 549-559. [CrossRef]

50. Chen, G.; Ouyang, Z.; Wang, F.; Wu, H.; Jia, B.; Chordia, M.D. Evaluation of Tc-99m-3PRGD2 Integrin Receptor Imaging in the Differential Diagnosis of Breast Lesions and Comparison With Mammography. Cancer Investig. 2017, 35, 108-115. [CrossRef]

51. Chen, Z.; Fu, F.; Li, F.; Zhu, Z.; Yang, Y.; Chen, X.; Jia, B.; Zheng, S.; Huang, C.; Miao, W. Comparison of $\left[{ }^{99 \mathrm{~m}} \mathrm{Tc}\right] 3 P R G D 2$ Imaging and $\left[{ }^{18} \mathrm{~F}\right] \mathrm{FDG}$ PET/CT in Breast Cancer and Expression of Integrin $\alpha \mathrm{V} \beta 3$ in Breast Cancer Vascular Endothelial Cells. Mol. Imaging Biol. 2018, 20, 846-856. [CrossRef] 
52. Yoon, H.J.; Kang, K.W.; Chun, I.K.; Cho, N.; Im, S.A.; Jeong, S.; Lee, S.; Jung, K.C.; Lee, Y.S.; Jeong, J.M.; et al. Correlation of breast cancer subtypes, based on estrogen receptor, progesterone receptor, and HER2, with functional imaging parameters from ${ }^{68} \mathrm{Ga}-\mathrm{RGD}$ PET/CT and ${ }^{18} \mathrm{~F}-\mathrm{FDG}$ PET/CT. Eur. J. Nucl. Med. Mol. Imaging 2014, 41, 1534-1543. [CrossRef]

53. Wu, J.; Wang, S.; Zhang, X.; Teng, Z.; Wang, J.; Yung, B.C.; Niu, G.; Zhu, H.; Lu, G.; Chen, X. ${ }^{18}$ F-Alfatide II PET/CT for Identification of Breast Cancer: A Preliminary Clinical Study. J. Nucl. Med. 2018, 59, 1809-1816. [CrossRef]

54. Zhang, J.; Niu, G.; Lang, L.; Li, F.; Fan, X.; Yan, X.; Yao, S.; Yan, W.; Huo, L.; Chen, L.; et al. Clinical Translation of a Dual Integrin $\alpha v \beta 3$ - and Gastrin-Releasing Peptide Receptor-Targeting PET Radiotracer, ${ }^{68} \mathrm{Ga}-\mathrm{BBN}-\mathrm{RGD}$. J. Nucl. Med. 2017, 58, 228-234. [CrossRef]

55. Zhang, J.; Mao, F.; Niu, G.; Peng, L.; Lang, L.; Li, F.; Ying, H.; Wu, H.; Pan, B.; Zhu, Z.; et al. ${ }^{68}$ Ga-BBN-RGD PET/CT for GRPR and Integrin $\alpha v \beta 3$ Imaging in Patients with Breast Cancer. Theranostics 2018, 8, 1121-1130. [CrossRef]

56. Li, L.; Ma, L.; Shang, D.; Liu, Z.; Yu, Q.; Wang, S.; Teng, X.; Zhang, Q.; Hu, X.; Zhao, W.; et al. Pretreatment $\mathrm{PET} / \mathrm{CT}$ imaging of angiogenesis based on ${ }^{18} \mathrm{~F}-\mathrm{RGD}$ tracer uptake may predict antiangiogenic response. Eur. J. Nucl. Med. Mol. Imaging 2019, 46, 940-947. [CrossRef]

57. Hausner, S.H.; Bold, R.; Cheuy, L.Y.; Chew, H.K.; Daly, M.E.; Davis, R.A.; Foster, C.C.; Kim, E.J.; Sutcliffe, J.L. Preclinical Development and First-in-Human Imaging of the Integrin $\alpha v \beta 6$ with $\left[{ }^{18} \mathrm{~F}\right] \alpha v \beta 6$-Binding Peptide in Metastatic Carcinoma. Clin. Cancer Res. 2019, 25, 1206-1215. [CrossRef]

58. Nimmagadda, S.; Pullambhatla, M.; Stone, K.; Green, G.; Bhujwalla, Z.M.; Pomper, M.G. Molecular imaging of CXCR4 receptor expression in human cancer xenografts with $\left[{ }^{64} \mathrm{Cu}\right] \mathrm{AMD} 3100$ positron emission tomography. Cancer Res. 2010, 70, 3935-3944. [CrossRef]

59. Zhao, Y.; Detering, L.; Sultan, D.; Cooper, M.L.; You, M.; Cho, S.; Meier, S.L.; Luehmann, H.; Sun, G.; Rettig, M.; et al. Gold Nanoclusters Doped with (64)Cu for CXCR4 Positron Emission Tomography Imaging of Breast Cancer and Metastasis. ACS Nano 2016, 10, 5959-5970. [CrossRef]

60. Vag, T.; Gerngross, C.; Herhaus, P.; Eiber, M.; Philipp-Abbrederis, K.; Graner, F.P.; Ettl, J.; Keller, U.; Wester, H.J.; Schwaiger, M. First Experience with Chemokine Receptor CXCR4-Targeted PET Imaging of Patients with Solid Cancers. J. Nucl. Med. 2016, 57, 741-746. [CrossRef]

61. Vag, T.; Steiger, K.; Rossmann, A.; Keller, U.; Noske, A.; Herhaus, P.; Ettl, J.; Niemeyer, M.; Wester, H.J.; Schwaiger, M. PET imaging of chemokine receptor CXCR4 in patients with primary and recurrent breast carcinoma. EJNMMI Res. 2018, 8, 90. [CrossRef] [PubMed]

62. Brickute, D.; Braga, M.; Kaliszczak, M.A.; Barnes, C.; Lau, D.; Carroll, L.; Stevens, E.; Trousil, S.; Alam, I.S.; Nguyen, Q.D.; et al. Development and Evaluation of an ${ }^{18} \mathrm{~F}$-Radiolabeled Monocyclam Derivative for Imaging CXCR4 Expression. Mol. Pharm. 2019, 16, 2106-2117. [CrossRef] [PubMed]

63. Azad, B.B.; Lisok, A.; Chatterjee, S.; Poirier, J.T.; Pullambhatla, M.; Luker, G.D.; Pomper, M.G.; Nimmagadda, S. Targeted Imaging of the Atypical Chemokine Receptor 3 (ACKR3/CXCR7) in Human Cancer Xenografts. J. Nucl. Med. 2016, 57, 981-988. [CrossRef] [PubMed]

64. Heskamp, S.; Hobo, W.; Molkenboer-Kuenen, J.D.; Olive, D.; Oyen, W.J.; Dolstra, H.; Boerman, O.C. Noninvasive Imaging of Tumor PD-L1 Expression Using Radiolabeled Anti-PD-L1 Antibodies. Cancer Res. 2015, 75, 2928-2936. [CrossRef]

65. Josefsson, A.; Nedrow, J.R.; Park, S.; Banerjee, S.R.; Rittenbach, A.; Jammes, F.; Tsui, B.; Sgouros, G. Imaging, Biodistribution, and Dosimetry of Radionuclide-Labeled PD-L1 Antibody in an Immunocompetent Mouse Model of Breast Cancer. Cancer Res. 2016, 76, 472-479. [CrossRef]

66. Lesniak, W.G.; Chatterjee, S.; Gabrielson, M.; Lisok, A.; Wharram, B.; Pomper, M.G.; Nimmagadda, S. PD-L1 Detection in Tumors Using [(64)Cu]Atezolizumab with PET. Bioconjug. Chem. 2016, 27, 2103-2110. [CrossRef] [PubMed]

67. Chatterjee, S.; Lesniak, W.G.; Gabrielson, M.; Lisok, A.; Wharram, B.; Sysa-Shah, P.; Azad, B.B.; Pomper, M.G.; Nimmagadda, S. A humanized antibody for imaging immune checkpoint ligand PD-L1 expression in tumors. Oncotarget 2016, 7, 10215-10227. [CrossRef]

68. Heskamp, S.; Wierstra, P.J.; Molkenboer-Kuenen, J.D.M.; Sandker, G.W.; Thordardottir, S.; Cany, J.; Olive, D.; Bussink, J.; Boerman, O.C.; Dolstra, H.; et al. PD-L1 microSPECT/CT Imaging for Longitudinal Monitoring of PD-L1 Expression in Syngeneic and Humanized Mouse Models for Cancer. Cancer Immunol. Res. 2019, 7 150-161. [CrossRef] 
69. Kumar, D.; Lisok, A.; Dahmane, E.; McCoy, M.; Shelake, S.; Chatterjee, S.; Allaj, V.; Sysa-Shah, P.; Wharram, B.; Lesniak, W.G.; et al. Peptide-based PET quantifies target engagement of PD-L1 therapeutics. J. Clin. Investig. 2019, 129, 616-630. [CrossRef]

70. Bensch, F.; van der Veen, E.L.; Lub-de Hooge, M.N.; Jorritsma-Smit, A.; Boellaard, R.; Kok, I.C.; Oosting, S.F.; Schröder, C.P.; Hiltermann, T.J.N.; van der Wekken, A.J.; et al. ${ }^{89} \mathrm{Zr}$-atezolizumab imaging as a non-invasive approach to assess clinical response to PD-L1 blockade in cancer. Nat. Med. 2018, 24, 1852-1858. [CrossRef]

71. Chereau, E.; Durand, L.; Frati, A.; Prignon, A.; Talbot, J.N.; Rouzier, R. Correlation of immunohistopathological expression of somatostatin receptor- 2 in breast cancer and tumor detection with ${ }^{68} \mathrm{Ga}$-DOTATOC and ${ }^{18} \mathrm{~F}-\mathrm{FDG}$ PET imaging in an animal model. Anticancer Res. 2013, 33, 3015-3019. [PubMed]

72. Cescato, R.; Waser, B.; Fani, M.; Reubi, J.C. Evaluation of 177Lu-DOTA-sst2 antagonist versus 177Lu-DOTA-sst2 agonist binding in human cancers in vitro. J. Nucl. Med. 2011, 52, 1886-1890. [CrossRef] [PubMed]

73. Dude, I.; Zhang, Z.; Rousseau, J.; Hundal-Jabal, N.; Colpo, N.; Merkens, H.; Lin, K.S.; Bénard, F. Evaluation of agonist and antagonist radioligands for somatostatin receptor imaging of breast cancer using positron emission tomography. EJNMMI Radiopharm. Chem. 2017, 2, 4. [CrossRef] [PubMed]

74. Maina, T.; Bergsma, H.; Kulkarni, H.R.; Mueller, D.; Charalambidis, D.; Krenning, E.P.; Nock, B.A.; de Jong, M.; Baum, R.P. Preclinical and first clinical experience with the gastrin-releasing peptide receptor-antagonist $\left[{ }^{68} \mathrm{Ga}\right] \mathrm{SB} 3$ and PET/CT. Eur. J. Nucl. Med. Mol. Imaging 2016, 43, 964-973. [CrossRef]

75. Stoykow, C.; Erbes, T.; Maecke, H.R.; Bulla, S.; Bartholomä, M.; Mayer, S.; Drendel, V.; Bronsert, P.; Werner, M.; Gitsch, G.; et al. Gastrin-releasing Peptide Receptor Imaging in Breast Cancer Using the Receptor Antagonist (68)Ga-RM2 And PET. Theranostics 2016, 6, 1641-1650. [CrossRef]

76. Zang, J.; Mao, F.; Wang, H.; Zhang, J.; Liu, Q.; Peng, L.; Li, F.; Lang, L.; Chen, X.; Zhu, Z. ${ }^{68}$ Ga-NOTA-RM26 PET/CT in the Evaluation of Breast Cancer: A Pilot Prospective Study. Clin. Nucl. Med. 2018, 43, 663-669. [CrossRef]

77. Kaloudi, A.; Lymperis, E.; Giarika, A.; Dalm, S.; Orlandi, F.; Barbato, D.; Tedesco, M.; Maina, T.; de Jong, M.; Nock, B.A. NeoBOMB1, a GRPR-Antagonist for Breast Cancer Theragnostics: First Results of a Preclinical Study with $\left[{ }^{67} \mathrm{Ga}\right.$ ]NeoBOMB1 in T-47D Cells and Tumor-Bearing Mice. Molecules 2017, 22, 1950. [CrossRef]

78. Khan, I.U.; Zwanziger, D.; Böhme, I.; Javed, M.; Naseer, H.; Hyder, S.W.; Beck-Sickinger, A.G. Breast-cancer diagnosis by neuropeptide $\mathrm{Y}$ analogues: From synthesis to clinical application. Angew. Chem. Int. Ed. 2010, 49, 1155-1158. [CrossRef]

79. Hofmann, S.; Maschauer, S.; Kuwert, T.; Beck-Sickinger, A.G.; Prante, O. Synthesis and in vitro and in vivo evaluation of an (18)F-labeled neuropeptide $\mathrm{Y}$ analogue for imaging of breast cancer by PET. Mol. Pharm. 2015, 12, 1121-1130. [CrossRef]

80. Kuukasjärvi, T.; Kononen, J.; Helin, H.; Holli, K.; Isola, J. Loss of estrogen receptor in recurrent breast cancer is associated with poor response to endocrine therapy. J. Clin. Oncol. 1996, 14, 2584-2589. [CrossRef]

81. Fuqua, S.A.; Cui, Y.; Lee, A.V.; Osborne, C.K.; Horwitz, K.B. Insights into the role of progesterone receptors in breast cancer. J. Clin. Oncol. 2005, 23, 932-933. [CrossRef] [PubMed]

82. Beauregard, J.M.; Croteau, E.; Ahmed, N.; van Lier, J.E.; Bénard, F. Assessment of human biodistribution and dosimetry of 4-fluoro-11beta-methoxy-16alpha- ${ }^{18}$ F-fluoroestradiol using serial whole-body PET/CT. J. Nucl. Med. 2009, 50, 100-107. [CrossRef] [PubMed]

83. Dehdashti, F.; Laforest, R.; Gao, F.; Aft, R.L.; Dence, C.S.; Zhou, D.; Shoghi, K.I.; Siegel, B.A.; Katzenellenbogen, J.A.; Welch, M.J. Assessment of progesterone receptors in breast carcinoma by PET with $21-{ }^{18}$ F-fluoro-16 $\alpha, 17 \alpha$-[(R)-(1'- $\alpha$-furylmethylidene)dioxy]-19-norpregn-4-ene-3,20-dione. J. Nucl. Med. 2012, 53, 363-370. [CrossRef] [PubMed]

84. Wei, W.; Ni, D.; Ehlerding, E.B.; Luo, Q.Y.; Cai, W. PET Imaging of Receptor Tyrosine Kinases in Cancer. Mol. Cancer Ther. 2018, 17, 1625-1636. [CrossRef]

85. Gala, K.; Chandarlapaty, S. Molecular pathways: HER3 targeted therapy. Clin. Cancer Res. 2014, 20, 1410-1416. [CrossRef]

86. Rapisarda, A.; Melillo, G. Role of the VEGF/VEGFR axis in cancer biology and therapy. Adv. Cancer Res. 2012, 114, 237-267.

87. Li, L.; Liu, S.; Liu, L.; Ma, Z.; Feng, M.; Ye, C.; Zhou, W.; Wang, Y.; Liu, L.; Wang, F.; et al. Impact of phosphorylated insulin-like growth factor-1 receptor on the outcome of breast cancer patients 
and the prognostic value of its alteration during neoadjuvant chemotherapy. Exp. Ther. Med. 2018, 16, 2949-2959. [CrossRef]

88. Camorani, S.; Hill, B.S.; Fontanella, R.; Greco, A.; Gramanzini, M.; Auletta, L.; Gargiulo, S.; Albanese, S.; Lucarelli, E.; Cerchia, L.; et al. Inhibition of Bone Marrow-Derived Mesenchymal Stem Cells Homing Towards Triple-Negative Breast Cancer Microenvironment Using an Anti-PDGFR $\beta$ Aptamer. Theranostics 2017, 7, 3595-3607. [CrossRef]

89. Camorani, S.; Hill, B.S.; Collina, F.; Gargiulo, S.; Napolitano, M.; Cantile, M.; Di Bonito, M.; Botti, G.; Fedele, M.; Zannetti, A.; et al. Targeted imaging and inhibition of triple-negative breast cancer metastases by a PDGFR $\beta$ aptamer. Theranostics 2018, 8, 5178-5199. [CrossRef]

90. Camorani, S.; Fedele, M.; Zannetti, A.; Cerchia, L. TNBC Challenge: Oligonucleotide Aptamers for New Imaging and Therapy Modalities. Pharmaceuticals 2018, 11, 123. [CrossRef]

91. Desgrosellier, J.S.; Cheresh, D.A. Integrins in cancer: Biological implications and therapeutic opportunities. Nat. Rev. Cancer. 2010, 10, 9-22. [CrossRef] [PubMed]

92. Soung, Y.H.; Clifford, J.L.; Chung, J. Crosstalk between integrin and receptor tyrosine kinase signaling in breast carcinoma progression. BMB Rep. 2010, 43, 311-318. [CrossRef] [PubMed]

93. Stewart, R.L.; O'Connor, K.L. Clinical significance of the integrin $\alpha 6 \beta 4$ in human malignancies. Lab. Investig. 2015, 95, 976-986. [CrossRef] [PubMed]

94. Camorani, S.; Crescenzi, E.; Gramanzini, M.; Fedele, M.; Zannetti, A.; Cerchia, L. Aptamer-mediated impairment of EGFR-integrin $\alpha \mathrm{v} \beta 3$ complex inhibits vasculogenic mimicry and growth of triple-negative breast cancers. Sci. Rep. 2017, 7, 46659. [CrossRef] [PubMed]

95. Avraamides, C.J.; Garmy-Susini, B.; Varner, J.A. Integrins in angiogenesis and lymphangiogenesis. Nat. Rev. Cancer 2008, 8, 604-617. [CrossRef] [PubMed]

96. Chen, X. Integrin Targeted Imaging and Therapy. Theranostics 2011, 2011, 28-29. [CrossRef]

97. Chen, H.; Niu, G.; Wu, H.; Chen, X. Clinical Application of Radiolabeled RGD Peptides for PET Imaging of Integrin $\alpha v \beta 3$. Theranostics 2016, 6, 78-92. [CrossRef]

98. Zannetti, A.; Del Vecchio, S.; Iommelli, F.; Del Gatto, A.; De Luca, S.; Zaccaro, L.; Papaccioli, A.; Sommella, J.; Panico, M.; Speranza, A.; et al. Imaging of alpha(v)beta(3) expression by a bifunctional chimeric RGD peptide not cross-reacting with alpha(v)beta(5). Clin. Cancer Res. 2009, 15, 5224-5233. [CrossRef]

99. Bolzati, C.; Salvarese, N.; Carpanese, D.; Seraglia, R.; Meléndez-Alafort, L.; Rosato, A.; Capasso, D.; Saviano, M.; Del Gatto, A.; Comegna, D.; et al. [ $\left.{ }^{99 \mathrm{~m}} \mathrm{Tc}\right][\mathrm{Tc}(\mathrm{N})$ PNP43]-Labeled RGD Peptides As New Probes for a Selective Detection of $\alpha \mathrm{v} \beta 3$ Integrin: Synthesis, Structure-Activity and Pharmacokinetic Studies. J. Med. Chem. 2018, 61, 9596-9610. [CrossRef]

100. Hill, B.S.; Sarnella, A.; Capasso, D.; Comegna, D.; Del Gatto, A.; Gramanzini, M.; Albanese, S.; Saviano, M.; Zaccaro, L.; Zannetti, A. Therapeutic Potential of a Novel $\alpha v \beta_{3}$ Antagonist to Hamper the Aggressiveness of Mesenchymal Triple Negative Breast Cancer Sub-Type. Cancers 2019, 11, 139. [CrossRef]

101. Beer, A.J.; Kessler, H.; Wester, H.J.; Schwaiger, M. PET Imaging of Integrin $\alpha$ V $\beta 3$ Expression. Theranostics 2011, 1, 48-57. [CrossRef] [PubMed]

102. Stone, M.J.; Hayward, J.A.; Huang, C.; Huma, Z.E.; Sanchez, J. Mechanisms of Regulation of the Chemokine-Receptor Network. Int. J. Mol. Sci. 2017, 18, 342. [CrossRef] [PubMed]

103. Palacios-Arreola, M.I.; Nava-Castro, K.E.; Castro, J.I.; García-Zepeda, E.; Carrero, J.C.; Morales-Montor, J. The role of chemokines in breast cancer pathology and its possible use as therapeutic targets. J. Immunol. Res. 2014, 2014, 849720. [CrossRef] [PubMed]

104. King, J.; Mir, H.; Singh, S. Association of Cytokines and Chemokines in Pathogenesis of Breast Cancer. Prog. Mol. Biol. Transl. Sci. 2017, 151, 113-136. [PubMed]

105. Xu, C.; Zhao, H.; Chen, H.; Yao, Q. CXCR4 in breast cancer: Oncogenic role and therapeutic targeting. Drug Des. Devel. Ther. 2015, 9, 4953-4964. [PubMed]

106. Buck, A.K.; Stolzenburg, A.; Hänscheid, H.; Schirbel, A.; Lückerath, K.; Schottelius, M.; Wester, H.J.; Lapa, C. Chemokine receptor-Directed imaging and therapy. Methods 2017, 130, 63-71. [CrossRef]

107. Allinen, M.; Beroukhim, R.; Cai, L.; Brennan, C.; Lahti-Domenici, J.; Huang, H.; Porter, D.; Hu, M.; Chin, L.; Richardson, A.; et al. Molecular characterization of the tumor microenvironment in breast cancer. Cancer Cell 2004, 6, 17-32. [CrossRef]

108. Weiss, I.D.; Jacobson, O. Molecular imaging of chemokine receptor CXCR4. Theranostics 2013, 3, 76-84. [CrossRef] 
109. Santagata, S.; Portella, L.; Napolitano, M.; Greco, A.; D'Alterio, C.; Barone, M.V.; Luciano, A.; Gramanzini, M.; Auletta, L.; Arra, C.; et al. A novel CXCR4-targeted near-infrared (NIR) fluorescent probe (Peptide R-NIR750) specifically detects CXCR4 expressing tumors. Sci. Rep. 2017, 7, 2554. [CrossRef]

110. Donzella, G.A.; Schols, D.; Lin, S.W.; Esté, J.A.; Nagashima, K.A.; Maddon, P.J.; Allaway, G.P.; Sakmar, T.P.; Henson, G.; De Clercq, E.; et al. AMD3100, a small molecule inhibitor of HIV-1 entry via the CXCR4 co-receptor. Nat. Med. 1998, 4, 72-77. [CrossRef]

111. Pernas, S.; Martin, M.; Kaufman, P.A.; Gil-Martin, M.; Gomez Pardo, P.; Lopez-Tarruella, S.; Manso, L.; Ciruelos, E.; Perez-Fidalgo, J.A.; Hernando, C.; et al. Balixafortide plus eribulin in HER2-negative metastatic breast cancer: A phase 1, single-arm, dose-escalation trial. Lancet Oncol. 2018, 19, 812-824. [CrossRef]

112. Rhodes, L.V.; Short, S.P.; Neel, N.F.; Salvo, V.A.; Zhu, Y.; Elliott, S.; Wei, Y.; Yu, D.; Sun, M.; Muir, S.E.; et al. Cytokine receptor CXCR4 mediates estrogen-independent tumorigenesis, metastasis, and resistance to endocrine therapy in human breast cancer. Cancer Res. 2011, 71, 603-613. [CrossRef] [PubMed]

113. Miao, Z.; Luker, K.E.; Summers, B.C.; Berahovich, R.; Bhojani, M.S.; Rehemtulla, A.; Kleer, C.G.; Essner, J.J.; Nasevicius, A.; Luker, G.D.; et al. CXCR7 (RDC1) promotes breast and lung tumor growth in vivo and is expressed on tumor-associated vasculature. Proc. Natl. Acad. Sci. USA 2007, 104, 15735-15740. [CrossRef] [PubMed]

114. Wani, N.; Nasser, M.W.; Ahirwar, D.K.; Zhao, H.; Miao, Z.; Shilo, K.; Ganju, R.K. C-X-C motif chemokine 12/C-X-C chemokine receptor type 7 signaling regulates breast cancer growth and metastasis by modulating the tumor microenvironment. Breast Cancer Res. 2014, 16, R54. [CrossRef] [PubMed]

115. Sun, C.; Mezzadra, R.; Schumacher, T.N. Regulation and Function of the PD-L1 Checkpoint. Immunity 2018, 48, 434-452. [CrossRef]

116. Zak, K.M.; Grudnik, P.; Magiera, K.; Dömling, A.; Dubin, G.; Holak, T.A. Structural Biology of the Immune Checkpoint Receptor PD-1 and Its Ligands PD-L1/PD-L2. Structure 2017, 25, 1163-1174. [CrossRef]

117. Sun, Z.; Fourcade, J.; Pagliano, O.; Chauvin, J.M.; Sander, C.; Kirkwood, J.M.; Zarour, H.M. IL10 and PD-1 Cooperate to Limit the Activity of Tumor-Specific CD8+ T Cells. Cancer Res. 2015, 75, 1635-1644. [CrossRef]

118. Kryczek, I.; Wei, S.; Gong, W.; Shu, X.; Szeliga, W.; Vatan, L.; Chen, L.; Wang, G.; Zou, W. Cutting edge: IFN-gamma enables APC to promote memory Th17 and abate Th1 cell development. J. Immunol. 2008, 181, 5842-5846. [CrossRef]

119. Butte, M.J.; Keir, M.E.; Phamduy, T.B.; Sharpe, A.H.; Freeman, G.J. PD-L1 interacts specifically with B7-1 to inhibit T cell proliferation. Immunity 2007, 27, 111-122. [CrossRef]

120. Sabatier, R.; Finetti, P.; Mamessier, E.; Adelaide, J.; Chaffanet, M.; Ali, H.R.; Viens, P.; Caldas, C.; Birnbaum, D.; Bertucci, F. Prognostic and predictive value of PDL1 expression in breast cancer. Oncotarget 2015, 6, 5449-5464. [CrossRef]

121. Muenst, S.; Soysal, S.D.; Gao, F.; Obermann, E.C.; Oertli, D.; Gillanders, W.E. The presence of programmed death 1 (PD-1)-positive tumor-infiltrating lymphocytes is associated with poor prognosis in human breast cancer. Breast Cancer Res. Treat. 2013, 139, 667-676. [CrossRef] [PubMed]

122. Force, J.; Leal, J.H.S.; McArthur, H.L. Checkpoint Blockade Strategies in the Treatment of Breast Cancer: Where We Are and Where We Are Heading. Curr. Treat. Options Oncol. 2019, 20, 35. [CrossRef] [PubMed]

123. Reubi, J.; Waser, B.; Foekens, J.A.; Klijn, J.G.; Lamberts, S.W.; Laissue, J. Somatostatin receptor incidence and distribution in breast cancer using receptor autoradiography: Relationship to EGF receptors. Int. J. Cancer 1990, 46, 416-420. [CrossRef] [PubMed]

124. Orlando, C.; Raggi, C.C.; Bianchi, S.; Distante, V.; Simi, L.; Vezzosi, V.; Gelmini, S.; Pinzani, P.; Smith, M.C.; Buonamano, A.; et al. Measurement of somatostatin receptor subtype 2 mRNA in breast cancer and corresponding normal tissue. Endocr.-Relat. Cancer 2004, 11, 323-332. [CrossRef] [PubMed]

125. Kumar, U.; Grigorakis, S.I.; Watt, H.L.; Sasi, R.; Snell, L.; Watson, P.; Chaudhari, S. Somatostatin receptors in primary human breast cancer: Quantitative analysis of mRNA for subtypes 1-5 and correlation with receptor protein expression and tumor pathology. Breast Cancer Res. Treat. 2005, 92, 175-186. [CrossRef]

126. Van Den Bossche, B.; D’haeninck, E.; De Vos, F.; Dierckx, R.A.; Van Belle, S.; Bracke, M.; Van de Wiele, C. Estrogen-mediated regulation of somatostatin receptor expression in human breast cancer cell lines assessed with 99mTc-depreotide. Eur. J. Nucl. Med. Mol. Imaging 2004, 31, 1022-1030. [CrossRef]

127. Dalm, S.U.; Martens, J.W.; Sieuwerts, A.M.; van Deurzen, C.H.; Koelewijn, S.J.; de Blois, E.; Maina, T.; Nock, B.A.; Brunel, L.; Fehrentz, J.A.; et al. In vitro and in vivo application of radiolabeled gastrin-releasing peptide receptor ligands in breast cancer. J. Nucl. Med. 2015, 56, 752-757. [CrossRef] 
128. Fani, M.; Nicolas, G.P.; Wild, D. Somatostatin Receptor Antagonists for Imaging and Therapy. J. Nucl. Med. 2017, 58, 61S-66S. [CrossRef]

129. Sollini, M.; Erba, P.A.; Fraternali, A.; Casali, M.; Di Paolo, M.L.; Froio, A.; Frasoldati, A.; Versari, A. PET and PET/CT with 68gallium-labeled somatostatin analogues in Non GEP-NETs Tumors. Sci. World J. 2014, 194123.

130. Morgat, C.; MacGrogan, G.; Brouste, V.; Vélasco, V.; Sévenet, N.; Bonnefoi, H.; Fernandez, P.; Debled, M.; Hindié, E. Expression of Gastrin-Releasing Peptide Receptor in Breast Cancer and Its Association with Pathologic, Biologic, and Clinical Parameters: A Study of 1432 Primary Tumors. J. Nucl. Med. 2017, 58, 1401-1407. [CrossRef]

131. Morgat, C.; Schollhammer, R.; Macgrogan, G.; Barthe, N.; Vélasco, V.; Vimont, D.; Cazeau, A.L.; Fernandez, P.; Hindié, E. Comparison of the binding of the gastrin-releasing peptide receptor (GRP-R) antagonist ${ }^{68} \mathrm{Ga}-\mathrm{RM} 2$ and ${ }^{18}$ F-FDG in breast cancer samples. PLoS ONE 2019, 14, e0210905. [CrossRef] [PubMed]

132. Nock, B.A.; Kaloudi, A.; Lymperis, E.; Giarika, A.; Kulkarni, H.R.; Klette, I.; Singh, A.; Krenning, E.P.; de Jong, M.; Maina, T.; et al. Theranostic perspectives in prostate cancer with the gastrin-releasing peptide receptor antagonist NeoBOMB1: Preclinical and first clinical results. J. Nucl. Med. 2017, 58, 75-80. [CrossRef] [PubMed]

133. Dalm, S.U.; Bakker, I.L.; de Blois, E.; Doeswijk, G.N.; Konijnenberg, M.W.; Orlandi, F.; Barbato, D.; Tedesco, M.; Maina, T.; Nock, B.A.; et al. ${ }^{68} \mathrm{Ga} / 177 \mathrm{Lu}-\mathrm{NeoBOMB1}$, a novel radiolabeled GRPR antagonist for theranostic use in oncology. J. Nucl. Med. 2017, 58, 293-299. [CrossRef] [PubMed]

134. Reubi, J.C.; Gugger, M.; Waser, B.; Schaer, J.C. Y(1)-mediated effect of neuropeptide Y in cancer: Breast carcinomas as targets. Cancer Res. 2001, 61, 4636-4641.

135. Körner, M.; Reubi, J.C. NPY receptors in human cancer: A review of current knowledge. Peptides 2007, 28, 419-425. [CrossRef]

136. Amlal, H.; Faroqui, S.; Balasubramaniam, A.; Sheriff, S. Estrogen up-regulates neuropeptide Y Y1 receptor expression in a human breast cancer cell line. Cancer Res. 2006, 66, 3706-3714. [CrossRef]

137. Memminger, M.; Keller, M.; Lopuch, M.; Pop, N.; Bernhardt, G.; von Angerer, E.; Buschauer, A. The neuropeptide y y(1) receptor: A diagnostic marker? Expression in mcf-7 breast cancer cells is down-regulated by antiestrogens in vitro and in xenografts. PLoS ONE 2012, 12, e51032. [CrossRef]

138. Farolfi, A.; Lima, G.M.; Oyen, W.; Fanti, S. Molecular Imaging and Theranostics-A Multidisciplinary Approach. Semin. Nucl. Med. 2019, 49, 247-254. [CrossRef]

139. Del Vecchio, S.; Zannetti, A.; Fonti, R.; Pace, L.; Salvatore, M. Nuclear imaging in cancer theranostics. Q. J. Nucl. Med. Mol. Imaging 2007, 51, 152-163.

(C) 2019 by the authors. Licensee MDPI, Basel, Switzerland. This article is an open access article distributed under the terms and conditions of the Creative Commons Attribution (CC BY) license (http://creativecommons.org/licenses/by/4.0/). 


\title{
Theragnostic Use of Radiolabelled Dota-Peptides in Meningioma: From Clinical Demand to Future Applications
}

\author{
Riccardo Laudicella ${ }^{1,+}$, Domenico Albano ${ }^{2,+}$, Salvatore Annunziata ${ }^{3,+}$, Diletta Calabrò ${ }^{4,+}$,
} Giovanni Argiroffi ${ }^{5,+}$, Elisabetta Abenavoli ${ }^{6,+}$, Flavia Linguanti ${ }^{6,+}$, Domenico Albano ${ }^{7,8, \ddagger}$,

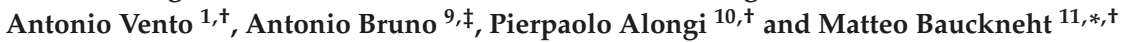

1 Department of Biomedical and Dental Sciences and of Morpho-Functional Imaging, Nuclear Medicine Unit, University of Messina, 98125 Messina, Italy

2 Department of Nuclear Medicine, University of Brescia and Spedali Civili Brescia, 25123 Brescia, Italy

3 Institute of Nuclear Medicine, Università Cattolica del Sacro Cuore, 00168 Roma, Italy

4 Nuclear Medicine, DIMES University of Bologna, S. Orsola-Malpighi Hospital, 40138 Bologna, Italy

5 Department of Health Sciences, University of Milan, 20142 Milan, Italy

6 Nuclear Medicine Unit, Department of Experimental and Clinical Biomedical Sciences "Mario Serio", University of Florence, 50134 Florence, Italy

7 IRCCS Istituto Ortopedico Galeazzi, Unità di Radiologia Diagnostica ed Interventistica, 20161 Milano, Italy

8 Sezione di Scienze Radiologiche, Dipartimento di Biomedicina, Neuroscienze e Diagnostica Avanzata, Università degli Studi di Palermo, 90127 Palermo, Italy

9 Department of Experimental, Diagnostic and Specialty Medicine-DIMES, University of Bologna, S

Orsola-Malpighi Hospital, 40138 Bologna, Italy

10 Unit of Nuclear Medicine, Fondazione Istituto G. Giglio, 90015 Cefalù, Italy

11 Nuclear Medicine Unit, IRCCS Ospedale Policlinico San Martino, 16132 Genoa, Italy

* Correspondence: matteo.bauckneht@hsanmartino.it; Tel.: +39-0105553038

+ These authors are on the behalf of Young AIMN Working Group.

$\ddagger$ These authors are on the behalf of Young SIRM Working Group.

Received: 3 September 2019; Accepted: 19 September 2019; Published: 22 September 2019

\begin{abstract}
Meningiomas account for approximately $30 \%$ of all new diagnoses of intracranial masses. The 2016 World Health Organization's (WHO) classification currently represents the clinical standard for meningioma's grading and prognostic stratification. However, watchful waiting is frequently the chosen treatment option, although this means the absence of a certain histological diagnosis. Consequently, MRI (or less frequently CT) brain imaging currently represents the unique available tool to define diagnosis, grading, and treatment planning in many cases. Nonetheless, these neuroimaging modalities show some limitations, particularly in the evaluation of skull base lesions. The emerging evidence supporting the use of radiolabelled somatostatin receptor analogues (such as dota-peptides) to provide molecular imaging of meningiomas might at least partially overcome these limitations. Moreover, their potential therapeutic usage might enrich the current clinical offering for these patients. Starting from the strengths and weaknesses of structural and functional neuroimaging in meningiomas, in the present article we systematically reviewed the published studies regarding the use of radiolabelled dota-peptides in surgery and radiotherapy planning, in the restaging of treated patients, as well as in peptide-receptor radionuclide therapy of meningioma.
\end{abstract}

Keywords: meningioma; somatostatin receptor; neuroimaging; positron emission tomography; radionuclide therapy 


\section{Introduction}

Meningioma is the most common primary brain tumour, accounting for $36 \%$ of all intracranial tumours [1]. Its clinical detection is usually related to the occurrence of symptoms when it reaches a large size [2]. However, with the increasing availability of neuroimaging, incidentally discovered meningiomas are becoming more frequent [3]. The preferred clinical management is heavily based on clinical profile and histopathology. In particular, according to the World Health Organization (WHO) histopathological grading, meningiomas can be classified as benign (WHO I), showing slow growth and a low recurrence rate, atypical (WHO II), or malignant (WHO III), showing a more aggressive clinical behaviour [4-6]. The most common treatment options are represented by neurosurgery and, in cases of contraindication, various radiotherapy options, including the emerging heavy particle radiotherapy [7]. However, it has been reported that after gross total resection, $12-19 \%$ of all tumours, including WHO grade I, recur within the first two decades [8,9]. Similarly, a tumour control rate varying from $85 \%$ to $100 \%$ at 5 years has been reported after radiation therapy as a first-line treatment in patients not eligible for surgery, regardless of WHO grading $[10,11]$. This situation is presumably related to the existence of unfavourable biological variables which, in turn, potentially instigate tumour recurrence, regardless of the histopathological grading. A representative example is represented by radiation-induced meningiomas, in which a higher frequency of malignant features at histopathology or multifocality, as well as higher recurrent-rates, have been reported concerning spontaneous meningioma [12,13]. On the other hand, in many cases, clinical observation with serial surveillance neuroimaging remains the preferred choice, particularly in asymptomatic slow-growing meningioma [3]. However, there is no class I or II evidence to support this convention. Moreover, delaying therapeutic intervention implies renouncing the obtaining of a definitive histopathological diagnosis, at least in the early phases of the clinical assessment, making neuroimaging the only source of information able to impact on clinical decision making.

For the above-mentioned reasons, in the last years, several molecular, genetic, and neuroimaging biomarkers have been developed, completing the histopathological classification and giving new insights into prognosis and treatment options [14]. Among them, the somatostatin receptor (SSTR) type 2 expression on meningioma cellular surface has recently emerged, with the consequent clinical implication related to the potential use of radiolabelled somatostatin receptor analogues (such as dota-peptides) for both diagnostic and therapeutic purposes. However, SSTR-targeted radionuclide imaging and therapy are, to date, still far from being included in the standard of care of meningioma, as the European Association of Neuro-Oncology (EANO) guidelines have included these approaches only in the "future directions" of meningioma's clinical practice [15]. In particular, the additive value of SSTR functional imaging with morphological neuroimaging technologies and the exact location of SSTR-targeted radionuclide therapy in the therapeutic flow-chart of meningiomas are still not fully defined. Starting from the strengths and weaknesses of morphological and functional neuroimaging in meningiomas, in the present article we systematically reviewed the published evidence regarding the use of radiolabelled dota-peptides in surgery and radiotherapy planning, in the restaging of treated patients, as well as in peptide-receptor radionuclide therapy.

\section{Methods}

We searched the PubMed, PMC, Scopus, Google Scholar, Embase, Web of Science and Cochrane library databases (up to June 2019), using the following as both text and as MeSH terms: "dota-peptides", "somatostatin receptor", "CT", "MRI", "Positron emission tomography" (PET), "PET", "PET/CT", "PET/MRI", and "meningioma". No language restriction was applied to the search, but only articles in English were reviewed. A further literature search focused on the application of radiolabelled dota-peptides for therapeutic purposes was also performed through the same databases using the

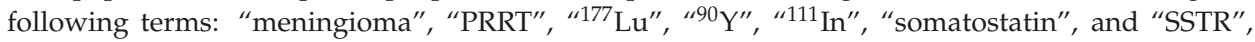
singularly and in cross-reference. Due to the large existing literature about CT and MRI in the diagnosis of meningioma, these articles were not systematically reviewed. Therefore, the corresponding section 
represents a narrative description of the radiological background for PET imaging in this field. Similarly, a narrative description of PET tracers, other than SSTR-targeted, was included. The systematic literature search returned 354 articles for PET imaging and 270 articles for Peptide Receptor Radionuclide Therapy (PRRT). According to PRISMA flow-chart, after duplicate removal, 24 and 27 articles for PET imaging and PRRT have been considered, respectively. These articles were fully read and analysed according to the title and abstract, as previously described [16]: this approach led to the exclusion of 573 articles for reasons reported in Supplementary Figures S1 and S2. Obtained articles were enough to be discussed separately by the clinical indication.

\section{Diagnostic Challenges for MRI Imaging in Meningioma}

MRI is the modality of choice to image meningiomas, due to its striking contrast resolution and multiparametric characteristics. In addition to MRI, CT has a well-established role in specific cases where calcification and bone changes adjacent to the lesion are present. Several MRI features of meningioma can be helpful to reach the diagnosis, although atypical imaging findings and pitfalls related to artifacts may lead to a wrong interpretation of images, with the diagnosis becoming challenging in some settings. Furthermore, it should be considered that several neoplastic and non-neoplastic conditions may resemble meningioma.

Meningiomas typically appear as extra-axial round or lobulated lesions with well-defined margins and broad dural base. These lesions can be observed along any of the outer surfaces of the brain and within the ventricular system, where they arise from the choroid plexus. These lesions are more frequently located in the parasagittal aspect of brain convexity, the convexity of the lateral hemisphere, the sphenoid wing, the middle cranial fossa, and the olfactory sulcus [17]. Meningiomas generally show homogeneous signal intensity, although many variants with different appearances can be encountered. The typical MRI signal consists of iso-hypointensity relative to grey matter on T1-weighted images and variable intensity on T2-weighted images (ranging from iso to hyperintense). However, they can also appear hypointense on T2-weighted images due to the fibro-collagenous matrix or calcific deposits [17]. In MRI, calcifications are best identified on susceptibility-weighted images as areas of low signal intensity. Nevertheless, calcifications can also be detected on T2-weighted and gradient-echo sequences as intralesional hypointense foci.

Meningioma may occasionally present an aggressive pattern with infiltrating growth over the dura ("en plaque" meningioma), which most commonly occurs along the sphenoid ridge or the convexity. After injection of gadolinium-based contrast agent, MRI images demonstrate strong and homogeneous enhancement of the lesion, which occasionally shows areas of central necrosis or calcifications without post-contrast enhancement. The contrast is especially helpful to delineate "en plaque" meningiomas [18], which generally appear as thickened asymmetric sheets of enhancing dura. Among the MRI findings of meningiomas that lead to the suspect of an extra-axial mass, the "dural tail" sign and the "fluid cleft" are the most relevant. The former results from a thickening of the dura mater, resembling a tail and extending from the mass with homogenous enhancement after contrast media injection [19]. This sign can be helpful, but it is not specific for meningiomas, being also observed in different lesions, such as metastatic lesions, glial tumours, and lymphoma $[19,20]$. In several cases, especially when meningiomas are located at the skull base, it can be challenging to differentiate the normal aspect of the dura from neoplastic tissue, due to the strong contrast enhancement of both [21]. The latter imaging feature derives from a cleft of cerebrospinal fluid or vessels observed between the tumour and the cerebral cortex. Further, meningiomas are often associated with adjacent bone changes, including osteolysis and hyperostosis [19] with the latter being more frequently observed, especially in the "en plaque form". In this setting, CT can be more helpful to better depict bone changes related to meningiomas [22]. Nevertheless, it can be challenging to clearly define margins and depth of bone infiltration, despite using CT. Pieper et al. reported that MRI is not sufficiently accurate to demonstrate bone involvement [23]. On the other hand, despite the benign nature of the WHO grade I meningiomas, tumour cells can microscopically infiltrate the adjacent structures, such as vessels and 
cranial nerves [24,25]. Similarly, primary intraosseous meningioma is a rare benign tumour, mostly arising from the skull bones, but also reported in the nasopharynx or neck [26]. This lesion can be confused with metastasis or other malignant bone tumours, also because its MRI features are relatively non-specific. The lesion appears hypo-isointense on T1-weighted images with strong enhancement in post-contrast images [27].

Besides bone involvement, MRI has some limitations in clearly defining the margins of meningiomas, particularly in those lesions with complex geometry and those located in the skull base [15]. In fact, the MRI evaluation of meningiomas can be hampered by artifacts, which are especially encountered in the base of the skull and can partly hide the lesion due to image distortion. Local magnetic inhomogeneities, called "susceptibility gradients", are more frequently observed in the skull base, where air, bones, and brain are closely associated. These susceptibility artifacts, observed at soft tissue and bone (petrous temporal bones) and air (paranasal sinuses) interfaces, are generally flame-shaped and determine regional image distortion with increased signal intensity [28]. Susceptibility artifacts can also be related to metal bodies and other foreign objects, which can be located "on" and "in" patients' bodies. These objects can mimic or, conversely, hide pathology, variably reducing images quality. The main problems arise when dealing with those foreign bodies that cannot be removed, especially when located precisely in the site of the lesion (e.g. surgical clips or staplers, endoprosthesis). Other artifacts responsible for a dramatic decrease of image quality can be related to orthodontic appliances and ventricular shunt valves [29].

Another potential source of MRI pitfall is reported as "anatomical blind spots": normal brain regions that are challenging sites to be evaluated with MRI, and where pathologic disorders may be barely detected in imaging examinations [30]. These blind spots include cerebral sulci, dural sinuses, orbits, cavernous sinuses, clivus, Meckel's cave, brainstem, skull base, and parapharyngeal soft tissues. In these anatomic districts, it is possible to observe densely compact anatomy with the juxtaposition of neural, vascular, bone, and soft tissue structures. In this setting, the detection of small meningiomas can be highly difficult, especially when dealing with small lesions without clear mass effects on the surrounding structures. Clearly, infiltrative lesions, such as small en plaque meningiomas, may also become a diagnostic challenge in these sites.

Finally, another challenging aspect is represented by the post-treatment evaluation, with MRI still having some limitations in the differentiation between residual tumour and recurrent tissue after surgery, as well as between residual tumour and vital tumour from scar tissue after radiotherapy. Accordingly, in the first six months after surgical resection of meningioma, MRI has a decreased accuracy in identifying residual tumour tissue [31].

\section{Diagnostic Challenges for PET Imaging in Meningioma}

Functional studies using FDG-PET/CT showed that meningiomas generally have a low FDG metabolic rate [32]. However, the potential application of FDG-PET imaging to describe meningioma aggressivity, leading to a non-invasive "metabolic grading" has been proposed. In particular, Di Chiro et al. [33] previously reported that FDG accumulation is correlated with the degree of malignancy in meningioma, highly reproducing Thallium-201 accumulation pattern, as depicted by SPECT imaging [34]. Furthermore, Cremerius et al. demonstrated high sensitivity (89\%) and specificity (88\%) of FDG-PET in detecting high-grade meningiomas [35]. Similarly, Kado et al. previously showed that FDG uptake was high in a case of highly aggressive radiation-induced meningioma [36]. On the clinical ground, FDG positivity in meningioma might become a predictive factor of tumour recurrence at the single-patient level [37]. However, other studies have showed opposite results. Iuchi et al. reported that meningioma FDG uptake was not significantly correlated with Ki-67 index or clinical malignancy [38]. Also, Park et al., who showed a correlation between FDG accumulation and tumour proliferative activity, did not find any capability of FDG-PET to predict tumour grade [39]. For these reasons, further studies are needed to verify the potential clinical application of this tool in the clinical setting. 
Because of its low physiological uptake by normal brain, amino acid imaging modalities, such as 11C-methionine (MET) and 18F-fluoroethyl-L-tyrosine (FET), are used for the detection of brain tumours enabling a high tumour/non-tumour contrast. However, the clinical role of these PET tracers in patients with meningioma is still not clear and warrants further investigation. Compared to FDG, MET showed higher detection rates of meningioma both in qualitative and semiquantitative images analysis [40,41]. Its diagnostic applications might be extended to tumour volume delineation for radiotherapy planning [42], as well as to the monitoring of treatment efficacy [43,44]. However, few studies are currently available on the topic. Moreover, while some studies demonstrated a fair correlation between MET uptake and meningioma's proliferative activity (as assessed by the Ki-67 index [38]), this finding was not subsequently confirmed [45]. Similarly, opposite results were obtained about the correlation between MET uptake and high WHO grade [43,45]. For these reasons, the pathophysiological meaning of MET accumulation in meningiomas, together with its clinical value, still needs to be clarified. Similarly, few studies are currently available on the role of FET-PET in meningioma, including in some cases direct comparisons with SSTR-targeted PET imaging, which shows a more favourable target-to-background ratio offered by the latter approach [46]. However, interesting preliminary data have been published about its potential application in tumour grading [47]. Moreover, differently from MET and SSTR ligands, FET does not accumulate in the pituitary gland, making this tracer potentially superior in detecting intrasellar invasion of meningioma $[48,49]$.

Few other tracers have been tested in meningioma, including 11C/18F-choline [50], 18F-Fluoride [49,51], and 11C-Acetate [52]. Again, the small available literature highly prevents their current application in the clinical setting.

\section{SSTR-PET in the Definition of Tumour Growth and Tumour Extent for Surgery Planning}

A crucial issue in meningioma management is the detection of tumour growth and extent, which are potentially related to the risk of recurrence and disease progression.

The assessment of meningioma's growth rate is critical to select the appropriate time point for therapy initiation and for the treatment choice. In the clinical practice, the temporal evolution of meningioma, assessed by serial MRI scans, together with the patient's clinical profile usually guide the surgical approach [15]. Indeed, therapy for patients with meningioma needs to be individualised because of the nature of meningiomas and the potential consequences of different treatments for different patients may vary greatly. However, in some cases, the combination of MRI data and clinic is not enough to define the appropriate treatment strategy. For instance, the growth rates and dynamics among the group of benign meningiomas have been shown to display a huge variability $[53,54]$ and long-term analyses revealed changing growth dynamics in benign meningioma (WHO grade I) with periods of exponential, linear, or no growth, whereas most atypical meningiomas display an exponential growth pattern [55-57]. In this scenario, the sole longitudinal observation by successive MRI studies might potentially delay the identification of exponential growth of benign meningioma postponing the timing of surgical resection. As a consequence, several studies have tried to identify novel molecular or imaging biomarkers able to potentially define the tumour growth pattern at baseline, since the time of meningioma identification. Oya et al. [58] identified that the male sex, baseline tumour diameter higher than $25 \mathrm{~mm}$, oedema on MRI, absence of calcifications, presence of symptoms, and T2 signal hyperintensity at MRI, were associated with increased tumour growth in a cohort of 273 intracranial meningiomas. The possible role of SSTR-PET in this field was investigated only in a few papers with promising results but without shared consensus. Sommerauer et al. [59] studied 23 patients with 64 meningioma who underwent 68Ga-DOTATATE PET/CT showing that tracer uptake (measured as the maximum standardized uptake value, SUVmax) is significantly correlated with tumour growth rate in WHO grades I and II meningioma, whereas no association was observed in WHO grade III lesions (perhaps due to the dedifferentiation phenomenon of aggressive meningioma cells that lost the expression of somatostatin receptors, Figure 1). Using multivariate analysis, SUVmax was the strongest predictor of the subsequent tumour growth even including baseline tumour diameter. 
These results demonstrate that SSTR2 expression predicts tumour growth in well-differentiated meningioma, suggesting the potential role for SSTR-PET in planning the better time point for surgical resection. This could be clinically relevant in meningioma diagnosed in critical sites in which SSTR positivity might guide the surgical resection, even in small, asymptomatic, well-differentiated lesions.

Transosseus meningioma (WHO I/II)
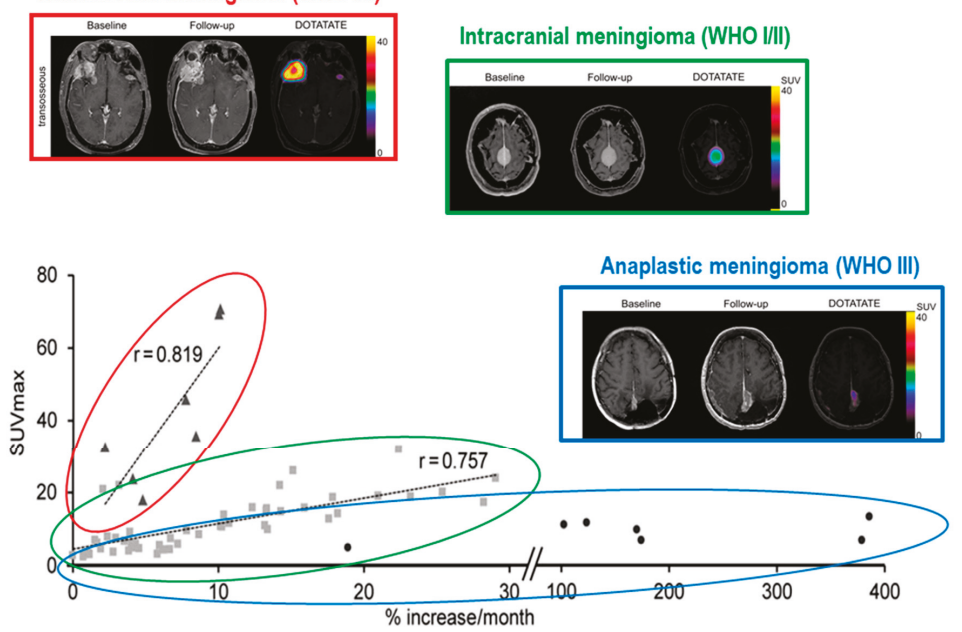

Figure 1. Correlation between SSTR-PET positivity and growth rate in meningioma. The tumour growth rate is directly correlated with SUVmax in grade I and II intracranial meningiomas (green), while this correlation is weak for grade III lesions (blue). As a consequence, high expression of SSTR2 seems to predict faster growth only in well-differentiated meningiomas. Moreover, meningiomas with transosseous growth (red) elicited considerably higher 68Ga-DOTATATE binding. Adapted with permission from Sommerauer et al. [59].

On the other hand, in the same study, higher SSTR positivity was found in transosseousvs. intracranial-growing lesions [59]. This finding configures the possible role for SSTR-PET as a further guide to surgical resection, by defining the exact tumour extent. Similarly, Kunz et al. [60] studied the osseous involvement with pathology-proven bone infiltration as the standard of reference, with 68Ga-DOTATATE PET/CT and contrast enhanced MRI both qualitatively and quantitatively. Using PET quantitative analysis, the authors confirmed the adequate distribution of the tracer in intraosseous components of meningiomas, with significantly higher tumour-to-background signals than MRI. Moreover, PET/CT provided a significantly larger volume estimate for the intraosseous extent, while the estimated volumes for extraosseous meningiomas did not differ significantly between the two imaging techniques. But, the high sensitivity of SSTR-PET imaging in the definition of tumour extent is not restricted to the bone invasion. Afshar-Oromieh et al. [61], studied 134 patients with 190 meningioma undergoing both 68Ga-DOTATOC PET/CT and MRI, showing that SSTR-PET provides better sensitivity in the overall detection of meningioma, especially in case of tumours adjacent to the falx cerebri, at the skull base, or in presence of calcifications, which reduce MRI accuracy, as stated above. Rachinger et al. [62] found that the threshold for best discrimination between tumour and tumour-free tissue as an SUVmax equal to 2.3 by spatially precise neuro-navigated tissue-sampling during surgical resection in 21 adult patients with primary or recurrent meningiomas, prospectively enrolled. Using this cut off, tumour tissue was identified with higher sensitivity than MRI (90\% vs. $79 \%$ ), without risking overtreatment, an aspect desirable in an imaging modality used for treatment decision making. Altogether, these data strongly support the use of SSTR-PET imaging for tumour delineation in meningiomas with suspected osseous infiltration, as well as in regions such as the skull 
base, orbits, or cavernous sinuses, where biopsy has a high haemorrhagic risk, and radiological images are often unclear. However, a limitation related to the use of PET SSTR-ligands may be the study of parasellar region, because the pituitary gland is the intracranial site showing higher physiologic SSTR2 expression. Although Henze et al. [63] showed a divergent dynamic uptake between meningiomas and pituitary gland after injection of 68Ga-DOTATOC, SSTR-PET accuracy might be reduced in this site.

\section{SSTR Radio-Guided Surgery}

Radio-guided surgery (RGS) offers several theoretical advantages in meningiomas, providing a more accurate detection of tumour lesions and of the total tumour burden "marked" by the radiopharmaceutical. Moreover, since radical resection is crucial for prognosis, RGS might allow confirmation of margins negativity. This is potentially relevant in the case of meningiomas "en plaque" and cranial base meningiomas that often involve cranial base bone [64]. However, up to now, few studies have evaluated the potential clinical benefit of RGS in meningiomas.

Gay et al. [65] showed that SSTR-targeted RGS is feasible and could increase the probability of complete meningioma resection in a cohort of 18 patients with "en plaque" meningiomas who underwent pre- and post-operative SSTRs imaging. Gamma probe intraoperative detection was performed $24 \mathrm{~h}$ after the intravenous administration of 111In-DTPA octreotide. In a subgroup of patients, detection of dural or periorbital involvement of "en plaque" sphenoid wing meningiomas was more difficult due to technical reasons, mainly related to the size of the device. Eight patients underwent postoperative scintigraphy, which resulted in a negative, confirming the radicality of the surgical resection. However, in two cases a slight detection three months after the surgical removal was observed, potentially related to post-surgical inflammatory tissue [65].

$\beta$ - emitting RGS represents a potential further development: $\beta$-radiation millimetric tissue penetration, with essentially no $\gamma$ contamination, better delineates the margins of the lesion, requiring lower activity than traditional $\gamma$-RGS and so rendering medical personnel radiation exposure negligible [66]. Collamati et al. used a dedicated $\beta$ - probe and demonstrated a high target-to-background (TBR) in 10 of 11 patients with meningioma using RGS with $\beta$ - emitters (TBR > 10 in almost all cases, and usually above 20). These results suggest that the technique can work even with administered activities much smaller than those needed for diagnostic PET scans [67].

Although promising, RGS is not routinely used and further studies are needed to better understand its clinical impact and feasibility of beta-probes employment.

\section{SSTR-PET in Radiotherapy Planning}

In the multimodal approach to meningioma, radiotherapy (RT) is a central treatment alternative to surgery [15]. Since surgery can result intricate due to the complex anatomy of the skull-base region, RT on the surgery bed, residual lesions or not resectable lesions has been associated with good local control and low toxicity. Recently, more and more high-precision RT techniques have been developed, like fractionated stereotactic radiotherapy (FSRT), intensity-modulated radiotherapy (IMRT), stereotactic radiosurgery (SRS), and particle therapy with protons or carbon ions. Current standard imaging for meningioma RT planning is contrast-enhanced MRI co-registered with CT. Post-contrast T1-weighted MRI is usually applied to delineate meningioma lesions and soft tissue, associated with CT scan for better bone assessment. However, the more precise RT techniques have become, the more precise tumour delineation must be. Defining gross tumour volume (GTV) can be challenging in those cases of postoperative settings, equivocal bone thickening, or enhancing dura tails. Thanks to the high tumour-to-background contrast over the past ten years, several studies have reported about integrating SSTR-PET in RT-planning, resulting in additional information on tumour extension, reduction on dose absorbed to organs-at-risk (OAR), and modification in final volume treated.

The first experience was provided by Milker-Zabel and colleagues in a study conducted on a population of 26 patients with WHO grade I to III, most of who had a recurring lesion or received 
previous radiotherapy treatment. Applying $68 \mathrm{Ga}$-DOTATOC-PET/CT to the planning target volume (PTV) for FSRT caused significant modifications in $73 \%$ of patients, leading to a larger final PTV in 10 out of 26 patients and a smaller one in 9 out of 26 patients [68]. These data were subsequently confirmed by Gehler et al. in a retrospective analysis on 26 patients selected for skull base meningioma IMRT planning. PET imaging provided additional information in 17 patients (65\%). Overall GTV based on MRI/CT was larger than GTV-PET in 10 patients and smaller in 13 patients. Since a validated cut-off value for the SUV of DOTATOC was missing, PET window levels were adjusted so that the PET tumour delineation optimally matched with a discernible tumour on MRI/CT [69]. The same approach was adopted by Nyuyki et al. on a definitive population of 39 meningioma patients, where SSTR-PET led to a modification in 28 out of 39 cases (GTV-PET larger than GTV-MRI/CT in 49\%, smaller in 23\%) [70]. Graf et al. retrospectively evaluated the impact of DOTATOC PET in 48 skull base meningioma patients, integrating PET information in FSRT planning, and showing that PET resulted in major changes in 32 cases (67\%). Again, final GTV was smaller than conventional GTV-MRI-CT in 40 meningiomas (83\%) and larger in 6 [71].

About particle RT, Combs et al. prospectively planned with MRI and DOTATOC PET, proton and carbon ion therapy of 70 patients with meningioma of heterogeneous pathological grading. The addition of PET provided valuable information in all patients and a reduction in GTV final volume in $40 \%$ of cases. These volumes in MRI were extending into soft tissue, like pterygoid muscles, pharyngeal structures. By contrast, PET volume contouring in smaller lesions of the skull base or located within the cavernous sinus or spheno-orbital bony region led to an increase in GTV [72]. Madani et al. investigated the feasibility of dose painting in IMPT based on 68Ga-DOTATATE-PET/CT in 5 patients treated with proton beam-therapy. In this experiment, GTV-PET and GTV-MRI/CT were determined by auto-segmentation with a 50\% threshold SUVmax on PET scans and traditional manual contouring on MRI/CT respectively, resulting in a quite small PTV-PET [73].

Stade et al. [74] focused their study on the adsorbed dose to OAR. A retrospective analysis was performed on 10 patients randomly selected from an institutional database. Both treatment plan for IMRT and proton therapy were evaluated on an MRI/CT and 68Ga-DOTATOC PET basis. Doses to relevant OAR were calculated (brain stem, optic chiasm, left and right optic nerve). Implementing PET in planning definition achieved a relevant decrease of the extension of target volumes compared to MRI/CT volume definition. More importantly, OAR doses were significantly reduced by PET, especially in the IMRT setting [74] (Figure 2).

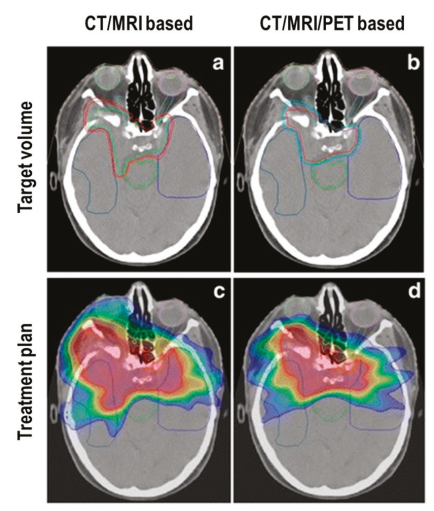

Figure 2. Reduction of IMRT target volume provided by adding 68Ga-DOTATOC-PET to CT and MRI. Panel (a) and (b) display the target volume based on CT and MRI and CT, MRI as well as 68Ga-DOTATOC-PET, respectively in a patient treated for a skull base meningioma. Panel (c) and (d) show the corresponding IMRT treatment plans. The present case shows the reduction of dose to OAR by adding SSTR-PET imaging to treatment planning. Adapted with permission from Stade et al. [74]. 
Altogether, the above-mentioned experiences suggest that DOTATOC-PET scans have high meningioma-to-background contrast, and that functional imaging provides a better delineation between an area of normal tissue and meningioma residual, bone infiltration, or dural tail leading to additional information or modification on RT-planning, especially for skull base meningiomas. However, a limitation when comparing results of those publications is caused by interobserver variability due to different methods applied on tumour contouring and windowing. Since a validated SUV cut-off value was missing, PET window levels were adjusted so that the PET tumour delineation optimal matched with a discernible tumour on MRI/CT by most of the authors, whereas others decided to apply an arbitrary percentage threshold to SUVmax. Based on these discrepancies, Maclean et al. tested the interobserver variability in a pilot study conducted on 10 patients who underwent simultaneous 68Ga-DOTATATE PET/MRI followed by PET/CT. They were selected as it was anticipated that target volume definition in their cases would be particularly challenging. Three radiotherapists applied an agreed protocol to contour target volumes. Statistical evaluation resulted in significant interobserver variability, particularly in bone followed by dural tail, postoperative bed, and venous sinuses. Nevertheless, absolute volumes contoured between different observers were often similar. Tumour edges evaluated with PET/MRI were sharper than PET/CT ones, but this did not affect contouring. Authors' advice is that PET-based RT contouring may have a role only in selected cases of meningioma where tumour boundaries are unclear on MRI and CT [75]. Conversely, the preliminary results of the group of Acker et al. on the evaluation of PET/MRI in SRS planning, suggest a significant impact in contouring lesions, particularly when the operator is at the beginning of his learning curve. Authors retrospectively selected 10 patients of which SRS-planning was done by 3 radiosurgeons (RS0, RS1, RS2), one of them (RS2) less experienced than the others. One of the most relevant analyses was the difference between the percentage of the volume defined by RS0 that would not have been treated if RS1 and RS2 had contoured without PET, 19\% and 40.2\% respectively, suggesting how the extent was highly dependent on operator experience [76]. However, further investigations conducted on larger populations are needed to establish a standardized protocol for windowing PET images over MRI in the definition of target volume contouring in order to harmonise the inter-centre analyses.

\section{SSTR-PET in Restaging of Treated Patients}

Although meningiomas are usually histologically benign, recurrence and progression are not infrequent. Indeed, distinguishing viable tumour from scar tissue by CT or MRI alone could be challenging, especially in cases of scar formation after surgery or radiation therapy [77], where MRI fails to differentiate between radiation-induced necrosis and recurrent disease in many cases. In fact, the main features of recurrent brain tumours are typically intravenous contrast enhancement, mass effect, and associated vasogenic oedema. On the other hand, post-treatment necrosis has similar characteristics, making it difficult to reliably distinguish from tumour recurrence. In this context, SSTR-PET imaging can play a fundamental role in the visualization of residual meningioma tissue, considering the high levels of SSTR-2 expression in meningiomas and the favourable target-to-background ratio [78]. Indeed, Rachinger and colleagues demonstrated the high sensitivity of 68Ga-DOTATATE in the discrimination between scar tissue and residual tumour due to low uptake in bone and healthy brain tissue. In particular, PET imaging showed a higher sensitivity $(90 \%$ vs. $79 \%)$, with specificity and positive predictive values similar to MR imaging, for both de novo and recurrent tumours. The combination of superior sensitivity with a non-inferior specificity proved that SSTR-PET provides additional useful information that might help to overcome the MRI critical limitations in the discrimination between scar tissue and recurrent tumour tissue [62]. More recently, in a retrospective study of 20 patients, Ivanidze et al. [79] demonstrated superior PET/MRI soft-tissue resolution, during the simultaneous acquisition of PET and MRI imaging, especially for the clinical purposes of differentiating recurrent meningioma from post-treatment changes. They used the superior sagittal sinus as a background reference region, given its absence of SSTR2. They showed a significant difference in the meningioma/reference ratio compared to posttreatment-change/reference ratio. 
Another limitation of morphological imaging is the identification of the transosseous extension of meningiomas, which is of utmost importance in the restaging of treated patients. As previously mentioned, contrast-enhanced MRI has limited sensitivity in the diagnosis of intracranial meningioma bone involvement, where the detection of osseous infiltration relies on few and non-specific morphologic features, such as hyperostosis or intraosseous contrast enhancement. Kunz et al., in a recent study, demonstrated the limited sensitivity of contrast-enhanced MRI to detect bone involvement in intracranial meningiomas in both pre- and post-operative phases, compared to the 68Ga-DOTATATE PET study. They showed the better diagnostic performance of SSTR-PET for treated lesions compared to standard MRI (sensitivity, $97 \%$ vs. $54 \%$; specificity, $100 \%$ vs. $83 \%$; positive predictive value, $100 \%$ vs. $95 \%$; and negative predictive value, $86 \%$ vs. $23 \%$ ) [60]. A representative case of improved restaging by SSTR-PET with respect to MRI is represented in Figure 3.

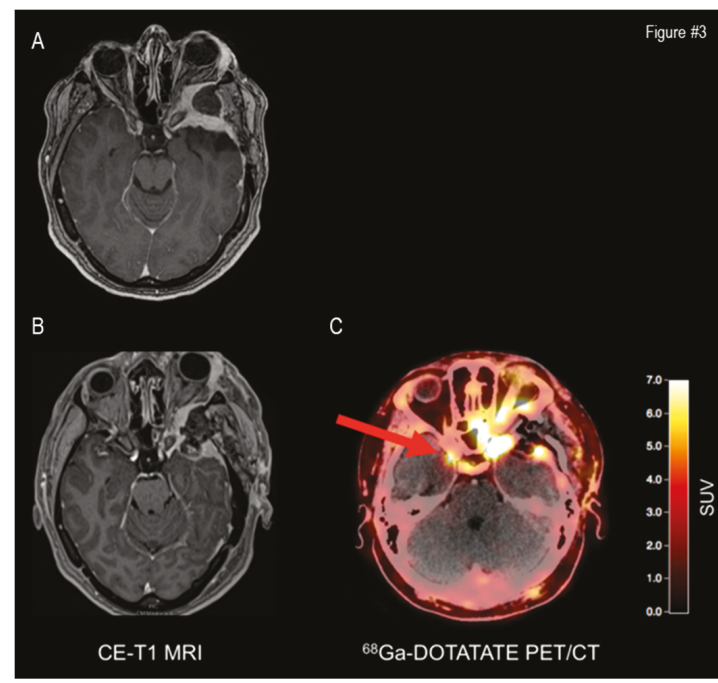

Figure 3. Additive value of SSTR-PET to MRI in restaging. A 42-year-old patient with a history of a left sphenoid skull base meningioma. Preoperative MRI shows tumour recurrence (Panel A). Postoperative MRI (Panel B) shows an incomplete resection of the tumour, necessitating adjuvant radiotherapy. 68Ga-DOTATATE PET/CT reveals an additional meningioma located at the tip of the right sphenoid wing (Panel C). Reprinted with permission from Galldiks et al. [78].

\section{Radionuclide Therapy in Meningioma}

As stated before, the first therapeutic choice in meningioma patients is represented by the surgical resection. However, for several reasons, complete resection is not always possible. Thus, other therapeutic opportunities in such patients include radiation therapy, SRS, heavy particle therapy, chemotherapy, immunotherapy, somatostatin therapy, and PRRT [80]. The molecular target used for PRRT is usually also labelled with a diagnostic radionuclide, or administered at different doses, to image patients before (demonstrating an adequate lesion's SSTR expression, that should be greater than the hepatic background uptake), during and after treatment [81]. The aim of creating such a "theragnostic" agent, is to couple the targeted therapy with diagnostic imaging using scintigraphy, SPECT, SPECT/CT, PET/CT and/or PET/MR to quantify the target expression in order to select patients that might most likely benefit from this treatment, also enabling the biodistribution assessment after each cycle of PRRT.

The origin of PRRT dates back to 1992, when, for the first time, a patient with a primary pancreatic islet cell tumour (glucagonoma) expressing SSTRs was successfully treated with high doses 
of 111Indium(111In)-penteotride, using the specific physical characteristics of the Auger's emission and conversion electrons of 111In [82]. However, due to its short tissue's range resulting in modest tumour shrinkage, it became clear that 111In was not the most suitable radionuclide for PRRT. During the same period, the dota-chelated peptides started becoming available, making it easier to label beta-emitter conjugates $[83,84]$. Thus, currently, the most used radiometals for therapy are beta-emitters 90 -Yttrium (90Y) and 177-Lutetium (177Lu), typically administered in depot formulations every 6 to 10 weeks. Beta-emitters have a short linear energy transfer (LET) and a long range of tissues' penetration, therefore increasing the average dose delivered to the tumour, but also to the surrounding healthy tissues (90Y more suitable for large-volume disease, 177Lu for small-volume lesions) with a low-incidence of mostly reversible and mild secondary effects, mainly hematological (more frequent with 177Lu) and renal. Namely, in the literature there is an overall risk of $2 \%$ for developing myeloproliferative disorders [85]; however, due to the mainly renal excretion of these tracers, renal toxicity is the most limiting factor over time, even if aminoacidic infusion notably reduces the radiopharmaceutical's renal uptake [86,87], thus narrowing this event to other therapies (i.e., chemotherapy, chemoembolization, radioembolization, and so on). In order to reduce toxicity while preserving the absorbed-effective dose, two principal strategies have been described using a variable activity in four cycles to reach the dose limit [88] or a fixed 7.4 GBq for each cycle until the achievement of the biological effective dose (BED) limit for at-risk organs [89].

To date, 177Lu radionuclide therapy is mainly used to treat patients with neuro-endocrine tumours (NET) targeting SSTRs [90], and patients with metastatic castration resistant prostate cancer targeting prostate-specific membrane antigen (PSMA), which is expressed at high levels on the surface of prostate cancer cells (radioligand therapy, RTL) [91]. However, with their localization outside of the blood-brain barrier and high SSTRs expression, meningioma also represents ideal targets for PRRT, that in this setting has been mainly used on a compassionate basis [67,92]. Despite the heterogeneity of the studies in the literature, PRRT in such patients reached a condition of stable disease (SD) in most WHO grade I patients, with a 6-months progression-free-survival (PFS) ranging from $55 \%$ to $100 \%$, and variable results in WHO grade II and III. In this paragraph, we focused our attention on the main results of the major studies published for each radiopharmaceutical related to this topic (see also Table 1 for the main characteristics of PRRT radiopharmaceuticals and Table 2 for the main characteristics of the analysed papers).

Table 1. Characteristics of PRRT Main Radiopharmaceuticals.

\begin{tabular}{|c|c|c|c|c|c|c|c|}
\hline Radionuclide & $\begin{array}{l}\text { Physical } \\
\text { Half-Life }\end{array}$ & $\begin{array}{c}\text { Emission } \\
\text { Peaks Energy }\end{array}$ & $\begin{array}{c}\text { Main } \\
\text { Applications }\end{array}$ & $\begin{array}{c}\text { Decay } \\
\text { (Abundance) }\end{array}$ & $\begin{array}{l}\text { Penetration } \\
\text { Range }\end{array}$ & $\begin{array}{l}\text { Source of } \\
\text { Production }\end{array}$ & $\begin{array}{l}\text { Specific } \\
\text { Activity }\end{array}$ \\
\hline $\begin{array}{l}\text { 111-Indium } \\
\left({ }^{111} \mathrm{In}\right)\end{array}$ & $67.9 \mathrm{~h}$ & $\begin{array}{l}\gamma(173 \mathrm{keV}) \\
\gamma(247 \mathrm{keV})\end{array}$ & Imaging & $\begin{array}{c}\text { Electron } \\
\text { Capture }(100 \%)\end{array}$ & $0.002-0.5 \mathrm{~mm}$ & Cyclotron & Medium \\
\hline $\begin{array}{c}90-Y \text { ttrium } \\
\left({ }^{90} \mathrm{Y}\right)\end{array}$ & $64 \mathrm{~h}$ & $\beta^{-}(2288 \mathrm{keV})$ & Therapy & $\beta^{-}(100 \%)$ & $4-8 \mathrm{~mm}$ & $\begin{array}{l}\text { Generator } \\
\text { or reactor }\end{array}$ & High \\
\hline $\begin{array}{l}\text { 177-Lutetium } \\
\quad\left({ }^{177} \mathrm{Lu}\right)\end{array}$ & 6.7 days & $\begin{array}{c}\beta^{-}(500 \mathrm{keV}) \\
\gamma(208 \mathrm{keV}) \\
\gamma(113 \mathrm{keV})\end{array}$ & $\begin{array}{l}\text { Only in } \\
\text { therapy }\end{array}$ & $\begin{array}{c}\beta^{-}(100 \%) \\
\gamma(27 \%)\end{array}$ & $1-2 \mathrm{~mm}$ & Reactor & Medium/high \\
\hline
\end{tabular}




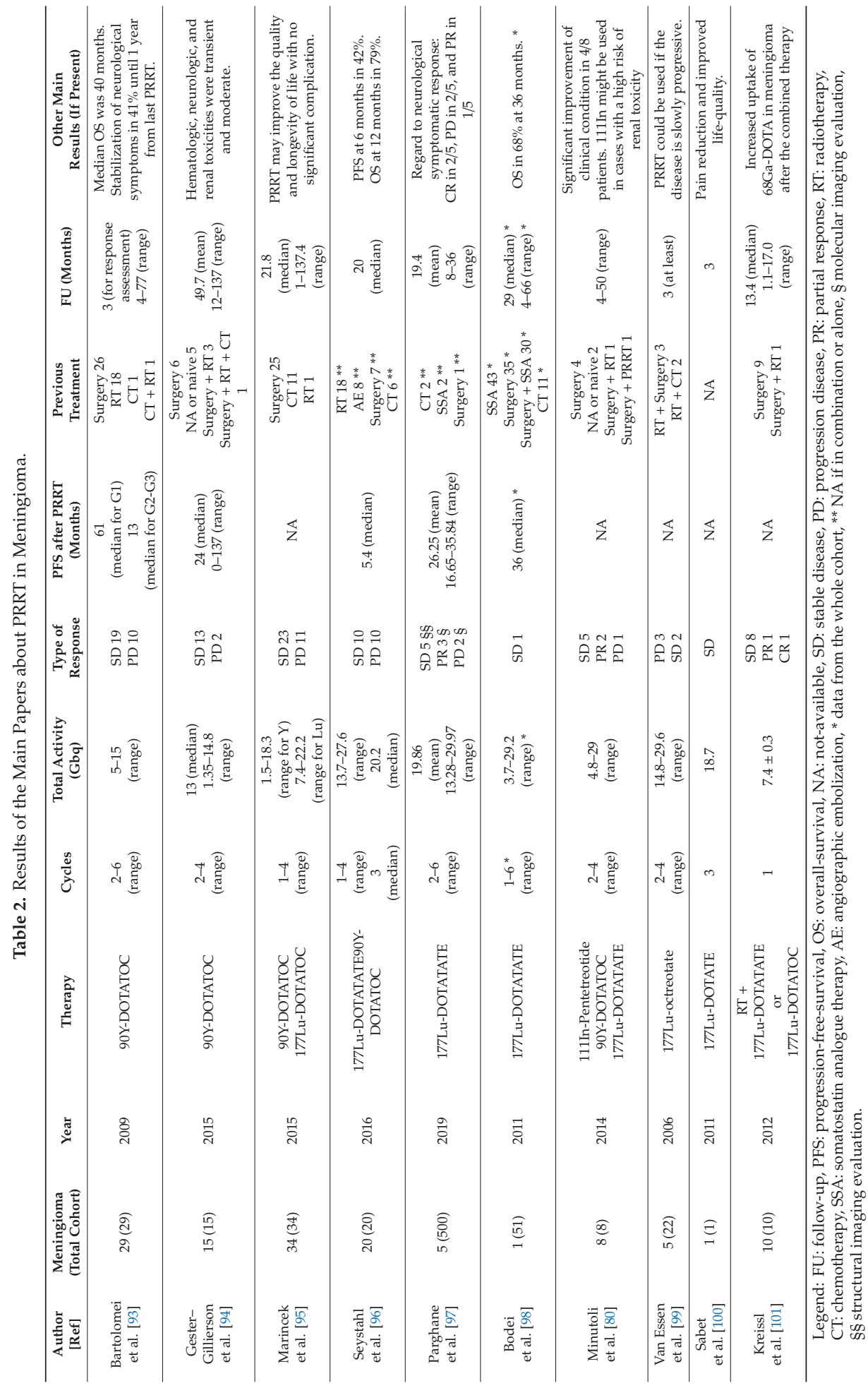




\subsection{0-Yttrium}

For many years, 90Y-octreotide was widely used as the radiometal of choice for PRRT [102]. One of the first experiments using 90Y-DOTATOC was done in Basel in 1999 in a cohort of 29 patients ( 3 of them affected by meningioma): they were treated with four or more doses of 90Y-DOTATOC with ascending activity at intervals of approximately 6 weeks (cumulative dose $6.12 \pm 1.3 \mathrm{MBq} / \mathrm{m}^{2}$ ). Treatment was monitored by CT and 111In-DOTATOC scintigraphy. According to 111In-DOTATOC scintigraphic uptake, 20 out of 29 patients showed SD ( 2 out of 20, meningioma patients), 2 out of 29 showed partial remission (PR) (decrease of $\geq 50 \%$ in tumour volume on CT scans), 4 a reduction in tumour mass $<50 \%$ ( 1 of them affected by meningioma), and 3 developed a progressive disease (PD) [103]. More recent studies demonstrated that 90Y-PRRT can slow meningioma growth in the absence of any major adverse events. Bartolomei et al., in a cohort of 29 meningioma patients, despite a non-significative reduction in lesion size, reported (at 3 months after PRRT completion) SD in 19 out of 29 patients $(66 \%)$ and PD in 10/ out of $29(34 \%)$, with a median PFS of 61 months in WHO grade I vs. 13 months in WHO grade II and III groups. Moreover, the authors reported a median overall survival (OS) of 69 months for WHO grade I vs. 30.5 months for WHO grade II and III patients [93]. In another study on a population of 15 patients with recurrent or progressive meningioma (9 out of 15 WHO grade I) treated with 90Y-PRRT, a condition of SD was obtained in 13 patients $(86.7 \%$ ) and PD in 2 patients (13.3\%), with an overall median PFS of at least 24 months (range 0-137) [94].

\subsection{7-Lutetium}

In 2000 another radiometal became available: 177Lu-DOTATATE. Due to a lower tissue penetration range (lower dosimetry to kidneys) and its photon gamma co-emission (that allow having both scintigraphic images and dosimetric studies at the same time), 177Lu became the radiometal of choice over 90Y for PRRT. During the last decades throughout Europe, the number of patients treated with 177Lu-DOTATATE dramatically increased. One of the first experiments was described by the group of Van Essen et al. with 177Lu-DOTATATE therapy in 5 patients with high-grade cranial or cervical meningioma, reporting PD in 3 patients and SD in 2. Moreover, the authors affirmed that if PRRT is used earlier or in combination with other therapies, results might be better [99]. Sabet et al. described the effects of 177Lu-DOTATE PRRT in a patient with pulmonary metastasis from anaplastic meningioma, reaching a dramatic reduction of symptoms with an improvement in the quality of life (SD at 3 months) [100]. Furthermore, Kreissl et al. described their experience in 10 meningioma patients treated with either 177Lu-DOTATATE (4 out of 10) or 177Lu-DOTATOC (6 out of 10) and external beam-RT. The authors described complete response (CR) in 1 patient, SD in 8 patients, and PD in 1 patient. Further, they reported that lesion volume after therapy (as estimated by 68Ga-PET/CT imaging) became $81 \% \pm 21 \%$ of the baseline volume [101]. In a recent study about a small cohort of meningioma patients treated with 177Lu-DOTATATE, Parghane et al. reported the occurrence of PR in 3 out of 5 and PD in 2 out of 5 at 68Ga-DOTATE PET/CT follow-up, while, at the structural imaging response evaluation, the authors observed a condition of SD in $100 \%$ of patients (5 out of 5), with a mean PFS of 26.25 months (in absence of any major adverse events) [97].

\subsection{Miscellaneous}

Minutoli et al. reported their experience in 8 patients treated with PRRT with 111In-Pentetreotide alone and in association with $90 \mathrm{Y}$ or $177 \mathrm{Lu}$. In the absence of any major adverse events, the authors observed PR in 2 patients (25\%), SD in $5(62.5 \%)$, and PD in 1 (12.5\%) [80]. In a long-term study of a cohort of 34 meningioma patients treated with $90 \mathrm{Y}$ and $177 \mathrm{Lu}$, Marincek et al. described a condition of SD in the $68 \%(23 / 34)$ with a mean survival of 8.6 years, considering the time of recruitment. Furthermore, SD after treatment and high tumour uptake were significantly associated with longer survival [95]. In the study by Seystahl et al., PRRT (with 177Lu and/or 90Y) led to SD in 50\% of population (10 of 20 meningioma patients), with, respectively, a median PFS of 32.2, 7.2, and 2.1 
months for G1, G2 and G3, and a median OS of 17.2 months in G3 patients (not reached for G1-2). Furthermore, the authors affirmed that in a 68Ga-PET/CT lesions-based analysis pre-PRRT, SUVmax and SUVmean were significantly higher in those lesions with radiological stability after 6 months and SSTRs expression (at immunohistochemistry) was related with a PFS of $>6$ months [96].

\section{Conclusions and Future Perspectives}

Thanks to the high sensitivity of SSTR detection, SSTR-targeted molecular imaging might improve several clinical challenges in meningioma from the definition of tumour growth rate and tumour extent, to the guide of therapy and, finally, to the restaging of treated patients, extending beyond the limitations of morphological imaging in these settings. Similarly, SSTR molecular imaging represents the fundamental prerequisite for SSTR-directed PRRT, which has emerged as a well-tolerated treatment option in patients with advanced recurrent or refractory meningioma, stabilizing or decelerating tumour growth.

However, several issues should be solved in the near future to further validate the additive value of these approaches in the clinical setting. First, in many cases, analysed studies were conducted on small populations. Larger prospective studies are needed to further verify the capability of SSTR-targeted imaging to significantly impact clinical decision making with respect to conventional imaging. Second, the integration between SSTR-imaging with MRI is still to be explored in the clinical setting. In particular, it could be foreseen that the introduction of the hybrid SSTR-PET/MRI approach will represent the ideal combination of radiological and nuclear medicine strengths, leading to high sensitivity, specificity, and diagnostic accuracy for meningioma, but further studies are needed to confirm or controvert it. In a pivotal study by Afshar-Oromieh et al. [104] the feasibility of 68Ga-DOTATOC PET/MRI for the detection of intracranial meningiomas was analysed in a cohort of 15 meningioma patients. The authors demonstrated that $68 \mathrm{Ga}$-DOTATOC PET/MRI could represent the ideal combination of sensitivity and specificity, offering the best morphological visualization together with SSTR expression status. However, the authors also showed the absence of superiority in treatment planning with respect to the current diagnostic gold standard. Similarly, if the global reduction in the final radiation exposure represents an acknowledged advantage provided by PET/MRI (MR-based attenuation correction allows to eliminate the patient's radiation exposure deriving from CT [79]), financial benefits, both for the patient and the health service, still need to be demonstrated.

Supplementary Materials: The following are available online at http://www.mdpi.com/2072-6694/11/10/1412/s1, Figure S1: PRISMA flow-chart for dota-peptide's diagnostic use, Figure S2: PRISMA flow-chart for dota-peptide's therapeutic use.

Author Contributions: Conceptualization of the study, M.B.; methodology, M.B. and R.L; literature search and systematic review of literature R.L., D.A., S.A., D.C., G.A., E.A., F.L., D.A., A.V., A.B; writing-original draft preparation, all co-authors; writing - review and editing, M.B., P.A., and R.L. All the authors approved the final version of the manuscript.

Funding: This research received no external funding.

Conflicts of Interest: The authors declare no conflict of interest.

\section{References}

1. Dolecek, T.A.; Propp, J.M.; Stroup, N.E.; Kruchko, C. CBTRUS statistical report: Primary brain and central nervous system tumors diagnosed in the United States in 2005-2009. Neuro-Oncology 2012, 14 (Suppl. 5), v1-v49. [CrossRef] [PubMed]

2. Ostrom, Q.T.; Gittleman, H.; Fulop, J.; Liu, M.; Blanda, R.; Kromer, C.; Wolinsky, Y.; Kruchko, C.; Barnholtz-Sloan, J.S. CBTRUS Statistical Report: Primary Brain and Central Nervous System Tumors Diagnosed in the United States in 2008-2012. Neuro-Oncology 2015, 17 (Suppl. 4), iv1-iv62. [CrossRef] [PubMed] 
3. Vernooij, M.W.; Ikram, M.A.; Tanghe, H.L.; Vincent, A.J.; Hofman, A.; Krestin, G.P.; Niessen, W.J.; Breteler, M.M.; van der Lugt, A. Incidental findings on brain MRI in the general population. N. Engl. J. Med. 2007, 357, 1821-1828. [CrossRef] [PubMed]

4. Louis, D.N.; Perry, A.; Reifenberger, G.; von Deimling, A.; Figarella-Branger, D.; Cavenee, W.K.; Ohgaki, H.; Wiestler, O.D.; Kleihues, P.; Ellison, D.W. The 2016 World Health Organization Classification of Tumors of the Central Nervous System: A summary. Acta Neuropathol. 2016, 131, 803-820. [CrossRef] [PubMed]

5. Riemenschneider, M.J.; Perry, A.; Reifenberger, G. Histological classification and molecular genetics of meningiomas. Lancet Neurol. 2006, 5, 1045-1054. [CrossRef]

6. Rogers, L.; Gilbert, M.; Vogelbaum, M.A. Intracranial meningiomas of atypical (WHO grade II) histology. J. Neurooncol. 2010, 99, 393-405. [CrossRef] [PubMed]

7. Wu, A.; Jin, M.C.; Meola, A.; Wong, H.N.; Chang, S.D. Efficacy and toxicity of particle radiotherapy in WHO grade II and grade III meningiomas: A systematic review. Neurosurg. Focus 2019, 46, E12. [CrossRef] [PubMed]

8. Stafford, S.L.; Perry, A.; Suman, V.J.; Meyer, F.B.; Scheithauer, B.W.; Lohse, C.M.; Shaw, E.G. Primarily resected meningiomas: Outcome and prognostic factors in 581 Mayo Clinic patients, 1978 through 1988. Mayo Clin. Proc. 1998, 73, 936-942. [CrossRef]

9. Jääskeläinen, J.; Haltia, M.; Laasonen, E.; Wahlström, T.; Valtonen, S. The growth rate of intracranial meningiomas and its relation to histology. An analysis of 43 patients. Surg. Neurol. 1985, 24, 165-172. [CrossRef]

10. Starke, R.M.; Przybylowski, C.J.; Sugoto, M.; Fezeu, F.; Awad, A.J.; Ding, D.; Nguyen, J.H.; Sheehan, J.P. Gamma Knife radiosurgery of large skull base meningiomas. J. Neurosurg. 2015, 122, 363-372. [CrossRef]

11. Patibandla, M.R.; Lee, C.C.; Sheehan, J. Stereotactic Radiosurgery of Central Skull Base Meningiomas-Volumetric Evaluation and Long-Term Outcomes. World Neurosurg. 2017, 108, 176-184. [CrossRef] [PubMed]

12. Rubinstein, A.B.; Shalit, M.N.; Cohen, M.L.; Zandbank, U.; Reichenthal, E. Radiation-induced cerebral meningioma: A recognizable entity. J. Neurosurg. 1984, 61, 966-971. [CrossRef] [PubMed]

13. Yamanaka, R.; Hayano, A.; Kanayama, T. Radiation-Induced Meningiomas: An Exhaustive Review of the Literature. World Neurosurg. 2017, 97, 635-644. [CrossRef] [PubMed]

14. Proctor, D.T.; Ramachandran, S.; Lama, S.; Sutherland, G.R. Towards Molecular Classification of Meningioma: Evolving Treatment and Diagnostic Paradigms. World Neurosurg. 2018, 119, 366-373. [CrossRef] [PubMed]

15. Goldbrunner, R.; Minniti, G.; Preusser, M.; Jenkinson, M.D.; Sallabanda, K.; Houdart, E.; von Deimling, A.; Stavrinou, P.; Lefranc, F.; Lund-Johansen, M.; et al. EANO guidelines for the diagnosis and treatment of meningiomas. Lancet Oncol. 2016, 17, e383-e391. [CrossRef]

16. Burford, B.J.; Welch, V.; Waters, E.; Tugwell, P.; Moher, D.; O’Neill, J.; Koehlmoos, T.; Petticrew, M. Testing the PRISMA-Equity 2012 reporting guideline: The perspectives of systematic review authors. PLoS ONE 2013, 8, e75122. [CrossRef] [PubMed]

17. Buetow, M.P.; Buetow, P.C.; Smirniotopoulos, J.G. Typical, atypical, and misleading features in meningioma. Radiographics 1991, 11, 1087-1106. [CrossRef] [PubMed]

18. Whittle, I.R.; Smith, C.; Navoo, P.; Collie, D. Meningiomas. Lancet 2004, 363, 1535-1543. [CrossRef]

19. O'Leary, S.; Adams, W.M.; Parrish, R.W.; Mukonoweshuro, W. Atypical imaging appearances of intracranial meningiomas. Clin. Radiol. 2007, 62, 10-17. [CrossRef]

20. Hakyemez, B.; Yildirim, N.; Erdoğan, C.; Kocaeli, H.; Korfali, E.; Parlak, M. Meningiomas with conventional MRI findings resembling intraaxial tumors: Can perfusion-weighted MRI be helpful in differentiation? Neuroradiology 2006, 48, 695-702. [CrossRef]

21. Guermazi, A.; Lafitte, F.; Miaux, Y.; Adem, C.; Bonneville, J.F.; Chiras, J. The dural tail sign-beyond meningioma. Clin. Radiol. 2005, 60, 171-188. [CrossRef] [PubMed]

22. Watts, J.; Box, G.; Galvin, A.; Brotchie, P.; Trost, N.; Sutherland, T. Magnetic resonance imaging of meningiomas: A pictorial review. Insights Imaging 2014, 5, 113-122. [CrossRef] [PubMed]

23. Pieper, D.R.; Al-Mefty, O.; Hanada, Y.; Buechner, D. Hyperostosis associated with meningioma of the cranial base: Secondary changes or tumor invasion. Neurosurgery 1999, 44, 742-747. [CrossRef] [PubMed]

24. Kotapka, M.J.; Kalia, K.K.; Martinez, A.J.; Sekhar, L.N. Infiltration of the carotid artery by cavernous sinus meningioma. J. Neurosurg. 1994, 81, 252-255. [CrossRef] [PubMed] 
25. Larson, J.J.; van Loveren, H.R.; Balko, M.G.; Tew, J.M., Jr. Evidence of meningioma infiltration into cranial nerves: Clinical implications for cavernous sinus meningiomas. J. Neurosurg. 1995, 83, 596-599. [CrossRef] [PubMed]

26. Lang, F.F.; Macdonald, O.K.; Fuller, G.N.; DeMonte, F. Primary extradural meningiomas: A report on nine cases and review of the literature from the era of computerized tomography scanning. J. Neurosurg. 2000, 93, 940-950. [CrossRef] [PubMed]

27. Kim, H.S.; Kim, S.H.; Kim, H.J.; Kang, S.W.; Kim, S.J.; Lee, J.H.; Hong, S.P.; Cho, Y.S.; Choi, J.Y. Primary Benign Intraosseous Meningioma on (18)F-FDG PET/CT Mimicking Malignancy. Nucl. Med. Mol. Imaging 2014, 48, 153-156. [CrossRef] [PubMed]

28. Albano, D.; La Grutta, L.; Grassedonio, E.; Patti, C.; Lagalla, R.; Midiri, M.; Galia, M. Pitfalls in whole body MRI with diffusion weighted imaging performed on patients with lymphoma: What radiologists should know. Magn. Reson. Imaging 2016, 34, 922-931. [CrossRef]

29. Krupa, K.; Bekiesińska-Figatowska, M. Artifacts in magnetic resonance imaging. Pol. J. Radiol. 2015, 80, 93-106.

30. Bahrami, S.; Yim, C.M. Quality initiatives: Blind spots at brain imaging. Radiographics 2009, 29, 1877-1896. [CrossRef]

31. Valotassiou, V.; Leondi, A.; Angelidis, G.; Psimadas, D.; Georgoulias, P. SPECT and PET imaging of meningiomas. Sci. World J. 2012, 2012, 412580. [CrossRef] [PubMed]

32. Ericson, K.; Lilja, A.; Bergstrom, M.; Collins, V.P.; Eriksson, L.; Ehrin, E.; von Holst, H.; Lundqvist, H.; Långsrom, B.B.; Mosskin, M. Positron emission tomography with ([11C]methyl)-L-methionine, [11C] D-glucose and [68Ga]EDTA in supratentorial tumors. J. Comput. Assist. Tomogr. 1985, 9, 683-689. [CrossRef]

33. Di Chiro, G.; Hatazawa, J.; David, A.K.; Rizzoli, H.V.; De Michele, D.J. Glucose utilization by intracranial meningiomas as an index of tumor aggressivity and probability of recurrence: A PET study. Radiology 1987, 164, 521-526. [CrossRef] [PubMed]

34. Okuchi, S.; Okada, T.; Yamamoto, A.; Kanagaki, M.; Fushimi, Y.; Okada, T.; Yamauchi, M.; Kataoka, M.; Arakawa, Y.; Takahashi, J.C.; et al. Grading meningioma: A comparative study of thallium-SPECT and FDG-PET. Medicine (Baltim.) 2015, 94, e549. [CrossRef] [PubMed]

35. Cremerius, U.; Bares, R.; Weis, J.; Sabri, O.; Mull, M.; Schröder, J.M.; Gilsbach, J.M.; Buell, U. Fasting improves discrimination of grade 1 and atypical or malignant meningioma in FDG-PET. J. Nucl. Med. 1997, 38, 26-30.

36. Kado, H.; Ogawa, T.; Hatazawa, J.; Iwase, M.; Kanno, I.; Okudera, T.; Uemura, K. Radiation-induced meningioma evaluated with positron emission tomography with fludeoxyglucose F 18. Am. J. Neuroradiol. 1996, 17, 937-938. [PubMed]

37. Lee, J.W.; Kang, K.W.; Park, S.H.; Lee, S.M.; Paeng, J.C.; Chung, J.K.; Lee, M.C.; Lee, D.S. 18F-FDG PET in the assessment of tumor grade and prediction of tumor recurrence in intracranial meningioma. Eur J. Nucl. Med. Mol. Imaging 2009, 36, 1574-1582. [CrossRef]

38. Iuchi, T.; Iwasate, Y.; Namba, H.; Osato, K.; Saeki, N.; Yamaura, A.; Uchida, Y. Glucose and methionine uptake and proliferative activity in meningiomas. Neurol. Res. 1999, 21, 640-644.

39. Park, Y.S.; Jeon, B.C.; Oh, H.S.; Lee, S.M.; Chun, B.K.; Chang, H.K. FDG PET/CT assessment of the biological behavior of meningiomas. J. Korean Neurosurg. Soc. 2006, 40, 428-433.

40. Mitamura, K.; Yamamoto, Y.; Norikane, T.; Hatakeyama, T.; Okada, M.; Nishiyama, Y. Correlation of 18F-FDG and 11C-methionine uptake on PET/CT with Ki-67 immunohistochemistry in newly diagnosed intracranial meningiomas. Ann. Nucl. Med. 2018, 32, 627-633. [CrossRef]

41. Tomura, N.; Saginoya, T.; Goto, H. 11C-Methionine Positron Emission Tomography/Computed Tomography Versus 18F-Fluorodeoxyglucose Positron Emission Tomography/Computed Tomography in Evaluation of Residual or Recurrent World Health Organization Grades II and III Meningioma After Treatment. J. Comput. Assist. Tomogr. 2018, 42, 517-521. [CrossRef] [PubMed]

42. Astner, S.T.; Dobrei-Ciuchendea, M.; Essler, M.; Bundschuh, R.A.; Sai, H.; Schwaiger, M.; Molls, M.; Weber, W.A.; Grosu, A.L. Effect of 11C-methionine-positron emission tomography on gross tumor volume delineation in stereotactic radiotherapy of skull base meningiomas. Int J. Radiat. Oncol. Biol. Phys. 2008, 72 , 1161-1167. [CrossRef] [PubMed]

43. Ikeda, H.; Tsuyuguchi, N.; Kunihiro, N.; Ishibashi, K.; Goto, T.; Ohata, K. Analysis of progression and recurrence of meningioma using (11)C-methionine PET. Ann. Nucl. Med. 2013, 27, 772-780. [CrossRef] [PubMed] 
44. Ryttefors, M.; Danfors, T.; Lantini, F.; Montelius, A.; Blomquist, E.; Gudjonsson, O. Long-term evaluation of the effect of hypofractionated high-energy proton treatment of benign meningiomas by means of (11)C-L-methionine positron emission tomography. Eur. J. Nucl. Med. Imaging 2016, 43, 1432-1443. [CrossRef] [PubMed]

45. Arita, H.; Kinoshita, M.; Okita, Y.; Hirayama, R.; Watabe, T.; Ishohashi, K.; Kijima, N.; Kagawa, N.; Fujimoto, Y.; Kishima, H.; et al. Clinical characteristics of meningiomas assessed by 11C-methionine and 18F-fluorodeoxyglucose positron-emission tomography. J. Neurooncol. 2012, 107, 379-386. [CrossRef] [PubMed]

46. Dittmar, J.O.; Kratochwil, C.; Dittmar, A.; Welzel, T.; Habermehl, D.; Rieken, S.; Giesel, F.L.; Haberkorn, U.; Debus, J.; Combs, S.E. First intraindividual comparison of contrast-enhanced MRI, FET- and DOTATOC-PET in patients with intracranial meningiomas. Radiat. Oncol. 2017, 12, 169. [CrossRef] [PubMed]

47. Cornelius, J.F.; Stoffels, G.; Filß, C.; Galldiks, N.; Slotty, P.; Kamp, M.; el Khatib, M.; Hänggi, D.; Sabel, M.; Felsberg, J.; et al. Uptake and tracer kinetics of O-(2-(18)F-fluoroethyl)-L-tyrosine in meningiomas: Preliminary results. Eur. J. Nucl. Med. Mol. Imaging 2015, 42, 459-467. [CrossRef]

48. Cornelius, J.F.; Langen, K.J.; Stoffels, G.; Hänggi, D.; Sabel, M.; Jakob Steiger, H. Positron emission tomography imaging of meningioma in clinical practice: Review of literature and future directions. Neurosurgery 2012, 70 , 1033-1041. [CrossRef]

49. Tateishi, U.; Tateishi, K.; Hino-Shishikura, A.; Torii, I.; Inoue, T.; Kawahara, N. Multimodal approach to detect osseous involvement in meningioma: Additional value of (18)F-fluoride PET/CT for conventional imaging. Radiology 2014, 273, 521-528. [CrossRef]

50. Giovacchini, G.; Fallanca, F.; Landoni, C.; Gianolli, L.; Picozzi, P.; Attuati, L.; Terreni, M.; Picchio, M.; Messa, C.; Fazio, F. C-11 choline versus F-18fluorodeoxyglucose for imaging meningiomas: An initial experience. Clin. Nucl. Med. 2009, 34, 7-10. [CrossRef]

51. Tateishi, U.; Tateishi, K.; Shizukuishi, K.; Shishikura, A.; Murata, H.; Inoue, T.; Kawahara, N. 18F-fluoride $\mathrm{PET} / \mathrm{CT}$ allows detection of hyperostosis and osseous involvement in meningioma: Initial experience. Clin. Nucl. Med. 2013, 38, e125-e131. [CrossRef] [PubMed]

52. Liu, R.S.; Chang, C.P.; Guo, W.Y.; Pan, D.H.; Ho, D.M.; Chang, C.W.; Yang, B.H.; Wu, L.C.; Yeh, S.H. 11C-acetate versus 18F-FDG PET in detection of meningioma and monitoring the effect of gamma-knife radiosurgery. J. Nucl. Med. 2010, 51, 883-891. [CrossRef] [PubMed]

53. Nakamura, M.; Roser, F.; Michel, J.; Jacobs, C.; Samii, M. Volumetric analysis of the growth rate of incompletely resected intracranial meningiomas. Zent. Neurochir. 2005, 66, 17-23. [CrossRef] [PubMed]

54. Fountain, D.M.; Soon, W.C.; Matys, T.; Guilfoyle, M.R.; Kirollos, R.; Santarius, T. Volumetric growth rates of meningioma and its correlation with histological diagnosis and clinical outcome: A systematic review. Acta Neurochir. (Wien) 2017, 159, 435-445. [CrossRef] [PubMed]

55. Nakasu, S.; Fukami, T.; Nakajima, M.; Watanabe, K.; Ichikawa, M.; Matsuda, M. Growth pattern changes of meningiomas: Long-term analysis. Neurosurgery 2005, 56, 946-955. [PubMed]

56. Nakasu, S.; Nakasu, Y.; Fukami, T.; Jito, J.; Nozaki, K. Growth curve analysis of asymptomatic and symptomatic meningiomas. J. Neurooncol. 2011, 102, 303-310. [CrossRef]

57. Lee, E.J.; Kim, J.H.; Park, E.S.; Kim, Y.H.; Lee, J.K.; Hong, S.H.; Cho, Y.H.; Kim, C.J. A novel weighted scoring system for estimating the risk of rapid growth in untreated intracranial meningiomas. J. Neurosurg. 2017, 127, 971-980. [CrossRef]

58. Oya, S.; Kim, S.H.; Sade, B.; Lee, J.H. The natural history of intracranial meningiomas. J. Neurosurg. 2011, 114, 1250-1256. [CrossRef]

59. Sommerauer, M.; Burkhardt, J.K.; Frontzek, K.; Rushing, E.; Buck, A.; Krayenbuehl, N.; Weller, M.; Schaefer, N.; Kuhn, F.P. 68Gallium-DOTATATE PET in meningioma: A reliable predictor of tumor growth rate? Neuro. Oncol. 2016, 18, 1021-1027. [CrossRef]

60. Kunz, W.G.; Jungblut, L.M.; Kazmierczak, P.M.; Vettermann, F.J.; Bollenbacher, A.; Tonn, J.C.; Schichor, C.; Rominger, A.; Albert, N.L.; Bartenstein, P.; et al. Improved Detection of Transosseous Meningiomas Using 68Ga-DOTATATE PET/CT Compared with Contrast-Enhanced MRI. J. Nucl. Med. 2017, 58, 1580-1587. [CrossRef]

61. Afshar-Oromieh, A.; Giesel, F.L.; Linhart, H.G.; Haberkorn, U.; Haufe, S.; Combs, S.E.; Podlesek, D.; Eisenhut, M.; Kratochwil, C. Detection of cranial meningiomas: Comparison of ${ }^{68}$ Ga-DOTATOC PET/CT and contrast-enhanced MRI. Eur. J. Nucl. Med. Mol. Imaging 2012, 39, 1409-1415. [CrossRef] [PubMed] 
62. Rachinger, W.; Stoecklein, V.M.; Terpolilli, N.A.; Haug, A.R.; Ertl, L.; Pöschl, J.; Schüller, U.; Schichor, C.; Thon, N.; Tonn, J.C. Increased 68Ga-DOTATATE uptake in PET imaging discriminates meningioma and tumor-free tissue. J. Nucl. Med. 2015, 56, 347-353. [CrossRef] [PubMed]

63. Henze, M.; Schuhmacher, J.; Hipp, P.; Kowalski, J.; Becker, D.W.; Doll, J.; Mäcke, H.R.; Hofmann, M.; Debus, J.; Haberkorn, U. PET imaging of somatostatin receptors using [68GA]DOTA-D-Phe1-Tyr3-octreotide: First results in patients with meningiomas. J. Nucl. Med. 2001, 42, 1053-1056. [PubMed]

64. Dammers, R.; Hsu, S.P.; Krisht, A.F. Radioguided improved resection of a cranial base meningioma. Neurosurgery 2009, 64, onse84-onse85. [CrossRef] [PubMed]

65. Gay, E.; Vuillez, J.P.; Palombi, O.; Brard, P.Y.; Bessou, P.; Passagia, J.G. Intraoperative and postoperative gamma detection of somatostatin receptors in bone-invasive en plaque meningiomas. Neurosurgery 2005, 57, 107-113. [CrossRef] [PubMed]

66. Solfaroli Camillocci, E.; Schiariti, M.; Bocci, V.; Carollo, A.; Chiodi, G.; Colandrea, M.; Collamati, F.; Cremonesi, M.; Donnarumma, R.; Ferrari, M.E.; et al. First ex vivo validation of a radioguided surgery technique with $\beta$-radiation. Phys. Med. 2016, 32, 1139-1144. [CrossRef] [PubMed]

67. Collamati, F.; Pepe, A.; Bellini, F.; Bocci, V.; Chiodi, G.; Cremonesi, M.; De Lucia, E.; Ferrari, M.E.; Frallicciardi, P.M.; Grana, C.M.; et al. Toward radioguided surgery with $\beta$-decays: Uptake of a somatostatin analogue, DOTATOC, in meningioma and high-grade glioma. J. Nucl. Med. 2015, 56, 3-8. [CrossRef]

68. Milker-Zabel, S.; Zabel-du Bois, A.; Henze, M.; Huber, P.; Schulz-Ertner, D.; Hoess, A.; Haberkorn, U.; Debus, J. Improved target volume definition for fractionated stereotactic radiotherapy in patients with intracranial meningiomas by correlation of CT, MRI, and [68Ga]-DOTATOC-PET. Int J. Radiat. Oncol. Biol. Phys. 2006, 65, 222-227. [CrossRef]

69. Gehler, B.; Paulsen, F.; Oksüz, M.O.; Hauser, T.K.; Eschmann, S.M.; Bares, R.; Pfannenberg, C.; Bamberg, M.; Bartenstein, P.; Belka, C.; et al. [68Ga]-DOTATOC-PET/CT for meningioma IMRT treatment planning. Radiat. Oncol. 2009, 4, 56. [CrossRef]

70. Nyuyki, F.; Plotkin, M.; Graf, R.; Michel, R.; Steffen, I.; Denecke, T.; Geworski, L.; Fahdt, D.; Brenner, W.; Wurm, R. Potential impact of 68Ga-DOTATOC PET/CT on stereotactic radiotherapy planning of meningiomas. Eur. J. Nucl. Med. Mol. Imaging 2010, 37, 310-318. [CrossRef]

71. Graf, R.; Nyuyki, F.; Steffen, I.G.; Michel, R.; Fahdt, D.; Wust, P.; Brenner, W.; Budach, V.; Wurm, R.; Plotkin, M. Contribution of 68Ga-DOTATOC PET/CT to target volume delineation of skull base meningiomas treated with stereotactic radiation therapy. Int J. Radiat. Oncol. Biol. Phys. 2013, 85, 68-73. [CrossRef] [PubMed]

72. Combs, S.E.; Welzel, T.; Habermehl, D.; Rieken, S.; Dittmar, J.O.; Kessel, K.; Jäkel, O.; Haberkorn, U.; Debus, J. Prospective evaluation of early treatment outcome in patients with meningiomas treated with particle therapy based on target volume definition with MRI and 68Ga-DOTATOC-PET. Acta Oncol. 2013, 52, 514-520. [CrossRef] [PubMed]

73. Madani, I.; Lomax, A.J.; Albertini, F.; Trnková, P.; Weber, D.C. Dose-painting intensity-modulated proton therapy for intermediate- and high-risk meningioma. Radiat. Oncol. 2015, 10, 72. [CrossRef] [PubMed]

74. Stade, F.; Dittmar, J.O.; Jäkel, O.; Kratochwil, C.; Haberkorn, U.; Debus, J.; Combs, S.E. Influence of 68Ga-DOTATOC on sparing of normal tissue for radiation therapy of skull base meningioma: Differential impact of photon and proton radiotherapy. Radiat. Oncol. 2018, 13, 58. [CrossRef] [PubMed]

75. Maclean, J.; Fersht, N.; Sullivan, K.; Kayani, I.; Bomanji, J.; Dickson, J.; O’Meara, C.; Short, S. Simultaneous 68Ga DOTATATE Positron Emission Tomography/Magnetic Resonance Imaging in Meningioma Target Contouring: Feasibility and Impact Upon Interobserver Variability Versus Positron Emission Tomography/Computed Tomography and Computed Tomography/Magnetic Resonance Imaging. Clin. Oncol. (R. Coll. Radiol.) 2017, 29, 448-458. [PubMed]

76. Acker, G.; Kluge, A.; Lukas, M.; Conti, A.; Pasemann, D.; Meinert, F.; Anh Nguyen, P.T.; Jelgersma, C.; Loebel, F.; Budach, V.; et al. Impact of 68Ga-DOTATOC PET/MRI on robotic radiosurgery treatment planning in meningioma patients: First experiences in a single institution. Neurosurg. Focus 2019, 46, E9. [CrossRef]

77. Jaeckle, K.A. Neuroimaging for central nervous system tumors. Semin. Oncol. 1991, 18, 150-157. [PubMed]

78. Galldiks, N.; Albert, N.L.; Sommerauer, M.; Grosu, A.L.; Ganswindt, U.; Law, I.; Preusser, M.; Le Rhun, E.; Vogelbaum, M.A.; Zadeh, G.; et al. PET imaging in patients with meningioma-report of the RANO/PET Group. Neuro-Oncology 2017, 19, 1576-1587. [CrossRef] [PubMed] 
79. Ivanidze, J.; Roytman, M.; Lin, E.; Magge, R.S.; Pisapia, D.J.; Liechty, B.; Karakatsanis, N.; Ramakrishna, R.; Knisely, J.; Schwartz, T.H.; et al. Gallium-68 DOTATATE PET in the Evaluation of Intracranial Meningiomas. J. Neuroimaging 2019, in press. [CrossRef]

80. Minutoli, F.; Amato, E.; Sindoni, A.; Cardile, D.; Conti, A.; Herberg, A.; Baldari, S. Peptide receptor radionuclide therapy in patients with inoperable meningiomas: Our experience and review of the literature. Cancer Biother. Radiopharm. 2014, 29, 193-199. [CrossRef]

81. Kwekkeboom, D.J.; de Herder, W.W.; Kam, B.L.; van Eijck, C.H.; van Essen, M.; Kooij, P.P.; Feelders, R.A.; van Aken, M.O.; Krenning, E.P. Treatment with the radiolabeled somatostatin analog [177 Lu-DOTA 0,Tyr3] octreotate: Toxicity, efficacy, and survival. J. Clin. Oncol. 2008, 26, 2124-2130. [CrossRef] [PubMed]

82. Krenning, E.P.; Kooij, P.P.; Bakker, W.H.; Breeman, W.A.; Postema, P.T.; Kwekkeboom, D.J.; Oei, H.Y.; de Jong, M.; Visser, T.J.; Reijs, A.E.; et al. Radiotherapy with a radiolabeled somatostatin analogue, [111In-DTPA-D-Phe1]-octreotide. A case history. Ann. N. Y. Acad. Sci. 1994, 733, 496-506. [CrossRef] [PubMed]

83. Otte, A.; Jermann, E.; Behe, M.; Goetze, M.; Bucher, H.C.; Roser, H.W.; Heppeler, A.; Mueller-Brand, J.; Maecke, H.R. DOTATOC: A powerful new tool for receptor-mediated radionuclide therapy. Eur. J. Nucl. Med. 1997, 24, 792-795. [PubMed]

84. Albert, R.; Smith-Jones, P.; Stolz, B.; Simeon, C.; Knecht, H.; Bruns, C.; Pless, J. Direct synthesis of [DOTA-DPhe1]-octreotide and [DOTA-DPhe1,Tyr3]-octreotide (SMT487): Two conjugates for systemic delivery of radiotherapeutical nuclides to somatostatin receptor positive tumors in man. Bioorg. Med. Chem. Lett. 1998, 8, 1207-1210. [CrossRef]

85. Bodei, L.; Kidd, M.; Paganelli, G.; Grana, C.M.; Drozdov, I.; Cremonesi, M.; Lepensky, C.; Kwekkebom, D.J.; Baum, R.P.; Krenning, E.P.; et al. Long-term tolerability of PRRT in 807 patients with neuroendocrine tumours: The value and limitations of clinical factors. Eur. J. Nucl. Med. Mol. Imaging 2015, 42, 5-19. [CrossRef] [PubMed]

86. Rolleman, E.J.; Valkema, R.; de Jong, M.; Kooij, P.P.; Krenning, E.P. Safe and effective inhibition of renal uptake of radiolabeled octreotide by a combination of lysine and arginine. Eur. J. Nucl. Med. Mol. Imaging 2003, 30, 9-15. [CrossRef]

87. Bushnell, D.; Menda, Y.; O’Dorisio, T.; Madsen, M.; Miller, S.; Carlisle, T.; Squires, S.; Kahn, D.; Walkner, W.; Connolly, M.; et al. Effects of intravenous amino acid administration with Y-90 DOTA-Phe1-Tyr3-Octreotide (SMT487[OctreoTher) treatment. Cancer Biother. Radiopharm. 2004, 19, 35-41. [CrossRef] [PubMed]

88. Del Prete, M.; Buteau, F.A.; Beauregard, J.M. Personalized 177Lu-octreotate peptide receptor radionuclide therapy of neuroendocrine tumours: A simulation study. Eur. J. Nucl. Med. Mol. Imaging 2017, 44, 1490-1500. [CrossRef]

89. Sundlöv, A.; Sjögreen-Gleisner, K.; Svensson, J.; Ljungberg, M.; Olsson, T.; Bernhardt, P.; Tennvall, J. Individualised 177Lu-DOTATATE treatment of neuroendocrine tumours based on kidney dosimetry. Eur. J. Nucl. Med. Mol. Imaging 2017, 44,1480-1489. [CrossRef]

90. FDA Approves Lutathera for GEP NET Therapy. J. Nucl. Med. 2018, 59, 9N.

91. Kratochwil, C.; Fendler, W.P.; Eiber, M.; Baum, R.; Bozkurt, M.F.; Czernin, J.; Delgado Bolton, R.C.; Ezzidin, S.; Forrer, F.; Hicks, R.J.; et al. EANM procedure guidelines for radionuclide therapy with (177)Lu-labelled PSMA ligands ((177Lu)Lu-PSMA-RLT). Eur. J. Nucl. Med. Mol. Imaging 2019, in press. [CrossRef] [PubMed]

92. Durand, A.; Champier, J.; Jouvet, A.; Labrousse, F.; Honnorat, J.; Guyotat, J.; Fèvre-Montange, M. Expression of c-Myc, neurofibromatosis Type 2, somatostatin receptor 2 and erb-B2 in human meningiomas: Relation to grades or histotypes. Clin. Neuropathol. 2008, 27, 334-345. [CrossRef] [PubMed]

93. Bartolomei, M.; Bodei, L.; De Cicco, C.; Grana, C.M.; Cremonesi, M.; Botteri, E.; Baio, S.M.; Aricò, D.; Sansovini, M.; Paganelli, G. Peptide receptor radionuclide therapy with (90)Y-DOTATOC in recurrent meningioma. Eur. J. Nucl. Med. Mol. Imaging 2009, 36, 1407-1416. [CrossRef] [PubMed]

94. Gerster-Gilliéron, K.; Forrer, F.; Maecke, H.; Mueller-Brand, J.; Merlo, A.; Cordier, D. 90Y-DOTATOC as a Therapeutic Option for Complex Recurrent or Progressive Meningiomas. J. Nucl. Med. 2015, 56, 1748-1751. [CrossRef] [PubMed]

95. Marincek, N.; Radojewski, P.; Dumont, R.A.; Brunner, P.; Müller-Brand, J.; Maecke, H.R.; Briel, M.; Walter, M.A. Somatostatin receptor-targeted radiopeptide therapy with 90Y-DOTATOC and 177Lu-DOTATOC in progressive meningioma: Long-term results of a phase II clinical trial. J. Nucl. Med. 2015, 56, 171-176. [CrossRef] [PubMed] 
96. Seystahl, K.; Stoecklein, V.; Schüller, U.; Rushing, E.; Nicolas, G.; Schäfer, N.; Ilhan, H.; Pangalu, A.; Weller, M.; Tonn, J.C.; et al. Somatostatin receptor-targeted radionuclide therapy for progressive meningioma: Benefit linked to 68Ga-DOTATATE/-TOC uptake. Neuro-Oncology 2016, 18, 1538-1547. [CrossRef] [PubMed]

97. Parghane, R.V.; Talole, S.; Basu, S. Prevalence of hitherto unknown brain meningioma detected on 68Ga-DOTATATE positron-emission tomography/computed tomography in patients with metastatic neuroendocrine tumor and exploring potential of 177Lu-DOTATATE peptide receptor radionuclide therapy as single-shot treatment approach targeting both tumors. World J. Nucl. Med. 2019, 18, 160-170.

98. Bodei, L.; Cremonesi, M.; Grana, C.M.; Fazio, N.; Iodice, S.; Baio, S.M.; Bartolomei, M.; Lombardo, D.; Ferrari, M.E.; Sansovini, M.; et al. Peptide receptor radionuclide therapy with ${ }^{177}$ Lu-DOTATATE: The IEO phase I-II study. Eur. J. Nucl. Med. Mol. Imaging 2011, 38, 2125-2135. [CrossRef]

99. van Essen, M.; Krenning, E.P.; Kooij, P.P.; Bakker, W.H.; Feelders, R.A.; de Herder, W.W.; Wolbers, J.G.; Kwekkeboom, D.J. Effects of therapy with [177Lu-DOTA0, Tyr3] octreotate in patients with paraganglioma, meningioma, small cell lung carcinoma, and melanoma. J. Nucl. Med. 2006, 47, 1599-1606.

100. Sabet, A.; Ahmadzadehfar, H.; Herrlinger, U.; Wilinek, W.; Biersack, H.J.; Ezziddin, S. Successful radiopeptide targeting of metastatic anaplastic meningioma: Case report. Radiat. Oncol. 2011, 6, 94. [CrossRef]

101. Kreissl, M.C.; Hänscheid, H.; Löhr, M.; Verburg, F.A.; Schiller, M.; Lassmann, M.; Reiners, C.; Samnick, S.S.; Buck, A.K.; Flentje, M.; et al. Combination of peptide receptor radionuclide therapy with fractionated external beam radiotherapy for treatment of advanced symptomatic meningioma. Radiat. Oncol. 2012, 7, 99. [CrossRef] [PubMed]

102. Paganelli, G.; Zoboli, S.; Cremonesi, M.; Bodei, L.; Ferrari, M.; Grana, C.; Bartolomei, M.; Orsi, F.; De Cicco, C.; Mäcke, H.R.; et al. Receptor-mediated radiotherapy with 90Y-DOTA-D-Phe1-Tyr3-octreotide. Eur. J. Nucl. Med. 2001, 28, 426-434. [CrossRef] [PubMed]

103. Otte, A.; Herrmann, R.; Heppeler, A.; Behe, M.; Jermann, E.; Powell, P.; Maecke, H.R.; Muller, J. Yttrium-90 DOTATOC: First clinical results. Eur. J. Nucl. Med. 1999, 26, 1439-1447. [CrossRef] [PubMed]

104. Afshar-Oromieh, A.; Wolf, M.B.; Kratochwil, C.; Giesel, F.L.; Combs, S.E.; Dimitrakopoulou-Strauss, A.; Gnirs, R.; Roethke, M.C.; Schlemmer, H.P.; Haberkorn, U. Comparison of ${ }^{68}$ Ga-DOTATOC-PET/CT and PET/MRI hybrid systems in patients with cranial meningioma: Initial results. Neuro-Oncology 2015, 17, 312-319. [CrossRef] [PubMed] 


\title{
${ }^{18}$ F-Facbc in Prostate Cancer: A Systematic Review and Meta-Analysis
}

\author{
Riccardo Laudicella ${ }^{1}$, Domenico Albano ${ }^{2}$, Pierpaolo Alongi ${ }^{3}$, Giovanni Argiroffi ${ }^{4}$, \\ Matteo Bauckneht ${ }^{5}$, Sergio Baldari ${ }^{1}$, Francesco Bertagna ${ }^{2}$, Michele Boero ${ }^{6}$, \\ Giuseppe De Vincentis ${ }^{7}$, Angelo Del Sole ${ }^{4}$, Giuseppe Rubini ${ }^{8}$, Lorenzo Fantechi ${ }^{9}$, \\ Viviana Frantellizzi ${ }^{10}$, Gloria Ganduscio ${ }^{3}$, Priscilla Guglielmo ${ }^{6}$, Anna Giulia Nappi ${ }^{8}$, \\ Laura Evangelista ${ }^{11, *}$ and on the behalf of Young AIMN Working Group ${ }^{\dagger}$
}

1 Department of Biomedical and Dental Sciences and of Morpho-functional Imaging, Nuclear Medicine Unit, University of Messina, 98125 Messina, Italy; riclaudi@hotmail.it (R.L.); sergio.baldari@unime.it (S.B.)

2 Department of Nuclear Medicine, University of Brescia and Spedali Civili Brescia, 25123 Brescia, Italy; Doalba87@libero.it (D.A.); francesco.bertagna@unibs.it (F.B.)

3 Unit of Nuclear Medicine, Fondazione Istituto G.Giglio, 90015 Cefalù, Italy; pierpaolo.alongi@hsrgiglio.it (P.A.); gloria.ganduscio@gmail.com (G.G.)

4 Department of Health Sciences, University of Milan, 20142 Milan, Italy; giovanni.argiroffi@gmail.com (G.A.); angelo.delsole@unimi.it (A.D.S.)

5 Nuclear Medicine Unit, IRCCS Policlinico San Martino, 16132 Genoa, Italy; matteo.bauckneht@gmail.com

6 Nuclear Medicine Unit, AO Brotzu, 09134 Cagliari, Italy; micboero@gmail.com (M.B.); priscilla.guglielmo@yahoo.it (P.G.)

7 Department of Radiological Sciences, Oncology and Anatomical Pathology, Sapienza University of Rome, 00161 Rome, Italy; giuseppe.devincentis@uniroma1.it

8 Nuclear Medicine Unit, Department of Interdisciplinary Medicine, University of Bari Aldo Moro, 70124 Bari, Italy; giuseppe.rubini@uniba.it (G.R.); anna.giulia.nappi@gmail.com (A.G.N.)

9 Department of New Technologies and Translational Research in Medicine and Surgery, Nuclear Medicine Unit, University of Pisa, 56126 Pisa, Italy; fantechi.lorenzo@gmail.com

10 Department of Molecular Medicine, Sapienza University of Rome, 00185 Rome, Italy; viviana.frantellizzi@uniroma1.it

11 Nuclear Medicine Unit, Veneto Institute of Oncology IOV - IRCCS, 35128 Padua, Italy

* Correspondence: laura.evangelista@iov.veneto.it; Tel.: +39-049-8217998; Fax: +39-049-8215508

+ Membership of Young AIMN Working Group is provided in the Acknowledgments.

Received: 11 July 2019; Accepted: 9 September 2019; Published: 11 September 2019

\begin{abstract}
Trans-1-amino-3- ${ }^{18} \mathrm{~F}$-fluorocyclobutanecarboxylic-acid (anti- $\left[{ }^{18} \mathrm{~F}\right]-\mathrm{FACBC}$ ) has been approved for the detection of prostate cancer (PCa) in patients with elevated prostate-specific-antigen following prior treatment. This review and meta-analysis aimed to investigate the diagnostic performance of ${ }^{18} \mathrm{~F}-\mathrm{FACBC}$ positron emission tomography/computed-tomography (PET/CT) in the detection of primary/recurrent $\mathrm{PCa}$. A bibliographic search was performed including several databases, using the following terms: "FACBC"/"fluciclovine" AND "prostate cancer"/"prostate" AND "PET"/"Positron Emission Tomography". Fifteen and 9 studies were included in the systematic reviews and meta-analysis, respectively. At patient-based analysis, the pooled sensitivity and specificity of ${ }^{18}$ F-FACBC-PET/CT for the assessment of PCa were $86.3 \%$ and $75.9 \%$, respectively. The pooled diagnostic odds-ratio value was 16.453 , with heterogeneity of $30 \%$. At the regional-based-analysis, the pooled sensitivity of ${ }^{18} \mathrm{~F}-\mathrm{FACBC}$-PET/CT for the evaluation of primary/recurrent disease in the prostatic bed was higher than in the extra-prostatic regions ( $90.4 \%$ vs. $76.5 \%$, respectively); conversely, the pooled specificity was higher for the evaluation of extra-prostatic region than the prostatic bed ( $89 \%$ vs. $45 \%$, respectively). ${ }^{18} \mathrm{~F}$-FACBC-PET/CT seems to be promising in recurrent PCa, particularly for the evaluation of the prostatic bed. Additional studies to evaluate its utility in clinical routine are mandatory.
\end{abstract}

Keywords: prostate cancer; ${ }^{18} \mathrm{~F}-\mathrm{FACBC}$; $\mathrm{PET} / \mathrm{CT}$; recurrence; meta-analysis; review 


\section{Introduction}

Prostate cancer $(\mathrm{PCa})$ is the most frequently detected type of cancer in men and constitutes a major healthcare problem in developed countries [1], remaining the second most common cause of cancer-related death in the Western world [2].

Following initial diagnosis, the majority of men receive several treatments, such as usually a radical prostatectomy \pm lymphadenectomy or radiation/brachytherapy in case of localized disease, and systemic therapy in case of widespread disease. Relapse remains common despite advances in primary treatment and improved overall survival (OS) with a biochemical recurrence developing in $20 \%$ to $40 \%$ of patients [3-6].

The management of primary and recurrent PCa patients has been completely changed after the inclusion of new imaging modalities, such as magnetic resonance imaging (MRI) and positron emission tomography (PET). MRI is a well-documented method to evaluate the extension of the primary tumor and to detect and localize recurrent cancer within the prostate [7-9]. However, routine multiparametric (mp) MRI is still limited by its poor specificity to differentiate significantly from indolent PCa [10].

In the last 10 years, PET/computed tomography (PET/CT) has gained an important role in the evaluation of patients with PCa. Radiolabeled choline PET/CT has demonstrated the ability to detect the presence of early recurrence of disease when conventional imaging resulted negative [11]. Furthermore, the recent introduction of radiolabeled prostate specific membrane antigen (PSMA), like ${ }^{68} \mathrm{Ga}-\mathrm{PSMA}$ and ${ }^{18}$ F-PSMA, has significantly improved the detection rate, also in case of early recurrence of disease (such as a prostate-specific antigen (PSA) $<0.5 \mathrm{ng} / \mathrm{mL}$ ) [12].

Trans-1-amino-3- ${ }^{18} \mathrm{~F}$-fluorocyclobutanecarboxylic acid (anti- $\left[{ }^{18} \mathrm{~F}\right]-\mathrm{FACBC}$ ) is an amino acid PET tracer that has shown to be promising for visualizing PCa. This tracer was developed for L-amino acid transport evaluation; it demonstrated favorable dosimetry with the liver being the critical organ [13]. Its safety, tracer stability, and uptake kinetics in patients have been reported in a phase I trial [14]. Nowadays, ${ }^{18} \mathrm{~F}-\mathrm{FACBC}$ is approved by the Food and Drug Administration (FDA) and the European Commission (EC) to detect PCa in patients with elevated PSA following prior treatment. Approval was based on encouraging diagnostic performance and histologically confirmed data on patients with biochemical recurrence [15]. Recently it was included in the National Comprehensive Cancer National (NCCN) guidelines for the management of recurrent PCa patients.

Until now, few pooled data have been published about the role of ${ }^{18} \mathrm{~F}-\mathrm{FACBC}$ PET/CT in patients with PCa. Ren et al. [16] collected data from six studies, published between 2011 and 2014 and including 251 patients that concluded for a good sensitivity of ${ }^{18} \mathrm{~F}-\mathrm{FACBC}$ PET/CT for the detection of PCa recurrence. In 2015, Yu et al. [17] published a critical analysis of the available tracers for PET/CT in PCa, collecting data for ${ }^{18} \mathrm{~F}$-FACBC from five studies ( $\mathrm{n}=84$ subjects), showing a limited detection rate of this imaging technique for the recurrence of post-prostatectomy $\mathrm{PCa}$ (detection rate $=40 \%$ ). However, in May 2016, ${ }^{18} \mathrm{~F}$-FACBC PET/CT received the approval by the Food and Drug administration for use in patients with suspected recurrent PCa [18]. In the last years, many prospective and retrospective experiences have been performed, and therefore, a new update of the recent findings seems necessary, not only in the restaging but also in the initial staging of disease.

Therefore, the present review and meta-analysis aimed to investigate the diagnostic performance of ${ }^{18} \mathrm{~F}-\mathrm{FACBC}$ in the detection of primary and recurrent $\mathrm{PC}$ a patients.

\section{Materials and Methods}

\subsection{Search Strategy and Study Selection}

A bibliographic search until 30 April 2019 was performed by including the following databases: Pubmed, Scopus, Embase, Web of Science, Cochrane library, and Google Scholar. The terms used were "FACBC" or "fluciclovine" AND "prostate cancer" or "prostate" AND "PET" or "Positron 
Emission Tomography". The search was carried out with and without the addition of filters (such as English language only; type of article: original article, research article; subjects: humans only). Three reviewers (Domenico Albano, Viviana Frantelizzi and Matteo Baucknhet) performed the literature search, and two independent reviewers (Priscilla Guglielmo and Lorenzo Fantechi) selected the study inclusion and data extraction in duplicate. Any discrepancies were resolved by a consensus, when necessary. All recognized records were combined, and the full texts were retrieved. Full texts were further evaluated by four reviewers (Giovanni Argiroffi, Riccardo Laudicella, Pierpaolo Alongi and Laura Evangelista). Moreover, a search across the databases was completed by another reviewer (Anna Giulia Nappi) checking the references of the studies included to further improve the eligibility.

This systematic review was carried out using established methods [19], and the presentation of results was made according to the PRISMA guidelines [20]. All studies that fulfilled the inclusion criteria were considered eligible for the systematic review and meta-analysis: (a) a sample size more than 10 patients; (b) the index test: ${ }^{18} \mathrm{~F}-\mathrm{FACBC}$ PET/CT; (d) the outcomes, such as detection rate (DR), true positive (TP), true negative (TN), false positive (FP), and false negative (FN), which allowed us to construct $2 \times 2$ contingency tables. Moreover, in the case of studies that included the same population, the report with the highest number of enrolled patients was considered for the meta-analysis. Conversely, reviews, clinical reports, meeting abstracts, and editor comments were excluded. The quality assessment included both the risk of bias assessment and applicability concerns by using QUADAS-2 evaluation [21].

\subsection{Data Extraction}

For each included study, general information was retrieved, such as basic data (authors, journal, year of publication, country and study design), patient characteristics (number of patients, mean or median age, Gleason score), type of treatment, mean or median PSA value at PET time, and PSA kinetic values.

\subsection{Statistical Method}

StatsDirect and Meta-Analyst (version Beta 3.13; [22]) were used to carry out the analysis. Heterogeneity was tested using the $\chi^{2}$ and the I2 tests. The $\chi^{2}$-test provided an estimate of the between-study variance and the I2 test measured the proportion of inconsistency in individual studies that cannot be explained by chance. According to Higgins et al. [19], the values of $25 \%, 50 \%$, and $75 \%$ for heterogeneity (I2) were considered low, moderate, and high, respectively. In accordance with the recommendation of the Cochrane Oral Health Group, the meta-analysis was carried out with the random-effect model as the number of studies was equal or superior to 4 .

Data on diagnostic performance such as pooled sensitivity, pooled specificity, positive and negative likelihood ratio (LR+ and LR-), diagnostic odds ratio (DOR) with 95\% confidence intervals (CIs) for the evaluation of primary and recurrent PCa, were assessed. A patient-based and a region-based meta-analyses were carried out in accordance with available data. Publication bias was assessed using a funnel plot. A symmetrical plot was indicative of the absence of publication bias.

\section{Results}

\subsection{Search Results}

The literature search revealed 40 articles published from 1 January 2007 to 30 April 2019. Reviewing titles and abstracts, we excluded 24 articles because these did not fit with the field of interest or because these papers were letters, editorials, reviews or due to the patient data overlap. Therefore, 15 studies were selected and included in the systematic reviews and 9 articles were considered for the meta-analysis (Figure 1). Also the papers by the developers of ${ }^{18} \mathrm{~F}-\mathrm{FACBC}$ were considered $[23,24]$. 


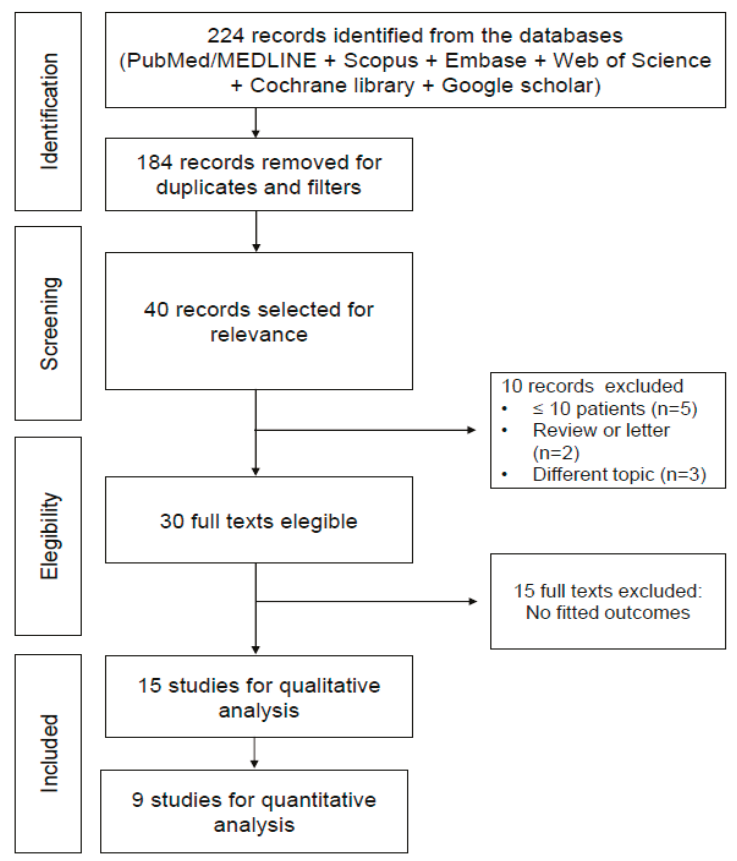

Figure 1. PRISMA flow-chart.

\subsection{Study Characteristics}

The basics characteristics of the included studies are reported in Table 1 [15,23-36]. The number of enrolled patients ranged from 15 to 596, and a total of 1226 PCa patients were included. The selected articles were published by researchers from Europe, USA, and Japan. Four studies were retrospective whereas 11 studies were prospective. ${ }^{18} \mathrm{~F}$-FACBC PET/CT was performed in the preoperative setting in 6 studies ( $n=178$ patients), for the detection of recurrence in patients with biochemical relapse after primary treatments in 8 studies ( $\mathrm{n}=1033$ patients) and in both settings in 1 study ( $n=15$ patients). In the restaging, the mean value of PSA ranged between 0.44 and $17.94 \mathrm{ng} / \mathrm{mL}$.

The mean and median age of the patients ranged from 42 to 90 years. The Gleason score (GS) was $\leq 6$ in $49(4 \%)$ patients, 7 in $376(30.6 \%)$ patients, $\geq 8$ in 142 (11.6\%) patients, not available in the remaining $659(53.8 \%)$. No significant adverse effects after the administration of ${ }^{18} \mathrm{~F}-\mathrm{FACBC}$ were reported. 


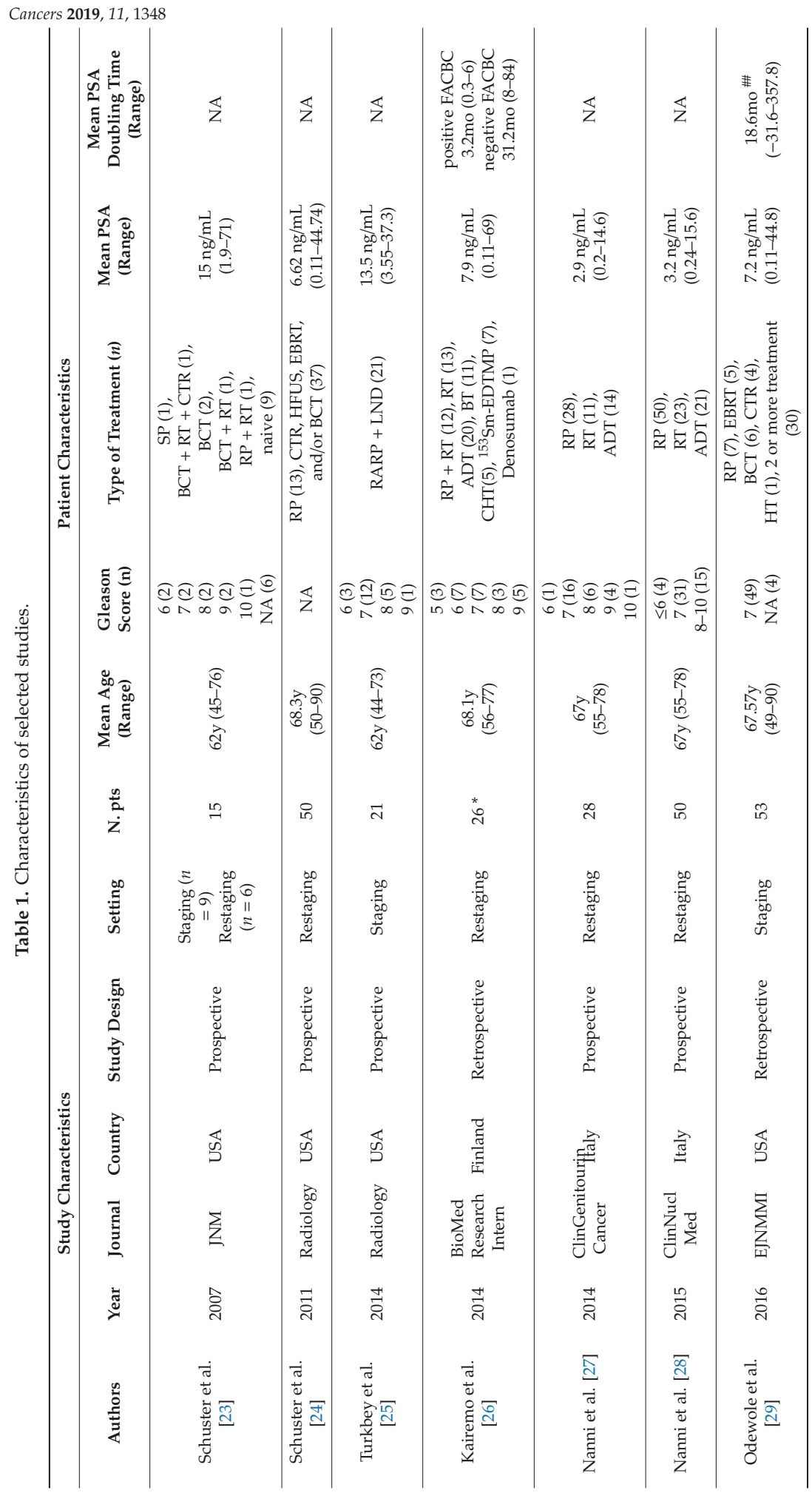




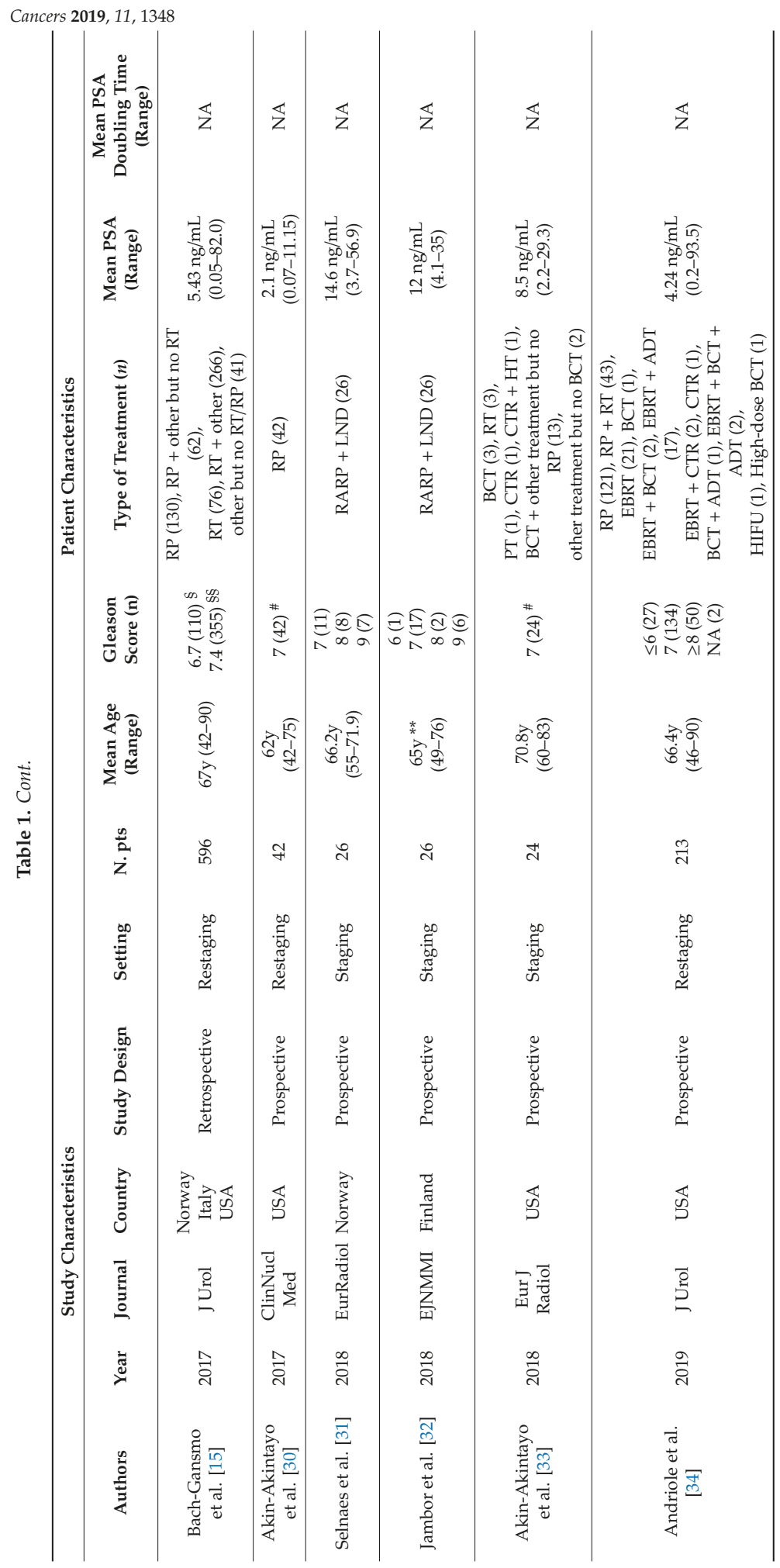




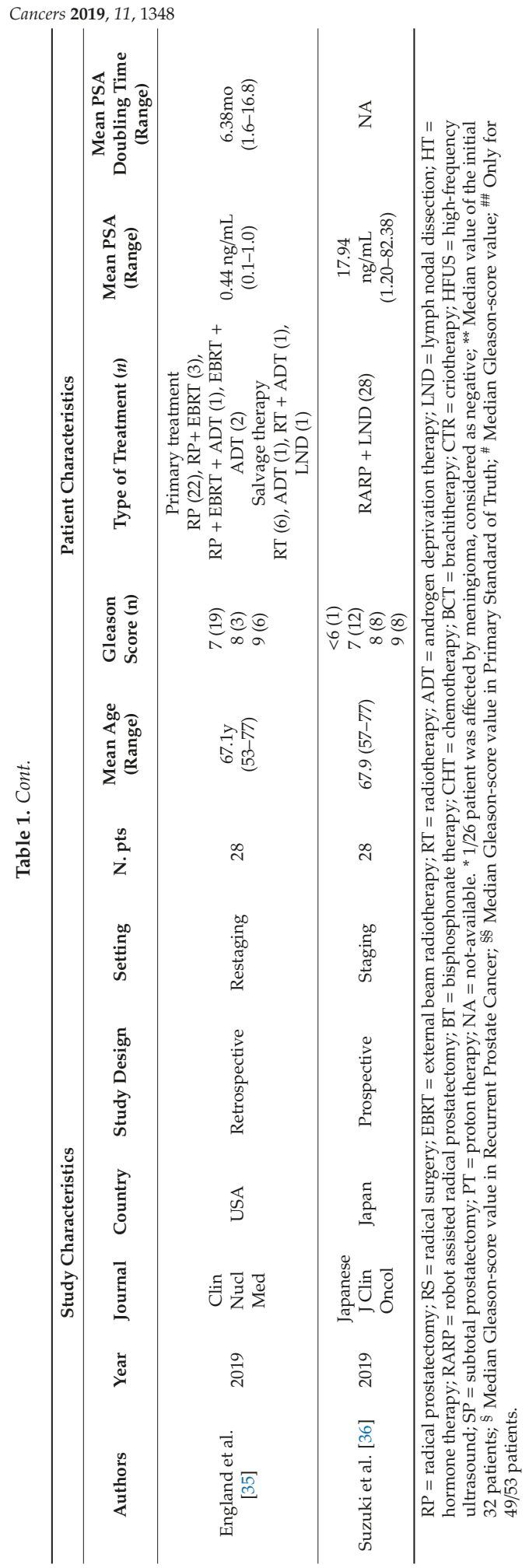




\subsection{Methodological Quality}

All 15 studies were evaluated qualitatively using the QUADAS-2 tool (Table S1; Figure 2). The risk of bias was unclear for patient selection in 1 study, which did not provide information regarding consecutive enrollment [15]. For the index test and reference standard, the risk of bias was low in 6 studies $[24,29,31-33,36]$. For flow and timing, many studies reported time intervals between PET/CT examinations and pathological or other imaging confirmations. The applicability of the included studies was adequate in the majority of reports, being unclear only in 1 study for the reference standard [30].

\section{Risk of Bias}

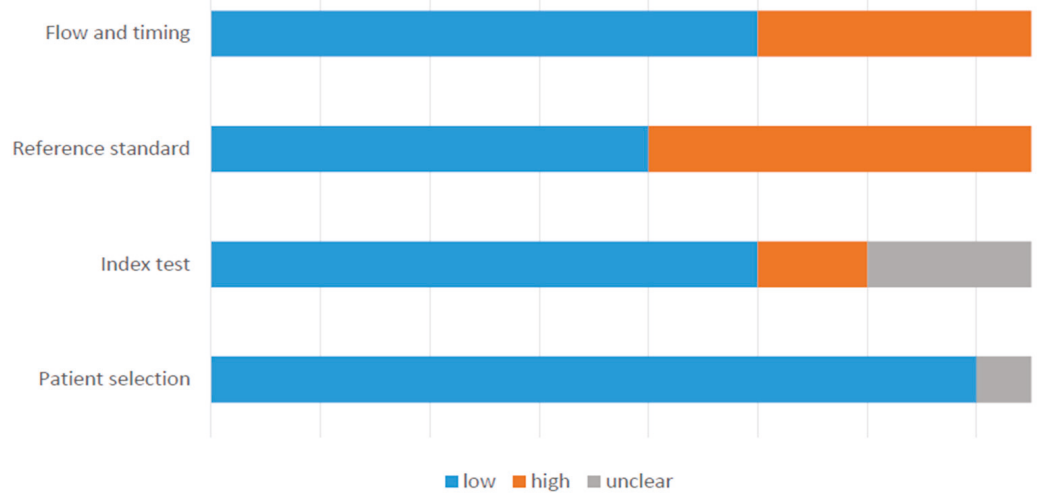

Applicability Concerns

Reference standard

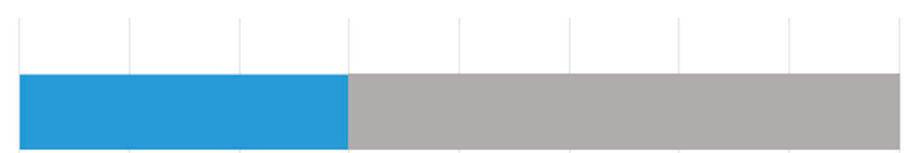

Index test
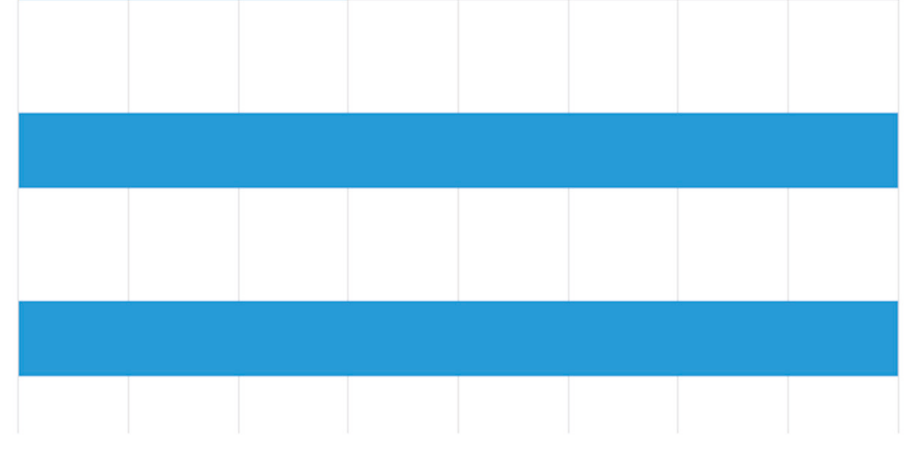

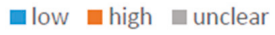

Figure 2. QUADAS 2 score of all included studies. 


\subsection{Qualitative Results}

PET/CT was employed in 14/15 studies, without CT contrast media injection, whereas PET/MRI was used in 2 studies [31,32]. The injected radiopharmaceutical activity and the time between radiotracer injection and image acquisition were similar across all studies.

Analysis of PET images was mostly performed using visual analysis; however, additional semi-quantitative criteria, i.e., maximal standardized uptake values (SUVmax), was performed in some reports [23,26-28]. ${ }^{18} \mathrm{~F}-\mathrm{FACBC}$ PET/CT or PET/MRI identified the presence of PCa in prostatic and extra-prostatic bed, such as in the regional, distant lymph nodes and bone. The DR was available in $9 / 15$ studies. It ranged between $36 \%$ and $90 \%$, being different in accordance with PSA serum levels (Table 2). Andriole et al. [34] demonstrated that DR was broadly proportional to pre-scan PSA: lesions were detected in $79 \%$ patients with PSA $\geq 1.0 \mathrm{ng} / \mathrm{mL}$ and in $84 \%$ with PSA $\geq 2.0 \mathrm{ng} / \mathrm{mL}$. On the other side, some authors found that there was no statistically significant difference in the PSA values and PSA doubling-time (PSAdt) between patients with positive and negative findings [26,35]. England et al. [35] reported that the DR was significantly higher for patients with GS $>7$ than those with a score equal to 7 .

The performance of ${ }^{18} \mathrm{~F}$-FACBC PET/CT was different based on the phase and the site of PCa (Table 3). In particular, in the initial staging, the sensitivity for the primary and lymph nodes metastasis was $71 \%$ [32] and $67 \%$ [36], respectively. In the restaging setting, the sensitivity for the prostatic bed and extra-prostatic bed recurrence was $89 \%$ [24] and 90\% [15], respectively. Interestingly, in the study by Turkbey et al. [25], ${ }^{18} \mathrm{~F}-\mathrm{FACBC}$ uptake in tumors was similar to that in benign prostatic hyperplasia $(\mathrm{BPH})$. However, Jambor et al. [32] reported that SUVmax in the primary tumor was statistically significantly higher for patients with GS $>7$ than GS $=6$ or BPH, thus underlying the importance of the patient selection.

Akin-Akintayo et al. [33] compared ${ }^{18} \mathrm{~F}-\mathrm{FACBC}$ PET/CT with mpMRI in patients with recurrent PCa showing a higher detection for the first modality (overall $94.7 \%$ vs. $36.8 \%$ ); Turkbey et al. [25], instead, performed a sector-based comparison with histopathologic analysis in patients with a recent diagnosis of PCa, revealing lower sensitivity and specificity for ${ }^{18} \mathrm{~F}-\mathrm{FACBC}$ PET/CT than for T2-weighted imaging ( $67 \%$ and $66 \%$ vs. $73 \%$ and $79 \%$, respectively), but combined modalities achieved a positive predictive value of $82 \%$ for tumor localization, which was higher than that with either modality alone. Another study proved higher positivity rates with ${ }^{18} \mathrm{~F}-\mathrm{FACBC}$ PET/CT than enhanced CT at all PSA levels, PSAdt and GS in patients with suspected recurrent PCa [29]. Furthermore, the performance of ${ }^{18} \mathrm{~F}$-FACBC PET/CT was superior to those of ${ }^{111} \mathrm{In}$-capromab SPECT/CT regarding sensitivity for prostatic and extra-prostatic bed ( $89 \%$ vs. $69 \%$ and $100 \%$ vs. 10\%, respectively) [24]. Finally, two studies directly compared ${ }^{18} \mathrm{~F}$-FACBC with ${ }^{11} \mathrm{C}$-Choline $\mathrm{PET} / \mathrm{CT}$, demonstrating a greater detection rate for ${ }^{18} \mathrm{~F}-\mathrm{FACBC}$ than $11 \mathrm{C}-\mathrm{Choline}$, either on a patient- and a lesion-based analysis and despite the PSA serum levels $[27,28]$.

The change of management with ${ }^{18} \mathrm{~F}-\mathrm{FACBC}$ PET/CT was reported by Andriole et al. [34], in 122 out of 213 patients (56\%); the most frequent change was to withhold planned salvage or non-curative systemic therapy in favor of watchful waiting. Moreover, Akin-Akintayo et al. [30] demonstrated that ${ }^{18} \mathrm{~F}-\mathrm{FACBC}$ PET/CT was able to modify the radiotherapy field and overall radiotherapy decision in $40.5 \%$ of patients with post-prostatectomy recurrent $\mathrm{PCa}$. 


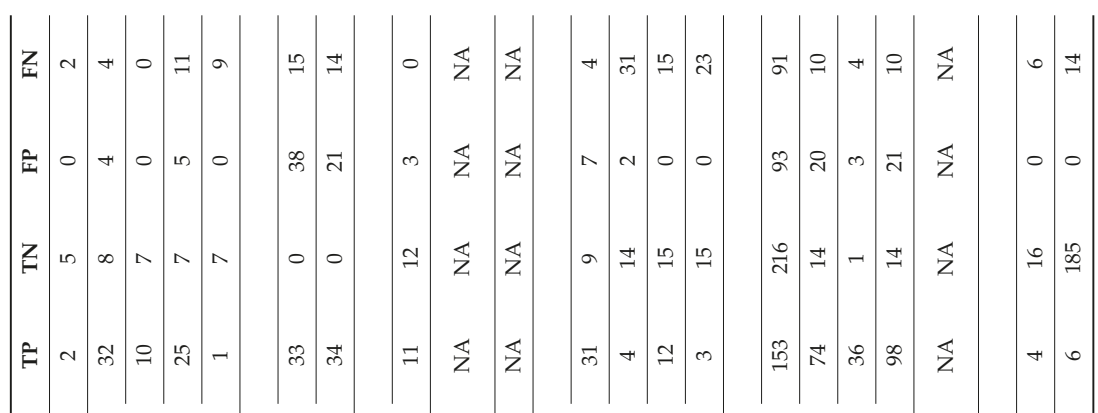

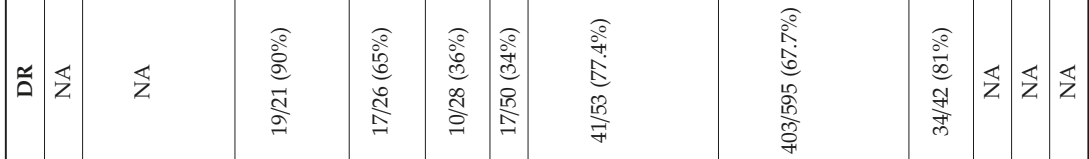

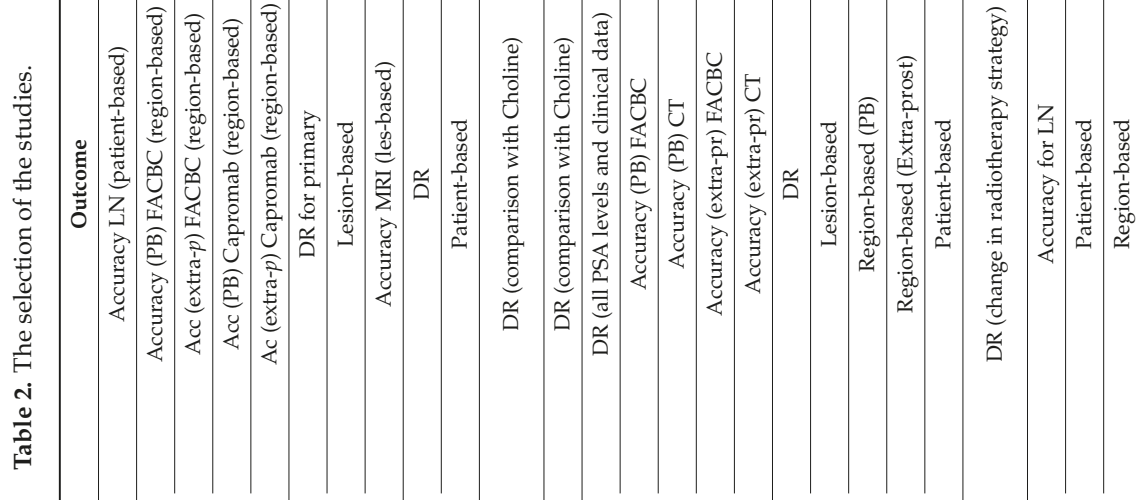

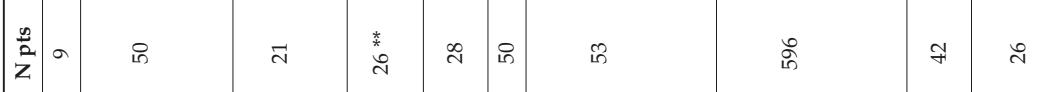

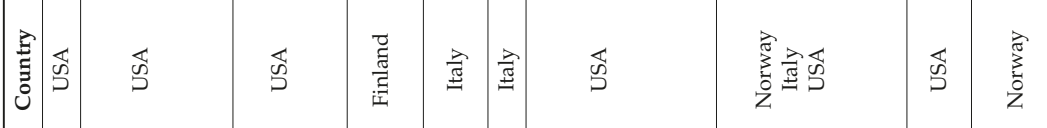

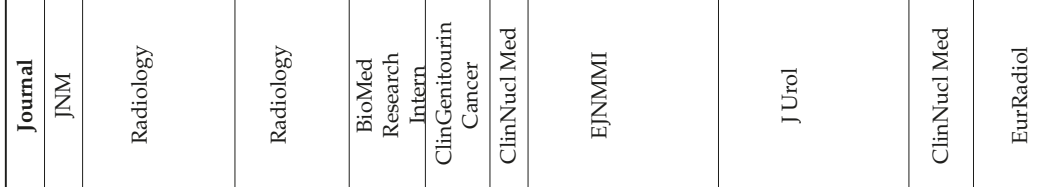

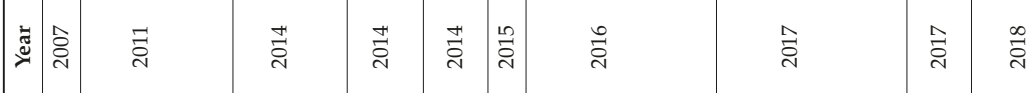

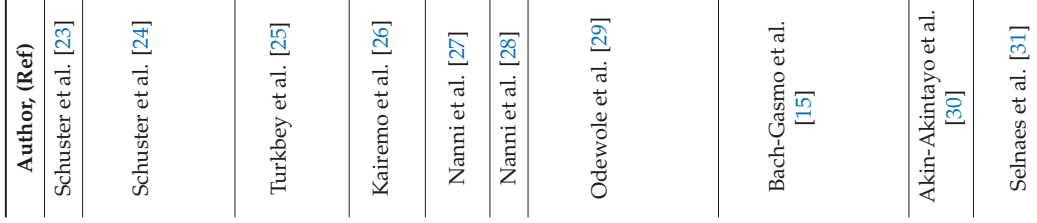


Cancers 2019, 11, 1348

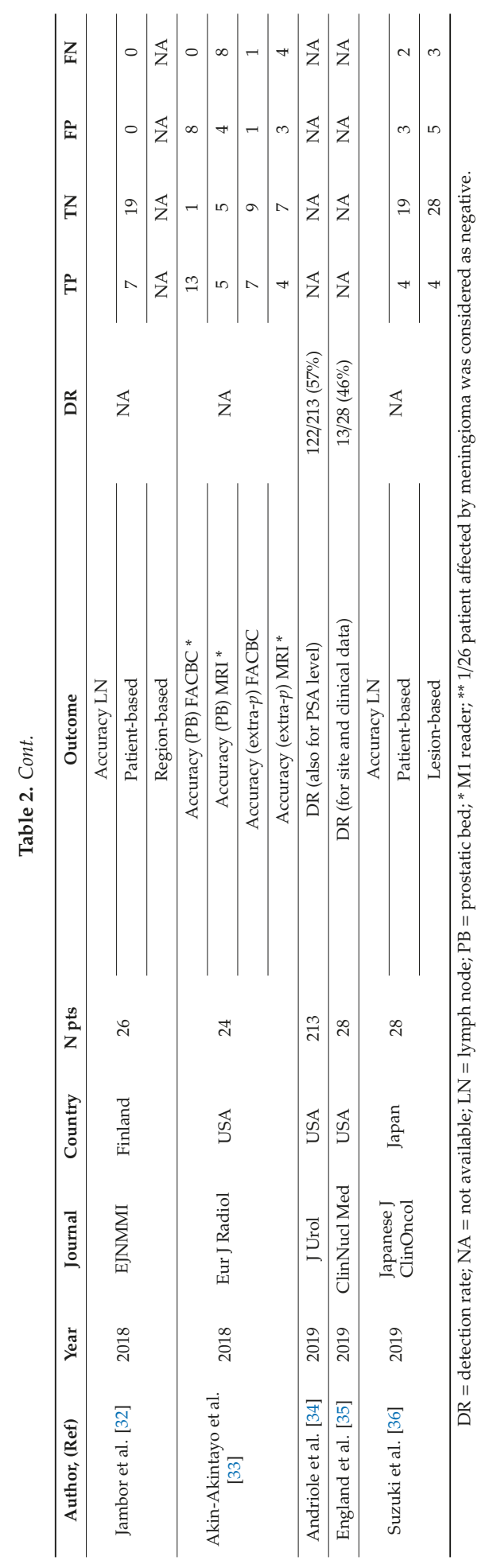




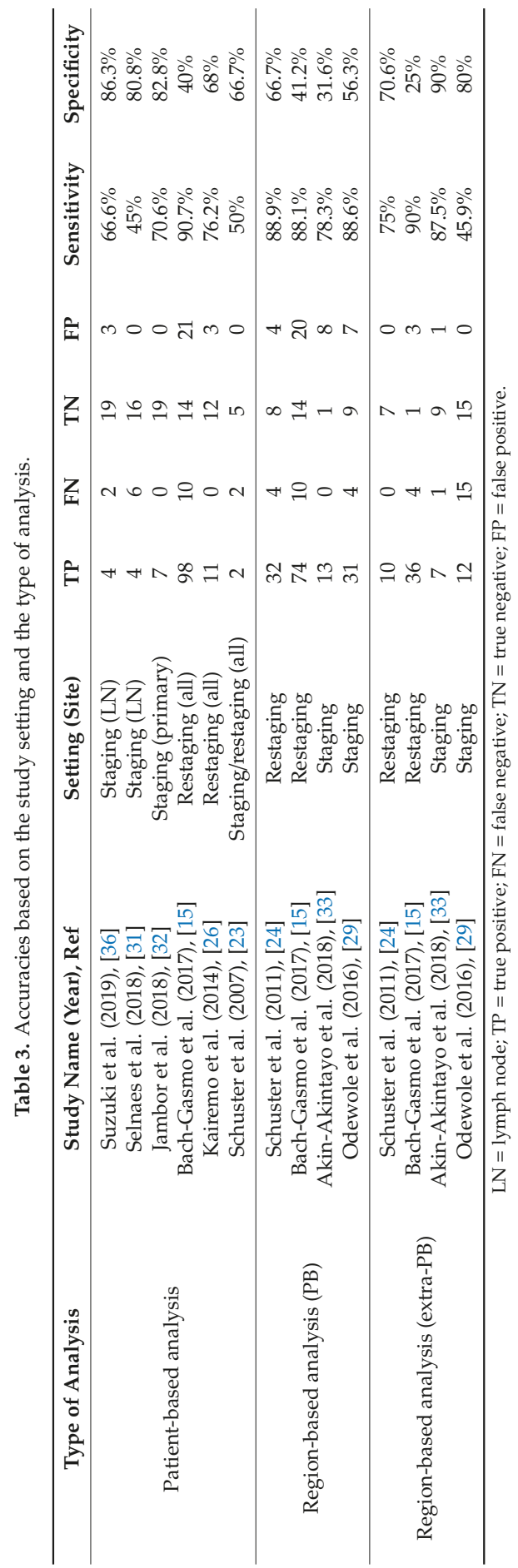




\subsection{Quantitative Results}

In accordance with the inclusion criteria, the quantitative assessment was available in 9 studies $[15,23,24,26,29,31-33,36]$ (Table 4$)$. At patient-based analysis ( $\mathrm{n}=6$ studies), the pooled sensitivity and specificity of ${ }^{18} \mathrm{~F}-\mathrm{FACBC}$ PET/CT scan for the assessment of primary and recurrent PCa were $86.3 \%$ (95\% CIs: $79.6-91.4 \%)$ and $75.9 \%(66.9-83.5 \%)$ with an heterogeneity of $78.6 \%$ and $88.7 \%$ (both $p<0.0001$ ), respectively. Moreover, the pooled DOR value was 16.453 (95\% CI: 5.241-51.646), with heterogeneity of $30 \%$. At the regional based-analysis ( $n=4$ studies), the pooled sensitivity of ${ }^{18} \mathrm{~F}$-FACBC PET/CT for the evaluation of primary and recurrent disease in the prostatic bed was higher than that in the extra-prostatic regions ( $90.4 \%$ vs. $76.5 \%$, respectively); conversely, the pooled specificity was higher for the evaluation of extra-prostatic region than the prostatic bed ( $89 \%$ vs. $45 \%$, respectively). Furthermore, LR+ was high in the extra-prostatic region, while LR- was low in prostatic bed, with heterogeneity of $0 \%$. No asymmetry in the forest plot was found; therefore, no publication bias was present across the studies.

Table 4 . The pooled diagnostic performance for ${ }^{18} \mathrm{~F}$-FACBC (independently from the clinical setting and site).

\begin{tabular}{|c|c|c|c|c|c|c|}
\hline \multirow{2}{*}{$\begin{array}{l}\text { Meta-Analysis } \\
\text { Results }\end{array}$} & \multicolumn{2}{|c|}{$\begin{array}{l}\text { Patient-Based Analysis } \\
(95 \% \mathrm{CI})\end{array}$} & \multicolumn{2}{|c|}{$\begin{array}{l}\text { Region-Based Analysis } \\
\text { (PB) }(95 \% \text { CI) }\end{array}$} & \multicolumn{2}{|c|}{$\begin{array}{c}\text { Region-Based Analysis } \\
\text { (ex-PB) }(95 \% \text { CI })\end{array}$} \\
\hline & Value & I2 & Value & I2 & Value & I2 \\
\hline Pooled sensitivity, $\%$ & $\begin{array}{c}86.3 \% \\
(79.6-91.4 \%)\end{array}$ & $78.6 \%$ & $\begin{array}{c}90.4 \% \\
(84.8-94.4 \%)\end{array}$ & $22.1 \%$ & $\begin{array}{c}76.5 \% \\
(66-85 \%)\end{array}$ & $87.3 \%$ \\
\hline Pooled specificity, \% & $\begin{array}{c}75.9 \% \\
(66.9-83.5 \%)\end{array}$ & $88.7 \%$ & $\begin{array}{c}45.1 \% \\
(33.2-57.3 \%)\end{array}$ & $63.3 \%$ & $\begin{array}{c}88.9 \% \\
(73.9-96.9 \%)\end{array}$ & $78.7 \%$ \\
\hline DOR & $\begin{array}{c}16.453 \\
(5.241-51.646)\end{array}$ & $29.9 \%$ & $\begin{array}{c}8.026 \\
(3.841-16.769)\end{array}$ & $3.5 \%$ & $\begin{array}{c}24.820 \\
(3.777-163.12)\end{array}$ & $36 \%$ \\
\hline LR+ & $\begin{array}{c}4.557 \\
(1.685-12.324)\end{array}$ & $72.9 \%$ & $\begin{array}{c}1.598 \\
(1.088-2.349)\end{array}$ & $70 \%$ & $\begin{array}{c}6.024 \\
(0.568-63.943)\end{array}$ & $85.6 \%$ \\
\hline LR- & $\begin{array}{c}0.337 \\
(0.166-0.681)\end{array}$ & $63.6 \%$ & $\begin{array}{c}0.221 \\
(0.130-0.375)\end{array}$ & $0 \%$ & $\begin{array}{c}0.251 \\
(0.058-1.090)\end{array}$ & $71.6 \%$ \\
\hline
\end{tabular}

$\mathrm{PB}=$ prostatic bed; $\mathrm{PPV}=$ positive predictive value; $\mathrm{NPV}=$ negative predictive value; $\mathrm{DOR}=$ diagnostic odds ratio; $\mathrm{LR}=$ likelihood ratio; $\mathrm{IC}=$ interval of confidence; $\mathrm{I} 2=$ inconsistency.

\section{Discussion}

As previously mentioned, the meta-analysis from Ren et al. [16] reported that 18F-FACBC PET/CT had a high sensitivity (pooled sensitivity $=87 \%$ ) and a moderate specificity (pooled specificity $=66 \%$ ), therefore it can be considered an useful non-invasive, metabolic imaging technique for the diagnostic workup of PCa relapse. In the present meta-analysis, performed in $1226 \mathrm{PCa}$, the pooled sensitivity and specificity were $86 \%$ and $76 \%$ respectively, thus showing a slight increase for the specificity.

Furthermore, in the analysis by Yu et al. [17], FACBC showed a detection rate ranged between $22 \%$ and $61 \%$ for prostatic disease and between $19 \%$ and $33 \%$ for extra-prostatic disease, in accordance with the primary treatments (radical prostatectomy or radiotherapy). In our meta-analysis, we did not evaluate the pooled detection rate, but we calculated the pooled sensitivity and specificity. As illustrated in Table 4 , the sensitivity of $18 \mathrm{~F}-\mathrm{FACBC}$ was equal to $90 \%$ for the identification of disease in the prostatic bed and $77 \%$ for extra-prostatic organs.

However, in the last years, PSMA-PET has rapidly been introduced in clinical practice for the management of patients with recurrent PCa, particularly in case of low PSA levels [37]. Already, the study by Yu C-Y et al. [17] reported that 18F-FACBC, Choline and Acetate-PET have similar detection rate for overall site of disease after radical prostatectomy or radiotherapy (ranged between $40 \%$ and $81 \%$ ), but PSMA was able to reach a detection rate ranged between $82 \%$ and $96 \%$ in the same setting. 
Two recent papers about a head-to-head comparison between 18F-FACBC and 68Ga-PSMA PET/CT have been published. The data are controversial. In the study by Pernthaler et al. [38] involving 58 patients with recurrent PCa with a PSA level ranged between 0.2 and $230 \mathrm{ng} / \mathrm{mL}, 18 \mathrm{~F}-\mathrm{FACBC}$ detected more accurately the presence of a local recurrence than 68Ga-PSMA, due to its favorable biodistribution. Furthermore, the authors found that $18 \mathrm{~F}-\mathrm{FACBC}$ is almost equivalent to 68Ga-PSMA-11 in detecting distant metastases of PCa recurrence. Conversely, in the study by Calais et al. [39] enrolling 50 patients with recurrent PCa, the detection rate of PSMA-PET was significantly higher than $18 \mathrm{~F}-\mathrm{FACBC}$ ( $56 \%$ vs. $26 \%$, respectively) in case of a PSA level $<1 \mathrm{ng} / \mathrm{mL}$. However, the authors found that the detection rate for the local recurrence was higher for 18F-FACBC than 68Ga-PSMA PET/CT (38\% vs. 14\%, respectively). The missing data about the diagnostic performance, in terms of sensitivity and specificity in both the above-mentioned papers, represent a great limitation for the final conclusion on "the best radiopharmaceutical agent". A recent paper by Lawhn-Heath et al. [40] reported that the sensitivity and specificity of 68Ga-PSMA-11 for recurrent PCa are equal to $89.1 \%$ and $31.2 \%$, thus registering a high rate of false positivity.

From the present systematic review and meta-analysis arise some considerations:

1. ${ }^{18} \mathrm{~F}$-FACBC is more performant than ${ }^{111} \mathrm{In}$-capromab SPECT/CT and ${ }^{11} \mathrm{C}$-Choline for the detection of PCa recurrence. Therefore, if available it should be preferred in patients with a PSA increase, after primary treatments. However, data about the comparison with ${ }^{18} \mathrm{~F}-\mathrm{Choline} \mathrm{PET/CT}$ are missing and should be explored, also considering the radioisotope properties.

2. The combination of ${ }^{18}$ F-FACBC PET/CT with mpMRI (or with a PET/MRI) seems useful for the detection of primary $\mathrm{PCa}$, and therefore, it would be suggested in case of undetectable tumors in patients with a negative biopsy but a persistent PSA level increase. However, the interpretation of this sophisticated imaging required a great experience and a significant learning curve.

3. The sensitivity for the evaluation of lymph node metastasis in the initial staging of disease is moderate (45\%-66\%; [31,36]), like for the other radiopharmaceuticals (radiolabeled PSMA and Choline; [41,42]). Probably the recent introduction of new imaging modalities, such as digital PET/CT or PET/MRI that has a higher spatial resolution, would improve the pathological lymph node detection.

4. The pooled sensitivity for the identification of recurrence in prostate bed is high, being $>90 \%$ with a limited pooled specificity (about 45\%), probably due to the FP findings in case of inflamed cells, as reported by Oka et al. [43]. However, the absent uptake of radiopharmaceutical in the bladder represents a great advantage for the identification of peri-anastomotic PCa recurrence. Further data about the complementary role of ${ }^{18} \mathrm{~F}-\mathrm{FACBC}$ and $\mathrm{MRI}$ are required for the assessment of prostatic bed recurrence, at different PSA levels.

5. The recurrence in the extra-prostatic site may be assessed by ${ }^{18}$ F-FACBC PET/CT with a moderate sensitivity and specificity, independently from the PSA levels. However, the correlation with PSA kinetics is warranted in a selected large cohort of patients, thus testing the final impact on the patient management.

6. Despite some articles have defined a potential impact of ${ }^{18}$ F-FACBC PET/CT on therapeutic management, there is still a lack information with regard to its role in radiotherapy planning and other adapted therapy.

\section{Future Researches}

More data about the correlation between the detection rate of ${ }^{18} \mathrm{~F}-\mathrm{FACBC}$ PET/CT or PET/MRI and the PSA kinetics are warranted, particularly by a site and lesion-based analysis. The complementary role of ${ }^{18}$ F-FACBC PET/CT and mpMRI for the evaluation of the prostatic bed should be largely explored. A head-to-head comparison with ${ }^{18} \mathrm{~F}$-Choline would be used in order to definitely assess its advantages in clinical routine. Data about the utility of ${ }^{18} \mathrm{~F}-\mathrm{FACBC}$ PET/CT in patients undergoing or not hormonal therapy are required. The evaluation of response to therapy (chemotherapy or 
new hormonal agents) by ${ }^{18}$ F-FACBC PET/CT should be assessed. Finally, additional data about the effect of ${ }^{18} \mathrm{~F}-\mathrm{FACBC}$ PET/CT on patient management is required, by considering both PSA levels and histopathological PCa characteristics.

\section{Conclusions}

${ }^{18} \mathrm{~F}$-FACBC PET/CT seems to be promising in recurrent PCa, particularly for the evaluation of the prostatic bed. However, additional studies are mandatory in order to evaluate its utility in clinical routine.

Supplementary Materials: The following are available online at http://www.mdpi.com/2072-6694/11/9/1348/s1, Table S1: QUADAS 2 score for each selected study (green smile = low risk; red smile = high risk; yellow question mark = unclear). Figure S1: Forest-plots for the patient-based and region-based analysis. Figure S2: ROC curves for patient-based and region-based analyses.

Author Contributions: Substantial contributions to the conception or design of the work; or the acquisition, analysis, or interpretation of data for the work: Priscilla Guglielmo, Lorenzo Fantechi, Giovanni Argiroffi, Riccardo Laudicella, Pierpaolo Alongi and Laura Evangelista. Drafting the work or revising it critically for important intellectual content: all authors. Final approval of the version to be published: all authors.

Funding: This research received no external funding.

Acknowledgments: Agreement to be accountable for all aspects of the work in ensuring that questions related to the accuracy or integrity of any part of the work are appropriately investigated and resolved.: all authors.

Conflicts of Interest: The authors declare no conflict of interest.

\section{References}

1. Torre, L.A.; Bray, F.; Siegel, R.L.; Ferlay, J; Lortet-Tieulent, J.; Jemal, A. Global cancer statistics, 2012. CA Cancer J. Clin. 2015, 65, 87-108. [CrossRef] [PubMed]

2. Siegel, R.L.; Miller, K.D.; Jemal, A. Cancer statistics, 2016. CA Cancer J. Clin. 2016, 66, 7-30. [CrossRef] [PubMed]

3. Kim, M.M.; Hoffman, K.E.; Levy, L.B.; Frank, S.J.; Pugh, T.J.; Choi, S.; Nguyen, Q.N.; McGuire, S.E.; Lee, A.K.; Kuban, D.A. Improvement in prostate cancer survival over time: A 20-year analysis. Cancer J. 2012, 18, 1-8. [CrossRef] [PubMed]

4. Bruce, J.Y.; Lang, J.M.; McNeel, D.G.; Liu, G. Current controversies in the management of biochemical failure in prostate cancer. Clin. Adv. Hematol. Oncol. 2012, 10, 716-722. [PubMed]

5. Roehl, K.A.; Han, M.; Ramos, C.G.; Antenor, J.A.V.; Catalona, W.J. Cancer progression and survival rates following anatomical radical retropubic prostatectomy in 3478 consecutive patients: Long-term results. J. Urol. 2004, 172, 910-914. [CrossRef] [PubMed]

6. Simmons, M.N.; Stephenson, A.J.; Klein, E.A. Natural history of biochemical recurrence after radical prostatectomy: Risk assessment for secondary therapy. Eur. Urol. 2007, 51, 1175-1184. [CrossRef] [PubMed]

7. Ahmed, H.U.; El-ShaterBosaily, A.; Brown, L.C.; Gabe, R.; Kaplan, R.; Parmar, M.K.; Collaco-Moraes, Y.; Ward, K.; Hindley, R.G.; Freeman, A.; et al. Diagnostic accuracy of multi-parametric MRI and TRUS biopsy in prostate cancer (PROMIS): A paired validating confirmatory study. Lancet (Lond. Engl.) 2017, 389, 815-822. [CrossRef]

8. Panebianco, V.; Barchetti, F.; Grompone, M.D.; Colarieti, A.; Salvo, V.; Cardone, G.; Catalano, C. Magnetic resonance imaging for localization of prostate cancer in the setting of biochemical recurrence. Urol. Oncol. Semin. Orig. Investig. 2016, 34, 303-310. [CrossRef] [PubMed]

9. Abd-Alazeez, M.; Ramachandran, N.; Dikaios, N.; Ahmed, H.U.; Emberton, M.; Kirkham, A.; Arya, M.; Taylor, S.; Halligan, S.; Punwani, S. Multiparametric MRI for detection of radiorecurrent prostate cancer: Added value of apparent diffusion coefficient maps and dynamic contrast-enhanced images. Prostate Cancer Prostatic Dis. 2015, 18, 128-136. [CrossRef]

10. Johnson, D.C.; Reiter, R.E. Multi-parametric magnetic resonance imaging as a management decision tool. Transl. Androl. Urol. 2017, 6, 472-482. [CrossRef]

11. Wallitt, K.L.; Khan, S.R.; Dubash, S.; Tam, H.H.; Khan, S.; Barwick, T.D. Clinical PET Imaging in Prostate Cancer. Radiographics 2017, 37, 1512-1536. [CrossRef] [PubMed] 
12. Farolfi, A.; Ceci, F.; Castellucci, P.; Graziani, T.; Siepe, G.; Lambertini, A.; Schiavina, R.; Lodi, F.; Morganti, A.G.; Fanti, S. 68Ga-PSMA-11 PET/CT in prostate cancer patients with biochemical recurrence after radical prostatectomy and PSA. Eur. J. Nucl. Med. Mol. Imaging 2019, 46, 11-19. [CrossRef] [PubMed]

13. Nye, J.A.; Schuster, D.M.; Yu, W.; Camp, V.M.; Goodman, M.M.; Votaw, J.R. Biodistribution and radiation dosimetry of the synthetic nonmetabolized amino acid analogue anti-18F-FACBC in humans. J. Nucl. Med. 2007, 48, 1017-1020. [CrossRef] [PubMed]

14. Sörensen, J.; Owenius, R.; Lax, M.; Johansson, S. Regional distribution and kinetics of [18F] fluciclovine (anti-[18F]FACBC), a tracer of amino acid transport, in subjects with primary prostate cancer. Eur. J. Nucl. Med. Mol. Imaging 2013, 40, 394-402. [CrossRef] [PubMed]

15. Bach-Gansmo, T.; Nanni, C.; Nieh, P.T.; Zanoni, L.; Bogsrud, T.V.; Sletten, H.; Korsan, K.A.; Kieboom, J.; Tade, F.I.; Odewole, O; et al. Multisite Experience of the Safety, Detection Rate and Diagnostic Performance of Fluciclovine (18F) Positron Emission Tomography/Computerized Tomography Imaging in the Staging of Biochemically Recurrent Prostate Cancer. J. Urol. 2017, 197, 676-683. [CrossRef]

16. Ren, J.; Yuan, L.; Wen, G.; Yang, J. The value of anti-1-amino-3-18F-fluorocyclobutane-1-carboxylic acid $\mathrm{PET} / \mathrm{CT}$ in the diagnosis of recurrent prostate carcinoma: A meta-analysis. Actaradiologica 2016, 57, 487-493. [CrossRef]

17. Yu, C.-Y.; Desai, B.; Ji, L.; Groshen, S.; Jadvar, H. Comparative performance of PET tracers in biochemical recurrence of prostate cancer: A critical analysis of literature. Am. J. Nucl. Med. Mol. Imaging 2014, 4, 580.

18. DA Approves 18F-Fluciclovine and 68Ga-DOTATATE Products. Available online: http://jnm.snmjournals. org/content/57/8/9N.full.pdf (accessed on 1 September 2019).

19. Higgins, J.P.T.; Thompson, S.G.; Deeks, J.J.; Altman, D.G. Measuring inconsistency in meta-analyses. BMJ 2003, 327, 557-560. [CrossRef]

20. Moher, D.; Liberati, A.; Tetzlaff, J.; Altman, D.G.; PRISMA Group. Preferred reporting items for systematic reviews and meta-analyses: The PRISMA statement. Ann. Intern. Med. 2009, 151, 264-269. [CrossRef]

21. Whiting, P.F.; Rutjes, A.W.S.; Westwood, M.E.; Mallett, S.; Deeks, J.J.; Reitsma, J.B.; Leeflang, M.M.G.; Sterne, J.A.C.; Bossuyt, P.M.M.; QUADAS-2 Group. QUADAS-2: A revised tool for the quality assessment of diagnostic accuracy studies. Ann. Intern. Med. 2011, 155, 529-536. [CrossRef]

22. Wallace, B.C.; Schmid, C.H.; Lau, J.; Trikalinos, T.A. Meta-Analyst: Software for meta-analysis of binary, continuous and diagnostic data. BMC Med. Res. Methodol. 2009, 9, 80. [CrossRef] [PubMed]

23. Schuster, D.M.; Votaw, J.R.; Nieh, P.T.; Yu, W.; Nye, J.A.; Master, V.; Bowman, F.D.; Issa, M.M.; Goodman, M.M. Initial experience with the radiotracer anti-1-amino-3-18F-fluorocyclobutane-1-carboxylic acid with PET/CT in prostate carcinoma. J. Nucl. Med. 2007, 48, 56-63. [PubMed]

24. Schuster, D.M.; Savir-Baruch, B.; Nieh, P.T.; Master, V.A.; Halkar, R.K.; Rossi, P.J.; Lewis, M.M.; Nye, J.A.; $\mathrm{Yu}$, W.; Bowman, F.D.; et al. Detection of recurrent prostate carcinoma with anti-1-amino-3-18Ffluorocyclobutane-1-carboxylic acid PET/CT and 111In-capromab pendetide SPECT/CT. Radiology 2011, 259, 852-861. [CrossRef] [PubMed]

25. Turkbey, B.; Mena, E.; Shih, J.; Pinto, P.A.; Merino, M.J.; Lindenberg, M.L.; Bernardo, M.; McKinney, Y.L.; Adler, S.; Owenius, R.; et al. Localized prostate cancer detection with 18F FACBC PET/CT: Comparison with MR imaging and histopathologic analysis. Radiology 2014, 270, 849-856. [CrossRef] [PubMed]

26. Kairemo, K.; Rasulova, N.; Partanen, K.; Joensuu, T. Preliminary clinical experience of trans-1-Amino-3-(18)Ffluorocyclobutanecarboxylic Acid (anti-(18)F-FACBC) PET/CT imaging in prostate cancer patients. BioMed Res. Int. 2014, 2014, 305182. [CrossRef] [PubMed]

27. Nanni, C.; Schiavina, R.; Brunocilla, E.; Borghesi, M.; Ambrosini, V.; Zanoni, L.; Gentile, G.; Vagnoni, V.; Romagnoli, D.; Martorana, G.; et al. 18F-FACBC compared with 11C-choline PET/CT in patients with biochemical relapse after radical prostatectomy: A prospective study in 28 patients. Clin. Genitourin. Cancer 2014, 12, 106-110. [CrossRef] [PubMed]

28. Nanni, C.; Schiavina, R.; Brunocilla, E.; Boschi, S.; Borghesi, M.; Zanoni, L.; Pettinato, C.; Martorana, G.; Fanti, S. 18F-Fluciclovine PET/CT for the Detection of Prostate Cancer Relapse: A Comparison to 11C-Choline PET/CT. Clin. Nucl. Med. 2015, 40, e386-e391. [CrossRef] [PubMed]

29. Odewole, O.A.; Tade, F.I.; Nieh, P.T.; Savir-Baruch, B.; Jani, A.B.; Master, V.A.; Rossi, P.J.; Halkar, R.K.; Osunkoya, A.O.; Akin-Akintayo, O.; et al. Recurrent prostate cancer detection with anti-3-[(18)F]FACBC PET/CT: Comparison with CT. Eur. J. Nucl. Med. Mol. Imaging 2016, 43, 1773-1783. [CrossRef] [PubMed] 
30. Akin-Akintayo, O.O.; Jani, A.B.; Odewole, O.; Tade, F.I.; Nieh, P.T.; Master, V.A.; Bellamy, L.M.; Halkar, R.K.; Zhang, C.; Chen, Z.; et al. Change in Salvage Radiotherapy Management Based on Guidance With FACBC (Fluciclovine) PET/CT in Postprostatectomy Recurrent Prostate Cancer. Clin. Nucl. Med. 2017, 42, e22-e28. [CrossRef]

31. Selnæs, K.M.; Krüger-Stokke, B.; Elschot, M.; Willoch, F.; Størkersen, Ø.; Sandsmark, E.; Moestue, S.A.; Tessem, M.-B.; Halvorsen, D.; Kjøbli, E.; et al. 18F-Fluciclovine PET/MRI for preoperative lymph node staging in high-risk prostate cancer patients. Eur. Radiol. 2018, 28, 3151-3159. [CrossRef]

32. Jambor, I.; Kuisma, A.; Kähkönen, E.; Kemppainen, J.; Merisaari, H.; Eskola, O.; Teuho, J.; Perez, I.M.; Pesola, M.; Aronen, H.J.; et al. Prospective evaluation of 18F-FACBC PET/CT and PET/MRI versus multiparametric MRI in intermediate- to high-risk prostate cancer patients (FLUCIPRO trial). Eur. J. Nucl. Med. Mol. Imaging 2018, 45, 355-364. [CrossRef] [PubMed]

33. Akin-Akintayo, O.; Tade, F.; Mittal, P.; Moreno, C.; Nieh, P.T.; Rossi, P.; Patil, D.; Halkar, R.; Fei, B.; Master, V.; et al. Prospective evaluation of fluciclovine (18F) PET-CT and MRI in detection of recurrent prostate cancer in non-prostatectomy patients. Eur. J. Radiol. 2018, 102, 1-8. [CrossRef] [PubMed]

34. Andriole, G.L.; Kostakoglu, L.; Chau, A.; Duan, F.; Mahmood, U.; Mankoff, D.A.; Schuster, D.M.; Siegel, B.A.; LOCATE Study Group. The Impact of Positron Emission Tomography with 18F-Fluciclovine on the Treatment of Biochemical Recurrence of Prostate Cancer: Results from the LOCATE Trial. J. Urol. 2019, 201, 322-331. [CrossRef] [PubMed]

35. England, J.R.; Paluch, J.; Ballas, L.K.; Jadvar, H. 18F-Fluciclovine PET/CT Detection of Recurrent Prostate Carcinoma in Patients With Serum PSA $\leq 1 \mathrm{ng} / \mathrm{mL}$ After Definitive Primary Treatment. Clin. Nucl. Med. 2019, 44, e128-e132. [CrossRef]

36. Suzuki, H.; Jinnouchi, S.; Kaji, Y.; Kishida, T.; Kinoshita, H.; Yamaguchi, S.; Tobe, T.; Okamura, T.; Kawakita, M.; Furukawa, J.; et al. Diagnostic performance of 18F-fluciclovine PET/CT for regional lymph node metastases in patients with primary prostate cancer: A multicenter phase II clinical trial. Jpn. J. Clin. Oncol. 2019. Epub ahead of print. Available online: https://academic.oup.com/jjco/advance-article/doi/10.1093/jjco/hyz072/ 5490163 (accessed on 1 September 2019).

37. Mottet, N.; Bellmunt, J.; Bolla, M.; Briers, E.; Cumberbatch, M.G.; De Santis, M.; Fossati, N.; Gross, T.; Henry, A.M.; Joniau, S.; et al. EAU-ESTRO-SIOG Guidelines on Prostate Cancer. Part 1: Screening, Diagnosis, and Local Treatment with Curative Intent. Eur Urol. 2017, 71, 618-629. [CrossRef] [PubMed]

38. Pernthaler, B.; Kulnik, R.; Gstettner, C.; Salamon, S.; Aigner, R.M.; Kvaternik, H. A Prospective Head-to-Head Comparison of 18F-Fluciclovine with 68Ga-PSMA-11 in Biochemical Recurrence of Prostate Cancer in PET/CT. Clin. Nucl. Med. 2019. Epub ahead of print. [CrossRef] [PubMed]

39. Calais, J.; Ceci, F.; Eiber, M.; Hope, T.A.; Hofman, M.S.; Rischpler, C.; Bach-Gansmo, T.; Nanni, C.; Savir-Baruch, B.; Elashoff, D.; et al. 18F-fluciclovine PET-CT and 68Ga-PSMA-11 PET-CT in patients with early biochemical recurrence after prostatectomy: A prospective, single-centre, single-arm, comparative imaging trial. Lancet Oncol. 2019. Epub ahead of print. [CrossRef]

40. Lawhn-Heath, C.; Flavell, R.R.; Behr, S.C.; Yohannan, T.; Greene, K.L.; Feng, F.; Carroll, P.R.; Hope, T.A. SingleCenter Prospective Evaluation of 68Ga-PSMA-11 PET in Biochemical Recurrence of Prostate Cancer. AJR Am. J. Roentgenol. 2019, 1-8, Epub ahead of print. [CrossRef]

41. Evangelista, L.; Guttilla, A.; Zattoni, F.; Muzzio, P.C.; Zattoni, F. Utility of choline positron emission tomography/computed tomography for lymph node involvement identification in intermediate- to high-risk prostate cancer: A systematic literature review and meta-analysis. Eur. Urol. 2013, 63, 1040-1048. [CrossRef]

42. Yaxley, J.W.; Raveenthiran, S.; Nouhaud, F.X.; Samartunga, H.; Yaxley, A.J.; Coughlin, G.; Delahunt, B.; Egevad, L.; McEwan, L.; Wong, D. Outcomes of Primary Lymph Node Staging of Intermediate and High Risk Prostate Cancer with 68 Ga-PSMA Positron Emission Tomography/Computerized Tomography Compared to Histological Correlation of Pelvic Lymph Node Pathology. J. Urol. 2019, 201, 815-820. [CrossRef]

43. Oka, S.; Hattori, R.; Kurosaki, F.; Toyama, M.; Williams, L.A.; Yu, W.; Votaw, J.R.; Yoshida, Y.; Goodman, M.M.; Ito, O. A preliminary study of Anti-1-amino-3-18F-fluorocyclobutyl-1-carboxylic acid for the detection of prostate cancer. J. Nucl. Med. 2007, 48, 46-55. [PubMed]

(C) 2019 by the authors. Licensee MDPI, Basel, Switzerland. This article is an open access article distributed under the terms and conditions of the Creative Commons Attribution (CC BY) license (http://creativecommons.org/licenses/by/4.0/). 

Review

\title{
Detection Rate of ${ }^{18}$ F-Labeled PSMA PET/CT in Biochemical Recurrent Prostate Cancer: A Systematic Review and a Meta-Analysis
}

\author{
Giorgio Treglia ${ }^{1,2,3, *}$, Salvatore Annunziata ${ }^{4}$, Daniele A. Pizzuto ${ }^{5}$, Luca Giovanella ${ }^{1,5}$, \\ John O. Prior ${ }^{3}$ and Luca Ceriani ${ }^{1,5}$ \\ 1 Clinic of Nuclear Medicine and Molecular Imaging, Imaging Institute of Southern Switzerland, CH-6500 \\ Bellinzona, Switzerland; luca.giovanella@eoc.ch (L.G.); luca.ceriani@eoc.ch (L.C.) \\ 2 Health Technology Assessment Unit, Ente Ospedaliero Cantonale, CH-6500 Bellinzona, Switzerland \\ 3 Department of Nuclear Medicine and Molecular Imaging, Lausanne University Hospital and University of \\ Lausanne, CH-1011 Lausanne, Switzerland; john.prior@chuv.ch \\ 4 Nuclear Medicine Unit, IFO Regina Elena National Cancer Institute, IT-00144 Rome, Italy; \\ salvatoreannunziata@live.it \\ 5 Department of Nuclear Medicine, University Hospital of Zürich, CH-8091 Zürich, Switzerland; \\ dapizzuto@gmail.com \\ * Correspondence: giorgio.treglia@eoc.ch; Tel.: +41-91-811-8919
}

Received: 1 May 2019; Accepted: 22 May 2019; Published: 23 May 2019

\begin{abstract}
Background: The use of radiolabeled prostate-specific membrane antigen positron emission tomography/computed tomography (PSMA PET/CT) for biochemical recurrent prostate cancer (BRPCa) is increasing worldwide. Recently, ${ }^{18} \mathrm{~F}$-labeled PSMA agents have become available. We performed a systematic review and meta-analysis regarding the detection rate (DR) of ${ }^{18} \mathrm{~F}$-labeled PSMA PET/CT in BRPCa to provide evidence-based data in this setting. Methods: A comprehensive literature search of PubMed/MEDLINE, EMBASE, and Cochrane Library databases through 23 April 2019 was performed. Pooled DR was calculated on a per-patient basis, with pooled proportion and 95\% confidence interval (95\% CI). Furthermore, pooled DR of ${ }^{18}$ F-PSMA PET/CT using different cut-off values of prostate-specific antigen (PSA) was obtained. Results: Six articles (645 patients) were included in the meta-analysis. The pooled DR of ${ }^{18} \mathrm{~F}$-labeled PSMA PET/CT in BRPCa was $81 \%$ (95\% CI: $71-88 \%$ ). The pooled DR was $86 \%$ for PSA $\geq 0.5 \mathrm{ng} / \mathrm{mL}$ (95\% CI: $78-93 \%$ ) and $49 \%$ for PSA $<0.5 \mathrm{ng} / \mathrm{mL}$ (95\% CI: 23-74\%). Statistical heterogeneity was found. Conclusions: ${ }^{18} \mathrm{~F}-\mathrm{labeled}$ PSMA PET/CT demonstrated a good DR in BRPCa. DR of ${ }^{18} \mathrm{~F}$-labeled PSMA PET/CT is related to PSA values with significant lower DR in patients with PSA $<0.5 \mathrm{ng} / \mathrm{mL}$. Prospective multicentric trials are needed to confirm these findings.
\end{abstract}

Keywords: PET; PSMA; prostate; DCFPyL; DCFBC; PSMA-1007

\section{Introduction}

The recent development of metabolic imaging methods has been aimed at improving diagnosis of prostate cancer (PCa), both at staging and in biochemical recurrent prostate cancer (BRPCa) when an increase of prostate-specific antigen (PSA) serum values is detected following curative primary treatments as radical prostatectomy or radiation therapy [1,2]. In patients with low but rising PSA serum values after definitive local therapy, it is important to identify the sites of recurrence early to maximize the effects of treatment; localizing the PCa recurrence can impact treatment decisions as local recurrence can be treated with focal radiation therapy, whereas distant metastases require more systemic therapies [1]. To this regard, radiolabeled prostate-specific membrane antigen (PSMA) positron emission tomography/computed tomography (PET/CT) is emerging as a very useful imaging 
method for detecting tumor lesions in BRPCa patients, with higher DR compared to other imaging modalities [1-5].

The PSMA is overexpressed in the majority of PCa cells but its overexpression has not been found in benign prostatic diseases; however, PSMA is not prostate specific and this protein may be expressed in other tissues and tumors beyond PCa [3-5].

Several PSMA ligands, differing slightly in chemical structure, are commercially available and they may be radiolabeled with different positron-emitters isotopes as Gallium-68 $\left({ }^{68} \mathrm{Ga}\right)$, Fluorine-18 $\left({ }^{18} \mathrm{~F}\right)$, or Copper-64 $\left({ }^{64} \mathrm{Cu}\right)$ to obtain PET radiopharmaceuticals which could be used in clinical practice [4-8]. ${ }^{68}$ Ga-labeled PSMA tracers are currently the most used PSMA agents for PET imaging of BRPCa patients. More recently, PSMA ligands had been labeled with other isotopes with more favorable physical characteristics, such as ${ }^{18} \mathrm{~F}$ or ${ }^{64} \mathrm{Cu}[6-8]$. Several ${ }^{18} \mathrm{~F}$-labeled PSMA agents have become available $\left({ }^{18} \mathrm{~F}-\mathrm{PSMA}-1007,{ }^{18} \mathrm{~F}-\mathrm{DCFPyL}\right.$, and ${ }^{18} \mathrm{~F}$-DCFBC). Labeling of PSMA agents with ${ }^{18} \mathrm{~F}$ may offer numerous advantages, including longer half-life and improved image resolution. Due to the lower positron energy, the theoretical achievable resolution of ${ }^{18} \mathrm{~F}$ is slightly better in comparison to ${ }^{68} \mathrm{Ga}[7,8]$. To date, several evidence-based articles evaluated the detection rate (DR) of ${ }^{68} \mathrm{Ga}$-labeled PSMA PET/CT in BRPCa patients [9-15]. Conversely, we aimed to perform a meta-analysis about the DR of ${ }^{18}$ F-labeled PSMA PET/CT in BRPCa patients to add evidence-based data in this setting.

\section{Methods}

Reporting of this systematic review and meta-analysis conforms to the "Preferred Reporting Items for a Systematic Review and Meta-Analysis of Diagnostic Test Accuracy Studies" (PRISMA-DTA statement) which describes an evidence-based minimum set of items for reporting in systematic reviews and meta-analyses of diagnostic studies [16,17].

\subsection{Search Strategy}

Three authors (G.T., S.A., D.A.P.) performed a comprehensive computer literature search of PubMed/MEDLINE, EMBASE and Cochrane library databases to find relevant published articles on the DR of PET/CT using ${ }^{18} \mathrm{~F}$-labeled PSMA-agents in patients with BRPCa.

A search algorithm based on a combination of these terms was used: (A) "PSMA" AND (B) "DCFPyL" OR "DCFBC" OR "1007". No beginning date limit and language restrictions were used, and the literature search was updated until 23 April 2019. To expand our search, references of the retrieved articles were also screened for additional studies.

\subsection{Study Selection}

Studies or subsets of studies investigating the DR of ${ }^{18} \mathrm{~F}$-labeled PSMA PET/CT in patients with BRPCa were eligible for inclusion in the qualitative (systematic review) and quantitative analysis (meta-analysis). The exclusion criteria for the systematic review were: (a) articles not within the field of interest of this review; (b) review articles, editorials or letters, comments, conference proceedings; (c) case reports or small case series. For the meta-analysis, articles with possible patient data overlap were excluded; in this case, articles with more complete information were included in the meta-analysis.

Titles and abstracts were independently reviewed by three researchers applying the selected inclusion and exclusion criteria. Disagreements were solved in a consensus meeting.

\subsection{Data Extraction}

For each eligible article, information was collected concerning basic study (authors, year of publication, country of origin, study design), patient characteristics (type and number of patients evaluated, mean age, Gleason score, mean/median PSA serum values, and PSA doubling time before ${ }^{18}$ F-PSMA PET/CT), technical aspects (radiotracer used, hybrid imaging modality, mean radiotracer injected activity, time interval between radiotracer injection and image acquisition, image analysis and other imaging modalities performed for comparison). For articles included in the meta-analysis, 
information was collected about DR values of ${ }^{18}$ F-PSMA PET/CT (overall and at different PSA cut-off values) on a per patient-based analysis, mean PSA serum values in patients with positive and negative ${ }^{18} \mathrm{~F}-\mathrm{PSMA} \mathrm{PET} / \mathrm{CT}$, percentage of change of management by using ${ }^{18} \mathrm{~F}-\mathrm{PSMA}$ PET/CT in BRPCa.

\subsection{Quality Assessment}

The overall quality of the studies included in the meta-analysis was critically appraised based on the revised "Quality Assessment of Diagnostic Accuracy Studies" tool (QUADAS-2) [18]. This tool comprises four domains (patient selection, index test, reference standard, and flow and timing) and each domain was assessed in terms of risk of bias, and the first three domains were also assessed in terms of concerns regarding applicability [18].

\subsection{Statistical Analysis}

The DR of ${ }^{18}$ F-PSMA PSMA PET/CT was defined as the ratio between the number of patients with at least one suspected lesion detected by PET/CT and the total number of BRPCa patients who underwent the scan. Pooled analyses about DR of ${ }^{18} \mathrm{~F}-\mathrm{PSMA}$ PET/CT were performed using data retrieved from the selected studies and subgroup analyses taking into account different PSA serum values or different radiotracers were planned. Furthermore, a pooled analysis about the mean difference of PSA serum values in patients with positive and negative ${ }^{18} \mathrm{~F}-\mathrm{PSMA}$ PET/CT was carried out.

A random-effects model was used for statistical pooling of the data, taking into account the heterogeneity between studies. The different weight of each study in the pooled analysis was related to the different sample size. Pooled data were presented with their respective $95 \%$ confidence interval $(95 \%$ $\mathrm{CI})$ values, and data were displayed using plots. Heterogeneity was estimated using the I-square index $\left(\mathrm{I}^{2}\right)$, which describes the percentage of variation across studies that was due to the heterogeneity rather than chance [19], whereas the publication bias was assessed through the Egger's test [20]. Statistical analyses were performed using the StatsDirect software version 3 (StatsDirect Ltd., Cambridge, UK).

\section{Results}

\subsection{Literature Search}

Literature search results are reported in Figure 1. 


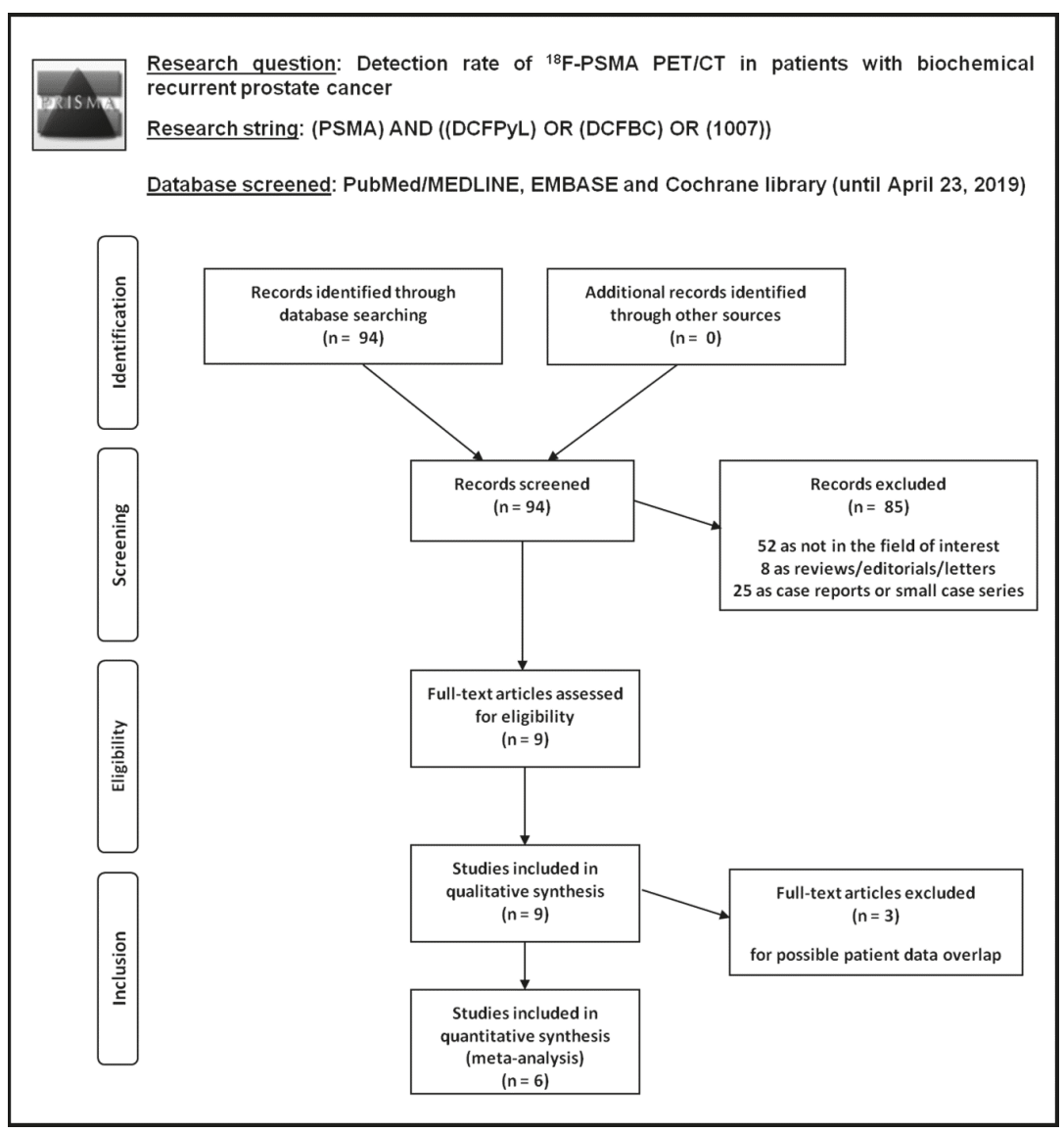

Figure 1. Flow chart of the search for eligible studies on the detection rate of ${ }^{18}$ F-PSMA PET/CT in patients with biochemical recurrent prostate cancer.

Ninety-four records were identified from the literature search of PubMed/MEDLINE, EMBASE, and Cochrane library databases. Screening titles and abstracts, 85 records were excluded: 52 because they were not in the field of interest; 8 as they were reviews, editorials or letters; and 25 as they were case reports. Nine articles were selected and retrieved [21-29]. No additional records were found screening the references of these articles. Therefore, 9 articles were eligible for the qualitative analysis (systematic review). Three articles were excluded from the meta-analysis for possible patient data overlap [21,25,26]; finally, 6 articles including 645 patients with BRPCa were included in the quantitative analysis (meta-analysis) [22-24,27-29]. The characteristics of the studies selected for the systematic review are presented in Tables 1 and 2. The main findings of the articles included in the meta-analysis are shown in Table 3, whereas the overall quality assessment of the studies is reported in Figure 2. 


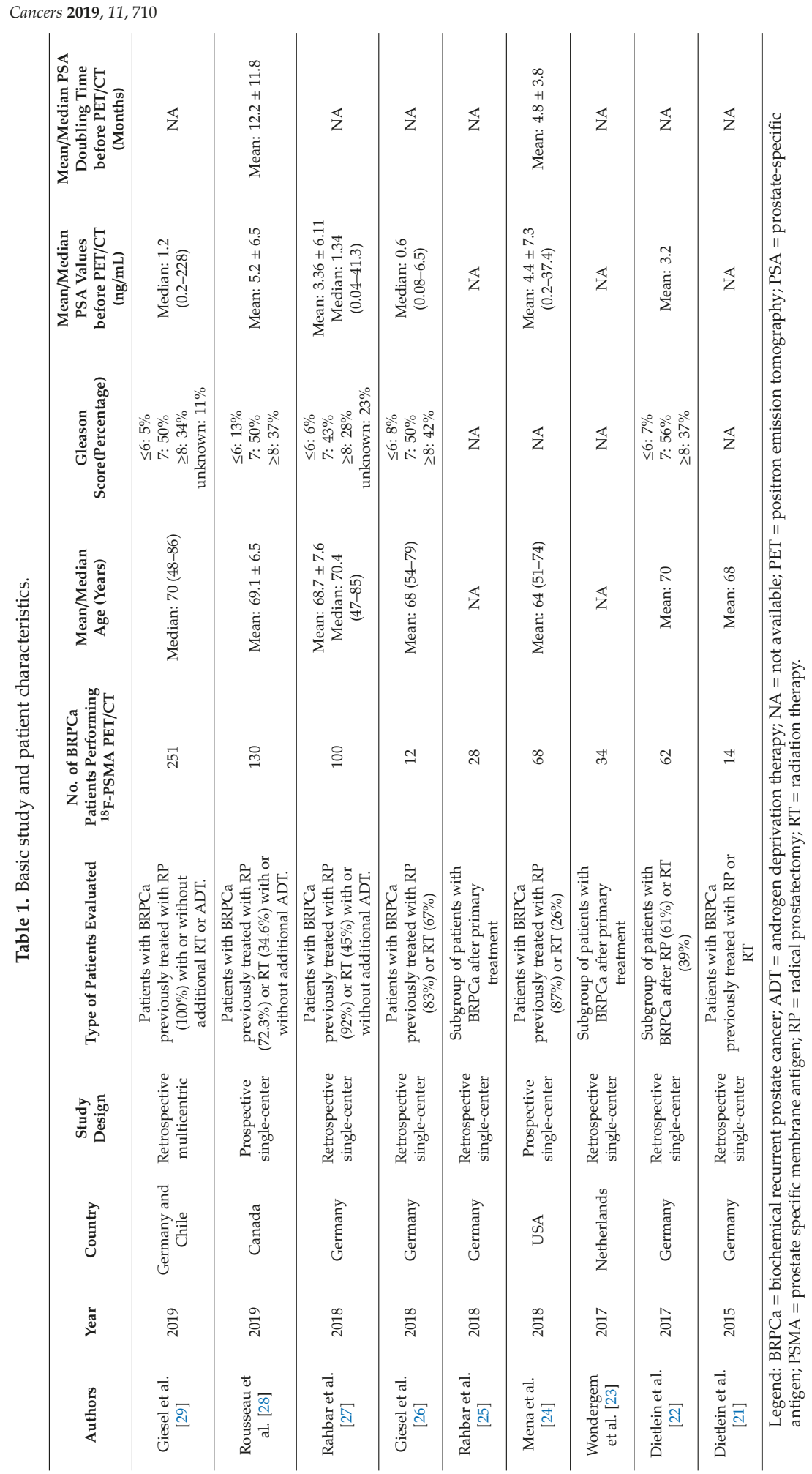




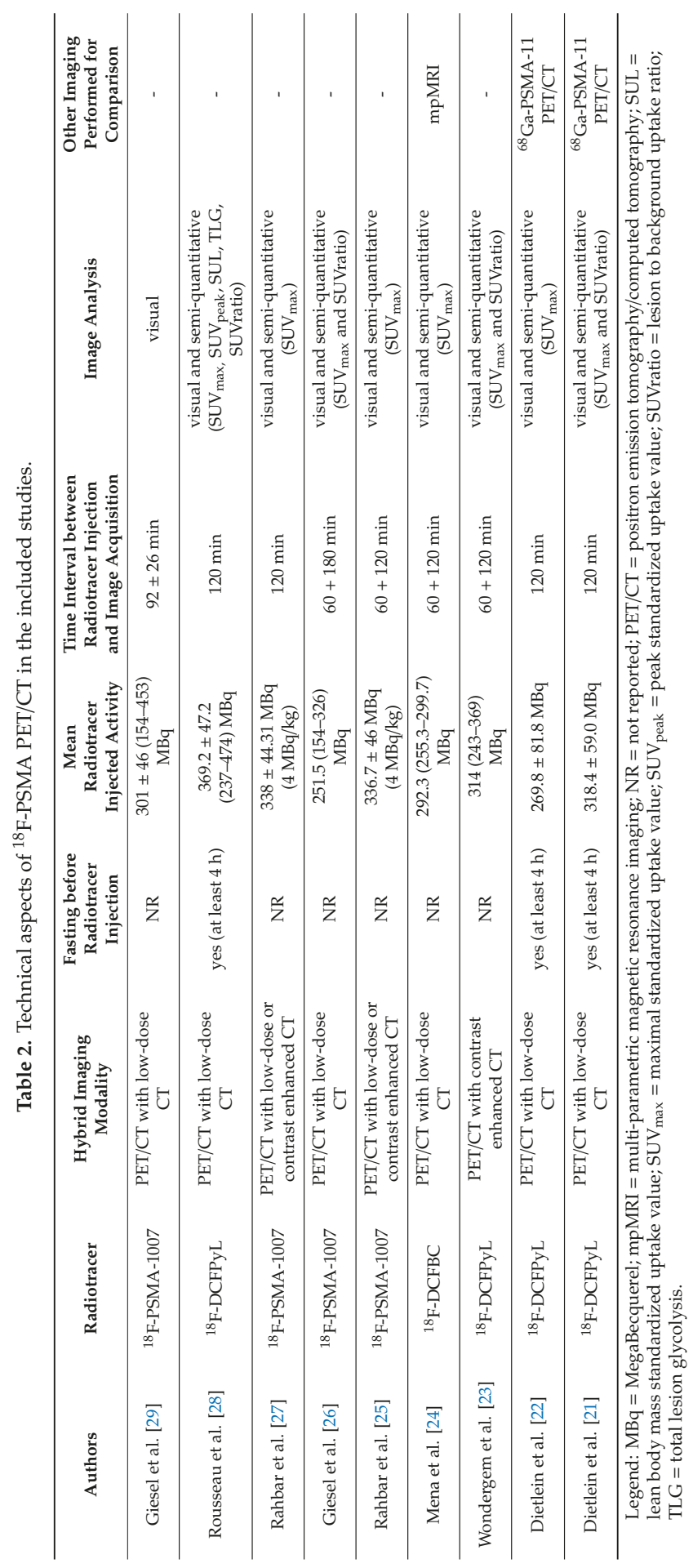




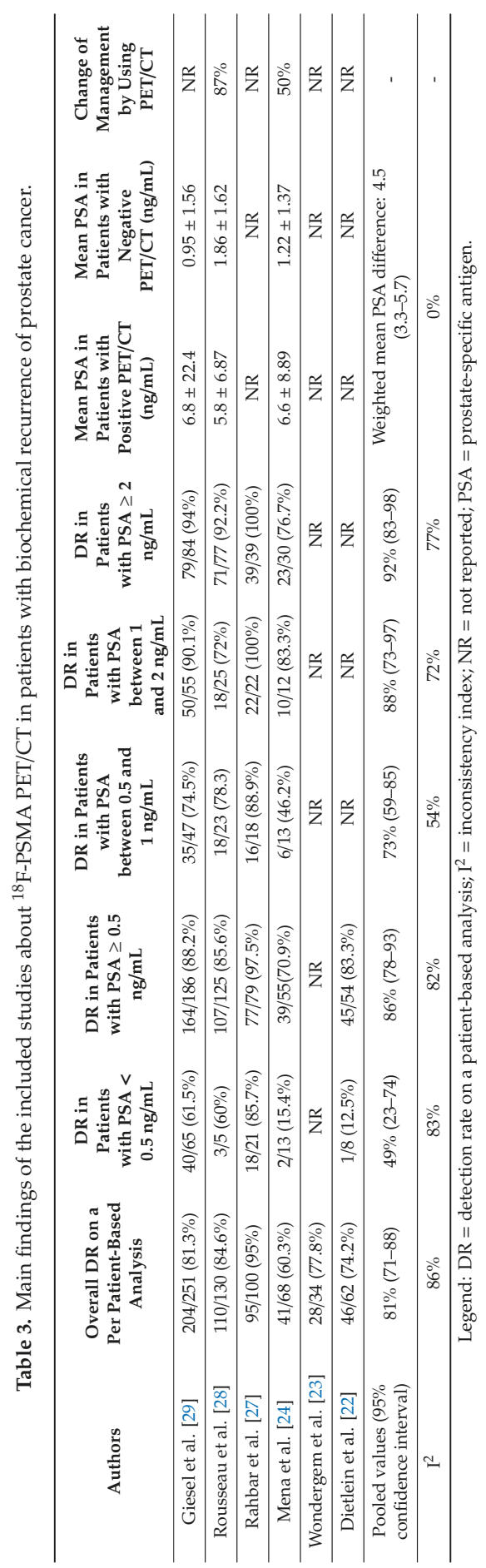




\section{QUADAS 2: Risk of bias}

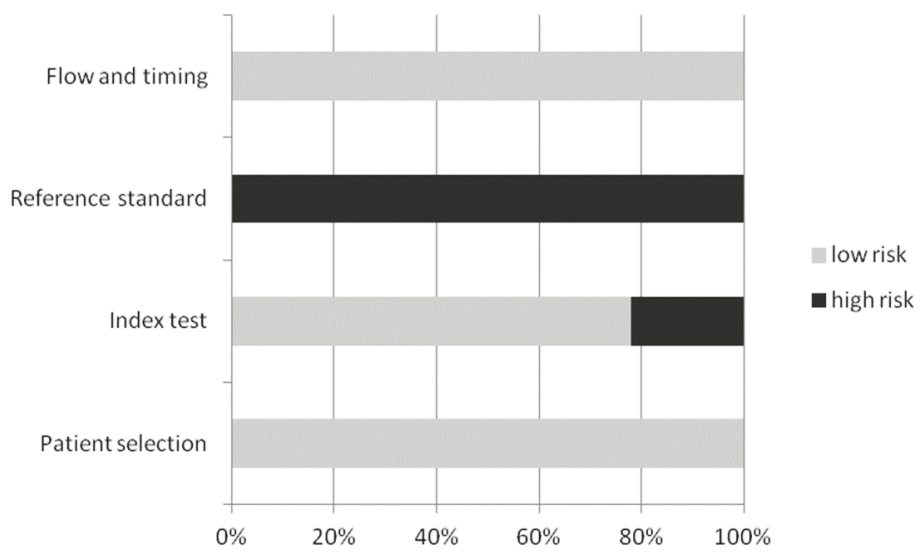

\section{QUADAS 2: Applicability concerns}

\section{Reference standard}

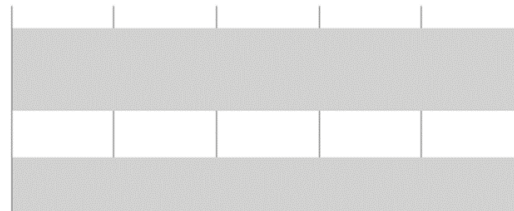

Patient selection

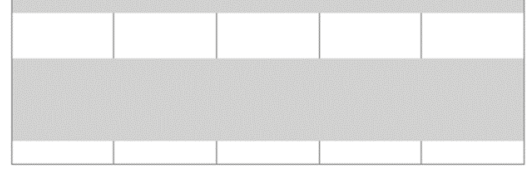

wigh risk

$0 \%$

Figure 2. Overall quality assessment of the studies included in the systematic review according to the QUADAS-2 tool.

\subsection{Qualitative Analysis (Systematic Review)}

\subsubsection{Basic Study and Patient Characteristics}

Nine articles evaluating the DR of ${ }^{18}$ F-PSMA PET/CT in BRPCa patients were selected (Table 1) [2129]. The selected articles were published in the last five years by researchers from Europe and America; only two out of nine studies were prospective studies (22\%). Mean and median age of the patients included in these studies ranged from 64 to 70 years. The Gleason score was 7 in $43-56 \%, \leq 6$ in $5-13 \%$, and $\geq 8$ in $28-42 \%$ of cases. Mean and median PSA serum values before PET/CT among the included BRPCa patients ranged from 0.6 to $5.2 \mathrm{ng} / \mathrm{mL}$. 


\subsubsection{Technical Aspects}

Technical aspects of the included studies are reported in Table 2. The ${ }^{18} \mathrm{~F}$-labeled PSMA agent used was ${ }^{18} \mathrm{~F}$-PSMA-1007 in four studies, ${ }^{18} \mathrm{~F}$-DCFPyL in four studies, and ${ }^{18} \mathrm{~F}$-DCFBC in one study only. The hybrid imaging modality was always PET/CT, mainly performed without CT contrast media injection. The injected radiopharmaceutical activity and the time between radiotracer injection and image acquisition were quite heterogeneous; in four studies a dual time point PET/CT imaging was performed. Analysis of PET images was performed using qualitative criteria (visual analysis) in all the studies and additional semi-quantitative criteria, i.e., calculating the maximal standardized uptake values $\left(\mathrm{SUV}_{\max }\right)$, in most of the studies. Areas of increased radiopharmaceutical uptake greater than the surrounding tissue that could not be explained by physiological activity were judged as positive findings at visual analysis. A clear reference standard was not specified in the included studies.

\subsubsection{Main Findings}

Most of the included studies demonstrated a good DR of ${ }^{18} \mathrm{~F}$-PSMA PET/CT in BRPCa patients which was dependent on PSA serum values: the proportion of positive scans increased with PSA levels [21-29]. Conversely, no significant correlation between PSA doubling time and DR of ${ }^{18}$ F-PSMA PET/CT was found [24]. The higher DR values were obtained using ${ }^{18}$ F-PSMA-1007 or ${ }^{18} \mathrm{~F}-\mathrm{DCFPyL}$ as radiotracers (Table 3 ).

Most frequent sites of lesions detected by ${ }^{18} \mathrm{~F}-\mathrm{PSMA}$ PET/CT in BRPCa were regional and distant lymph nodal metastases, local relapse, and bone metastases [21-29].

In three studies, a statistically significant difference of PSA serum values in patients with positive ${ }^{18} \mathrm{~F}-\mathrm{PSMA}$ PET/CT compared to patients with negative ${ }^{18} \mathrm{~F}$-PSMA PET/CT was found, but with a large overlap in PSA values across these two categories [24,28,29].

In studies performing a dual time point ${ }^{18} \mathrm{~F}$-PSMA PET/CT, a significant increased lesion uptake and higher lesion-to-background uptake ratios were observed at a second time point (120 or $180 \mathrm{~min}$ after radiotracer injection) compared to the first time point (60 min after radiotracer injection) [23-26].

No significant adverse effects of ${ }^{18} \mathrm{~F}-\mathrm{PSMA}$ PET/CT were reported [21,24,27-29]. The change of management by using ${ }^{18} \mathrm{~F}$-PSMA PET/CT in BRPCa ranged from 50 to $87 \%$ of cases [24,28].

Two articles compared the DR of ${ }^{18} \mathrm{~F}-\mathrm{DCFPyL}$ PET/CT with ${ }^{18} \mathrm{Ga}-\mathrm{PSMA}-11$ PET/CT in BRPCa patients. The ${ }^{18} \mathrm{~F}$-DCFPyL PET/CT detected additional lesions compared to ${ }^{18} \mathrm{Ga}-\mathrm{PSMA}-11$ PET/CT (in particular for PSA values between 0.5 and $3.5 \mathrm{ng} / \mathrm{mL}$ ) and the mean $\mathrm{SUV}_{\max }$ of ${ }^{18} \mathrm{~F}-\mathrm{DCFPyL}$ PSMA-positive lesions was significantly higher as compared to ${ }^{18} \mathrm{Ga}$-PSMA-11 positive lesions [21,22].

Several discordant findings were found when ${ }^{18} \mathrm{~F}-\mathrm{PSMA}$ PET/CT was compared to multi-parametric MRI, demonstrating the complementary role of these imaging methods in BRPCa patients [24].

\subsection{Quantitative Analysis (Meta-Analysis)}

Six studies (645 BRPCa patients) were selected for the pooled analysis [22-24,27-29]. The overall DR of ${ }^{18}$ F-PSMA PET/CT on a per patient-based analysis ranged from $60 \%$ to $95 \%$, with a pooled value of $81 \%$ ( $95 \%$ CI: $71-88 \%$ ) (Figure 3 and Table 3). We have detected a significant heterogeneity among the selected studies $\left(\mathrm{I}^{2}=86 \%\right)$, whereas a publication bias was not revealed $(p=0.16)$.

Performing a sub-group analysis taking into account different PSA cut-off values (Table 3 and Figure 4), we found a statistical significant difference in DR of ${ }^{18} \mathrm{~F}-\mathrm{PSMA}$ PET/CT in BRPCa patients with PSA $\geq 0.5 \mathrm{ng} / \mathrm{mL}$ (pooled DR: $86 \%$; 95\% CI: 78-93\%) compared to patients with PSA $<0.5 \mathrm{ng} / \mathrm{mL}$ (pooled DR: $49 \%$; $95 \%$ CI: $23-74 \%$ ).

Performing a sub-group analysis taking into account different radiotracers, the pooled DR of ${ }^{18}$ F-PSMA-1007, ${ }^{18}$ F-DCFPyL, and ${ }^{18} \mathrm{~F}-\mathrm{DCFBC}$ PET/CT were 89\% (95\% CI: 72-98\%), 81\% (95\% CI: $74-87 \%)$ and $60 \%$ (95\% CI: $48-72 \%)$, respectively. 
Weighted mean difference of PSA values among patients with positive ${ }^{18} \mathrm{~F}-\mathrm{PSMA}$ PET/CT and patients with negative ${ }^{18}$ F-PSMA PET/CT was 4.5 (95\% CI: 3.3-5.7) without significant heterogeneity $\left(\mathrm{I}^{2}=0 \%\right)$.

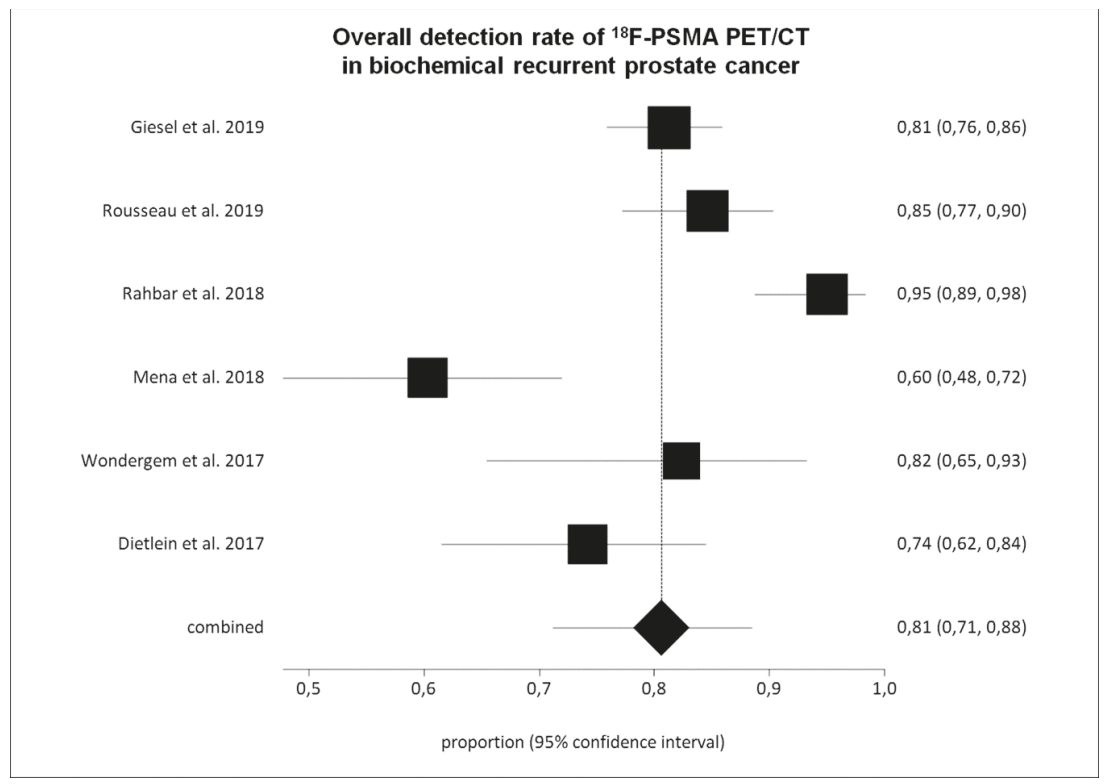

Figure 3. Plots of individual studies and pooled detection rate of ${ }^{18} \mathrm{~F}-\mathrm{PSMA}$ PET/CT in biochemical recurrent prostate cancer on a per patient-based analysis, including 95\% confidence intervals (95\% CI). The size of the squares indicates the weight of each study.

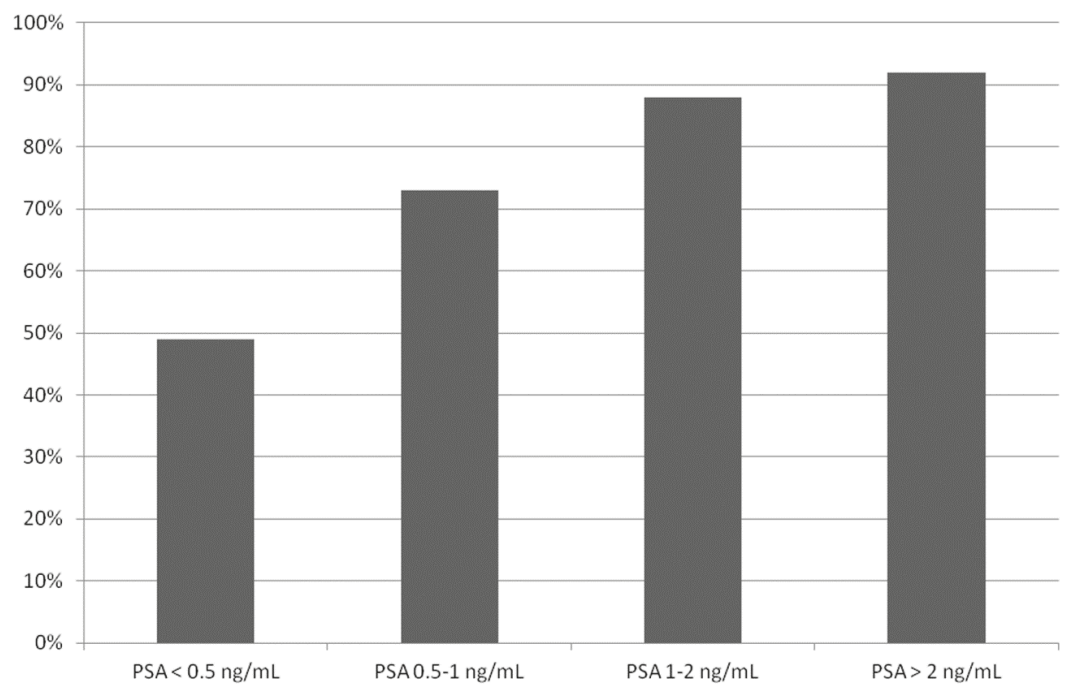

Figure 4. Bar graph showing the pooled detection rate of ${ }^{18}$ F-PSMA PET/CT in biochemical recurrent prostate cancer on a per patient-based analysis, according to different PSA serum values. 


\section{Discussion}

Recently, some studies have evaluated the diagnostic performance of ${ }^{18} \mathrm{~F}$-PSMA PET/CT in BRPCa patients [21-29]; as these studies have limited power, due to the relatively small number of patients enrolled and assessed, we have pooled data reported in published studies to derive more robust estimates on the DR of ${ }^{18}$ F-PSMA PET/CT in this setting.

Overall, our systematic review and meta-analysis indicates a good DR of ${ }^{18} \mathrm{~F}-\mathrm{PSMA}$ PET/CT in BRPCa patients, in particular using ${ }^{18} \mathrm{~F}-\mathrm{PSMA}-1007$ and ${ }^{18} \mathrm{~F}-\mathrm{DCFPyL}$. The DR was dependent on PSA serum values [21-29]: using the PSA cut-off of $0.5 \mathrm{ng} / \mathrm{mL}$, the pooled DR of ${ }^{18} \mathrm{~F}-\mathrm{PSMA}$ PET/CT was $86 \%$ in BRPCa patients with PSA $\geq 0.5 \mathrm{ng} / \mathrm{mL}$ and $49 \%$ in patients with PSA $<0.5 \mathrm{ng} / \mathrm{mL}$. Therefore, accurate timing of ${ }^{18} \mathrm{~F}$-PSMA PET/CT, based on PSA values, substantially affects its diagnostic value in BRPCa patients, and monitoring of PSA values could be useful for accurate timing of ${ }^{18}$ F-PSMA $\mathrm{PET} / \mathrm{CT}$.

Beyond the PSA serum values, low PSMA expression (i.e., due to the tumor heterogeneity) might cause false-negative ${ }^{18} \mathrm{~F}-\mathrm{PSMA}$ PET/CT findings in some PCa patients [21-29]. About the pooled DR of ${ }^{18} \mathrm{~F}-\mathrm{PSMA} \mathrm{PET} / \mathrm{CT}$ in BRPCa, we found similar results compared to those reported in the literature with ${ }^{68}$ Ga-labeled PSMA PET/CT [9-15]. Compared to PET/CT with ${ }^{68}$ Ga-labeled PSMA, the longer half-life and higher injected activities of ${ }^{18} \mathrm{~F}$-PSMA allow high-quality delayed images, higher lesion uptake, and superior clearance of background activity [21-29]. Two studies only reported a comparison of ${ }^{18} \mathrm{~F}-\mathrm{PSMA}$ and ${ }^{68} \mathrm{Ga}$-PSMA PET/CT in BRPCa patients with a trend towards a higher DR of ${ }^{18} \mathrm{~F}$-PSMA compared to ${ }^{68} \mathrm{Ga}$-PSMA PET/CT, but the acquisition protocols used in these studies included different tracer uptake time periods for ${ }^{18} \mathrm{~F}$-PSMA (120 $\left.\mathrm{min}\right)$ and ${ }^{68} \mathrm{Ga}$-PSMA (60 $\mathrm{min}$ ) before image acquisition and different activity used for these radiopharmaceuticals $\left({ }^{18} \mathrm{~F}-\mathrm{PSMA}>{ }^{68} \mathrm{Ga}\right.$-PSMA), which could explain these results [21,22]. Assuming similar DR, the real additional value of ${ }^{18} \mathrm{~F}-\mathrm{PSMA}$ tracers might be the possibility of large-scale batch production in a cyclotron and satellite-center supply due to the longer half-life [21-29].

As a significant increased ${ }^{18} \mathrm{~F}$-PSMA uptake over time was demonstrated in PCa lesions with a higher contrast at delayed PET/CT time points compared to early PET/CT time points [23-26], it is not recommended to perform ${ }^{18} \mathrm{~F}$-PSMA PET/CT at $60 \mathrm{~min}$ after radiotracer injection (which is the common imaging time point for ${ }^{68} \mathrm{Ga}$-PSMA PET/CT). However, imaging at late time points may be a logistic challenge for PET/CT centers.

Only two articles assessed the change of management that can be obtained by using ${ }^{18} \mathrm{~F}-\mathrm{PSMA}$ PET/CT in patients with BRPCa $[24,28]$, reporting a significant change of management ranging from 50 to $87 \%$ of cases, in line with literature data about the change of management obtained by using ${ }^{18}$ Ga-PSMA PET/CT in this setting [30].

Some limitations and biases of our meta-analysis should be taken into account. First of all, a limited number of studies were available for the meta-analysis. The major limitation of the included studies was that not all positive PET/CT findings were confirmed by histology (verification bias). Confirmation was impaired by the small volume of individual lesions and the high number of biopsy-inaccessible lesions. In absence of histological validation, it cannot be excluded that some lesions detected by ${ }^{18}$ F-PSMA PET/CT may represent false-positive findings. Nevertheless, if modern imaging methods are performed in BRPCa patients, then confirmation of positive findings are needed only in highly selected cases and with a biopsy when findings are equivocal [1]. Even in the absence of histological confirmation, clinical follow-up or decline of PSA after therapy can be helpful.

A possible limitation of our meta-analysis is the detected heterogeneity, likely due to the different characteristics of patients, methods, and quality of included studies. We tried to explain this heterogeneity performing sub-group analyses taking into account different PSA cut-off values and different radiotracers. Some differences of DR were found using various ${ }^{68} \mathrm{~F}-\mathrm{PSMA}$ agents, but studies performing a head-to-head comparison of these tracers in BRPCa setting are not available yet. We found a lower DR value using ${ }^{18} \mathrm{~F}-\mathrm{DCFBC}$ compared with second-generation ${ }^{68} \mathrm{~F}$-PSMA agents $\left({ }^{18} \mathrm{~F}-\mathrm{PSMA}-1007\right.$ and $\left.{ }^{18} \mathrm{~F}-\mathrm{DCFPyL}\right)$, likely because of the higher background signal of ${ }^{18} \mathrm{~F}-\mathrm{DCFBC}$ 
due to the considerable blood-pool activity, which could limit the DR of pelvic and retroperitoneal lymph node metastases [24]. In a recent pilot prospective study comparing ${ }^{18}$ F-PSMA-1007 and ${ }^{18} \mathrm{~F}-\mathrm{DCFPyL}$ in the setting of PCa staging, similar DR were found using these radiopharmaceuticals [31]. Non-urinary excretion of ${ }^{18}$ F-PSMA-1007 might present some advantages with regard to delineation of local recurrence or pelvic lymph node metastases in selected patients; the lower hepatic background might favor ${ }^{18} \mathrm{~F}-\mathrm{DCFPyL}$ in late stages, when rare cases of liver metastases can occur [31].

Diagnostic accuracy of a test is not a measure of clinical effectiveness and high DR values do not necessarily result in improved patient outcomes. Other factors beyond the DR should influence the choice of an imaging modality in patients with BRPCa (i.e., availability, radiation dose, safety, examination time, legal, organization, economic aspects, and cost-effectiveness). Overall, our systematic review and meta-analysis demonstrated a good DR of ${ }^{18} \mathrm{~F}-\mathrm{PSMA}$ PET/CT in patients with BRPCa, but large prospective multi-center studies, and in particular, cost-effectiveness analyses comparing ${ }^{18}$ F-PSMA to other PET radiopharmaceuticals are warranted.

\section{Conclusions}

- $\quad{ }^{18}$ F-labeled PSMA PET/CT demonstrated a good DR in BRPCa, in particular using ${ }^{18}$ F-PSMA-1007 and ${ }^{18} \mathrm{~F}-\mathrm{DCFPyL}$, with similar results compared to those reported in the literature with ${ }^{68} \mathrm{Ga}$-labeled PSMA PET/CT.

- The DR of ${ }^{18}$ F-labeled PSMA PET/CT is related to PSA values with significant lower DR in patients with PSA $<0.5 \mathrm{ng} / \mathrm{mL}$.

- Prospective multicentric trials are needed to confirm these findings; nevertheless, ${ }^{18} \mathrm{~F}$-labeled PSMA PET/CT seems to be a promising cost-effective alternative to ${ }^{68} \mathrm{Ga}$-labeled PSMA PET/CT in BRPCa.

Author Contributions: Conceptualization, G.T. and L.C.; methodology, G.T.; software, G.T.; validation, J.O.P. and L.C., formal analysis, G.T., S.A. and D.A.P.; resources, G.T.; data curation, G.T., S.A. and D.A.P.; writing-original draft preparation, G.T.; writing—review and editing, S.A., D.A.P.; supervision, J.O.P., L.C. and L.G.

Funding: This research received no external funding.

Conflicts of Interest: The authors declare no conflict of interest.

\section{References}

1. Fanti, S.; Minozzi, S.; Antoch, G.; Banks, I.; Briganti, A.; Carrio, I.; Chiti, A.; Clarke, N.; Eiber, M.; De Bono, J.; et al. Consensus on molecular imaging and theranostics in prostate cancer. Lancet Oncol. 2018, 19, e696-e708. [CrossRef]

2. Tangel, M.R.; Rastinehad, A.R. Advances in prostate cancer imaging. F1000Research 2018, 7. [CrossRef] [PubMed]

3. O'Keefe, D.S.; Bacich, D.J.; Huang, S.S.; Heston, W.D.W. A Perspective on the Evolving Story of PSMA Biology, PSMA-Based Imaging, and Endoradiotherapeutic Strategies. J. Nucl. Med. 2018, 59, 1007-1013. [CrossRef] [PubMed]

4. Ceci, F.; Castellucci, P.; Fanti, S. Current application and future perspectives of PSMA PET imaging in prostate cancer. Q. J. Nucl. Med. Mol. Imaging 2019, 63, 7-18. [CrossRef]

5. Giovacchini, G.; Giovannini, E.; Riondato, M.; Ciarmiello, A. PET/CT With (68)Ga-PSMA in Prostate Cancer: Radiopharmaceutical Background and Clinical Implications. Curr. Radiopharm. 2018, 11, 4-13. [CrossRef]

6. Gourni, E.; Henriksen, G. Metal-Based PSMA Radioligands. Molecules 2017, 22, 523. [CrossRef]

7. Czarniecki, M.; Mena, E.; Lindenberg, L.; Cacko, M.; Harmon, S.; Radtke, J.P.; Giesel, F.; Turkbey, B.; Choyke, P.L. Keeping up with the prostate-specific membrane antigens (PSMAs): An introduction to a new class of positron emission tomography (PET) imaging agents. Transl. Androl. Urol. 2018, 7, 831-843. [CrossRef]

8. $\quad$ Eiber, M.; Fendler, W.P.; Rowe, S.P.; Calais, J.; Hofman, M.S.; Maurer, T.; Schwarzenboeck, S.M.; Kratowchil, C.; Herrmann, K.; Giesel, F.L. Prostate-Specific Membrane Antigen Ligands for Imaging and Therapy. J. Nucl. Med. 2017, 58, 67S-76S. [CrossRef] 
9. Tan, N.; Bavadian, N.; Calais, J.; Oyoyo, U.; Kim, J.; Turkbey, I.B.; Mena, E.; Davenport, M.S. Imaging of PSMA-targeted Radiotracers for the Detection of Prostate Cancer Biochemical Recurrence After Definitive Therapy: A Systematic Review and Meta-analysis. J. Urol. 2019. [CrossRef]

10. Perera, M.; Papa, N.; Roberts, M.; Williams, M.; Udovicich, C.; Vela, I.; Christidis, D.; Bolton, D.; Hofman, M.S.; Lawrentschuk, N.; et al. Gallium-68 Prostate-specific Membrane Antigen Positron Emission Tomography in Advanced Prostate Cancer-Updated Diagnostic Utility, Sensitivity, Specificity, and Distribution of Prostate-specific Membrane Antigen-avid Lesions: A Systematic Review and Meta-analysis. Eur. Urol. 2019. [CrossRef]

11. Pereira Mestre, R.; Treglia, G.; Ferrari, M.; Pascale, M.; Mazzara, C.; Azinwi, N.C.; Llado', A.; Stathis, A.; Giovanella, L.; Roggero, E. Correlation between PSA kinetics and PSMA-PET in prostate cancer restaging: A meta-analysis. Eur. J. Clin. Investig. 2019, 49, e13063. [CrossRef]

12. Hope, T.A.; Goodman, J.Z.; Allen, I.E.; Calais, J.; Fendler, W.P.; Carroll, P.R. Meta-analysis of (68)Ga-PSMA-11 PET Accuracy for the Detection of Prostate Cancer Validated by Histopathology. J. Nucl. Med. 2018. [CrossRef]

13. Sathianathen, N.J.; Butaney, M.; Konety, B.R. The utility of PET-based imaging for prostate cancer biochemical recurrence: A systematic review and meta-analysis. World J. Urol. 2018. [CrossRef]

14. Eissa, A.; Elsherbiny, A.; Coelho, R.F.; Rassweiler, J.; Davis, J.W.; Porpiglia, F.; Patel, V.R.; Prandini, N.; Micali, S.; Sighinolfi, M.C.; et al. The role of 68Ga-PSMA PET/CT scan in biochemical recurrence after primary treatment for prostate cancer: A systematic review of the literature. Minerva Urol. Nefrol. 2018, 70, 462-478. [CrossRef]

15. von Eyben, F.E.; Picchio, M.; von Eyben, R.; Rhee, H.; Bauman, G. (68)Ga-Labeled Prostate-specific Membrane Antigen Ligand Positron Emission Tomography/Computed Tomography for Prostate Cancer: A Systematic Review and Meta-analysis. Eur. Urol. Focus 2018, 4, 686-693. [CrossRef]

16. McInnes, M.D.F.; Moher, D.; Thombs, B.D.; McGrath, T.A.; Bossuyt, P.M.; the PRISMA-DTA Group; Clifford, T.; Cohen, J.F.; Deeks, J.J.; Gatsonis, C.; et al. Preferred Reporting Items for a Systematic Review and Meta-analysis of Diagnostic Test Accuracy Studies: The PRISMA-DTA Statement. JAMA 2018, 319, 388-396. [CrossRef]

17. Sadeghi, R.; Treglia, G. Systematic reviews and meta-analyses of diagnostic studies: A practical guideline. Clin. Transl. Imaging 2017, 5, 83-87. [CrossRef]

18. Whiting, P.F.; Rutjes, A.W.; Westwood, M.E.; Mallett, S.; Deeks, J.J.; Reitsma, J.B.; Leeflang, M.M.; Sterne, J.A.; Bossuyt, P.M. QUADAS-2 Group. QUADAS-2: A revised tool for the quality assessment of diagnostic accuracy studies. Ann. Intern. Med. 2011, 155, 529-536. [CrossRef]

19. Higgins, J.P.; Thompson, S.G. Quantifying heterogeneity in a meta-analysis. Stat. Med. 2002, 21, 1539-1558. [CrossRef]

20. Harbord, R.M.; Egger, M.; Sterne, J.A. A modified test for small-study effects in meta-analyses of controlled trials with binary endpoints. Stat. Med. 2006, 25, 3443-3457. [CrossRef]

21. Dietlein, M.; Kobe, C.; Kuhnert, G.; Stockter, S.; Fischer, T.; Schomäcker, K.; Schmidt, M.; Dietlein, F.; Zlatopolskiy, B.D.; Krapf, P.; et al. Comparison of [(18)F]DCFPyL and [(68)Ga]Ga-PSMA-HBED-CC for PSMA-PET Imaging in Patients with Relapsed Prostate Cancer. Mol. Imaging Biol. 2015, 17, 575-584. [CrossRef]

22. Dietlein, F.; Kobe, C.; Neubauer, S.; Schmidt, M.; Stockter, S.; Fischer, T.; Schomäcker, K.; Heidenreich, A.; Zlatopolskiy, B.D.; Neumaier, B.; et al. PSA-Stratified Performance of (18)F- and (68)Ga-PSMA PET in Patients with Biochemical Recurrence of Prostate Cancer. J. Nucl. Med. 2017, 58, 947-952. [CrossRef]

23. Wondergem, M.; van der Zant, F.M.; Knol, R.J.J.; Lazarenko, S.V.; Pruim, J.; de Jong, I.J. (18)F-DCFPyL PET/CT in the Detection of Prostate Cancer at 60 and 120 Minutes: Detection Rate, Image Quality, Activity Kinetics, and Biodistribution. J. Nucl. Med. 2017, 58, 1797-1804. [CrossRef] [PubMed]

24. Mena, E.; Lindenberg, M.L.; Shih, J.H.; Adler, S.; Harmon, S.; Bergvall, E.; Citrin, D.; Dahut, W.; Ton, A.T.; McKinney, Y; et al. Clinical impact of PSMA-based (18)F-DCFBC PET/CT imaging in patients with biochemically recurrent prostate cancer after primary local therapy. Eur. J. Nucl. Med. Mol. Imaging 2018, 45, 4-11. [CrossRef] [PubMed]

25. Rahbar, K.; Afshar-Oromieh, A.; Bögemann, M.; Wagner, S.; Schäfers, M.; Stegger, L.; Weckesser, M. (18)F-PSMA-1007 PET/CT at 60 and 120 minutes in patients with prostate cancer: Biodistribution, tumour detection and activity kinetics. Eur. J. Nucl. Med. Mol. Imaging 2018, 45, 1329-1334. [CrossRef] [PubMed] 
26. Giesel, F.L.; Will, L.; Kesch, C.; Freitag, M.; Kremer, C.; Merkle, J.; Neels, O.C.; Cardinale, J.; Hadaschik, B.; Hohenfellner, M.; et al. Biochemical Recurrence of Prostate Cancer: Initial Results with [(18)F]PSMA-1007 PET/CT. J. Nucl. Med. 2018, 59, 632-635. [CrossRef]

27. Rahbar, K.; Afshar-Oromieh, A.; Seifert, R.; Wagner, S.; Schäfers, M.; Bögemann, M.; Weckesser, M. Diagnostic performance of (18)F-PSMA-1007 PET/CT in patients with biochemical recurrent prostate cancer. Eur. J. Nucl. Med. Mol. Imaging 2018, 45, 2055-2061. [CrossRef]

28. Rousseau, E.; Wilson, D.; Lacroix-Poisson, F.; Krauze, A.; Chi, K.; Gleave, M.; McKenzie, M.; Tyldesley, S.; Goldenberg, S.L.; Bénard, F. A Prospective Study on (18)F-DCFPyL PSMA PET/CT Imaging in Biochemical Recurrence of Prostate Cancer. J. Nucl. Med. 2019. [CrossRef] [PubMed]

29. Giesel, F.L.; Knorr, K.; Spohn, F.; Will, L.; Maurer, T.; Flechsig, P.; Neels, O.; Schiller, K.; Amaral, H.; Weber, W.A.; et al. Detection Efficacy of (18)F-PSMA-1007 PET/CT in 251 Patients with Biochemical Recurrence of Prostate Cancer After Radical Prostatectomy. J. Nucl. Med. 2019, 60, 362-368. [CrossRef] [PubMed]

30. Han, S.; Woo, S.; Kim, Y.J.; Suh, C.H. Impact of (68)Ga-PSMA PET on the Management of Patients with Prostate Cancer: A Systematic Review and Meta-analysis. Eur. Urol. 2018, 74, 179-190. [CrossRef] [PubMed]

31. Giesel, F.L.; Will, L.; Lawal, I.; Lengana, T.; Kratochwil, C.; Vorster, M.; Neels, O.; Reyneke, F.; Haberkon, U.; Kopka, K.; et al. Intraindividual Comparison of (18)F-PSMA-1007 and (18)F-DCFPyL PET/CT in the Prospective Evaluation of Patients with Newly Diagnosed Prostate Carcinoma: A Pilot Study. J. Nucl. Med. 2018, 59, 1076-1080. [CrossRef]

(C) 2019 by the authors. Licensee MDPI, Basel, Switzerland. This article is an open access article distributed under the terms and conditions of the Creative Commons Attribution (CC BY) license (http://creativecommons.org/licenses/by/4.0/). 
Brief Report

\title{
${ }^{99} \mathrm{~m}$ Tc-A1 as a Novel Imaging Agent Targeting Mesothelin-Expressing Pancreatic Ductal Adenocarcinoma
}

\author{
Christopher Montemagno ${ }^{1,2}$, Shamir Cassim ${ }^{2}$, Dimitry Trichanh ${ }^{1}$, Clara Savary ${ }^{3}$, \\ Jacques Pouyssegur ${ }^{2,4}$, Gilles Pagès ${ }^{2,4}$, Daniel Fagret ${ }^{1}$, Alexis Broisat ${ }^{1, *}$ and Catherine Ghezzi ${ }^{1}$ \\ 1 LRB, CHU Grenoble Alpes, INSERM U1039, Université Grenoble Alpes, 38000 Grenoble, France; \\ montemagno.chris@gmail.com (C.M.); dim.trichanh@gmail.com (D.T.); DFagret@chu-grenoble.fr (D.F.); \\ catherine.ghezzi@univ-grenoble-alpes.fr (C.G.) \\ 2 Biomedical Department, Centre Scientifique de Monaco, 98000 Monaco, Monaco; \\ shamir_cassim@yahoo.fr (S.C.); Jacques.POUYSSEGUR@unice.fr (J.P.); Gilles.Pages@unice.fr (G.P.) \\ 3 IGDR (Institut de génétique et développement de Rennes), UMR CNRS 6290, Université de Rennes, \\ 35000 Rennes, France; clara.savary@univ-rennes1.fr \\ 4 Institute for Research on Cancer and Aging of Nice, Centre Antoine Lacassagne, CNRS UMR 7284, INSERM \\ U1081, Université Côte d'Azur, 06200 Nice, France \\ * Correspondence: alexis.broisat@inserm.fr; Tel.: +33-4-76-63-71-02
}

Received: 20 September 2019; Accepted: 8 October 2019; Published: 10 October 2019

\begin{abstract}
Mesothelin is a membrane-associated protein overexpressed in pancreatic ductal adenocarcinoma (PDAC). Some mesothelin-targeted therapies are in clinical development but the identification of patients eligible for such therapies is still challenging. The objective of this study was to perform the imaging of mesothelin in mice models of PDAC with a technetium-labeled anti-mesothelin single-domain antibody ( $\left.{ }^{99 \mathrm{~m}} \mathrm{Tc}-\mathrm{A} 1\right)$. Methods: The Cancer Genomic Atlas (TCGA) database was used to determine the prognostic role of mesothelin in PDAC. ${ }^{99 \mathrm{~m}} \mathrm{Tc}-\mathrm{A} 1$ was evaluated both in vitro in PDAC cells (SW1990 and AsPC-1) and in vivo in an experimental model of mesothelin-expressing PDAC (AsPC-1) in mice. Results: TCGA analysis showed that PDAC patients with high mesothelin expression had a shorter overall survival $(\mathrm{P}=0.00066)$. The binding of ${ }^{99 \mathrm{~m}} \mathrm{Tc}-\mathrm{A} 1 \mathrm{was} 2.1$-fold greater in high-mesothelin-expressing AsPC-1 cells when compared to moderate-mesothelin-expressing SW1990 cells $(p<0.05)$. In vivo, the ${ }^{99 \mathrm{~m}} \mathrm{Tc}-\mathrm{A} 1$ uptake was 3.5-fold higher in AsPC-1-derived tumors as compared to a technetium-labeled irrelevant antibody $\left({ }^{99 \mathrm{~m}} \mathrm{Tc}-\mathrm{Ctl}\right)(p<0.01)$. Conclusions: ${ }^{99 \mathrm{~m}} \mathrm{Tc}-\mathrm{A} 1$ accurately allows imaging of mesothelin-expressing experimental PDAC tumors. Our experiments paved the way for the development of a companion test for mesothelin-targeted therapies.
\end{abstract}

Keywords: PDAC; Mesothelin; noninvasive imaging

\section{Introduction}

Pancreatic ductal adenocarcinoma (PDAC) is one of the most aggressive tumors, representing the fourth leading cause of cancer-related deaths worldwide in 2018 [1]. By the year 2030, PDAC is projected to surpass breast, colorectal, and prostate cancer and to become the second most deadly malignancy [2]. Despite intense clinical research, the five-year survival rate remains just around 5-7\% and one-year survival is achieved in less than $20 \%$ of cases [3]. Moreover, resistance to chemotherapy and lack of effective therapies contribute to the pejorative prognosis [4]. Finally, the majority of PDAC is diagnosed at advanced stages, thereby limiting therapeutic windows to manage patients [5].

The mesothelin gene encodes a $71 \mathrm{kDa}$ precursor protein. It is processed in a shed form known as Megakaryocyte Potentiating Factor, and a $40 \mathrm{kDa}$ GPI-anchored membrane form, which is the mesothelin protein itself [6]. Mesothelin expression is limited to mesothelial cells of the pleura, 
peritoneum, and pericardium. In normal tissues, the expression of mesothelin is very weak. However, its overexpression has been evidenced in several types of cancers including ovarian cancers and mesothelioma. Hence, mesothelin has been identified as a diagnostic marker and a relevant therapeutic target. As much as $80-85 \%$ of PDAC displayed greater levels of mesothelin [6,7]. No significant elevation of the shed form was detected in the serum of PDAC patients. The major form in cancers is membrane-associated mesothelin [8]. Mesothelin-targeted therapies are currently on clinical evaluation for the management of PDAC patients. However, the identification of patients eligible for such therapies still remains a challenging issue [6].

We recently validated ${ }^{99} \mathrm{~m}$ Tc-A1 as a single-domain-based imaging agent used for the phenotypic imaging of membrane mesothelin-expressing breast cancer [9]. In this study, we tested the ability of ${ }^{99} \mathrm{~m}$ Tc-A1 in imaging mesothelin-expressing PDAC tumors.

\section{Results}

\subsection{Overexpression of Mesothelin in PDAC Patients Is Associated with Poor Clinical Outcomes}

Tumoral PDAC-derived specimens demonstrated significant increased levels of mesothelin $(M S L N)$ when compared to peritumoral (nontumoral) pancreatic tissues (Figure 1A) $(\mathrm{n}=179$ and 171, respectively; $p<0.05)$. PDAC patients with high MSLN tumoral gene expression had a significant decreased overall survival when compared to patients with low expression (Figure 1B) ( $\mathrm{n}=177$; $\mathrm{P}=0.00066$; HR: 2.05). Moreover, an elevated expression pattern was only observed in advanced stages (comparison of stages I and II to stages III and IV, $\operatorname{Pr}(>\mathrm{F})=0.00881$ ) (Figure $1 \mathrm{C}$ ). To further validate our PDAC in silico dataset study model, greater expression of proliferative markers MKI67, CCNB1, and PCNA were only depicted in tumoral PDAC-derived specimens (Figure S1A, $p<0.05$ ) and their overexpressions were associated with a shorter overall survival (Figure S1B, $p<0.01$ ).

A

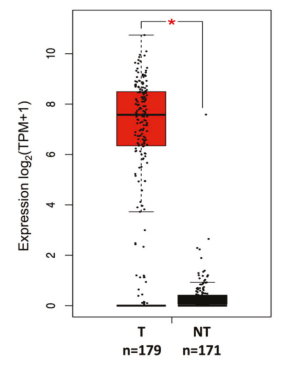

B

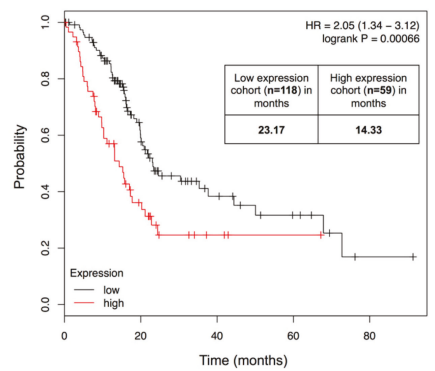

C

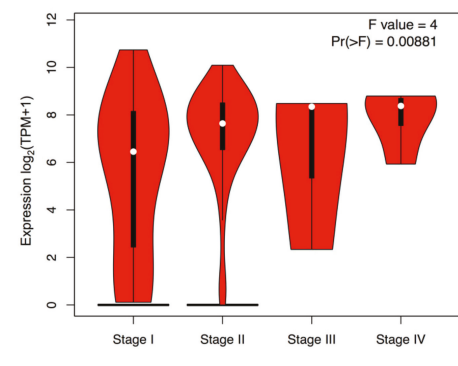

Figure 1. Prognostic value of mesothelin expression by pancreatic ductal adenocarcinoma (PDAC) patients for survival. (A) Expression of mesothelin in tumoral (T) and nontumoral (NT) pancreatic tissues from The Cancer Genomic Atlas (TCGA) and Genomic Tissue-Expression (GTEx) datasets. The red and gray boxes represent PDAC and nontumoral-derived tissues, respectively (T: $\mathrm{n}=179$ and NT: $\mathrm{n}=171$ ). (B) Kaplan-Meier plots of overall survival probability (plotted on Y-axis) of PDAC cancer patients is shown (TCGA data, $\mathrm{n}=177$ ). Patients have been stratified into high (red lines, $\mathrm{n}=59$ ) or low (black lines, $\mathrm{n}=118$ ) expression-based "risk-groups" by their gene expression of mesothelin. The patient follow-up is indicated in months on the X-axis. Respective log-rank test $p$-value, HR, and computed median survivals of low and high expression cohorts in months are shown and were calculated at the best auto-selected cut-off. (C) Violin plot showing the average gene expression levels of mesothelin at early (I and II) and advanced (III and IV) cancer stages of PDAC patients (TCGA database, $\mathrm{n}=179)$. ${ }^{*} p<0.05$. 


\section{2. ${ }^{99 m} \mathrm{Tc}-A 1$ Binding on Mesothelin-Expressing PDAC Cell Lines}

Through an unbiased in silico approach, mesothelin expression was assessed in 20 PDAC cell lines. An increased, moderated, and reduced mRNA expression of mesothelin was evidenced in AsPC-1, SW1990, and MIAPaCa-2, respectively (Figure 2A). Based on this observation, high-, medium-, and low-MSLN-expressing PDAC cell lines were selected for in vitro characterization. Mesothelin protein was expressed by AsPC-1 and SW1990 but not by MIAPaCa-2 cells (Figure 2B, Figure S2). ${ }^{99 \mathrm{~m}} \mathrm{Tc}-\mathrm{A} 1$ binding was then assessed on these cell lines (Figure 2C). ${ }^{99 \mathrm{~m}} \mathrm{Tc}-\mathrm{A} 1$ binding was 2.1 -fold higher in AsPC-1 as compared to SW1990 cells $(p<0.05)$.

A

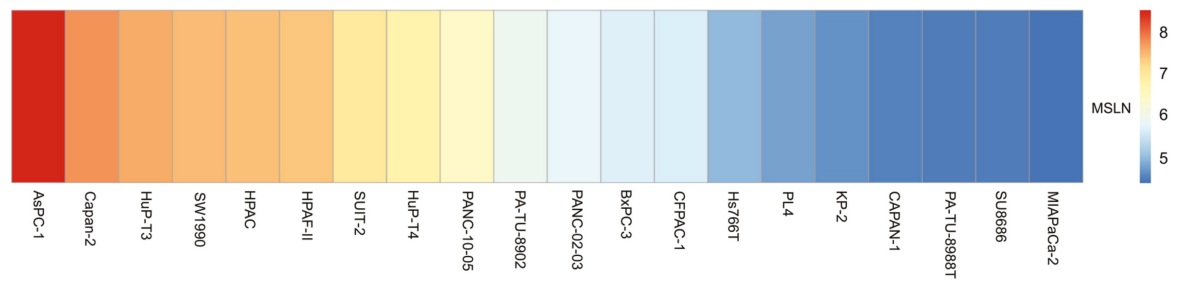

B

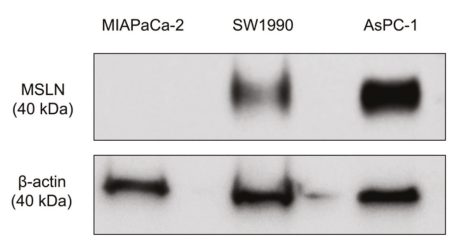

C

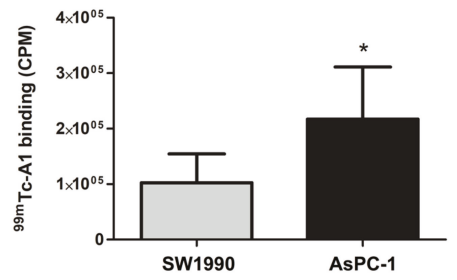

Figure 2. ${ }^{99 \mathrm{~m}} \mathrm{Tc}-\mathrm{A} 1$ binds to mesothelin-expressing cells in vitro. (A) Heatmap displaying MSLN gene expression levels across 20 PDAC cell lines. (B) Mesothelin expression of MIAPaCa-2, SW1990, AsPC-1 cells was assessed by Western blot. (C) Binding of ${ }^{99 m}$ Tc-A1 to SW1990 and AsPC-1 cells ( $n=6$ per condition). Results were expressed in counts per minute (CPM). ${ }^{*} p<0.05$ vs. SW1990.

\subsection{SPECT-CT Imaging of Mesothelin in Subcutaneous Tumor Model}

Coronal and transversal views of fused Single Photon Emission Computed Tomography (SPECT-CT) images are shown in Figure 3A. ${ }^{99 \mathrm{~m}}$ Tc-A1 uptake in mesothelin-positive AsPC-1 cells was readily identifiable, whereas a weak signal was detected using the irrelevant control sdAb (Figure $3 \mathrm{~A}$ ). This observation was further confirmed by image quantification showing that ${ }^{99 \mathrm{~m}} \mathrm{Tc}-\mathrm{A} 1$ uptake was 3.5 -fold higher than ${ }^{99 \mathrm{~m}} \mathrm{Tc}-\mathrm{Ctl}$ uptake in AsPC-1 tumor-bearing mice $\left(2.4 \pm 0.6 \mathrm{vs} .0 .7 \pm 0.2 \% \mathrm{ID} / \mathrm{cm}^{3}\right.$, $\mathrm{P}<0.01$ ) (Figure 3B). This result was then confirmed by ex vivo gamma-well counting showing that the ${ }^{99 \mathrm{~m}} \mathrm{Tc}-\mathrm{A} 1$ condition displayed a significant greater uptake $(\mathrm{P}<0.01)$ (Figure $\left.3 \mathrm{C}\right)$. Linear regression analysis confirmed the observations from both in vivo and ex vivo quantifications $(\mathrm{Y}=1.25 \times \mathrm{X}+0.04$, $r^{2}=0.98, P<0.001$ ) (Figure 3D). Thus, these results validate the use of ${ }^{99 m} \mathrm{Tc}-\mathrm{A} 1$ in assessing in vivo MSLN expression in PDAC. 
A

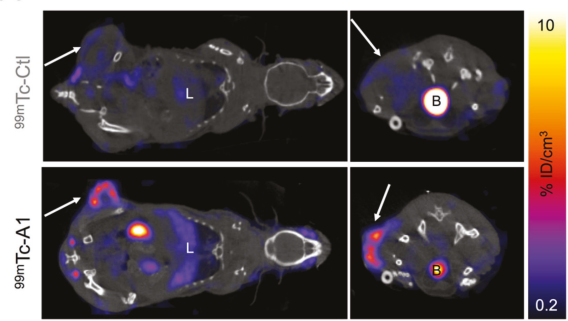

C

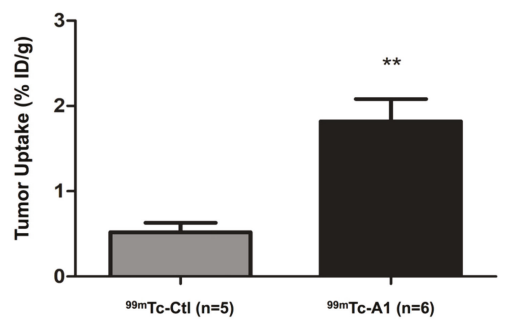

B

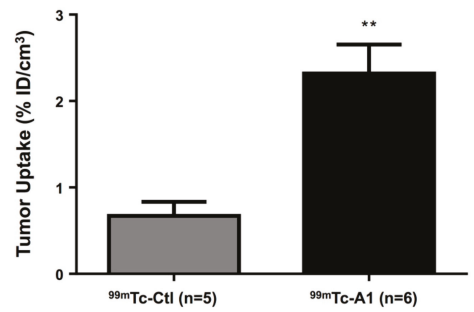

D

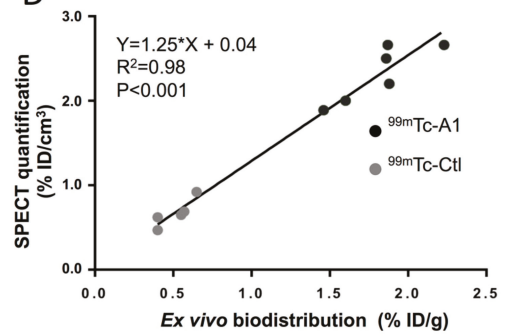

Figure 3. ${ }^{99 \mathrm{~m}} \mathrm{Tc}-\mathrm{A} 1$ binds to AsPC-1 tumor in vivo. (A) Representative coronal and transversal views of fused SPECT-CT images of AsPC-1 tumor-bearing mice one hour after IV injection of ${ }^{99 \mathrm{~m}} \mathrm{Tc}-\mathrm{Ctl}$ $(n=5)$ or ${ }^{99 m}$ Tc-A1 $(n=6)$. B: bladder and L: liver. Tumor is indicated by the white arrow. (B) In vivo quantification of ${ }^{99} \mathrm{~m}$ Tc-A1 and ${ }^{99} \mathrm{~m}$ Tc-A1 tumor uptake from SPECT images. (C) Ex vivo quantification of ${ }^{99 \mathrm{~m}} \mathrm{Tc}-\mathrm{A} 1$ tumor uptake from postmortem analysis. (D) Correlation between tumor uptake assessed by SPECT and biodistribution. ${ }^{* *} p<0.01$ vs. ${ }^{99 \mathrm{~m}} \mathrm{Tc}-\mathrm{Ctl}$.

\section{Discussion}

Despite intense clinical efforts, PDAC remains one of the most aggressive malignancies with a five-year survival rate around 5-7\% [3]. The advanced stages of PDAC at the diagnosis and the intrinsic resistance to standard therapies give rise to this dismal prognosis [7]. Until today, most clinical trials have failed to demonstrate significant improvement in patient survival [10]. Identification of targetable molecules for PDAC early diagnosis and treatment thus represents an urgent need. Among the potential targets, current evidence includes mesothelin as a PDAC biomarker. Mesothelin expression is restricted to mesothelial cells (pleura, peritoneum, and pericardium) and its physiological function still remains unknown. Indeed, mesothelin seems to be nonessential in normal tissues [11]. However, mesothelin is present in a wide range of tumors including ovarian and lung cancers (60-65\%), and also PDAC-derived tumors (80-85\%) [12]. Several studies demonstrated a crucial role of mesothelin in cell survival, proliferation, and resistance to chemotherapy $[7,13]$.

Using TCGA datasets, we observed an overexpression of mesothelin only in PDAC-derived specimens in comparison to nontumoral samples. In agreement with other previous studies, high expression of mesothelin predicted shorter overall survival of PDAC patients [7,14]. The increase of mesothelin expression in the advanced disease stages of PDAC suggests its relevance as a disease progression biomarker. Accumulating evidences suggest its role as a diagnostic marker and a therapeutic target [7]. Several strategies including monoclonal antibodies (mAbs), vaccines, or immunotoxins are currently under clinical evaluation [7]. Importantly, soluble mesothelin assays failed in validating their diagnostic potential for PDAC detection despite overexpression of the membrane-bound form $[15,16]$. No significant variation of serum mesothelin levels could be evidenced regardless of the different stages of PDAC progression [17]. Nevertheless, identification of patients eligible for such anti-mesothelin therapies still remains a perplexing issue. 
Nuclear imaging represents a highly sensitive and noninvasive imaging modality that could address this challenge. Indeed, we previously validated ${ }^{99 \mathrm{~m}} \mathrm{Tc}-\mathrm{A} 1$, which is a single-domain antibody-derived imaging agent, as an efficient probe in accurately targeting mesothelin-expressing triple-negative breast cancer [9]. Herein, we evaluated the potential of ${ }^{99 \mathrm{~m}} \mathrm{Tc}-\mathrm{A} 1 \mathrm{as}$ an imaging probe of mesothelin-expressing PDAC. Our in vivo experiments showed that ${ }^{99 \mathrm{~m}} \mathrm{Tc}-\mathrm{A} 1$ enabled the noninvasive visualization of AsPC-1-derived tumors by SPECT imaging at early time points. These results reinforce our study on triple-negative breast cancer, despite the lower ${ }^{99 \mathrm{~m}} \mathrm{Tc}-\mathrm{A} 1$ uptake of PDAC mesothelin-positive tumors [9]. In addition to triple-negative breast cancer and PDAC, ${ }^{99 \mathrm{~m}} \mathrm{Tc}-\mathrm{A} 1$ imaging represents a relevant option to visualize other aggressive cancers overexpressing mesothelin such as ovarian and lung cancers, for which the imaging modalities are mainly based on the use of conventional antibodies. However, further in vivo competition experiments using unlabeled A1 would confirm the specificity of ${ }^{99 \mathrm{~m}} \mathrm{Tc}-\mathrm{A} 1$ uptake by PDAC-derived tumors as previously established in breast cancer [9]. To our knowledge, all mesothelin-targeted radiotracers indeed rely on the production of mAbs or single-chain variable fragments (scFv) [18-20]. Their hepatic elimination and their slow blood clearance are the main limitations for their use as imaging probes. The smaller size of sdAb-based imaging agents allows: (1) a fast blood clearance and (2) image acquisitions with high target-to-background ratios as early as one hour following administration. Future perspectives of this work include evaluation of the sensitivity of ${ }^{99 \mathrm{~m}} \mathrm{Tc}-\mathrm{A} 1$ and its ability to phenotype mesothelin-expressing tumors in an orthotopic model of PDAC. These results support further preclinical development of ${ }^{99 \mathrm{~m}} \mathrm{Tc}-\mathrm{A} 1$ and translation to human applications for cancers that overexpress mesothelin.

Future directions of this work would therefore include clinical translation of ${ }^{99 \mathrm{~m}} \mathrm{Tc}-\mathrm{A} 1$ for the identification of mesothelin-expressing PDAC that would allow selection of patients who might benefit from mesothelin-targeted therapies that are currently undergoing clinical trials [6].

\section{Materials and Methods}

\subsection{Patients Online Datasets}

Gene expression levels of mesothelin (MSLN), MKI67, CCNB1, and PCNA were analyzed and compared in tumor (T) and normal tissue (NT) from PDAC-derived specimens using PAAD (Pancreatic adenocarcinoma), TCGA (The Cancer Genome Atlas), and GTEx datasets (T: $n=179$ and NT: $n=171$ ) through the available interface Gene Expression Profiling Interactive Analysis (GEPIA) [13]. The results published here are based upon data generated by the TCGA Research Network [21]. Survival analysis was performed using the Kaplan-Meier (KM) Plotter Database as already described [22-24]. The gene expression was extracted from the TCGA database, with at least five-year follow-up data from PDAC patients $(\mathrm{n}=177)$. The data were not adjusted for clinical status and to analyze the prognostic value of MSLN, MKI67, CCNB1, and PCNA genes, the KM method was used to estimate survival curves. Hazard ratio (HR) and overall survival were calculated at the best auto-selected cut-off. $p$-values were calculated using the log-rank test to compare survival curves of high and low gene expression groups and a $p$-value below 0.05 was considered to be statistically significant.

\subsection{Cell Lines Gene Expression Data and Visualization}

Preprocessed microarray gene expression data deposited in ArrayExpress (E-MTAB-3610, EMBL-EBI, Cambridgeshire, UK) were downloaded from the Genomics of Drug Sensitivity in Cancer (GDSC) web page [25]. The gene symbols were mapped to Ensembl gene IDs by using the R package biomaRt v.2.40.3 with the human genome version GRCh37.p13. The R package pheatmap v.1.0.12 was used to visualize the gene expression data using hierarchical clustering with Euclidean distance and "complete" agglomeration method. 


\subsection{Cell Lines and Culture Conditions}

AsPC-1, SW1990, and MIAPaCa-2 cell lines were maintained at $37^{\circ} \mathrm{C}$ and $5 \% \mathrm{CO}_{2}$ and cultured in DMEM medium (4.5 g/L glucose, 2 mM L-glutamine, 1 mM Sodium Pyruvate) supplemented with $10 \%$ fetal bovine serum and $1 \%$ penicillin-streptomycin.

\subsection{Immunoblotting}

Total proteins from AsPC-1, SW1990, and MIAPaCa-2 cells were extracted using RIPA buffer (Thermo Fisher Scientific, Illkirch, France). After migration in 12\% SDS-polyacrylamide gel, proteins were transferred to nitrocellulose membranes (90 min, $100 \mathrm{~V}$ ). They were saturated in PBS-Tween $0.1 \%$ containing $2 \%$ milk at room temperature for $1 \mathrm{~h}$ and then probed overnight with the antimesothelin antibody (1/2000, Boster Immunoleader, Pleasanton, CA, USA). Membranes were then stripped during $10 \mathrm{~min}(0.1 \%$ SDS, $1.5 \%$ glycin, $1 \%$ Tween, $\mathrm{pH} 2.2)$ for $\beta$-actin detection $(1 / 10000$, Beckton Dickinson, Le Pont de Claix, France).

\subsection{In Vitro Binding Studies}

A1 and irrelevant control single-domain antibody (sdAb) were radiolabeled with technetium-99m $\left({ }^{99 \mathrm{~m}} \mathrm{Tc}\right)$ using the tricarbonyl method as previously described [9]. For in vitro studies, 200,000 AsPC-1, SW1990, and MIAPaCa-2 cells were incubated with $40 \mathrm{nM}$ of ${ }^{99 \mathrm{~m}} \mathrm{Tc}-\mathrm{A} 1$ for $1 \mathrm{~h}$ at $4{ }^{\circ} \mathrm{C}$. After five washes in cold PBS, the radioactivity was determined using a $\gamma$-counter (Wizard ${ }^{2}$, Perkin Elmer, Courtaboeuf, France). Unspecific binding of ${ }^{99} \mathrm{~m}$ Tc-A1 was determined on MIAPaCa-2 cells and was subtracted from ${ }^{99 \mathrm{~m}}$ Tc-A1 binding on SW1990 and AsPC-1 cells. Results were expressed in counts per minute (CPM).

\subsection{Tumor Model, SPECT-CT Imaging, and Postmortem Analysis}

All animal procedures conformed to French government guidelines (Articles R214-87 to R214-126; European directive 2010/63/UE). They were performed in an approved facility (C385161 0005) under permit APAFIS\#3690-2016011916045217 v4 from the French Ministry of Research. Four million AsPC-1 cells were subcutaneously injected into the left flank of five-week-old female Swiss Nude immunodeficient mice $(\mathrm{n}=11)$, in a $2 / 1(\mathrm{v} / \mathrm{v})$ PBS/Matrigel (Corning) mix. Tumors were allowed to grow until they reached $200 \mathrm{~mm}^{3}$. AsPC-1-tumor-bearing mice were either injected with irrelevant control ${ }^{99 \mathrm{~m}} \mathrm{Tc}-\mathrm{Ctl}(\mathrm{n}=5)$ or ${ }^{99 \mathrm{~m}} \mathrm{Tc}-\mathrm{A} 1(\mathrm{n}=6)$. SPECT-CT acquisitions were performed one hour after injection of $42.1 \pm 9.0 \mathrm{MBq}$ of ${ }^{99 \mathrm{~m}} \mathrm{Tc}-\mathrm{sd} \mathrm{Abs}$. ${ }^{99 \mathrm{~m}} \mathrm{Tc}-\mathrm{Ctl}$ and ${ }^{99 \mathrm{~m}} \mathrm{Tc}-\mathrm{A} 1$ tumor uptake was expressed in $\% \mathrm{ID} / \mathrm{cm}^{3}$. Two hours after injection and immediately following SPECT-CT image acquisitions, anesthetized mice were euthanized using $\mathrm{CO}_{2}$ and tumors were harvested and weighed, and tracer activity was determined with a $\gamma$-counter (Wizard ${ }^{2}$, Perkin). Results were corrected for decay, injected dose, and tumor weight, and expressed as \% ID/g.

\subsection{Statistics}

Results are expressed as means \pm standard deviation and analyzed with GraphPad Prism software (Version 6, software, San Diego, CA, USA). Differences between groups were analyzed using an unpaired Mann-Whitney test for intergroup analysis. Significance of linear correlations was assessed using a Pearson's test. A $p$ value below 0.05 was considered significant $\left({ }^{*} \mathrm{P}<0.05\right.$, ${ }^{*} \mathrm{P}<0.01$, *** $\mathrm{P}<0.001)$.

\section{Conclusions}

${ }^{99 m}$ Tc-A1 allows imaging of mesothelin-expressing PDAC. Our study represents the first step to using this technology as a companion test to select patients eligible for mesothelin-targeted therapies.

Supplementary Materials: The following are available online at http://www.mdpi.com/2072-6694/11/10/1531/s1, Figure S1: Markers of proliferation are associated with decreased overall survival in patients with PDAC. Figure S2: Mesothelin expression of MIAPaCa-2, SW1990 and AsPC-1 was assessed by Western Blot. 
Author Contributions: Conceptualization, C.M., A.B., C.G., D.F; methodology, C.M., S.C., A.B., C.G., D.F.; formal analysis, C.M., S.C., D.T., C.S., J.P., G.P., A.B., C.G., D.F; investigation, C.M., S.C., D.T., C.S., J.P., G.P., A.B., C.G., D.F.; resources, C.M., S.C., D.T., C.S., J.P., G.P., A.B., C.G., D.F.; writing-original draft preparation, C.M., S.C.; writing-review and editing, C.M., S.C., D.T., C.S., J.P., G.P., A.B., C.G., D.F.; visualization, C.M., A.B., C.G., D.F.; supervision, J.P., G.P., A.B., D.F., C.G.; funding acquisition, A.B., C.G., D.F.

Funding: This work was partly funded by France Life Imaging, grant "ANR-11-INBS-0006".

Acknowledgments: We thank Gael Roth (Université Grenoble Alpes, Inserm, CNRS, IAB, 38000 Grenoble) who kindly provided MIAPaCa-2, SW1990 and AsPC-1 cell lines.

Conflicts of Interest: The authors declare no conflict of interest.

\section{References}

1. Bray, F.; Ferlay, J.; Soerjomataram, I.; Siegel, R.L.; Torre, L.A.; Jemal, A. Global cancer statistics 2018: GLOBOCAN estimates of incidence and mortality worldwide for 36 cancers in 185 countries. CA A Cancer J. Clin. 2018, 68, 394-424. [CrossRef] [PubMed]

2. Rahib, L.; Smith, B.D.; Aizenberg, R.; Rosenzweig, A.B.; Fleshman, J.M.; Matrisian, L.M. Projecting Cancer Incidence and Deaths to 2030: The Unexpected Burden of Thyroid, Liver, and Pancreas Cancers in the United States. Cancer Res. 2014, 74, 2913-2921. [CrossRef] [PubMed]

3. Kleeff, J.; Korc, M.; Apte, M. Pancreatic cancer. Nat. Rev. Dis. Primers 2016, 2, 16022. [CrossRef] [PubMed]

4. Aslan, M.; Shahbazi, R.; Ulubayram, K.; Ozpolat, B. Targeted Therapies for Pancreatic Cancer and Hurdles Ahead. Anticancer Res. 2018, 38, 6591-6606. [CrossRef] [PubMed]

5. Ryan, D.P.; Hong, T.S.; Bardeesy, N. Pancreatic adenocarcinoma. N. Engl. J. Med. 2014, 371, 1039-1049. [CrossRef] [PubMed]

6. Hassan, R.; Thomas, A.; Alewine, C.; Le, D.T.; Jaffee, E.M.; Pastan, I. Mesothelin Immunotherapy for Cancer: Ready for Prime Time? J. Clin. Oncol. 2016, 34, 4171-4179. [CrossRef] [PubMed]

7. Nichetti, F.; Marra, A.; Corti, F. The Role of Mesothelin as a Diagnostic and Therapeutic Target in Pancreatic Ductal Adenocarcinoma: A Comprehensive Review. Targ Oncol. 2018, 13, 333-351. [CrossRef]

8. Sharon, E.; Zhang, J.; Hollevoet, K.; Steinberg, S.M.; Pastan, I.; Onda, M.; Gaedcke, J.; Ghadimi, B.M.; Ried, T.; Hassan, R. Serum mesothelin and megakaryocyte potentiating factor in pancreatic and biliary cancers. Clin. Chem. Lab. Med. 2012, 50, 721-725. [CrossRef]

9. Montemagno, C.; Bacot, S.; Ahmadi, M.; Kerfelec, B.; Baty, D.; Debiossat, M.; Soubies, A.; Perret, P.; Riou, L.; Fagret, D.; et al. Preclinical Evaluation of Mesothelin-Specific Ligands for SPECT Imaging of Triple-Negative Breast Cancer. J. Nucl. Med. 2018, 59, 1056-1062. [CrossRef]

10. Adamska, A.; Domenichini, A.; Falasca, M. Pancreatic Ductal Adenocarcinoma: Current and Evolving Therapies. Int. J. Mol. Sci. 2017, 18, 1338. [CrossRef]

11. Bera, T.K.; Pastan, I. Mesothelin Is Not Required for Normal Mouse Development or Reproduction. Mol. Cell. Boil. 2000, 20, 2902-2906. [CrossRef] [PubMed]

12. Morello, A.; Sadelain, M.; Adusumilli, P.S. Mesothelin-Targeted CARs: Driving T Cells to Solid Tumors. Cancer Discov. 2016, 6, 133-146. [CrossRef] [PubMed]

13. Tang, Z.; Li, C.; Kang, B.; Gao, G.; Li, C.; Zhang, Z. GEPIA: A web server for cancer and normal gene expression profiling and interactive analyses. Nucleic Acids Res. 2017, 45, W98-W102. [CrossRef] [PubMed]

14. Argani, P.; Iacobuzio-Donahue, C.; Ryu, B.; Rosty, C.; Goggins, M.; Wilentz, R.E.; Murugesan, S.R.; Leach, S.D.; Jaffee, E.; Yeo, C.J.; et al. Mesothelin is overexpressed in the vast majority of ductal adenocarcinomas of the pancreas: Identification of a new pancreatic cancer marker by serial analysis of gene expression (SAGE). Clin. Cancer Res. 2001, 7, 3862-3868. [PubMed]

15. Zhu, L.; Liu, Y.; Chen, G. Diagnostic value of mesothelinin pancreatic cancer: A meta-analysis. Int. J. Clin. Exp. Med. 2014, 7, 4000-4007.

16. Johnston, F.M.; Tan, M.C.; Tan, B.R.; Porembka, M.R.; Brunt, E.M.; Linehan, D.C.; Simon, P.O.; Plambeck-Suess, S.; Eberlein, T.J.; Hellstrom, K.E.; et al. Circulating mesothelin protein and cellular antimesothelin immunity in patients with pancreatic cancer. Clin. Cancer Res. 2009, 15, 6511-6518. [CrossRef]

17. Kendrick, Z.W.; Firpo, M.A.; Repko, R.C.; Scaife, C.L.; Adler, D.G.; Boucher, K.M.; Mulvihill, S.J. Serum IGFBP2 and MSLN as diagnostic and prognostic biomarkers for pancreatic cancer. HPB 2014, 16, 670-676. [CrossRef] 
18. Ter Weele, E.J.; Terwisscha van Scheltinga, A.G.T.; Kosterink, J.G.W. Imaging the distribution of an antibody-drug conjugate constituent targeting mesothelin with $89 \mathrm{Zr}$ and IRDye 800CW in mice bearing human pancreatic tumor xenografts. Oncotarget 2015, 6, 42081-42090. [CrossRef]

19. Yakushiji, H.; Kobayashi, K.; Takenaka, F.; Kishi, Y.; Shinohara, M.; Akehi, M.; Sasaki, T.; Ohno, E.; Matsuura, E. Novel single-chain variant of antibody against mesothelin established by phage library. Cancer Sci. 2019, 110, 2722-2733. [CrossRef]

20. Kobayashi, K.; Sasaki, T.; Takenaka, F.; Yakushiji, H.; Fujii, Y.; Kishi, Y.; Kita, S.; Shen, L.; Kumon, H.; Matsuura, E. A Novel PET Imaging Using 64Cu-Labeled Monoclonal Antibody against Mesothelin Commonly Expressed on Cancer Cells. J. Immunol. Res. 2015, 2015, 1-15. [CrossRef]

21. The Cancer Genome Atlas. Available online: https://www.cancer.gov/tcga (accessed on 8 October 2019).

22. Györffy, B.; Lanczky, A.; Eklund, A.C. An online survival analysis tool to rapidly assess the effect of 22,277 genes on breast cancer prognosis using microarray data of 1,809 patients. Breast Cancer Res. Treat. 2010, 123, 725-731. [CrossRef] [PubMed]

23. Győrffy, B.; Lánczky, A.; Szállási, Z. Implementing an online tool for genome-wide validation of survival-associated biomarkers in ovarian-cancer using microarray data from 1287 patients. Endocr. Related Cancer 2012, 19, 197-208. [CrossRef] [PubMed]

24. Szász, A.M.; Lánczky, A.; Nagy, Á.; Förster, S.; Hark, K.; Green, J.E.; Boussioutas, A.; Busuttil, R.; Szabó, A.; Győrffy, B. Cross-validation of survival associated biomarkers in gastric cancer using transcriptomic data of 1,065 patients. Oncotarget 2016, 7, 49322-49333. [CrossRef] [PubMed]

25. The Genomics of Drug Sensitivity in Cancer (GDSC). Available online: ftp://ftp.sanger.ac.uk/pub/project/ cancerrxgene/releases/release-7.0/ (accessed on 8 October 2019).

(C) 2019 by the authors. Licensee MDPI, Basel, Switzerland. This article is an open access article distributed under the terms and conditions of the Creative Commons Attribution (CC BY) license (http://creativecommons.org/licenses/by/4.0/). 


\title{
FDG-PET/CT for Response Monitoring in Metastatic Breast Cancer: Today, Tomorrow, and Beyond
}

\author{
Malene Grubbe Hildebrandt ${ }^{1,2,3, *}$, Jeppe Faurholdt Lauridsen ${ }^{4}$, Marianne Vogsen ${ }^{1,2,5}$, \\ Jorun Holm ${ }^{1}$, Mie Holm Vilstrup ${ }^{1}$, Poul-Erik Braad ${ }^{1,2}$, Oke Gerke ${ }^{1,2}$, Mads Thomassen ${ }^{2,6}$, \\ Marianne Ewertz ${ }^{2,5}$, Poul Flemming Høilund-Carlsen ${ }^{1,2}$ and The Centre for Personalized \\ Response Monitoring in Oncology (PREMIO) \\ 1 Department of Nuclear Medicine, Odense University Hospital, 5000 Odense, Denmark \\ 2 Department of Clinical Research, University of Southern Denmark, 5230 Odense, Denmark \\ 3 Centre for Innovative Medical Technology, Odense University Hospital, 5000 Odense, Denmark \\ 4 Department of Nuclear Medicine, Lillebaelt Hospital, 7100 Vejle, Denmark \\ 5 Department of Oncology, Odense University Hospital, 5000 Odense, Denmark \\ 6 Department of Clinical Genetics, Odense University Hospital, 5000 Odense, Denmark \\ * Correspondence: Malene.grubbe.hildebrandt@rsyd.dk; Tel.: +45-3017-1888
}

Received: 20 July 2019; Accepted: 14 August 2019; Published: 15 August 2019

\begin{abstract}
While current international guidelines include imaging of the target lesion for response monitoring in metastatic breast cancer, they do not provide specific recommendations for choice of imaging modality or response criteria. This is important as clinical decisions may vary depending on which imaging modality is used for monitoring metastatic breast cancer. FDG-PET/CT has shown high accuracy in diagnosing metastatic breast cancer, and the Positron Emission Tomography Response Criteria in Solid Tumors (PERCIST) have shown higher predictive values than the CT-based Response Evaluation Criteria in Solid Tumors (RECIST) for prediction of progression-free survival. No studies have yet addressed the clinical impact of using different imaging modalities or response evaluation criteria for longitudinal response monitoring in metastatic breast cancer. We present a case study of a patient with metastatic breast cancer who was monitored first with conventional CT and then with FDG-PET/CT. We retrospectively applied PERCIST to evaluate the longitudinal response to treatment. We used the one-lesion PERCIST model measuring SULpeak in the hottest metastatic lesion on consecutive scans. This model provides a continuous variable that allows graphical illustration of disease fluctuation along with response categories. The one-lesion PERCIST approach seems able to reflect molecular changes and has the potential to support clinical decision-making. Prospective clinical studies addressing the clinical impact of PERCIST in metastatic breast cancer are needed to establish evidence-based recommendations for response monitoring in this disease.
\end{abstract}

Keywords: precision oncology; FDG-PET/CT; PERCIST; metastatic breast cancer

\section{Introduction}

Breast cancer is the most frequent malignant disease among women. In developed countries, more than $90 \%$ are diagnosed with operable breast cancer, while $5-10 \%$ have advanced or metastatic disease at diagnosis. The prognosis is generally good, with 10 -year survival rates of about $80 \%$ [1]. Survival with metastatic cancer is improving along with the rapid development of new treatments, such as HER2-targeting therapies [2]. Breast cancer is thus emerging as a chronic disease, where survivors can live for long periods of time.

In randomized clinical trials of new drugs, response to therapy is monitored using the New Response Evaluation Criteria in Solid Tumors (RECIST) approach [3], but current guidelines do not provide specific recommendations for response monitoring for patients with metastatic breast 
cancer outside clinical trials [4]. There is limited evidence supporting the clinical use of molecular imaging with 2-deoxy-2[18F] fluoroglucose-positron emission tomography/computed tomography (FDG-PET/CT) and the related PET Response Criteria in Solid Tumors (PERCIST) [5,6] for response monitoring in metastatic breast cancer. Due to the heterogeneity of breast cancer within and between patients, response monitoring has become a complex area, and specialized knowledge of the metastatic process and the molecular basis for response prediction and monitoring is required to enable the guidelines to be updated.

In this perspective paper, we address the potential of using PERCIST for longitudinal response monitoring in metastatic breast cancer and illustrate this with a case study. We reflect on the advantages of using PERCIST and suggest ways to improve and innovate the approach based on our experiences of using FDG-PET/CT for response monitoring in metastatic breast cancer. In addition, we relate advancements in molecular imaging technology to current knowledge about the biology of metastases and the evolving field of precision oncology.

\section{Metastasis, Progression, and Precision Oncology}

Treatment recommendations for primary breast cancer are based on the pathological molecular profile of the cancer's dominant cell clone [7]. Primary tumors are often heterogeneous, however, and may present with minor components of differing tumor cell types, i.e., cells with differing molecular profiles [8]. When a cancer spreads to other tissues, the metastases can be of a different type to the primary cancer, and sometimes the metastases are even different to each other $[9,10]$. This may be due to heterogeneity in the primary tumor that comprises more than one cell clone [9]. Similarly, if a tumor progresses during oncologic treatment, we may see sufficient treatment effect on the dominant clone but synchronous growth of a minor clone that does not respond to the chosen therapy [8].

This heterogeneity of metastatic breast cancer has stimulated the development of new treatment approaches such as estrogen- and HER2-receptor targeting therapies. The prolonged survival of patients with metastatic breast cancer is strongly related to estrogen- and HER2-positive tumors and is most likely due to these targeting therapies [2,11]. Revolutionary approaches for future cancer treatment include therapies targeting specific receptors on cancer cells or genetic mutations in gene clusters that encode so-called 'hub proteins'. As these mutations may be common across several cancer forms, future treatments could address clusters of genes or proteins rather than use the traditional categorization according to the originating tissue [12]. The next generation sequencing of tumor DNA and RNA appear to be relevant tools in future precision oncology as they might enable categorization of patients into different intervention 'baskets' with associated evidence-based recommendations for clinical management [13].

Precision medicine approaches, such as liquid biopsies that analyze circulating tumor DNA (ctDNA), may be able to accurately present the disease biology and potentially predict treatment response and prognosis [14]. However, potential drawbacks of sequencing ctDNA are a relatively low sensitivity and lack of visualization of the metastatic sites. These drawbacks could be reduced by the addition of molecular imaging to further support clinical decision-making. In the future, therefore, we expect that an optimal approach for response prediction and monitoring in metastatic cancers may be the combination of molecular imaging with genomic profiling using ctDNA.

\section{Response Prediction and Response Monitoring}

Most cancer treatments are still directed against the classic hallmarks of cancer and thus aim to suppress cancer growth and proliferation, cell division, and angiogenesis. While precision oncology approaches such as liquid biopsy $[8,15]$ have created high expectations for the ability to predict treatment effect and monitor disease, the methodology for analyzing liquid biopsies and gene mutations is not yet available for clinical purposes. In the meantime, we need to address currently available methods to improve clinical decision-making for systemic treatment of metastatic breast cancer. A first step should be to use molecular imaging methods based on glycosylation in cancer [16]. 
Munkley et al. proposed that glycosylation is closely associated with all the hallmarks of cancer and that glycans play a major role in cancer growth [16]. A key feature is the shift from oxidative phosphorylation to aerobic glycolysis (the Warburg effect). The increased glucose uptake in cancer cells is used in the molecular imaging modality of FDG-PET/CT to enable prognosis and response monitoring of a range of oncologic diseases despite their varying molecular profiles. Another developing area involves theranostic approaches such as targeting radionuclides for combined PET-imaging and targeted radioisotope therapies. FDG-PET/CT imaging reflects glucose metabolism in cancer, and the level of FDG-uptake mirrors the aggressiveness of cancer cells [17]. Early papers have shown a clear relationship between FDG uptake (measured as the standardized uptake value, SUV) and disease prognosis-a relationship that was clearly demonstrated for malignant mesothelioma in 1999 [18]. The prognostic value of the maximum SUV (SUVmax) and the metabolic tumor volume (MTV) was also recently demonstrated for primary breast cancer [19], further supporting the central role of glycosylation in cancer. As recurrent and metastatic breast cancers are FDG-avid diseases [20,21], FDG-PET/CT may be a valuable tool for response monitoring. Morphologic response monitoring tools such as the Response Evaluation Criteria in Solid Tumors (RECIST) and RECIST 1.1 have traditionally been used for this purpose [3]. However, the emerging molecular-directed treatments mean that we need to consider a shift towards molecular-based criteria. A recent retrospective study of patients with metastatic breast cancer showed that response prediction of progression-free and disease-specific survival was superior with PERCIST compared to RECIST [22]. In the following, we present a case study to illustrate the potential of FDG-PET/CT and PERCIST in longitudinal response monitoring of metastatic breast cancer.

\section{A Case Study of Longitudinal Response Monitoring in Metastatic Breast Cancer}

A 47-year-old woman presented in January 2010 with locally advanced estrogen-positive, HER2-normal carcinoma in the right breast. She gave written informed consent for using her data for research purposes. Figure 1 shows FDG-PET/CT images at the time of diagnosis of primary breast cancer, where a metastasis in a small osteolytic lesion in her left trochanter major was also suggested. This was not visible with conventional X-rays, and thus neoadjuvant chemotherapy was initiated with cyclophosphamide/doxorubicin followed by docetaxel. The patient underwent mastectomy and axillary lymph node dissection and then received adjuvant radiotherapy and endocrine therapy. The small osteolytic lesion in the left trochanter major might have been overlooked or considered a benign lesion by a less sensitive reader. Today, when equivocal bone lesions are observed on FDG-PET/CT, it would be relevant to consider providing a better $\mathrm{CT}$ window for evaluation of bone structures or performing additional imaging such as Magnetic Resonance Imaging (MRI) or PET/MRI in order to increase the diagnostic accuracy [23].

Fifteen months later, the patient experienced lower back pain. As shown in Figure 2, FDG-PET/CT revealed a focal intense FDG uptake in the left trochanter major (A) whereas no changes were identified on CT. Bone metastasis was verified by biopsy. Treatment with capecitabine and ibandronate was initiated, and the treatment effect was monitored for the first year using contrast-enhanced CT. The last of four conventional CT scans detected an osteolytic lesion in vertebra L3, while FDG-PET/CT shortly after revealed unexpected massive progression to the skeleton (B). Treatment was changed to navelbine, and monitoring with FDG-PET/CT was started, using qualitative assessment of response. When progression occurred after four months $(C)$, treatment was changed to eribulin, and subsequent disease regression was seen. The disease progressed again after five months (D) and treatment was altered to gemcitabine, resulting in several months without FDG-avid metastases. This sequence of progression, treatment change, and subsequent regression repeated itself, during which the patient received paclitaxel (2-3 months) (E); fulvestrant (5 months) (F); doxorubicin (10 months) (G); no treatment (4 months) (H), irinotecan (5 months) (I); and cyclophosphamide-methotrexate-fluorouracil (J). She passed away in February 2018. 
a

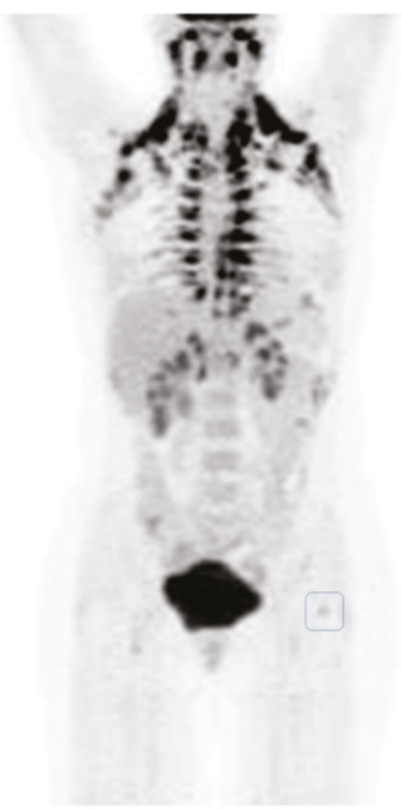

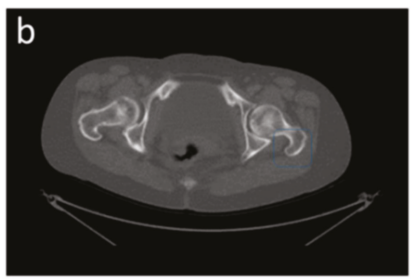

C
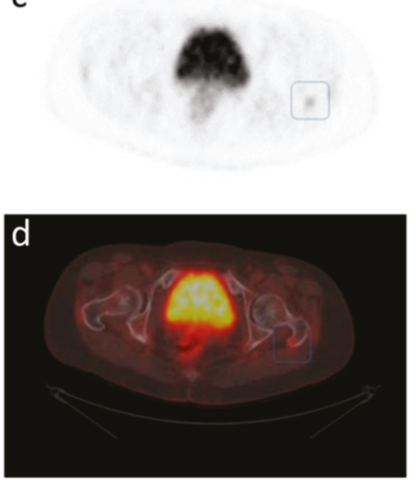

Figure 1. FDG-PET/CT performed for the first time in February 2010. Left (a): Maximum-intensity projection image showing an FDG-positive lesion in the left trochanter major, outlined by the blue square, suspicious of bone metastasis. High FDG uptake is seen in activated physiological brown fat tissue, but FDG uptake could not be seen in the primary tumor in the right breast. Right: Axial images of the pelvic region: (b) CT alone, (c) FDG-PET alone), and (d) fused FDG-PET/CT.

In this patient case, conventional $\mathrm{CT}$ in the first year after initiation of systemic treatment for metastatic breast cancer showed complete response (CR) in three initial scans and then progressive disease (PD) on a fourth CT scan in April 2012. A supplemental FDG-PET/CT performed four days later revealed massive progression (Figure 2B; April 12), indicating that conventional CT detects progression later than FDG-PET/CT does. We need prospective trials, however, to investigate whether this could influence clinical decisions or patient survival and quality of life.

As the maximum intensity projection (MIP) images from scans are easily interpreted, they are a useful collaborative tool in clinical decision-making. Current guidelines emphasize that patients and their families should participate in decision-making processes, and MIP or 'MIP-like' images can be relevant here as well [4]. A further option is standardized semi-quantitative assessment using PERCIST. This may offer extra advantages and is addressed in the next section. 

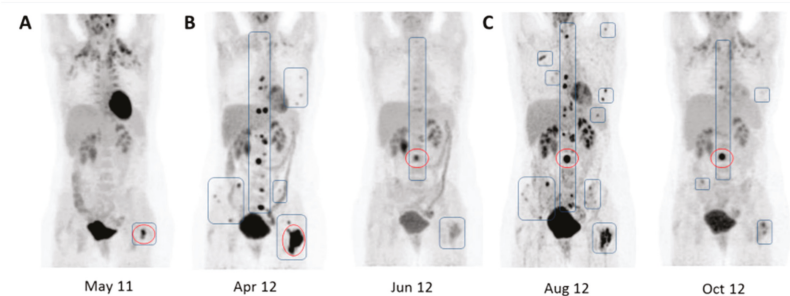

Ibandronate + Capecitabine Navelbine

D
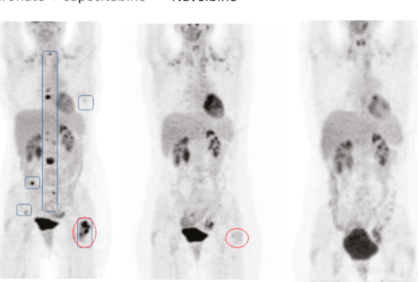

Aug 12

Oct 12

Jan 13

Mar 13

Jun 13
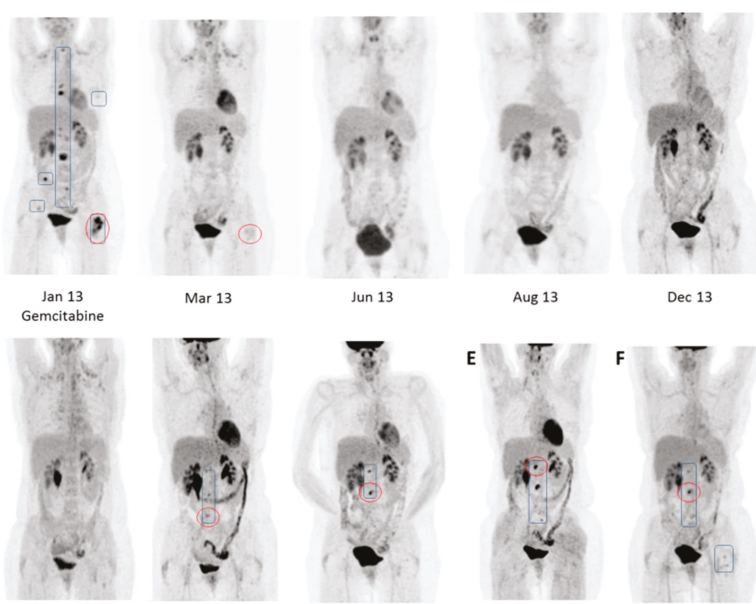

Aug 13

Dec 13

Mar 14

Jun 14
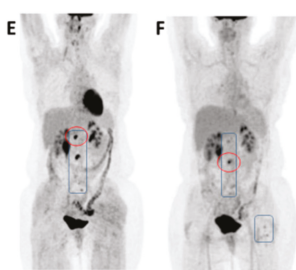

Oct 14

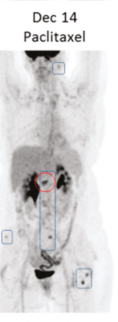

Feb 15
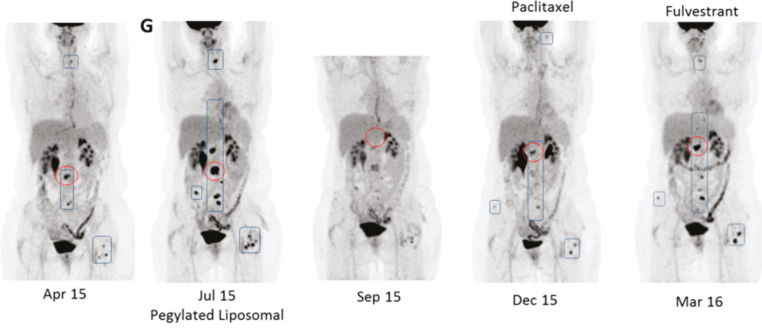

H

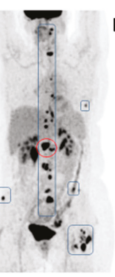

Doxorubicin
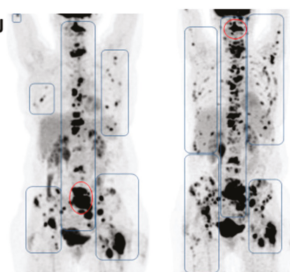

Apr 17

CyclophosphamideMethotrexate-
Fluorouracil

Mar 16

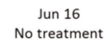

Oct 16
Irinotecan

$\operatorname{Jan} 17$

Figure 2. Maximum intensity projection images of a patient with metastatic breast cancer monitored longitudinally with FDG-PET/CT. Baseline scan (A) and pretreatment scans (B-J). Blue squares outline metastatic lesions. Red circles outline the hottest lesion representing a shifting target lesion for which SULpeak was measured using PERCIST 1.0. 


\section{PERCIST 1.0 for Response Monitoring in Metastatic Breast Cancer}

Figure 3 illustrates the treatment course of the patient in the case study based on PERCIST 1.0. We used the one-lesion method according to Wahl et al. [6] and O et al. [5] (Table 1) and thus used SULpeak of the hottest lesion, which may differ in consecutive scans. SULpeak represents the highest mean standardized uptake value (SUV) in a volume of interest of $1 \mathrm{~cm}^{3}$ corrected for lean body mass. The reference value in healthy liver tissue was measured in a volume of interest with $3 \mathrm{~cm}$ diameter to check for assessability in follow-up scans.

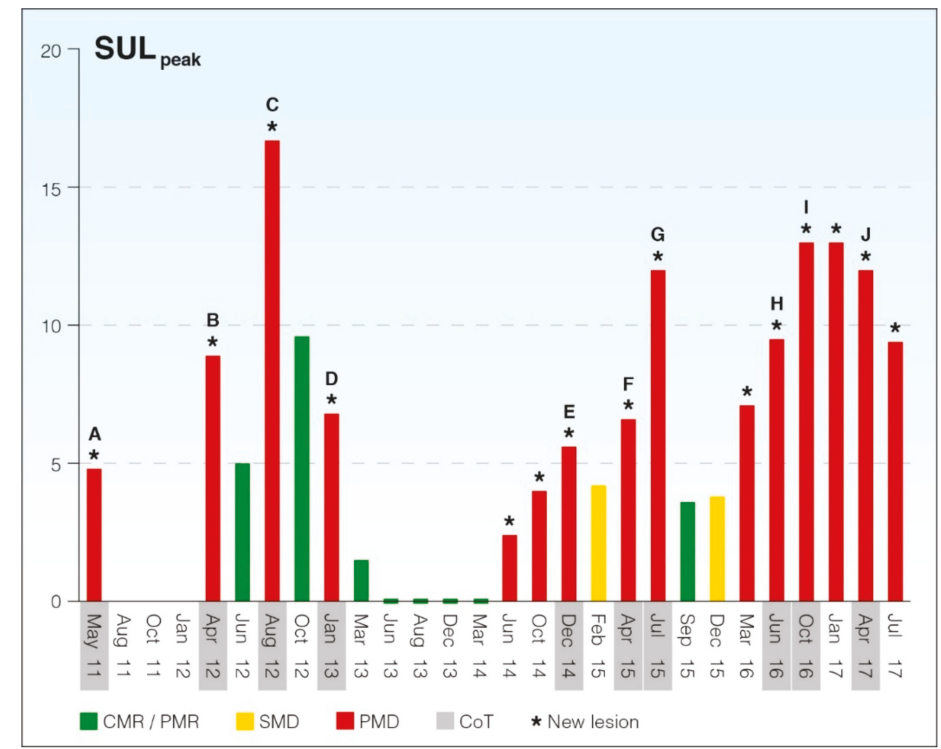

Figure 3. Graphical illustration of changes in PERCIST one-lesion SULpeak in a patient with metastatic breast cancer monitored for more than six years with FDG-PET/CT. Colored response categories: complete metabolic response (CMR), partial metabolic response (PMR), stable metabolic disease (SMD), and progressive metabolic disease (PMD). Grey-toned months represent time points for change of treatment (CoT). Conventional CT was performed Aug 11, Oct 11, and Jan 12. * Indicates scans with detection of new lesions.

To illustrate some aspects related to the assessibility criteria listed in Table 1, we present data for the PERCIST response categorization in Table 2. In June 2013, we changed to a new scanner with matrix size $256 \times 256$ instead of $128 \times 128$. The ordered subset expectation maximization (OSEM) reconstruction algorithm was used for all scans. Although serum glucose levels were not available retrospectively, patients in our institution are asked to fast for six hours prior to the scan, so we consider this criterion to be met sufficiently. The injection-to-scan time was higher than $70 \mathrm{~min}$ on three occasions and differed on several occasions more than $15 \mathrm{~min}$ from baseline or pretreatment scans. The standard FDG dose applied at our institution is $4 \mathrm{MBq}$ per kilogram, which we believe better accommodates potential weight changes than the dose proposed by PERCIST. SULmean in the liver differed more than 0.3 SUL units on several occasions (Table 2). 
Table 1. Specifications for the PERCIST 1.0 approach $[5,6]$.

\begin{tabular}{|c|c|c|}
\hline Quantification Measure & $\begin{array}{l}\text { Suggested } \\
\text { quantification measure } \\
\text { Suggested } \\
\text { quantification measure }\end{array}$ & $\begin{array}{l}\text { SUL } L_{\text {peak }} \text { for the hottest tumor, which is not necessarily the same } \\
\text { tumor on scans over time (one-lesion method) } \\
\text { SUL } \\
\text { per of up to five target lesions in hottest tumors, up to two } \\
\text { per organe-lesions method) }\end{array}$ \\
\hline $\begin{array}{l}\text { Measurability Criteria at } \\
\text { Baseline }\end{array}$ & & $\begin{array}{l}\text { Disease under study must be FDG-avid, meaning that } \mathrm{SUL}_{\text {peak }} \\
\text { should be } \geq 1 \text { and } \geq 1.5 \times \mathrm{SUL}_{\text {mean, liver }}+2 \mathrm{SD} \mathrm{SUL}_{\text {mean, liver }}\end{array}$ \\
\hline \multirow{6}{*}{$\begin{array}{l}\text { Assessabilty Criteria at } \\
\text { Follow-Up }\end{array}$} & Scanner criteria & $\begin{array}{l}\text { Same scanner or scanner model at same site should be used } \\
\text { Same reconstruction should be applied } \\
\text { Properly calibrated scanners should be used }\end{array}$ \\
\hline & Patient criteria & Patients should fast for at least $4 \mathrm{~h}$ \\
\hline & & Difference in injected dose should be $\leq 20 \%$ \\
\hline & FDG criteria & \\
\hline & & Injection-to-scan-time should be within $50-70 \mathrm{~min}$ \\
\hline & $\begin{array}{l}\text { Background activity } \\
\text { criteria }\end{array}$ & Difference in $\mathrm{SUL}_{\text {mean, liver }}<20 \%$ and $<0.3 \mathrm{SUL}$ units \\
\hline \multicolumn{2}{|c|}{ Response Categories at Follow-Up } & $\begin{array}{l}\text { Follow-up scans are suggested to be compared to the } \\
\text { baseline/pretreatment scan }\end{array}$ \\
\hline \multicolumn{2}{|c|}{ Complete Metabolic Response } & $\begin{array}{l}\text { Complete resolution of cancer-suspect lesions. Any previous } \\
\text { lesion should have FDG uptake less than SUL } \text { mean, liver and be }_{\text {indistinguishable from background. No new lesions appeared. }}\end{array}$ \\
\hline \multicolumn{2}{|c|}{ Partial Metabolic Response } & $\begin{array}{l}\text { Decrease in } \mathrm{SUL}_{\text {peak }} \text { of hottest tumor } \geq 30 \% \text { and at least } 0.8 \mathrm{SUL} \\
\text { units. No new lesions appeared. No increase in size greater than } \\
30 \% \text {. No unequivocal progression of a non-target lesion (increase } \\
\text { in SUL peak or size greater than } 30 \% \text { ). }\end{array}$ \\
\hline \multicolumn{2}{|c|}{ Stable Metabolic Disease } & $\begin{array}{l}\text { Increase or decrease of } S U L_{\text {peak }} \text { of less than } 30 \% \text {. No new lesions } \\
\text { appeared. No increase in size greater than } 30 \% \text {. No unequivocal } \\
\text { progression of a non-target lesion. }\end{array}$ \\
\hline \multicolumn{2}{|c|}{ Progressive Metabolic Disease } & $\begin{array}{c}\text { Increase in SUL peak of hottest tumor of } \geq 30 \% \text { and of at least } 0.8 \\
\text { SUL units, or development of one or more new lesions in a pattern } \\
\text { suspect of cancer. }\end{array}$ \\
\hline
\end{tabular}

$\mathrm{SUL}_{\text {mean, liver }}$ : mean standardized uptake value in liver, corrected for lean body mass; $\mathrm{SUL}_{\text {peak: }}$ mean standardized uptake value in a one $\mathrm{cm}^{3}$ volume of interest; SD: standard deviation.

The software tool PET VCAR (AW server, version 3.2, Ext. 1.0, GE Healthcare, Waukesha, Milwaukee, WI, USA) was used for semi-automated measurement of the SULpeak for the hottest lesion at consecutive FDG-PET/CT scans. At the baseline scan in May 2011, SULpeak was $4.8 \mathrm{~g} / \mathrm{mL}$, SULmean in the liver was $1.5 \mathrm{~g} / \mathrm{mL}$, and the standard deviation of SULmean in the liver was $0.15 \mathrm{~g} / \mathrm{mL}$. This means that a SULpeak of a potential target lesion of $2.55 \mathrm{~g} / \mathrm{mL}$ would have been sufficient for meeting measurability criteria of PERCIST 1.0. We used SULpeak values for the hottest lesion at consecutive scans, and PERCIST response categories were applied in all scans (Table 2).

We used the baseline or pretreatment scan as reference and, although it was not specified in PERCIST 1.0, we also used the nadir scan as reference in treatment intervals with decreasing SULpeak values in order to obtain clinically relevant response categories. As shown in Figure 3, we used color coding of response categories to graphically illustrate the long-term course. This approach can show decreasing or increasing trends in SULpeak before a change is detected by response categories or qualitative assessment. In doing this, we did not meet several specifications by PERCIST 1.0, but the depicted SULpeak fluctuations and PERCIST response categories appeared to mirror the disease activity in a timely manner. Thus, when PMD did not give rise to a change of treatment, we could still observe PMD on the following scan. When PMD did trigger a change of treatment, then PMR was observed four times (B-D, G), SMD once (E), and PMD three times (F, I, J), suggesting that the disease was more responsive to new treatment during its initial phase. 
Table 2. Data for PERCIST response categorization for the treatment course presented.

\begin{tabular}{|c|c|c|c|c|c|c|c|}
\hline $\begin{array}{c}\text { Scan } \\
\text { Month }\end{array}$ & $\begin{array}{c}\text { Baseline/Pre- } \\
\text { Treatment Scan }\left(^{*}\right)\end{array}$ & $\mathrm{SUL}_{\text {mean, liver }}$ & ITST/Min & $\begin{array}{c}\text { Matrix } \\
\text { Size }\end{array}$ & $\begin{array}{c}\text { New } \\
\text { Lesion }\end{array}$ & $\begin{array}{c}\text { SUL }_{\text {peak }} \text { Target } \\
\text { Lesion }\end{array}$ & $\begin{array}{l}\text { Response } \\
\text { Category }\end{array}$ \\
\hline May 11 & Yes (A) & 1.51 & 64 & 128 & Yes & 4.78 & $\mathrm{BL}$ \\
\hline Apr 12 & Yes (B) & 2.03 & 60 & 128 & Yes & 8.89 & PMD \\
\hline Jun 12 & & 1.66 & 82 & 128 & No & 4.99 & PMR \\
\hline Aug 12 & Yes (C) & 1.92 & 74 & 128 & Yes & 16.67 & PMD \\
\hline Oct 12 & & 1.35 & 73 & 128 & No & 9.58 & PMR \\
\hline Jan 13 & Yes (D) & 1.68 & 61 & 128 & Yes & 6.81 & PMD \\
\hline Mar 13 & & 1.68 & 64 & 128 & No & 1.47 & PMR \\
\hline Jun 13 & & 1.85 & 68 & 128 & No & - & CMR \\
\hline Aug 13 & & 1.92 & 58 & 256 & No & - & CMR \\
\hline Dec 13 & & 2.07 & 58 & 256 & No & - & CMR \\
\hline Mar 14 & & 1.80 & 111 & 256 & No & - & CMR \\
\hline Jun 14 & & 1.95 & 58 & 256 & Yes & 2.42 & PMD \\
\hline Oct 14 & & 1.93 & 67 & 256 & Yes & 4.03 & PMD \\
\hline Dec 14 & Yes (E) & 1.82 & 59 & 256 & Yes & 5.59 & PMD \\
\hline Feb 15 & & 1.94 & 62 & 256 & No & 4.16 & PMR \\
\hline Apr 15 & Yes (F) & 2.04 & 66 & 256 & Yes & 6.59 & PMD \\
\hline Jul 15 & Yes $(G)$ & 2.06 & 58 & 256 & Yes & 12.01 & PMD \\
\hline Sep 15 & & 1.91 & 70 & 256 & No & 3.59 & PMR \\
\hline Dec 15 & & 1.99 & 54 & 256 & No & 3.82 & PMR \\
\hline Mar 16 & & 1.80 & 57 & 256 & Yes & 7.08 & PMD \\
\hline Jun 16 & Yes $(\mathrm{H})$ & 1.81 & 66 & 256 & Yes & 9.46 & PMD \\
\hline Oct 16 & Yes (I) & 1.71 & 60 & 256 & Yes & 13.07 & PMD \\
\hline Jan 17 & & 1.93 & 65 & 256 & Yes & 13.09 & PMD \\
\hline Apr 17 & Yes (J) & 1.44 & 64 & 256 & Yes & 11.93 & PMD \\
\hline Jul 17 & & 2.03 & 61 & 256 & Yes & 9.44 & PMD \\
\hline
\end{tabular}

BL: Baseline; PMD: progressive metabolic disease; PMR: partial metabolic response; CMR: complete metabolic response; * Treatment transitions $\mathrm{A}-\mathrm{J}$, as described in text; $\mathrm{SUL}_{\text {mean, liver: }}$ mean standardized uptake value in liver, corrected for lean body mass $(\mathrm{g} / \mathrm{mL})$; ITST: injection to scan time; SUL peak: mean standardized uptake value in a $1 \mathrm{~cm}^{3}$ volume of interest $(\mathrm{g} / \mathrm{mL})$.

The graphical display provides an overview of treatment lines and impact of treatment change using SULpeak as a surrogate marker. A potential pitfall of the proposed PERCIST 1.0 is the lack of attention to the nadir level of SULpeak, which will be clinically relevant for monitoring cancer lesions that present with decreasing SULpeak values. However, this can be easily detected when using the graphical illustration (Figure 3).

Monitoring progression of metastatic cancer in this way has the potential to detect upcoming tumor clones that are resistant to ongoing oncologic treatment. Along with an increase or decrease of SULpeak in the target lesion, it can detect new suspicious lesions or unequivocal progression in non-target lesions, both of which would indicate progression. We consider PERCIST well suited for monitoring the biological processes in cancer progression and find it highly relevant for monitoring metastatic cancers such as breast cancer.

In the present case, it would have been valuable to retrospectively compare RECIST with PERCIST evaluation to estimate the degree of agreement/disagreement in response categorization $[3,6]$. Our FDG-PET/CT scans used low-dose CT, however, which prevented us from applying the RECIST evaluation. Our case study shows that the patient had 'bone metastases only' for a long time until June 2016, meaning that RECIST would predominantly have evaluated the disease as being non-measurable. It is our impression that the PERCIST criteria would have been suitable for response monitoring in this patient and potentially also in other patients with metastatic breast cancer. PERCIST appears to provide more information about the disease and its fluctuation, a higher degree of interrater agreement [24], a means to improve between-institution comparisons, and an integrative tool for shared clinical decision-making. Prospective studies are needed, however, to provide further clinical evidence.

\section{Today}

Current guidelines for management of advanced breast cancer recommend hormone-directed treatments as first-line therapy for estrogen- and HER2-receptor positive disease, whereas chemotherapy is recommended for receptor-negative disease. A biopsy of the metastatic lesion should thus be carried 
out before the patient starts treatment for metastatic breast cancer [4]. Evaluation of response to therapy is recommended every 2-4 months for endocrine therapy or after 2-4 cycles of chemotherapy. However, current guidelines do not recommend which imaging modality should be used for response evaluation [4].

A relevant issue to consider when using FDG-PET/CT for response evaluation or response monitoring in metastatic breast cancer is the optimal timing of PET/CT. Vach et al. concluded that schedule-optimizing studies should be performed [25]. While current guidelines do recommend regular time intervals for response evaluation, these should be reconsidered in light of the rapid shift in oncologic therapies and further development of imaging modalities [4,25].

A recent systematic review of PET/CT-based response evaluation [26] found that of 122 published papers, 112 (90\%) were accuracy and/or prognostic studies. No randomized controlled trials were found, and only some studies included more than one post-baseline scan (31\%). Further studies of response evaluation with PET/CT are thus needed to address issues of timing, repeat scans, and patient-relevant outcome measures. Well-planned multicenter trials will be required as large patient samples are needed to demonstrate significant outcomes.

Recent retrospective evidence showed that the PERCIST 1.0 one-lesion method was a better predictor of progression-free survival than RECIST [22]. The issue of the number of lesions to be quantified is relevant. In a recent study comparing the use of one-lesion versus five-lesion PERCIST for response prediction in metastatic breast cancer, Pinker et al. found no major impact on the prognostic value of the PERCIST approach [27]. A comprehensive review of the clinical impact of FDG-PET/CT for patients with breast cancer concluded that PET/CT is better at evaluating treatment response than conventional CT scan [28]. Several issues are still unanswered, however, and need further attention.

\section{Tomorrow}

There is no simple answer to the issue of optimal time interval in response monitoring for patients with metastatic breast cancer. The relevant interval will vary from cancer to cancer, from one therapy to another, from patient to patient. Imaging is indispensable, however, for characterizing the individual patient's cancer and for choosing therapy.

Another question is how the individual scans should be compared to determine whether or not an actual change has occurred over time. PERCIST recommends comparing all successive scans to the baseline scan. This may not always be optimal, as reflected in our case study. In cases of metabolic regression, SULpeak will decrease and may have reached a nadir level that makes comparison to baseline misleading. In some cases, metabolic regression can be detected by comparison to the baseline scan, but in principle progression is observed by comparison to the nadir. This issue is relevant in clinical practice when several treatment lines are available, and especially with the development of targeting therapies. The nadir concept has previously been emphasized in the RECIST criteria [3].

A further issue is the standardization of acquisition and quantification procedures. This has been addressed by several authors in papers about PERCIST $[5,6,29,30]$. Scanners and reconstructions methods are under continuous technical improvement [29] to improve not only the quality of imaging but also the quantitative measures. We support the use of SULpeak as suggested in PERCIST 1.0, but we recognize that other measures and concepts are relevant and should be explored prospectively in future studies. These include total lesion glycolysis, metabolic tumor volume, the five-lesion method of PERCIST, and the use of PERCIST in initially non-measurable tumors. Other relevant explorative analyses would be to investigate the optimal limits of percentage increase and decrease for determining partial metabolic response or progressive metabolic disease. Limits of $30 \%$ have been suggested in PERCIST 1.0, but this needs to be confirmed in clinical trials.

There may be situations where qualitative evaluation of FDG-PET/CT results in a 'mixed response'. This may refer to growth of a minor resistant clone alongside simultaneous response to a major and previously dominant clone. In such situations, PERCIST will most likely categorize this as progressive disease, consistent with the biology of cancer progression. The clinical decision for this may be 
equivocal, however, as it is very difficult to question the relevance of ongoing treatment that is having a positive effect on the initial dominant clone. If a shift in treatment is decided, however, this could potentially treat both clones. Clinical trials using patient-relevant outcome measures are thus highly relevant and should ideally include liquid biopsies.

\section{Beyond}

Artificial intelligence is an emerging field in which medical imaging such as FDG-PET/CT has an enormous potential by providing reproducible, observer-independent measures of cancer disease and its extent in the body. The development of radiomic tools for clinical decision support systems requires standardized and reproducible procedures, standardized data collection, and evaluation criteria [31]. Modelling of radiomic workflows is likely to be suitable for PERCIST and other analyses, providing a powerful tool in future medical treatment.

Another evolving field is the involvement of patients in treatment decisions, as emphasized in current clinical guidelines [4]. Shared decision-making facilitates patient engagement via collaboration between the clinician and the patient and includes elicitation of patient preferences and decision-making tools. Most patients with metastatic breast cancer want to be involved in decisions about their own treatment, and this appears to have positive effects [32,33]. Tools for shared decision-making in management of metastatic breast cancer have been developed, but recent papers call for more innovative and developmental research in the field [33,34]. None of the proposed tools include PET/CT scans or their results. As these play a crucial role in clinical decision-making, it is likely that greater communication between patients and health professionals about the timing and implications of scan results would contribute to shared decision-making.

In this perspective paper, we have dealt with FDG as the only PET tracer for response monitoring in metastatic breast cancer. Its huge importance is indisputable, not least because it typically shows uptake in different clones of breast cancer, and it is valuable even in long patient courses as the one described in our case study. However, it is worth investigating alternatives, especially more specific tracers that could characterize the individual patient and her cancer more accurately. Candidates include tracers targeting 18F-fluorotymidine, HER2, estrogen receptor, and gastrin-releasing peptide receptor [35-38]. Such specific tracers used alone or in combination may better predict treatment response. In a study by Ulaner et al., they found positive lesions on 89Zr-Trastuzumab PET/CT in six of 20 patients with HER2-negative primary breast cancer, and three of them had biopsy verified HER2-positive metastases [39]. This finding substantiates the theory of the heterogeneity of cancer and appoints opportunities for prediction of targeting therapy. Hence, when therapeutic ligands are developed, the theranostic potential represents a unique aspect of precision oncology that can benefit future patients [40].

Finally, the rapidly growing field of genomic analyses in precision oncology will without doubt become a major player in future research and clinical practice. We believe that molecular imaging will integrate with genomic analyses to provide improved treatment regimens that collaborative clinical teams can use for the benefit of patients with metastatic breast cancer.

\section{Conclusions}

Precision oncology is rapidly evolving, and targeted therapeutic options are already available for treatment of metastatic breast cancer. There is a gap between advanced modern therapy and contemporary monitoring of its effects, however, and current guidelines contain few recommendations for response evaluation criteria when monitoring response to oncologic treatment. The molecular imaging modality FDG-PET/CT may fill the gap as it reflects the central cancer hallmark of glycosylation, and it has proven clinical relevance in the diagnosis of metastatic breast cancer. There is evidence that the PET-based response evaluation criteria, PERCIST, can improve prediction in molecular-based treatment regimens compared to RECIST. We believe that PERCIST has the potential to provide 
greater information about disease fluctuation, thus better supporting clinical decision-making and patient empowerment.

Author Contributions: Conceptualization, M.G.H., J.F.L., J.H. and M.H.V.; methodology, M.G.H., J.H., M.H.V. and P.-E.B.; software, M.G.H., J.H. and M.H.V.; formal analysis, O.G.; data curation, M.G.H.; writing-original draft preparation, M.G.H., M.V.; writing—review and editing, M.T., M.E., P.F.H.C.; visualization, J.F.L., supervision, P.F.H.C.; funding acquisition, M.G.H.

Funding: This research was funded by independent Research Fund, Denmark.

Acknowledgments: We thank Claire Gudex for language editing.

Conflicts of Interest: The authors declare no conflict of interest.

\section{References}

1. Ferlay, J.; Colombet, M.; Soerjomataram, I.; Mathers, C.; Parkin, D.M.; Pineros, M.; Znaor, A.; Bray, F. Estimating the Global Cancer Incidence and Mortality in 2018: Globocan Sources and Methods. Int. J. Cancer 2019, 144, 1941-1953. [CrossRef] [PubMed]

2. Swain, S.M.; Baselga, J.; Kim, S.B.; Ro, J.; Semiglazov, V.; Campone, M.; Ciruelos, E.; Ferrero, J.M.; Schneeweiss, A.; Heeson, S.; et al. Pertuzumab, Trastuzumab, and Docetaxel in Her2-Positive Metastatic Breast Cancer. N. Engl. J. Med. 2015, 372, 724-734. [CrossRef] [PubMed]

3. Eisenhauer, E.A.; Therasse, P.; Bogaerts, J.; Schwartz, L.H.; Sargent, D.; Ford, R.; Dancey, J.; Arbuck, S.; Gwyther, S.; Mooney, M.; et al. New Response Evaluation Criteria in Solid Tumours: Revised Recist Guideline (Version 1.1). Eur. J. Cancer 2009, 45, 228-247. [CrossRef] [PubMed]

4. Cardoso, F.; Senkus, E.; Costa, A.; Papadopoulos, E.; Aapro, M.; Andre, F.; Harbeck, N.; Aguilar Lopez, B.; Barrios, C.H.; Bergh, J.; et al. 4th Eso-Esmo International Consensus Guidelines for Advanced Breast Cancer (Abc 4) Dagger. Ann. Oncol. 2018, 29, 1634-1657. [CrossRef] [PubMed]

5. O, J.H.; Lodge, M.A.; Wahl, R.L. Practical PERCIST: A Simplified Guide to PET Response Criteria in Solid Tumors 1.0. Radiology 2016, 2, 576. [CrossRef] [PubMed]

6. Wahl, R.L.; Jacene, H.; Kasamon, Y.; Lodge, M.A. From RECIST to PERCIST: Evolving Considerations for Pet Response Criteria in Solid Tumors. J. Nucl. Med. 2009, 50, 122S-150S. [CrossRef] [PubMed]

7. Cardoso, F.; Kyriakides, S.; Ohno, S.; Penault-Llorca, F.; Poortmans, P.; Rubio, I.T.; Zackrisson, S.; Senkus, E. Early Breast Cancer: ESMO Clinical Practice Guidelines for Diagnosis, Treatment and Follow-Up. Ann. Oncol. 2019, mdz189. [CrossRef]

8. Alimirzaie, S.; Bagherzadeh, M.; Akbari, M.R. Liquid Biopsy in Breast Cancer: A Comprehensive Review. Clin. Genet. 2019, 95, 643-660. [CrossRef]

9. Kroigard, A.B.; Larsen, M.J.; Thomassen, M.; Kruse, T.A. Molecular Concordance between Primary Breast Cancer and Matched Metastases. Breast J. 2016, 22, 420-430. [CrossRef]

10. Gundem, G.; Van Loo, P.; Kremeyer, B.; Alexandrov, L.B.; Tubio, J.M.C.; Papaemmanuil, E.; Brewer, D.S.; Kallio, H.M.L.; Hognas, G.; Annala, M.; et al. The Evolutionary History of Lethal Metastatic Prostate Cancer. Nature 2015, 520, 353-357. [CrossRef]

11. Weide, R.; Feiten, S.; Friesenhahn, V.; Heymanns, J.; Kleboth, K.; Thomalla, J.; van Roye, C.; Koppler, H. Metastatic Breast Cancer: Prolongation of Survival in Routine Care Is Restricted to Hormone-Receptor- and Her2-Positive Tumors. Springerplus 2014, 3, 535. [CrossRef] [PubMed]

12. Goh, K.I.; Cusick, M.E.; Valle, D.; Childs, B.; Vidal, M.; Barabasi, A.L. The Human Disease Network. Proc. Natl. Acad. Sci. USA 2007, 104, 8685-8690. [CrossRef] [PubMed]

13. Horak, P.; Klink, B.; Heining, C.; Groschel, S.; Hutter, B.; Frohlich, M.; Uhrig, S.; Hubschmann, D.; Schlesner, M.; Eils, R.; et al. Precision Oncology Based on Omics Data: The Nct Heidelberg Experience. Int. J. Cancer 2017, 141, 877-886. [CrossRef] [PubMed]

14. Buono, G.; Gerratana, L.; Bulfoni, M.; Provinciali, N.; Basile, D.; Giuliano, M.; Corvaja, C.; Arpino, G.; Del Mastro, L.; De Placido, S.; et al. Circulating Tumor DNA Analysis in Breast Cancer: Is It Ready for Prime-Time? Cancer Treat. Rev. 2019, 73, 73-83. [CrossRef] [PubMed]

15. Kroigard, A.B.; Larsen, M.J.; Brasch-Andersen, C.; Laenkholm, A.V.; Knoop, A.S.; Jensen, J.D.; Bak, M.; Mollenhauer, J.; Thomassen, M.; Kruse, T.A. Genomic Analyses of Breast Cancer Progression Reveal Distinct Routes of Metastasis Emergence. Sci. Rep. 2017, 7, 43813. [CrossRef] [PubMed] 
16. Munkley, J.; Elliott, D.J. Hallmarks of Glycosylation in Cancer. Oncotarget 2016, 7, 35478-35489. [CrossRef] [PubMed]

17. Kwee, T.C.; Basu, S.; Saboury, B.; Ambrosini, V.; Torigian, D.A.; Alavi, A. A New Dimension of FDG-PET Interpretation: Assessment of Tumor Biology. Eur. J. Nucl. Med. Mol. Imaging 2011, 38, 1158-1170. [CrossRef]

18. Benard, F.; Sterman, D.; Smith, R.J.; Kaiser, L.R.; Albelda, S.M.; Alavi, A. Prognostic Value of FDG PET Imaging in Malignant Pleural Mesothelioma. J. Nucl. Med. 1999, 40, 1241-1245.

19. Kitajima, K.; Miyoshi, Y.; Yamano, T.; Odawara, S.; Higuchi, T.; Yamakado, K. Prognostic Value of FDG-PET and DWI in Breast Cancer. Ann. Nucl. Med. 2018, 32, 44-53. [CrossRef]

20. Baun, C.; Falch, K.; Gerke, O.; Hansen, J.; Nguyen, T.; Alavi, A.; Hoilund-Carlsen, P.F.; Hildebrandt, M.G. Quantification of FDG-PET/CT with Delayed Imaging in Patients with Newly Diagnosed Recurrent Breast Cancer. BMC Med. Imaging 2018, 18, 11. [CrossRef]

21. Hildebrandt, M.G.; Gerke, O.; Baun, C.; Falch, K.; Hansen, J.A.; Farahani, Z.A.; Petersen, H.; Larsen, L.B.; Duvnjak, S.; Buskevica, I.; et al. [18f]Fluorodeoxyglucose (FDG)-Positron Emission Tomography (PET)/Computed Tomography (CT) in Suspected Recurrent Breast Cancer: A Prospective Comparative Study of Dual-Time-Point FDG-PET/CT, Contrast-Enhanced CT, and Bone Scintigraphy. J. Clin. Oncol. 2016, 34, 1889-1897. [CrossRef] [PubMed]

22. Riedl, C.C.; Pinker, K.; Ulaner, G.A.; Ong, L.T.; Baltzer, P.; Jochelson, M.S.; McArthur, H.L.; Gonen, M.; Dickler, M.; Weber, W.A. Comparison of FDG-PET/CT and Contrast-Enhanced CT for Monitoring Therapy Response in Patients with Metastatic Breast Cancer. Eur. J. Nucl. Med. Mol. Imaging 2017, 44, 1428-1437. [CrossRef] [PubMed]

23. Catalano, O.A.; Nicolai, E.; Rosen, B.R.; Luongo, A.; Catalano, M.; Iannace, C.; Guimaraes, A.; Vangel, M.G.; Mahmood, U.; Soricelli, A.; et al. Comparison of CE-FDG-PET/CT with CE-FDG-PET/MR in the evaluation of osseous metastases in breast cancer patients. Br. J. Cancer 2015, 112, 1452-1460. [CrossRef] [PubMed]

24. Fledelius, J.; Khalil, A.; Hjorthaug, K.; Frokiaer, J. Inter-observer agreement improves with PERCIST 1.0 as opposed to qualitative evaluation in non-small cell lung cancer patients evaluated with F-18-FDG PET/CT early in the course of chemo-radiotherapy. EJNMMI Res. 2016, 6, 71. [CrossRef] [PubMed]

25. Vach, W.; Hoilund-Carlsen, P.F.; Fischer, B.M.; Gerke, O.; Weber, W. How to Study Optimal Timing of PET/CT for Monitoring of Cancer Treatment. Am. J. Nucl. Med. Mol. Imaging 2011, 1, 54-62. [PubMed]

26. Gerke, O.; Ehlers, K.; Motschall, E.; Hoilund-Carlsen, P.F.; Vach, W. PET/CT-Based Response Evaluation in Cancer-a Systematic Review of Design Issues. Mol. Imaging Biol. 2019. [CrossRef] [PubMed]

27. Pinker, K.; Riedl, C.C.; Ong, L.; Jochelson, M.; Ulaner, G.A.; McArthur, H.; Dickler, M.; Gonen, M.; Weber, W.A. The Impact That Number of Analyzed Metastatic Breast Cancer Lesions Has on Response Assessment by 18f-FDG PET/CT Using PERCIST. J. Nucl. Med. 2016, 57, 1102-1104. [CrossRef]

28. Ulaner, G.A. PET/CT for Patients with Breast Cancer: Where Is the Clinical Impact? AJR Am. J. Roentgenol. 2019, 213, 254-265. [CrossRef]

29. Devriese, J.; Beels, L.; Maes, A.; Van de Wiele, C.; Pottel, H. Impact of Pet Reconstruction Protocols on Quantification of Lesions That Fulfil the Percist Lesion Inclusion Criteria. EJNMMI Phys. 2018, 5, 35. [CrossRef]

30. O, J.H.; Wahl, R.L. PERCIST in Perspective. Nucl. Med. Mol. Imaging 2018, 52, 1-4. [CrossRef]

31. Lambin, P.; Leijenaar, R.T.H.; Deist, T.M.; Peerlings, J.; de Jong, E.E.C.; van Timmeren, J.; Sanduleanu, S.; Larue, R.; Even, A.J.G.; Jochems, A.; et al. Radiomics: The Bridge between Medical Imaging and Personalized Medicine. Nat. Rev. Clin. Oncol. 2017, 14, 749-762. [CrossRef] [PubMed]

32. Sepucha, K.R.; Ozanne, E.M.; Partridge, A.H.; Moy, B. Is There a Role for Decision Aids in Advanced Breast Cancer? Med. Decis. Making 2009, 29, 475-482. [CrossRef] [PubMed]

33. Spronk, I.; Burgers, J.S.; Schellevis, F.G.; van Vliet, L.M.; Korevaar, J.C. The Availability and Effectiveness of Tools Supporting Shared Decision Making in Metastatic Breast Cancer Care: A Review. BMC Palliat. Care 2018, 17, 74. [CrossRef] [PubMed]

34. Niranjan, S.J.; Wallace, A.; Williams, B.R.; Turkman, Y.; Williams, C.P.; Bhatia, S.; Knight, S.; Rocque, G.B. Trust but Verify: Exploring the Role of Treatment-Related Information and Patient-Physician Trust in Shared Decision Making among Patients with Metastatic Breast Cancer. J. Cancer Educ. 2019. [CrossRef] [PubMed] 
35. Gebhart, G.L.; Lamberts, E.; Wimana, Z.; Garcia, C.; Emonts, P.; Ameye, L.; Stroobants, S.; Huizing, M.; Aftimos, P.; Tol, J.; et al. Molecular Imaging as a Tool to Investigate Heterogeneity of Advanced HER2-Positive Breast Cancer and to Predict Patient Outcome under Trastuzumab Emtansine (T-Dm1): The Zephir Trial. Ann. Oncol. 2016, 4, 619-624. [CrossRef]

36. Gong, C.; Yang, Z.; Sun, Y.; Zhang, J.; Zheng, C.; Wang, L.; Zhang, Y.; Xue, J.; Yao, Z.; Pan, H.; et al. A Preliminary Study of (18)F-FES PET/CT in Predicting Metastatic Breast Cancer in Patients Receiving Docetaxel or Fulvestrant with Docetaxel. Sci. Rep. 2017, 7, 6584. [CrossRef] [PubMed]

37. Pantaleo, M.A.; Nannini, M.; Maleddu, A.; Fanti, S.; Ambrosini, V.; Nanni, C.; Boschi, S.; Biasco, G. Conventional and Novel PET Tracers for Imaging in Oncology in the Era of Molecular Therapy. Cancer Treat. Rev. 2008, 34, 103-121. [CrossRef]

38. Dalm, S.; Verzijlbergen, U.J.F.; De Jong, M. Review: Receptor Targeted Nuclear Imaging of Breast Cancer. Int. J. Mol. Sci. 2017, 18, 260. [CrossRef]

39. Ulaner, G.A.; Hyman, D.M.; Lyashchenko, S.K.; Lewis, J.S.; Carrasquillo, J.A. 89Zr-Trastuzumab PET/CT for Detection of Human Epidermal Growth Factor Receptor 2-Positive Metastases in Patients With Human Epidermal Growth Factor Receptor 2-Negative Primary Breast Cancer. Clin. Nucl. Med. 2017, 42, 912-917. [CrossRef]

40. Yordanova, A.; Eppard, E.; Kurpig, S.; Bundschuh, R.A.; Schonberger, S.; Gonzalez-Carmona, M.; Feldmann, G.; Ahmadzadehfar, H.; Essler, M. Theranostics in Nuclear Medicine Practice. Onco. Targets Ther. 2017, 10, 4821-4828. [CrossRef]

(C) 2019 by the authors. Licensee MDPI, Basel, Switzerland. This article is an open access article distributed under the terms and conditions of the Creative Commons Attribution (CC BY) license (http://creativecommons.org/licenses/by/4.0/). 

MDPI

St. Alban-Anlage 66

4052 Basel

Switzerland

Tel. +41616837734

Fax +41 613028918

www.mdpi.com

Cancers Editorial Office

E-mail: cancers@mdpi.com www.mdpi.com/journal/cancers

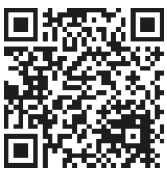



MDPI

St. Alban-Anlage 66

4052 Basel

Switzerland

Tel: +41 616837734

Fax: +41 613028918

www.mdpi.com 